\title{
AGR-1 Depletion Benchmark
}

James W. Sterbentz Joshua J. Cogliati

July 2018

The INL is a

U.S. Department of Energy National Laboratory

operated by

Battelle Energy Alliance

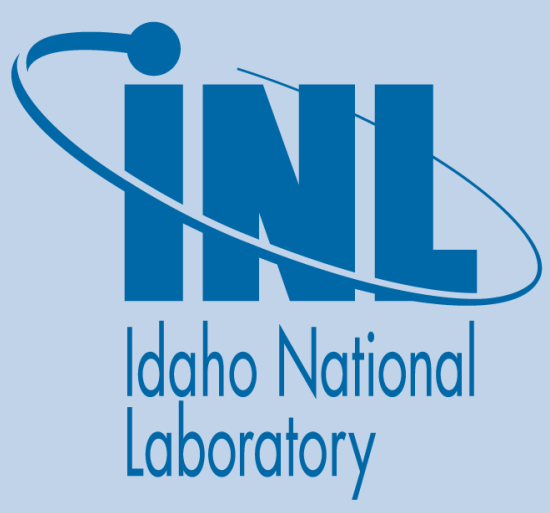




\section{DISCLAIMER}

This information was prepared as an account of work sponsored by an agency of the U.S. Government. Neither the U.S. Government nor any agency thereof, nor any of their employees, makes any warranty, expressed or implied, or assumes any legal liability or responsibility for the accuracy, completeness, or usefulness, of any information, apparatus, product, or process disclosed, or represents that its use would not infringe privately owned rights. References herein to any specific commercial product, process, or service by trade name, trade mark, manufacturer, or otherwise, does not necessarily constitute or imply its endorsement, recommendation, or favoring by the U.S. Government or any agency thereof. The views and opinions of authors expressed herein do not necessarily state or reflect those of the U.S. Government or any agency thereof. 
INL/EXT-18-

Revision 0

\title{
AGR-1 Depletion Benchmark
}

James W. Sterbentz

Joshua J. Cogliati

July 2018

\begin{abstract}
Idaho National Laboratory
INL ART Program

Idaho Falls, Idaho 83415
\end{abstract}

http://www.inl.gov

Prepared for the

U.S. Department of Energy

Office of Nuclear Energy

Under DOE Idaho Operations Office

Contract DE-AC07-05ID14517 



\section{INL ART Program \\ AGR-1 Depletion Benchmark}

INL/EXT-18-

Revision 0

July 2018

Authors:

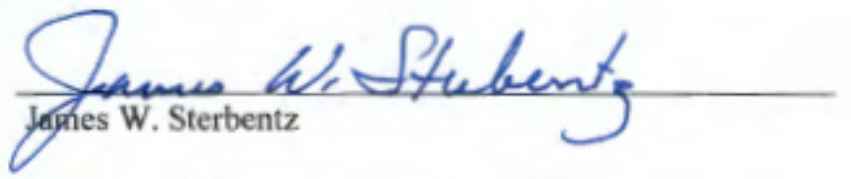

June 28,2018
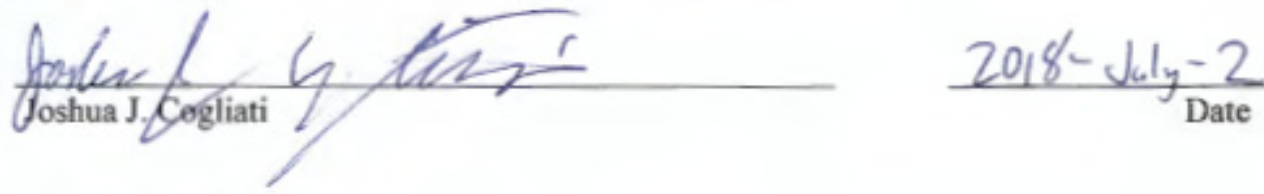

Technical Reviewer:

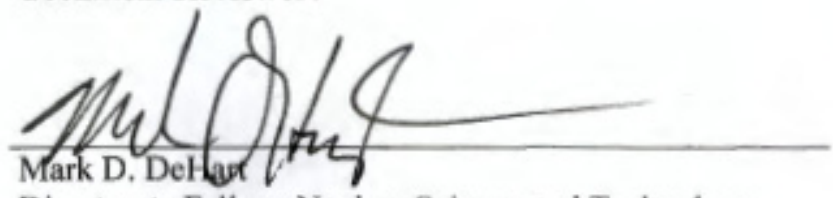

Directorate Fellow, Nuclear Science and Technology

Directorate

\section{Approvers:}
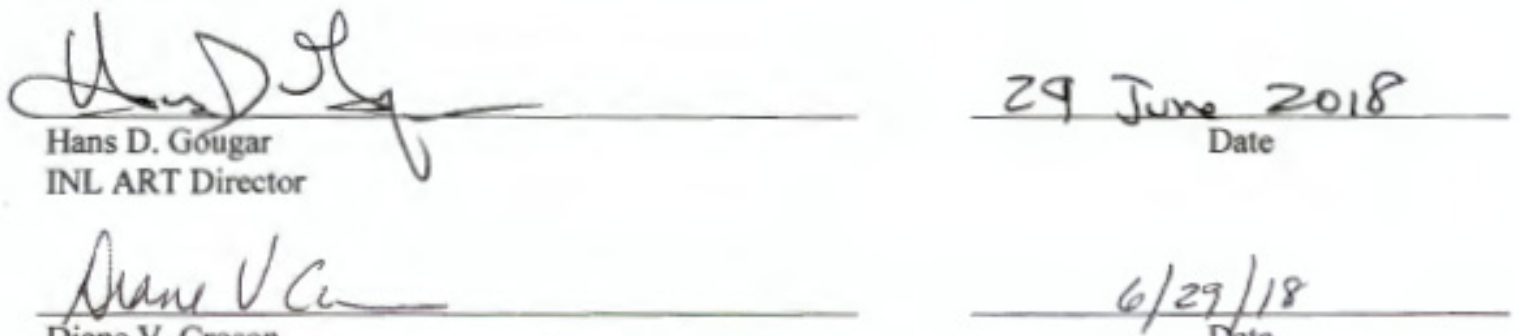

Diane V. Croson

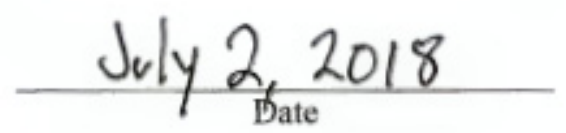

INL ART Deputy Director
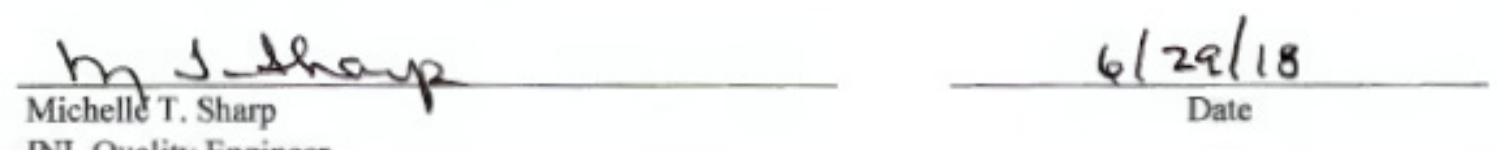

INL Quality Engineer 



\section{REVISION LOG}

\begin{tabular}{|c|c|c|c|}
\hline Rev. & Date & Affected Pages & Revision Description \\
\hline 0 & $07 / 02 / 2018$ & All & New document. \\
\hline & & & \\
\hline & & & \\
\hline & & & \\
\hline & & & \\
\hline & & & \\
\hline & & & \\
\hline & & & \\
\hline & & & \\
\hline & & & \\
\hline & & & \\
\hline & & & \\
\hline & & & \\
\hline & & & \\
\hline & & & \\
\hline & & & \\
\hline & & & \\
\hline & & & \\
\hline
\end{tabular}





\begin{abstract}
The AGR-1 TRISO particle irradiation test was the first Advanced Gas Reactor test irradiated in the Advanced Test Reactor (ATR) at the Idaho National Laboratory. The TRISO particle fuel was $350 \mu \mathrm{m}$ diameter uranium oxy-carbide (UCO) kernels enriched to $19.75 \mathrm{wt} \% \mathrm{U}-235$. The particles were embedded in cylindrical graphite compacts, placed in test capsules, and irradiated over a period of three years or 13 ATR power cycles. The fuel compacts achieved burnups approaching 20\% FIMA and accumulated fast fluence up to $4.25 \mathrm{E}+25$ $\mathrm{n} / \mathrm{m}^{2}\left(\mathrm{E}_{\mathrm{n}}>0.18 \mathrm{MeV}\right)$. This report describes the AGR-1 test train, capsules, compacts, and TRISO particle fuel in sufficient detail for a physics analyst to model the AGR-1 experiment as part of a larger ATR neutron transport model and perform a depletion calculation. The intent of the data herein is to define a practical numerical TRISO particle depletion benchmark problem for the AGR-1 irradiation test.
\end{abstract}




\section{CONTENTS}

ABSTRACT vii

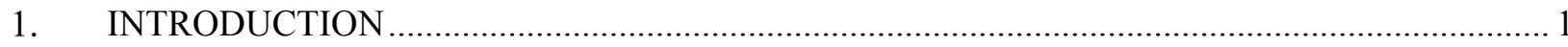

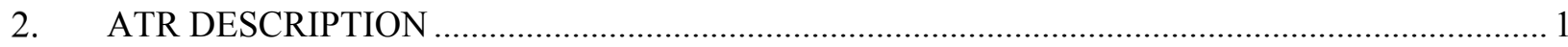

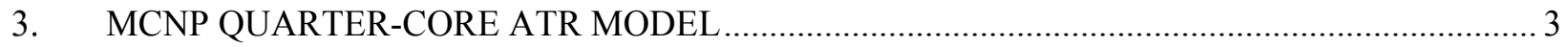

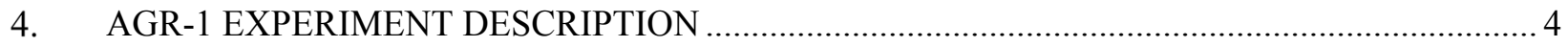

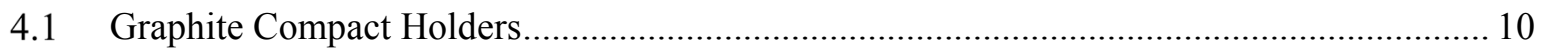

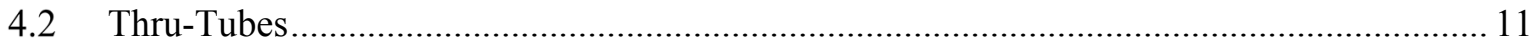

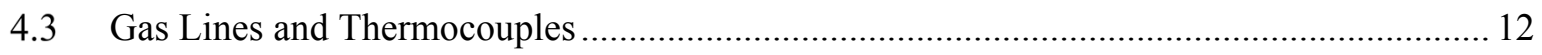

4.4 Capsule

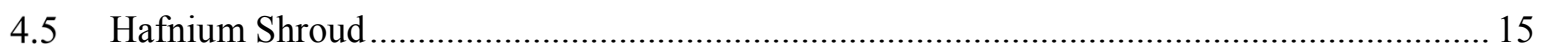

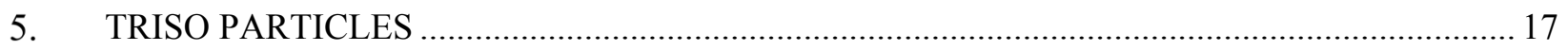

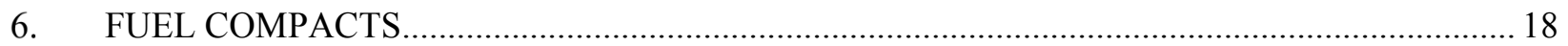

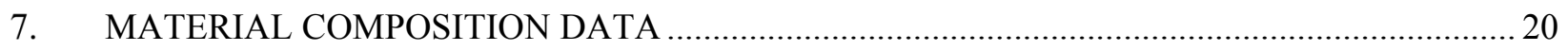

8. OUTER SHIM CONTROL CYLINDER MODELING............................................................... 22

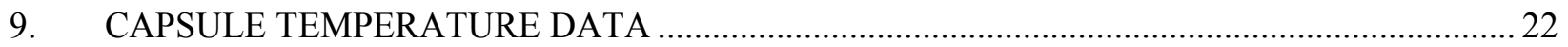

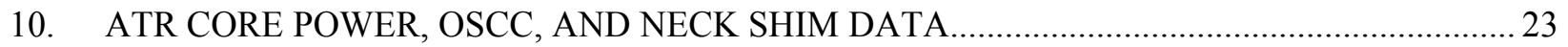

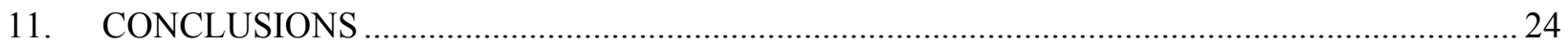

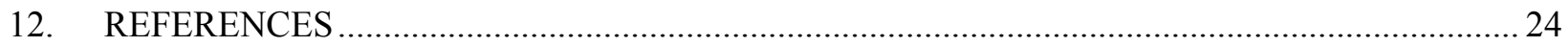

Appendix A ATR Lobe and Total Core Power History by Cycle and Timestep ....................................26

Appendix B ATR Outer Shim Control Cylinder Positions by Cycle and Timestep ...............................45

Appendix C ATR Neck Shim Positions by Cycle and Timestep ....................................................... 64

Appendix D MCNP Quarter-Core ATR Model Input File Listing ..................................................... 79

\section{FIGURES}

Figure 1. Cross section view of the ATR core showing the B-10 irradiation test facility along with $3 \times 3$ array of flux traps (southeast flux trap is indicated), serpentine driver core, and east outer shim control cylinders (E1, E2, E3, and E4) .............................................................

Figure 2. Cross section view of the MCNP quarter-core model (MCNP plot)........................................ 4 
Figure 3. Axial view of the in-core section of the AGR-1 test train assembly, showing the six capsules, compacts, spacers, and gas plenums (MCNP plot) ............................................... 6

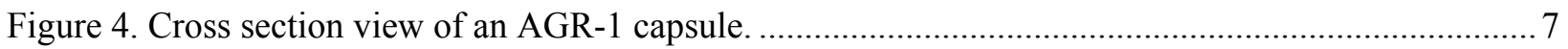

Figure 5. Three-dimensional cutaway of a single AGR-1 capsule. .......................................................... 8

Figure 6. Axial view of capsule with internal components labeled. ....................................................... 9

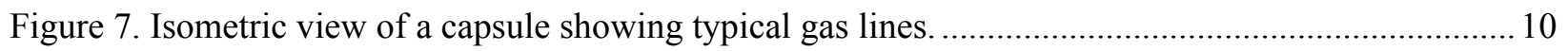

Figure 8. Cross section view of each capsule showing type, position, and insertion depth of the

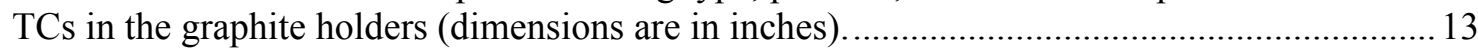

Figure 9. Four hafnium shroud azimuthal sectors for 240-degree hafnium shroud, plus 120-degree

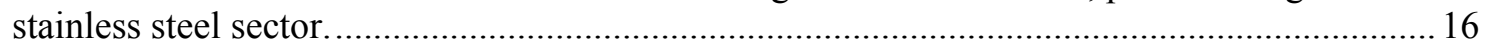

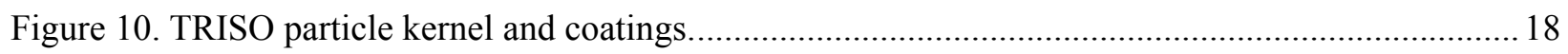

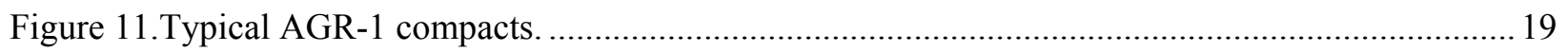

Figure 12.Axial radiograph of a typical compact showing the TRISO particles (small dark circles) and the matrix and top and bottom graphite endcaps (gray)

\section{TABLES}

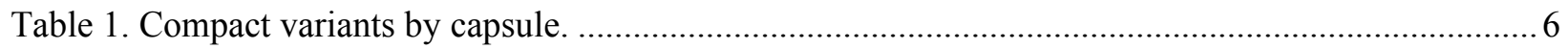

Table 2. Compact holder and embedded component materials and dimensions. ..................................... 11

Table 3. Compact stack $x-y$ coordinates in the graphite holders. ......................................................... 11

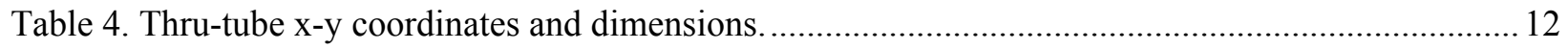

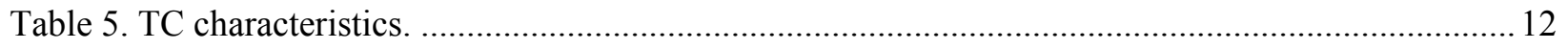

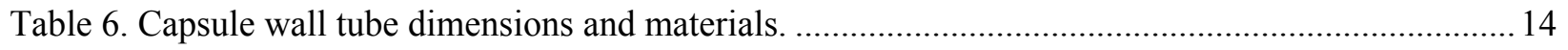

Table 7. Axial plane elevations for component pieces between capsules.............................................. 14

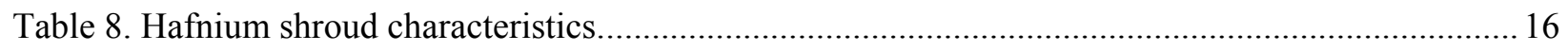

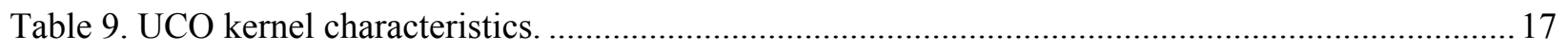

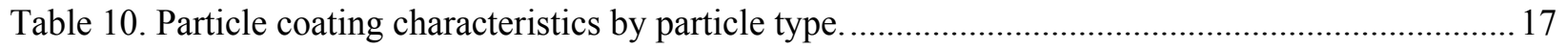

Table 11. Compact dimensions, loadings, and density characteristics for the four particle types............. 19

Table 12. Stainless steel 316L (SS316L) composition $\left(8.03 \mathrm{~g} / \mathrm{cm}^{3}\right)$................................................ 20

Table 13. Borated graphite holder number densities (4.76 atom percent boron)....................................2 21

Table 14. Borated graphite holder number densities (6.05 atom percent boron)...................................2 21

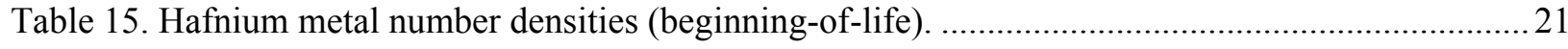

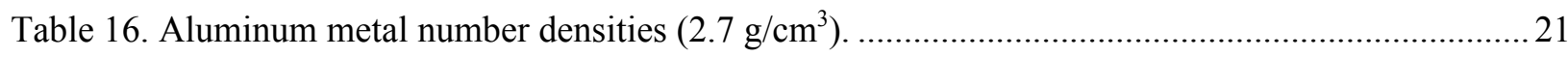

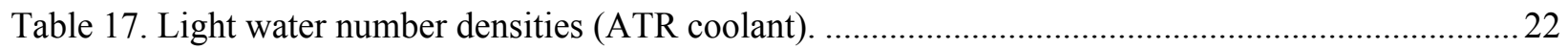

Table 18. Formulas for modeling OSCC hafnium plate positions or rotations........................................22 
Table 19. Fuel compact temperature data for the six AGR-1 capsules...................................................23

Table 20. Shutdown periods between AGR-1 power cycles................................................................... 24 


\section{ACRONYMS}

AGR Advanced Gas Reactor

ART Advanced Reactor Technologies

ASTM American Society for Testing and Materials

ATR Advanced Test Reactor

FIMA fissions per initial metal atom

HTGR high-temperature, gas-cooled reactor

ID inner diameter

INL Idaho National Laboratory

IPyC inner pyrolytic carbon

IR inner radius

MCNP Monte Carlo N-Particle Transport Code

NGNP Next Generation Nuclear Plant

OD outer diameter

OPyC outer pyrolytic carbon

OR outer radius

OSCC outer shim control cylinder

$\mathrm{SiC} \quad$ silicon carbide

TAVA time-averaged, volume-averaged

TC thermocouple

TRISO tristructural isotropic

UCO uranium oxy-carbide 


\section{AGR-1 Depletion Benchmark \\ 1. INTRODUCTION}

The first Advanced Gas Reactor (AGR) irradiation test or AGR-1 was a tristructural isotropic (TRISO) particle irradiation test in the Advanced Test Reactor (ATR) at the Idaho National Laboratory. The AGR-1 experiment was the first in a series of four TRISO particle fuel irradiations as part of the Department of Energy Advanced Gas Reactor Fuel Development and Qualification Program to support the development of the Next Generation Nuclear Plant. The Next Generation Nuclear Plant was proposed to be high-temperature, gas-cooled reactor (HTGR) fueled with the new high-performance TRISO fuel developed on the AGR program.

The AGR experiments have multiple specific goals that include: (1) provide irradiation performance data to support the fuel development process, (2) provide irradiated fuel and materials for post-irradiation examination and safety testing, (3) validate fuel performance and fission product transport models and codes, and (4) qualify fuel for normal operating conditions in a high-temperature, gas-cooled demonstration reactor, either a prismatic-block or pebble-bed reactor.

The AGR-1 test was a very successful test with no observed fuel particle failures despite maximum compact burnups up to $20 \%$ heavy metal fissions per initial atom (FIMA) and cumulative fast fluences approaching $4.5 \mathrm{E}+25 \mathrm{n} / \mathrm{m}^{2}\left(\mathrm{E}_{\mathrm{n}}>0.18 \mathrm{MeV}\right)$. The AGR-1 test was irradiated in ATR over a period of three years, corresponding to 13 power cycles, for a total of approximately 662 irradiation days.

The following data has been compiled to develop a benchmark model of the AGR-1 experiment. The data includes material descriptions and component dimensions for the AGR-1 test train, test capsules, fuel compacts, and TRISO particles. There should be sufficient data to enable a physics analyst to build a neutron transport model of the AGR-1 experiment to any desired level of rigor and perform a depletion calculation with the goal to benchmark HTGR computer codes, models, and modelling assumptions.

Due to the geometrical complexity of the ATR core, a Monte Carlo N-Particle transport code (MCNP) quarter-core model of ATR is provided separately, along with the benchmark data in this report. In the supplied quarter-core model, the B-10 irradiation test position or test facility is purposely voided to allow the physics analyst to build the AGR-1 test train, capsules, and fuel compacts as they see fit and per their own modelling assumptions.

Three supplimental reports may be useful to the physics analyst as guides to modelling the AGR-1 depletion benchmark problem and providing expectations for the calculated results. Reference [1] gives calculated results for fuel compact burnup, fast fluence, and end-of-irradiation isotopic concentrations using the MCNP quarter-core ATR model. Results are compared for a variety of modelling assumptions to show sensitivities. Reference [2] is similar to the quarter-core work, but is a more rigorous AGR-1 depletion calculation using a full-core MCNP ATR model. Reference [3] provides a comparison of the Reference [2] calculated results to actual post-irradiation examination measured data. The physics analyst should be able to gain insight into how the AGR-1 model can be constructed, the impact of certain modeling assumptions, and the type of results to expect from these three reports.

\section{ATR DESCRIPTION}

ATR is a compact, geometrically-complex reactor core with a serpentine driver core and a beryllium reflector (Reference [4]). Forty high-enriched driver fuel elements form the serpentine or cloverleaf core that naturally creates a $3 \times 3$ array of large-volume flux traps for experiment irradiations (Figure 1). The driver fuel elements consist of 19 curved plates with high-enriched (93\%) uranium, aluminum cladding, and light-water coolant. The active core fuel height is $121.92 \mathrm{~cm}$ (48 in.). The light-water coolant and beryllium metal reflector produce a predominantly thermal neutron spectrum. 
In addition to the 9 flux traps, the beryllium reflector has additional irradiation test positions, including the large B-10 position where the AGR-1 test train was located (Figure 1). The beryllium reflector also contains 16 outer shim control cylinders (OSCC) that are beryllium cylinders (drums) with partial-circumferential hafnium plates on the surface. The hafnium plates on the OSCCs are not discernable in Figure 1, but in Figure 2 below the hafnium plates are visible. The hafnium plate on the OSCCs can be rotated either toward or away from the driver core to adjust core reactivity. The depletion benchmark data includes OSCC cycle rotation data so that the reactivity effects of the OSCCs can be simulated in the depletion calculation at each timestep.

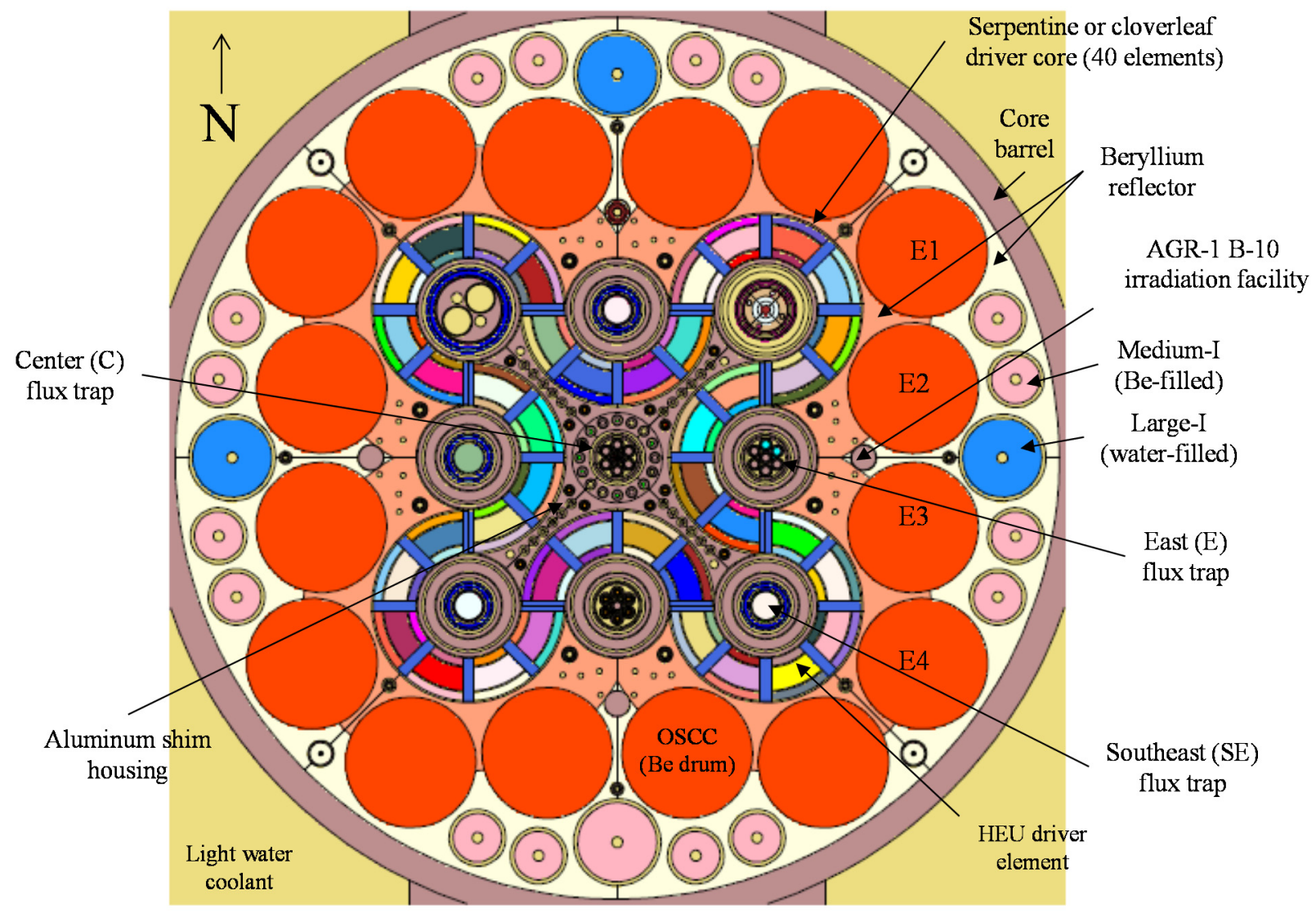

Figure 1 . Cross section view of the ATR core showing the B-10 irradiation test facility along with $3 \times 3$ array of flux traps (southeast flux trap is indicated), serpentine driver core, and east outer shim control cylinders (E1, E2, E3, and E4).

ATR typically runs at a total core power of between 100-110 MWth. At these power levels, intense neutron irradiation fluxes can be generated to accelerate irradiation testing. The five core lobes, northeast (NE), southeast (SE), southwest (SW), northwest (NW), and center (C) consist of 8 driver fuel elements each and typically operate a different power levels in the 14-22 MW range. The lobe powers are adjusted through the 16 OSCCs in the reflector. Each ATR power cycle typically lasts for 40-60 days.

Small core reactivity and lobe power adjustments can also be made through the insertion or withdrawal of the 24 hafnium neck shim rods located in the aluminum shim housing between the inboard flux traps (northeast, southeast, southwest, and northwest) and the center flux trap. Figure 2 shows the aluminum housing and neck shims. With the exception of the regulating rod (SE4), the neck shim rods are either fully withdrawn or fully inserted. Typically, at beginning-of-cycle most of the shim rods are fully inserted into the core. When a neck is withdrawn, the cell volume is then filled by an aluminum follower rod. 
As previously mentioned, the beryllium reflector also contains a number of smaller diameter irradiation test positions. One of those positions is the B-10 position where the AGR-1 experiment was placed and underwent 13 ATR power cycles over a three-year period.

\section{MCNP QUARTER-CORE ATR MODEL}

Due to the complexity of the ATR core, an MCNP quarter-core model of the ATR is provided with this benchmark problem (Appendix D). The quarter-core model is intended to reduce the size of the benchmark depletion model without significant loss of numerical accuracy and allow the physics analyst to focus more on the modelling of the AGR-1 experiment in the B-10 test position.

The MCNP quarter-core model represents the east core quadrant of the ATR core. The east quadrant includes the east outboard flux trap, one-half of the southeast and northeast flux traps, and one-quarter of the center flux trap as shown in Figure 2 (MCNP plot). The quarter-core model also includes ATR driver fuel elements 6 through 15, the beryllium reflector (green and orange), beryllium reflector irradiation positions (medium I-4, I-5, I-7, and I-8 in blue and large I-6 in yellow), outer shim control cylinders (E1, E2, E3, and E4), one-half the six hafnium neck shims rods in both the southeast and northeast aluminum neck shim housing, core barrel, and outer light-water radiation shield. The driver fuel in the model is for the beginning-of-cycle 145A and is assumed to be appropriate for beginning-of-cycle for the other 12 cycles as well. The northeast and the southeast radial sides that intersect at the core center or center of the center flux trap have a reflective boundary condition applied along these planes to reflect neutrons back into the model.

The B-10 test position, where the AGR-1 experiment is placed, is completely voided in the model and left to the physics analyst to develop the AGR-1 experiment geometry and material model based on the AGR-1 test data that follows. In Figure 2, the B-10 test position is voided (white circle). 


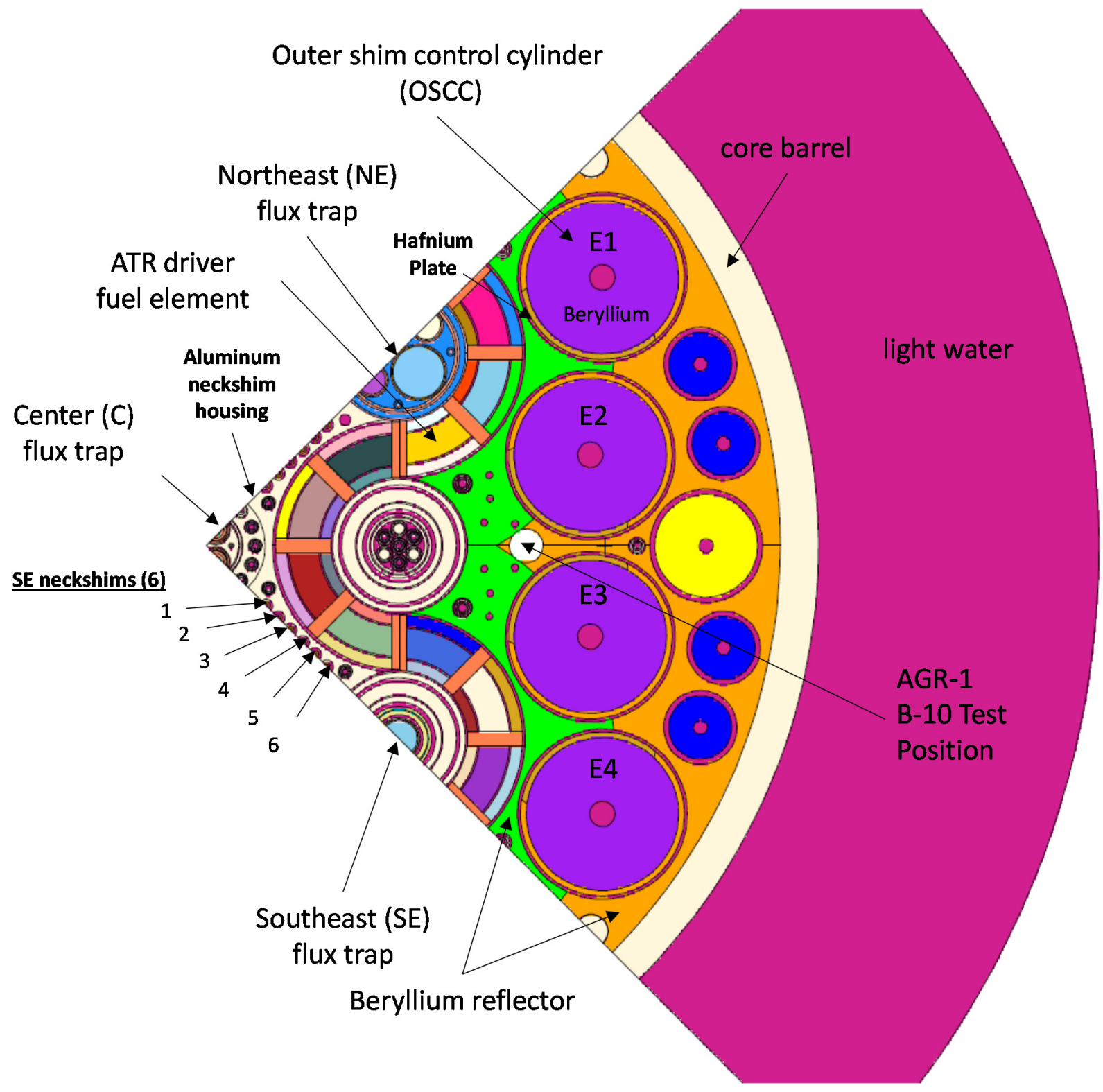

Figure 2. Cross section view of the MCNP quarter-core model (MCNP plot).

\section{AGR-1 EXPERIMENT DESCRIPTION}

This section will describe the AGR-1 experiment in some detail with the goal to help the physics analyst to better understand the AGR-1 experiment and therefore aid in the development of the AGR-1 experiment for the MCNP quarter-core model.

The AGR-1 experiment consisted of six cylindrical test capsules containing a total of 72 total TRISO particle fuel compacts. The capsules were stacked vertically as part of the AGR-1 experiment test train in the active core region of ATR. The test train was located in the B-10 test position (Figure 1). The B-10 test position has a $3.81 \mathrm{~cm}$ (1.5 in.) diameter vertical bore hole in the beryllium side reflector of the ATR core. The cylindrical test train was lowered into the B-10 test hole and positioned such that the axial centerline between capsules 3 and 4 coincided with the ATR core midplane. The ATR core midplane is the axial center or midpoint of the ATR fuel (axial elevation of $\mathrm{z}=60.96 \mathrm{~cm}$ in the MCNP ATR quarter- 
core model). The zero $\mathrm{cm}$ elevation $(\mathrm{z}=0)$ in the quarter-core model is then at the bottom of the active core, or bottom of the driver fuel meat. The ATR active core height is $121.96 \mathrm{~cm}$ (48 in.).

The capsules were stacked end-to-end with each capsule separated by graphite spacers on top and bottom of each capsule with a gas plenum in between the spacers. The top (upper) and bottom (lower) graphite spacers are pure graphite (no boron). The upper graphite spacers have a density of $0.95 \mathrm{~g} / \mathrm{cm}^{3}$ and the lower graphite spacers have a density of $1.015 \mathrm{~g} / \mathrm{cm}^{3}$. Figure 3 is an MCNP plot and shows the six capsules in an axial view. Capsule 1 was at the bottom of the test train, and capsule 6 at the top. Each capsule contained a borated graphite cylindrical holder. Each graphite holder had three equally-spaced vertical bore holes arranged on a triangular pitch. Each of the three holder holes contained a stack of four compacts, or 12 compacts per capsule.

Figure 3 shows the six AGR-1 capsules stacked vertically (z-direction) inside the test train. Capsule 1 is on the bottom of the stack and Capsule 6 is on the top. The ATR core midplane is exactly between Capsules 3 and 4 or through the middle of the gas plenum. The colored squares in the expanded view of Capsule 4 show two stacks of compacts, where each compact has been subdivided axially into two pieces, or the 8 colored squares represent 4 cylindrical compacts in a capsule stack. The compact subdivision was needed in Reference [2] to increase the resolution of the compact heat rates for the thermal analyses. The graphite holder, top and bottom graphite spacers, capsule lids, and gas plenums are also shown. The ATR light-water coolant (dark blue) outside the test train. The axial view here slices through 2 of the 3 compact stacks. The combined length of the six capsules is approximately $90 \mathrm{~cm}$ and the outer diameter of the capsule wall is approximately $3.57 \mathrm{~cm}$. 


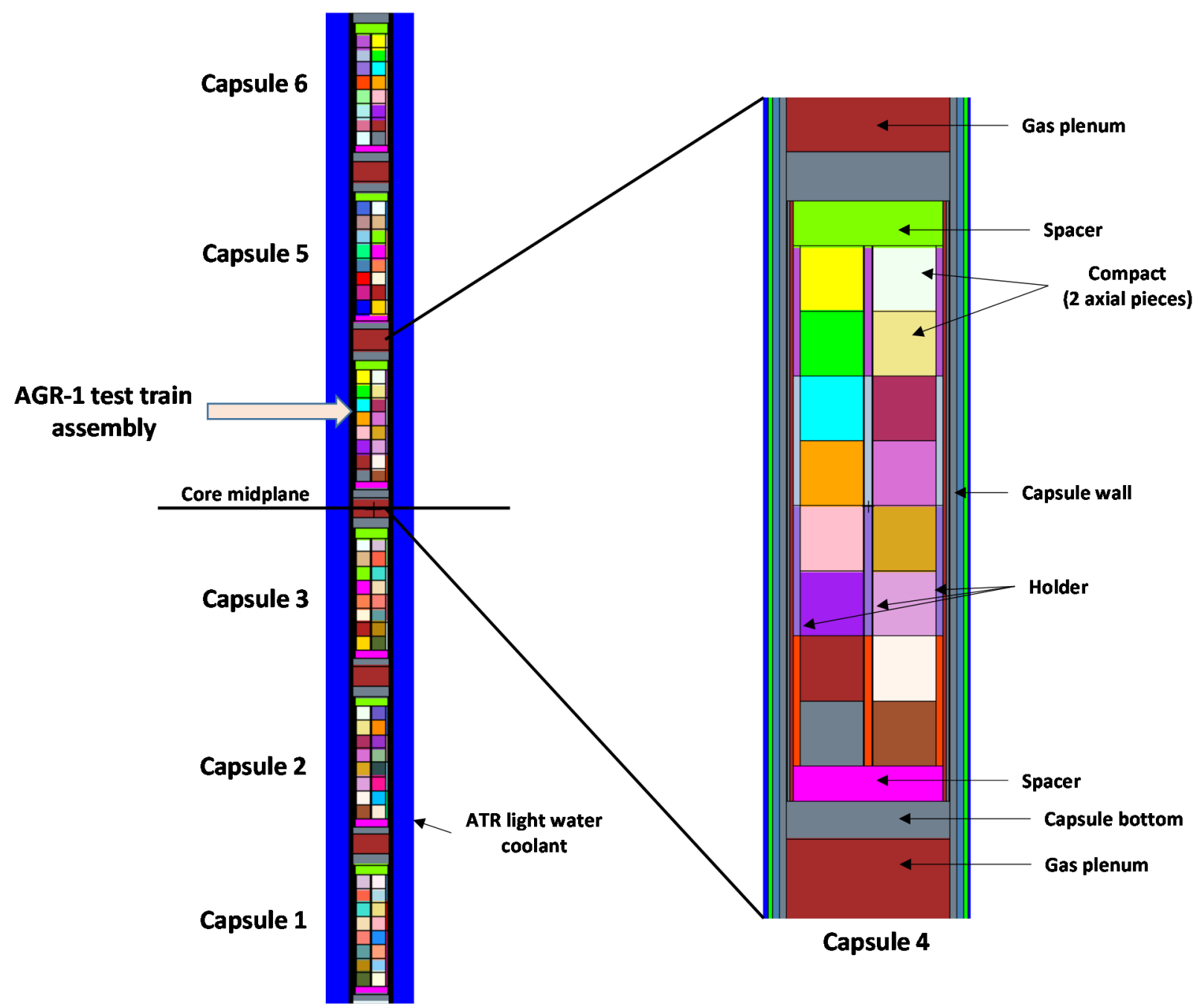

Figure 3. Axial view of the in-core section of the AGR-1 test train assembly, showing the six capsules, compacts, spacers, and gas plenums (MCNP plot).

In each capsule, there were 12 compacts. Since there were six capsules, AGR-1 contained a total of 72 fuel compacts. AGR-1 had four different types of compacts: (1) Baseline, (2) Variant 1, (3) Variant 2, and (4) Variant 3.

Differences between the baseline and variants 1, 2, and 3 were attributed primarily to the TRISO particle coatings, with relatively small differences in coating thicknesses, densities, and chemistry. Table 1 shows which capsules the four different TRISO particle types were placed. Each capsule contained only one type of particle.

Table 1. Compact variants by capsule.

\begin{tabular}{|l|l|}
\hline \multicolumn{1}{|c|}{ Location } & \multicolumn{1}{|c|}{ TRISO Particle Type } \\
\hline Capsule 6 & Baseline \\
\hline Capsule 5 & Variant 1 \\
\hline Capsule 4 & Variant 3 \\
\hline Capsule 3 & Baseline \\
\hline Capsule 2 & Variant 2 \\
\hline Capsule 1 & Variant 3 \\
\hline
\end{tabular}


From a neutronic standpoint, these TRISO particle differences are insignificant. However, there are differences in the number of particles per compact that do need to be considered in the benchmark model. The number of particles per compact are given in Table 11 below. Each compact had a nominal average measured length of $2.54 \mathrm{~cm}$ and diameter of $1.27 \mathrm{~cm}$.

Figure 4 shows a cross section view of Capsule 6 . In this figure, the orienting vector or North direction is up or vertically up the page, or the same direction as arrow in Figure 1. The three compact stacks are labeled 1, 2, and 3. Stack 1 and Stack 3 face the ATR core center as shown. Stack 2 is behind these two stacks and partially shielded by these two stacks from the inherent fast and thermal neutron flux gradients produced by the ATR driver core (flux gradient vectors are directed radially inward toward the core center), Stack 2 compacts therefore have lower burnups relative to Stacks 1 and 3. Due to their similar positions, Stacks 1 and 3 will have comparable burnups, but with some slight differences due to rotational differences in local outer shim control cylinders (E2 and E3) next to the B-10 facility. The E2 and E3 OSCCs are on separate control banks. Note: the compact stacks and thru-tube centers (x-y coordinates) are the same for all six capsules.

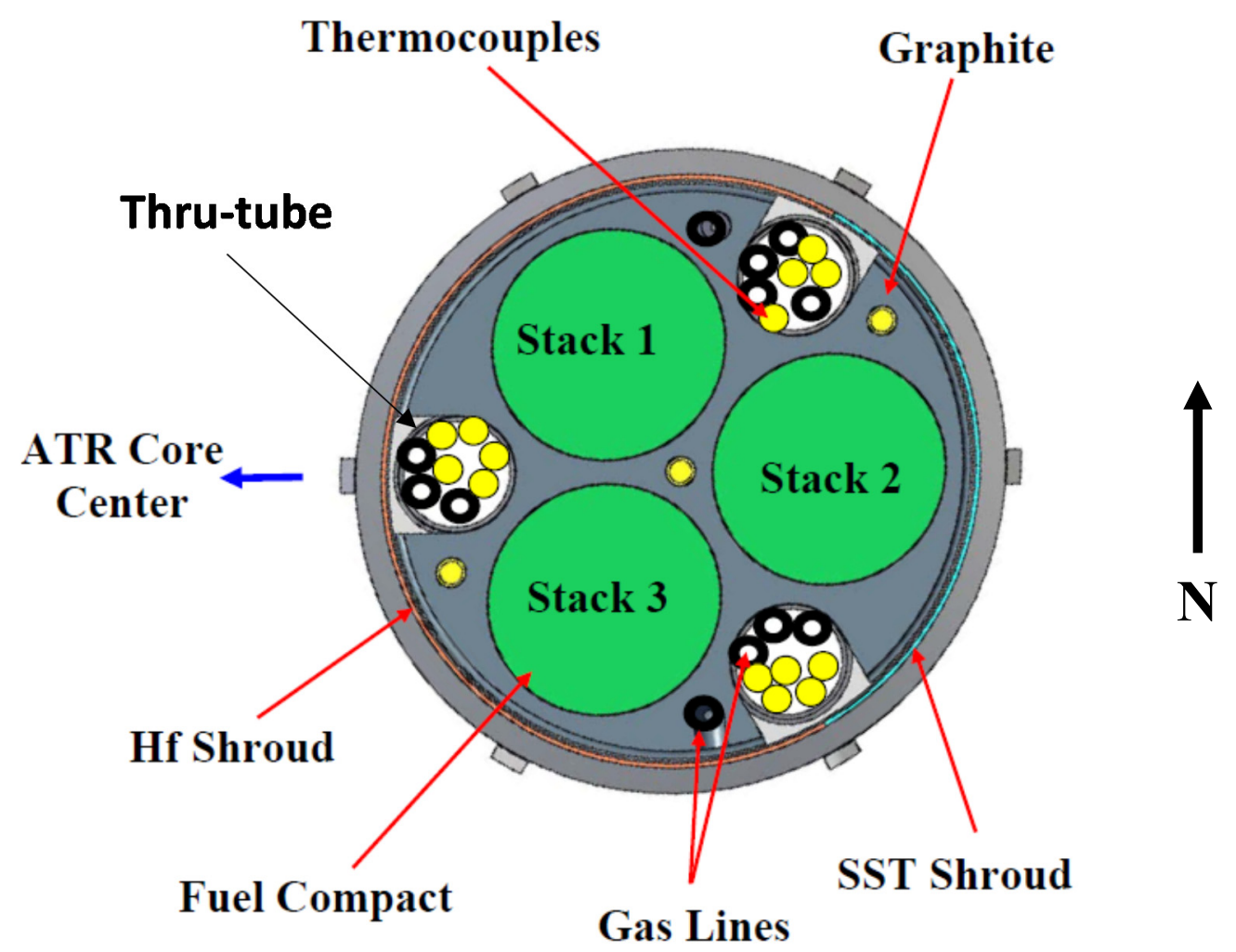

Figure 4. Cross section view of an AGR-1 capsule.

Figure 5 is a three-dimensional cutaway of a single AGR-1 capsule and Figure 6 is an axial cross section view with internal capsule components identified. Figure 7 is an isometric view of a capsule. 


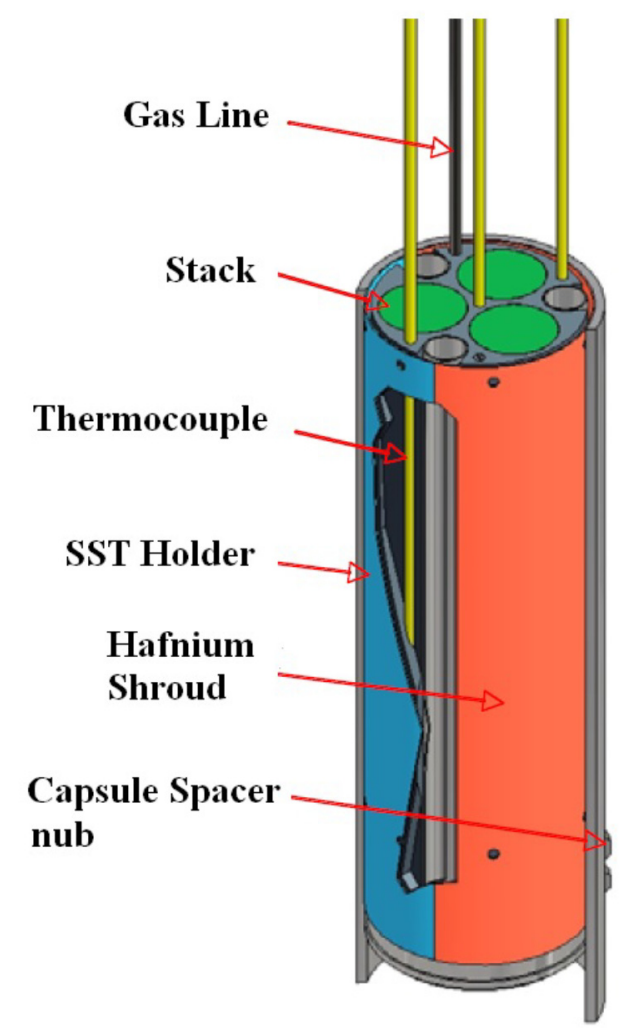

Figure 5. Three-dimensional cutaway of a single AGR-1 capsule. 


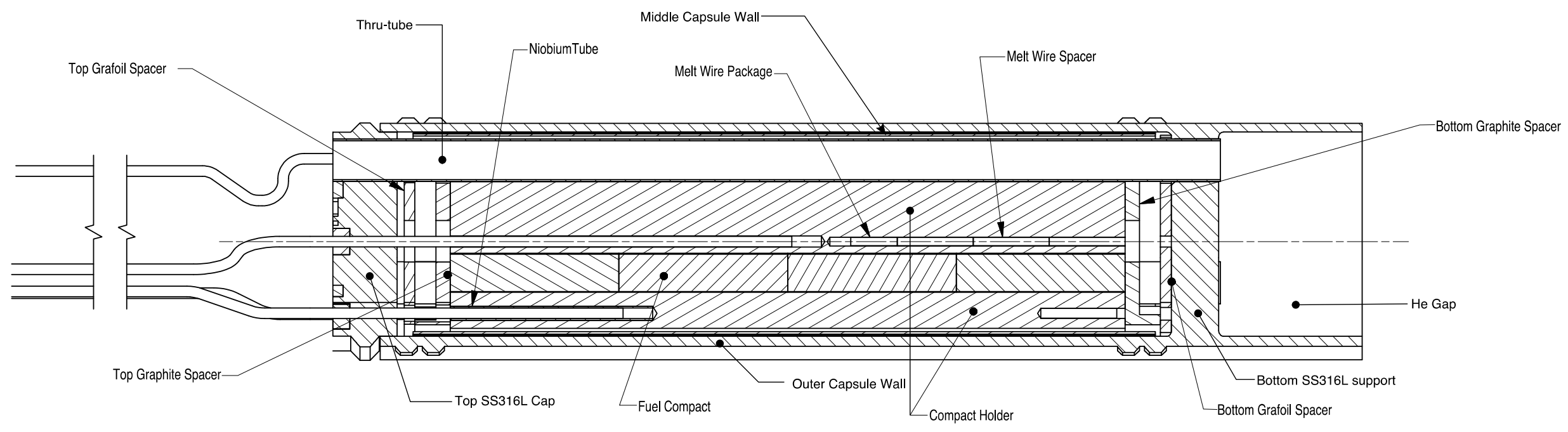

Figure 6. Axial view of capsule with internal components labeled. 


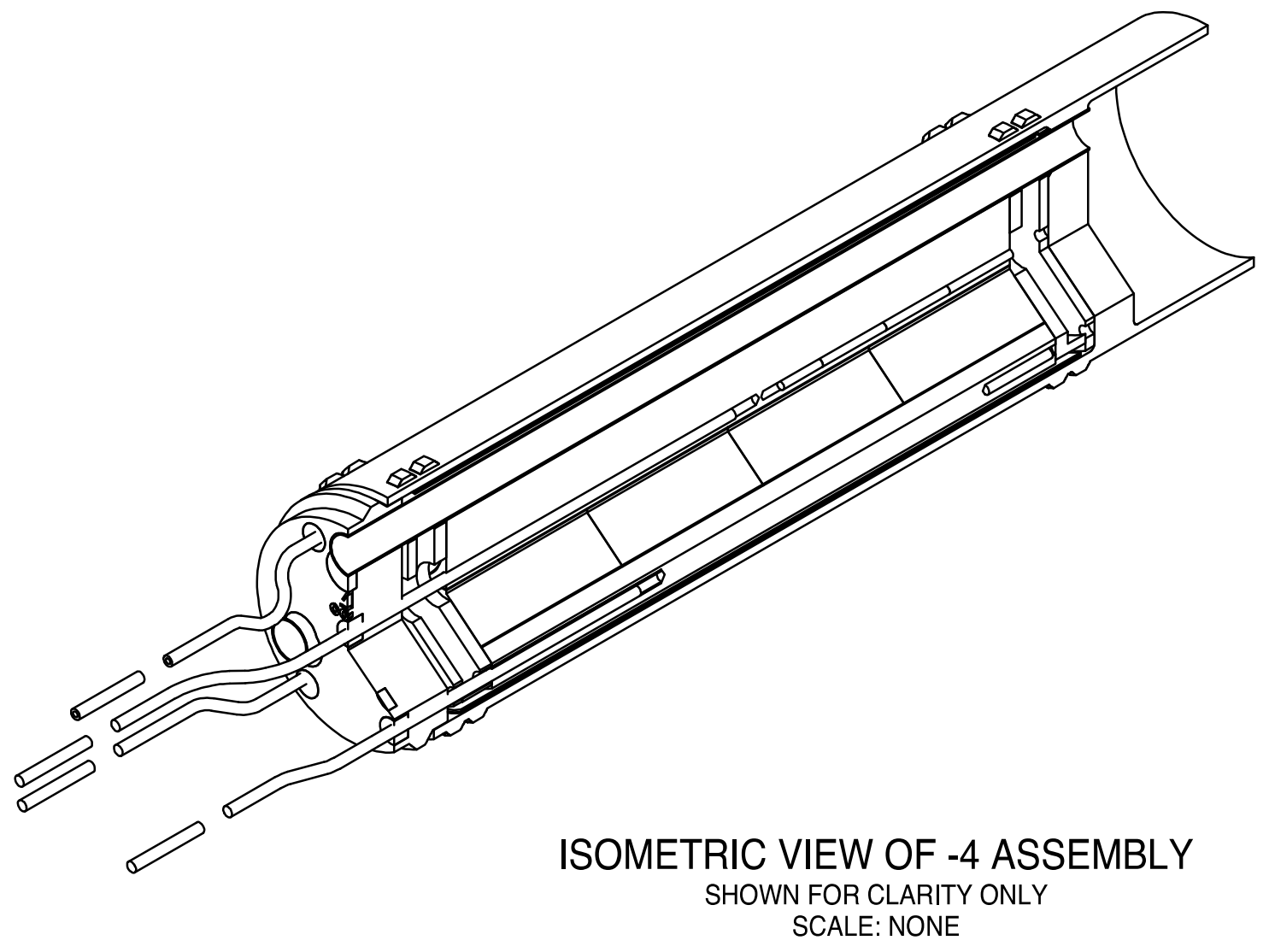

Figure 7. Isometric view of a capsule showing typical gas lines.

In addition to the fuel compacts, there are other components inside each capsule which include: (1) the borated graphite compact holders, (2) thru-tubes, (3) gas lines, (4) thermocouples (TC), (5) graphite spacers, and (6) gas plenums. The cylindrical capsule wall contained the graphite holders with a small helium-neon gas gap between them. The capsule wall was stainless steel with an embedded hafnium metal shroud. The boron in the graphite holders were designed reduce the thermal neutron flux and hence the compact fission rates, in particular for the first several ATR power cycles; the hafnium foil in the capsule wall was designed to further reduce and flatten the thermal and epithermal neutron flux gradient across the train (flux gradient existed radially outward from the core center). The 240-degree circumferential hafnium foil depleted slowly over the 13 power cycles and provided continuous flux gradient flattening.

\subsection{Graphite Compact Holders}

Table 2 gives material properties and dimensions for the graphite compact holders by capsule, along with internal capsule components that axially penetrate or traverse the holders. The compact holders are cylindrical rods of borated graphite, each nominally $3.03826 \mathrm{~cm}$ in diameter and $10.16 \mathrm{~cm}$ (4 in.) in length. There are six compact holders, one in each of the six capsules. Each compact holder contains three stacks of TRISO particle compacts, or 4 compacts per stack, each compact nominally $2.54 \mathrm{~cm}(1 \mathrm{in}$.) in 
length. The axially drilled holes in the holders contain the compacts plus have an associated gas gap between the compact and holder with a thickness on the order of $0.00635 \mathrm{~cm}$. The drilled holes for the compact stacks are equidistant from each other with a $1.405611 \mathrm{~cm}$ triangular pitch.

Table 2. Compact holder and embedded component materials and dimensions.

\begin{tabular}{|c|c|c|c|c|c|c|c|c|}
\hline Component & Capsule & Material & $\begin{array}{l}\text { Density } \\
\left(\mathrm{g} / \mathrm{cm}^{3}\right)\end{array}$ & $\begin{array}{l}\text { Total No. } \\
\text { Density } \\
\text { (a/b/cm) }\end{array}$ & $\begin{array}{c}\text { Boron } \\
(\mathrm{a} / \mathrm{o})\end{array}$ & $\begin{array}{c}\text { B-10 } \\
\text { Enrich } \\
(\mathrm{a} / \mathrm{o})\end{array}$ & $\begin{array}{c}\text { Outer } \\
\text { Diameter } \\
(\mathrm{cm})\end{array}$ & $\begin{array}{l}\text { Length } \\
(\mathrm{cm})\end{array}$ \\
\hline \multirow{6}{*}{$\begin{array}{l}\text { Compact } \\
\text { holders }\end{array}$} & 6 & borated graphite & 1.7695 & 0.089145 & 4.76 & 19.9 & 3.03826 & 10.16 \\
\hline & 5 & borated graphite & 1.7788 & 0.089728 & 6.05 & 19.9 & 3.03826 & 10.16 \\
\hline & 4 & borated graphite & 1.7788 & 0.089728 & 6.05 & 19.9 & 3.03826 & 10.16 \\
\hline & 3 & borated graphite & 1.7788 & 0.089728 & 6.05 & 19.9 & 3.03826 & 10.16 \\
\hline & 2 & borated graphite & 1.7788 & 0.089728 & 6.05 & 19.9 & 3.03826 & 10.16 \\
\hline & 1 & borated graphite & 1.7695 & 0.089145 & 4.76 & 19.9 & 3.03826 & 10.16 \\
\hline $\begin{array}{l}\text { Compact } \\
\text { gas gap }\end{array}$ & $1-6$ & $\mathrm{He}-\mathrm{Ne}$ & - & - & - & - & 1.2467 & 10.16 \\
\hline Thru-tubes & $2-6$ & $\begin{array}{c}\text { Molybdenum } \\
\text { Type } 361 \\
\text { ASTM B387 }\end{array}$ & 10.1390 & 0.063659 & - & - & $\begin{array}{c}0.6350 \text { OD } \\
0.5842 \text { ID }\end{array}$ & $\begin{array}{l}\text { Through } \\
\text { capsules } \\
2,3,4,5,6\end{array}$ \\
\hline Gas Lines & $2-6$ & Niobium & 8.57 & 0.05555 & - & - & 0.15875 & -- \\
\hline TCs & $1-6$ & Table 5 & Table 5 & Table 5 & - & - & Table 5 & Table 5 \\
\hline
\end{tabular}

Table 3 gives the $x-y$ coordinate centers of each stack relative to the center of the ATR core $(x=0$, $\mathrm{y}=0$ ), which is also the central point of the center flux trap. Coordinates are also given for the MCNP model. Note the ATR east directional vector runs equidistant between Stacks 1 and 3 and directly through the center of Stack 2 and the B-10 irradiation facility. The x-y coordinates of the centers of the three compact stacks are the same in each capsule. Note that in the MCNP model, the x-y coordinates $(\mathrm{cm})$ corresponding to the center of the B-10 test hole is $(+25.337,-25.337)$ and in the ATR coordinate system $(35.8319,0.0)$ are the $\mathrm{x}-\mathrm{y}$ coordinates. The original MCNP models, and all subsequent models, including the MCNP quarter-core model here, were unfortunately constructed with a 45-degree rotation relative to the actual ATR core reference north direction.

Table 3. Compact stack x-y coordinates in the graphite holders.

\begin{tabular}{|c|c|c|c|c|c|}
\hline Stack & $\begin{array}{c}\text { ATR } \\
\text { x-coordinate } \\
(\mathrm{cm})\end{array}$ & $\begin{array}{c}\text { ATR } \\
\text { y-coordinate } \\
(\mathrm{cm})\end{array}$ & $\begin{array}{c}\text { Pitch } \\
(\mathrm{cm})\end{array}$ & $\begin{array}{c}\text { MCNP model } \\
\text { x-coordinate } \\
(\mathrm{cm})\end{array}$ & $\begin{array}{c}\text { MCNP model } \\
\text { y-coordinate } \\
(\mathrm{cm})\end{array}$ \\
\hline 1 & 35.426164 & 0.702805 & 1.405611 & 25.547039 & -24.553123 \\
\hline 2 & 35.426164 & -0.702805 & 1.405611 & 24.553123 & -25.547039 \\
\hline 3 & 36.643459 & 0.000000 & 1.405611 & 25.910838 & -25.910838 \\
\hline Holder Center & 35.8319 & 0.0 & Not applicable & 25.337 & -25.337 \\
\hline
\end{tabular}

\subsection{Thru-Tubes}

Other vertically-drilled holes in the graphite holders include three locations for the thru-tubes that house the gas lines and thermocouples for each capsule. The thru-tube holes in the graphite holders are $0.6858 \mathrm{~cm}$ in diameter. The molybdenum thru-tubes themselves are $0.635 \mathrm{~cm}$ in diameter with a wall thickness of 0.0254 $\mathrm{cm}$. Table 4 gives the $\mathrm{x}-\mathrm{y}$ coordinates for the thru-tubes relative to the ATR core center and for the MCNP model. 
Table 4. Thru-tube $\mathrm{x}-\mathrm{y}$ coordinates and dimensions.

\begin{tabular}{|c|c|c|c|c|c|}
\hline $\begin{array}{c}\text { Thru-Tube } \\
\text { Location }\end{array}$ & $\begin{array}{c}\text { ATR } \\
\text { x-coordinate } \\
(\mathrm{cm})\end{array}$ & $\begin{array}{c}\text { ATR } \\
\text { y-coordinate } \\
(\mathrm{cm})\end{array}$ & $\begin{array}{c}\text { Tube IR/OR } \\
(\mathrm{cm})\end{array}$ & $\begin{array}{c}\text { MCNP model } \\
\text { x-coordinate } \\
(\mathrm{cm})\end{array}$ & $\begin{array}{c}\text { MCNP model } \\
\text { y-coordinate } \\
(\mathrm{cm})\end{array}$ \\
\hline West side & 34.6076 & 0.0000 & $0.2921 / 0.3175$ & 24.4713 & -24.4713 \\
\hline Northeast side & 36.4441 & 1.0603 & $0.2921 / 0.3175$ & 26.5196 & -25.0201 \\
\hline Southeast side & 36.4441 & -1.0603 & $0.2921 / 0.3175$ & 25.0201 & -26.5196 \\
\hline
\end{tabular}

\subsection{Gas Lines and Thermocouples}

Inside the thru-tubes are both gas lines and thermocouples. Gas lines were located in capsules 2-6. Capsule $1 \mathrm{did}$ not have thru-tubes or gas lines that penetrated axially through the holders. Each gas line had an outer diameter of $0.15875 \mathrm{~cm}(0.0625 \mathrm{in}$.), a wall thickness of $0.0254 \mathrm{~cm}(0.01 \mathrm{in}$.), and a $50 \% \mathrm{He}-$ $50 \% \mathrm{Ne}$ gas mixture inside. Capsule 6 (top capsule) had 10 gas lines, capsule 5 had 8 gas lines, capsule 4 had 6 gas lines, capsule 3 had 4 gas lines, and capsule 2 had 2 gas lines.

Thermocouple modeling can be somewhat tedious. There were three different types of thermocouples (TC) in AGR-1. Table 5 lists the characteristics of these TCs. Figure 8 shows the TC type, position, and insertion depth in each graphite holder/capsule. Depending on the level of detail desired in the MCNP model, the TCs can either be homogenized or explicitly modelled. Note in Figure 8 that the number of gas lines and TCs in the thru-tubes appear to be the same for all capsules.

Table 5. TC characteristics.

\begin{tabular}{|c|c|c|c|}
\hline Type & Thermo-elements & Insulation & Sheath \\
\hline$N(3 / 32)$ & $\begin{array}{l}\text { Nisil \& Nicrosil } 0.0381 \mathrm{~cm} \\
\text { (0.015 in.) OD each }\end{array}$ & $\mathrm{A} 12 \mathrm{O} 3$ & $\begin{array}{l}\text { Molybdenum } 0.023368 \mathrm{~cm}(0.092 \text { in.) OD } \times \\
0.14224 \mathrm{~cm}(0.056 \text { in.) ID }\end{array}$ \\
\hline $\mathrm{N}(1 / 16)$ & $\begin{array}{l}\text { Nisil \& Nicrosil } 0.0254 \mathrm{~cm} \\
\text { (0.010 in.) OD each }\end{array}$ & $\mathrm{MgO}$ & $\begin{array}{l}\text { Inconel-600 } 0.15748 \mathrm{~cm}(0.062 \text { in. }) \\
O D \times 0.10668 \mathrm{~cm}(0.042 \text { in. }) \text { ID }\end{array}$ \\
\hline INL Type 50 & $\begin{array}{l}\text { Nb1Zr } 0.021082 \mathrm{~cm}(0.0083 \\
\text { in.) OD and Mo } 0.023368 \mathrm{~cm} \\
(0.0092 \text { in. }) \text { OD }\end{array}$ & $\mathrm{HfO} 2$ & $\begin{array}{l}\mathrm{Nb} 1 \mathrm{Zr} 0.15748 \mathrm{~cm}(0.062 \mathrm{in} .) \\
\mathrm{OD} \times 0.105918 \mathrm{~cm}(0.0417 \text { in. }) \mathrm{ID}\end{array}$ \\
\hline
\end{tabular}



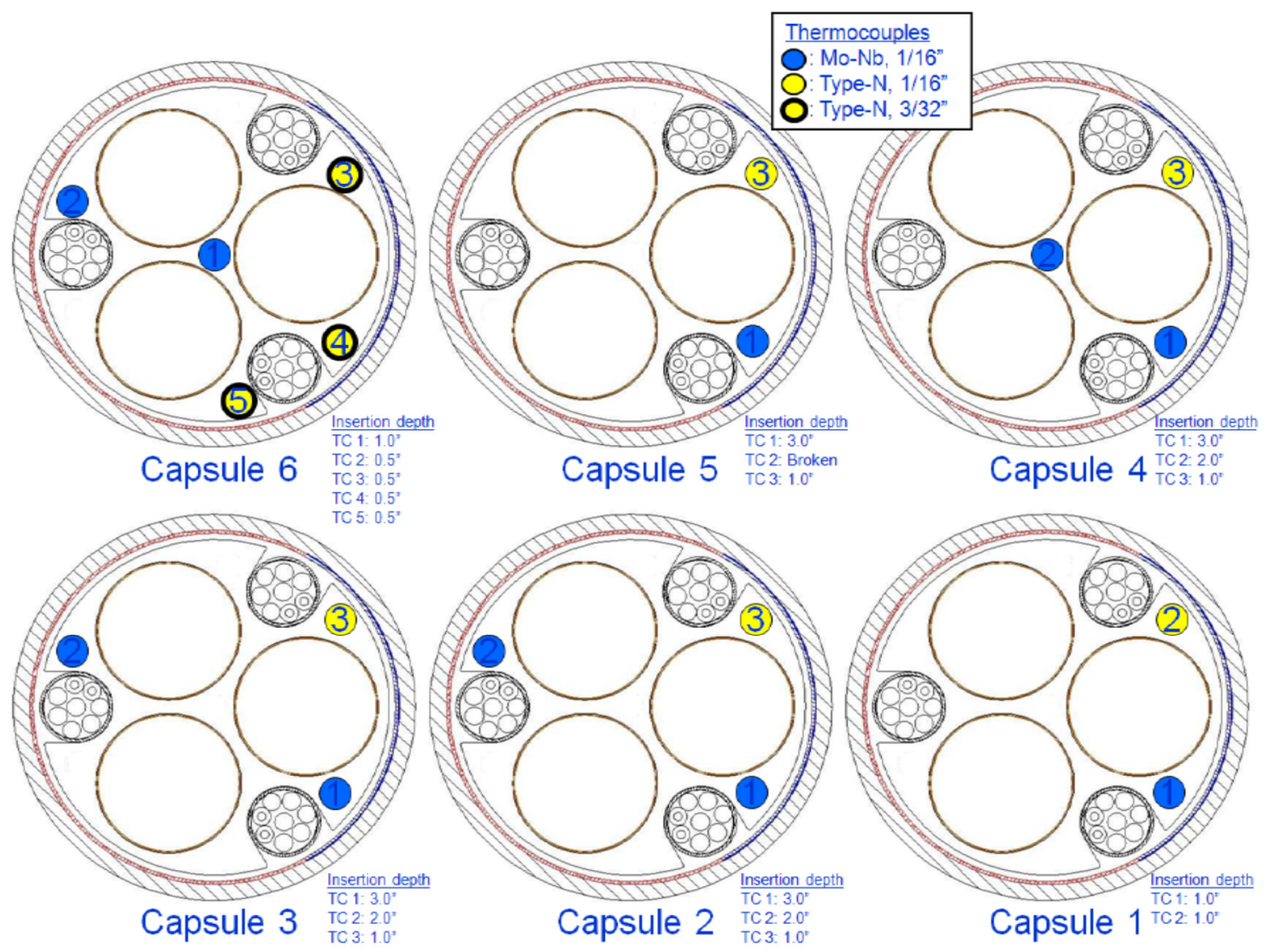

Figure 8. Cross section view of each capsule showing type, position, and insertion depth of the TCs in the graphite holders (dimensions are in inches).

\subsection{Capsule}

Table 6 gives the capsule wall tube dimensions and materials. The capsule wall is composed of three annular tubes pressed together. The three walls are: (1) an inner stainless steel tube, (2) a middle tube composed of hafnium and stainless steel (shroud or neutron filter), and (3) an outer stainless steel tube. The middle tube is composed of natural hafnium foil which extends $240^{\circ}$ azimuthally around the circumference of the tube; the remaining 120-degree sector of the middle tube is stainless steel of comparable thickness to the hafnium foil. The center of the 240-degree hafnium sector is centered and pointed directly at the ATR core center. As mentioned previously, the hafnium foil absorbs and reduces the intensity of thermal and epi-thermal neutron current emanating from the direction of the core center, thus mitigating the fission density in the compacts. There are six hafnium shrouds, one shroud per capsule centered about the compact stacks. Each hafnium shroud is $11.176 \mathrm{~cm}$ in axial height and is axiallylocated between the SS316L cap and the bottom SS316L support. The outer stainless steel tube is relatively thick and is basically the main pressure-bearing wall of the capsule wall (pressure vessel). The gas in the gas gap between the borated graphite holders and the inside of the capsule wall was composed of a mixture of helium-neon, which can be assumed to have a gas composition of either $100 \%$ helium, or a more accurate $50 \% \mathrm{He}$ and $50 \% \mathrm{Ne}$ mixture by volume. Note a second gas gap exists between the middle and outer capsule walls as well (Table 6). 
Table 6. Capsule wall tube dimensions and materials.

\begin{tabular}{|l|c|c|c|c|c|c|}
\hline \multicolumn{1}{|c|}{ Component } & Material & $\begin{array}{c}\text { IR } \\
(\mathrm{cm})\end{array}$ & $\begin{array}{c}\text { OR } \\
(\mathrm{cm})\end{array}$ & $\begin{array}{c}\text { Length } \\
(\mathrm{cm})\end{array}$ & $\begin{array}{c}\text { Azimuthal } \\
\text { Extent } \\
(\text { degrees })\end{array}$ & $\begin{array}{c}\text { Total Number } \\
\text { Density } \\
(\mathrm{a} / \mathrm{b} / \mathrm{cm})\end{array}$ \\
\hline Gas gap & $\mathrm{He}$ & 1.51913 & 1.5875 & 90.0 & 360 & $1.24931 \mathrm{E}-04$ \\
\hline Inner Capsule wall & $\mathrm{SS316L}$ & 1.5875 & 1.62179 & 90.0 & 360 & 0.087000 \\
\hline Middle Capsule wall & $\mathrm{Hf}$ & 1.62179 & 1.64719 & 90.0 & 240 & $13.36 \mathrm{~g} / \mathrm{cm}^{3}$ \\
\hline Middle Capsule wall & $\mathrm{SS316L}$ & 1.62179 & 1.64719 & 90.0 & 120 & 0.087000 \\
\hline Gas gap & $\mathrm{He}$ & 1.64719 & 1.64846 & 90.0 & 360 & $1.24931 \mathrm{E}-04$ \\
\hline Outer Capsule wall & $\mathrm{SS} 316 \mathrm{~L}$ & 1.64846 & 1.78562 & 90.0 & 360 & 0.087000 \\
\hline $\begin{array}{l}\text { ATR light water coolant } \\
\text { outside capsule wall }\end{array}$ & $\mathrm{H}_{2} \mathrm{O}$ & 1.78562 & 1.905 & 121.92 & 360 & 0.100127 \\
\hline
\end{tabular}

Radially outside the capsule wall is ATR light water coolant which extends out to $1.905 \mathrm{~cm}$ or the inner radius of the drilled bole hole in the beryllium reflector block that is the B-10 irradiation position.

Table 7 data provides the physics analyst with axial elevations of the major components in each of the six capsules. Note that the elevations are axial heights above the bottom of the ATR active core or the bottom of the ATR fuel. The bottom of the ATR fuel is the designated reference elevation at zero centimeters $(\mathrm{z}=0.0 \mathrm{~cm})$ in the MCNP model. The capsule top cap and bottom support components are solid cylindrical stainless steel pieces with a radius equal to the graphite holders $(1.51913 \mathrm{~cm})$. The top of the compact stack coincides with the top of the graphite holders, and above the graphite holders is an upper graphite spacer (solid graphite cylinder) with a radius equal to the graphite holder below it with a thickness of $0.86868 \mathrm{~cm}(0.342 \mathrm{in}$.) and is made of pure graphite (no boron) with a total number density of $4.76735 \mathrm{E}-02 \mathrm{a} / \mathrm{b} / \mathrm{cm}\left(0.95 \mathrm{~g} / \mathrm{cm}^{3}\right)$. Above the upper graphite spacer is a stainless steel top cap (solid cylinder) with a thickness of $0.9652 \mathrm{~cm}$ (0.38 in.). Above the stainless steel top cap is a helium gap separating the capsules. The helium gap has a radius equal to the capsule inner wall radius $(1.5875 \mathrm{~cm})$, thickness of $1.8161 \mathrm{~cm}(0.715 \mathrm{in})$, and an all-helium number density of $1.24931 \mathrm{E}-04 \mathrm{a} / \mathrm{b} / \mathrm{cm}$. Above the helium gap is the bottom stainless steel support piece and above this support piece is the lower graphite spacer. The top of this lower graphite spacer coincides with the bottom of the graphite holder and compact stack of the capsule above. These component pieces between capsules or between the graphite compact holders are repeated and the same for all capsules.

Table 7. Axial plane elevations for component pieces between capsules.

\begin{tabular}{|c|r|r|r|}
\hline Capsule & \multicolumn{1}{|c|}{ Component } & \multicolumn{1}{|c|}{$\begin{array}{c}\text { Elevation } \\
(\mathrm{cm})\end{array}$} & $\begin{array}{c}\text { Elevation } \\
\text { (in.) }\end{array}$ \\
\hline 6 & Top of graphite spacer/bottom of SS316L cap & 104.98328 & 41.712 \\
\hline 6 & top of compact stack & 104.11460 & 40.990 \\
\hline 6 & midplane of compact stack & 99.03460 & 38.990 \\
\hline 6 & Top of lower graphite spacer/bottom of compact stack & 93.95460 & 36.990 \\
\hline 6 & top of SS316L support/bottom of lower graphite spacer & 93.26118 & 36.717 \\
\hline 6 & Top of helium gap/bottom of SS316L support & 92.53728 & 36.432 \\
\hline 6 & top SS316L cap & 90.72118 & 35.717 \\
\hline 5 & Top of graphite spacer/bottom of SS316L cap & 89.75598 & 35.337 \\
\hline 5 & top of compact stack & 88.88730 & 34.995 \\
\hline 5 & midplane of compact stack & 83.80730 & 32.995 \\
\hline 5 & Top of lower graphite spacer/bottom of compact stack & 78.72730 & 30.995 \\
\hline 5 & top of SS316L support/bottom of lower graphite spacer & 78.03388 & 30.722 \\
\hline 5 & Top of helium gap/bottom of SS316L support & 77.30998 & 30.437 \\
\hline 5 & &
\end{tabular}


Table 7. (continued).

\begin{tabular}{|c|c|c|c|}
\hline 4 & top SS316L cap & 75.49388 & 29.722 \\
\hline 4 & Top of graphite spacer/bottom of SS316L cap & 74.52868 & 29.342 \\
\hline 4 & top of compact stack & 73.66000 & 29.000 \\
\hline 4 & midplane of compact stack & 68.58000 & 27.000 \\
\hline 4 & Top of lower graphite spacer/bottom of compact stack & 63.50000 & 25.000 \\
\hline 4 & top of SS316L support/bottom of lower graphite spacer & 62.80658 & 24.727 \\
\hline 4 & Top of helium gap/bottom of SS316L support & 62.08268 & 24.442 \\
\hline 3 & top SS316L cap & 60.26658 & 23.727 \\
\hline 3 & Top of graphite spacer/bottom of SS316L cap & 59.30138 & 23.347 \\
\hline 3 & top of compact stack & 58.43270 & 23.005 \\
\hline 3 & midplane of compact stack & 53.35270 & 21.005 \\
\hline 3 & Top of lower graphite spacer/bottom of compact stack & 48.27270 & 19.005 \\
\hline 3 & top of SS316L support/bottom of lower graphite spacer & 47.57928 & 18.732 \\
\hline 3 & Top of helium gap/bottom of SS316L support & 46.85538 & 18.447 \\
\hline 2 & top SS316L cap & 45.03928 & 17.732 \\
\hline 2 & Top of graphite spacer/bottom of SS316L cap & 44.07408 & 17.352 \\
\hline 2 & top of compact stack & 43.20540 & 17.010 \\
\hline 2 & midplane of compact stack & 38.12540 & 15.010 \\
\hline 2 & Top of lower graphite spacer/bottom of compact stack & 33.04540 & 13.010 \\
\hline 2 & top of SS316L support/bottom of lower graphite spacer & 32.35198 & 12.737 \\
\hline 2 & Top of helium gap/bottom of SS316L support & 31.62808 & 12.452 \\
\hline 1 & top SS316L cap & 29.81198 & 11.737 \\
\hline 1 & Top of graphite spacer/bottom of SS316L cap & 28.84678 & 11.357 \\
\hline 1 & top of compact stack & 27.97810 & 11.015 \\
\hline 1 & midplane of compact stack & 22.89810 & 9.015 \\
\hline 1 & Top of lower graphite spacer/bottom of compact stack & 17.81810 & 7.015 \\
\hline 1 & top of SS316L support/bottom of lower graphite spacer & 17.12468 & 6.742 \\
\hline \multirow[t]{3}{*}{1} & bottom of SS316L support & 16.40078 & 6.457 \\
\hline & graphite spacer & 14.67358 & 5.777 \\
\hline & bottom of SS316L capsule support & 13.65758 & 5.377 \\
\hline
\end{tabular}

The length of the B-10 test volume in the quarter-core model extends from $\mathrm{z}=-2.54 \mathrm{~cm}$ up to $\mathrm{z}=+127.0 \mathrm{~cm}$. In Table 7, the bottom elevation is $\mathrm{z}=+13.65758 \mathrm{~cm}$ and the top elevation is $\mathrm{z}=+105.94848 \mathrm{~cm}$. For the volume above $+105.84848 \mathrm{~cm}$ up to $+127.0 \mathrm{~cm}$, the capsule wall and thrutubes can simply be extended up to $+127.0 \mathrm{~cm}$ and the rest of the space filled with air. For the volume below $+13.65758 \mathrm{~cm}$ down to $-2.54 \mathrm{~cm}$, the capsule wall can again be extended down to $-2.54 \mathrm{~cm}$ and filled with air. One can also replace the air between -2.54 to $0.0 \mathrm{~cm}(1 \mathrm{in}$.) inside the capsule with a solid stainless steel wafer to represent the bottom dome of the test train.

\subsection{Hafnium Shroud}

As mentioned above, the hafnium shroud(s) are composed of natural hafnium foil which extends 240degrees azimuthally around the circumference of the tube; the remaining 120-degree sector of the middle tube is stainless steel of comparable thickness to the hafnium foil. The center of the 240-degree hafnium sector is centered and pointed directly at the ATR core center (Figure 9). There are six hafnium shrouds, one shroud per capsule centered about the compact stacks. Each hafnium shroud is $11.176 \mathrm{~cm}$ in axial height and is axially-located between the SS316L cap and the bottom SS316L support. Table 6 provides addition hafnium shroud dimension and material data. 


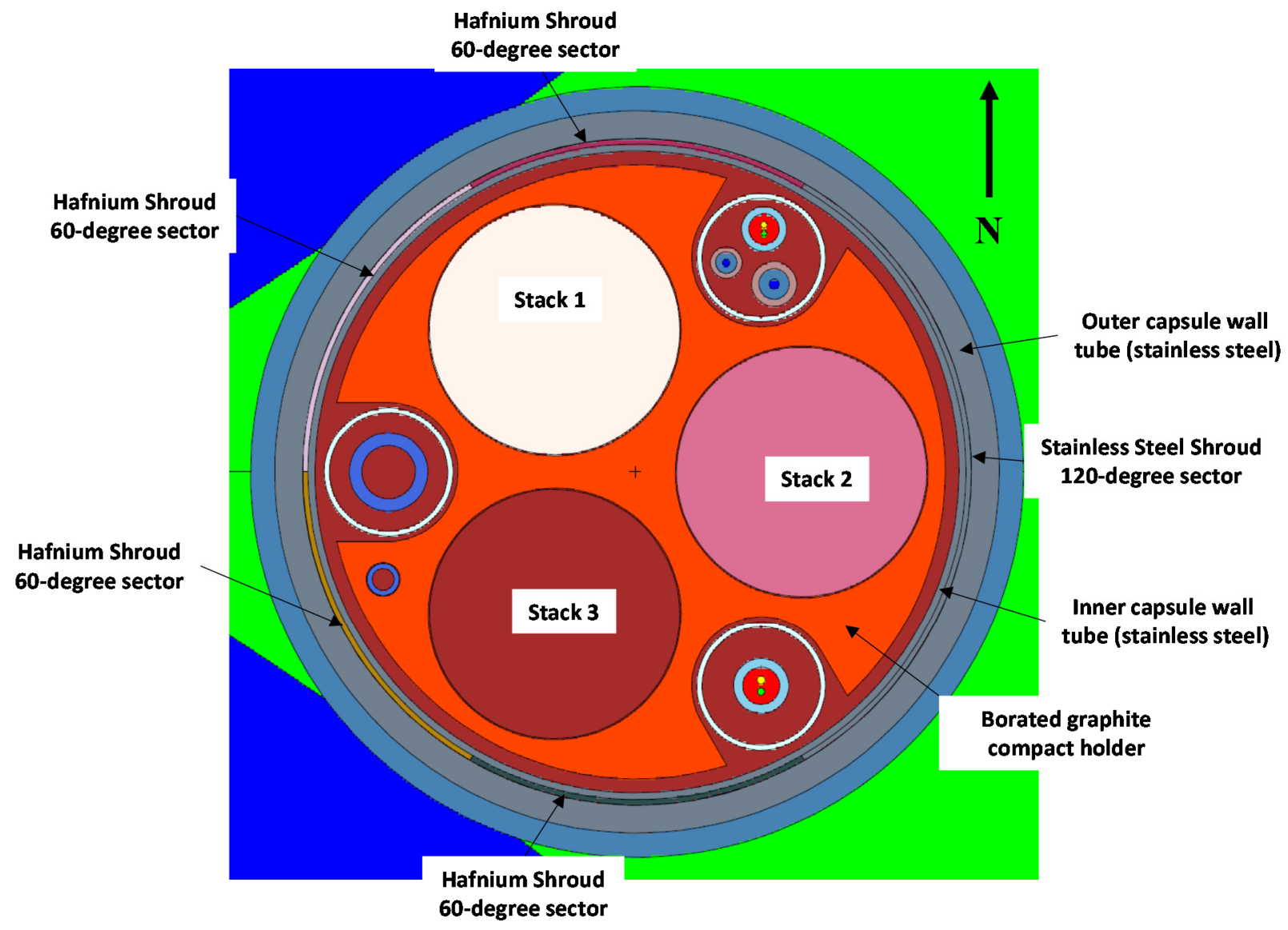

Figure 9. Four hafnium shroud azimuthal sectors for 240-degree hafnium shroud, plus 120-degree stainless steel sector.

Table 8. Hafnium shroud characteristics.

\begin{tabular}{|c|c|c|c|c|c|c|}
\hline Capsule & $\begin{array}{c}\text { Shroud } \\
\text { Axial } \\
\text { Edge }\end{array}$ & $\begin{array}{l}\text { Shroud } \\
\text { Elevation } \\
(\mathrm{cm})\end{array}$ & $\begin{array}{l}\text { Axial Length } \\
(\mathrm{cm})\end{array}$ & $\begin{array}{c}\text { Hf Foil } \\
\text { IR } \\
(\mathrm{cm})\end{array}$ & $\begin{array}{c}\text { Hf Foil } \\
\text { OR } \\
(\mathrm{cm})\end{array}$ & $\begin{array}{l}\text { Hf Foil } \\
\text { Density } \\
\left(\mathrm{g} / \mathrm{cm}^{3}\right)\end{array}$ \\
\hline $\begin{array}{l}6 \\
6\end{array}$ & $\begin{array}{l}\text { Top } \\
\text { Bottom }\end{array}$ & $\begin{array}{c}104.98328 \\
93.26118\end{array}$ & 11.176 & 1.62179 & 1.64719 & 12.8081 \\
\hline $\begin{array}{l}5 \\
5\end{array}$ & $\begin{array}{l}\text { Top } \\
\text { Bottom }\end{array}$ & $\begin{array}{l}89.75598 \\
78.03388\end{array}$ & 11.176 & 1.62179 & 1.64719 & 12.8081 \\
\hline $\begin{array}{l}4 \\
4\end{array}$ & $\begin{array}{l}\text { Top } \\
\text { Bottom }\end{array}$ & $\begin{array}{l}74.52868 \\
62.80658\end{array}$ & 11.176 & 1.62179 & 1.64719 & 12.8081 \\
\hline $\begin{array}{l}3 \\
3\end{array}$ & $\begin{array}{l}\text { Top } \\
\text { Bottom }\end{array}$ & $\begin{array}{l}59.30138 \\
47.57928\end{array}$ & 11.176 & 1.62179 & 1.64719 & 12.8081 \\
\hline $\begin{array}{l}2 \\
2 \\
\end{array}$ & $\begin{array}{l}\text { Top } \\
\text { Bottom }\end{array}$ & $\begin{array}{l}44.07408 \\
32.35198 \\
\end{array}$ & 11.176 & 1.62179 & 1.64719 & 12.8081 \\
\hline $\begin{array}{l}1 \\
1\end{array}$ & $\begin{array}{l}\text { Top } \\
\text { Bottom }\end{array}$ & $\begin{array}{l}28.84678 \\
17.12468\end{array}$ & 11.176 & 1.62179 & 1.64719 & 12.8081 \\
\hline
\end{tabular}




\section{TRISO PARTICLES}

The AGR-1 experiment included a baseline particle type along with three particle variants. Most of the differences associated with the four particle types involved the coating thicknesses and densities. All four particles have a spherical uranium oxy-carbide (UCO) kernel and four outer spherical coatings. The four coatings include: (1) low density graphite buffer, (2) inner pyrolithic graphite (IPyC) layer, (3) silicon carbide ( $\mathrm{SiC})$ layer, and (4) outer pyrolithic graphite (OPyC) layer. Table 9 (References [5] and [6]) gives the overall UCO kernel diameter, density, uranium enrichment, and carbon-to-uranium ratio and oxygen-to-uranium ratio in the uranium oxy-carbide (UCO) kernel. Table 10 gives the spherical particle coating thicknesses and densities (Reference [7]). Figure 10 shows schematically a TRISO particle with the kernel at the center of the particle and four particle coatings.

Table 9. UCO kernel characteristics.

\begin{tabular}{|l|c|}
\hline \multicolumn{1}{|c|}{ Property } & Mean Value (measured) \\
\hline Diameter $(\mu \mathrm{m})$ & 349.7 \\
\hline Density $\left(\mathrm{g} / \mathrm{cm}^{3}\right)$ & 10.924 \\
\hline $\mathrm{U}-234$ enrichment $(\mathrm{wt} \%)$ & 0.3294 \\
\hline $\mathrm{U}-235$ enrichment $(\mathrm{wt} \%)$ & 19.7624 \\
\hline $\mathrm{U}-236$ enrichment $(\mathrm{wt} \%)$ & 0.0192 \\
\hline $\mathrm{U}-238$ enrichment $(\mathrm{wt} \%)$ & 79.8890 \\
\hline Carbon/uranium (atomic ratio) & 0.3253 \\
\hline Oxygen/uranium (atomic ratio) & 1.3613 \\
\hline Total uranium $(\mathrm{wt} \%)$ & 90.059 \\
\hline
\end{tabular}

Table 10. Particle coating characteristics by particle type.

\begin{tabular}{|l|c|c|c|c|}
\hline \multirow{2}{*}{\multicolumn{1}{|c|}{ Property }} & \multicolumn{4}{|c|}{ Mean Value (measured) } \\
\cline { 2 - 5 } & Baseline & Variant 1 & Variant 2 & Variant 3 \\
\hline Buffer Thickness $(\mu \mathrm{m})$ & 103.5 & 102.5 & 102.9 & 104.2 \\
\hline IPyC Thickness $(\mu \mathrm{m})$ & 39.4 & 40.5 & 40.1 & 38.8 \\
\hline SiC Thickness $(\mu \mathrm{m})$ & 35.3 & 35.7 & 35.0 & 35.9 \\
\hline OPyC Thickness $(\mu \mathrm{m})$ & 41.0 & 41.1 & 39.8 & 39.3 \\
\hline Buffer Density $\left(\mathrm{g} / \mathrm{cm}^{3}\right)$ & 1.10 & 1.10 & 1.10 & 1.10 \\
\hline IPyC Density $\left(\mathrm{g} / \mathrm{cm}^{3}\right)$ & 1.904 & 1.853 & 1.912 & 1.904 \\
\hline SiC Density $\left(\mathrm{g} / \mathrm{cm}^{3}\right)$ & 3.208 & 3.206 & 3.207 & 3.205 \\
\hline OPyC Density $\left(\mathrm{g} / \mathrm{cm}^{3}\right)$ & 1.907 & 1.898 & 1.901 & 1.911 \\
\hline
\end{tabular}




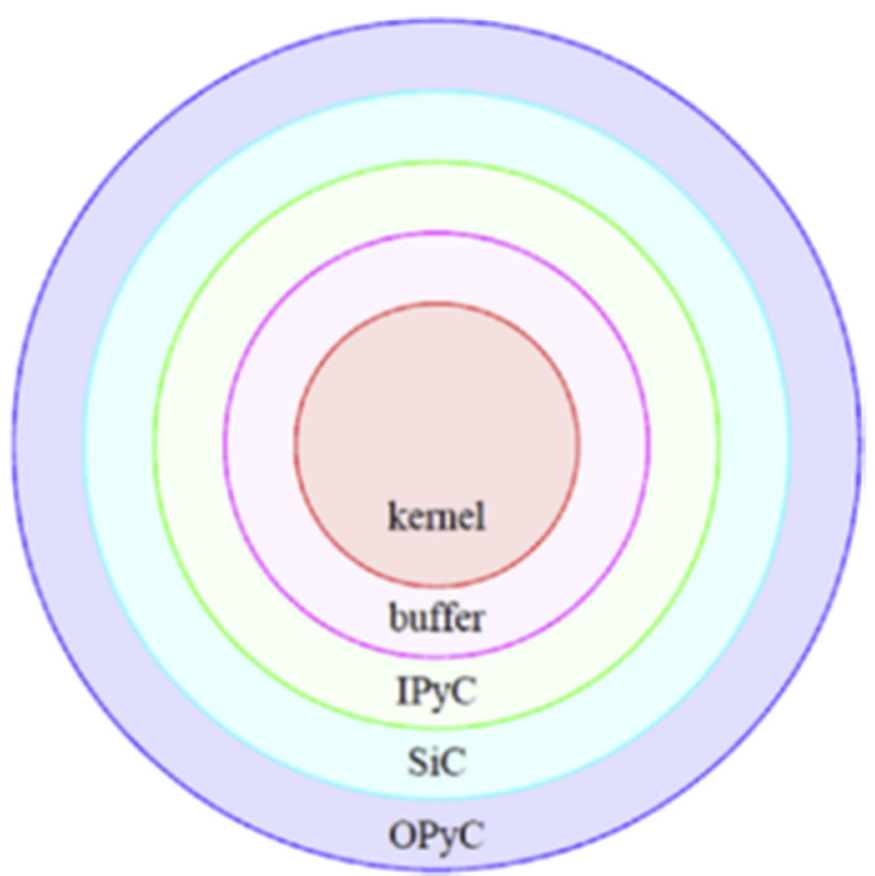

Figure 10. TRISO particle kernel and coatings.

\section{FUEL COMPACTS}

A typical AGR-1 compact is shown in Figure 11. The compacts were cylindrical rodlets that contained the TRISO particles. Each compact was nominally $1.27 \mathrm{~cm}(0.5$ in.) in diameter and $2.54 \mathrm{~cm}$ (1.0 in.) in length. Specific dimensions and compact characteristics are given in Table 11 per Reference [5]. 


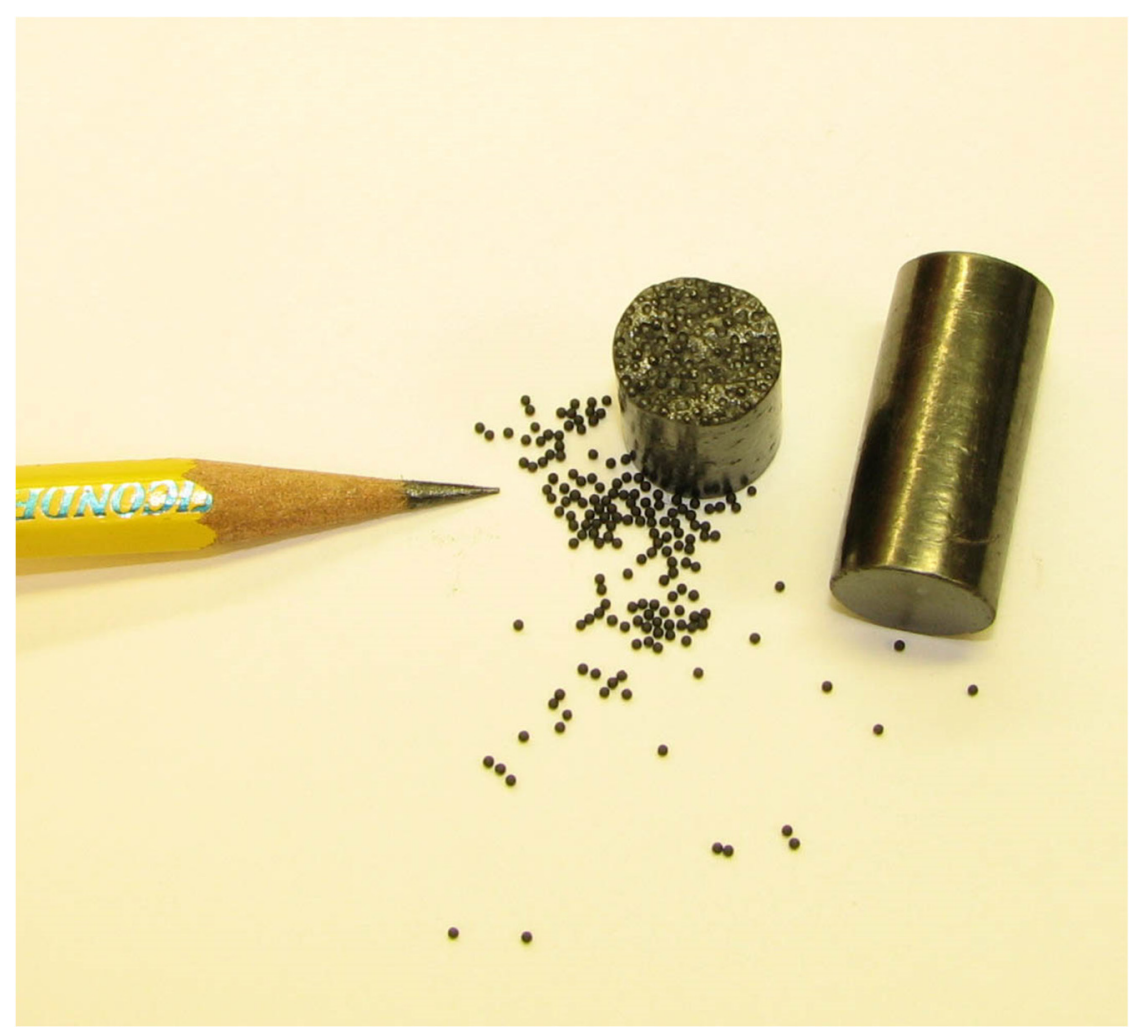

Figure 11.Typical AGR-1 compacts.

Table 11. Compact dimensions, loadings, and density characteristics for the four particle types.

\begin{tabular}{|l|c|c|c|c|}
\hline \multirow{2}{*}{ Property } & \multicolumn{4}{c|}{ Mean Value } \\
\cline { 2 - 5 } & Baseline & Variant 1 & Variant 2 & Variant 3 \\
\hline Diameter $(\mathrm{mm})$ & 12.36 & 12.36 & 12.36 & 12.34 \\
\hline Length $(\mathrm{mm})$ & 25.066 & 25.123 & 25.077 & 25.227 \\
\hline Number of particles per compact & 4154 & 4145 & 4095 & 4132 \\
\hline Average particle packing fraction (\%) & 36.99 & 37.42 & 36.26 & 36.04 \\
\hline Mean uranium loading (g U/compact) & 0.917 & 0.915 & 0.904 & 0.912 \\
\hline Compact mass $(\mathrm{g})$ & 5.4789 & 5.3371 & 5.3736 & 5.5930 \\
\hline Compact matrix density $\left(\mathrm{g} / \mathrm{cm}^{3}\right)$ & 1.297 & 1.219 & 1.256 & 1.344 \\
\hline Effective overall compact density $\left.\mathrm{g} / \mathrm{cm}^{3}\right)$ & 1.822 & 1.771 & 1.786 & 1.854 \\
\hline
\end{tabular}

Each AGR-1 compact had protective graphite endcaps on the top and bottom (Figure 12). The top endcap was nominally $0.16 \mathrm{~cm}$ in axial length and the bottom endcap $0.20 \mathrm{~cm}$. Both endcaps were graphite with a density equal to the compact matrix density given in Table 11 . The endcaps contained no TRISO particles so the effective compact packing fraction of particles in the middle portion of the compact will be slightly larger than the Table 11 average values. These endcaps were added to protect the TRISO particles in the compact and are unique to the AGR-1 compacts. 


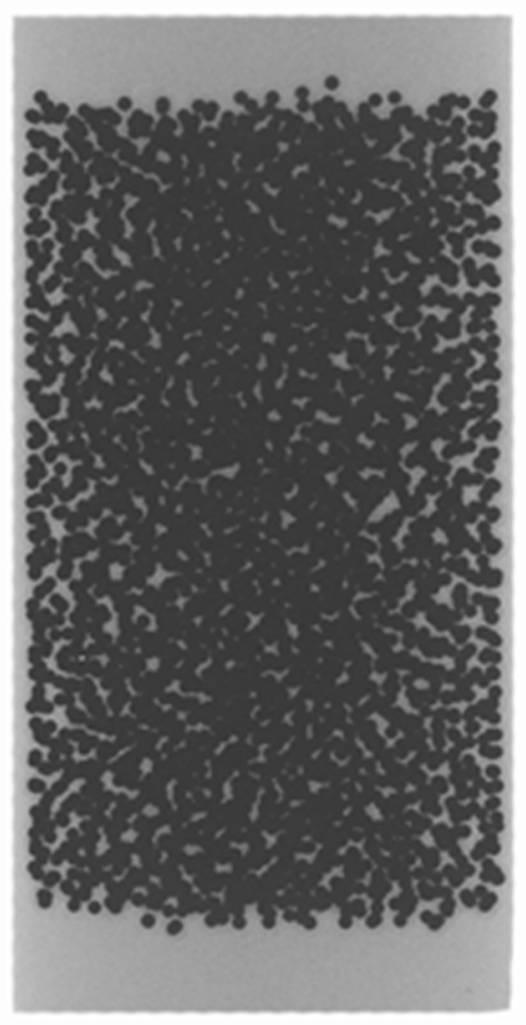

Figure 12.Axial radiograph of a typical compact showing the TRISO particles (small dark circles) and the matrix and top and bottom graphite endcaps (gray).

\section{MATERIAL COMPOSITION DATA}

Tables 12 - 17 provide additional elemental and isotopic composition descriptions for materials in the AGR-1 test train and the ATR quarter-core model. These materials include: (1) stainless steel 316L, (2) the two AGR-1 borated graphite holder materials, (3) hafnium metal (used in the capsule shroud, neck shim rods, and OSCCs), (4) aluminum-6061, and (4) ATR light water coolant.

Table 12. Stainless steel 316L (SS316L) composition $\left(8.03 \mathrm{~g} / \mathrm{cm}^{3}\right)$.

\begin{tabular}{|c|c|}
\hline Element & $\begin{array}{c}\text { Weight Fraction } \\
(w \mathrm{w} \%)\end{array}$ \\
\hline $\mathrm{C}$ & 0.030 \\
\hline $\mathrm{Si}$ & 0.750 \\
\hline $\mathrm{Cr}$ & 17.000 \\
\hline $\mathrm{Mn}$ & 2.000 \\
\hline $\mathrm{Fe}$ & 65.545 \\
\hline $\mathrm{Co}$ & 0.000 \\
\hline $\mathrm{Ni}$ & 12.000 \\
\hline $\mathrm{Cu}$ & 0.000 \\
\hline $\mathrm{N}$ & 0.100 \\
\hline $\mathrm{S}$ & 0.030 \\
\hline
\end{tabular}


Table 12. (continued).

\begin{tabular}{|c|c|}
\hline $\mathrm{P}$ & 0.045 \\
\hline Mo & 2.500 \\
\hline
\end{tabular}

Table 13. Borated graphite holder number densities (4.76 atom percent boron).

\begin{tabular}{|c|c|}
\hline Element & $\begin{array}{c}\text { No. density } \\
(\mathrm{a} / \mathrm{b} / \mathrm{cm})\end{array}$ \\
\hline C-12 & $8.4900 \mathrm{E}-02$ \\
\hline B-10 & $8.4496 \mathrm{E}-04$ \\
\hline B-11 & $3.4003 \mathrm{E}-03$ \\
\hline
\end{tabular}

Table 14. Borated graphite holder number densities (6.05 atom percent boron).

\begin{tabular}{|c|c|}
\hline Element & $\begin{array}{c}\text { No. density } \\
(\mathrm{a} / \mathrm{b} / \mathrm{cm})\end{array}$ \\
\hline C-12 & $8.4300 \mathrm{E}-02$ \\
\hline B-10 & $1.0804 \mathrm{E}-03$ \\
\hline B-11 & $4.3476 \mathrm{E}-03$ \\
\hline
\end{tabular}

Table 15. Hafnium metal number densities (beginning-of-life).

\begin{tabular}{|c|c|}
\hline Isotope/Element & $\begin{array}{c}\text { No. density } \\
(\mathrm{a} / \mathrm{b} / \mathrm{cm})\end{array}$ \\
\hline O-16 & $1.3500 \mathrm{E}-04$ \\
\hline C-12 & $4.4300 \mathrm{E}-05$ \\
\hline Si-nat & $6.8700 \mathrm{E}-06$ \\
\hline Zr-nat & $1.9900 \mathrm{E}-03$ \\
\hline Hf-174 & $6.7512 \mathrm{E}-05$ \\
\hline Hf-176 & $2.1934 \mathrm{E}-03$ \\
\hline Hf-177 & $7.8473 \mathrm{E}-03$ \\
\hline Hf-178 & $1.1431 \mathrm{E}-02$ \\
\hline Hf-179 & $5.7955 \mathrm{E}-03$ \\
\hline Hf-180 & $1.4845 \mathrm{E}-02$ \\
\hline
\end{tabular}

Table 16. Aluminum metal number densities $\left(2.7 \mathrm{~g} / \mathrm{cm}^{3}\right)$.

\begin{tabular}{|c|c|}
\hline Isotope/Element & $\begin{array}{c}\text { No. density } \\
(\mathrm{a} / \mathrm{b} / \mathrm{cm})\end{array}$ \\
\hline Al-27 & $5.90699 \mathrm{E}-02$ \\
\hline Cr-nat & $6.25887 \mathrm{E}-05$ \\
\hline Si-nat & $3.47620 \mathrm{E}-04$ \\
\hline Mg-nat & $6.69484 \mathrm{E}-04$ \\
\hline Cu-nat & $6.40160 \mathrm{E}-05$ \\
\hline
\end{tabular}


Table 17. Light water number densities (ATR coolant).

\begin{tabular}{|c|c|}
\hline Element & $\begin{array}{c}\text { No. density } \\
(\mathrm{a} / \mathrm{b} / \mathrm{cm})\end{array}$ \\
\hline $\mathrm{H}$ & $6.68506-2$ \\
\hline $\mathrm{O}-16$ & $3.34253-2$ \\
\hline
\end{tabular}

The ATR light water coolant is an average temperature of approximately $62^{\circ} \mathrm{C}(335 \mathrm{~K})$ and $2.5 \mathrm{MPa}$.

\section{OUTER SHIM CONTROL CYLINDER MODELING}

The beryllium OSCC drums each have a hafnium plate attached to their outer surface. The hafnium plate circumferentially covers only approximately 90 -degrees of the full 360-degrees of the cylinder surface. The hafnium plate can be rotated to absorb more or less neutrons and change core reactivity. The MCNP quarter-core model represents the OSCC rotation by moving a cylinder that is intersected with other cylinders to simulate rotating the hafnium metal plate on the drum. The formula for the $\mathrm{x}$ and $\mathrm{y}$ coordinates for the moving cylinders (E1, E2, E3, and E4 OSCCs) are given in Table 18. The "NE" and the "SE" variables correspond to the angles in Appendix B. These angles are put into the formulas to calculate and $\mathrm{x}$ and $\mathrm{y}$ coordinate. Note that cosine $(\cos )$ and sine $(\sin )$ arguments are in degrees. Also, in Appendix B, the "NW" and "SW" data is not used in the quarter-core model.

Table 18. Formulas for modeling OSCC hafnium plate positions or rotations.

\begin{tabular}{|c|c|c|c|}
\hline \multirow{2}{*}{ OSCC } & MCNP surface plane no. & $\begin{array}{c}\text { Coordinate } \\
(\mathrm{cm})\end{array}$ & Formula \\
\hline \multirow{2}{*}{ E1 } & \multirow{2}{*}{981} & $\mathrm{X}$ & $52.596-9.195^{*} \cos (\mathrm{NE}-24.72)$ \\
\cline { 3 - 4 } & \multirow{2}{*}{ E2 } & $\mathrm{Y}$ & $-10.157-9.195 * \sin (\mathrm{NE}-24.72)$ \\
\hline \multirow{2}{*}{ E3 } & \multirow{2}{*}{982} & $\mathrm{X}$ & $37.61-9.195 * \cos (\mathrm{NE}-38.06)$ \\
\cline { 3 - 4 } & \multirow{2}{*}{ E4 } & $\mathrm{Y}$ & $-23.228-9.195 * \sin (\mathrm{NE}-38.06)$ \\
\hline \multirow{2}{*}{ E4 } & \multirow{2}{*}{984} & $\mathrm{X}$ & $23.228+9.195^{*} \sin (\mathrm{SE}-38.06)$ \\
\hline & & $\mathrm{X}$ & $-37.61+9.195^{*} \cos (\mathrm{SE}-38.06)$ \\
\hline & & $\mathrm{Y}$ & $10.157+9.195 * \sin (\mathrm{SE}-24.72)$ \\
\hline
\end{tabular}

Examples of the OSCC surface card descriptions using the Table 18 formulas are as follows:

$\begin{array}{cccccl} & & \mathrm{X} & \mathrm{Y} & \mathrm{R} & \\ 981 & \mathrm{c} / \mathrm{z} & 46.648 & -17.169 & 9.195 & \text { \$E1: NE shim position=74.410 degrees } \\ 982 & \mathrm{c} / \mathrm{z} & 30.204 & -28.678 & 9.195 & \text { \$E2: NE shim position=74.410 degrees } \\ 983 & \mathrm{c} / \mathrm{z} & 29.892 & -31.275 & 9.195 & \text { \$E3: SE shim position=84.510 degrees } \\ 984 & \mathrm{c} / \mathrm{z} & 18.103 & -47.969 & 9.195 & \text { \$E4: SE shim position=84.510 degrees }\end{array}$

Note that the variable " $R$ " above is the cylinder radius in centimeters.

\section{CAPSULE TEMPERATURE DATA}

Fuel compact temperatures in each capsule over the 13 ATR power cycles are provided in this section (Reference [8]). Table 19 compact temperatures specifically include: (1) time-average minimum temperature, (2) time-averaged, volume-averaged (TAVA) temperature, and (3) time-average maximum temperature. Other capsule component temperatures can be extracted from Reference [8], as desired. 
Table 19. Fuel compact temperature data for the six AGR-1 capsules.

\begin{tabular}{|l|c|c|c|}
\hline Capsule & $\begin{array}{c}\text { Time-Average } \\
\text { Minimum } \\
\left({ }^{\circ} \mathrm{C}\right)\end{array}$ & $\begin{array}{c}\text { TAVA } \\
\left({ }^{\circ} \mathrm{C}\right)\end{array}$ & $\begin{array}{c}\text { Time-Average } \\
\text { Maximum } \\
\left({ }^{\circ} \mathrm{C}\right)\end{array}$ \\
\hline Capsule 6 & 885 & 1088 & 1197 \\
\hline Capsule 5 & 818 & 1023 & 1144 \\
\hline Capsule 4 & 866 & 1070 & 1187 \\
\hline Capsule 3 & 828 & 1028 & 1147 \\
\hline Capsule 2 & 800 & 1002 & 1124 \\
\hline Capsule 1 & 854 & 1054 & 1167 \\
\hline
\end{tabular}

The AGR-1 neutron spectrum is predominantly determined by the (1) ATR driver fuel, (2) volume of light water around the AGR-1 test train, (3) hafnium shroud, and (4) boron in the graphite holders. Temperature of the fuel compacts and capsule components have only a very minor impact on the local neutron spectrum and exact temperatures may not be required in the AGR-1 depletion calculation. A more complete MCNP model however can include these average temperatures for the fuel compacts for adjusting the compact and capsule cell temperatures (Free Gas Scattering Temperature card or TMP card in MCNP) and selecting appropriate $S(\alpha, \beta)$ thermal neutron scattering libraries for cells with graphite (bound carbon atoms).

\section{ATR CORE POWER, OSCC, AND NECK SHIM DATA}

As-run ATR measured test data for the 13 ATR power cycles (138B, 139A, 139B, 140A, 140B, 141A, 142a, 142B, 143A, 143B, 144A, 144B, and 145A) are contained in Appendices A, B, and C. These cycles were the 13 cycles AGR-1 under went irradiation in ATR. The three appendices contain the following as-run data:

Appendix A: ATR Lobe and Total Core Power History by Cycle and Timestep

Appendix B: ATR Outer Shim Control Cylinder (OSCC) Positions by Cycle and Timestep

\section{Appendix C: ATR Neck Shim Positions by Cycle and Timestep}

These as-run data are intended as input data for the MCNP quarter-core model, although not all the data is needed for the quarter-core model. For example, the northwest and southwest lobe powers are not needed as the quarter-core model does not include these ATR lobes.

Appendix A contains as-run ATR total and lobe power data for the 13 power cycles in which the AGR-1 test train was irradiated in the B-10 irradiation test facility. These data include the ATR power cycle number (138B through 145A), number of timesteps and duration of each timestep per cycle, the five ATR lobe powers by timestep, and the total ATR core power (far right column of the Appendix A table). For tally normalization, the B-10 irradiation test facility tally data is typically normalized to an "east lobe power" defined as one-third the sum of the northeast (NE), center (C), and southeast (SE) lobe powers. These same lobe powers can alternatively be weighted based on the number of nearest ATR driver elements by lobe, for example, $40 \%$ southeast, $20 \%$ center, and $40 \%$ northeast lobe power, instead of $33 \%$ southeast, center, northeast lobe power. Note: an ATR lobe contains 8 driver fuel elements.

There are a total of 662 timesteps over the 13 ATR power cycles. The majority of the timesteps are 24-hour increments. Those timesteps that are less than 24 hours are typically partial-day durations in which the reactor was either coming up in power (power-up) or going down in power (power-down). Power-ups are typically either beginning-of-cycle cold startup or mid-cycle startup after a scram. Power- 
downs in mid-cycle are reactor scrams and at end-of-cycle reactor shutdown. Mid-cycle timesteps greater than 24 hours are primarily scram periods with the reactor power at zero power.

Appendix B and $\mathrm{C}$ contain corresponding outer shim control cylinder and neck shim position data by timestep for the 13 cycles.

Between power cycles, ATR is shutdown. The duration of these shutdown periods (decay periods) are given Table 20.

Table 20. Shutdown periods between AGR-1 power cycles.

\begin{tabular}{|c|c|c|}
\hline \multicolumn{2}{|c|}{ Between Cycles } & $\begin{array}{c}\text { Shutdown Duration } \\
\text { (days) }\end{array}$ \\
\hline $138 \mathrm{~B}$ & $139 \mathrm{~A}$ & 15 \\
\hline $139 \mathrm{~A}$ & $139 \mathrm{~B}$ & 95 \\
\hline $139 \mathrm{~B}$ & $140 \mathrm{~A}$ & 15 \\
\hline $140 \mathrm{~A}$ & $140 \mathrm{~B}$ & 9 \\
\hline $140 \mathrm{~B}$ & $141 \mathrm{~A}$ & 56 \\
\hline $141 \mathrm{~A}$ & $142 \mathrm{~A}$ & 14 \\
\hline $142 \mathrm{~A}$ & $142 \mathrm{~B}$ & 24 \\
\hline $142 \mathrm{~B}$ & $143 \mathrm{~A}$ & 16 \\
\hline $143 \mathrm{~A}$ & $143 \mathrm{~B}$ & 20 \\
\hline $143 \mathrm{~B}$ & $144 \mathrm{~A}$ & 15 \\
\hline $144 \mathrm{~A}$ & $144 \mathrm{~B}$ & 62 \\
\hline $144 \mathrm{~B}$ & $145 \mathrm{~A}$ & EOC Nov. 6, 2009 \\
\hline $145 \mathrm{~A}$ & & \multicolumn{2}{c|}{} \\
\hline & & \\
\hline
\end{tabular}

\section{CONCLUSIONS}

This document provides the necessary benchmark data needed to model the AGR-1 experiment in an MCNP quarter-core model of the ATR. The MCNP quarter-core ATR model input file listing is provided in Appendix D in this report. The AGR-1 data herein can be used to generate MCNP surface, cell, and material cards for the AGR-1 test train, capsule, fuel compacts, and TRISO particles. These MCNP cards can then be integrated into the quarter-core model. The dimension and material data provided herein is for beginning-of-life or pre-irradiation data. The model together with the ATR as-run data can then be used to perform a depletion calculation of the AGR-1 TRISO particle compacts. Calculated estimates of compact burnup (\% FIMA), fast fluence $\left(\mathrm{n} / \mathrm{cm}^{2}\right)$, and isotopic concentration inventories as a function of burnup can be compared to several other reference calculations and measured data. For example, calculated data can be compared to: (1) quarter-core model depletion calculation (Reference [1]), (2) full-core model depletion calculation (Reference [2]), and/or (3) measured data (Reference [3]). Good result comparisons can provide a verification and validation basis for computer codes and models to be used for HTGR design and analysis.

\section{REFERENCES}

1. J.J. Cogliati and J.W. Sterbentz, "MCNP Results for the AGR-1 Depletion Benchmark," INL/LTD-1845646, Idaho National Laboratory, July 2018.

2. J.W. Sterbentz, "JMOCUP As-Run Daily Depletion Calculation for the AGR-1 Experiment in ATR B-10 Test Position," Engineering Calculations and Analysis Report, ECAR-958, Rev.3, Idaho National Laboratory, September 3, 2013. 
3. J.W. Sterbentz, et al., "Validation of the Physics Analysis used to Characterize the AGR-1 TRISO Fuel Irradiation Test," INL/CON-14-33982 and proceedings of ICAPP 2015, Nice, France, paper 15497, May 3-6, 2015.

4. “Advanced Test Reactor National Scientific User Facility: Users' Guide," Idaho National Laboratory, INL/EXT-07-13577 (2008).

5. “AGR-1 Irradiation Experiment Test Plan," Idaho National Laboratory, Idaho Falls, ID, INL/EXT05-00593, Rev. 3, October 20, 2009.

6. BWXT Nuclear Products Division G73 Lot G73D-20-69302, "Chemistry Analysis" sheets.

7. “AGR-1 Irradiation Test Final As-Run Report," Blaise P. Collin, INL-EXT-10-18097 rev 3, January 2015.

8. G.L Hawkes, “AGR-1 Daily As-run Thermal Analyses,” INL/MIS-11-23669, Rev. 4, September 3, 2014. 


\section{Appendix A}

\section{ATR Lobe and Total Core Power History by Cycle and Timestep}

\begin{tabular}{|c|c|c|c|c|c|c|c|c|c|}
\hline Cycle & Timestep & $\begin{array}{l}\text { Cumulative } \\
\text { Timestep }\end{array}$ & $\begin{array}{c}\text { Time } \\
\text { Interval } \\
\text { (hrs) }\end{array}$ & $\begin{array}{l}\text { NW } \\
\text { Lobe } \\
\text { Power } \\
(\mathrm{MW}) \\
\end{array}$ & $\begin{array}{c}\mathrm{NE} \\
\text { Lobe } \\
\text { Power } \\
\text { (MW) }\end{array}$ & $\begin{array}{c}\text { C } \\
\text { Lobe } \\
\text { Power } \\
(\mathrm{MW})\end{array}$ & $\begin{array}{c}\text { SW } \\
\text { Lobe } \\
\text { Power } \\
(\mathrm{MW})\end{array}$ & $\begin{array}{c}\text { SE } \\
\text { Lobe } \\
\text { Power } \\
(\mathrm{MW})\end{array}$ & $\begin{array}{l}\text { Total } \\
\text { Core } \\
\text { Power } \\
(\mathrm{MW}) \\
\end{array}$ \\
\hline $138 \mathrm{~B}$ & 1 & 1 & 9 & 1.4611 & 1.4761 & 1.8833 & 2.0861 & 2.1028 & 9.0094 \\
\hline 138B & 2 & 2 & 24 & 4.3281 & 4.3510 & 5.0875 & 6.1204 & 6.2483 & 26.1354 \\
\hline 138B & 3 & 3 & 24 & 17.2033 & 17.1969 & 19.4444 & 21.9544 & 23.8315 & 99.6304 \\
\hline $138 \mathrm{~B}$ & 4 & 4 & 24 & 17.9902 & 18.0056 & 22.1019 & 23.0196 & 25.0567 & 106.1740 \\
\hline $138 \mathrm{~B}$ & 5 & 5 & 24 & 18.0575 & 18.0433 & 22.1181 & 22.9727 & 25.0910 & 106.2827 \\
\hline $138 \mathrm{~B}$ & 6 & 6 & 24 & 18.0715 & 17.9946 & 21.9185 & 22.9433 & 25.1115 & 106.0394 \\
\hline $138 \mathrm{~B}$ & 7 & 7 & 24 & 18.0627 & 18.0896 & 22.5400 & 23.0081 & 25.0560 & 106.7565 \\
\hline $138 \mathrm{~B}$ & 8 & 8 & 24 & 18.1790 & 18.1825 & 22.5865 & 23.0735 & 25.2979 & 107.3194 \\
\hline $138 \mathrm{~B}$ & 9 & 9 & 24 & 18.0865 & 18.2785 & 23.0002 & 23.0731 & 25.1902 & 107.6285 \\
\hline $138 \mathrm{~B}$ & 10 & 10 & 24 & 18.1913 & 18.3413 & 23.1858 & 23.1723 & 25.1800 & 108.0706 \\
\hline $138 \mathrm{~B}$ & 11 & 11 & 24 & 18.1504 & 18.1279 & 23.3069 & 23.1213 & 25.1781 & 107.8846 \\
\hline $138 \mathrm{~B}$ & 12 & 12 & 24 & 18.2460 & 18.2440 & 23.5031 & 23.2946 & 25.2638 & 108.5515 \\
\hline $138 \mathrm{~B}$ & 13 & 13 & 24 & 18.1640 & 18.1731 & 23.0742 & 23.1794 & 25.1408 & 107.7315 \\
\hline 138B & 14 & 14 & 24 & 18.3108 & 18.3550 & 23.5188 & 23.3733 & 25.3648 & 108.9227 \\
\hline $138 \mathrm{~B}$ & 15 & 15 & 24 & 18.2871 & 18.3279 & 23.4992 & 23.3060 & 25.2477 & 108.6679 \\
\hline $138 \mathrm{~B}$ & 16 & 16 & 24 & 18.2215 & 18.2733 & 23.4185 & 23.2515 & 25.3398 & 108.5046 \\
\hline $138 \mathrm{~B}$ & 17 & 17 & 24 & 18.1758 & 18.1671 & 22.8590 & 23.2079 & 25.1621 & 107.5719 \\
\hline $138 \mathrm{~B}$ & 18 & 18 & 24 & 18.1221 & 18.1879 & 22.7592 & 23.0750 & 25.0869 & 107.2310 \\
\hline $138 \mathrm{~B}$ & 19 & 19 & 24 & 18.0960 & 18.0908 & 23.0235 & 23.0679 & 25.0729 & 107.3513 \\
\hline $138 \mathrm{~B}$ & 20 & 20 & 24 & 18.0940 & 18.0446 & 22.9885 & 23.0550 & 25.0829 & 107.2650 \\
\hline $138 \mathrm{~B}$ & 21 & 21 & 24 & 18.0960 & 18.1373 & 23.2446 & 22.9790 & 25.0381 & 107.4950 \\
\hline $138 \mathrm{~B}$ & 22 & 22 & 24 & 18.1094 & 18.0979 & 22.9383 & 23.0263 & 25.0267 & 107.1985 \\
\hline $138 \mathrm{~B}$ & 23 & 23 & 24 & 18.1775 & 18.2092 & 23.0529 & 23.1935 & 25.1019 & 107.7350 \\
\hline $138 \mathrm{~B}$ & 24 & 24 & 24 & 18.1335 & 18.1646 & 23.2175 & 23.1002 & 25.1090 & 107.7248 \\
\hline $138 \mathrm{~B}$ & 25 & 25 & 24 & 17.9775 & 18.0969 & 23.1796 & 22.9185 & 24.9642 & 107.1367 \\
\hline $138 \mathrm{~B}$ & 26 & 26 & 24 & 18.0075 & 17.9792 & 23.2758 & 23.0152 & 24.9119 & 107.1896 \\
\hline $138 \mathrm{~B}$ & 27 & 27 & 24 & 18.1098 & 18.1125 & 23.3548 & 23.1446 & 25.0744 & 107.7960 \\
\hline $138 \mathrm{~B}$ & 28 & 28 & 24 & 18.1902 & 18.1765 & 23.3658 & 23.1888 & 25.1515 & 108.0727 \\
\hline $138 \mathrm{~B}$ & 29 & 29 & 24 & 18.1604 & 18.1496 & 23.5248 & 23.1381 & 25.1002 & 108.0731 \\
\hline $138 \mathrm{~B}$ & 30 & 30 & 24 & 18.1283 & 18.1231 & 24.0544 & 23.0796 & 25.0948 & 108.4802 \\
\hline $138 \mathrm{~B}$ & 31 & 31 & 24 & 18.1319 & 18.1946 & 23.8523 & 23.1090 & 25.1190 & 108.4067 \\
\hline
\end{tabular}




\begin{tabular}{|c|c|c|c|c|c|c|c|c|c|}
\hline Cycle & Timestep & $\begin{array}{l}\text { Cumulative } \\
\text { Timestep } \\
\end{array}$ & \begin{tabular}{|c|} 
Time \\
Interval \\
$(\mathrm{hrs})$
\end{tabular} & $\begin{array}{l}\text { NW } \\
\text { Lobe } \\
\text { Power } \\
(\mathrm{MW})\end{array}$ & $\begin{array}{c}\text { NE } \\
\text { Lobe } \\
\text { Power } \\
(\mathrm{MW})\end{array}$ & $\begin{array}{c}\text { C } \\
\text { Lobe } \\
\text { Power } \\
(\mathrm{MW}) \\
\end{array}$ & $\begin{array}{c}\text { SW } \\
\text { Lobe } \\
\text { Power } \\
\text { (MW) }\end{array}$ & $\begin{array}{c}\text { SE } \\
\text { Lobe } \\
\text { Power } \\
(\mathrm{MW}) \\
\end{array}$ & $\begin{array}{l}\text { Total } \\
\text { Core } \\
\text { Power } \\
(\mathrm{MW}) \\
\end{array}$ \\
\hline $138 \mathrm{~B}$ & 32 & 32 & 24 & 18.1508 & 18.2592 & 23.7390 & 23.2388 & 25.2023 & 108.5900 \\
\hline $138 \mathrm{~B}$ & 33 & 33 & 24 & 18.0558 & 18.1783 & 23.6569 & 23.2340 & 25.0938 & 108.2188 \\
\hline $138 \mathrm{~B}$ & 34 & 34 & 24 & 18.1556 & 18.2013 & 23.8108 & 23.2138 & 25.1765 & 108.5579 \\
\hline $138 \mathrm{~B}$ & 35 & 35 & 24 & 18.1085 & 18.1679 & 23.6260 & 23.1242 & 25.1125 & 108.1392 \\
\hline $138 \mathrm{~B}$ & 36 & 36 & 24 & 18.1465 & 18.1646 & 23.6319 & 23.1365 & 25.0694 & 108.1488 \\
\hline $138 \mathrm{~B}$ & 37 & 37 & 24 & 18.1523 & 18.2085 & 24.2633 & 23.2225 & 25.1881 & 109.0348 \\
\hline $138 \mathrm{~B}$ & 38 & 38 & 24 & 18.1271 & 18.1377 & 24.4344 & 23.1023 & 25.1073 & 108.9088 \\
\hline $138 \mathrm{~B}$ & 39 & 39 & 24 & 18.0650 & 18.1308 & 24.1665 & 23.0415 & 25.1117 & 108.5154 \\
\hline $138 \mathrm{~B}$ & 40 & 40 & 24 & 18.1563 & 18.3010 & 24.4973 & 23.1204 & 25.2096 & 109.2846 \\
\hline $138 \mathrm{~B}$ & 41 & 41 & 24 & 18.2523 & 18.3358 & 24.4163 & 23.3406 & 25.2525 & 109.5975 \\
\hline $138 \mathrm{~B}$ & 42 & 42 & 24 & 18.2635 & 18.2756 & 24.6056 & 23.2815 & 25.2419 & 109.6681 \\
\hline $138 \mathrm{~B}$ & 43 & 43 & 24 & 18.2229 & 18.2565 & 24.5585 & 23.2444 & 25.3108 & 109.5931 \\
\hline $138 \mathrm{~B}$ & 44 & 44 & 24 & 18.4092 & 18.4888 & 24.8844 & 23.4742 & 25.4335 & 110.6900 \\
\hline $138 \mathrm{~B}$ & 45 & 45 & 24 & 18.3998 & 18.4271 & 24.4771 & 23.4331 & 25.4525 & 110.1896 \\
\hline $138 \mathrm{~B}$ & 46 & 46 & 24 & 18.3233 & 18.3400 & 23.8204 & 23.3060 & 25.3152 & 109.1050 \\
\hline $138 \mathrm{~B}$ & 47 & 47 & 24 & 18.2585 & 18.3538 & 23.7048 & 23.3092 & 25.3071 & 108.9333 \\
\hline $138 \mathrm{~B}$ & 48 & 48 & 24 & 18.2879 & 18.2781 & 23.6496 & 23.3098 & 25.3092 & 108.8346 \\
\hline $138 \mathrm{~B}$ & 49 & 49 & 9 & 18.3189 & 18.3694 & 23.9450 & 23.3928 & 25.3933 & 109.4194 \\
\hline $139 \mathrm{~A}$ & 1 & 50 & 8 & 5.2931 & 5.2006 & 6.9469 & 6.8475 & 7.4444 & 31.7325 \\
\hline $139 \mathrm{~A}$ & 2 & 51 & 24 & 17.9554 & 17.9506 & 22.5175 & 22.8456 & 24.7823 & 106.0515 \\
\hline 139A & 3 & 52 & 24 & 18.3754 & 18.3533 & 23.2496 & 23.3375 & 25.3523 & 108.6681 \\
\hline $139 \mathrm{~A}$ & 4 & 53 & 24 & 18.3458 & 18.3360 & 23.9098 & 23.3273 & 25.3154 & 109.2344 \\
\hline $139 \mathrm{~A}$ & 5 & 54 & 24 & 18.2538 & 18.2658 & 24.1850 & 23.2923 & 25.2388 & 109.2356 \\
\hline 139A & 6 & 55 & 24 & 18.4325 & 18.4281 & 24.0956 & 23.4548 & 25.4833 & 109.8944 \\
\hline 139A & 7 & 56 & 24 & 18.2798 & 18.3544 & 23.9396 & 23.4002 & 25.3860 & 109.3600 \\
\hline $139 \mathrm{~A}$ & 8 & 57 & 24 & 18.3854 & 18.4017 & 24.4523 & 23.4898 & 25.3644 & 110.0935 \\
\hline $139 \mathrm{~A}$ & 9 & 58 & 24 & 18.4304 & 18.4415 & 24.2800 & 23.4581 & 25.4402 & 110.0502 \\
\hline $139 \mathrm{~A}$ & 10 & 59 & 24 & 18.3721 & 18.4100 & 24.0260 & 23.3967 & 25.3563 & 109.5610 \\
\hline $139 \mathrm{~A}$ & 11 & 60 & 24 & 18.4225 & 18.4183 & 23.9127 & 23.4188 & 25.4223 & 109.5946 \\
\hline $139 \mathrm{~A}$ & 12 & 61 & 24 & 18.4060 & 18.4146 & 23.9465 & 23.4575 & 25.4521 & 109.6767 \\
\hline $139 \mathrm{~A}$ & 13 & 62 & 24 & 18.3525 & 18.4129 & 23.9973 & 23.4819 & 25.4563 & 109.7008 \\
\hline $139 \mathrm{~A}$ & 14 & 63 & 24 & 18.2963 & 18.3817 & 23.7613 & 23.3221 & 25.3869 & 109.1481 \\
\hline 139A & 15 & 64 & 24 & 18.3098 & 18.3492 & 24.3242 & 23.4163 & 25.3742 & 109.7735 \\
\hline $139 \mathrm{~A}$ & 16 & 65 & 24 & 18.3119 & 18.3756 & 24.2692 & 23.3377 & 25.4508 & 109.7452 \\
\hline $139 \mathrm{~A}$ & 17 & 66 & 24 & 18.3838 & 18.3167 & 24.1610 & 23.3829 & 25.4217 & 109.6660 \\
\hline 139A & 18 & 67 & 24 & 18.3915 & 18.3792 & 24.0321 & 23.4060 & 25.4238 & 109.6325 \\
\hline
\end{tabular}




\begin{tabular}{|c|c|c|c|c|c|c|c|c|c|}
\hline Cycle & Timestep & $\begin{array}{l}\text { Cumulative } \\
\text { Timestep }\end{array}$ & \begin{tabular}{|c|} 
Time \\
Interval \\
$($ hrs $)$
\end{tabular} & $\begin{array}{l}\text { NW } \\
\text { Lobe } \\
\text { Power } \\
\text { (MW) }\end{array}$ & $\begin{array}{c}\text { NE } \\
\text { Lobe } \\
\text { Power } \\
(\mathrm{MW}) \\
\end{array}$ & $\begin{array}{c}\text { C } \\
\text { Lobe } \\
\text { Power } \\
(\mathrm{MW}) \\
\end{array}$ & $\begin{array}{c}\text { SW } \\
\text { Lobe } \\
\text { Power } \\
(\mathrm{MW})\end{array}$ & $\begin{array}{c}\text { SE } \\
\text { Lobe } \\
\text { Power } \\
\text { (MW) }\end{array}$ & $\begin{array}{l}\text { Total } \\
\text { Core } \\
\text { Power } \\
(\mathrm{MW}) \\
\end{array}$ \\
\hline 139A & 19 & 68 & 24 & 18.3573 & 18.4571 & 24.2404 & 23.4448 & 25.4358 & 109.9354 \\
\hline $139 \mathrm{~A}$ & 20 & 69 & 24 & 18.4040 & 18.3946 & 24.6627 & 23.4963 & 25.4242 & 110.3817 \\
\hline $139 \mathrm{~A}$ & 21 & 70 & 24 & 18.4525 & 18.4865 & 24.6160 & 23.5494 & 25.4290 & 110.5333 \\
\hline $139 \mathrm{~A}$ & 22 & 71 & 24 & 18.4363 & 18.5342 & 24.2560 & 23.4567 & 25.4927 & 110.1758 \\
\hline $139 \mathrm{~A}$ & 23 & 72 & 24 & 6.1640 & 6.1769 & 8.0715 & 7.8121 & 8.5281 & 36.7525 \\
\hline $139 \mathrm{~A}$ & 24 & 73 & 24 & 0.0001 & 0.0001 & 0.0001 & 0.0001 & 0.0001 & 0.0005 \\
\hline $139 \mathrm{~A}$ & 25 & 74 & 24 & 0.0001 & 0.0001 & 0.0001 & 0.0001 & 0.0001 & 0.0005 \\
\hline $139 \mathrm{~A}$ & 26 & 75 & 24 & 15.8846 & 15.8833 & 21.8650 & 20.2838 & 21.9600 & 95.8767 \\
\hline $139 \mathrm{~A}$ & 27 & 76 & 24 & 18.3881 & 18.4083 & 24.2977 & 23.4069 & 25.3813 & 109.8823 \\
\hline $139 \mathrm{~A}$ & 28 & 77 & 24 & 18.2469 & 18.2652 & 24.1023 & 23.2513 & 25.2792 & 109.1448 \\
\hline 139A & 29 & 78 & 24 & 18.3077 & 18.3821 & 24.4958 & 23.4150 & 25.2890 & 109.8896 \\
\hline $139 \mathrm{~A}$ & 30 & 79 & 24 & 18.2383 & 18.3140 & 24.1138 & 23.2808 & 25.2821 & 109.2290 \\
\hline $139 \mathrm{~A}$ & 31 & 80 & 24 & 18.2192 & 18.2644 & 23.9813 & 23.2517 & 25.2254 & 108.9419 \\
\hline $139 \mathrm{~A}$ & 32 & 81 & 24 & 18.3119 & 18.3594 & 24.1031 & 23.2656 & 25.3302 & 109.3702 \\
\hline $139 \mathrm{~A}$ & 33 & 82 & 24 & 18.4319 & 18.4908 & 24.2250 & 23.4069 & 25.4558 & 110.0104 \\
\hline $139 \mathrm{~A}$ & 34 & 83 & 24 & 18.5442 & 18.6229 & 24.3681 & 23.5771 & 25.6425 & 110.7548 \\
\hline $139 \mathrm{~A}$ & 35 & 84 & 24 & 18.5060 & 18.5596 & 23.9729 & 23.6021 & 25.5502 & 110.1908 \\
\hline 139A & 36 & 85 & 24 & 18.5156 & 18.5700 & 23.8919 & 23.6188 & 25.4623 & 110.0585 \\
\hline 139A & 37 & 86 & 24 & 18.4356 & 18.4988 & 23.9731 & 23.5583 & 25.4594 & 109.9252 \\
\hline 139A & 38 & 87 & 24 & 18.4575 & 18.5390 & 23.7142 & 23.4931 & 25.5000 & 109.7038 \\
\hline $139 \mathrm{~A}$ & 39 & 88 & 24 & 18.3906 & 18.5252 & 23.9177 & 23.4029 & 25.3702 & 109.6067 \\
\hline $139 \mathrm{~A}$ & 40 & 89 & 24 & 18.4783 & 18.5304 & 24.0100 & 23.6606 & 25.5633 & 110.2427 \\
\hline $139 \mathrm{~A}$ & 41 & 90 & 24 & 18.3835 & 18.3802 & 23.7308 & 23.5360 & 25.3527 & 109.3833 \\
\hline $139 \mathrm{~A}$ & 42 & 91 & 24 & 18.3392 & 18.3742 & 23.8146 & 23.3779 & 25.2656 & 109.1715 \\
\hline $139 \mathrm{~A}$ & 43 & 92 & 24 & 18.4340 & 18.4377 & 23.6573 & 23.4281 & 25.3613 & 109.3183 \\
\hline 139A & 44 & 93 & 24 & 18.4104 & 18.4033 & 23.5433 & 23.4192 & 25.3815 & 109.1577 \\
\hline 139A & 45 & 94 & 24 & 18.3885 & 18.4310 & 23.9563 & 23.4213 & 25.3644 & 109.5615 \\
\hline $139 \mathrm{~A}$ & 46 & 95 & 24 & 18.4013 & 18.4285 & 23.9265 & 23.4502 & 25.3371 & 109.5435 \\
\hline $139 \mathrm{~A}$ & 47 & 96 & 24 & 18.4767 & 18.5117 & 24.4106 & 23.5385 & 25.5038 & 110.4413 \\
\hline $139 \mathrm{~A}$ & 48 & 97 & 24 & 18.4779 & 18.4783 & 24.3356 & 23.5817 & 25.4742 & 110.3477 \\
\hline $139 \mathrm{~A}$ & 49 & 98 & 24 & 18.4581 & 18.4890 & 24.5452 & 23.5529 & 25.4765 & 110.5217 \\
\hline $139 \mathrm{~A}$ & 50 & 99 & 24 & 18.4698 & 18.6375 & 24.9296 & 23.5473 & 25.4479 & 111.0321 \\
\hline $139 \mathrm{~A}$ & 51 & 100 & 24 & 18.5219 & 18.6910 & 25.1260 & 23.5867 & 25.5373 & 111.4629 \\
\hline $139 \mathrm{~A}$ & 52 & 101 & 24 & 18.5108 & 18.6015 & 24.9300 & 23.6013 & 25.5723 & 111.2158 \\
\hline $139 \mathrm{~A}$ & 53 & 102 & 24 & 18.4683 & 18.4921 & 24.4213 & 23.5273 & 25.4829 & 110.3919 \\
\hline $139 \mathrm{~A}$ & 54 & 103 & 24 & 18.4819 & 18.5708 & 24.3196 & 23.6221 & 25.5025 & 110.4969 \\
\hline
\end{tabular}




\begin{tabular}{|c|c|c|c|c|c|c|c|c|c|}
\hline Cycle & Timestep & $\begin{array}{l}\text { Cumulative } \\
\text { Timestep } \\
\end{array}$ & \begin{tabular}{|c|} 
Time \\
Interval \\
$(\mathrm{hrs})$
\end{tabular} & $\begin{array}{l}\text { NW } \\
\text { Lobe } \\
\text { Power } \\
(\mathrm{MW})\end{array}$ & $\begin{array}{c}\text { NE } \\
\text { Lobe } \\
\text { Power } \\
(\mathrm{MW})\end{array}$ & $\begin{array}{c}\text { C } \\
\text { Lobe } \\
\text { Power } \\
\text { (MW) }\end{array}$ & $\begin{array}{c}\text { SW } \\
\text { Lobe } \\
\text { Power } \\
\text { (MW) }\end{array}$ & $\begin{array}{c}\text { SE } \\
\text { Lobe } \\
\text { Power } \\
(\mathrm{MW}) \\
\end{array}$ & $\begin{array}{l}\text { Total } \\
\text { Core } \\
\text { Power } \\
(\mathrm{MW}) \\
\end{array}$ \\
\hline $139 \mathrm{~A}$ & 55 & 104 & 24 & 18.4340 & 18.5881 & 24.1950 & 23.4573 & 25.4315 & 110.1058 \\
\hline 139A & 56 & 105 & 9 & 18.3589 & 18.4689 & 24.0117 & 23.3867 & 25.4078 & 109.6339 \\
\hline 139B & 1 & 106 & 8 & 5.5281 & 5.4563 & 7.2319 & 7.3244 & 7.4425 & 32.9831 \\
\hline 139B & 2 & 107 & 24 & 17.5258 & 17.5000 & 21.0285 & 22.4321 & 22.3821 & 100.8685 \\
\hline 139B & 3 & 108 & 24 & 17.7960 & 17.7746 & 22.0979 & 22.7873 & 22.6885 & 103.1444 \\
\hline 139B & 4 & 109 & 24 & 18.1858 & 18.2050 & 22.4596 & 23.1563 & 23.1477 & 105.1544 \\
\hline 139B & 5 & 110 & 24 & 18.2608 & 18.2758 & 22.4967 & 23.2540 & 23.2823 & 105.5696 \\
\hline $139 \mathrm{~B}$ & 6 & 111 & 24 & 18.3450 & 18.4546 & 23.3006 & 23.3690 & 23.4085 & 106.8777 \\
\hline 139B & 7 & 112 & 24 & 18.2108 & 18.3221 & 23.1546 & 23.2846 & 23.2704 & 106.2425 \\
\hline 139B & 8 & 113 & 24 & 18.3071 & 18.3469 & 23.1717 & 23.3508 & 23.2738 & 106.4502 \\
\hline 139B & 9 & 114 & 24 & 18.3504 & 18.3600 & 23.2508 & 23.4677 & 23.3865 & 106.8154 \\
\hline 139B & 10 & 115 & 24 & 18.3358 & 18.3467 & 23.3092 & 23.3581 & 23.3329 & 106.6827 \\
\hline 139B & 11 & 116 & 24 & 18.2742 & 18.3406 & 23.4108 & 23.2373 & 23.3738 & 106.6367 \\
\hline 139B & 12 & 117 & 24 & 18.1971 & 18.1896 & 23.1113 & 23.2506 & 23.2063 & 105.9548 \\
\hline 139B & 13 & 118 & 24 & 18.0765 & 18.1502 & 22.7838 & 23.1631 & 23.1363 & 105.3098 \\
\hline 139B & 14 & 119 & 24 & 18.1200 & 18.1465 & 22.9606 & 23.1792 & 23.1692 & 105.5754 \\
\hline 139B & 15 & 120 & 24 & 18.2140 & 18.1735 & 23.0235 & 23.2510 & 23.2104 & 105.8725 \\
\hline 139B & 16 & 121 & 24 & 18.1621 & 18.1981 & 23.0608 & 23.2306 & 23.1652 & 105.8169 \\
\hline 139B & 17 & 122 & 24 & 18.1104 & 18.1746 & 22.9077 & 23.1706 & 23.1110 & 105.4744 \\
\hline 139B & 18 & 123 & 24 & 18.1633 & 18.1085 & 23.4867 & 23.1608 & 23.1917 & 106.1110 \\
\hline 139B & 19 & 124 & 24 & 18.1977 & 18.2000 & 23.3098 & 23.1373 & 23.2348 & 106.0796 \\
\hline 139B & 20 & 125 & 24 & 18.1819 & 18.1506 & 23.6115 & 23.2171 & 23.1675 & 106.3285 \\
\hline 139B & 21 & 126 & 24 & 18.1365 & 18.2265 & 23.6071 & 23.3038 & 23.1569 & 106.4306 \\
\hline 139B & 22 & 127 & 24 & 18.1238 & 18.0948 & 23.3519 & 23.1879 & 23.1102 & 105.8685 \\
\hline 139B & 23 & 128 & 24 & 18.3102 & 18.3302 & 23.7046 & 23.4415 & 23.5046 & 107.2910 \\
\hline 139B & 24 & 129 & 24 & 18.4152 & 18.4367 & 23.4740 & 23.4210 & 23.4254 & 107.1723 \\
\hline 139B & 25 & 130 & 24 & 18.3398 & 18.3990 & 23.8392 & 23.2602 & 23.2773 & 107.1154 \\
\hline 139B & 26 & 131 & 24 & 18.1833 & 18.2146 & 23.7763 & 23.1927 & 23.1858 & 106.5527 \\
\hline 139B & 27 & 132 & 24 & 18.2683 & 18.2433 & 23.6590 & 23.2708 & 23.2804 & 106.7219 \\
\hline 139B & 28 & 133 & 24 & 18.2438 & 18.2377 & 23.7117 & 23.3410 & 23.3073 & 106.8415 \\
\hline 139B & 29 & 134 & 24 & 18.2300 & 18.2379 & 23.7121 & 23.3144 & 23.2821 & 106.7765 \\
\hline 139B & 30 & 135 & 24 & 18.2021 & 18.2404 & 23.5800 & 23.2804 & 23.2396 & 106.5425 \\
\hline 139B & 31 & 136 & 24 & 18.2335 & 18.2377 & 23.6610 & 23.2294 & 23.1977 & 106.5594 \\
\hline 139B & 32 & 137 & 24 & 18.1802 & 18.2696 & 24.1015 & 23.2317 & 23.2027 & 106.9856 \\
\hline 139B & 33 & 138 & 7 & 18.1857 & 18.2536 & 23.9307 & 23.2929 & 23.2471 & 106.9100 \\
\hline 139B & 34 & 139 & 1044 & 0.0100 & 0.0100 & 0.0100 & 0.0100 & 0.0100 & 0.0500 \\
\hline
\end{tabular}




\begin{tabular}{|c|c|c|c|c|c|c|c|c|c|}
\hline Cycle & Timestep & $\begin{array}{l}\text { Cumulative } \\
\text { Timestep }\end{array}$ & $\begin{array}{c}\text { Time } \\
\text { Interval } \\
\text { (hrs) }\end{array}$ & $\begin{array}{l}\text { NW } \\
\text { Lobe } \\
\text { Power } \\
(\mathrm{MW}) \\
\end{array}$ & $\begin{array}{c}\text { NE } \\
\text { Lobe } \\
\text { Power } \\
\text { (MW) }\end{array}$ & $\begin{array}{c}\text { C } \\
\text { Lobe } \\
\text { Power } \\
(\mathrm{MW}) \\
\end{array}$ & $\begin{array}{c}\text { SW } \\
\text { Lobe } \\
\text { Power } \\
\text { (MW) }\end{array}$ & $\begin{array}{c}\text { SE } \\
\text { Lobe } \\
\text { Power } \\
(\mathrm{MW})\end{array}$ & $\begin{array}{l}\text { Total } \\
\text { Core } \\
\text { Power } \\
(\mathrm{MW}) \\
\end{array}$ \\
\hline 139B & 35 & 140 & 13 & 0.3108 & 0.2923 & 0.4600 & 0.3869 & 0.3808 & 1.8308 \\
\hline 139B & 36 & 141 & 24 & 15.6479 & 15.5990 & 20.9117 & 19.9977 & 20.0317 & 92.1879 \\
\hline 139B & 37 & 142 & 24 & 17.9638 & 18.0271 & 23.9738 & 22.9429 & 22.9240 & 105.8315 \\
\hline 139B & 38 & 143 & 24 & 18.0563 & 18.0521 & 24.0385 & 22.9883 & 23.0238 & 106.1590 \\
\hline 139B & 39 & 144 & 24 & 17.9113 & 17.8775 & 23.8208 & 22.8663 & 22.8610 & 105.3369 \\
\hline 139B & 40 & 145 & 24 & 17.8552 & 17.8160 & 23.7177 & 22.8669 & 22.8331 & 105.0890 \\
\hline 139B & 41 & 146 & 24 & 17.8633 & 17.8215 & 23.8483 & 22.8683 & 22.8546 & 105.2560 \\
\hline 139B & 42 & 147 & 24 & 17.9379 & 17.9310 & 24.1148 & 22.9333 & 22.9565 & 105.8735 \\
\hline 139B & 43 & 148 & 24 & 17.9781 & 18.0721 & 24.0706 & 23.0210 & 23.0098 & 106.1517 \\
\hline 139B & 44 & 149 & 24 & 18.0217 & 17.9081 & 23.8810 & 23.0133 & 23.0335 & 105.8577 \\
\hline 139B & 45 & 150 & 24 & 18.0006 & 17.9267 & 24.0652 & 22.9225 & 22.9783 & 105.8933 \\
\hline 139B & 46 & 151 & 24 & 18.0044 & 17.9758 & 24.1283 & 22.9690 & 22.9508 & 106.0283 \\
\hline 139B & 47 & 152 & 24 & 18.0765 & 18.0802 & 24.0125 & 23.0292 & 22.9983 & 106.1967 \\
\hline 139B & 48 & 153 & 24 & 18.0752 & 18.0540 & 23.8631 & 23.0013 & 23.0633 & 106.0569 \\
\hline 139B & 49 & 154 & 24 & 17.9977 & 17.9463 & 23.3710 & 22.9681 & 22.9346 & 105.2177 \\
\hline 139B & 50 & 155 & 24 & 17.9394 & 17.9048 & 22.5429 & 22.8635 & 22.9117 & 104.1623 \\
\hline 139B & 51 & 156 & 24 & 17.9483 & 17.9506 & 22.5035 & 23.0033 & 22.8965 & 104.3023 \\
\hline 139B & 52 & 157 & 24 & 18.0435 & 18.0165 & 22.4910 & 23.0167 & 23.0606 & 104.6283 \\
\hline 139B & 53 & 158 & 24 & 17.9506 & 17.9838 & 22.0596 & 22.9188 & 22.9158 & 103.8285 \\
\hline 139B & 54 & 159 & 24 & 17.9577 & 18.0563 & 22.1690 & 22.9271 & 22.9960 & 104.1060 \\
\hline 139B & 55 & 160 & 21 & 17.9257 & 17.9569 & 21.8081 & 23.0460 & 22.9098 & 103.6464 \\
\hline $140 \mathrm{~A}$ & 1 & 161 & 8 & 4.7250 & 4.5281 & 5.6556 & 6.2550 & 6.2831 & 27.4469 \\
\hline $140 \mathrm{~A}$ & 2 & 162 & 24 & 17.5063 & 17.4758 & 19.9173 & 22.3383 & 22.2494 & 99.4871 \\
\hline $140 \mathrm{~A}$ & 3 & 163 & 24 & 18.9165 & 19.0085 & 22.0500 & 23.9619 & 23.8863 & 107.8231 \\
\hline $140 \mathrm{~A}$ & 4 & 164 & 24 & 18.7071 & 18.8427 & 22.0029 & 23.7169 & 23.6563 & 106.9258 \\
\hline $140 \mathrm{~A}$ & 5 & 165 & 24 & 18.5554 & 18.6590 & 21.9254 & 23.6354 & 23.5425 & 106.3177 \\
\hline $140 \mathrm{~A}$ & 6 & 166 & 24 & 18.4506 & 18.4988 & 22.6792 & 23.5063 & 23.4215 & 106.5563 \\
\hline $140 \mathrm{~A}$ & 7 & 167 & 24 & 18.3963 & 18.4965 & 22.2681 & 23.3667 & 23.3654 & 105.8929 \\
\hline $140 \mathrm{~A}$ & 8 & 168 & 24 & 18.5054 & 18.6438 & 21.7967 & 23.5071 & 23.4685 & 105.9215 \\
\hline $140 \mathrm{~A}$ & 9 & 169 & 24 & 18.5169 & 18.6110 & 21.7569 & 23.5129 & 23.5156 & 105.9133 \\
\hline $140 \mathrm{~A}$ & 10 & 170 & 24 & 18.1729 & 18.2044 & 21.4483 & 23.2458 & 23.2263 & 104.2977 \\
\hline $140 \mathrm{~A}$ & 11 & 171 & 24 & 18.0742 & 18.0735 & 21.7981 & 23.0954 & 23.1140 & 104.1552 \\
\hline $140 \mathrm{~A}$ & 12 & 172 & 24 & 18.0902 & 18.0665 & 22.1550 & 23.0750 & 23.0833 & 104.4700 \\
\hline $140 \mathrm{~A}$ & 13 & 173 & 24 & 18.0385 & 18.0685 & 21.9313 & 23.1210 & 23.1583 & 104.3177 \\
\hline $140 \mathrm{~A}$ & 14 & 174 & 24 & 18.0635 & 18.1515 & 21.9571 & 23.0942 & 23.1131 & 104.3794 \\
\hline $140 \mathrm{~A}$ & 15 & 175 & 24 & 18.2552 & 18.1923 & 22.0313 & 23.1256 & 23.2406 & 104.8450 \\
\hline
\end{tabular}




\begin{tabular}{|c|c|c|c|c|c|c|c|c|c|}
\hline Cycle & Timestep & $\begin{array}{l}\text { Cumulative } \\
\text { Timestep }\end{array}$ & \begin{tabular}{|c|} 
Time \\
Interval \\
$($ hrs $)$
\end{tabular} & $\begin{array}{l}\text { NW } \\
\text { Lobe } \\
\text { Power } \\
(\mathrm{MW})\end{array}$ & $\begin{array}{c}\text { NE } \\
\text { Lobe } \\
\text { Power } \\
(\mathrm{MW}) \\
\end{array}$ & $\begin{array}{c}\text { C } \\
\text { Lobe } \\
\text { Power } \\
(\mathrm{MW}) \\
\end{array}$ & $\begin{array}{c}\text { SW } \\
\text { Lobe } \\
\text { Power } \\
(\mathrm{MW})\end{array}$ & $\begin{array}{c}\text { SE } \\
\text { Lobe } \\
\text { Power } \\
\text { (MW) }\end{array}$ & $\begin{array}{l}\text { Total } \\
\text { Core } \\
\text { Power } \\
(\mathrm{MW}) \\
\end{array}$ \\
\hline $140 \mathrm{~A}$ & 16 & 176 & 24 & 18.1225 & 18.1406 & 21.9104 & 23.1940 & 23.1488 & 104.5163 \\
\hline $140 \mathrm{~A}$ & 17 & 177 & 24 & 18.1238 & 18.1331 & 21.9483 & 23.1690 & 23.1863 & 104.5604 \\
\hline $140 \mathrm{~A}$ & 18 & 178 & 24 & 18.1267 & 18.0752 & 21.7027 & 23.2563 & 23.1102 & 104.2710 \\
\hline $140 \mathrm{~A}$ & 19 & 179 & 24 & 18.1696 & 18.1469 & 21.4873 & 23.1969 & 23.2106 & 104.2113 \\
\hline $140 \mathrm{~A}$ & 20 & 180 & 24 & 18.0769 & 18.1198 & 21.4108 & 23.0921 & 23.1050 & 103.8046 \\
\hline $140 \mathrm{~A}$ & 21 & 181 & 24 & 18.0971 & 18.1273 & 22.0150 & 23.1104 & 23.1040 & 104.4538 \\
\hline $140 \mathrm{~A}$ & 22 & 182 & 24 & 18.0671 & 18.1027 & 21.6596 & 23.1302 & 23.0658 & 104.0254 \\
\hline $140 \mathrm{~A}$ & 23 & 183 & 24 & 18.1660 & 18.1560 & 21.6933 & 23.1275 & 23.1946 & 104.3375 \\
\hline $140 \mathrm{~A}$ & 24 & 184 & 24 & 18.1921 & 18.2469 & 21.7194 & 23.3181 & 23.2229 & 104.6994 \\
\hline $140 \mathrm{~A}$ & 25 & 185 & 24 & 18.1354 & 18.1817 & 21.4488 & 23.2490 & 23.2358 & 104.2506 \\
\hline $140 \mathrm{~A}$ & 26 & 186 & 24 & 18.0079 & 17.9700 & 21.5896 & 23.0656 & 22.9894 & 103.6225 \\
\hline $140 \mathrm{~A}$ & 27 & 187 & 24 & 17.9544 & 17.9506 & 21.6560 & 23.0469 & 23.0198 & 103.6277 \\
\hline $140 \mathrm{~A}$ & 28 & 188 & 24 & 17.9973 & 18.0217 & 21.3902 & 22.9698 & 22.9946 & 103.3735 \\
\hline $140 \mathrm{~A}$ & 29 & 189 & 24 & 18.0227 & 17.9846 & 21.8838 & 23.0258 & 23.0544 & 103.9713 \\
\hline $140 \mathrm{~A}$ & 30 & 190 & 24 & 17.9892 & 17.9794 & 22.0765 & 23.0371 & 23.0092 & 104.0913 \\
\hline $140 \mathrm{~A}$ & 31 & 191 & 24 & 17.9546 & 17.9060 & 21.8927 & 23.0271 & 22.9915 & 103.7719 \\
\hline $140 \mathrm{~A}$ & 32 & 192 & 24 & 17.9081 & 17.9221 & 21.7658 & 22.9552 & 22.9040 & 103.4552 \\
\hline $140 \mathrm{~A}$ & 33 & 193 & 24 & 17.9979 & 17.9623 & 22.0598 & 22.9733 & 22.9390 & 103.9323 \\
\hline $140 \mathrm{~A}$ & 34 & 194 & 24 & 18.0185 & 17.9504 & 21.9350 & 22.9983 & 23.0254 & 103.9277 \\
\hline $140 \mathrm{~A}$ & 35 & 195 & 24 & 17.9608 & 17.9608 & 22.1731 & 23.0319 & 22.9963 & 104.1229 \\
\hline $140 \mathrm{~A}$ & 36 & 196 & 24 & 18.0373 & 18.0356 & 22.1754 & 23.0302 & 23.0363 & 104.3148 \\
\hline $140 \mathrm{~A}$ & 37 & 197 & 24 & 17.8940 & 17.8644 & 21.7838 & 22.7096 & 22.8275 & 103.0792 \\
\hline $140 \mathrm{~A}$ & 38 & 198 & 24 & 17.8088 & 17.8335 & 21.8963 & 22.7994 & 22.8460 & 103.1840 \\
\hline $140 \mathrm{~A}$ & 39 & 199 & 24 & 17.8423 & 17.8302 & 21.8235 & 22.8090 & 22.8148 & 103.1198 \\
\hline $140 \mathrm{~A}$ & 40 & 200 & 24 & 17.8490 & 17.8285 & 22.1425 & 22.8446 & 22.8727 & 103.5373 \\
\hline $140 \mathrm{~A}$ & 41 & 201 & 24 & 17.9265 & 17.9600 & 21.7940 & 22.8988 & 22.8654 & 103.4446 \\
\hline $140 \mathrm{~A}$ & 42 & 202 & 24 & 17.9550 & 17.9975 & 21.9694 & 22.9063 & 22.9644 & 103.7925 \\
\hline $140 \mathrm{~A}$ & 43 & 203 & 24 & 17.9327 & 17.8783 & 22.2044 & 22.9690 & 22.8992 & 103.8835 \\
\hline $140 \mathrm{~A}$ & 44 & 204 & 24 & 17.9702 & 17.9167 & 21.9402 & 22.9415 & 22.9463 & 103.7148 \\
\hline $140 \mathrm{~A}$ & 45 & 205 & 24 & 17.9771 & 17.9398 & 22.0650 & 22.9463 & 22.8621 & 103.7902 \\
\hline $140 \mathrm{~A}$ & 46 & 206 & 24 & 17.9094 & 17.9079 & 22.0690 & 22.9840 & 22.8854 & 103.7556 \\
\hline $140 \mathrm{~A}$ & 47 & 207 & 24 & 17.9110 & 17.9992 & 21.9740 & 23.0085 & 22.9354 & 103.8281 \\
\hline $140 \mathrm{~A}$ & 48 & 208 & 11 & 17.9527 & 17.8900 & 21.7218 & 23.0305 & 22.9555 & 103.5505 \\
\hline $140 \mathrm{~B}$ & 1 & 209 & 8 & 0.2650 & 0.2688 & 0.3075 & 0.3375 & 0.3650 & 1.5438 \\
\hline $140 \mathrm{~B}$ & 2 & 210 & 24 & 12.9713 & 13.0158 & 14.1060 & 16.5525 & 16.7419 & 73.3875 \\
\hline 140B & 3 & 211 & 24 & 17.6521 & 17.6908 & 20.5602 & 22.5633 & 22.5923 & 101.0588 \\
\hline
\end{tabular}




\begin{tabular}{|c|c|c|c|c|c|c|c|c|c|}
\hline Cycle & Timestep & $\begin{array}{l}\text { Cumulative } \\
\text { Timestep }\end{array}$ & \begin{tabular}{|c|} 
Time \\
Interval \\
$($ hrs $)$
\end{tabular} & $\begin{array}{l}\text { NW } \\
\text { Lobe } \\
\text { Power } \\
(\mathrm{MW})\end{array}$ & $\begin{array}{c}\text { NE } \\
\text { Lobe } \\
\text { Power } \\
(\mathrm{MW}) \\
\end{array}$ & $\begin{array}{c}\text { C } \\
\text { Lobe } \\
\text { Power } \\
(\mathrm{MW}) \\
\end{array}$ & $\begin{array}{c}\text { SW } \\
\text { Lobe } \\
\text { Power } \\
(\mathrm{MW})\end{array}$ & $\begin{array}{c}\text { SE } \\
\text { Lobe } \\
\text { Power } \\
\text { (MW) }\end{array}$ & $\begin{array}{l}\text { Total } \\
\text { Core } \\
\text { Power } \\
(\mathrm{MW}) \\
\end{array}$ \\
\hline $140 \mathrm{~B}$ & 4 & 212 & 24 & 17.7023 & 17.6438 & 21.0492 & 22.6127 & 22.6835 & 101.6915 \\
\hline $140 \mathrm{~B}$ & 5 & 213 & 24 & 17.6621 & 17.5427 & 20.7190 & 22.4998 & 22.5450 & 100.9685 \\
\hline $140 \mathrm{~B}$ & 6 & 214 & 24 & 17.6488 & 17.5508 & 20.7394 & 22.5231 & 22.6100 & 101.0721 \\
\hline $140 \mathrm{~B}$ & 7 & 215 & 24 & 17.6373 & 17.5560 & 20.5779 & 22.5165 & 22.6183 & 100.9060 \\
\hline $140 \mathrm{~B}$ & 8 & 216 & 24 & 17.5538 & 17.4873 & 20.2498 & 22.5458 & 22.5654 & 100.4021 \\
\hline 140B & 9 & 217 & 24 & 17.5915 & 17.5481 & 20.7381 & 22.4500 & 22.5779 & 100.9056 \\
\hline 140B & 10 & 218 & 24 & 17.5963 & 17.5619 & 21.0425 & 22.5308 & 22.5550 & 101.2865 \\
\hline $140 \mathrm{~B}$ & 11 & 219 & 24 & 17.6394 & 17.5771 & 21.1640 & 22.5646 & 22.6346 & 101.5796 \\
\hline $140 \mathrm{~B}$ & 12 & 220 & 24 & 17.6340 & 17.6652 & 20.9381 & 22.5633 & 22.5938 & 101.3944 \\
\hline $140 \mathrm{~B}$ & 13 & 221 & 24 & 17.5148 & 17.5038 & 20.9196 & 22.4825 & 22.5300 & 100.9506 \\
\hline $140 \mathrm{~B}$ & 14 & 222 & 24 & 17.5721 & 17.5742 & 20.8848 & 22.4752 & 22.5446 & 101.0508 \\
\hline $140 \mathrm{~B}$ & 15 & 223 & 24 & 17.5435 & 17.5373 & 20.8338 & 22.4508 & 22.5492 & 100.9146 \\
\hline $140 \mathrm{~B}$ & 16 & 224 & 24 & 17.5315 & 17.5083 & 20.5821 & 22.4596 & 22.5125 & 100.5940 \\
\hline 140B & 17 & 225 & 24 & 17.5617 & 17.5452 & 20.3992 & 22.4563 & 22.5088 & 100.4710 \\
\hline $140 \mathrm{~B}$ & 18 & 226 & 24 & 17.5527 & 17.5529 & 20.3850 & 22.4342 & 22.5873 & 100.5121 \\
\hline $140 \mathrm{~B}$ & 19 & 227 & 24 & 17.5831 & 17.5133 & 20.6081 & 22.4579 & 22.5519 & 100.7144 \\
\hline $140 \mathrm{~B}$ & 20 & 228 & 24 & 17.5350 & 17.5079 & 20.3835 & 22.4215 & 22.4875 & 100.3354 \\
\hline $140 \mathrm{~B}$ & 21 & 229 & 24 & 17.5083 & 17.4604 & 20.0492 & 22.3348 & 22.4413 & 99.7940 \\
\hline $140 \mathrm{~B}$ & 22 & 230 & 24 & 17.5204 & 17.5363 & 20.3594 & 22.5533 & 22.6002 & 100.5696 \\
\hline $140 \mathrm{~B}$ & 23 & 231 & 24 & 17.5827 & 17.5544 & 20.1496 & 22.5098 & 22.6223 & 100.4188 \\
\hline $140 \mathrm{~B}$ & 24 & 232 & 24 & 17.5967 & 17.6023 & 19.9940 & 22.4990 & 22.5563 & 100.2481 \\
\hline $140 \mathrm{~B}$ & 25 & 233 & 24 & 17.5775 & 17.7281 & 19.8598 & 22.2981 & 22.5942 & 100.0577 \\
\hline $140 \mathrm{~B}$ & 26 & 234 & 19 & 17.4903 & 17.4842 & 21.5989 & 22.1189 & 22.2716 & 100.9639 \\
\hline $140 \mathrm{~B}$ & 27 & 235 & 185 & 0.1000 & 0.1000 & 0.1000 & 0.1000 & 0.1000 & 0.5000 \\
\hline $140 \mathrm{~B}$ & 28 & 236 & 11 & 18.3141 & 18.4332 & 23.8514 & 23.3414 & 23.3800 & 107.3200 \\
\hline $140 \mathrm{~B}$ & 29 & 237 & 24 & 18.2567 & 18.3348 & 23.7371 & 23.3204 & 23.2565 & 106.9054 \\
\hline $140 \mathrm{~B}$ & 30 & 238 & 24 & 18.2775 & 18.3323 & 24.0029 & 23.2488 & 23.2985 & 107.1600 \\
\hline $140 \mathrm{~B}$ & 31 & 239 & 24 & 18.3465 & 18.3421 & 24.0385 & 23.3479 & 23.3154 & 107.3904 \\
\hline $140 \mathrm{~B}$ & 32 & 240 & 24 & 18.3927 & 18.4346 & 24.2806 & 23.3690 & 23.4052 & 107.8821 \\
\hline $140 \mathrm{~B}$ & 33 & 241 & 24 & 18.4177 & 18.4563 & 24.7279 & 23.4727 & 23.4494 & 108.5240 \\
\hline $140 \mathrm{~B}$ & 34 & 242 & 24 & 18.3629 & 18.4077 & 24.4644 & 23.3031 & 23.3788 & 107.9169 \\
\hline $140 \mathrm{~B}$ & 35 & 243 & 24 & 18.4535 & 18.4858 & 24.4508 & 23.5035 & 23.3896 & 108.2833 \\
\hline $140 \mathrm{~B}$ & 36 & 244 & 24 & 18.4498 & 18.5058 & 24.1715 & 23.4283 & 23.4919 & 108.0473 \\
\hline $140 \mathrm{~B}$ & 37 & 245 & 13 & 17.0458 & 17.0742 & 22.2881 & 21.6938 & 21.7265 & 99.8285 \\
\hline $141 \mathrm{~A}$ & 1 & 246 & 8 & 8.2213 & 8.2031 & 10.2344 & 10.4494 & 10.6113 & 47.7194 \\
\hline $141 \mathrm{~A}$ & 2 & 247 & 24 & 18.4094 & 18.4373 & 22.4488 & 23.5071 & 23.4608 & 106.2633 \\
\hline
\end{tabular}




\begin{tabular}{|c|c|c|c|c|c|c|c|c|c|}
\hline Cycle & Timestep & $\begin{array}{l}\text { Cumulative } \\
\text { Timestep }\end{array}$ & \begin{tabular}{|c|} 
Time \\
Interval \\
$($ hrs $)$
\end{tabular} & $\begin{array}{l}\text { NW } \\
\text { Lobe } \\
\text { Power } \\
\text { (MW) }\end{array}$ & $\begin{array}{c}\text { NE } \\
\text { Lobe } \\
\text { Power } \\
\text { (MW) }\end{array}$ & $\begin{array}{c}\text { C } \\
\text { Lobe } \\
\text { Power } \\
(\mathrm{MW}) \\
\end{array}$ & $\begin{array}{c}\text { SW } \\
\text { Lobe } \\
\text { Power } \\
(\mathrm{MW})\end{array}$ & $\begin{array}{c}\text { SE } \\
\text { Lobe } \\
\text { Power } \\
(\mathrm{MW}) \\
\end{array}$ & $\begin{array}{l}\text { Total } \\
\text { Core } \\
\text { Power } \\
(\mathrm{MW}) \\
\end{array}$ \\
\hline $141 \mathrm{~A}$ & 3 & 248 & 24 & 18.2981 & 18.2442 & 22.4758 & 23.2363 & 23.2029 & 105.4573 \\
\hline $141 \mathrm{~A}$ & 4 & 249 & 24 & 18.4835 & 18.5283 & 23.6810 & 23.5719 & 23.4825 & 107.7473 \\
\hline $141 \mathrm{~A}$ & 5 & 250 & 24 & 18.4156 & 18.4152 & 23.4517 & 23.5683 & 23.4348 & 107.2856 \\
\hline $141 \mathrm{~A}$ & 6 & 251 & 24 & 18.3510 & 18.3881 & 23.2988 & 23.4440 & 23.3440 & 106.8258 \\
\hline $141 \mathrm{~A}$ & 7 & 252 & 24 & 18.4721 & 18.5525 & 24.0392 & 23.5990 & 23.5058 & 108.1685 \\
\hline $141 \mathrm{~A}$ & 8 & 253 & 24 & 18.5260 & 18.5610 & 23.9708 & 23.6285 & 23.5775 & 108.2640 \\
\hline $141 \mathrm{~A}$ & 9 & 254 & 24 & 18.5590 & 18.6308 & 23.8900 & 23.6271 & 23.6004 & 108.3073 \\
\hline $141 \mathrm{~A}$ & 10 & 255 & 24 & 18.5958 & 18.6583 & 23.9940 & 23.7138 & 23.6369 & 108.5988 \\
\hline $141 \mathrm{~A}$ & 11 & 256 & 24 & 18.6465 & 18.6631 & 24.0517 & 23.7546 & 23.6756 & 108.7915 \\
\hline $141 \mathrm{~A}$ & 12 & 257 & 24 & 18.6313 & 18.5954 & 23.9735 & 23.7642 & 23.6635 & 108.6279 \\
\hline $141 \mathrm{~A}$ & 13 & 258 & 24 & 18.6600 & 18.6727 & 24.0800 & 23.7913 & 23.7044 & 108.9083 \\
\hline $141 \mathrm{~A}$ & 14 & 259 & 24 & 18.5375 & 18.5925 & 22.9044 & 23.6271 & 23.6163 & 107.2777 \\
\hline $141 \mathrm{~A}$ & 15 & 260 & 24 & 18.5790 & 18.8075 & 22.4808 & 22.4188 & 23.6200 & 105.9060 \\
\hline $141 \mathrm{~A}$ & 16 & 261 & 24 & 18.6925 & 18.7021 & 23.7948 & 23.8740 & 23.7671 & 108.8304 \\
\hline $141 \mathrm{~A}$ & 17 & 262 & 24 & 18.7596 & 18.6627 & 23.7788 & 23.8325 & 23.8242 & 108.8577 \\
\hline $141 \mathrm{~A}$ & 18 & 263 & 24 & 18.7679 & 18.8123 & 23.6202 & 23.9048 & 23.8204 & 108.9256 \\
\hline $141 \mathrm{~A}$ & 19 & 264 & 24 & 18.7319 & 18.7583 & 24.1863 & 23.8690 & 23.8171 & 109.3625 \\
\hline $141 \mathrm{~A}$ & 20 & 265 & 24 & 18.5550 & 18.5652 & 23.9690 & 23.6473 & 23.6058 & 108.3423 \\
\hline $141 \mathrm{~A}$ & 21 & 266 & 24 & 18.6150 & 18.5969 & 24.4221 & 23.7898 & 23.6623 & 109.0860 \\
\hline $141 \mathrm{~A}$ & 22 & 267 & 24 & 18.6754 & 18.6967 & 24.2444 & 23.7419 & 23.7160 & 109.0744 \\
\hline $141 \mathrm{~A}$ & 23 & 268 & 24 & 18.6419 & 18.7527 & 23.9000 & 23.7013 & 23.6629 & 108.6588 \\
\hline $141 \mathrm{~A}$ & 24 & 269 & 24 & 18.7300 & 18.7998 & 24.2181 & 23.7523 & 23.7608 & 109.2610 \\
\hline $141 \mathrm{~A}$ & 25 & 270 & 24 & 18.7504 & 18.8833 & 24.2040 & 23.7988 & 23.8271 & 109.4635 \\
\hline $141 \mathrm{~A}$ & 26 & 271 & 24 & 18.7085 & 18.7898 & 23.8585 & 23.8050 & 23.7563 & 108.9181 \\
\hline $141 \mathrm{~A}$ & 27 & 272 & 24 & 18.7718 & 18.7864 & 23.6432 & 23.8655 & 23.7623 & 108.8291 \\
\hline $141 \mathrm{~A}$ & 28 & 273 & 24 & 18.6852 & 18.7140 & 23.8035 & 23.7671 & 23.6940 & 108.6638 \\
\hline $141 \mathrm{~A}$ & 29 & 274 & 24 & 18.5435 & 18.6798 & 23.7590 & 23.6056 & 23.5994 & 108.1873 \\
\hline $141 \mathrm{~A}$ & 30 & 275 & 24 & 18.6238 & 18.6981 & 24.0569 & 23.7669 & 23.6396 & 108.7852 \\
\hline $141 \mathrm{~A}$ & 31 & 276 & 24 & 18.6321 & 18.6823 & 24.2631 & 23.6648 & 23.6873 & 108.9296 \\
\hline $141 \mathrm{~A}$ & 32 & 277 & 24 & 18.6167 & 18.6360 & 23.9979 & 23.8373 & 23.7569 & 108.8448 \\
\hline $141 \mathrm{~A}$ & 33 & 278 & 24 & 18.5552 & 18.6544 & 24.1940 & 23.6756 & 23.6315 & 108.7106 \\
\hline $141 \mathrm{~A}$ & 34 & 279 & 7 & 18.5121 & 18.5279 & 24.1107 & 23.7057 & 23.5207 & 108.3771 \\
\hline $142 \mathrm{~A}$ & 1 & 280 & 8 & 8.0300 & 6.3188 & 9.3931 & 8.1069 & 8.0456 & 39.8944 \\
\hline $142 \mathrm{~A}$ & 2 & 281 & 24 & 23.3765 & 18.4021 & 25.2127 & 23.4231 & 23.3633 & 113.7777 \\
\hline $142 \mathrm{~A}$ & 3 & 282 & 24 & 23.5592 & 18.6002 & 24.8750 & 23.6894 & 23.6517 & 114.3754 \\
\hline $142 \mathrm{~A}$ & 4 & 283 & 24 & 23.3542 & 18.3660 & 24.1696 & 23.4321 & 23.3631 & 112.6850 \\
\hline
\end{tabular}




\begin{tabular}{|c|c|c|c|c|c|c|c|c|c|}
\hline Cycle & Timestep & $\begin{array}{l}\text { Cumulative } \\
\text { Timestep } \\
\end{array}$ & \begin{tabular}{|c|} 
Time \\
Interval \\
$($ hrs $)$
\end{tabular} & $\begin{array}{l}\text { NW } \\
\text { Lobe } \\
\text { Power } \\
\text { (MW) }\end{array}$ & $\begin{array}{c}\text { NE } \\
\text { Lobe } \\
\text { Power } \\
(\mathrm{MW}) \\
\end{array}$ & $\begin{array}{c}\text { C } \\
\text { Lobe } \\
\text { Power } \\
(\mathrm{MW}) \\
\end{array}$ & $\begin{array}{c}\text { SW } \\
\text { Lobe } \\
\text { Power } \\
(\mathrm{MW}) \\
\end{array}$ & $\begin{array}{c}\text { SE } \\
\text { Lobe } \\
\text { Power } \\
\text { (MW) }\end{array}$ & $\begin{array}{l}\text { Total } \\
\text { Core } \\
\text { Power } \\
(\mathrm{MW}) \\
\end{array}$ \\
\hline $142 \mathrm{~A}$ & 5 & 284 & 24 & 23.2483 & 18.2265 & 24.0923 & 25.0223 & 23.2510 & 113.8404 \\
\hline $142 \mathrm{~A}$ & 6 & 285 & 24 & 23.2225 & 18.2700 & 24.0723 & 25.3063 & 23.2933 & 114.1644 \\
\hline $142 \mathrm{~A}$ & 7 & 286 & 25 & 23.2079 & 18.2542 & 24.1100 & 25.2288 & 23.2731 & 114.0740 \\
\hline $142 \mathrm{~A}$ & 8 & 287 & 24 & 23.2740 & 18.3440 & 24.1694 & 25.2635 & 23.2683 & 114.3192 \\
\hline $142 \mathrm{~A}$ & 9 & 288 & 24 & 23.3177 & 18.3783 & 24.1421 & 25.2942 & 23.3288 & 114.4610 \\
\hline $142 \mathrm{~A}$ & 10 & 289 & 24 & 23.4546 & 18.4906 & 24.4433 & 25.5215 & 23.4740 & 115.3840 \\
\hline $142 \mathrm{~A}$ & 11 & 290 & 24 & 23.3442 & 18.3467 & 24.3927 & 25.4304 & 23.3600 & 114.8740 \\
\hline $142 \mathrm{~A}$ & 12 & 291 & 24 & 23.2898 & 18.1952 & 24.2073 & 25.2688 & 23.2394 & 114.2004 \\
\hline $142 \mathrm{~A}$ & 13 & 292 & 24 & 23.2963 & 18.2496 & 23.9527 & 25.2902 & 23.2625 & 114.0513 \\
\hline $142 \mathrm{~A}$ & 14 & 293 & 24 & 23.2752 & 18.3098 & 23.7727 & 25.3323 & 23.3621 & 114.0521 \\
\hline $142 \mathrm{~A}$ & 15 & 294 & 24 & 23.3748 & 18.3023 & 24.2642 & 25.3625 & 23.3238 & 114.6275 \\
\hline $142 \mathrm{~A}$ & 16 & 295 & 24 & 23.2713 & 18.1948 & 24.0769 & 25.3344 & 23.2633 & 114.1406 \\
\hline $142 \mathrm{~A}$ & 17 & 296 & 24 & 23.3256 & 18.3433 & 24.5965 & 25.2527 & 23.2031 & 114.7213 \\
\hline $142 \mathrm{~A}$ & 18 & 297 & 24 & 23.2825 & 18.2554 & 24.9602 & 25.3421 & 23.3221 & 115.1623 \\
\hline $142 \mathrm{~A}$ & 19 & 298 & 24 & 23.2285 & 18.2852 & 24.6910 & 25.2713 & 23.2375 & 114.7135 \\
\hline $142 \mathrm{~A}$ & 20 & 299 & 24 & 23.2250 & 18.2329 & 24.4983 & 25.1946 & 23.2796 & 114.4304 \\
\hline $142 \mathrm{~A}$ & 21 & 300 & 24 & 23.1733 & 18.1992 & 24.6388 & 25.2235 & 23.1823 & 114.4171 \\
\hline $142 \mathrm{~A}$ & 22 & 301 & 24 & 23.2781 & 18.2300 & 24.4715 & 25.2227 & 23.1806 & 114.3829 \\
\hline $142 \mathrm{~A}$ & 23 & 302 & 24 & 23.2252 & 18.2508 & 24.6433 & 25.2471 & 23.2279 & 114.5944 \\
\hline $142 \mathrm{~A}$ & 24 & 303 & 24 & 23.2135 & 18.2790 & 24.9854 & 25.3138 & 23.2325 & 115.0242 \\
\hline $142 \mathrm{~A}$ & 25 & 304 & 24 & 23.3017 & 18.2838 & 25.1585 & 25.3448 & 23.3381 & 115.4269 \\
\hline $142 \mathrm{~A}$ & 26 & 305 & 24 & 23.4167 & 18.4238 & 25.1990 & 25.4744 & 23.4119 & 115.9256 \\
\hline $142 \mathrm{~A}$ & 27 & 306 & 24 & 23.4796 & 18.3985 & 24.8238 & 25.4865 & 23.4767 & 115.6650 \\
\hline $142 \mathrm{~A}$ & 28 & 307 & 24 & 23.4510 & 18.4473 & 24.6515 & 25.4804 & 23.4869 & 115.5171 \\
\hline $142 \mathrm{~A}$ & 29 & 308 & 24 & 23.5913 & 18.5260 & 24.8352 & 25.6494 & 23.5856 & 116.1875 \\
\hline $142 \mathrm{~A}$ & 30 & 309 & 24 & 23.4960 & 18.5948 & 25.3204 & 25.5748 & 23.5273 & 116.5133 \\
\hline $142 \mathrm{~A}$ & 31 & 310 & 25 & 23.6460 & 18.7042 & 25.3563 & 25.6396 & 23.6529 & 116.9990 \\
\hline $142 \mathrm{~A}$ & 32 & 311 & 24 & 23.6671 & 18.6669 & 25.5427 & 25.7067 & 23.5700 & 117.1533 \\
\hline $142 \mathrm{~A}$ & 33 & 312 & 20 & 21.1790 & 16.7478 & 22.7615 & 23.0325 & 21.2435 & 104.9643 \\
\hline $142 \mathrm{~A}$ & 34 & 313 & 17 & 0.1000 & 0.1000 & 0.1000 & 0.1000 & 0.1000 & 0.5000 \\
\hline $142 \mathrm{~A}$ & 35 & 314 & 11 & 18.2895 & 14.3923 & 21.9641 & 19.8273 & 18.2845 & 92.7577 \\
\hline $142 \mathrm{~A}$ & 36 & 315 & 24 & 23.5790 & 18.5848 & 25.5360 & 25.6327 & 23.5896 & 116.9221 \\
\hline $142 \mathrm{~A}$ & 37 & 316 & 24 & 23.4279 & 18.4460 & 25.1846 & 25.4579 & 23.4100 & 115.9265 \\
\hline $142 \mathrm{~A}$ & 38 & 317 & 24 & 23.4390 & 18.4825 & 25.2846 & 25.4717 & 23.4244 & 116.1021 \\
\hline $142 \mathrm{~A}$ & 39 & 318 & 24 & 23.4913 & 18.5823 & 25.4492 & 25.5575 & 23.5729 & 116.6531 \\
\hline $142 \mathrm{~A}$ & 40 & 319 & 24 & 23.4513 & 18.4875 & 25.3540 & 25.5283 & 23.4946 & 116.3156 \\
\hline
\end{tabular}




\begin{tabular}{|c|c|c|c|c|c|c|c|c|c|}
\hline Cycle & Timestep & $\begin{array}{l}\text { Cumulative } \\
\text { Timestep } \\
\end{array}$ & \begin{tabular}{|c|} 
Time \\
Interval \\
$($ hrs $)$
\end{tabular} & $\begin{array}{l}\text { NW } \\
\text { Lobe } \\
\text { Power } \\
\text { (MW) }\end{array}$ & $\begin{array}{c}\text { NE } \\
\text { Lobe } \\
\text { Power } \\
(\mathrm{MW}) \\
\end{array}$ & $\begin{array}{c}\text { C } \\
\text { Lobe } \\
\text { Power } \\
\text { (MW) }\end{array}$ & $\begin{array}{c}\text { SW } \\
\text { Lobe } \\
\text { Power } \\
(\mathrm{MW})\end{array}$ & $\begin{array}{c}\text { SE } \\
\text { Lobe } \\
\text { Power } \\
(\mathrm{MW}) \\
\end{array}$ & $\begin{array}{l}\text { Total } \\
\text { Core } \\
\text { Power } \\
(\mathrm{MW}) \\
\end{array}$ \\
\hline $142 \mathrm{~A}$ & 41 & 320 & 24 & 23.5242 & 18.5265 & 25.6215 & 25.5910 & 23.5392 & 116.8023 \\
\hline $142 \mathrm{~A}$ & 42 & 321 & 24 & 23.5981 & 18.6140 & 25.5206 & 25.5638 & 23.6942 & 116.9906 \\
\hline $142 \mathrm{~A}$ & 43 & 322 & 24 & 23.4429 & 18.5069 & 25.6667 & 25.4938 & 23.5171 & 116.6273 \\
\hline $142 \mathrm{~A}$ & 44 & 323 & 24 & 23.3921 & 18.4673 & 25.8269 & 25.4467 & 23.4290 & 116.5619 \\
\hline $142 \mathrm{~A}$ & 45 & 324 & 24 & 23.3825 & 18.3588 & 25.7588 & 25.4029 & 23.4544 & 116.3573 \\
\hline $142 \mathrm{~A}$ & 46 & 325 & 24 & 23.3848 & 18.4615 & 25.6881 & 25.4483 & 23.3840 & 116.3667 \\
\hline $142 \mathrm{~A}$ & 47 & 326 & 24 & 23.5027 & 18.5115 & 25.7858 & 25.5133 & 23.5242 & 116.8375 \\
\hline $142 \mathrm{~A}$ & 48 & 327 & 24 & 23.4456 & 18.5490 & 25.5448 & 25.4450 & 23.5225 & 116.5069 \\
\hline $142 \mathrm{~A}$ & 49 & 328 & 24 & 23.3833 & 18.4033 & 25.6427 & 25.4329 & 23.4231 & 116.2854 \\
\hline $142 \mathrm{~A}$ & 50 & 329 & 24 & 23.4775 & 18.4596 & 25.8615 & 25.4754 & 23.4713 & 116.7452 \\
\hline $142 \mathrm{~A}$ & 51 & 330 & 15 & 23.5610 & 18.5870 & 26.1203 & 25.5057 & 23.5680 & 117.3420 \\
\hline 142B & 1 & 331 & 8 & 7.4138 & 5.8700 & 9.0088 & 8.0969 & 8.0331 & 38.4225 \\
\hline 142B & 2 & 332 & 24 & 21.6702 & 16.9098 & 23.1467 & 23.6000 & 21.6931 & 107.0198 \\
\hline 142B & 3 & 333 & 24 & 2.9015 & 2.3169 & 3.1106 & 3.1783 & 3.0679 & 14.5752 \\
\hline $142 B$ & 4 & 334 & 45 & 0.0200 & 0.0200 & 0.0200 & 0.0200 & 0.0200 & 0.1000 \\
\hline $142 B$ & 5 & 335 & 13 & 15.4000 & 12.1831 & 19.0596 & 16.7558 & 16.6312 & 80.0296 \\
\hline $142 B$ & 6 & 336 & 24 & 23.7775 & 18.8117 & 26.0094 & 25.8721 & 25.8000 & 120.2706 \\
\hline 142B & 7 & 337 & 24 & 23.6752 & 18.7388 & 25.2963 & 25.7102 & 25.7121 & 119.1325 \\
\hline 142B & 8 & 338 & 24 & 23.5619 & 18.6448 & 24.9635 & 25.5535 & 25.6048 & 118.3285 \\
\hline 142B & 9 & 339 & 24 & 23.5329 & 18.5302 & 25.2463 & 25.5529 & 25.5667 & 118.4290 \\
\hline $142 \mathrm{~B}$ & 10 & 340 & 24 & 23.5350 & 18.5490 & 25.0496 & 25.6223 & 25.5798 & 118.3356 \\
\hline 142B & 11 & 341 & 24 & 23.5573 & 18.5821 & 25.1104 & 25.6954 & 25.6298 & 118.5750 \\
\hline 142B & 12 & 342 & 24 & 23.6046 & 18.7317 & 25.2248 & 25.7702 & 25.6881 & 119.0194 \\
\hline 142B & 13 & 343 & 24 & 23.6706 & 18.7419 & 24.7704 & 25.8060 & 25.7183 & 118.7073 \\
\hline 142B & 14 & 344 & 24 & 23.4488 & 18.5946 & 24.5229 & 25.6279 & 25.6229 & 117.8171 \\
\hline $142 B$ & 15 & 345 & 24 & 23.5090 & 18.5290 & 25.8588 & 25.6600 & 25.5494 & 119.1060 \\
\hline 142B & 16 & 346 & 24 & 23.6058 & 18.5588 & 26.0329 & 25.8679 & 25.7642 & 119.8296 \\
\hline 142B & 17 & 347 & 24 & 23.6288 & 18.7104 & 26.2875 & 25.7992 & 25.6858 & 120.1117 \\
\hline 142B & 18 & 348 & 24 & 23.5402 & 18.6275 & 26.0629 & 25.6931 & 25.5771 & 119.5008 \\
\hline 142B & 19 & 349 & 24 & 23.5358 & 18.4742 & 25.6215 & 25.6925 & 25.5515 & 118.8754 \\
\hline 142B & 20 & 350 & 24 & 23.4863 & 18.4988 & 25.5131 & 25.6852 & 25.5581 & 118.7415 \\
\hline 142B & 21 & 351 & 24 & 23.4892 & 18.5477 & 25.5042 & 25.6085 & 25.4971 & 118.6467 \\
\hline 142B & 22 & 352 & 24 & 23.5388 & 18.5348 & 25.4277 & 25.6208 & 25.5275 & 118.6496 \\
\hline $142 B$ & 23 & 353 & 24 & 23.4813 & 18.5127 & 25.4831 & 25.6050 & 25.5081 & 118.5902 \\
\hline $142 B$ & 24 & 354 & 24 & 23.6110 & 18.5983 & 25.8998 & 25.5365 & 25.6740 & 119.3196 \\
\hline 142B & 25 & 355 & 24 & 23.5521 & 18.5469 & 25.5977 & 25.6704 & 25.6171 & 118.9842 \\
\hline
\end{tabular}




\begin{tabular}{|c|c|c|c|c|c|c|c|c|c|}
\hline Cycle & Timestep & $\begin{array}{l}\text { Cumulative } \\
\text { Timestep }\end{array}$ & \begin{tabular}{|c|} 
Time \\
Interval \\
$($ hrs $)$
\end{tabular} & $\begin{array}{l}\text { NW } \\
\text { Lobe } \\
\text { Power } \\
(\mathrm{MW})\end{array}$ & $\begin{array}{c}\text { NE } \\
\text { Lobe } \\
\text { Power } \\
\text { (MW) }\end{array}$ & $\begin{array}{c}\text { C } \\
\text { Lobe } \\
\text { Power } \\
(\mathrm{MW}) \\
\end{array}$ & $\begin{array}{c}\text { SW } \\
\text { Lobe } \\
\text { Power } \\
(\mathrm{MW})\end{array}$ & $\begin{array}{c}\text { SE } \\
\text { Lobe } \\
\text { Power } \\
(\mathrm{MW}) \\
\end{array}$ & $\begin{array}{l}\text { Total } \\
\text { Core } \\
\text { Power } \\
(\mathrm{MW}) \\
\end{array}$ \\
\hline $142 \mathrm{~B}$ & 26 & 356 & 24 & 23.5171 & 18.5554 & 25.7698 & 25.5967 & 25.5558 & 118.9948 \\
\hline $142 \mathrm{~B}$ & 27 & 357 & 24 & 23.5206 & 18.5871 & 25.5635 & 25.5890 & 25.4927 & 118.7529 \\
\hline $142 \mathrm{~B}$ & 28 & 358 & 24 & 23.4390 & 18.5988 & 26.0238 & 25.5319 & 25.3952 & 118.9885 \\
\hline $142 \mathrm{~B}$ & 29 & 359 & 24 & 23.4600 & 18.5771 & 25.9296 & 25.5408 & 25.5608 & 119.0683 \\
\hline $142 \mathrm{~B}$ & 30 & 360 & 24 & 23.5140 & 18.5590 & 25.8596 & 25.5706 & 25.5200 & 119.0231 \\
\hline 142B & 31 & 361 & 24 & 23.4867 & 18.4758 & 25.4154 & 25.6158 & 25.5227 & 118.5165 \\
\hline 142B & 32 & 362 & 31 & 23.4615 & 18.4015 & 25.0632 & 25.5718 & 25.4802 & 117.9781 \\
\hline $142 \mathrm{~B}$ & 33 & 363 & 46 & 0.0200 & 0.0200 & 0.0200 & 0.0200 & 0.0200 & 0.1000 \\
\hline $142 B$ & 34 & 364 & 11 & 19.9091 & 15.6723 & 24.9150 & 21.7405 & 21.5914 & 103.8282 \\
\hline $142 B$ & 35 & 365 & 24 & 23.3910 & 18.4044 & 26.5463 & 26.3667 & 25.4406 & 120.1490 \\
\hline $142 B$ & 36 & 366 & 24 & 23.3325 & 18.3517 & 25.5365 & 26.2565 & 25.3742 & 118.8513 \\
\hline $142 \mathrm{~B}$ & 37 & 367 & 24 & 23.2196 & 18.2438 & 25.2515 & 26.1583 & 25.3002 & 118.1733 \\
\hline $142 \mathrm{~B}$ & 38 & 368 & 24 & 23.2006 & 18.2898 & 25.1158 & 26.2281 & 25.2506 & 118.0850 \\
\hline $142 \mathrm{~B}$ & 39 & 369 & 24 & 23.3358 & 18.3758 & 24.9756 & 26.2494 & 25.2981 & 118.2348 \\
\hline $142 \mathrm{~B}$ & 40 & 370 & 24 & 23.3217 & 18.3502 & 24.9127 & 26.3413 & 25.2750 & 118.2008 \\
\hline $142 \mathrm{~B}$ & 41 & 371 & 24 & 23.3150 & 18.3790 & 25.6463 & 26.3913 & 25.3296 & 119.0610 \\
\hline $142 \mathrm{~B}$ & 42 & 372 & 24 & 23.3433 & 18.4158 & 25.4358 & 26.2821 & 25.3727 & 118.8498 \\
\hline $142 \mathrm{~B}$ & 43 & 373 & 24 & 23.3913 & 18.3404 & 25.0142 & 26.3194 & 25.3660 & 118.4313 \\
\hline $142 \mathrm{~B}$ & 44 & 374 & 24 & 23.3917 & 18.4379 & 26.2844 & 26.3379 & 25.3738 & 119.8256 \\
\hline $142 \mathrm{~B}$ & 45 & 375 & 24 & 23.3113 & 18.3404 & 26.2379 & 26.2913 & 25.3365 & 119.5173 \\
\hline $142 \mathrm{~B}$ & 46 & 376 & 24 & 23.4677 & 18.4883 & 26.5517 & 26.4602 & 25.4950 & 120.4629 \\
\hline $142 \mathrm{~B}$ & 47 & 377 & 24 & 23.4515 & 18.5042 & 26.2088 & 26.4577 & 25.4933 & 120.1154 \\
\hline $142 \mathrm{~B}$ & 48 & 378 & 24 & 23.4446 & 18.3921 & 26.0260 & 26.4381 & 25.4858 & 119.7867 \\
\hline $142 \mathrm{~B}$ & 49 & 379 & 24 & 23.4265 & 18.4290 & 25.7046 & 26.4146 & 25.5173 & 119.4919 \\
\hline $142 B$ & 50 & 380 & 24 & 23.4790 & 18.5715 & 25.6596 & 26.5738 & 25.5246 & 119.8083 \\
\hline $142 \mathrm{~B}$ & 51 & 381 & 24 & 23.4831 & 18.6404 & 25.7508 & 26.5140 & 25.5060 & 119.8944 \\
\hline $142 \mathrm{~B}$ & 52 & 382 & 24 & 23.4194 & 18.4875 & 25.7127 & 26.4546 & 25.4579 & 119.5321 \\
\hline $142 \mathrm{~B}$ & 53 & 383 & 24 & 23.4117 & 18.4940 & 26.6373 & 26.4496 & 25.4890 & 120.4815 \\
\hline $142 \mathrm{~B}$ & 54 & 384 & 24 & 23.4527 & 18.6540 & 27.6181 & 26.6365 & 25.5381 & 121.8994 \\
\hline $142 \mathrm{~B}$ & 55 & 385 & 24 & 23.5610 & 18.5815 & 27.4229 & 26.5581 & 25.5960 & 121.7196 \\
\hline $142 \mathrm{~B}$ & 56 & 386 & 24 & 23.5681 & 18.6531 & 26.8021 & 26.4804 & 25.5869 & 121.0906 \\
\hline $142 B$ & 57 & 387 & 21 & 23.6850 & 18.7450 & 26.7836 & 26.5638 & 25.6340 & 121.4114 \\
\hline $143 \mathrm{~A}$ & 1 & 388 & 8 & 10.6913 & 10.5900 & 15.3406 & 14.7863 & 14.6944 & 66.1025 \\
\hline $143 \mathrm{~A}$ & 2 & 389 & 24 & 18.0748 & 17.8308 & 23.4185 & 25.9913 & 25.0077 & 110.3231 \\
\hline $143 \mathrm{~A}$ & 3 & 390 & 24 & 18.2983 & 18.3335 & 23.8910 & 27.2754 & 25.2279 & 113.0263 \\
\hline $143 \mathrm{~A}$ & 4 & 391 & 24 & 18.3808 & 18.3615 & 24.0940 & 27.2708 & 25.3123 & 113.4194 \\
\hline
\end{tabular}




\begin{tabular}{|c|c|c|c|c|c|c|c|c|c|}
\hline Cycle & Timestep & $\begin{array}{l}\text { Cumulative } \\
\text { Timestep } \\
\end{array}$ & \begin{tabular}{|c|} 
Time \\
Interval \\
$(\mathrm{hrs})$
\end{tabular} & $\begin{array}{l}\text { NW } \\
\text { Lobe } \\
\text { Power } \\
\text { (MW) }\end{array}$ & $\begin{array}{c}\text { NE } \\
\text { Lobe } \\
\text { Power } \\
(\mathrm{MW}) \\
\end{array}$ & $\begin{array}{c}\text { C } \\
\text { Lobe } \\
\text { Power } \\
(\mathrm{MW}) \\
\end{array}$ & $\begin{array}{c}\text { SW } \\
\text { Lobe } \\
\text { Power } \\
(\mathrm{MW}) \\
\end{array}$ & $\begin{array}{c}\text { SE } \\
\text { Lobe } \\
\text { Power } \\
\text { (MW) }\end{array}$ & $\begin{array}{l}\text { Total } \\
\text { Core } \\
\text { Power } \\
(\mathrm{MW}) \\
\end{array}$ \\
\hline $143 \mathrm{~A}$ & 5 & 392 & 24 & 18.2192 & 18.2492 & 24.4560 & 27.2354 & 25.3042 & 113.4640 \\
\hline $143 \mathrm{~A}$ & 6 & 393 & 24 & 18.2575 & 18.3100 & 24.5435 & 27.2677 & 25.3617 & 113.7404 \\
\hline $143 \mathrm{~A}$ & 7 & 394 & 24 & 18.2342 & 18.2494 & 24.2946 & 27.2738 & 25.3196 & 113.3715 \\
\hline $143 \mathrm{~A}$ & 8 & 395 & 24 & 18.3067 & 18.3283 & 24.1610 & 27.3010 & 25.3485 & 113.4456 \\
\hline $143 \mathrm{~A}$ & 9 & 396 & 24 & 18.1098 & 18.1596 & 24.3538 & 27.0858 & 25.0788 & 112.7877 \\
\hline $143 \mathrm{~A}$ & 10 & 397 & 24 & 18.1440 & 18.1938 & 24.4073 & 27.1413 & 25.1594 & 113.0456 \\
\hline $143 \mathrm{~A}$ & 11 & 398 & 24 & 18.0750 & 18.0808 & 24.0713 & 27.0325 & 25.0396 & 112.2992 \\
\hline $143 \mathrm{~A}$ & 12 & 399 & 24 & 18.0285 & 17.9844 & 23.9642 & 26.9913 & 25.0356 & 112.0040 \\
\hline $143 \mathrm{~A}$ & 13 & 400 & 24 & 17.9400 & 18.0642 & 23.7113 & 26.8456 & 24.9958 & 111.5569 \\
\hline $143 \mathrm{~A}$ & 14 & 401 & 24 & 17.9779 & 18.0002 & 23.7538 & 26.9708 & 25.0196 & 111.7223 \\
\hline $143 \mathrm{~A}$ & 15 & 402 & 24 & 18.0394 & 18.1000 & 23.7048 & 27.0573 & 25.0856 & 111.9871 \\
\hline $143 \mathrm{~A}$ & 16 & 403 & 24 & 18.0571 & 18.1106 & 23.5256 & 27.0363 & 25.2277 & 111.9573 \\
\hline $143 \mathrm{~A}$ & 17 & 404 & 24 & 18.1106 & 18.0777 & 23.4475 & 27.0427 & 25.2035 & 111.8821 \\
\hline $143 \mathrm{~A}$ & 18 & 405 & 24 & 18.0390 & 18.0048 & 23.5642 & 26.9660 & 25.1110 & 111.6850 \\
\hline $143 \mathrm{~A}$ & 19 & 406 & 24 & 18.0504 & 18.0356 & 23.8152 & 27.0613 & 25.0663 & 112.0288 \\
\hline $143 \mathrm{~A}$ & 20 & 407 & 24 & 18.1444 & 18.1104 & 23.6202 & 27.0521 & 25.1352 & 112.0623 \\
\hline $143 \mathrm{~A}$ & 21 & 408 & 24 & 18.0798 & 18.1550 & 23.6875 & 27.1006 & 25.1988 & 112.2217 \\
\hline $143 \mathrm{~A}$ & 22 & 409 & 24 & 18.1269 & 18.1785 & 23.9206 & 27.0794 & 25.1385 & 112.4440 \\
\hline $143 \mathrm{~A}$ & 23 & 410 & 24 & 18.1098 & 18.1667 & 23.6894 & 27.1458 & 25.1463 & 112.2579 \\
\hline $143 \mathrm{~A}$ & 24 & 411 & 15 & 18.1730 & 18.2337 & 24.1910 & 27.1537 & 25.1203 & 112.8717 \\
\hline $143 \mathrm{~A}$ & 25 & 412 & 449 & 0.0100 & 0.0100 & 0.0100 & 0.0100 & 0.0100 & 0.0500 \\
\hline $143 \mathrm{~A}$ & 26 & 413 & 24 & 15.2940 & 15.2731 & 21.1873 & 22.8210 & 21.1746 & 95.7500 \\
\hline $143 \mathrm{~A}$ & 27 & 414 & 24 & 18.0948 & 18.0196 & 24.3271 & 27.0719 & 25.0252 & 112.5385 \\
\hline $143 \mathrm{~A}$ & 28 & 415 & 24 & 18.1292 & 18.1308 & 24.4331 & 27.1575 & 25.0994 & 112.9500 \\
\hline $143 \mathrm{~A}$ & 29 & 416 & 24 & 18.0917 & 18.0775 & 24.3683 & 27.1390 & 25.0096 & 112.6860 \\
\hline $143 \mathrm{~A}$ & 30 & 417 & 24 & 18.1252 & 18.1640 & 24.2179 & 27.0925 & 25.0317 & 112.6313 \\
\hline $143 \mathrm{~A}$ & 31 & 418 & 24 & 18.1296 & 18.2004 & 24.4040 & 27.0321 & 25.0742 & 112.8402 \\
\hline $143 \mathrm{~A}$ & 32 & 419 & 24 & 18.2210 & 18.1731 & 24.4044 & 27.0998 & 25.2108 & 113.1092 \\
\hline $143 \mathrm{~A}$ & 33 & 420 & 24 & 18.1625 & 18.1494 & 24.6785 & 27.0688 & 25.0902 & 113.1494 \\
\hline $143 \mathrm{~A}$ & 34 & 421 & 24 & 18.2246 & 18.2335 & 24.7023 & 27.0802 & 25.2602 & 113.5008 \\
\hline $143 \mathrm{~A}$ & 35 & 422 & 24 & 18.1215 & 18.1354 & 24.7663 & 27.0771 & 25.2029 & 113.3031 \\
\hline $143 \mathrm{~A}$ & 36 & 423 & 24 & 18.2096 & 18.2040 & 24.7208 & 27.2235 & 25.2354 & 113.5933 \\
\hline $143 \mathrm{~A}$ & 37 & 424 & 24 & 18.2544 & 18.2069 & 24.7421 & 27.2800 & 25.2394 & 113.7227 \\
\hline $143 \mathrm{~A}$ & 38 & 425 & 24 & 18.2444 & 18.2171 & 24.6577 & 27.2067 & 25.2869 & 113.6127 \\
\hline $143 \mathrm{~A}$ & 39 & 426 & 24 & 18.2110 & 18.2560 & 24.7196 & 27.1829 & 25.2573 & 113.6269 \\
\hline $143 \mathrm{~A}$ & 40 & 427 & 24 & 18.1531 & 18.1448 & 24.6410 & 27.1071 & 25.1650 & 113.2110 \\
\hline
\end{tabular}




\begin{tabular}{|c|c|c|c|c|c|c|c|c|c|}
\hline Cycle & Timestep & \begin{tabular}{|c} 
Cumulative \\
Timestep \\
\end{tabular} & $\begin{array}{c}\text { Time } \\
\text { Interval } \\
\text { (hrs) }\end{array}$ & $\begin{array}{l}\text { NW } \\
\text { Lobe } \\
\text { Power } \\
(\mathrm{MW})\end{array}$ & $\begin{array}{c}\text { NE } \\
\text { Lobe } \\
\text { Power } \\
(\mathrm{MW}) \\
\end{array}$ & $\begin{array}{c}\text { C } \\
\text { Lobe } \\
\text { Power } \\
(\mathrm{MW}) \\
\end{array}$ & $\begin{array}{c}\text { SW } \\
\text { Lobe } \\
\text { Power } \\
(\mathrm{MW})\end{array}$ & $\begin{array}{c}\text { SE } \\
\text { Lobe } \\
\text { Power } \\
\text { (MW) }\end{array}$ & $\begin{array}{l}\text { Total } \\
\text { Core } \\
\text { Power } \\
(\mathrm{MW}) \\
\end{array}$ \\
\hline $143 \mathrm{~A}$ & 41 & 428 & 28 & 17.8218 & 17.8264 & 23.9716 & 26.6643 & 24.7614 & 111.0455 \\
\hline $143 \mathrm{~A}$ & 42 & 429 & 112 & 0.0100 & 0.0100 & 0.0100 & 0.0100 & 0.0100 & 0.0500 \\
\hline $143 \mathrm{~A}$ & 43 & 430 & 8 & 12.0919 & 12.0031 & 17.8688 & 17.4456 & 16.7938 & 76.2031 \\
\hline $143 \mathrm{~A}$ & 44 & 431 & 24 & 18.2258 & 18.1840 & 25.2496 & 27.1952 & 25.3338 & 114.1883 \\
\hline $143 \mathrm{~A}$ & 45 & 432 & 24 & 18.3352 & 18.3419 & 25.4381 & 27.2808 & 25.4223 & 114.8183 \\
\hline $143 \mathrm{~A}$ & 46 & 433 & 24 & 18.3533 & 18.4565 & 25.3031 & 27.3333 & 25.3998 & 114.8460 \\
\hline $143 \mathrm{~A}$ & 47 & 434 & 24 & 18.2523 & 18.1963 & 25.1079 & 27.1829 & 25.3063 & 114.0456 \\
\hline $143 \mathrm{~A}$ & 48 & 435 & 24 & 18.1927 & 18.2071 & 24.6885 & 27.1840 & 25.2575 & 113.5298 \\
\hline $143 \mathrm{~A}$ & 49 & 436 & 24 & 18.2250 & 18.2629 & 25.2408 & 27.2027 & 25.2588 & 114.1902 \\
\hline $143 \mathrm{~A}$ & 50 & 437 & 24 & 18.2733 & 18.3142 & 25.0948 & 27.2202 & 25.2785 & 114.1810 \\
\hline $143 \mathrm{~A}$ & 51 & 438 & 24 & 18.2804 & 18.2379 & 24.9383 & 27.1900 & 25.3308 & 113.9775 \\
\hline $143 \mathrm{~A}$ & 52 & 439 & 24 & 18.2460 & 18.2785 & 25.1196 & 27.1113 & 25.2129 & 113.9683 \\
\hline $143 \mathrm{~A}$ & 53 & 440 & 22 & 18.2718 & 18.2041 & 25.1470 & 27.0375 & 25.1252 & 113.7857 \\
\hline $143 \mathrm{~B}$ & 1 & 441 & 8 & 0.2138 & 0.2188 & 0.2913 & 0.2638 & 0.2725 & 1.2600 \\
\hline $143 \mathrm{~B}$ & 2 & 442 & 24 & 9.7135 & 9.7754 & 13.4425 & 13.3298 & 13.2273 & 59.4885 \\
\hline $143 \mathrm{~B}$ & 3 & 443 & 24 & 18.5788 & 18.6088 & 24.4527 & 25.6669 & 25.6204 & 112.9275 \\
\hline $143 \mathrm{~B}$ & 4 & 444 & 24 & 18.6935 & 18.7015 & 24.3708 & 25.6692 & 25.6513 & 113.0863 \\
\hline $143 \mathrm{~B}$ & 5 & 445 & 24 & 18.5244 & 18.5635 & 23.9783 & 25.5710 & 25.5546 & 112.1919 \\
\hline $143 \mathrm{~B}$ & 6 & 446 & 24 & 18.6175 & 18.6815 & 24.1533 & 25.7346 & 25.6304 & 112.8173 \\
\hline $143 \mathrm{~B}$ & 7 & 447 & 24 & 18.6825 & 18.7706 & 24.4083 & 25.9183 & 25.7827 & 113.5625 \\
\hline $143 \mathrm{~B}$ & 8 & 448 & 24 & 18.7417 & 18.8017 & 24.1240 & 25.8852 & 25.8260 & 113.3785 \\
\hline $143 \mathrm{~B}$ & 9 & 449 & 24 & 18.7142 & 18.8219 & 24.6702 & 25.9019 & 25.8608 & 113.9690 \\
\hline $143 \mathrm{~B}$ & 10 & 450 & 24 & 18.6746 & 18.7477 & 24.7213 & 25.8960 & 25.8479 & 113.8875 \\
\hline $143 \mathrm{~B}$ & 11 & 451 & 24 & 18.6285 & 18.6717 & 24.6525 & 25.7796 & 25.7948 & 113.5271 \\
\hline $143 \mathrm{~B}$ & 12 & 452 & 24 & 18.5394 & 18.6463 & 24.4533 & 25.8021 & 25.7406 & 113.1817 \\
\hline $143 \mathrm{~B}$ & 13 & 453 & 24 & 18.5817 & 18.6288 & 24.1721 & 25.7754 & 25.7608 & 112.9188 \\
\hline $143 \mathrm{~B}$ & 14 & 454 & 24 & 18.4429 & 18.5633 & 24.2817 & 25.6427 & 25.5850 & 112.5156 \\
\hline $143 \mathrm{~B}$ & 15 & 455 & 24 & 18.4848 & 18.5290 & 24.8073 & 26.8304 & 25.6079 & 114.2594 \\
\hline $143 \mathrm{~B}$ & 16 & 456 & 24 & 18.5198 & 18.5456 & 25.4773 & 28.0775 & 25.5835 & 116.2038 \\
\hline $143 \mathrm{~B}$ & 17 & 457 & 24 & 18.6000 & 18.7406 & 25.5802 & 28.1373 & 25.7004 & 116.7585 \\
\hline $143 \mathrm{~B}$ & 18 & 458 & 24 & 18.5625 & 18.7010 & 25.3333 & 28.3360 & 25.8244 & 116.7573 \\
\hline $143 \mathrm{~B}$ & 19 & 459 & 24 & 18.5279 & 18.5065 & 25.4175 & 28.1169 & 25.6240 & 116.1927 \\
\hline $143 \mathrm{~B}$ & 20 & 460 & 24 & 18.5960 & 18.6646 & 25.6527 & 28.2088 & 25.6773 & 116.7994 \\
\hline $143 \mathrm{~B}$ & 21 & 461 & 24 & 18.5885 & 18.5308 & 25.3656 & 28.1685 & 25.7098 & 116.3633 \\
\hline $143 \mathrm{~B}$ & 22 & 462 & 24 & 18.5344 & 18.5256 & 25.2196 & 28.2129 & 25.6181 & 116.1106 \\
\hline $143 \mathrm{~B}$ & 23 & 463 & 24 & 18.5540 & 18.6827 & 25.6890 & 28.2515 & 25.6444 & 116.8215 \\
\hline
\end{tabular}




\begin{tabular}{|c|c|c|c|c|c|c|c|c|c|}
\hline Cycle & Timestep & $\begin{array}{l}\text { Cumulative } \\
\text { Timestep } \\
\end{array}$ & \begin{tabular}{|c|} 
Time \\
Interval \\
$(\mathrm{hrs})$
\end{tabular} & $\begin{array}{l}\text { NW } \\
\text { Lobe } \\
\text { Power } \\
(\mathrm{MW})\end{array}$ & $\begin{array}{c}\text { NE } \\
\text { Lobe } \\
\text { Power } \\
(\mathrm{MW})\end{array}$ & $\begin{array}{c}\text { C } \\
\text { Lobe } \\
\text { Power } \\
(\mathrm{MW}) \\
\end{array}$ & $\begin{array}{c}\text { SW } \\
\text { Lobe } \\
\text { Power } \\
\text { (MW) }\end{array}$ & $\begin{array}{c}\text { SE } \\
\text { Lobe } \\
\text { Power } \\
(\mathrm{MW}) \\
\end{array}$ & $\begin{array}{l}\text { Total } \\
\text { Core } \\
\text { Power } \\
(\mathrm{MW}) \\
\end{array}$ \\
\hline $143 \mathrm{~B}$ & 24 & 464 & 24 & 18.6319 & 18.6717 & 25.7150 & 28.3090 & 25.7298 & 117.0573 \\
\hline $143 \mathrm{~B}$ & 25 & 465 & 24 & 18.6100 & 18.6406 & 25.8031 & 28.2654 & 25.6223 & 116.9415 \\
\hline $143 \mathrm{~B}$ & 26 & 466 & 24 & 18.5515 & 18.5813 & 25.7719 & 28.2004 & 25.5692 & 116.6742 \\
\hline $143 \mathrm{~B}$ & 27 & 467 & 24 & 18.5656 & 18.6692 & 25.8465 & 28.3138 & 25.6127 & 117.0077 \\
\hline $143 \mathrm{~B}$ & 28 & 468 & 24 & 18.5856 & 18.7238 & 25.9054 & 28.2165 & 25.5904 & 117.0217 \\
\hline $143 \mathrm{~B}$ & 29 & 469 & 53 & 4.0182 & 4.0655 & 5.6000 & 6.0443 & 5.4994 & 25.2275 \\
\hline $143 \mathrm{~B}$ & 30 & 470 & 24 & 17.0663 & 17.1613 & 25.2500 & 25.8492 & 23.5446 & 108.8713 \\
\hline $143 \mathrm{~B}$ & 31 & 471 & 24 & 18.7963 & 18.8727 & 26.0544 & 28.4885 & 25.9602 & 118.1721 \\
\hline $143 \mathrm{~B}$ & 32 & 472 & 24 & 18.7708 & 18.7377 & 25.9152 & 28.3119 & 25.8723 & 117.6079 \\
\hline $143 \mathrm{~B}$ & 33 & 473 & 24 & 18.6013 & 18.7127 & 25.4169 & 28.2779 & 25.7258 & 116.7346 \\
\hline $143 \mathrm{~B}$ & 34 & 474 & 24 & 18.6050 & 18.7215 & 25.4567 & 28.1813 & 25.6923 & 116.6567 \\
\hline $143 \mathrm{~B}$ & 35 & 475 & 24 & 18.4610 & 18.5092 & 25.4165 & 28.1075 & 25.6360 & 116.1302 \\
\hline $143 \mathrm{~B}$ & 36 & 476 & 24 & 18.5144 & 18.5550 & 25.2546 & 28.1675 & 25.6304 & 116.1219 \\
\hline $143 \mathrm{~B}$ & 37 & 477 & 24 & 18.5965 & 18.5823 & 25.5094 & 28.2221 & 25.6248 & 116.5350 \\
\hline $143 \mathrm{~B}$ & 38 & 478 & 24 & 18.6117 & 18.6738 & 25.6200 & 28.2277 & 25.7019 & 116.8350 \\
\hline $143 \mathrm{~B}$ & 39 & 479 & 24 & 18.6729 & 18.7606 & 25.7698 & 28.2694 & 25.7650 & 117.2377 \\
\hline $143 \mathrm{~B}$ & 40 & 480 & 24 & 18.6858 & 18.7875 & 25.9981 & 28.2804 & 25.7608 & 117.5127 \\
\hline $143 \mathrm{~B}$ & 41 & 481 & 24 & 18.6910 & 18.8008 & 26.0110 & 28.2648 & 25.7465 & 117.5142 \\
\hline $143 \mathrm{~B}$ & 42 & 482 & 24 & 18.5900 & 18.6115 & 25.9490 & 28.1213 & 25.5729 & 116.8446 \\
\hline $143 \mathrm{~B}$ & 43 & 483 & 24 & 18.5463 & 18.6129 & 25.5021 & 28.1700 & 25.6367 & 116.4679 \\
\hline $143 \mathrm{~B}$ & 44 & 484 & 24 & 18.6306 & 18.6413 & 25.1292 & 28.2521 & 25.8077 & 116.4608 \\
\hline $143 \mathrm{~B}$ & 45 & 485 & 24 & 18.6740 & 18.7808 & 24.9252 & 28.3396 & 25.7348 & 116.4544 \\
\hline $143 \mathrm{~B}$ & 46 & 486 & 24 & 18.5154 & 18.6017 & 24.8502 & 28.1529 & 25.5223 & 115.6425 \\
\hline $143 \mathrm{~B}$ & 47 & 487 & 24 & 18.5506 & 18.6092 & 25.1540 & 28.1538 & 25.6877 & 116.1552 \\
\hline $143 \mathrm{~B}$ & 48 & 488 & 24 & 18.7069 & 18.7969 & 25.2198 & 28.2944 & 25.7700 & 116.7879 \\
\hline $143 \mathrm{~B}$ & 49 & 489 & 24 & 18.6429 & 18.7088 & 25.2098 & 28.2288 & 25.7200 & 116.5102 \\
\hline $143 \mathrm{~B}$ & 50 & 490 & 24 & 18.6840 & 18.7615 & 25.5404 & 28.2925 & 25.7656 & 117.0440 \\
\hline $143 \mathrm{~B}$ & 51 & 491 & 24 & 18.7006 & 18.7860 & 25.2581 & 28.3623 & 25.8454 & 116.9525 \\
\hline $143 \mathrm{~B}$ & 52 & 492 & 24 & 18.8110 & 18.7806 & 25.2738 & 27.6310 & 25.9108 & 116.4073 \\
\hline $143 \mathrm{~B}$ & 53 & 493 & 24 & 18.6906 & 18.7406 & 25.1902 & 28.2944 & 25.8242 & 116.7400 \\
\hline $143 \mathrm{~B}$ & 54 & 494 & 24 & 18.6254 & 18.7804 & 25.1621 & 28.1550 & 25.7669 & 116.4898 \\
\hline $143 \mathrm{~B}$ & 55 & 495 & 24 & 18.7510 & 18.8290 & 25.5154 & 28.4027 & 25.8471 & 117.3452 \\
\hline $143 \mathrm{~B}$ & 56 & 496 & 24 & 18.8733 & 18.8402 & 25.8383 & 28.4294 & 25.8710 & 117.8523 \\
\hline $143 \mathrm{~B}$ & 57 & 497 & 24 & 18.8610 & 18.9777 & 25.9321 & 28.4665 & 25.8950 & 118.1323 \\
\hline $143 \mathrm{~B}$ & 58 & 498 & 24 & 18.8967 & 19.0148 & 25.5810 & 28.5383 & 26.0106 & 118.0415 \\
\hline $143 \mathrm{~B}$ & 59 & 499 & 24 & 18.8256 & 18.9171 & 24.9742 & 28.2025 & 25.9294 & 116.8488 \\
\hline
\end{tabular}




\begin{tabular}{|c|c|c|c|c|c|c|c|c|c|}
\hline Cycle & Timestep & \begin{tabular}{|c} 
Cumulative \\
Timestep \\
\end{tabular} & \begin{tabular}{|c|} 
Time \\
Interval \\
$(\mathrm{hrs})$
\end{tabular} & $\begin{array}{l}\text { NW } \\
\text { Lobe } \\
\text { Power } \\
(\mathrm{MW})\end{array}$ & $\begin{array}{c}\text { NE } \\
\text { Lobe } \\
\text { Power } \\
(\mathrm{MW}) \\
\end{array}$ & $\begin{array}{c}\text { C } \\
\text { Lobe } \\
\text { Power } \\
(\mathrm{MW}) \\
\end{array}$ & $\begin{array}{c}\text { SW } \\
\text { Lobe } \\
\text { Power } \\
(\mathrm{MW})\end{array}$ & $\begin{array}{c}\text { SE } \\
\text { Lobe } \\
\text { Power } \\
\text { (MW) }\end{array}$ & $\begin{array}{l}\text { Total } \\
\text { Core } \\
\text { Power } \\
(\mathrm{MW}) \\
\end{array}$ \\
\hline $143 \mathrm{~B}$ & 60 & 500 & 9 & 18.8839 & 18.8878 & 24.6917 & 27.9400 & 25.8506 & 116.2539 \\
\hline $144 \mathrm{~A}$ & 1 & 501 & 8 & 1.3456 & 1.3350 & 1.8681 & 1.5938 & 1.6500 & 7.7925 \\
\hline $144 \mathrm{~A}$ & 2 & 502 & 24 & 16.4298 & 16.4338 & 21.7763 & 20.9210 & 22.6396 & 98.2004 \\
\hline $144 \mathrm{~A}$ & 3 & 503 & 24 & 18.3696 & 18.4035 & 23.5519 & 23.3248 & 25.2854 & 108.9352 \\
\hline $144 \mathrm{~A}$ & 4 & 504 & 24 & 18.4452 & 18.3933 & 23.5360 & 23.3894 & 25.3875 & 109.1515 \\
\hline $144 \mathrm{~A}$ & 5 & 505 & 24 & 18.3610 & 18.4252 & 23.3346 & 23.4079 & 25.3825 & 108.9113 \\
\hline $144 \mathrm{~A}$ & 6 & 506 & 24 & 18.3650 & 18.3977 & 22.7419 & 23.4206 & 25.4133 & 108.3385 \\
\hline $144 \mathrm{~A}$ & 7 & 507 & 24 & 18.4306 & 18.4963 & 23.0817 & 23.4700 & 25.5260 & 109.0046 \\
\hline $144 \mathrm{~A}$ & 8 & 508 & 24 & 18.4177 & 18.4573 & 22.9535 & 23.5433 & 25.5077 & 108.8796 \\
\hline $144 \mathrm{~A}$ & 9 & 509 & 24 & 18.4271 & 18.3950 & 22.9335 & 23.4267 & 25.4117 & 108.5940 \\
\hline $144 \mathrm{~A}$ & 10 & 510 & 24 & 18.4265 & 18.4621 & 22.9721 & 23.3892 & 25.5029 & 108.7527 \\
\hline $144 \mathrm{~A}$ & 11 & 511 & 24 & 18.4263 & 18.4360 & 22.9200 & 23.4783 & 25.5473 & 108.8079 \\
\hline $144 \mathrm{~A}$ & 12 & 512 & 24 & 18.3546 & 18.3948 & 23.0033 & 23.4444 & 25.4198 & 108.6169 \\
\hline $144 \mathrm{~A}$ & 13 & 513 & 24 & 18.3744 & 18.3844 & 23.0031 & 23.4790 & 25.4485 & 108.6894 \\
\hline $144 \mathrm{~A}$ & 14 & 514 & 24 & 18.3779 & 18.3977 & 23.2669 & 23.3900 & 25.3781 & 108.8106 \\
\hline $144 \mathrm{~A}$ & 15 & 515 & 24 & 18.3604 & 18.4113 & 23.4398 & 23.4852 & 25.4071 & 109.1038 \\
\hline $144 \mathrm{~A}$ & 16 & 516 & 24 & 18.3954 & 18.4319 & 23.4138 & 23.4494 & 25.4598 & 109.1502 \\
\hline $144 \mathrm{~A}$ & 17 & 517 & 24 & 18.3919 & 18.4704 & 23.3250 & 23.5102 & 25.5765 & 109.2740 \\
\hline $144 \mathrm{~A}$ & 18 & 518 & 24 & 18.2835 & 18.3600 & 23.0902 & 23.3375 & 25.3977 & 108.4690 \\
\hline $144 \mathrm{~A}$ & 19 & 519 & 24 & 18.3590 & 18.4519 & 23.1585 & 23.5394 & 25.4667 & 108.9754 \\
\hline $144 \mathrm{~A}$ & 20 & 520 & 24 & 18.2833 & 18.2758 & 22.9317 & 23.3779 & 25.4238 & 108.2925 \\
\hline $144 \mathrm{~A}$ & 21 & 521 & 24 & 18.2938 & 18.3548 & 22.9923 & 23.3371 & 25.3973 & 108.3752 \\
\hline $144 \mathrm{~A}$ & 22 & 522 & 24 & 18.3725 & 18.4142 & 23.2131 & 23.3898 & 25.5277 & 108.9173 \\
\hline $144 \mathrm{~A}$ & 23 & 523 & 24 & 18.3969 & 18.4596 & 23.1673 & 23.4998 & 25.5504 & 109.0740 \\
\hline $144 \mathrm{~A}$ & 24 & 524 & 24 & 18.2952 & 18.2823 & 23.2567 & 23.3621 & 25.3438 & 108.5400 \\
\hline $144 \mathrm{~A}$ & 25 & 525 & 24 & 18.4023 & 18.3579 & 23.2765 & 23.3973 & 25.3890 & 108.8229 \\
\hline $144 \mathrm{~A}$ & 26 & 526 & 24 & 18.3079 & 18.3315 & 23.3673 & 23.3398 & 25.3315 & 108.6779 \\
\hline $144 \mathrm{~A}$ & 27 & 527 & 24 & 18.3135 & 18.3692 & 23.5373 & 23.3483 & 25.3596 & 108.9279 \\
\hline $144 \mathrm{~A}$ & 28 & 528 & 24 & 18.3683 & 18.3067 & 23.4025 & 23.3890 & 25.3506 & 108.8171 \\
\hline $144 \mathrm{~A}$ & 29 & 529 & 24 & 18.3092 & 18.3435 & 23.3900 & 23.3071 & 25.3496 & 108.6994 \\
\hline $144 \mathrm{~A}$ & 30 & 530 & 24 & 18.3540 & 18.3988 & 23.2615 & 23.3596 & 25.4079 & 108.7817 \\
\hline $144 \mathrm{~A}$ & 31 & 531 & 24 & 18.3523 & 18.4069 & 23.5679 & 23.3883 & 25.4610 & 109.1765 \\
\hline $144 \mathrm{~A}$ & 32 & 532 & 24 & 18.4013 & 18.4410 & 23.6633 & 23.3508 & 25.4100 & 109.2665 \\
\hline $144 \mathrm{~A}$ & 33 & 533 & 24 & 17.5694 & 18.5890 & 23.6133 & 23.5260 & 25.5960 & 108.8938 \\
\hline $144 \mathrm{~A}$ & 34 & 534 & 24 & 18.3102 & 18.3381 & 23.3548 & 23.3667 & 25.3231 & 108.6929 \\
\hline $144 \mathrm{~A}$ & 35 & 535 & 24 & 18.2871 & 18.2560 & 23.3390 & 23.3188 & 25.2748 & 108.4756 \\
\hline
\end{tabular}




\begin{tabular}{|c|c|c|c|c|c|c|c|c|c|}
\hline Cycle & Timestep & $\begin{array}{l}\text { Cumulative } \\
\text { Timestep } \\
\end{array}$ & \begin{tabular}{|c|} 
Time \\
Interval \\
$(\mathrm{hrs})$
\end{tabular} & $\begin{array}{l}\text { NW } \\
\text { Lobe } \\
\text { Power } \\
(\mathrm{MW})\end{array}$ & $\begin{array}{c}\text { NE } \\
\text { Lobe } \\
\text { Power } \\
(\mathrm{MW}) \\
\end{array}$ & $\begin{array}{c}\text { C } \\
\text { Lobe } \\
\text { Power } \\
\text { (MW) }\end{array}$ & $\begin{array}{c}\text { SW } \\
\text { Lobe } \\
\text { Power } \\
\text { (MW) }\end{array}$ & $\begin{array}{c}\text { SE } \\
\text { Lobe } \\
\text { Power } \\
\text { (MW) }\end{array}$ & $\begin{array}{l}\text { Total } \\
\text { Core } \\
\text { Power } \\
(\mathrm{MW}) \\
\end{array}$ \\
\hline $144 \mathrm{~A}$ & 36 & 536 & 24 & 18.2650 & 18.2771 & 23.1413 & 23.3752 & 25.3600 & 108.4185 \\
\hline $144 \mathrm{~A}$ & 37 & 537 & 24 & 18.4250 & 18.5360 & 23.1998 & 23.4521 & 25.5442 & 109.1571 \\
\hline $144 \mathrm{~A}$ & 38 & 538 & 24 & 18.4073 & 18.4419 & 23.2344 & 23.5402 & 25.4060 & 109.0298 \\
\hline $144 \mathrm{~A}$ & 39 & 539 & 24 & 18.3521 & 18.4165 & 23.7813 & 23.3860 & 25.4358 & 109.3717 \\
\hline $144 \mathrm{~A}$ & 40 & 540 & 24 & 18.5523 & 18.5983 & 24.0538 & 23.5179 & 25.5427 & 110.2650 \\
\hline $144 \mathrm{~A}$ & 41 & 541 & 24 & 18.5242 & 18.5850 & 23.7925 & 23.6092 & 25.5308 & 110.0417 \\
\hline $144 \mathrm{~A}$ & 42 & 542 & 24 & 18.5850 & 18.6115 & 23.5177 & 23.5690 & 25.5254 & 109.8085 \\
\hline $144 \mathrm{~A}$ & 43 & 543 & 24 & 18.5285 & 18.4594 & 23.3621 & 23.5367 & 25.4540 & 109.3406 \\
\hline $144 \mathrm{~A}$ & 44 & 544 & 24 & 18.5954 & 18.5342 & 23.2660 & 23.5008 & 25.5354 & 109.4319 \\
\hline $144 \mathrm{~A}$ & 45 & 545 & 18 & 17.5428 & 17.5464 & 21.8222 & 22.3025 & 24.1983 & 103.4122 \\
\hline $144 \mathrm{~B}$ & 1 & 546 & 8 & 6.5194 & 6.5713 & 9.3038 & 8.3206 & 8.3038 & 39.0188 \\
\hline 144B & 2 & 547 & 24 & 18.4650 & 18.4729 & 23.5979 & 23.4042 & 23.4348 & 107.3748 \\
\hline 144B & 3 & 548 & 24 & 18.4588 & 18.5142 & 23.1083 & 22.8331 & 23.4500 & 106.3644 \\
\hline 144B & 4 & 549 & 24 & 18.4758 & 18.5360 & 23.4142 & 23.5471 & 23.5148 & 107.4879 \\
\hline $144 \mathrm{~B}$ & 5 & 550 & 24 & 18.5244 & 18.5798 & 23.4510 & 23.5860 & 23.6171 & 107.7583 \\
\hline $144 \mathrm{~B}$ & 6 & 551 & 24 & 18.4600 & 18.4252 & 23.0079 & 23.5594 & 23.4185 & 106.8710 \\
\hline $144 \mathrm{~B}$ & 7 & 552 & 24 & 18.4973 & 18.5294 & 22.9081 & 23.5375 & 23.4004 & 106.8727 \\
\hline $144 \mathrm{~B}$ & 8 & 553 & 24 & 18.5377 & 18.5790 & 22.8863 & 23.6450 & 23.5827 & 107.2306 \\
\hline $144 \mathrm{~B}$ & 9 & 554 & 24 & 18.4856 & 18.5063 & 22.7935 & 23.5685 & 23.5252 & 106.8792 \\
\hline $144 \mathrm{~B}$ & 10 & 555 & 24 & 18.3563 & 18.3538 & 22.8002 & 23.4229 & 23.3988 & 106.3319 \\
\hline $144 \mathrm{~B}$ & 11 & 556 & 24 & 18.4358 & 18.4831 & 23.1610 & 23.4725 & 23.4248 & 106.9773 \\
\hline $144 \mathrm{~B}$ & 12 & 557 & 24 & 18.3463 & 18.5710 & 23.4046 & 23.3777 & 23.3938 & 107.0933 \\
\hline $144 \mathrm{~B}$ & 13 & 558 & 24 & 18.4638 & 18.6773 & 23.4569 & 23.5460 & 23.5298 & 107.6738 \\
\hline 144B & 14 & 559 & 24 & 18.4329 & 18.4721 & 23.0846 & 23.5377 & 23.4448 & 106.9721 \\
\hline $144 \mathrm{~B}$ & 15 & 560 & 24 & 18.4165 & 18.5277 & 23.0467 & 23.5050 & 23.5221 & 107.0179 \\
\hline $144 \mathrm{~B}$ & 16 & 561 & 24 & 18.3242 & 18.4146 & 22.7994 & 23.5044 & 23.3865 & 106.4290 \\
\hline $144 \mathrm{~B}$ & 17 & 562 & 24 & 18.2348 & 18.3213 & 22.3346 & 23.3388 & 23.2473 & 105.4767 \\
\hline $144 \mathrm{~B}$ & 18 & 563 & 24 & 18.3396 & 18.3779 & 22.7633 & 23.3998 & 23.3725 & 106.2531 \\
\hline $144 \mathrm{~B}$ & 19 & 564 & 24 & 18.3602 & 18.4058 & 22.4754 & 23.4475 & 23.4398 & 106.1288 \\
\hline $144 \mathrm{~B}$ & 20 & 565 & 24 & 18.4023 & 18.4448 & 22.3333 & 23.4890 & 23.3894 & 106.0588 \\
\hline $144 \mathrm{~B}$ & 21 & 566 & 24 & 18.2742 & 18.4440 & 22.2531 & 23.3340 & 23.3375 & 105.6427 \\
\hline $144 \mathrm{~B}$ & 22 & 567 & 19 & 18.4297 & 18.4550 & 22.4263 & 23.5453 & 23.5484 & 106.4047 \\
\hline 144B & 23 & 568 & 66 & 0.0100 & 0.0100 & 0.0100 & 0.0100 & 0.0100 & 0.0500 \\
\hline $144 \mathrm{~B}$ & 24 & 569 & 19 & 14.9555 & 14.9911 & 19.5121 & 18.7800 & 18.9979 & 87.2366 \\
\hline $144 \mathrm{~B}$ & 25 & 570 & 24 & 18.4873 & 18.5729 & 22.3056 & 23.7077 & 23.6588 & 106.7323 \\
\hline $144 \mathrm{~B}$ & 26 & 571 & 24 & 18.4435 & 18.5419 & 22.0506 & 23.4646 & 23.5571 & 106.0577 \\
\hline
\end{tabular}




\begin{tabular}{|c|c|c|c|c|c|c|c|c|c|}
\hline Cycle & Timestep & $\begin{array}{l}\text { Cumulative } \\
\text { Timestep }\end{array}$ & \begin{tabular}{|c|} 
Time \\
Interval \\
$($ hrs $)$
\end{tabular} & $\begin{array}{l}\text { NW } \\
\text { Lobe } \\
\text { Power } \\
\text { (MW) }\end{array}$ & $\begin{array}{c}\text { NE } \\
\text { Lobe } \\
\text { Power } \\
(\mathrm{MW}) \\
\end{array}$ & $\begin{array}{c}\text { C } \\
\text { Lobe } \\
\text { Power } \\
(\mathrm{MW}) \\
\end{array}$ & $\begin{array}{c}\text { SW } \\
\text { Lobe } \\
\text { Power } \\
(\mathrm{MW})\end{array}$ & $\begin{array}{c}\text { SE } \\
\text { Lobe } \\
\text { Power } \\
\text { (MW) }\end{array}$ & $\begin{array}{l}\text { Total } \\
\text { Core } \\
\text { Power } \\
(\mathrm{MW}) \\
\end{array}$ \\
\hline $144 \mathrm{~B}$ & 27 & 572 & 24 & 18.3354 & 18.4013 & 22.3060 & 23.4663 & 23.3790 & 105.8879 \\
\hline $144 \mathrm{~B}$ & 28 & 573 & 24 & 18.1956 & 18.2773 & 21.9121 & 23.5015 & 23.3492 & 105.2356 \\
\hline $144 \mathrm{~B}$ & 29 & 574 & 24 & 18.3560 & 18.4167 & 21.7644 & 23.4552 & 23.3738 & 105.3660 \\
\hline $144 \mathrm{~B}$ & 30 & 575 & 24 & 18.3033 & 18.4081 & 21.4823 & 23.3506 & 23.4042 & 104.9485 \\
\hline $144 \mathrm{~B}$ & 31 & 576 & 24 & 18.4204 & 18.4969 & 21.8623 & 23.4567 & 23.4056 & 105.6419 \\
\hline 144B & 32 & 577 & 24 & 18.3456 & 18.3579 & 21.9435 & 23.4171 & 23.3867 & 105.4508 \\
\hline 144B & 33 & 578 & 24 & 18.2592 & 18.3085 & 21.6848 & 23.3440 & 23.3152 & 104.9117 \\
\hline $144 \mathrm{~B}$ & 34 & 579 & 24 & 18.2683 & 18.3696 & 21.9660 & 23.3719 & 23.3098 & 105.2856 \\
\hline $144 \mathrm{~B}$ & 35 & 580 & 24 & 18.4525 & 18.4783 & 22.0104 & 23.5658 & 23.4933 & 106.0004 \\
\hline $144 \mathrm{~B}$ & 36 & 581 & 24 & 18.3479 & 18.4113 & 22.1294 & 23.3746 & 23.3933 & 105.6565 \\
\hline $144 \mathrm{~B}$ & 37 & 582 & 24 & 18.4196 & 18.4196 & 22.3506 & 23.4742 & 23.4073 & 106.0713 \\
\hline 144B & 38 & 583 & 24 & 18.5544 & 18.5648 & 22.2131 & 23.6763 & 23.6725 & 106.6810 \\
\hline $144 \mathrm{~B}$ & 39 & 584 & 24 & 18.2892 & 18.3060 & 22.5475 & 23.3844 & 23.2967 & 105.8238 \\
\hline 144B & 40 & 585 & 24 & 18.3713 & 18.4219 & 22.5485 & 23.4529 & 23.4292 & 106.2238 \\
\hline $144 \mathrm{~B}$ & 41 & 586 & 24 & 18.3415 & 18.3531 & 22.3115 & 23.3790 & 23.2858 & 105.6708 \\
\hline $144 \mathrm{~B}$ & 42 & 587 & 24 & 18.3242 & 18.3765 & 22.4758 & 23.4073 & 23.3808 & 105.9646 \\
\hline $144 \mathrm{~B}$ & 43 & 588 & 24 & 18.4127 & 18.4448 & 22.5440 & 23.5021 & 23.4756 & 106.3792 \\
\hline $144 \mathrm{~B}$ & 44 & 589 & 24 & 18.4540 & 18.5610 & 22.4258 & 23.5217 & 23.4719 & 106.4344 \\
\hline $144 \mathrm{~B}$ & 45 & 590 & 24 & 18.3063 & 18.3148 & 22.1877 & 23.3544 & 23.3208 & 105.4840 \\
\hline $144 \mathrm{~B}$ & 46 & 591 & 24 & 18.2702 & 18.3054 & 21.9775 & 23.2925 & 23.2410 & 105.0867 \\
\hline $144 \mathrm{~B}$ & 47 & 592 & 24 & 18.3079 & 18.3731 & 21.9890 & 23.3750 & 23.3540 & 105.3990 \\
\hline $144 \mathrm{~B}$ & 48 & 593 & 24 & 18.2227 & 18.2385 & 22.0342 & 23.2723 & 23.2058 & 104.9735 \\
\hline $144 \mathrm{~B}$ & 49 & 594 & 24 & 18.1865 & 18.2104 & 22.3083 & 23.2727 & 23.1344 & 105.1123 \\
\hline $144 B$ & 50 & 595 & 24 & 18.3152 & 18.3048 & 23.3429 & 23.3808 & 23.3092 & 106.6529 \\
\hline $144 \mathrm{~B}$ & 51 & 596 & 24 & 18.3338 & 18.3608 & 23.2556 & 23.3825 & 23.3090 & 106.6417 \\
\hline $144 \mathrm{~B}$ & 52 & 597 & 24 & 18.3538 & 18.3954 & 23.1198 & 23.4644 & 23.3798 & 106.7131 \\
\hline $144 \mathrm{~B}$ & 53 & 598 & 24 & 18.3763 & 18.4550 & 23.3621 & 23.4035 & 23.4796 & 107.0765 \\
\hline $144 \mathrm{~B}$ & 54 & 599 & 27 & 18.3665 & 18.4180 & 23.4115 & 23.4804 & 23.4254 & 107.1017 \\
\hline $145 \mathrm{~A}$ & 1 & 600 & 11 & 8.2073 & 8.2059 & 12.2091 & 10.5105 & 11.3686 & 50.5014 \\
\hline $145 \mathrm{~A}$ & 2 & 601 & 11 & 16.8845 & 16.8591 & 22.5741 & 21.4732 & 22.9573 & 100.7482 \\
\hline $145 \mathrm{~A}$ & 3 & 602 & 24 & 17.3775 & 16.8573 & 21.9288 & 22.4356 & 23.8429 & 102.4421 \\
\hline $145 \mathrm{~A}$ & 4 & 603 & 24 & 17.4738 & 16.8542 & 21.7892 & 22.4950 & 23.8156 & 102.4277 \\
\hline $145 \mathrm{~A}$ & 5 & 604 & 24 & 17.8163 & 17.5242 & 22.5133 & 23.0585 & 24.4242 & 105.3365 \\
\hline $145 \mathrm{~A}$ & 6 & 605 & 24 & 18.3544 & 18.4052 & 23.2869 & 23.3696 & 25.3454 & 108.7615 \\
\hline $145 \mathrm{~A}$ & 7 & 606 & 24 & 18.3767 & 18.4221 & 23.3173 & 23.4750 & 25.4363 & 109.0273 \\
\hline $145 \mathrm{~A}$ & 8 & 607 & 24 & 18.3196 & 18.2642 & 23.1944 & 23.4023 & 25.3681 & 108.5485 \\
\hline
\end{tabular}




\begin{tabular}{|c|c|c|c|c|c|c|c|c|c|}
\hline Cycle & Timestep & $\begin{array}{l}\text { Cumulative } \\
\text { Timestep }\end{array}$ & $\begin{array}{c}\text { Time } \\
\text { Interval } \\
\text { (hrs) }\end{array}$ & $\begin{array}{l}\text { NW } \\
\text { Lobe } \\
\text { Power } \\
(\mathrm{MW})\end{array}$ & $\begin{array}{c}\text { NE } \\
\text { Lobe } \\
\text { Power } \\
\text { (MW) }\end{array}$ & $\begin{array}{c}\text { C } \\
\text { Lobe } \\
\text { Power } \\
(\mathrm{MW}) \\
\end{array}$ & $\begin{array}{c}\text { SW } \\
\text { Lobe } \\
\text { Power } \\
(\mathrm{MW})\end{array}$ & $\begin{array}{c}\text { SE } \\
\text { Lobe } \\
\text { Power } \\
(\mathrm{MW}) \\
\end{array}$ & $\begin{array}{l}\text { Total } \\
\text { Core } \\
\text { Power } \\
(\mathrm{MW}) \\
\end{array}$ \\
\hline $145 \mathrm{~A}$ & 9 & 608 & 24 & 18.2829 & 18.2667 & 23.1271 & 23.2800 & 25.2760 & 108.2327 \\
\hline $145 \mathrm{~A}$ & 10 & 609 & 24 & 18.2096 & 18.2023 & 22.8823 & 23.3031 & 25.2690 & 107.8663 \\
\hline $145 \mathrm{~A}$ & 11 & 610 & 24 & 18.1965 & 18.2144 & 22.7310 & 23.3023 & 25.2954 & 107.7396 \\
\hline $145 \mathrm{~A}$ & 12 & 611 & 24 & 18.2496 & 18.2444 & 22.9583 & 23.3131 & 25.3167 & 108.0821 \\
\hline $145 \mathrm{~A}$ & 13 & 612 & 24 & 18.3283 & 18.3229 & 23.2283 & 23.9271 & 25.3738 & 109.1804 \\
\hline $145 \mathrm{~A}$ & 14 & 613 & 24 & 18.2396 & 18.2294 & 23.4169 & 24.3317 & 26.0244 & 110.2419 \\
\hline $145 \mathrm{~A}$ & 15 & 614 & 24 & 18.2873 & 18.2456 & 23.6129 & 24.2633 & 26.1865 & 110.5956 \\
\hline $145 \mathrm{~A}$ & 16 & 615 & 24 & 18.1102 & 18.0156 & 23.4831 & 24.1131 & 25.9785 & 109.7006 \\
\hline $145 \mathrm{~A}$ & 17 & 616 & 24 & 18.2083 & 18.2919 & 23.4519 & 24.2888 & 26.3817 & 110.6225 \\
\hline $145 \mathrm{~A}$ & 18 & 617 & 24 & 18.3263 & 18.2885 & 23.4390 & 24.3798 & 26.3029 & 110.7365 \\
\hline $145 \mathrm{~A}$ & 19 & 618 & 24 & 18.2577 & 18.2527 & 23.3358 & 24.3198 & 26.3267 & 110.4927 \\
\hline $145 \mathrm{~A}$ & 20 & 619 & 24 & 18.2363 & 18.2448 & 23.1856 & 24.3406 & 26.3692 & 110.3765 \\
\hline $145 \mathrm{~A}$ & 21 & 620 & 24 & 18.2221 & 18.2088 & 23.2398 & 24.2575 & 26.3271 & 110.2552 \\
\hline $145 \mathrm{~A}$ & 22 & 621 & 24 & 18.0890 & 18.1075 & 23.3263 & 24.2365 & 26.3144 & 110.0735 \\
\hline $145 \mathrm{~A}$ & 23 & 622 & 24 & 18.0623 & 18.0602 & 23.5329 & 24.1027 & 26.1983 & 109.9565 \\
\hline $145 \mathrm{~A}$ & 24 & 623 & 24 & 18.1681 & 18.1619 & 23.3946 & 24.2125 & 26.1140 & 110.0510 \\
\hline $145 \mathrm{~A}$ & 25 & 624 & 24 & 18.1106 & 18.1300 & 23.2031 & 24.0381 & 26.1667 & 109.6485 \\
\hline $145 \mathrm{~A}$ & 26 & 625 & 8 & 18.1925 & 18.1063 & 23.0813 & 24.1256 & 26.2394 & 109.7450 \\
\hline $145 \mathrm{~A}$ & 27 & 626 & 43 & 0.0020 & 0.0020 & 0.0020 & 0.0020 & 0.0020 & 0.0100 \\
\hline $145 \mathrm{~A}$ & 28 & 627 & 12 & 11.5442 & 11.5050 & 16.4392 & 15.3771 & 16.6463 & 71.5117 \\
\hline $145 \mathrm{~A}$ & 29 & 628 & 10 & 18.2125 & 18.2335 & 24.3385 & 24.3300 & 26.3255 & 111.4400 \\
\hline $145 \mathrm{~A}$ & 30 & 629 & 24 & 18.2808 & 18.2883 & 23.3917 & 24.3542 & 26.3654 & 110.6804 \\
\hline $145 \mathrm{~A}$ & 31 & 630 & 24 & 18.2315 & 18.2435 & 23.1875 & 24.2079 & 26.2692 & 110.1396 \\
\hline $145 \mathrm{~A}$ & 32 & 631 & 24 & 18.2288 & 18.2763 & 23.1708 & 24.1863 & 26.3300 & 110.1921 \\
\hline $145 \mathrm{~A}$ & 33 & 632 & 24 & 18.2000 & 18.2802 & 23.0754 & 24.2913 & 26.2756 & 110.1225 \\
\hline $145 \mathrm{~A}$ & 34 & 633 & 24 & 18.1367 & 18.1902 & 23.2165 & 24.1260 & 26.1856 & 109.8550 \\
\hline $145 \mathrm{~A}$ & 35 & 634 & 25 & 18.1690 & 18.1780 & 23.0098 & 24.1870 & 26.1410 & 109.6848 \\
\hline $145 \mathrm{~A}$ & 36 & 635 & 60 & 0.0020 & 0.0020 & 0.0020 & 0.0020 & 0.0020 & 0.0100 \\
\hline $145 \mathrm{~A}$ & 37 & 636 & 11 & 11.7750 & 11.7509 & 16.6941 & 15.6864 & 16.7682 & 72.6745 \\
\hline $145 \mathrm{~A}$ & 38 & 637 & 24 & 18.2290 & 18.2196 & 23.7106 & 24.3113 & 26.3056 & 110.7760 \\
\hline $145 \mathrm{~A}$ & 39 & 638 & 18 & 18.2678 & 18.2944 & 23.3197 & 24.3261 & 26.3267 & 110.5347 \\
\hline $145 \mathrm{~A}$ & 40 & 639 & 58 & 0.0020 & 0.0020 & 0.0020 & 0.0020 & 0.0020 & 0.0100 \\
\hline $145 \mathrm{~A}$ & 41 & 640 & 8 & 6.2444 & 6.3575 & 9.0081 & 8.2100 & 8.8625 & 38.6825 \\
\hline $145 \mathrm{~A}$ & 42 & 641 & 12 & 18.1792 & 18.2133 & 24.9471 & 24.2150 & 26.2325 & 111.7871 \\
\hline $145 \mathrm{~A}$ & 43 & 642 & 24 & 18.3694 & 18.4017 & 23.5015 & 24.4750 & 26.5096 & 111.2571 \\
\hline $145 \mathrm{~A}$ & 44 & 643 & 24 & 18.4263 & 18.4760 & 23.1971 & 24.5042 & 26.5260 & 111.1296 \\
\hline
\end{tabular}




\begin{tabular}{|c|c|c|c|c|c|c|c|c|c|}
\hline & & Cumulative & $\begin{array}{c}\text { Time } \\
\text { Interval } \\
\text { Cyrs })\end{array}$ & $\begin{array}{c}\text { NW } \\
\text { Pobe } \\
\text { Power } \\
\text { (MW) }\end{array}$ & $\begin{array}{c}\text { NE } \\
\text { Lobe } \\
\text { Power } \\
\text { (MW) }\end{array}$ & $\begin{array}{c}\text { C } \\
\text { Lobe } \\
\text { Power } \\
\text { (MW) }\end{array}$ & $\begin{array}{c}\text { SW } \\
\text { Lobe } \\
\text { Power } \\
\text { (MW) }\end{array}$ & $\begin{array}{c}\text { SE } \\
\text { Lobe } \\
\text { Power } \\
\text { (MW) }\end{array}$ & $\begin{array}{c}\text { Total } \\
\text { Core } \\
\text { Power } \\
\text { (MW) }\end{array}$ \\
\hline 145A & 45 & 644 & 24 & 18.3106 & 18.3417 & 22.8935 & 24.3981 & 26.3148 & 110.2588 \\
\hline $145 \mathrm{~A}$ & 46 & 645 & 24 & 18.2675 & 18.2538 & 22.8154 & 24.2827 & 26.2696 & 109.8890 \\
\hline $145 \mathrm{~A}$ & 47 & 646 & 24 & 18.2458 & 18.2769 & 22.9352 & 24.2242 & 26.3121 & 109.9942 \\
\hline $145 \mathrm{~A}$ & 48 & 647 & 24 & 18.3015 & 18.2815 & 23.0767 & 24.3027 & 26.2706 & 110.2329 \\
\hline $145 \mathrm{~A}$ & 49 & 648 & 24 & 18.2333 & 18.2725 & 23.2875 & 24.2377 & 26.2944 & 110.3254 \\
\hline $145 \mathrm{~A}$ & 50 & 649 & 24 & 18.2544 & 18.3077 & 23.2142 & 24.3075 & 26.4300 & 110.5138 \\
\hline $145 \mathrm{~A}$ & 51 & 650 & 24 & 18.2183 & 18.2363 & 23.4431 & 24.1825 & 26.2425 & 110.3227 \\
\hline $145 \mathrm{~A}$ & 52 & 651 & 24 & 18.2531 & 18.2377 & 23.3048 & 24.2640 & 26.2698 & 110.3294 \\
\hline $145 \mathrm{~A}$ & 53 & 652 & 24 & 18.2319 & 18.1958 & 23.3644 & 24.2269 & 26.1627 & 110.1817 \\
\hline $145 \mathrm{~A}$ & 54 & 653 & 24 & 18.2258 & 18.2385 & 23.6521 & 24.2258 & 26.2727 & 110.6150 \\
\hline $145 \mathrm{~A}$ & 55 & 654 & 24 & 18.2156 & 18.2440 & 23.7102 & 24.2792 & 26.3044 & 110.7533 \\
\hline $145 \mathrm{~A}$ & 56 & 655 & 24 & 18.2463 & 18.2385 & 23.5346 & 24.2288 & 26.2531 & 110.5013 \\
\hline $145 \mathrm{~A}$ & 57 & 656 & 24 & 18.2352 & 18.2371 & 23.7621 & 24.2294 & 26.2844 & 110.7481 \\
\hline $145 \mathrm{~A}$ & 58 & 657 & 24 & 18.2056 & 18.2490 & 23.6085 & 24.2129 & 26.3417 & 110.6177 \\
\hline $145 \mathrm{~A}$ & 59 & 658 & 24 & 18.0825 & 18.1085 & 23.2790 & 24.0456 & 26.1263 & 109.6419 \\
\hline $145 \mathrm{~A}$ & 60 & 659 & 24 & 18.2313 & 18.2133 & 23.8140 & 24.1817 & 26.1538 & 110.5940 \\
\hline $145 \mathrm{~A}$ & 61 & 660 & 24 & 18.1408 & 18.1413 & 23.7085 & 24.0531 & 26.1356 & 110.1794 \\
\hline $145 \mathrm{~A}$ & 62 & 661 & 24 & 18.1558 & 18.1425 & 23.7458 & 24.0515 & 26.2663 & 110.3619 \\
\hline $145 \mathrm{~A}$ & 63 & 662 & 29 & 18.2024 & 18.2159 & 23.6090 & 24.1162 & 26.2064 & 110.3498 \\
\hline
\end{tabular}




\section{Appendix B}

\section{ATR Outer Shim Control Cylinder Positions by Cycle and Timestep}

\begin{tabular}{|c|c|c|c|c|c|c|c|}
\hline Cycle & Timestep & $\begin{array}{c}\text { Cumulative } \\
\text { Timestep }\end{array}$ & $\begin{array}{c}\text { Time } \\
\text { Interval } \\
\text { (hrs) }\end{array}$ & $\begin{array}{c}\text { NW } \\
\text { OSCC } \\
\text { (degrees) }\end{array}$ & $\begin{array}{c}\text { SW } \\
\text { OSCC } \\
\text { (degrees) }\end{array}$ & $\begin{array}{c}\mathrm{NE} \\
\text { OSCC } \\
\text { (degrees) }\end{array}$ & $\begin{array}{c}\mathrm{SE} \\
\text { OSCC } \\
\text { (degrees) }\end{array}$ \\
\hline $138 \mathrm{~B}$ & 1 & 1 & 9 & 66.64 & 66.67 & 66.68 & 66.66 \\
\hline $138 \mathrm{~B}$ & 2 & 2 & 24 & 86.27 & 85.06 & 86.36 & 85.77 \\
\hline $138 \mathrm{~B}$ & 3 & 3 & 24 & 94.85 & 84.93 & 97.97 & 92.68 \\
\hline $138 \mathrm{~B}$ & 4 & 4 & 24 & 84.08 & 81.44 & 85.13 & 85.01 \\
\hline $138 \mathrm{~B}$ & 5 & 5 & 24 & 84.74 & 82.10 & 87.74 & 86.41 \\
\hline $138 \mathrm{~B}$ & 6 & 6 & 24 & 84.75 & 81.84 & 88.45 & 86.51 \\
\hline $138 \mathrm{~B}$ & 7 & 7 & 24 & 81.84 & 81.80 & 83.86 & 84.41 \\
\hline $138 \mathrm{~B}$ & 8 & 8 & 24 & 82.84 & 81.90 & 85.30 & 84.64 \\
\hline $138 \mathrm{~B}$ & 9 & 9 & 24 & 80.60 & 81.96 & 80.57 & 82.58 \\
\hline $138 \mathrm{~B}$ & 10 & 10 & 24 & 81.66 & 83.15 & 80.19 & 82.19 \\
\hline $138 \mathrm{~B}$ & 11 & 11 & 24 & 83.03 & 82.82 & 81.96 & 82.98 \\
\hline $138 \mathrm{~B}$ & 12 & 12 & 24 & 83.68 & 83.42 & 83.34 & 82.92 \\
\hline 138B & 13 & 13 & 24 & 84.98 & 83.97 & 84.71 & 84.06 \\
\hline 138B & 14 & 14 & 24 & 82.64 & 83.87 & 82.73 & 83.96 \\
\hline $138 \mathrm{~B}$ & 15 & 15 & 24 & 81.86 & 83.85 & 83.84 & 83.95 \\
\hline $138 \mathrm{~B}$ & 16 & 16 & 24 & 81.98 & 84.01 & 85.73 & 84.60 \\
\hline $138 \mathrm{~B}$ & 17 & 17 & 24 & 83.44 & 85.22 & 85.50 & 85.09 \\
\hline $138 \mathrm{~B}$ & 18 & 18 & 24 & 84.74 & 85.78 & 85.46 & 85.80 \\
\hline $138 \mathrm{~B}$ & 19 & 19 & 24 & 83.44 & 82.46 & 85.13 & 84.54 \\
\hline $138 \mathrm{~B}$ & 20 & 20 & 24 & 83.77 & 81.51 & 84.65 & 84.77 \\
\hline $138 \mathrm{~B}$ & 21 & 21 & 24 & 84.26 & 82.40 & 85.85 & 85.52 \\
\hline $138 \mathrm{~B}$ & 22 & 22 & 24 & 85.86 & 83.09 & 87.09 & 85.30 \\
\hline $138 \mathrm{~B}$ & 23 & 23 & 24 & 86.62 & 83.69 & 88.65 & 85.93 \\
\hline $138 \mathrm{~B}$ & 24 & 24 & 24 & 83.07 & 82.78 & 86.65 & 86.28 \\
\hline $138 \mathrm{~B}$ & 25 & 25 & 24 & 83.53 & 82.69 & 83.45 & 83.98 \\
\hline $138 \mathrm{~B}$ & 26 & 26 & 24 & 83.83 & 83.09 & 83.32 & 83.62 \\
\hline $138 \mathrm{~B}$ & 27 & 27 & 24 & 84.94 & 83.61 & 85.12 & 84.24 \\
\hline $138 \mathrm{~B}$ & 28 & 28 & 24 & 86.35 & 85.26 & 85.58 & 85.52 \\
\hline $138 \mathrm{~B}$ & 29 & 29 & 24 & 84.09 & 83.77 & 84.11 & 83.70 \\
\hline $138 \mathrm{~B}$ & 30 & 30 & 24 & 80.79 & 82.18 & 80.47 & 81.34 \\
\hline $138 \mathrm{~B}$ & 31 & 31 & 24 & 81.88 & 83.57 & 81.83 & 82.53 \\
\hline
\end{tabular}




\begin{tabular}{|c|c|c|c|c|c|c|c|}
\hline Cycle & Timestep & $\begin{array}{l}\text { Cumulative } \\
\text { Timestep }\end{array}$ & $\begin{array}{c}\text { Time } \\
\text { Interval } \\
\text { (hrs) }\end{array}$ & $\begin{array}{c}\text { NW } \\
\text { OSCC } \\
\text { (degrees) }\end{array}$ & $\begin{array}{c}\text { SW } \\
\text { OSCC } \\
\text { (degrees) }\end{array}$ & $\begin{array}{c}\mathrm{NE} \\
\text { OSCC } \\
\text { (degrees) }\end{array}$ & $\begin{array}{c}\mathrm{SE} \\
\text { OSCC } \\
\text { (degrees) }\end{array}$ \\
\hline $138 \mathrm{~B}$ & 32 & 32 & 24 & 83.52 & 85.09 & 82.80 & 83.19 \\
\hline $138 \mathrm{~B}$ & 33 & 33 & 24 & 84.44 & 85.96 & 83.61 & 83.99 \\
\hline $138 \mathrm{~B}$ & 34 & 34 & 24 & 84.35 & 84.16 & 83.99 & 84.75 \\
\hline $138 \mathrm{~B}$ & 35 & 35 & 24 & 85.19 & 83.62 & 84.39 & 85.41 \\
\hline $138 \mathrm{~B}$ & 36 & 36 & 24 & 86.78 & 84.53 & 85.41 & 85.88 \\
\hline $138 \mathrm{~B}$ & 37 & 37 & 24 & 82.86 & 83.23 & 82.77 & 85.00 \\
\hline $138 \mathrm{~B}$ & 38 & 38 & 24 & 81.96 & 82.38 & 81.07 & 82.87 \\
\hline $138 \mathrm{~B}$ & 39 & 39 & 24 & 83.44 & 83.91 & 83.31 & 84.54 \\
\hline $138 \mathrm{~B}$ & 40 & 40 & 24 & 85.04 & 85.25 & 84.31 & 85.30 \\
\hline $138 \mathrm{~B}$ & 41 & 41 & 24 & 86.73 & 86.84 & 84.49 & 86.60 \\
\hline $138 \mathrm{~B}$ & 42 & 42 & 24 & 86.10 & 84.58 & 82.98 & 86.44 \\
\hline $138 \mathrm{~B}$ & 43 & 43 & 24 & 87.21 & 86.58 & 84.83 & 87.84 \\
\hline $138 \mathrm{~B}$ & 44 & 44 & 24 & 89.83 & 88.20 & 88.45 & 88.60 \\
\hline $138 \mathrm{~B}$ & 45 & 45 & 24 & 90.96 & 89.57 & 89.44 & 89.72 \\
\hline $138 \mathrm{~B}$ & 46 & 46 & 24 & 93.67 & 91.11 & 92.47 & 92.32 \\
\hline $138 \mathrm{~B}$ & 47 & 47 & 24 & 95.46 & 92.41 & 94.21 & 94.42 \\
\hline $138 \mathrm{~B}$ & 48 & 48 & 24 & 97.77 & 94.33 & 96.37 & 95.80 \\
\hline $138 \mathrm{~B}$ & 49 & 49 & 9 & 99.03 & 95.53 & 98.19 & 96.94 \\
\hline $139 \mathrm{~A}$ & 1 & 50 & 8 & 64.73 & 64.01 & 65.53 & 64.14 \\
\hline $139 \mathrm{~A}$ & 2 & 51 & 24 & 81.48 & 83.14 & 86.35 & 80.91 \\
\hline $139 \mathrm{~A}$ & 3 & 52 & 24 & 85.42 & 88.01 & 88.58 & 88.73 \\
\hline $139 \mathrm{~A}$ & 4 & 53 & 24 & 81.51 & 85.98 & 83.30 & 84.19 \\
\hline $139 \mathrm{~A}$ & 5 & 54 & 24 & 80.06 & 85.47 & 81.44 & 82.35 \\
\hline $139 \mathrm{~A}$ & 6 & 55 & 24 & 80.67 & 86.06 & 82.04 & 83.17 \\
\hline $139 \mathrm{~A}$ & 7 & 56 & 24 & 80.71 & 87.24 & 83.47 & 83.63 \\
\hline $139 \mathrm{~A}$ & 8 & 57 & 24 & 79.14 & 83.92 & 82.95 & 81.48 \\
\hline $139 \mathrm{~A}$ & 9 & 58 & 24 & 80.41 & 83.35 & 84.74 & 82.05 \\
\hline $139 \mathrm{~A}$ & 10 & 59 & 24 & 81.75 & 84.15 & 84.89 & 82.84 \\
\hline $139 \mathrm{~A}$ & 11 & 60 & 24 & 82.90 & 85.23 & 85.05 & 83.20 \\
\hline $139 \mathrm{~A}$ & 12 & 61 & 24 & 83.23 & 86.37 & 85.00 & 83.52 \\
\hline $139 \mathrm{~A}$ & 13 & 62 & 24 & 83.51 & 86.65 & 85.55 & 83.70 \\
\hline $139 \mathrm{~A}$ & 14 & 63 & 24 & 84.62 & 87.24 & 85.89 & 84.02 \\
\hline $139 \mathrm{~A}$ & 15 & 64 & 24 & 82.68 & 82.87 & 84.60 & 82.44 \\
\hline $139 \mathrm{~A}$ & 16 & 65 & 24 & 83.06 & 82.51 & 85.00 & 82.50 \\
\hline $139 \mathrm{~A}$ & 17 & 66 & 24 & 83.88 & 82.51 & 85.84 & 82.50 \\
\hline $139 \mathrm{~A}$ & 18 & 67 & 24 & 84.90 & 83.10 & 87.50 & 82.60 \\
\hline $139 \mathrm{~A}$ & 19 & 68 & 24 & 84.52 & 83.24 & 84.37 & 81.61 \\
\hline
\end{tabular}




\begin{tabular}{|c|c|c|c|c|c|c|c|}
\hline Cycle & Timestep & $\begin{array}{l}\text { Cumulative } \\
\text { Timestep }\end{array}$ & $\begin{array}{c}\text { Time } \\
\text { Interval } \\
\text { (hrs) }\end{array}$ & $\begin{array}{c}\text { NW } \\
\text { OSCC } \\
\text { (degrees) }\end{array}$ & $\begin{array}{c}\text { SW } \\
\text { OSCC } \\
\text { (degrees) }\end{array}$ & $\begin{array}{c}\mathrm{NE} \\
\text { OSCC } \\
\text { (degrees) }\end{array}$ & $\begin{array}{c}\mathrm{SE} \\
\text { OSCC } \\
\text { (degrees) }\end{array}$ \\
\hline $139 \mathrm{~A}$ & 20 & 69 & 24 & 83.89 & 82.82 & 81.66 & 80.45 \\
\hline $139 \mathrm{~A}$ & 21 & 70 & 24 & 85.26 & 83.54 & 84.06 & 81.00 \\
\hline $139 \mathrm{~A}$ & 22 & 71 & 24 & 86.42 & 84.31 & 85.32 & 82.02 \\
\hline $139 \mathrm{~A}$ & 23 & 72 & 24 & 29.00 & 28.45 & 28.69 & 27.78 \\
\hline $139 \mathrm{~A}$ & 24 & 73 & 24 & 29.00 & 28.45 & 28.69 & 27.78 \\
\hline $139 \mathrm{~A}$ & 25 & 74 & 24 & 29.00 & 28.45 & 28.69 & 27.78 \\
\hline $139 \mathrm{~A}$ & 26 & 75 & 24 & 71.67 & 68.81 & 71.41 & 69.62 \\
\hline $139 \mathrm{~A}$ & 27 & 76 & 24 & 85.48 & 83.89 & 85.15 & 82.91 \\
\hline $139 \mathrm{~A}$ & 28 & 77 & 24 & 81.62 & 83.89 & 83.59 & 82.55 \\
\hline $139 \mathrm{~A}$ & 29 & 78 & 24 & 80.10 & 83.25 & 83.50 & 82.15 \\
\hline $139 \mathrm{~A}$ & 30 & 79 & 24 & 81.14 & 83.48 & 83.73 & 82.80 \\
\hline $139 \mathrm{~A}$ & 31 & 80 & 24 & 82.32 & 84.59 & 84.41 & 83.73 \\
\hline $139 \mathrm{~A}$ & 32 & 81 & 24 & 83.28 & 84.39 & 87.80 & 84.43 \\
\hline $139 \mathrm{~A}$ & 33 & 82 & 24 & 84.38 & 83.85 & 93.55 & 84.98 \\
\hline $139 \mathrm{~A}$ & 34 & 83 & 24 & 86.52 & 84.91 & 95.30 & 86.25 \\
\hline $139 \mathrm{~A}$ & 35 & 84 & 24 & 88.16 & 86.49 & 96.29 & 87.00 \\
\hline $139 \mathrm{~A}$ & 36 & 85 & 24 & 87.87 & 86.95 & 98.04 & 87.37 \\
\hline $139 \mathrm{~A}$ & 37 & 86 & 24 & 85.63 & 86.53 & 96.77 & 88.05 \\
\hline $139 \mathrm{~A}$ & 38 & 87 & 24 & 87.67 & 87.85 & 97.62 & 88.53 \\
\hline $139 \mathrm{~A}$ & 39 & 88 & 24 & 87.11 & 87.43 & 95.00 & 83.69 \\
\hline $139 \mathrm{~A}$ & 40 & 89 & 24 & 88.02 & 88.48 & 98.94 & 85.35 \\
\hline $139 \mathrm{~A}$ & 41 & 90 & 24 & 88.00 & 88.50 & 105.00 & 86.60 \\
\hline $139 \mathrm{~A}$ & 42 & 91 & 24 & 86.41 & 88.35 & 102.63 & 87.24 \\
\hline $139 \mathrm{~A}$ & 43 & 92 & 24 & 86.31 & 88.54 & 101.50 & 88.24 \\
\hline $139 \mathrm{~A}$ & 44 & 93 & 24 & 87.92 & 89.95 & 103.68 & 89.43 \\
\hline $139 \mathrm{~A}$ & 45 & 94 & 24 & 87.24 & 89.38 & 102.08 & 86.09 \\
\hline $139 \mathrm{~A}$ & 46 & 95 & 24 & 88.73 & 90.90 & 103.72 & 87.10 \\
\hline $139 \mathrm{~A}$ & 47 & 96 & 24 & 87.93 & 89.94 & 101.19 & 84.55 \\
\hline $139 \mathrm{~A}$ & 48 & 97 & 24 & 89.10 & 91.14 & 102.05 & 85.07 \\
\hline $139 \mathrm{~A}$ & 49 & 98 & 24 & 87.57 & 90.29 & 102.57 & 85.58 \\
\hline $139 \mathrm{~A}$ & 50 & 99 & 24 & 86.10 & 88.92 & 101.53 & 84.42 \\
\hline $139 \mathrm{~A}$ & 51 & 100 & 24 & 86.34 & 90.10 & 100.11 & 83.87 \\
\hline $139 \mathrm{~A}$ & 52 & 101 & 24 & 88.75 & 92.11 & 101.23 & 85.30 \\
\hline $139 \mathrm{~A}$ & 53 & 102 & 24 & 91.44 & 95.02 & 103.24 & 86.82 \\
\hline $139 \mathrm{~A}$ & 54 & 103 & 24 & 91.71 & 95.54 & 103.03 & 87.60 \\
\hline $139 \mathrm{~A}$ & 55 & 104 & 24 & 92.57 & 96.58 & 100.04 & 88.21 \\
\hline $139 \mathrm{~A}$ & 56 & 105 & 9 & 93.22 & 97.51 & 100.41 & 89.27 \\
\hline
\end{tabular}




\begin{tabular}{|c|c|c|c|c|c|c|c|}
\hline Cycle & Timestep & $\begin{array}{l}\text { Cumulative } \\
\text { Timestep }\end{array}$ & $\begin{array}{c}\text { Time } \\
\text { Interval } \\
\text { (hrs) }\end{array}$ & $\begin{array}{c}\text { NW } \\
\text { OSCC } \\
\text { (degrees) }\end{array}$ & $\begin{array}{c}\text { SW } \\
\text { OSCC } \\
\text { (degrees) }\end{array}$ & $\begin{array}{c}\mathrm{NE} \\
\text { OSCC } \\
\text { (degrees) }\end{array}$ & $\begin{array}{c}\mathrm{SE} \\
\text { OSCC } \\
\text { (degrees) }\end{array}$ \\
\hline 139B & 1 & 106 & 8 & 68.38 & 64.22 & 69.70 & 62.53 \\
\hline 139B & 2 & 107 & 24 & 91.03 & 83.89 & 94.36 & 76.57 \\
\hline 139B & 3 & 108 & 24 & 90.02 & 90.78 & 88.38 & 79.68 \\
\hline 139B & 4 & 109 & 24 & 89.36 & 90.12 & 87.60 & 80.32 \\
\hline 139B & 5 & 110 & 24 & 88.36 & 91.49 & 92.09 & 80.95 \\
\hline 139B & 6 & 111 & 24 & 82.01 & 87.80 & 89.39 & 78.93 \\
\hline $139 \mathrm{~B}$ & 7 & 112 & 24 & 81.19 & 85.82 & 89.81 & 78.93 \\
\hline $139 \mathrm{~B}$ & 8 & 113 & 24 & 82.35 & 86.74 & 90.69 & 79.73 \\
\hline 139B & 9 & 114 & 24 & 82.61 & 86.54 & 92.42 & 80.61 \\
\hline 139B & 10 & 115 & 24 & 84.11 & 85.46 & 93.79 & 81.16 \\
\hline 139B & 11 & 116 & 24 & 83.18 & 82.85 & 91.93 & 80.25 \\
\hline 139B & 12 & 117 & 24 & 84.42 & 83.93 & 92.15 & 81.12 \\
\hline 139B & 13 & 118 & 24 & 85.58 & 84.21 & 92.97 & 81.70 \\
\hline 139B & 14 & 119 & 24 & 86.71 & 84.63 & 93.78 & 82.46 \\
\hline 139B & 15 & 120 & 24 & 87.93 & 86.05 & 95.13 & 83.14 \\
\hline 139B & 16 & 121 & 24 & 84.65 & 85.12 & 93.59 & 83.12 \\
\hline 139B & 17 & 122 & 24 & 84.53 & 85.80 & 94.05 & 83.12 \\
\hline 139B & 18 & 123 & 24 & 82.81 & 81.62 & 93.85 & 81.73 \\
\hline 139B & 19 & 124 & 24 & 83.08 & 82.21 & 94.17 & 83.03 \\
\hline 139B & 20 & 125 & 24 & 80.10 & 81.64 & 93.00 & 82.68 \\
\hline 139B & 21 & 126 & 24 & 80.55 & 81.85 & 93.28 & 83.15 \\
\hline 139B & 22 & 127 & 24 & 82.16 & 82.13 & 93.98 & 82.86 \\
\hline 139B & 23 & 128 & 24 & 82.31 & 82.03 & 93.56 & 79.87 \\
\hline 139B & 24 & 129 & 24 & 84.28 & 83.15 & 95.46 & 80.67 \\
\hline 139B & 25 & 130 & 24 & 82.39 & 82.59 & 94.14 & 80.75 \\
\hline 139B & 26 & 131 & 24 & 81.04 & 82.54 & 92.33 & 81.02 \\
\hline 139B & 27 & 132 & 24 & 82.58 & 84.26 & 92.04 & 82.53 \\
\hline 139B & 28 & 133 & 24 & 83.57 & 85.62 & 93.22 & 83.46 \\
\hline 139B & 29 & 134 & 24 & 84.14 & 86.04 & 94.23 & 83.53 \\
\hline 139B & 30 & 135 & 24 & 84.27 & 84.73 & 93.50 & 79.68 \\
\hline 139B & 31 & 136 & 24 & 84.47 & 84.07 & 94.00 & 80.03 \\
\hline 139B & 32 & 137 & 24 & 82.66 & 82.02 & 92.93 & 79.12 \\
\hline 139B & 33 & 138 & 7 & 84.00 & 83.10 & 93.50 & 80.10 \\
\hline 139B & 34 & 139 & 1044 & 84.00 & 83.10 & 93.50 & 80.10 \\
\hline 139B & 35 & 140 & 13 & 54.90 & 54.94 & 54.74 & 54.48 \\
\hline 139B & 36 & 141 & 24 & 73.21 & 74.05 & 80.08 & 73.27 \\
\hline $139 \mathrm{~B}$ & 37 & 142 & 24 & 81.31 & 82.69 & 94.35 & 82.94 \\
\hline
\end{tabular}




\begin{tabular}{|c|c|c|c|c|c|c|c|}
\hline Cycle & Timestep & $\begin{array}{l}\text { Cumulative } \\
\text { Timestep }\end{array}$ & $\begin{array}{c}\text { Time } \\
\text { Interval } \\
\text { (hrs) }\end{array}$ & $\begin{array}{c}\text { NW } \\
\text { OSCC } \\
\text { (degrees) }\end{array}$ & $\begin{array}{c}\text { SW } \\
\text { OSCC } \\
\text { (degrees) }\end{array}$ & $\begin{array}{c}\mathrm{NE} \\
\text { OSCC } \\
\text { (degrees) }\end{array}$ & $\begin{array}{c}\mathrm{SE} \\
\text { OSCC } \\
\text { (degrees) }\end{array}$ \\
\hline 139B & 38 & 143 & 24 & 81.48 & 82.38 & 94.79 & 83.40 \\
\hline 139B & 39 & 144 & 24 & 82.00 & 82.73 & 94.50 & 83.97 \\
\hline 139B & 40 & 145 & 24 & 82.35 & 82.53 & 93.04 & 82.45 \\
\hline 139B & 41 & 146 & 24 & 83.03 & 83.45 & 94.17 & 82.23 \\
\hline 139B & 42 & 147 & 24 & 83.84 & 83.53 & 95.40 & 82.23 \\
\hline 139B & 43 & 148 & 24 & 84.35 & 84.18 & 94.53 & 81.43 \\
\hline $139 \mathrm{~B}$ & 44 & 149 & 24 & 87.01 & 85.91 & 95.50 & 83.33 \\
\hline $139 \mathrm{~B}$ & 45 & 150 & 24 & 86.57 & 85.23 & 96.59 & 82.00 \\
\hline 139B & 46 & 151 & 24 & 87.85 & 85.76 & 96.01 & 81.59 \\
\hline 139B & 47 & 152 & 24 & 90.29 & 87.78 & 97.85 & 83.76 \\
\hline 139B & 48 & 153 & 24 & 91.95 & 89.55 & 98.93 & 85.64 \\
\hline 139B & 49 & 154 & 24 & 94.64 & 93.39 & 102.53 & 88.31 \\
\hline 139B & 50 & 155 & 24 & 100.61 & 99.47 & 111.07 & 94.88 \\
\hline 139B & 51 & 156 & 24 & 102.08 & 101.64 & 113.07 & 96.22 \\
\hline 139B & 52 & 157 & 24 & 104.26 & 104.04 & 116.15 & 98.13 \\
\hline 139B & 53 & 158 & 24 & 106.94 & 106.53 & 117.93 & 100.48 \\
\hline 139B & 54 & 159 & 24 & 108.13 & 107.35 & 124.06 & 102.19 \\
\hline 139B & 55 & 160 & 21 & 110.80 & 110.72 & 124.60 & 104.17 \\
\hline $140 \mathrm{~A}$ & 1 & 161 & 8 & 70.10 & 69.58 & 71.02 & 69.47 \\
\hline $140 \mathrm{~A}$ & 2 & 162 & 24 & 84.75 & 86.89 & 93.16 & 83.09 \\
\hline $140 \mathrm{~A}$ & 3 & 163 & 24 & 86.06 & 91.72 & 91.23 & 86.81 \\
\hline $140 \mathrm{~A}$ & 4 & 164 & 24 & 85.52 & 93.08 & 91.86 & 88.47 \\
\hline $140 \mathrm{~A}$ & 5 & 165 & 24 & 85.88 & 93.30 & 91.87 & 89.20 \\
\hline $140 \mathrm{~A}$ & 6 & 166 & 24 & 82.93 & 89.81 & 88.44 & 88.95 \\
\hline $140 \mathrm{~A}$ & 7 & 167 & 24 & 83.48 & 89.53 & 88.93 & 89.96 \\
\hline $140 \mathrm{~A}$ & 8 & 168 & 24 & 84.32 & 92.04 & 87.56 & 91.58 \\
\hline $140 \mathrm{~A}$ & 9 & 169 & 24 & 84.38 & 92.43 & 87.51 & 92.88 \\
\hline $140 \mathrm{~A}$ & 10 & 170 & 24 & 85.97 & 93.08 & 88.52 & 93.63 \\
\hline $140 \mathrm{~A}$ & 11 & 171 & 24 & 85.36 & 90.90 & 88.81 & 89.78 \\
\hline $140 \mathrm{~A}$ & 12 & 172 & 24 & 84.22 & 88.20 & 87.40 & 86.11 \\
\hline $140 \mathrm{~A}$ & 13 & 173 & 24 & 85.50 & 88.57 & 89.07 & 86.79 \\
\hline $140 \mathrm{~A}$ & 14 & 174 & 24 & 87.20 & 88.96 & 89.89 & 87.24 \\
\hline $140 \mathrm{~A}$ & 15 & 175 & 24 & 88.00 & 89.42 & 90.35 & 87.80 \\
\hline $140 \mathrm{~A}$ & 16 & 176 & 24 & 88.61 & 90.76 & 90.70 & 88.06 \\
\hline $140 \mathrm{~A}$ & 17 & 177 & 24 & 90.96 & 90.47 & 92.15 & 89.15 \\
\hline $140 \mathrm{~A}$ & 18 & 178 & 24 & 92.00 & 91.20 & 93.36 & 89.50 \\
\hline $140 \mathrm{~A}$ & 19 & 179 & 24 & 92.88 & 92.00 & 94.24 & 90.03 \\
\hline
\end{tabular}




\begin{tabular}{|c|c|c|c|c|c|c|c|}
\hline Cycle & Timestep & $\begin{array}{l}\text { Cumulative } \\
\text { Timestep }\end{array}$ & $\begin{array}{c}\text { Time } \\
\text { Interval } \\
\text { (hrs) }\end{array}$ & $\begin{array}{c}\text { NW } \\
\text { OSCC } \\
\text { (degrees) }\end{array}$ & $\begin{array}{c}\text { SW } \\
\text { OSCC } \\
\text { (degrees) }\end{array}$ & $\begin{array}{c}\mathrm{NE} \\
\text { OSCC } \\
\text { (degrees) }\end{array}$ & $\begin{array}{c}\mathrm{SE} \\
\text { OSCC } \\
\text { (degrees) }\end{array}$ \\
\hline $140 \mathrm{~A}$ & 20 & 180 & 24 & 90.89 & 90.56 & 93.34 & 89.31 \\
\hline $140 \mathrm{~A}$ & 21 & 181 & 24 & 86.28 & 86.80 & 90.43 & 87.56 \\
\hline $140 \mathrm{~A}$ & 22 & 182 & 24 & 87.48 & 88.73 & 91.34 & 89.03 \\
\hline $140 \mathrm{~A}$ & 23 & 183 & 24 & 88.72 & 89.58 & 91.96 & 89.59 \\
\hline $140 \mathrm{~A}$ & 24 & 184 & 24 & 89.55 & 90.07 & 92.71 & 90.17 \\
\hline $140 \mathrm{~A}$ & 25 & 185 & 24 & 90.93 & 90.67 & 93.83 & 91.07 \\
\hline $140 \mathrm{~A}$ & 26 & 186 & 24 & 89.57 & 89.55 & 92.16 & 84.96 \\
\hline $140 \mathrm{~A}$ & 27 & 187 & 24 & 90.34 & 90.07 & 93.38 & 85.94 \\
\hline $140 \mathrm{~A}$ & 28 & 188 & 24 & 92.50 & 91.62 & 94.83 & 87.09 \\
\hline $140 \mathrm{~A}$ & 29 & 189 & 24 & 89.24 & 89.99 & 93.18 & 85.63 \\
\hline $140 \mathrm{~A}$ & 30 & 190 & 24 & 87.12 & 88.19 & 92.20 & 84.81 \\
\hline $140 \mathrm{~A}$ & 31 & 191 & 24 & 88.45 & 88.88 & 93.55 & 86.50 \\
\hline $140 \mathrm{~A}$ & 32 & 192 & 24 & 88.91 & 89.22 & 94.46 & 86.75 \\
\hline $140 \mathrm{~A}$ & 33 & 193 & 24 & 86.32 & 89.60 & 92.30 & 86.87 \\
\hline $140 \mathrm{~A}$ & 34 & 194 & 24 & 87.34 & 90.16 & 93.29 & 87.86 \\
\hline $140 \mathrm{~A}$ & 35 & 195 & 24 & 87.40 & 90.40 & 92.66 & 85.46 \\
\hline $140 \mathrm{~A}$ & 36 & 196 & 24 & 88.90 & 91.39 & 93.38 & 85.09 \\
\hline $140 \mathrm{~A}$ & 37 & 197 & 24 & 89.29 & 92.58 & 93.89 & 86.64 \\
\hline $140 \mathrm{~A}$ & 38 & 198 & 24 & 86.73 & 93.43 & 91.56 & 87.08 \\
\hline $140 \mathrm{~A}$ & 39 & 199 & 24 & 86.70 & 93.61 & 91.74 & 86.58 \\
\hline $140 \mathrm{~A}$ & 40 & 200 & 24 & 86.31 & 93.19 & 88.50 & 84.11 \\
\hline $140 \mathrm{~A}$ & 41 & 201 & 24 & 88.83 & 93.81 & 92.07 & 85.63 \\
\hline $140 \mathrm{~A}$ & 42 & 202 & 24 & 88.74 & 95.50 & 92.43 & 86.14 \\
\hline $140 \mathrm{~A}$ & 43 & 203 & 24 & 87.00 & 95.54 & 90.08 & 86.75 \\
\hline $140 \mathrm{~A}$ & 44 & 204 & 24 & 89.65 & 97.62 & 92.42 & 88.27 \\
\hline $140 \mathrm{~A}$ & 45 & 205 & 24 & 89.44 & 98.77 & 92.26 & 86.83 \\
\hline $140 \mathrm{~A}$ & 46 & 206 & 24 & 89.12 & 98.74 & 91.63 & 86.10 \\
\hline $140 \mathrm{~A}$ & 47 & 207 & 24 & 90.73 & 100.70 & 93.08 & 87.64 \\
\hline $140 \mathrm{~A}$ & 48 & 208 & 11 & 92.13 & 102.31 & 93.87 & 88.96 \\
\hline 140B & 1 & 209 & 8 & 79.11 & 79.13 & 79.14 & 79.14 \\
\hline 140B & 2 & 210 & 24 & 93.10 & 95.13 & 90.85 & 89.07 \\
\hline 140B & 3 & 211 & 24 & 90.93 & 93.68 & 89.14 & 90.28 \\
\hline 140B & 4 & 212 & 24 & 88.99 & 93.80 & 83.26 & 88.08 \\
\hline 140B & 5 & 213 & 24 & 89.28 & 95.30 & 83.54 & 88.74 \\
\hline 140B & 6 & 214 & 24 & 89.39 & 96.14 & 84.35 & 89.53 \\
\hline 140B & 7 & 215 & 24 & 90.99 & 97.04 & 86.49 & 91.23 \\
\hline $140 \mathrm{~B}$ & 8 & 216 & 24 & 92.17 & 98.93 & 88.22 & 92.26 \\
\hline
\end{tabular}




\begin{tabular}{|c|c|c|c|c|c|c|c|}
\hline Cycle & Timestep & $\begin{array}{c}\text { Cumulative } \\
\text { Timestep }\end{array}$ & $\begin{array}{c}\text { Time } \\
\text { Interval } \\
\text { (hrs) }\end{array}$ & $\begin{array}{c}\text { NW } \\
\text { OSCC } \\
\text { (degrees) }\end{array}$ & $\begin{array}{c}\text { SW } \\
\text { OSCC } \\
\text { (degrees) }\end{array}$ & $\begin{array}{c}\mathrm{NE} \\
\text { OSCC } \\
\text { (degrees) }\end{array}$ & $\begin{array}{c}\mathrm{SE} \\
\text { OSCC } \\
\text { (degrees) }\end{array}$ \\
\hline 140B & 9 & 217 & 24 & 89.02 & 97.26 & 85.58 & 87.34 \\
\hline 140B & 10 & 218 & 24 & 83.00 & 95.84 & 82.58 & 85.89 \\
\hline 140B & 11 & 219 & 24 & 82.69 & 96.50 & 81.58 & 86.88 \\
\hline 140B & 12 & 220 & 24 & 84.13 & 98.53 & 83.15 & 87.72 \\
\hline 140B & 13 & 221 & 24 & 84.13 & 98.40 & 81.64 & 85.35 \\
\hline 140B & 14 & 222 & 24 & 85.21 & 99.26 & 81.11 & 84.75 \\
\hline $140 \mathrm{~B}$ & 15 & 223 & 24 & 86.17 & 100.28 & 81.86 & 85.55 \\
\hline 140B & 16 & 224 & 24 & 87.18 & 101.56 & 83.51 & 86.88 \\
\hline 140B & 17 & 225 & 24 & 88.68 & 103.12 & 85.50 & 87.71 \\
\hline 140B & 18 & 226 & 24 & 89.73 & 104.29 & 85.74 & 88.73 \\
\hline $140 \mathrm{~B}$ & 19 & 227 & 24 & 85.77 & 102.62 & 82.90 & 88.46 \\
\hline 140B & 20 & 228 & 24 & 87.11 & 104.64 & 84.12 & 89.11 \\
\hline 140B & 21 & 229 & 24 & 88.30 & 106.28 & 85.48 & 90.72 \\
\hline 140B & 22 & 230 & 24 & 89.57 & 108.11 & 86.79 & 92.06 \\
\hline $140 \mathrm{~B}$ & 23 & 231 & 24 & 92.62 & 109.54 & 87.76 & 92.68 \\
\hline 140B & 24 & 232 & 24 & 93.30 & 112.56 & 89.05 & 94.07 \\
\hline 140B & 25 & 233 & 24 & 93.70 & 113.81 & 89.47 & 94.09 \\
\hline 140B & 26 & 234 & 19 & 93.39 & 109.03 & 95.22 & 94.21 \\
\hline 140B & 27 & 235 & 185 & 93.39 & 109.03 & 95.22 & 94.21 \\
\hline 140B & 28 & 236 & 11 & 93.31 & 109.36 & 94.80 & 94.32 \\
\hline $140 \mathrm{~B}$ & 29 & 237 & 24 & 93.42 & 108.95 & 94.32 & 93.41 \\
\hline $140 \mathrm{~B}$ & 30 & 238 & 24 & 92.62 & 107.28 & 92.91 & 90.71 \\
\hline 140B & 31 & 239 & 24 & 94.35 & 110.73 & 94.16 & 91.57 \\
\hline 140B & 32 & 240 & 24 & 92.83 & 111.72 & 95.09 & 92.67 \\
\hline 140B & 33 & 241 & 24 & 90.54 & 109.75 & 93.57 & 92.50 \\
\hline 140B & 34 & 242 & 24 & 90.79 & 110.89 & 91.78 & 90.43 \\
\hline 140B & 35 & 243 & 24 & 93.26 & 114.59 & 93.77 & 93.12 \\
\hline 140B & 36 & 244 & 24 & 95.00 & 117.35 & 94.87 & 94.89 \\
\hline $140 \mathrm{~B}$ & 37 & 245 & 13 & 96.56 & 120.37 & 97.30 & 96.48 \\
\hline $141 \mathrm{~A}$ & 1 & 246 & 8 & 75.64 & 75.84 & 76.34 & 71.63 \\
\hline $141 \mathrm{~A}$ & 2 & 247 & 24 & 87.58 & 89.87 & 89.99 & 85.06 \\
\hline $141 \mathrm{~A}$ & 3 & 248 & 24 & 88.79 & 93.75 & 92.25 & 87.89 \\
\hline $141 \mathrm{~A}$ & 4 & 249 & 24 & 84.76 & 88.26 & 84.41 & 82.53 \\
\hline $141 \mathrm{~A}$ & 5 & 250 & 24 & 86.93 & 90.80 & 87.51 & 84.84 \\
\hline $141 \mathrm{~A}$ & 6 & 251 & 24 & 86.97 & 90.12 & 88.69 & 84.96 \\
\hline $141 \mathrm{~A}$ & 7 & 252 & 24 & 85.21 & 88.84 & 87.80 & 84.53 \\
\hline $141 \mathrm{~A}$ & 8 & 253 & 24 & 86.57 & 88.81 & 89.81 & 85.18 \\
\hline
\end{tabular}




\begin{tabular}{|c|c|c|c|c|c|c|c|}
\hline Cycle & Timestep & $\begin{array}{l}\text { Cumulative } \\
\text { Timestep }\end{array}$ & $\begin{array}{c}\text { Time } \\
\text { Interval } \\
\text { (hrs) }\end{array}$ & $\begin{array}{c}\text { NW } \\
\text { OSCC } \\
\text { (degrees) }\end{array}$ & $\begin{array}{c}\text { SW } \\
\text { OSCC } \\
\text { (degrees) }\end{array}$ & $\begin{array}{c}\mathrm{NE} \\
\text { OSCC } \\
\text { (degrees) }\end{array}$ & $\begin{array}{c}\mathrm{SE} \\
\text { OSCC } \\
\text { (degrees) }\end{array}$ \\
\hline $141 \mathrm{~A}$ & 9 & 254 & 24 & 87.93 & 89.90 & 92.03 & 86.50 \\
\hline $141 \mathrm{~A}$ & 10 & 255 & 24 & 89.58 & 90.81 & 92.82 & 87.30 \\
\hline $141 \mathrm{~A}$ & 11 & 256 & 24 & 91.13 & 92.64 & 95.04 & 88.14 \\
\hline $141 \mathrm{~A}$ & 12 & 257 & 24 & 91.37 & 93.30 & 95.81 & 88.58 \\
\hline $141 \mathrm{~A}$ & 13 & 258 & 24 & 92.61 & 93.64 & 97.80 & 89.53 \\
\hline $141 \mathrm{~A}$ & 14 & 259 & 24 & 94.05 & 94.56 & 99.84 & 90.01 \\
\hline $141 \mathrm{~A}$ & 15 & 260 & 24 & 95.66 & 95.58 & 100.94 & 91.09 \\
\hline $141 \mathrm{~A}$ & 16 & 261 & 24 & 96.13 & 94.18 & 100.27 & 87.18 \\
\hline $141 \mathrm{~A}$ & 17 & 262 & 24 & 96.60 & 94.48 & 100.54 & 86.18 \\
\hline $141 \mathrm{~A}$ & 18 & 263 & 24 & 98.18 & 95.63 & 101.20 & 86.93 \\
\hline $141 \mathrm{~A}$ & 19 & 264 & 24 & 93.48 & 93.86 & 95.60 & 85.62 \\
\hline $141 \mathrm{~A}$ & 20 & 265 & 24 & 87.03 & 93.35 & 90.86 & 84.04 \\
\hline $141 \mathrm{~A}$ & 21 & 266 & 24 & 87.53 & 93.18 & 93.79 & 84.17 \\
\hline $141 \mathrm{~A}$ & 22 & 267 & 24 & 88.74 & 93.59 & 96.22 & 85.47 \\
\hline $141 \mathrm{~A}$ & 23 & 268 & 24 & 90.44 & 95.65 & 97.76 & 86.82 \\
\hline $141 \mathrm{~A}$ & 24 & 269 & 24 & 91.60 & 97.26 & 98.38 & 87.76 \\
\hline $141 \mathrm{~A}$ & 25 & 270 & 24 & 93.71 & 99.46 & 99.86 & 89.05 \\
\hline $141 \mathrm{~A}$ & 26 & 271 & 24 & 95.05 & 99.99 & 100.76 & 89.93 \\
\hline $141 \mathrm{~A}$ & 27 & 272 & 24 & 96.67 & 100.67 & 103.65 & 90.98 \\
\hline $141 \mathrm{~A}$ & 28 & 273 & 24 & 92.05 & 100.27 & 102.13 & 90.13 \\
\hline $141 \mathrm{~A}$ & 29 & 274 & 24 & 88.15 & 99.78 & 100.18 & 89.46 \\
\hline $141 \mathrm{~A}$ & 30 & 275 & 24 & 88.83 & 100.59 & 100.67 & 91.10 \\
\hline $141 \mathrm{~A}$ & 31 & 276 & 24 & 86.20 & 101.05 & 97.82 & 90.98 \\
\hline $141 \mathrm{~A}$ & 32 & 277 & 24 & 88.21 & 103.72 & 97.65 & 92.56 \\
\hline $141 \mathrm{~A}$ & 33 & 278 & 24 & 87.26 & 102.43 & 97.25 & 88.59 \\
\hline $141 \mathrm{~A}$ & 34 & 279 & 7 & 88.49 & 102.80 & 98.39 & 89.85 \\
\hline $142 \mathrm{~A}$ & 1 & 280 & 8 & 59.06 & 55.83 & 58.50 & 58.13 \\
\hline $142 \mathrm{~A}$ & 2 & 281 & 24 & 78.21 & 75.49 & 76.81 & 75.08 \\
\hline $142 \mathrm{~A}$ & 3 & 282 & 24 & 85.73 & 84.98 & 85.69 & 83.76 \\
\hline $142 \mathrm{~A}$ & 4 & 283 & 24 & 88.19 & 87.24 & 91.30 & 85.48 \\
\hline $142 \mathrm{~A}$ & 5 & 284 & 24 & 86.01 & 94.98 & 91.06 & 83.29 \\
\hline $142 \mathrm{~A}$ & 6 & 285 & 24 & 87.47 & 97.21 & 94.21 & 83.96 \\
\hline $142 \mathrm{~A}$ & 7 & 286 & 25 & 87.60 & 94.79 & 94.85 & 83.83 \\
\hline $142 \mathrm{~A}$ & 8 & 287 & 24 & 87.90 & 92.71 & 95.54 & 83.53 \\
\hline $142 \mathrm{~A}$ & 9 & 288 & 24 & 89.06 & 94.34 & 96.52 & 84.54 \\
\hline $142 \mathrm{~A}$ & 10 & 289 & 24 & 89.81 & 96.28 & 96.88 & 85.25 \\
\hline $142 \mathrm{~A}$ & 11 & 290 & 24 & 90.06 & 96.85 & 94.74 & 85.34 \\
\hline
\end{tabular}




\begin{tabular}{|c|c|c|c|c|c|c|c|}
\hline Cycle & Timestep & $\begin{array}{l}\text { Cumulative } \\
\text { Timestep }\end{array}$ & $\begin{array}{c}\text { Time } \\
\text { Interval } \\
\text { (hrs) }\end{array}$ & $\begin{array}{c}\text { NW } \\
\text { OSCC } \\
\text { (degrees) }\end{array}$ & $\begin{array}{c}\text { SW } \\
\text { OSCC } \\
\text { (degrees) }\end{array}$ & $\begin{array}{c}\mathrm{NE} \\
\text { OSCC } \\
\text { (degrees) }\end{array}$ & $\begin{array}{c}\mathrm{SE} \\
\text { OSCC } \\
\text { (degrees) }\end{array}$ \\
\hline $142 \mathrm{~A}$ & 12 & 291 & 24 & 90.63 & 97.25 & 93.05 & 85.37 \\
\hline $142 \mathrm{~A}$ & 13 & 292 & 24 & 91.40 & 98.21 & 94.97 & 86.15 \\
\hline $142 \mathrm{~A}$ & 14 & 293 & 24 & 91.96 & 99.38 & 96.65 & 87.50 \\
\hline $142 \mathrm{~A}$ & 15 & 294 & 24 & 90.83 & 94.29 & 96.15 & 86.27 \\
\hline $142 \mathrm{~A}$ & 16 & 295 & 24 & 90.84 & 93.28 & 96.66 & 86.36 \\
\hline $142 \mathrm{~A}$ & 17 & 296 & 24 & 89.84 & 92.90 & 90.71 & 85.29 \\
\hline $142 \mathrm{~A}$ & 18 & 297 & 24 & 87.77 & 88.78 & 88.39 & 84.78 \\
\hline $142 \mathrm{~A}$ & 19 & 298 & 24 & 88.85 & 90.35 & 91.29 & 86.35 \\
\hline $142 \mathrm{~A}$ & 20 & 299 & 24 & 88.97 & 91.09 & 89.23 & 85.80 \\
\hline $142 \mathrm{~A}$ & 21 & 300 & 24 & 88.45 & 91.96 & 86.99 & 85.48 \\
\hline $142 \mathrm{~A}$ & 22 & 301 & 24 & 89.83 & 93.06 & 87.48 & 87.00 \\
\hline $142 \mathrm{~A}$ & 23 & 302 & 24 & 89.36 & 92.90 & 87.98 & 86.70 \\
\hline $142 \mathrm{~A}$ & 24 & 303 & 24 & 88.91 & 90.54 & 87.18 & 86.17 \\
\hline $142 \mathrm{~A}$ & 25 & 304 & 24 & 88.58 & 89.33 & 88.17 & 85.90 \\
\hline $142 \mathrm{~A}$ & 26 & 305 & 24 & 88.48 & 88.55 & 88.69 & 85.73 \\
\hline $142 \mathrm{~A}$ & 27 & 306 & 24 & 90.27 & 90.18 & 90.04 & 87.31 \\
\hline $142 \mathrm{~A}$ & 28 & 307 & 24 & 91.30 & 92.02 & 91.74 & 88.60 \\
\hline $142 \mathrm{~A}$ & 29 & 308 & 24 & 92.48 & 94.03 & 93.15 & 90.03 \\
\hline $142 \mathrm{~A}$ & 30 & 309 & 24 & 91.15 & 94.36 & 90.91 & 89.25 \\
\hline $142 \mathrm{~A}$ & 31 & 310 & 25 & 91.60 & 95.48 & 90.26 & 89.77 \\
\hline $142 \mathrm{~A}$ & 32 & 311 & 24 & 88.61 & 95.44 & 88.84 & 89.38 \\
\hline $142 \mathrm{~A}$ & 33 & 312 & 20 & 85.20 & 92.27 & 85.29 & 86.90 \\
\hline $142 \mathrm{~A}$ & 34 & 313 & 17 & 85.20 & 92.27 & 85.29 & 86.90 \\
\hline $142 \mathrm{~A}$ & 35 & 314 & 11 & 67.54 & 72.35 & 64.30 & 68.35 \\
\hline $142 \mathrm{~A}$ & 36 & 315 & 24 & 87.00 & 94.59 & 86.70 & 88.97 \\
\hline $142 \mathrm{~A}$ & 37 & 316 & 24 & 91.87 & 99.58 & 90.06 & 89.21 \\
\hline $142 \mathrm{~A}$ & 38 & 317 & 24 & 92.73 & 101.03 & 92.58 & 90.75 \\
\hline $142 \mathrm{~A}$ & 39 & 318 & 24 & 90.61 & 101.90 & 91.04 & 91.03 \\
\hline $142 \mathrm{~A}$ & 40 & 319 & 24 & 90.65 & 103.61 & 91.00 & 92.11 \\
\hline $142 \mathrm{~A}$ & 41 & 320 & 24 & 91.37 & 103.09 & 91.34 & 90.34 \\
\hline $142 \mathrm{~A}$ & 42 & 321 & 24 & 93.05 & 104.94 & 93.04 & 92.01 \\
\hline $142 \mathrm{~A}$ & 43 & 322 & 24 & 89.06 & 105.04 & 89.71 & 90.53 \\
\hline $142 \mathrm{~A}$ & 44 & 323 & 24 & 88.81 & 106.06 & 85.87 & 90.00 \\
\hline $142 \mathrm{~A}$ & 45 & 324 & 24 & 90.19 & 107.88 & 85.77 & 90.05 \\
\hline $142 \mathrm{~A}$ & 46 & 325 & 24 & 90.68 & 109.65 & 85.63 & 88.87 \\
\hline $142 \mathrm{~A}$ & 47 & 326 & 24 & 92.85 & 113.58 & 87.46 & 92.24 \\
\hline $142 \mathrm{~A}$ & 48 & 327 & 24 & 93.49 & 116.18 & 88.19 & 93.25 \\
\hline
\end{tabular}




\begin{tabular}{|c|c|c|c|c|c|c|c|}
\hline Cycle & Timestep & $\begin{array}{l}\text { Cumulative } \\
\text { Timestep }\end{array}$ & $\begin{array}{c}\text { Time } \\
\text { Interval } \\
\text { (hrs) }\end{array}$ & $\begin{array}{c}\text { NW } \\
\text { OSCC } \\
\text { (degrees) }\end{array}$ & $\begin{array}{c}\text { SW } \\
\text { OSCC } \\
\text { (degrees) }\end{array}$ & $\begin{array}{c}\mathrm{NE} \\
\text { OSCC } \\
\text { (degrees) }\end{array}$ & $\begin{array}{c}\mathrm{SE} \\
\text { OSCC } \\
\text { (degrees) }\end{array}$ \\
\hline $142 \mathrm{~A}$ & 49 & 328 & 24 & 92.44 & 116.42 & 87.09 & 93.76 \\
\hline $142 \mathrm{~A}$ & 50 & 329 & 24 & 92.87 & 118.80 & 88.94 & 93.81 \\
\hline $142 \mathrm{~A}$ & 51 & 330 & 15 & 92.90 & 119.34 & 88.49 & 92.40 \\
\hline 142B & 1 & 331 & 8 & 57.44 & 56.56 & 55.38 & 57.04 \\
\hline 142B & 2 & 332 & 24 & 82.04 & 81.50 & 81.40 & 76.59 \\
\hline 142B & 3 & 333 & 24 & 88.23 & 87.90 & 86.70 & 88.77 \\
\hline 142B & 4 & 334 & 45 & 88.23 & 87.90 & 86.70 & 88.77 \\
\hline 142B & 5 & 335 & 13 & 58.05 & 55.90 & 52.08 & 59.13 \\
\hline 142B & 6 & 336 & 24 & 83.24 & 81.84 & 80.61 & 86.68 \\
\hline 142B & 7 & 337 & 24 & 89.90 & 88.12 & 87.49 & 91.22 \\
\hline 142B & 8 & 338 & 24 & 90.44 & 89.44 & 88.42 & 93.08 \\
\hline 142B & 9 & 339 & 24 & 90.54 & 88.00 & 85.14 & 88.74 \\
\hline 142B & 10 & 340 & 24 & 91.29 & 88.98 & 85.97 & 89.06 \\
\hline 142B & 11 & 341 & 24 & 92.05 & 89.82 & 88.39 & 89.69 \\
\hline 142B & 12 & 342 & 24 & 92.85 & 89.98 & 90.38 & 90.49 \\
\hline 142B & 13 & 343 & 24 & 93.82 & 90.91 & 91.43 & 90.48 \\
\hline 142B & 14 & 344 & 24 & 94.59 & 92.06 & 91.60 & 90.16 \\
\hline 142B & 15 & 345 & 24 & 86.46 & 82.60 & 83.06 & 82.45 \\
\hline 142B & 16 & 346 & 24 & 86.87 & 83.07 & 84.00 & 83.10 \\
\hline 142B & 17 & 347 & 24 & 82.88 & 81.67 & 82.65 & 81.53 \\
\hline 142B & 18 & 348 & 24 & 84.11 & 82.96 & 83.81 & 82.60 \\
\hline 142B & 19 & 349 & 24 & 84.91 & 82.66 & 84.80 & 83.39 \\
\hline 142B & 20 & 350 & 24 & 85.23 & 83.46 & 86.39 & 83.54 \\
\hline 142B & 21 & 351 & 24 & 85.85 & 84.16 & 86.33 & 84.19 \\
\hline 142B & 22 & 352 & 24 & 87.12 & 84.74 & 87.08 & 84.95 \\
\hline 142B & 23 & 353 & 24 & 86.84 & 84.05 & 85.95 & 84.74 \\
\hline 142B & 24 & 354 & 24 & 86.63 & 84.18 & 84.25 & 84.32 \\
\hline 142B & 25 & 355 & 24 & 86.29 & 85.28 & 85.05 & 84.40 \\
\hline 142B & 26 & 356 & 24 & 83.78 & 84.46 & 83.59 & 84.08 \\
\hline 142B & 27 & 357 & 24 & 84.26 & 85.16 & 83.28 & 84.13 \\
\hline 142B & 28 & 358 & 24 & 83.14 & 84.90 & 80.12 & 83.11 \\
\hline 142B & 29 & 359 & 24 & 84.21 & 85.89 & 80.89 & 84.18 \\
\hline 142B & 30 & 360 & 24 & 85.34 & 87.47 & 81.52 & 84.92 \\
\hline 142B & 31 & 361 & 24 & 86.79 & 88.21 & 82.50 & 86.71 \\
\hline 142B & 32 & 362 & 31 & 87.80 & 88.73 & 84.06 & 88.08 \\
\hline 142B & 33 & 363 & 46 & 77.85 & 77.95 & 77.75 & 77.50 \\
\hline 142B & 34 & 364 & 11 & 60.56 & 61.17 & 55.25 & 62.17 \\
\hline
\end{tabular}




\begin{tabular}{|c|c|c|c|c|c|c|c|}
\hline Cycle & Timestep & $\begin{array}{l}\text { Cumulative } \\
\text { Timestep }\end{array}$ & $\begin{array}{c}\text { Time } \\
\text { Interval } \\
\text { (hrs) }\end{array}$ & $\begin{array}{c}\text { NW } \\
\text { OSCC } \\
\text { (degrees) }\end{array}$ & $\begin{array}{c}\text { SW } \\
\text { OSCC } \\
\text { (degrees) }\end{array}$ & $\begin{array}{c}\mathrm{NE} \\
\text { OSCC } \\
\text { (degrees) }\end{array}$ & $\begin{array}{c}\mathrm{SE} \\
\text { OSCC } \\
\text { (degrees) }\end{array}$ \\
\hline 142B & 35 & 365 & 24 & 77.16 & 82.58 & 73.84 & 80.10 \\
\hline 142B & 36 & 366 & 24 & 82.88 & 88.05 & 80.44 & 85.66 \\
\hline 142B & 37 & 367 & 24 & 83.78 & 89.54 & 82.14 & 86.69 \\
\hline 142B & 38 & 368 & 24 & 84.70 & 91.24 & 83.78 & 87.25 \\
\hline 142B & 39 & 369 & 24 & 85.99 & 92.76 & 86.34 & 88.57 \\
\hline 142B & 40 & 370 & 24 & 87.28 & 93.60 & 87.84 & 89.92 \\
\hline 142B & 41 & 371 & 24 & 85.73 & 90.50 & 86.88 & 88.44 \\
\hline 142B & 42 & 372 & 24 & 87.57 & 92.61 & 88.30 & 90.33 \\
\hline 142B & 43 & 373 & 24 & 89.50 & 94.23 & 90.84 & 92.00 \\
\hline 142B & 44 & 374 & 24 & 86.25 & 88.62 & 85.41 & 85.75 \\
\hline 142B & 45 & 375 & 24 & 84.90 & 87.02 & 83.43 & 83.97 \\
\hline 142B & 46 & 376 & 24 & 86.70 & 89.73 & 85.43 & 85.50 \\
\hline 142B & 47 & 377 & 24 & 88.48 & 91.58 & 86.89 & 86.95 \\
\hline 142B & 48 & 378 & 24 & 90.46 & 92.66 & 90.37 & 89.16 \\
\hline 142B & 49 & 379 & 24 & 92.15 & 94.89 & 93.51 & 90.66 \\
\hline 142B & 50 & 380 & 24 & 93.87 & 98.11 & 96.17 & 92.45 \\
\hline 142B & 51 & 381 & 24 & 94.44 & 99.91 & 94.23 & 93.39 \\
\hline 142B & 52 & 382 & 24 & 93.22 & 100.60 & 89.15 & 93.08 \\
\hline 142B & 53 & 383 & 24 & 87.81 & 98.75 & 84.98 & 91.82 \\
\hline 142B & 54 & 384 & 24 & 84.46 & 97.94 & 80.78 & 89.41 \\
\hline 142B & 55 & 385 & 24 & 87.88 & 99.09 & 83.99 & 92.06 \\
\hline 142B & 56 & 386 & 24 & 90.06 & 101.58 & 86.39 & 94.38 \\
\hline 142B & 57 & 387 & 21 & 91.68 & 105.03 & 87.75 & 96.45 \\
\hline $143 \mathrm{~A}$ & 1 & 388 & 8 & 51.14 & 54.52 & 63.33 & 56.68 \\
\hline $143 \mathrm{~A}$ & 2 & 389 & 24 & 71.69 & 81.65 & 93.09 & 79.86 \\
\hline $143 \mathrm{~A}$ & 3 & 390 & 24 & 72.63 & 93.17 & 92.01 & 84.21 \\
\hline $143 \mathrm{~A}$ & 4 & 391 & 24 & 72.59 & 92.12 & 90.28 & 85.26 \\
\hline $143 \mathrm{~A}$ & 5 & 392 & 24 & 70.91 & 89.43 & 89.66 & 84.44 \\
\hline $143 \mathrm{~A}$ & 6 & 393 & 24 & 71.06 & 89.92 & 91.50 & 85.35 \\
\hline $143 \mathrm{~A}$ & 7 & 394 & 24 & 71.83 & 91.42 & 93.04 & 85.85 \\
\hline $143 \mathrm{~A}$ & 8 & 395 & 24 & 72.30 & 91.65 & 94.21 & 86.02 \\
\hline $143 \mathrm{~A}$ & 9 & 396 & 24 & 71.53 & 91.66 & 89.95 & 84.70 \\
\hline $143 \mathrm{~A}$ & 10 & 397 & 24 & 72.40 & 91.70 & 90.47 & 85.45 \\
\hline $143 \mathrm{~A}$ & 11 & 398 & 24 & 72.90 & 92.26 & 91.24 & 86.08 \\
\hline $143 \mathrm{~A}$ & 12 & 399 & 24 & 73.65 & 93.10 & 92.33 & 86.18 \\
\hline $143 \mathrm{~A}$ & 13 & 400 & 24 & 75.25 & 93.72 & 93.58 & 86.68 \\
\hline $143 \mathrm{~A}$ & 14 & 401 & 24 & 75.53 & 94.18 & 93.93 & 86.95 \\
\hline
\end{tabular}




\begin{tabular}{|c|c|c|c|c|c|c|c|}
\hline Cycle & Timestep & $\begin{array}{c}\text { Cumulative } \\
\text { Timestep }\end{array}$ & $\begin{array}{c}\text { Time } \\
\text { Interval } \\
\text { (hrs) }\end{array}$ & $\begin{array}{c}\text { NW } \\
\text { OSCC } \\
\text { (degrees) }\end{array}$ & $\begin{array}{c}\text { SW } \\
\text { OSCC } \\
\text { (degrees) }\end{array}$ & $\begin{array}{c}\mathrm{NE} \\
\text { OSCC } \\
\text { (degrees) }\end{array}$ & $\begin{array}{c}\mathrm{SE} \\
\text { OSCC } \\
\text { (degrees) }\end{array}$ \\
\hline $143 \mathrm{~A}$ & 15 & 402 & 24 & 76.16 & 94.00 & 95.55 & 87.79 \\
\hline $143 \mathrm{~A}$ & 16 & 403 & 24 & 77.70 & 94.33 & 96.25 & 88.05 \\
\hline $143 \mathrm{~A}$ & 17 & 404 & 24 & 79.15 & 95.38 & 97.38 & 88.55 \\
\hline $143 \mathrm{~A}$ & 18 & 405 & 24 & 78.52 & 95.49 & 95.16 & 87.81 \\
\hline $143 \mathrm{~A}$ & 19 & 406 & 24 & 77.53 & 95.48 & 92.77 & 86.51 \\
\hline $143 \mathrm{~A}$ & 20 & 407 & 24 & 78.76 & 95.93 & 94.48 & 87.80 \\
\hline $143 \mathrm{~A}$ & 21 & 408 & 24 & 79.99 & 96.66 & 96.43 & 88.88 \\
\hline $143 \mathrm{~A}$ & 22 & 409 & 24 & 81.42 & 97.27 & 98.36 & 89.43 \\
\hline $143 \mathrm{~A}$ & 23 & 410 & 24 & 82.41 & 98.79 & 100.31 & 89.00 \\
\hline $143 \mathrm{~A}$ & 24 & 411 & 15 & 80.60 & 98.18 & 93.97 & 86.67 \\
\hline $143 \mathrm{~A}$ & 25 & 412 & 449 & 80.60 & 98.18 & 93.97 & 86.67 \\
\hline $143 \mathrm{~A}$ & 26 & 413 & 24 & 69.04 & 84.24 & 77.36 & 76.38 \\
\hline $143 \mathrm{~A}$ & 27 & 414 & 24 & 80.60 & 96.08 & 96.01 & 89.77 \\
\hline $143 \mathrm{~A}$ & 28 & 415 & 24 & 80.92 & 94.83 & 97.53 & 89.80 \\
\hline $143 \mathrm{~A}$ & 29 & 416 & 24 & 81.49 & 95.21 & 99.31 & 89.35 \\
\hline $143 \mathrm{~A}$ & 30 & 417 & 24 & 82.40 & 96.51 & 101.61 & 90.06 \\
\hline $143 \mathrm{~A}$ & 31 & 418 & 24 & 81.11 & 93.08 & 100.28 & 90.33 \\
\hline $143 \mathrm{~A}$ & 32 & 419 & 24 & 81.89 & 94.49 & 101.08 & 90.85 \\
\hline $143 \mathrm{~A}$ & 33 & 420 & 24 & 80.74 & 92.35 & 101.27 & 89.98 \\
\hline $143 \mathrm{~A}$ & 34 & 421 & 24 & 81.80 & 91.85 & 101.81 & 90.73 \\
\hline $143 \mathrm{~A}$ & 35 & 422 & 24 & 80.97 & 92.07 & 100.66 & 86.19 \\
\hline $143 \mathrm{~A}$ & 36 & 423 & 24 & 82.53 & 93.84 & 103.16 & 87.29 \\
\hline $143 \mathrm{~A}$ & 37 & 424 & 24 & 81.59 & 92.16 & 103.94 & 86.73 \\
\hline $143 \mathrm{~A}$ & 38 & 425 & 24 & 82.00 & 92.44 & 105.31 & 87.73 \\
\hline $143 \mathrm{~A}$ & 39 & 426 & 24 & 82.93 & 92.99 & 105.88 & 86.21 \\
\hline $143 \mathrm{~A}$ & 40 & 427 & 24 & 83.44 & 93.29 & 105.43 & 84.86 \\
\hline $143 \mathrm{~A}$ & 41 & 428 & 28 & 85.37 & 95.01 & 107.38 & 86.18 \\
\hline $143 \mathrm{~A}$ & 42 & 429 & 112 & 85.37 & 95.01 & 107.38 & 86.18 \\
\hline $143 \mathrm{~A}$ & 43 & 430 & 8 & 60.72 & 66.22 & 68.05 & 64.24 \\
\hline $143 \mathrm{~A}$ & 44 & 431 & 24 & 80.94 & 92.68 & 99.98 & 82.30 \\
\hline $143 \mathrm{~A}$ & 45 & 432 & 24 & 82.95 & 97.06 & 106.95 & 85.76 \\
\hline $143 \mathrm{~A}$ & 46 & 433 & 24 & 84.27 & 98.08 & 107.92 & 86.21 \\
\hline $143 \mathrm{~A}$ & 47 & 434 & 24 & 83.52 & 97.41 & 105.52 & 84.00 \\
\hline $143 \mathrm{~A}$ & 48 & 435 & 24 & 84.72 & 99.66 & 108.09 & 85.56 \\
\hline $143 \mathrm{~A}$ & 49 & 436 & 24 & 84.20 & 100.05 & 106.37 & 84.02 \\
\hline $143 \mathrm{~A}$ & 50 & 437 & 24 & 86.08 & 104.03 & 107.61 & 84.50 \\
\hline $143 \mathrm{~A}$ & 51 & 438 & 24 & 87.05 & 107.70 & 108.62 & 85.68 \\
\hline
\end{tabular}




\begin{tabular}{|c|c|c|c|c|c|c|c|}
\hline Cycle & Timestep & $\begin{array}{l}\text { Cumulative } \\
\text { Timestep }\end{array}$ & $\begin{array}{c}\text { Time } \\
\text { Interval } \\
\text { (hrs) }\end{array}$ & $\begin{array}{c}\text { NW } \\
\text { OSCC } \\
\text { (degrees) }\end{array}$ & $\begin{array}{c}\text { SW } \\
\text { OSCC } \\
\text { (degrees) }\end{array}$ & $\begin{array}{c}\mathrm{NE} \\
\text { OSCC } \\
\text { (degrees) }\end{array}$ & $\begin{array}{c}\mathrm{SE} \\
\text { OSCC } \\
\text { (degrees) }\end{array}$ \\
\hline $143 \mathrm{~A}$ & 52 & 439 & 24 & 82.79 & 108.16 & 107.68 & 86.42 \\
\hline $143 \mathrm{~A}$ & 53 & 440 & 22 & 81.10 & 109.22 & 106.65 & 87.71 \\
\hline 143B & 1 & 441 & 8 & 53.76 & 53.78 & 53.74 & 53.66 \\
\hline 143B & 2 & 442 & 24 & 65.89 & 67.58 & 65.23 & 68.38 \\
\hline 143B & 3 & 443 & 24 & 84.94 & 89.17 & 82.98 & 87.21 \\
\hline 143B & 4 & 444 & 24 & 87.78 & 94.49 & 86.40 & 90.55 \\
\hline 143B & 5 & 445 & 24 & 87.29 & 95.66 & 86.72 & 91.69 \\
\hline 143B & 6 & 446 & 24 & 87.16 & 95.31 & 87.42 & 92.15 \\
\hline 143B & 7 & 447 & 24 & 85.35 & 90.69 & 88.41 & 90.57 \\
\hline 143B & 8 & 448 & 24 & 86.59 & 91.68 & 89.58 & 91.00 \\
\hline 143B & 9 & 449 & 24 & 84.29 & 86.29 & 88.88 & 89.27 \\
\hline 143B & 10 & 450 & 24 & 85.26 & 86.62 & 89.32 & 89.64 \\
\hline 143B & 11 & 451 & 24 & 86.50 & 86.87 & 90.91 & 90.50 \\
\hline 143B & 12 & 452 & 24 & 87.61 & 87.00 & 91.80 & 90.80 \\
\hline 143B & 13 & 453 & 24 & 88.84 & 87.35 & 93.29 & 91.28 \\
\hline 143B & 14 & 454 & 24 & 89.00 & 87.27 & 92.08 & 91.00 \\
\hline 143B & 15 & 455 & 24 & 86.63 & 89.75 & 88.48 & 88.46 \\
\hline 143B & 16 & 456 & 24 & 81.79 & 90.62 & 86.05 & 84.73 \\
\hline 143B & 17 & 457 & 24 & 81.60 & 91.50 & 86.00 & 85.50 \\
\hline 143B & 18 & 458 & 24 & 82.58 & 91.88 & 86.67 & 85.83 \\
\hline 143B & 19 & 459 & 24 & 81.15 & 87.28 & 86.02 & 83.22 \\
\hline $143 \mathrm{~B}$ & 20 & 460 & 24 & 82.07 & 87.85 & 86.18 & 83.53 \\
\hline $143 B$ & 21 & 461 & 24 & 82.81 & 88.51 & 87.24 & 84.15 \\
\hline 143B & 22 & 462 & 24 & 83.48 & 89.10 & 87.39 & 84.40 \\
\hline 143B & 23 & 463 & 24 & 82.85 & 89.22 & 85.10 & 83.86 \\
\hline 143B & 24 & 464 & 24 & 84.58 & 90.43 & 86.77 & 84.76 \\
\hline 143B & 25 & 465 & 24 & 84.02 & 88.57 & 86.99 & 84.13 \\
\hline 143B & 26 & 466 & 24 & 83.43 & 87.21 & 87.35 & 83.50 \\
\hline 143B & 27 & 467 & 24 & 85.18 & 88.03 & 88.64 & 84.14 \\
\hline 143B & 28 & 468 & 24 & 84.48 & 88.38 & 86.10 & 83.73 \\
\hline $143 \mathrm{~B}$ & 29 & 469 & 53 & 84.00 & 88.50 & 90.03 & 83.50 \\
\hline 143B & 30 & 470 & 24 & 72.63 & 77.84 & 72.85 & 74.34 \\
\hline 143B & 31 & 471 & 24 & 86.00 & 89.90 & 86.09 & 85.88 \\
\hline 143B & 32 & 472 & 24 & 83.93 & 90.59 & 85.65 & 86.42 \\
\hline 143B & 33 & 473 & 24 & 84.25 & 92.36 & 86.71 & 86.73 \\
\hline 143B & 34 & 474 & 24 & 85.20 & 93.93 & 88.33 & 87.87 \\
\hline $143 B$ & 35 & 475 & 24 & 84.05 & 94.36 & 83.56 & 87.33 \\
\hline
\end{tabular}




\begin{tabular}{|c|c|c|c|c|c|c|c|}
\hline Cycle & Timestep & $\begin{array}{l}\text { Cumulative } \\
\text { Timestep }\end{array}$ & $\begin{array}{c}\text { Time } \\
\text { Interval } \\
\text { (hrs) }\end{array}$ & $\begin{array}{c}\text { NW } \\
\text { OSCC } \\
\text { (degrees) }\end{array}$ & $\begin{array}{c}\text { SW } \\
\text { OSCC } \\
\text { (degrees) }\end{array}$ & $\begin{array}{c}\mathrm{NE} \\
\text { OSCC } \\
\text { (degrees) }\end{array}$ & $\begin{array}{c}\mathrm{SE} \\
\text { OSCC } \\
\text { (degrees) }\end{array}$ \\
\hline 143B & 36 & 476 & 24 & 86.40 & 95.78 & 85.24 & 88.54 \\
\hline 143B & 37 & 477 & 24 & 87.32 & 96.13 & 86.51 & 88.18 \\
\hline 143B & 38 & 478 & 24 & 87.12 & 96.69 & 87.13 & 87.00 \\
\hline 143B & 39 & 479 & 24 & 86.59 & 97.64 & 87.26 & 87.64 \\
\hline 143B & 40 & 480 & 24 & 84.53 & 97.19 & 86.08 & 86.78 \\
\hline 143B & 41 & 481 & 24 & 85.02 & 98.21 & 84.80 & 86.12 \\
\hline 143B & 42 & 482 & 24 & 84.98 & 98.64 & 83.45 & 84.06 \\
\hline 143B & 43 & 483 & 24 & 85.53 & 99.71 & 91.91 & 87.88 \\
\hline 143B & 44 & 484 & 24 & 88.83 & 101.92 & 97.30 & 90.65 \\
\hline 143B & 45 & 485 & 24 & 90.45 & 104.14 & 98.42 & 90.84 \\
\hline 143B & 46 & 486 & 24 & 89.53 & 105.05 & 94.78 & 91.07 \\
\hline 143B & 47 & 487 & 24 & 86.45 & 105.66 & 94.59 & 91.82 \\
\hline 143B & 48 & 488 & 24 & 89.05 & 108.54 & 95.90 & 93.03 \\
\hline 143B & 49 & 489 & 24 & 87.51 & 108.89 & 96.14 & 92.99 \\
\hline 143B & 50 & 490 & 24 & 86.45 & 109.74 & 93.52 & 93.63 \\
\hline 143B & 51 & 491 & 24 & 87.20 & 112.11 & 92.21 & 94.72 \\
\hline 143B & 52 & 492 & 24 & 89.58 & 115.06 & 94.58 & 96.54 \\
\hline 143B & 53 & 493 & 24 & 88.66 & 116.43 & 94.17 & 97.60 \\
\hline 143B & 54 & 494 & 24 & 87.64 & 118.22 & 92.92 & 97.84 \\
\hline 143B & 55 & 495 & 24 & 87.89 & 121.89 & 90.23 & 98.10 \\
\hline 143B & 56 & 496 & 24 & 88.66 & 124.11 & 89.21 & 98.60 \\
\hline 143B & 57 & 497 & 24 & 88.61 & 126.58 & 88.63 & 99.06 \\
\hline 143B & 58 & 498 & 24 & 90.80 & 133.94 & 90.30 & 101.57 \\
\hline 143B & 59 & 499 & 24 & 94.91 & 145.89 & 93.24 & 103.65 \\
\hline 143B & 60 & 500 & 9 & 97.50 & 150.00 & 94.80 & 105.80 \\
\hline $144 \mathrm{~A}$ & 1 & 501 & 8 & 58.55 & 58.63 & 58.62 & 58.58 \\
\hline $144 \mathrm{~A}$ & 2 & 502 & 24 & 72.98 & 82.45 & 78.94 & 86.51 \\
\hline $144 \mathrm{~A}$ & 3 & 503 & 24 & 84.31 & 93.12 & 91.71 & 96.79 \\
\hline $144 \mathrm{~A}$ & 4 & 504 & 24 & 84.67 & 94.38 & 92.35 & 97.20 \\
\hline $144 \mathrm{~A}$ & 5 & 505 & 24 & 85.00 & 94.88 & 93.30 & 98.03 \\
\hline $144 \mathrm{~A}$ & 6 & 506 & 24 & 85.99 & 96.89 & 95.37 & 99.82 \\
\hline $144 \mathrm{~A}$ & 7 & 507 & 24 & 85.34 & 96.57 & 92.66 & 97.69 \\
\hline $144 \mathrm{~A}$ & 8 & 508 & 24 & 86.15 & 97.45 & 92.36 & 97.56 \\
\hline $144 \mathrm{~A}$ & 9 & 509 & 24 & 87.12 & 96.36 & 95.00 & 98.00 \\
\hline $144 \mathrm{~A}$ & 10 & 510 & 24 & 87.39 & 96.85 & 95.91 & 98.20 \\
\hline $144 \mathrm{~A}$ & 11 & 511 & 24 & 87.49 & 96.33 & 96.41 & 97.85 \\
\hline $144 \mathrm{~A}$ & 12 & 512 & 24 & 85.88 & 91.56 & 95.99 & 96.43 \\
\hline
\end{tabular}




\begin{tabular}{|c|c|c|c|c|c|c|c|}
\hline Cycle & Timestep & $\begin{array}{l}\text { Cumulative } \\
\text { Timestep }\end{array}$ & $\begin{array}{c}\text { Time } \\
\text { Interval } \\
\text { (hrs) }\end{array}$ & $\begin{array}{c}\text { NW } \\
\text { OSCC } \\
\text { (degrees) }\end{array}$ & $\begin{array}{c}\text { SW } \\
\text { OSCC } \\
\text { (degrees) }\end{array}$ & $\begin{array}{c}\mathrm{NE} \\
\text { OSCC } \\
\text { (degrees) }\end{array}$ & $\begin{array}{c}\mathrm{SE} \\
\text { OSCC } \\
\text { (degrees) }\end{array}$ \\
\hline $144 \mathrm{~A}$ & 13 & 513 & 24 & 86.01 & 92.03 & 93.88 & 95.20 \\
\hline $144 \mathrm{~A}$ & 14 & 514 & 24 & 86.00 & 92.23 & 93.00 & 94.46 \\
\hline $144 \mathrm{~A}$ & 15 & 515 & 24 & 85.50 & 92.20 & 91.30 & 94.00 \\
\hline $144 \mathrm{~A}$ & 16 & 516 & 24 & 86.23 & 93.07 & 91.72 & 94.67 \\
\hline $144 \mathrm{~A}$ & 17 & 517 & 24 & 86.81 & 93.69 & 91.70 & 95.00 \\
\hline $144 \mathrm{~A}$ & 18 & 518 & 24 & 87.66 & 94.13 & 94.17 & 96.12 \\
\hline $144 \mathrm{~A}$ & 19 & 519 & 24 & 88.28 & 95.36 & 94.53 & 96.54 \\
\hline $144 \mathrm{~A}$ & 20 & 520 & 24 & 89.60 & 95.96 & 95.94 & 97.50 \\
\hline $144 \mathrm{~A}$ & 21 & 521 & 24 & 89.70 & 96.53 & 96.44 & 97.22 \\
\hline $144 \mathrm{~A}$ & 22 & 522 & 24 & 89.17 & 96.86 & 92.99 & 96.40 \\
\hline $144 \mathrm{~A}$ & 23 & 523 & 24 & 90.40 & 98.02 & 93.88 & 96.25 \\
\hline $144 \mathrm{~A}$ & 24 & 524 & 24 & 89.19 & 94.09 & 93.08 & 94.73 \\
\hline $144 \mathrm{~A}$ & 25 & 525 & 24 & 91.10 & 95.07 & 95.04 & 96.10 \\
\hline $144 \mathrm{~A}$ & 26 & 526 & 24 & 90.99 & 94.28 & 95.08 & 94.45 \\
\hline $144 \mathrm{~A}$ & 27 & 527 & 24 & 91.70 & 93.97 & 94.82 & 93.38 \\
\hline $144 \mathrm{~A}$ & 28 & 528 & 24 & 92.75 & 95.29 & 96.18 & 94.38 \\
\hline $144 \mathrm{~A}$ & 29 & 529 & 24 & 93.89 & 96.64 & 98.76 & 95.42 \\
\hline $144 \mathrm{~A}$ & 30 & 530 & 24 & 95.09 & 98.09 & 100.24 & 96.51 \\
\hline $144 \mathrm{~A}$ & 31 & 531 & 24 & 94.04 & 96.05 & 99.43 & 95.83 \\
\hline $144 \mathrm{~A}$ & 32 & 532 & 24 & 94.67 & 95.23 & 101.15 & 96.17 \\
\hline $144 \mathrm{~A}$ & 33 & 533 & 24 & 96.00 & 96.31 & 102.00 & 97.45 \\
\hline $144 \mathrm{~A}$ & 34 & 534 & 24 & 100.15 & 97.13 & 99.37 & 96.99 \\
\hline $144 \mathrm{~A}$ & 35 & 535 & 24 & 100.55 & 98.19 & 97.05 & 98.25 \\
\hline $144 \mathrm{~A}$ & 36 & 536 & 24 & 101.23 & 99.30 & 98.36 & 99.48 \\
\hline $144 \mathrm{~A}$ & 37 & 537 & 24 & 103.85 & 101.65 & 101.17 & 100.08 \\
\hline $144 \mathrm{~A}$ & 38 & 538 & 24 & 102.87 & 102.85 & 100.84 & 101.38 \\
\hline $144 \mathrm{~A}$ & 39 & 539 & 24 & 94.06 & 100.31 & 99.38 & 100.98 \\
\hline $144 \mathrm{~A}$ & 40 & 540 & 24 & 93.99 & 98.20 & 99.94 & 99.55 \\
\hline $144 \mathrm{~A}$ & 41 & 541 & 24 & 96.09 & 100.56 & 102.35 & 101.32 \\
\hline $144 \mathrm{~A}$ & 42 & 542 & 24 & 97.36 & 103.19 & 103.73 & 103.31 \\
\hline $144 \mathrm{~A}$ & 43 & 543 & 24 & 99.13 & 105.44 & 107.10 & 105.60 \\
\hline $144 \mathrm{~A}$ & 44 & 544 & 24 & 100.45 & 107.18 & 108.46 & 108.06 \\
\hline $144 \mathrm{~A}$ & 45 & 545 & 18 & 101.07 & 108.25 & 112.71 & 110.04 \\
\hline 144B & 1 & 546 & 8 & 61.05 & 60.14 & 60.31 & 60.65 \\
\hline 144B & 2 & 547 & 24 & 88.43 & 86.07 & 87.46 & 86.73 \\
\hline 144B & 3 & 548 & 24 & 94.52 & 94.82 & 95.09 & 98.15 \\
\hline 144B & 4 & 549 & 24 & 91.51 & 94.75 & 95.09 & 97.51 \\
\hline
\end{tabular}




\begin{tabular}{|c|c|c|c|c|c|c|c|}
\hline Cycle & Timestep & $\begin{array}{l}\text { Cumulative } \\
\text { Timestep }\end{array}$ & $\begin{array}{c}\text { Time } \\
\text { Interval } \\
\text { (hrs) }\end{array}$ & $\begin{array}{c}\text { NW } \\
\text { OSCC } \\
\text { (degrees) }\end{array}$ & $\begin{array}{c}\text { SW } \\
\text { OSCC } \\
\text { (degrees) }\end{array}$ & $\begin{array}{c}\mathrm{NE} \\
\text { OSCC } \\
\text { (degrees) }\end{array}$ & $\begin{array}{c}\mathrm{SE} \\
\text { OSCC } \\
\text { (degrees) }\end{array}$ \\
\hline 144B & 5 & 550 & 24 & 90.89 & 93.67 & 94.34 & 98.12 \\
\hline 144B & 6 & 551 & 24 & 92.16 & 94.68 & 95.90 & 99.16 \\
\hline 144B & 7 & 552 & 24 & 93.40 & 95.76 & 97.80 & 99.83 \\
\hline 144B & 8 & 553 & 24 & 94.80 & 97.19 & 99.33 & 100.68 \\
\hline 144B & 9 & 554 & 24 & 96.18 & 98.02 & 101.07 & 101.22 \\
\hline 144B & 10 & 555 & 24 & 95.83 & 96.60 & 99.25 & 97.00 \\
\hline 144B & 11 & 556 & 24 & 97.42 & 95.70 & 102.70 & 98.62 \\
\hline 144B & 12 & 557 & 24 & 92.35 & 95.26 & 90.00 & 94.91 \\
\hline 144B & 13 & 558 & 24 & 93.82 & 96.82 & 89.81 & 95.18 \\
\hline 144B & 14 & 559 & 24 & 95.52 & 97.33 & 90.63 & 96.76 \\
\hline 144B & 15 & 560 & 24 & 96.16 & 98.40 & 91.10 & 97.50 \\
\hline 144B & 16 & 561 & 24 & 97.79 & 99.29 & 92.85 & 97.95 \\
\hline 144B & 17 & 562 & 24 & 99.41 & 99.29 & 93.88 & 99.38 \\
\hline 144B & 18 & 563 & 24 & 100.52 & 100.53 & 95.04 & 99.91 \\
\hline 144B & 19 & 564 & 24 & 101.54 & 101.47 & 96.39 & 100.78 \\
\hline 144B & 20 & 565 & 24 & 103.13 & 102.49 & 97.95 & 101.81 \\
\hline 144B & 21 & 566 & 24 & 104.65 & 102.38 & 101.00 & 103.50 \\
\hline 144B & 22 & 567 & 19 & 106.77 & 103.41 & 101.71 & 104.21 \\
\hline 144B & 23 & 568 & 66 & 106.77 & 103.41 & 101.71 & 104.21 \\
\hline 144B & 24 & 569 & 19 & 80.82 & 80.63 & 77.20 & 80.90 \\
\hline 144B & 25 & 570 & 24 & 106.89 & 103.67 & 102.26 & 104.81 \\
\hline 144B & 26 & 571 & 24 & 110.71 & 107.11 & 105.40 & 107.82 \\
\hline 144B & 27 & 572 & 24 & 105.07 & 106.09 & 103.89 & 106.99 \\
\hline 144B & 28 & 573 & 24 & 106.01 & 106.90 & 105.13 & 107.86 \\
\hline 144B & 29 & 574 & 24 & 108.35 & 107.94 & 106.60 & 109.50 \\
\hline 144B & 30 & 575 & 24 & 110.03 & 109.56 & 109.05 & 111.35 \\
\hline 144B & 31 & 576 & 24 & 110.80 & 110.10 & 108.93 & 109.93 \\
\hline 144B & 32 & 577 & 24 & 110.63 & 110.23 & 106.89 & 107.21 \\
\hline 144B & 33 & 578 & 24 & 111.69 & 111.68 & 108.83 & 108.68 \\
\hline 144B & 34 & 579 & 24 & 107.58 & 111.49 & 108.67 & 108.88 \\
\hline 144B & 35 & 580 & 24 & 110.85 & 113.67 & 108.75 & 110.80 \\
\hline 144B & 36 & 581 & 24 & 109.43 & 105.88 & 108.98 & 108.68 \\
\hline 144B & 37 & 582 & 24 & 109.23 & 104.75 & 108.70 & 106.04 \\
\hline 144B & 38 & 583 & 24 & 110.97 & 107.67 & 110.55 & 106.82 \\
\hline 144B & 39 & 584 & 24 & 109.94 & 108.28 & 104.76 & 106.43 \\
\hline 144B & 40 & 585 & 24 & 112.39 & 109.49 & 107.02 & 108.84 \\
\hline 144B & 41 & 586 & 24 & 110.61 & 109.08 & 107.63 & 110.39 \\
\hline
\end{tabular}




\begin{tabular}{|c|c|c|c|c|c|c|c|}
\hline Cycle & Timestep & $\begin{array}{l}\text { Cumulative } \\
\text { Timestep }\end{array}$ & $\begin{array}{c}\text { Time } \\
\text { Interval } \\
\text { (hrs) }\end{array}$ & $\begin{array}{c}\text { NW } \\
\text { OSCC } \\
\text { (degrees) }\end{array}$ & $\begin{array}{c}\text { SW } \\
\text { OSCC } \\
\text { (degrees) }\end{array}$ & $\begin{array}{c}\mathrm{NE} \\
\text { OSCC } \\
\text { (degrees) }\end{array}$ & $\begin{array}{c}\mathrm{SE} \\
\text { OSCC } \\
\text { (degrees) }\end{array}$ \\
\hline 144B & 42 & 587 & 24 & 109.83 & 110.48 & 108.66 & 111.58 \\
\hline 144B & 43 & 588 & 24 & 112.06 & 112.75 & 111.06 & 114.09 \\
\hline 144B & 44 & 589 & 24 & 114.68 & 115.18 & 113.29 & 116.23 \\
\hline 144B & 45 & 590 & 24 & 114.80 & 115.28 & 111.14 & 114.03 \\
\hline 144B & 46 & 591 & 24 & 116.45 & 116.69 & 112.86 & 115.12 \\
\hline 144B & 47 & 592 & 24 & 118.52 & 119.89 & 114.96 & 118.62 \\
\hline 144B & 48 & 593 & 24 & 118.34 & 116.07 & 116.23 & 118.10 \\
\hline 144B & 49 & 594 & 24 & 113.18 & 114.22 & 114.49 & 118.93 \\
\hline 144B & 50 & 595 & 24 & 109.58 & 111.55 & 105.46 & 112.62 \\
\hline 144B & 51 & 596 & 24 & 110.78 & 114.19 & 106.67 & 113.85 \\
\hline 144B & 52 & 597 & 24 & 113.70 & 117.18 & 109.88 & 117.86 \\
\hline 144B & 53 & 598 & 24 & 115.38 & 116.43 & 112.25 & 119.82 \\
\hline 144B & 54 & 599 & 27 & 115.82 & 114.82 & 112.39 & 120.66 \\
\hline $145 \mathrm{~A}$ & 1 & 600 & 11 & 53.15 & 50.94 & 53.12 & 56.04 \\
\hline $145 \mathrm{~A}$ & 2 & 601 & 11 & 76.14 & 69.63 & 79.12 & 76.45 \\
\hline $145 \mathrm{~A}$ & 3 & 602 & 24 & 88.60 & 83.43 & 89.86 & 92.21 \\
\hline $145 \mathrm{~A}$ & 4 & 603 & 24 & 91.16 & 86.21 & 93.53 & 95.95 \\
\hline $145 \mathrm{~A}$ & 5 & 604 & 24 & 89.47 & 85.79 & 95.98 & 96.49 \\
\hline $145 \mathrm{~A}$ & 6 & 605 & 24 & 88.98 & 84.48 & 97.23 & 97.77 \\
\hline $145 \mathrm{~A}$ & 7 & 606 & 24 & 89.24 & 85.28 & 96.66 & 98.80 \\
\hline $145 \mathrm{~A}$ & 8 & 607 & 24 & 90.09 & 86.13 & 97.69 & 99.68 \\
\hline $145 \mathrm{~A}$ & 9 & 608 & 24 & 91.66 & 86.01 & 100.11 & 100.50 \\
\hline $145 \mathrm{~A}$ & 10 & 609 & 24 & 91.81 & 87.33 & 102.33 & 101.00 \\
\hline $145 \mathrm{~A}$ & 11 & 610 & 24 & 92.66 & 87.87 & 103.67 & 101.33 \\
\hline $145 \mathrm{~A}$ & 12 & 611 & 24 & 92.61 & 87.73 & 100.79 & 100.53 \\
\hline $145 \mathrm{~A}$ & 13 & 612 & 24 & 91.96 & 89.69 & 97.45 & 98.75 \\
\hline $145 \mathrm{~A}$ & 14 & 613 & 24 & 90.42 & 91.61 & 95.66 & 100.42 \\
\hline $145 \mathrm{~A}$ & 15 & 614 & 24 & 90.43 & 89.80 & 92.96 & 95.33 \\
\hline $145 \mathrm{~A}$ & 16 & 615 & 24 & 91.60 & 90.31 & 93.52 & 95.71 \\
\hline $145 \mathrm{~A}$ & 17 & 616 & 24 & 92.60 & 90.83 & 94.48 & 96.37 \\
\hline $145 \mathrm{~A}$ & 18 & 617 & 24 & 94.08 & 92.06 & 96.30 & 96.83 \\
\hline $145 \mathrm{~A}$ & 19 & 618 & 24 & 94.45 & 92.71 & 97.21 & 97.31 \\
\hline $145 \mathrm{~A}$ & 20 & 619 & 24 & 95.94 & 93.50 & 98.66 & 98.00 \\
\hline $145 \mathrm{~A}$ & 21 & 620 & 24 & 95.76 & 94.10 & 96.67 & 97.35 \\
\hline $145 \mathrm{~A}$ & 22 & 621 & 24 & 94.32 & 93.79 & 93.00 & 95.15 \\
\hline $145 \mathrm{~A}$ & 23 & 622 & 24 & 94.09 & 92.91 & 91.53 & 91.10 \\
\hline $145 \mathrm{~A}$ & 24 & 623 & 24 & 95.93 & 94.13 & 93.58 & 91.98 \\
\hline
\end{tabular}




\begin{tabular}{|c|c|c|c|c|c|c|c|}
\hline Cycle & Timestep & $\begin{array}{l}\text { Cumulative } \\
\text { Timestep }\end{array}$ & $\begin{array}{c}\text { Time } \\
\text { Interval } \\
\text { (hrs) }\end{array}$ & $\begin{array}{c}\text { NW } \\
\text { OSCC } \\
\text { (degrees) }\end{array}$ & $\begin{array}{c}\text { SW } \\
\text { OSCC } \\
\text { (degrees) }\end{array}$ & $\begin{array}{c}\mathrm{NE} \\
\text { OSCC } \\
\text { (degrees) }\end{array}$ & $\begin{array}{c}\mathrm{SE} \\
\text { OSCC } \\
\text { (degrees) }\end{array}$ \\
\hline $145 \mathrm{~A}$ & 25 & 624 & 24 & 97.88 & 94.25 & 94.85 & 93.25 \\
\hline $145 \mathrm{~A}$ & 26 & 625 & 8 & 98.14 & 95.43 & 95.91 & 93.91 \\
\hline $145 \mathrm{~A}$ & 27 & 626 & 43 & 98.14 & 95.43 & 95.91 & 93.91 \\
\hline $145 \mathrm{~A}$ & 28 & 627 & 12 & 75.81 & 77.28 & 72.68 & 76.04 \\
\hline $145 \mathrm{~A}$ & 29 & 628 & 10 & 85.64 & 85.41 & 83.44 & 83.03 \\
\hline $145 \mathrm{~A}$ & 30 & 629 & 24 & 98.55 & 95.80 & 96.22 & 93.12 \\
\hline $145 \mathrm{~A}$ & 31 & 630 & 24 & 98.04 & 96.87 & 98.45 & 94.63 \\
\hline $145 \mathrm{~A}$ & 32 & 631 & 24 & 97.90 & 97.47 & 98.80 & 95.30 \\
\hline $145 \mathrm{~A}$ & 33 & 632 & 24 & 99.15 & 99.10 & 100.67 & 95.97 \\
\hline $145 \mathrm{~A}$ & 34 & 633 & 24 & 97.67 & 93.67 & 101.04 & 95.33 \\
\hline $145 \mathrm{~A}$ & 35 & 634 & 25 & 99.39 & 95.34 & 102.95 & 96.50 \\
\hline $145 \mathrm{~A}$ & 36 & 635 & 60 & 99.39 & 95.34 & 102.95 & 96.50 \\
\hline $145 \mathrm{~A}$ & 37 & 636 & 11 & 69.07 & 70.00 & 68.36 & 69.72 \\
\hline $145 \mathrm{~A}$ & 38 & 637 & 24 & 92.92 & 91.23 & 93.24 & 91.65 \\
\hline $145 \mathrm{~A}$ & 39 & 638 & 18 & 100.19 & 97.37 & 99.78 & 97.30 \\
\hline $145 \mathrm{~A}$ & 40 & 639 & 58 & 100.19 & 97.37 & 99.78 & 97.30 \\
\hline $145 \mathrm{~A}$ & 41 & 640 & 8 & 66.89 & 67.56 & 65.99 & 67.28 \\
\hline $145 \mathrm{~A}$ & 42 & 641 & 12 & 79.95 & 80.01 & 78.40 & 79.49 \\
\hline $145 \mathrm{~A}$ & 43 & 642 & 24 & 99.20 & 97.17 & 99.12 & 95.79 \\
\hline $145 \mathrm{~A}$ & 44 & 643 & 24 & 104.41 & 101.39 & 104.28 & 99.19 \\
\hline $145 \mathrm{~A}$ & 45 & 644 & 24 & 106.61 & 103.06 & 105.82 & 100.94 \\
\hline $145 \mathrm{~A}$ & 46 & 645 & 24 & 105.18 & 103.49 & 106.32 & 102.25 \\
\hline $145 \mathrm{~A}$ & 47 & 646 & 24 & 102.75 & 104.34 & 104.75 & 102.46 \\
\hline $145 \mathrm{~A}$ & 48 & 647 & 24 & 102.42 & 106.42 & 102.02 & 103.10 \\
\hline $145 \mathrm{~A}$ & 49 & 648 & 24 & 100.72 & 101.33 & 102.90 & 103.49 \\
\hline $145 \mathrm{~A}$ & 50 & 649 & 24 & 102.55 & 102.68 & 104.50 & 104.61 \\
\hline $145 \mathrm{~A}$ & 51 & 650 & 24 & 101.90 & 103.48 & 100.19 & 103.33 \\
\hline $145 \mathrm{~A}$ & 52 & 651 & 24 & 104.10 & 105.84 & 102.05 & 105.34 \\
\hline $145 \mathrm{~A}$ & 53 & 652 & 24 & 104.80 & 105.42 & 102.87 & 106.60 \\
\hline $145 \mathrm{~A}$ & 54 & 653 & 24 & 103.80 & 102.89 & 102.20 & 105.46 \\
\hline $145 \mathrm{~A}$ & 55 & 654 & 24 & 104.39 & 104.02 & 102.51 & 104.92 \\
\hline $145 \mathrm{~A}$ & 56 & 655 & 24 & 106.80 & 106.39 & 104.81 & 106.83 \\
\hline $145 \mathrm{~A}$ & 57 & 656 & 24 & 106.23 & 105.66 & 103.84 & 105.48 \\
\hline $145 \mathrm{~A}$ & 58 & 657 & 24 & 108.69 & 108.25 & 105.94 & 106.74 \\
\hline $145 \mathrm{~A}$ & 59 & 658 & 24 & 111.18 & 110.81 & 108.58 & 108.68 \\
\hline $145 \mathrm{~A}$ & 60 & 659 & 24 & 110.07 & 107.30 & 109.02 & 108.09 \\
\hline $145 \mathrm{~A}$ & 61 & 660 & 24 & 109.40 & 108.26 & 110.78 & 109.94 \\
\hline
\end{tabular}




\begin{tabular}{|c|c|c|c|c|c|c|c|}
\hline Cycle & Timestep & $\begin{array}{c}\text { Cumulative } \\
\text { Timestep }\end{array}$ & $\begin{array}{c}\text { Time } \\
\text { Interval } \\
\text { (hrs) }\end{array}$ & $\begin{array}{c}\text { NW } \\
\text { OSCC } \\
\text { (degrees) }\end{array}$ & $\begin{array}{c}\text { SW } \\
\text { OSCC } \\
\text { (degrees) }\end{array}$ & $\begin{array}{c}\text { NE } \\
\text { OSCC } \\
\text { (degrees) }\end{array}$ & $\begin{array}{c}\text { SE } \\
\text { OSCC } \\
\text { (degrees) }\end{array}$ \\
\hline $145 \mathrm{~A}$ & 62 & 661 & 24 & 108.68 & 109.64 & 112.41 & 112.99 \\
\hline $145 \mathrm{~A}$ & 63 & 662 & 29 & 110.66 & 112.77 & 115.78 & 114.42 \\
\hline
\end{tabular}




\section{Appendix C}

\section{ATR Neck Shim Positions by Cycle and Timestep}

Note: " 1 " indicates the neck shim rod is fully inserted into the ATR core (aluminum neck shim housing) and " 0 " indicates the neck shim rod is fully withdrawn (replaced with an aluminum rod follower section).

\begin{tabular}{|c|c|c|c|c|c|c|c|c|c|c|c|c|c|c|c|c|c|c|c|c|c|c|c|c|c|c|c|}
\hline \multirow[b]{2}{*}{ Cycle } & \multirow[b]{2}{*}{ Timestep } & \multirow{2}{*}{$\begin{array}{c}\text { Cumulative } \\
\text { Timestep }\end{array}$} & \multirow{2}{*}{$\begin{array}{c}\text { Time } \\
\text { Interval } \\
(\mathrm{hrs})\end{array}$} & \multicolumn{6}{|c|}{$\begin{array}{c}\text { NW } \\
\text { Neck Shims }\end{array}$} & \multicolumn{6}{|c|}{$\begin{array}{c}\text { NE } \\
\text { Neck Shims }\end{array}$} & \multicolumn{6}{|c|}{$\begin{array}{c}\text { SW } \\
\text { Neck Shims }\end{array}$} & \multicolumn{6}{|c|}{$\begin{array}{c}\text { SE } \\
\text { Neck Shims }\end{array}$} \\
\hline & & & & 1 & 2 & 3 & 4 & \begin{tabular}{|l|}
5 \\
\end{tabular} & 6 & \begin{tabular}{l|l}
1 \\
\end{tabular} & 2 & 3 & 4 & 5 & 6 & 1 & \begin{tabular}{|l|}
2 \\
\end{tabular} & 3 & 4 & 5 & 6 & 1 & 2 & 3 & \begin{tabular}{|l|l}
4 \\
\end{tabular} & 5 & 6 \\
\hline $138 \mathrm{~B}$ & 1 & 1 & 9 & 1 & 1 & 1 & 1 & 1 & 1 & 1 & 1 & 1 & 1 & 1 & 1 & 1 & 1 & 1 & 1 & \begin{tabular}{|l|}
1 \\
\end{tabular} & 1 & 1 & 1 & 1 & 1 & 1 & 1 \\
\hline $138 \mathrm{~B}$ & 2 & 2 & 24 & 1 & 1 & 1 & 1 & 1 & 1 & 1 & 1 & 1 & 1 & 1 & 1 & 1 & 1 & 1 & 1 & 1 & 1 & 1 & 1 & 1 & 1 & 1 & 1 \\
\hline $138 \mathrm{~B}$ & 3 & 3 & 24 & 1 & 1 & 1 & 1 & 0 & 0 & 1 & 1 & 0 & 0 & 0 & 0 & 1 & 1 & 1 & 1 & 1 & 0 & 1 & 1 & 1 & 1 & 0 & 0 \\
\hline 138B & 4 & 4 & 24 & 1 & 1 & 1 & 1 & 0 & 0 & 1 & 1 & 0 & 0 & 0 & 0 & 1 & 1 & 1 & 1 & 1 & 0 & 1 & 1 & 1 & 1 & 0 & 0 \\
\hline $138 \mathrm{~B}$ & 5 & 5 & 24 & 1 & 1 & 1 & 1 & 0 & 0 & 1 & 1 & 0 & 0 & 0 & 0 & 1 & 1 & 1 & 1 & 1 & 0 & 1 & 1 & 1 & 1 & 0 & 0 \\
\hline 138B & 6 & 6 & 24 & 1 & 1 & 1 & 1 & 0 & 0 & 1 & 1 & 0 & 0 & 0 & 0 & 1 & 1 & 1 & 1 & 1 & 0 & 1 & 1 & 1 & 1 & 0 & 0 \\
\hline $138 \mathrm{~B}$ & 7 & 7 & 24 & 1 & 1 & 1 & 1 & 0 & 0 & 1 & 0 & 0 & 0 & 0 & 0 & 1 & 1 & 1 & 1 & 1 & 0 & 1 & 1 & 1 & 1 & 0 & 0 \\
\hline 138B & 8 & 8 & 24 & 1 & 1 & 1 & 1 & 0 & 0 & \begin{tabular}{|l|}
1 \\
\end{tabular} & 0 & 0 & 0 & 0 & 0 & 1 & \begin{tabular}{|l|}
1 \\
\end{tabular} & 1 & 1 & 1 & 0 & 1 & 1 & 1 & 1 & 0 & 0 \\
\hline 138B & 9 & 9 & 24 & 1 & 1 & 1 & 1 & 0 & 0 & \begin{tabular}{l|l|}
0 \\
\end{tabular} & 0 & 0 & 0 & 0 & 0 & 1 & 1 & 1 & 1 & 1 & 0 & 1 & 1 & 1 & 1 & 0 & 0 \\
\hline 138B & 10 & 10 & 24 & 1 & 1 & 1 & 1 & 0 & 0 & 0 & 0 & 0 & 0 & 0 & 0 & 1 & 1 & 1 & 1 & 1 & 0 & 1 & 1 & 1 & 1 & 0 & 0 \\
\hline $138 \mathrm{~B}$ & 11 & 11 & 24 & 1 & 1 & 1 & 1 & 0 & 0 & 0 & 0 & 0 & 0 & 0 & 0 & 1 & 1 & 1 & 1 & 11 & 0 & 1 & 1 & 1 & 1 & 0 & 0 \\
\hline 138B & 12 & 12 & 24 & 1 & 1 & 1 & 1 & 0 & 0 & 0 & 0 & 0 & 0 & 0 & 0 & 1 & 1 & 1 & 1 & 1 & 0 & 1 & 1 & 1 & 1 & 0 & 0 \\
\hline $138 \mathrm{~B}$ & 13 & 13 & 24 & 1 & 1 & 1 & 1 & 0 & 0 & 0 & 0 & \begin{tabular}{l|l}
0 \\
\end{tabular} & 0 & 0 & 0 & 1 & 1 & 1 & 1 & 1 & 0 & 1 & 1 & 1 & 1 & 0 & 0 \\
\hline $138 \mathrm{~B}$ & 14 & 14 & 24 & 1 & 1 & 1 & 0 & 0 & 0 & 0 & 0 & 0 & 0 & 0 & 0 & 1 & 1 & 1 & 1 & 1 & 0 & 1 & 1 & 1 & 1 & 0 & 0 \\
\hline 138B & 15 & 15 & 24 & 1 & 1 & 1 & 0 & 0 & 0 & 0 & 0 & 0 & 0 & 0 & 0 & 1 & 1 & 1 & 1 & 1 & 0 & 1 & 1 & 1 & 1 & 0 & 0 \\
\hline $138 \mathrm{~B}$ & 16 & 16 & 24 & 1 & 1 & 1 & 0 & 0 & 0 & \begin{tabular}{l|l}
0 \\
\end{tabular} & 0 & 0 & 0 & 0 & 0 & 1 & 1 & 1 & 1 & 1 & 0 & 1 & 1 & 1 & 1 & 0 & 0 \\
\hline $138 \mathrm{~B}$ & 17 & 17 & 24 & 1 & 1 & 1 & 0 & 0 & 0 & \begin{tabular}{l|l|}
0 \\
\end{tabular} & 0 & 0 & 0 & 0 & 0 & 1 & 1 & 1 & 1 & \begin{tabular}{|l|}
1 \\
\end{tabular} & 0 & 1 & 1 & 1 & 1 & 0 & 0 \\
\hline 138B & 18 & 18 & 24 & 1 & 1 & 1 & 0 & 0 & 0 & 0 & 0 & 0 & 0 & 0 & 0 & 1 & 1 & 1 & 1 & 1 & 0 & 1 & 1 & 1 & 1 & 0 & 0 \\
\hline $138 \mathrm{~B}$ & 19 & 19 & 24 & \begin{tabular}{|l|}
1 \\
\end{tabular} & 1 & 1 & 0 & 0 & 0 & 0 & 0 & 0 & 0 & 0 & 0 & 1 & \begin{tabular}{|l|}
1 \\
\end{tabular} & 1 & 1 & \begin{tabular}{|l|}
0 \\
\end{tabular} & \begin{tabular}{|l|}
0 \\
\end{tabular} & 1 & 1 & 1 & 1 & 0 & 0 \\
\hline 138B & 20 & 20 & 24 & 1 & 1 & 1 & 0 & 0 & 0 & \begin{tabular}{l|l|}
0 \\
\end{tabular} & 0 & \begin{tabular}{l|l}
0 \\
\end{tabular} & 0 & 0 & 0 & 1 & 1 & 1 & 1 & 0 & 0 & 1 & 1 & 1 & 1 & 0 & 0 \\
\hline 138B & 21 & 21 & 24 & 1 & 1 & 1 & 0 & 0 & 0 & 0 & 0 & 0 & 0 & 0 & 0 & 1 & 1 & 1 & 1 & 0 & 0 & 1 & 1 & 1 & 1 & 0 & 0 \\
\hline $138 \mathrm{~B}$ & 22 & 22 & 24 & 1 & 1 & 1 & 0 & 0 & 0 & 0 & 0 & 0 & 0 & 0 & 0 & 1 & 1 & 1 & 1 & 0 & 0 & 1 & 1 & 1 & 1 & 0 & 0 \\
\hline $138 \mathrm{~B}$ & 23 & 23 & 24 & 1 & 1 & 0 & 0 & 0 & 0 & \begin{tabular}{|l|l|l|} 
& \\
\end{tabular} & 0 & 0 & 0 & 0 & 0 & 1 & 1 & 1 & 1 & 0 & 0 & 1 & 1 & 1 & 1 & 0 & 0 \\
\hline 138B & 24 & 24 & 24 & 1 & 1 & 0 & 0 & 0 & 0 & 0 & 0 & 0 & 0 & 0 & 0 & 1 & 1 & 1 & 1 & 0 & 0 & 1 & 1 & 1 & 1 & 0 & 0 \\
\hline 138B & 25 & 25 & 24 & 11 & 1 & 0 & 0 & 0 & 0 & 0 & 0 & 0 & 0 & 0 & 0 & 1 & 1 & 1 & 1 & 0 & 0 & 1 & 1 & 0 & 1 & 0 & 0 \\
\hline $138 \mathrm{~B}$ & 26 & 26 & 24 & 1 & 1 & 0 & 0 & 0 & 0 & 0 & 0 & 0 & 0 & 0 & 0 & 1 & 1 & 1 & 1 & \begin{tabular}{|l|}
0 \\
\end{tabular} & 0 & 1 & 1 & 0 & 1 & 0 & 0 \\
\hline 138B & 27 & 27 & 24 & 1 & 1 & 0 & 0 & 0 & 0 & 0 & 0 & 0 & 0 & 0 & 0 & 1 & 1 & 1 & 1 & 0 & 0 & 1 & 1 & 0 & 1 & 0 & 0 \\
\hline 138B & 28 & 28 & 24 & 1 & 1 & 0 & 0 & 0 & 0 & \begin{tabular}{l|l|}
0 \\
\end{tabular} & 0 & 0 & 0 & 0 & 0 & 1 & 1 & 1 & 1 & 0 & 0 & 1 & 1 & 0 & 1 & 0 & 0 \\
\hline 138B & 29 & 29 & 24 & 1 & 0 & 0 & 0 & 0 & 0 & 0 & 0 & 0 & 0 & 0 & 0 & 1 & 1 & 1 & 1 & 0 & 0 & 1 & 0 & 0 & 1 & 0 & 0 \\
\hline 138B & 30 & 30 & 24 & 1 & 0 & 0 & 0 & 0 & 0 & 0 & 0 & 0 & 0 & 0 & 0 & 1 & 1 & 1 & 1 & 0 & 0 & 1 & 0 & 0 & 1 & 0 & 0 \\
\hline 138B & 31 & 31 & 24 & 1 & 0 & 0 & 0 & 0 & 0 & 0 & 0 & 0 & 0 & 0 & 0 & 1 & 1 & 1 & 1 & 0 & 0 & 1 & 0 & 0 & 1 & 0 & 0 \\
\hline 138B & 32 & 32 & 24 & 1 & 0 & 0 & 0 & 0 & 0 & \begin{tabular}{l|l}
0 \\
\end{tabular} & 0 & 0 & 0 & 0 & 0 & 1 & 1 & 1 & 1 & \begin{tabular}{|l|}
0 \\
\end{tabular} & 0 & 1 & \begin{tabular}{|l|}
0 \\
\end{tabular} & 0 & 1 & 0 & 0 \\
\hline 138B & 33 & 33 & 24 & 1 & 0 & 0 & 0 & 0 & 0 & 0 & 0 & 0 & 0 & 0 & 0 & 1 & 1 & 1 & 1 & 0 & 0 & 1 & 0 & 0 & 1 & 0 & 0 \\
\hline $138 \mathrm{~B}$ & 34 & 34 & 24 & 1 & 0 & 0 & 0 & 0 & 0 & 0 & 0 & \begin{tabular}{l|l}
0 \\
\end{tabular} & 0 & 0 & 0 & 1 & 1 & 0 & 1 & 0 & 0 & 1 & 0 & 0 & 1 & 0 & 0 \\
\hline $138 \mathrm{~B}$ & 35 & 35 & 24 & \begin{tabular}{|l|}
1 \\
\end{tabular} & 0 & 0 & 0 & 0 & 0 & 0 & 0 & 0 & 0 & 0 & 0 & 1 & \begin{tabular}{|l|}
1 \\
\end{tabular} & 0 & 1 & \begin{tabular}{|l|}
0 \\
\end{tabular} & 0 & 1 & 0 & 0 & 1 & 0 & 0 \\
\hline 138B & 36 & 36 & 24 & 0 & 0 & 0 & 0 & 0 & 0 & 0 & 0 & 0 & 0 & 0 & 0 & 1 & 1 & 0 & 1 & 0 & 0 & 1 & 0 & 0 & 1 & 0 & 0 \\
\hline $138 \mathrm{~B}$ & 37 & 37 & 24 & 0 & 0 & 0 & 0 & \begin{tabular}{|l|l|}
0 \\
\end{tabular} & 0 & \begin{tabular}{|l|l|}
0 \\
\end{tabular} & 0 & \begin{tabular}{l|l|}
0 \\
\end{tabular} & 0 & 0 & 0 & 1 & \begin{tabular}{|l|}
1 \\
\end{tabular} & 0 & 1 & \begin{tabular}{|l|}
0 \\
\end{tabular} & \begin{tabular}{|l|}
0 \\
\end{tabular} & 0 & 0 & 0 & 1 & 0 & 0 \\
\hline
\end{tabular}




\begin{tabular}{|c|c|c|c|c|c|c|c|c|c|c|c|c|c|c|c|c|c|c|c|c|c|c|c|c|c|c|c|}
\hline \multirow[b]{2}{*}{ Cycle } & \multirow[b]{2}{*}{ Timestep } & \multirow{2}{*}{$\begin{array}{l}\text { Cumulative } \\
\text { Timestep }\end{array}$} & \multirow{2}{*}{$\begin{array}{l}\text { Time } \\
\text { Interval } \\
(\mathrm{hrs})\end{array}$} & \multicolumn{6}{|c|}{$\begin{array}{c}\text { NW } \\
\text { Neck Shims }\end{array}$} & \multicolumn{6}{|c|}{$\begin{array}{c}\text { NE } \\
\text { Neck Shims }\end{array}$} & \multicolumn{6}{|c|}{$\begin{array}{c}\text { SW } \\
\text { Neck Shims }\end{array}$} & \multicolumn{6}{|c|}{$\begin{array}{c}\text { SE } \\
\text { Neck Shims }\end{array}$} \\
\hline & & & & \begin{tabular}{|l|}
1 \\
\end{tabular} & 2 & \begin{tabular}{|l|l|}
3 \\
\end{tabular} & 4 & 5 & 6 & 1 & \begin{tabular}{|l|}
2 \\
\end{tabular} & 3 & \begin{tabular}{|l|}
4 \\
\end{tabular} & \begin{tabular}{|l|}
5 \\
\end{tabular} & 6 & 1 & \begin{tabular}{|l|l|}
2 \\
\end{tabular} & 3 & \begin{tabular}{|l|}
4 \\
\end{tabular} & 5 & 6 & 1 & 2 & \begin{tabular}{|l|}
3 \\
\end{tabular} & 4 & & 6 \\
\hline $138 \mathrm{~B}$ & 38 & 38 & 24 & 0 & 0 & 0 & 0 & 0 & 0 & 0 & 0 & 0 & 0 & 0 & 0 & 1 & 1 & 0 & 1 & 0 & 0 & 0 & 0 & 0 & 1 & 0 & 0 \\
\hline $138 \mathrm{~B}$ & 39 & 39 & 24 & 0 & 0 & 0 & 0 & 0 & 0 & 0 & 0 & 0 & 0 & 0 & 0 & 1 & 1 & 0 & 1 & 0 & 0 & 0 & 0 & 0 & 1 & 0 & 0 \\
\hline $138 \mathrm{~B}$ & 40 & 40 & 24 & 0 & 0 & 0 & 0 & 0 & 0 & 0 & 0 & 0 & 0 & 0 & 0 & 1 & 1 & 0 & 1 & 0 & 0 & 0 & 0 & 0 & 1 & 0 & 0 \\
\hline 138B & 41 & 41 & 24 & 0 & 0 & 0 & 0 & 0 & 0 & 0 & 0 & 0 & 0 & 0 & 0 & 0 & 1 & 0 & 1 & 0 & 0 & 0 & 0 & 0 & 1 & 0 & 0 \\
\hline $138 \mathrm{~B}$ & 42 & 42 & 24 & 0 & 0 & 0 & 0 & 0 & 0 & 0 & 0 & 0 & 0 & 0 & 0 & 0 & 1 & 0 & 1 & 0 & 0 & 0 & 0 & 0 & 1 & 0 & 0 \\
\hline 138B & 43 & 43 & 24 & 0 & 0 & 0 & 0 & 0 & 0 & 0 & 0 & 0 & 0 & 0 & 0 & 0 & 1 & 0 & 1 & 0 & 0 & 0 & 0 & 0 & 1 & 0 & 0 \\
\hline 138B & 44 & 44 & 24 & 0 & 0 & 0 & 0 & 0 & 0 & 0 & 0 & 0 & 0 & 0 & 0 & 0 & 1 & 0 & 1 & 0 & 0 & 0 & 0 & 0 & 1 & 0 & 0 \\
\hline $138 \mathrm{~B}$ & 45 & 45 & 24 & 0 & 0 & 0 & 0 & 0 & 0 & 0 & 0 & 0 & 0 & 0 & 0 & 0 & 1 & 0 & 1 & 0 & 0 & 0 & 0 & 0 & 1 & 0 & 0 \\
\hline $138 \mathrm{~B}$ & 46 & 46 & 24 & 0 & 0 & 0 & 0 & 0 & 0 & 0 & 0 & 0 & 0 & 0 & 0 & 0 & 1 & 0 & 1 & 0 & 0 & 0 & 0 & 0 & 1 & 0 & 0 \\
\hline 138B & 47 & 47 & 24 & 0 & 0 & 0 & 0 & 0 & 0 & 0 & 0 & 0 & 0 & 0 & 0 & 0 & 1 & 0 & 1 & 0 & 0 & 0 & 0 & 0 & 1 & 0 & 0 \\
\hline $138 \mathrm{~B}$ & 48 & 48 & 24 & 0 & 0 & 0 & 0 & 0 & 0 & 0 & 0 & 0 & 0 & 0 & 0 & 0 & 1 & 0 & 1 & 0 & 0 & 0 & 0 & 0 & 1 & 0 & 0 \\
\hline 138B & 49 & 49 & 9 & 0 & 0 & 0 & 0 & 0 & 0 & 0 & 0 & 0 & 0 & 0 & 0 & 0 & 1 & 0 & 1 & 0 & 0 & 0 & 0 & 0 & 1 & 0 & 0 \\
\hline $139 \mathrm{~A}$ & 1 & 50 & 8 & 1 & 1 & 1 & 1 & 1 & 1 & 1 & 1 & 1 & 1 & 1 & 1 & 1 & 1 & 1 & 1 & 1 & 1 & 1 & 1 & 1 & 1 & 1 & 1 \\
\hline $139 \mathrm{~A}$ & 2 & 51 & 24 & 1 & 1 & 1 & 1 & 1 & 1 & 1 & 1 & 0 & 0 & 0 & 0 & 1 & 1 & 1 & 1 & 1 & 0 & 1 & 1 & 1 & 1 & 1 & 1 \\
\hline $139 \mathrm{~A}$ & 3 & 52 & 24 & 1 & 1 & 1 & 1 & 1 & 1 & 1 & 1 & 0 & 0 & 0 & 0 & 1 & 1 & 1 & 1 & 0 & 0 & 1 & 1 & 1 & 1 & 1 & 1 \\
\hline 139A & 4 & 53 & 24 & 1 & 1 & 1 & 1 & 1 & 1 & 1 & 0 & 0 & 0 & 0 & 0 & 1 & 1 & 1 & 1 & 0 & 0 & 1 & 1 & 1 & 1 & 1 & 0 \\
\hline $139 \mathrm{~A}$ & 5 & 54 & 24 & 1 & 1 & 1 & 1 & 1 & 1 & 1 & 0 & 0 & 0 & 0 & 0 & 1 & 1 & 1 & 1 & 0 & 0 & 1 & 1 & 1 & 1 & 1 & 0 \\
\hline 139A & 6 & 55 & 24 & 1 & 1 & 1 & 1 & 1 & 1 & 1 & 0 & 0 & 0 & 0 & 0 & 1 & 1 & 1 & 1 & 0 & 0 & 1 & 1 & 1 & 1 & 1 & 0 \\
\hline $139 \mathrm{~A}$ & 7 & 56 & 24 & 1 & 1 & 1 & 1 & 1 & 1 & 1 & 0 & 0 & 0 & 0 & 0 & 1 & 1 & 1 & 1 & 0 & 0 & 1 & 1 & 1 & 1 & 1 & 0 \\
\hline $139 \mathrm{~A}$ & 8 & 57 & 24 & 1 & 1 & 1 & 1 & 1 & 1 & 1 & 0 & 0 & 0 & 0 & 0 & 1 & 1 & 0 & 1 & 0 & 0 & 1 & 1 & 1 & 1 & 1 & 0 \\
\hline $139 \mathrm{~A}$ & 9 & 58 & 24 & 1 & 1 & 1 & 1 & 1 & 1 & 1 & 0 & 0 & 0 & 0 & 0 & 1 & 1 & 0 & 1 & 0 & 0 & 1 & 1 & 1 & 1 & 1 & 0 \\
\hline $139 \mathrm{~A}$ & 10 & 59 & 24 & 1 & 1 & 1 & 1 & 1 & 1 & 1 & 0 & 0 & 0 & 0 & 0 & 1 & 1 & 0 & 1 & 0 & 0 & 1 & 1 & 1 & 1 & 1 & 0 \\
\hline 139A & 11 & 60 & 24 & 1 & 1 & 1 & 1 & 1 & 1 & 1 & 0 & 0 & 0 & 0 & 0 & 1 & 1 & 0 & 1 & 0 & 0 & 1 & 1 & 1 & 1 & 1 & 0 \\
\hline 139A & 12 & 61 & 24 & 1 & 1 & 1 & 1 & 1 & 1 & 1 & 0 & 0 & 0 & 0 & 0 & 1 & 1 & 0 & 1 & 0 & 0 & 1 & 1 & 1 & 1 & 1 & 0 \\
\hline 139A & 13 & 6 & 24 & 1 & 1 & 1 & 1 & 1 & 1 & 1 & 0 & 0 & 0 & 0 & 0 & 1 & 1 & 0 & 1 & 0 & 0 & 1 & 1 & 1 & 1 & 1 & 0 \\
\hline $139 \mathrm{~A}$ & 14 & 63 & 24 & 1 & 1 & 1 & 1 & 1 & 1 & 1 & 0 & 0 & 0 & 0 & 0 & 1 & 1 & 0 & 1 & 0 & 0 & 1 & 1 & 1 & 1 & 1 & 0 \\
\hline 139A & 15 & 64 & 24 & 1 & 1 & 1 & 1 & 1 & 1 & 1 & 0 & 0 & 0 & 0 & 0 & 1 & 0 & 0 & 1 & 0 & 0 & 1 & 1 & 1 & 1 & 1 & 0 \\
\hline $139 \mathrm{~A}$ & 16 & 65 & 24 & 1 & 1 & 1 & 1 & 1 & 1 & 1 & 0 & 0 & 0 & 0 & 0 & 1 & 0 & 0 & 1 & 0 & 0 & 1 & 1 & 1 & 1 & 1 & 0 \\
\hline 139A & 17 & 66 & 24 & 1 & 1 & 1 & 1 & 1 & 1 & 1 & 0 & 0 & 0 & 0 & 0 & 1 & 0 & 0 & 1 & 0 & 0 & 1 & 1 & 1 & 1 & 1 & 0 \\
\hline $139 \mathrm{~A}$ & 18 & 67 & 24 & 1 & 1 & 1 & 1 & 1 & 1 & 1 & 0 & 0 & 0 & 0 & 0 & 1 & 0 & 0 & 1 & 0 & 0 & 1 & 1 & 1 & 1 & 1 & 0 \\
\hline $139 \mathrm{~A}$ & 19 & 68 & 24 & 1 & 1 & 1 & 1 & 1 & 1 & 0 & 0 & 0 & 0 & 0 & 0 & 1 & 0 & 0 & 1 & 0 & 0 & 1 & 1 & 1 & 1 & 1 & 0 \\
\hline $139 \mathrm{~A}$ & 20 & 69 & 24 & 1 & 1 & 1 & 1 & 1 & 1 & 0 & 0 & 0 & 0 & 0 & 0 & 1 & 0 & 0 & 1 & 0 & 0 & 1 & 1 & 1 & 1 & 1 & 0 \\
\hline 139A & 21 & 70 & 24 & 1 & 1 & 1 & 1 & 1 & 1 & 0 & 0 & 0 & 0 & 0 & 0 & 1 & 0 & 0 & 1 & 0 & 0 & 1 & 1 & 1 & 1 & 1 & 0 \\
\hline $139 \mathrm{~A}$ & 22 & 71 & 24 & 1 & 1 & 1 & 1 & 1 & 1 & 0 & 0 & 0 & 0 & 0 & 0 & 1 & 0 & 0 & 1 & 0 & 0 & 1 & 1 & 1 & 1 & 1 & 0 \\
\hline $139 \mathrm{~A}$ & 23 & 72 & 24 & 1 & 1 & 1 & 1 & 1 & 1 & 1 & 1 & 1 & 1 & 1 & 1 & 1 & 1 & 1 & 1 & 1 & 1 & 1 & 1 & 1 & 1 & 1 & 1 \\
\hline 139A & 24 & 73 & 24 & 1 & 1 & 1 & 1 & 1 & 1 & 1 & 1 & 1 & 1 & 1 & 1 & 1 & 1 & 1 & 1 & 1 & 1 & 1 & 1 & 1 & 1 & 1 & 1 \\
\hline $139 \mathrm{~A}$ & 25 & 74 & 24 & 1 & 1 & 1 & 1 & 1 & 1 & 0 & 0 & 0 & 0 & 0 & 0 & 1 & 0 & 0 & 1 & 0 & 0 & 1 & 1 & 1 & 1 & 1 & 0 \\
\hline 139A & 26 & 75 & 24 & 1 & 1 & 1 & 1 & 1 & 1 & 1 & 0 & 0 & 0 & 0 & 0 & 1 & 0 & 0 & 1 & 0 & 0 & 1 & 1 & 1 & 1 & 1 & 0 \\
\hline $139 \mathrm{~A}$ & 27 & 76 & 24 & 1 & 1 & 1 & 1 & 1 & 0 & 0 & 0 & 0 & 0 & 0 & 0 & 1 & 0 & 0 & 1 & 0 & 0 & 1 & 1 & 1 & 1 & 1 & 0 \\
\hline 139A & 28 & 77 & 24 & 1 & 1 & 1 & 1 & 0 & 0 & 0 & 0 & 0 & 0 & 0 & 0 & 1 & 0 & 0 & 1 & 0 & 0 & 1 & 1 & 1 & 1 & 1 & 0 \\
\hline 139A & 29 & 78 & 24 & 1 & 1 & 1 & 1 & 0 & 0 & 0 & 0 & 0 & 0 & 0 & 0 & 1 & 0 & 0 & 1 & 0 & 0 & 1 & 1 & 1 & 1 & 1 & 0 \\
\hline $139 \mathrm{~A}$ & 30 & 79 & 24 & 1 & 1 & 1 & 1 & 0 & 0 & 0 & 0 & 0 & 0 & 0 & 0 & 1 & 0 & 0 & 1 & 0 & 0 & 1 & 1 & 1 & 1 & 1 & 0 \\
\hline $139 \mathrm{~A}$ & 31 & 80 & 24 & 1 & 1 & 1 & 1 & 0 & 0 & 0 & 0 & 0 & 0 & 0 & 0 & 1 & 0 & 0 & 1 & 0 & 0 & 1 & 1 & 1 & 1 & 1 & 0 \\
\hline $139 \mathrm{~A}$ & 32 & 81 & 24 & 1 & 1 & 1 & 1 & 0 & 0 & 0 & 0 & 0 & 0 & 0 & 1 & 0 & 0 & 0 & 1 & 0 & 0 & 1 & 1 & 1 & 1 & 1 & 0 \\
\hline 139A & 33 & 82 & 24 & 1 & 1 & 1 & 1 & 0 & 0 & 0 & \begin{tabular}{|l|}
0 \\
\end{tabular} & 0 & \begin{tabular}{|l|}
0 \\
\end{tabular} & 0 & 1 & 0 & 0 & 0 & 1 & 0 & 0 & 1 & 1 & 1 & 1 & 1 & 0 \\
\hline
\end{tabular}




\begin{tabular}{|c|c|c|c|c|c|c|c|c|c|c|c|c|c|c|c|c|c|c|c|c|c|c|c|c|c|c|c|}
\hline \multirow[b]{2}{*}{ Cycle } & \multirow[b]{2}{*}{ Timestep } & \multirow{2}{*}{$\begin{array}{l}\text { Cumulative } \\
\text { Timestep }\end{array}$} & \multirow{2}{*}{$\begin{array}{c}\text { Time } \\
\text { Interval } \\
\text { (hrs) }\end{array}$} & \multicolumn{6}{|c|}{$\begin{array}{c}\text { NW } \\
\text { Neck Shims } \\
\end{array}$} & \multicolumn{6}{|c|}{$\begin{array}{c}\text { NE } \\
\text { Neck Shims }\end{array}$} & \multicolumn{6}{|c|}{$\begin{array}{c}\text { SW } \\
\text { Neck Shims }\end{array}$} & \multicolumn{6}{|c|}{$\begin{array}{c}\text { SE } \\
\text { Neck Shims }\end{array}$} \\
\hline & & & & \begin{tabular}{|l|}
1 \\
\end{tabular} & \begin{tabular}{|l|}
2 \\
\end{tabular} & \begin{tabular}{|l|l|}
3 \\
\end{tabular} & 4 & 5 & 6 & 1 & \begin{tabular}{|l|}
2 \\
\end{tabular} & 3 & \begin{tabular}{|l|}
4 \\
\end{tabular} & \begin{tabular}{|l|}
5 \\
\end{tabular} & 6 & 1 & \begin{tabular}{|l|l|}
2 \\
\end{tabular} & 3 & \begin{tabular}{|l|}
4 \\
\end{tabular} & 5 & 6 & 1 & \begin{tabular}{|l|}
2 \\
\end{tabular} & & \begin{tabular}{l|l}
4 \\
\end{tabular} & & 6 \\
\hline $139 \mathrm{~A}$ & 34 & 83 & 24 & 1 & 1 & 1 & 1 & 0 & 0 & 0 & 0 & 0 & 0 & 0 & 1 & 0 & 0 & 0 & 1 & 0 & 0 & 1 & 1 & 1 & 1 & 1 & 0 \\
\hline 139A & 35 & 84 & 24 & 1 & 1 & 1 & 1 & 0 & 0 & 0 & 0 & 0 & 0 & 0 & 1 & 0 & 0 & 0 & 1 & 0 & 0 & 1 & 1 & 1 & 1 & 1 & 0 \\
\hline $139 \mathrm{~A}$ & 36 & 85 & 24 & 1 & 1 & 1 & 0 & 0 & 0 & 0 & 0 & 0 & 0 & 0 & 1 & 0 & 0 & 0 & 1 & 0 & 0 & 1 & \begin{tabular}{|l|}
1 \\
\end{tabular} & 1 & 1 & 1 & 0 \\
\hline 139A & 37 & 86 & 24 & 1 & 1 & 1 & 0 & 0 & 0 & 0 & 0 & 0 & 0 & 0 & 1 & 0 & 0 & 0 & 1 & 0 & 0 & 1 & 1 & 1 & 1 & 1 & 0 \\
\hline $139 \mathrm{~A}$ & 38 & 87 & 24 & 1 & 1 & 1 & 0 & 0 & 0 & 0 & 0 & 0 & 0 & 0 & 1 & 0 & 0 & 0 & 1 & 0 & 0 & 1 & \begin{tabular}{|l|}
1 \\
\end{tabular} & 1 & 1 & 0 & 0 \\
\hline $139 \mathrm{~A}$ & 39 & 88 & 24 & 1 & 1 & 1 & 0 & 0 & 0 & 0 & 0 & 0 & 0 & 0 & 1 & 0 & 0 & 0 & 1 & 0 & 0 & 1 & 1 & 1 & 1 & 0 & 0 \\
\hline $139 \mathrm{~A}$ & 40 & 89 & 24 & 1 & 1 & 0 & 0 & 0 & 0 & 0 & 0 & 0 & 1 & 0 & 1 & 0 & 0 & 0 & 1 & 0 & 0 & 1 & \begin{tabular}{|l|}
1 \\
\end{tabular} & 1 & 1 & 0 & 0 \\
\hline $139 \mathrm{~A}$ & 41 & 90 & 24 & 1 & 1 & 0 & 0 & 0 & 0 & 0 & 0 & 0 & 1 & 0 & 1 & 0 & 0 & 0 & 1 & 0 & 0 & 1 & \begin{tabular}{|l|}
1 \\
\end{tabular} & 1 & 1 & 0 & 0 \\
\hline 139A & 42 & 91 & 24 & 1 & 0 & 0 & 0 & 0 & \begin{tabular}{|l|}
0 \\
\end{tabular} & 0 & 0 & 0 & 1 & 0 & 1 & 0 & 0 & 0 & 1 & 0 & 0 & 1 & 1 & 1 & 1 & 0 & 0 \\
\hline $139 \mathrm{~A}$ & 43 & 92 & 24 & 1 & 0 & 0 & 0 & 0 & 0 & 0 & 0 & 0 & 1 & 0 & 1 & 0 & 0 & 0 & 1 & 0 & 0 & 1 & 1 & 1 & 1 & 0 & 0 \\
\hline 139A & 44 & 93 & 24 & 1 & 0 & 0 & 0 & 0 & 0 & 0 & 0 & 0 & 1 & 0 & 11 & 0 & 0 & 0 & 1 & 0 & 0 & 1 & 1 & 1 & 1 & 0 & 0 \\
\hline $139 \mathrm{~A}$ & 45 & 94 & 24 & 1 & 0 & 0 & 0 & 0 & 0 & 0 & 0 & 0 & 1 & 0 & 1 & 0 & 0 & 0 & 1 & 0 & 0 & 1 & 1 & 0 & 1 & 0 & 0 \\
\hline $139 \mathrm{~A}$ & 46 & 95 & 24 & 1 & 0 & 0 & 0 & 0 & 0 & 0 & 0 & 0 & 1 & 0 & 1 & 0 & 0 & 0 & 1 & 0 & 0 & 1 & \begin{tabular}{|l|}
1 \\
\end{tabular} & 0 & 1 & 0 & 0 \\
\hline $139 \mathrm{~A}$ & 47 & 96 & 24 & 1 & 0 & 0 & 0 & 0 & 0 & 0 & 0 & 0 & 1 & 0 & 1 & 0 & 0 & 0 & 1 & 0 & 0 & 1 & \begin{tabular}{|l|}
0 \\
\end{tabular} & 0 & 1 & 0 & 0 \\
\hline 139A & 48 & 97 & 24 & 1 & 0 & 0 & 0 & 0 & 0 & 0 & 0 & 0 & 1 & 0 & 1 & 0 & 0 & 0 & 1 & 0 & 0 & 1 & \begin{tabular}{|l|}
0 \\
\end{tabular} & 0 & 1 & 0 & 0 \\
\hline 139A & 49 & 98 & 24 & 0 & 0 & 0 & 0 & 0 & 0 & 0 & 0 & 0 & 0 & 0 & 0 & 0 & 0 & 0 & 1 & 0 & 0 & 0 & 0 & 0 & 1 & 0 & 0 \\
\hline 139A & 50 & 99 & 24 & 0 & 0 & 0 & 0 & 0 & 0 & 0 & 0 & 0 & 1 & 0 & 1 & 0 & 0 & 0 & 1 & 0 & 0 & 0 & \begin{tabular}{|l|}
0 \\
\end{tabular} & 0 & 1 & 0 & 0 \\
\hline 139A & 51 & 100 & 24 & 0 & 0 & 0 & 0 & 0 & 0 & 0 & 0 & 0 & 1 & 0 & 1 & 0 & 0 & 0 & 1 & 0 & 0 & 0 & 0 & 0 & 1 & 0 & 0 \\
\hline $139 \mathrm{~A}$ & 52 & 101 & 24 & 0 & 0 & 0 & 0 & 0 & 0 & 0 & 0 & 0 & 1 & 0 & 1 & 0 & 0 & 0 & 1 & 0 & 0 & 0 & \begin{tabular}{|l|}
0 \\
\end{tabular} & 0 & 1 & 0 & 0 \\
\hline $139 \mathrm{~A}$ & 53 & 102 & 24 & 0 & 0 & 0 & 0 & 0 & 0 & 0 & 0 & 0 & 1 & 0 & 1 & 0 & 0 & 0 & 1 & 0 & 0 & 0 & \begin{tabular}{|l|}
0 \\
\end{tabular} & 0 & 1 & 0 & 0 \\
\hline $139 \mathrm{~A}$ & 54 & 103 & 24 & 0 & 0 & 0 & 0 & 0 & 0 & 0 & 0 & 0 & 1 & 0 & 0 & 0 & 0 & 0 & 1 & 0 & 0 & 0 & 0 & 0 & 1 & 0 & 0 \\
\hline $139 \mathrm{~A}$ & 55 & 104 & 24 & 0 & 0 & 0 & 0 & 0 & 0 & 0 & 0 & 0 & 1 & 0 & 0 & 0 & 0 & 0 & 1 & 0 & 0 & 0 & \begin{tabular}{|l|}
0 \\
\end{tabular} & 0 & 1 & 0 & 0 \\
\hline 139A & 56 & 105 & 9 & 0 & 0 & 0 & 0 & 0 & 0 & 0 & 0 & 0 & 1 & 0 & 0 & 0 & 0 & 0 & 1 & 0 & 0 & 0 & \begin{tabular}{|l|}
0 \\
\end{tabular} & 0 & 1 & 0 & 0 \\
\hline 139B & 1 & 106 & 8 & 1 & 1 & 1 & 1 & 1 & 1 & 1 & 1 & 1 & 1 & 1 & 1 & 1 & 1 & 1 & 1 & 1 & 1 & 1 & 1 & 1 & 1 & 1 & 1 \\
\hline 139B & 2 & 10 & 24 & 1 & 1 & 1 & 1 & 1 & 0 & 1 & 0 & 0 & 0 & 0 & 0 & 1 & 1 & 1 & 1 & 1 & 1 & 1 & 1 & 1 & 1 & 1 & 1 \\
\hline 139B & 3 & 108 & 24 & 1 & 1 & 1 & 1 & 1 & 0 & 0 & 0 & 0 & 0 & 0 & 0 & 1 & 1 & 1 & 1 & 1 & 1 & 1 & 1 & 1 & 1 & 1 & 1 \\
\hline 139B & 4 & 109 & 24 & 1 & 1 & 1 & 1 & 1 & 0 & 0 & 0 & 0 & 0 & 0 & 0 & 1 & 1 & 1 & 1 & 1 & 1 & 1 & 1 & 1 & 1 & 1 & 1 \\
\hline 139B & 5 & 110 & 24 & 1 & 1 & 1 & 1 & 0 & 0 & 0 & 0 & 0 & 0 & 0 & 0 & 1 & 1 & 1 & 1 & 1 & 1 & 1 & 1 & 1 & 1 & 1 & 1 \\
\hline 139B & 6 & 111 & 24 & 1 & 1 & 1 & 1 & 0 & 0 & 0 & 0 & 0 & 0 & 0 & 0 & 1 & 1 & 1 & 1 & 1 & 0 & 1 & 1 & 1 & 1 & 1 & 1 \\
\hline 139B & 7 & 112 & 24 & 1 & 1 & 1 & 1 & 0 & 0 & 0 & 0 & 0 & 0 & 0 & 0 & 1 & 1 & 1 & 1 & 1 & 0 & 1 & 1 & 1 & 1 & 1 & 1 \\
\hline 139B & 8 & 113 & 24 & 1 & 1 & 1 & 1 & 0 & 0 & 0 & 0 & 0 & 0 & 0 & 0 & 1 & 1 & 1 & 1 & 1 & 0 & 1 & 1 & 1 & 1 & 1 & 1 \\
\hline 139B & 9 & 114 & 24 & 1 & 1 & 1 & 1 & 0 & 0 & 0 & 0 & 0 & 0 & 0 & 0 & 1 & 1 & 1 & 1 & 1 & 0 & 1 & 1 & 1 & 1 & 1 & 1 \\
\hline 139B & 10 & 115 & 24 & 1 & 1 & 1 & 1 & 0 & 0 & 0 & 0 & 0 & 0 & 0 & 0 & 1 & 1 & 1 & 1 & 0 & 0 & 1 & 1 & 1 & 1 & 1 & 1 \\
\hline 139B & 11 & 116 & 24 & 1 & 1 & 1 & 1 & 0 & 0 & 0 & 0 & 0 & 0 & 0 & 0 & 1 & 1 & 1 & 1 & 0 & 0 & 1 & 1 & 1 & 1 & 1 & 1 \\
\hline 139B & 12 & 117 & 24 & 1 & 1 & 1 & 1 & 0 & 0 & 0 & 0 & 0 & 0 & 0 & 0 & 1 & 1 & 1 & 1 & 0 & 0 & 1 & 1 & 1 & 1 & 1 & 1 \\
\hline 139B & 13 & 118 & 24 & 1 & 1 & 1 & 1 & 0 & 0 & 0 & 0 & 0 & 0 & 0 & 0 & 1 & 1 & 1 & 1 & 0 & 0 & 1 & 1 & 1 & 1 & 1 & 1 \\
\hline 139B & 14 & 119 & 24 & 1 & 1 & 1 & 1 & 0 & 0 & 0 & 0 & 0 & 0 & 0 & 0 & 1 & 1 & 1 & 1 & 0 & 0 & 1 & 1 & 1 & 1 & 1 & 1 \\
\hline 139B & 15 & 120 & 24 & 1 & 1 & 1 & 1 & 0 & 0 & 0 & 0 & 0 & 0 & 0 & 0 & 1 & 1 & 1 & 1 & 0 & 0 & 1 & 1 & 1 & 1 & 1 & 1 \\
\hline 139B & 16 & 121 & 24 & 1 & 1 & 1 & 0 & 0 & 0 & 0 & 0 & 0 & 0 & 0 & 0 & 1 & 1 & 1 & 1 & 0 & 0 & 1 & 1 & 1 & 1 & 1 & 1 \\
\hline 139B & 17 & 122 & 24 & 1 & 1 & 1 & 0 & 0 & 0 & 0 & 0 & 0 & 0 & 0 & 0 & 1 & 1 & 1 & 1 & 0 & 0 & 1 & 1 & 1 & 1 & 1 & 1 \\
\hline 139B & 18 & 123 & 24 & 1 & 1 & 1 & 0 & 0 & 0 & 0 & 0 & 0 & 0 & 0 & 0 & 1 & 1 & 0 & 1 & 0 & 0 & 1 & 1 & 1 & 1 & 1 & 1 \\
\hline 139B & 19 & 124 & 24 & 1 & 1 & 0 & 0 & 0 & 0 & 0 & 0 & 0 & 0 & 0 & 0 & 1 & 1 & 0 & 1 & 0 & 0 & 1 & 1 & 1 & 1 & 1 & 1 \\
\hline 139B & 20 & 125 & 24 & 1 & 1 & 0 & 0 & 0 & 0 & 0 & 0 & 0 & 0 & 0 & 0 & 1 & 1 & 0 & 1 & 0 & 0 & 1 & \begin{tabular}{|l|}
1 \\
\end{tabular} & 1 & 1 & 1 & 1 \\
\hline 139B & 21 & 126 & 24 & 1 & 1 & 0 & 0 & 0 & 0 & 0 & 0 & 0 & 0 & 0 & 0 & 1 & 1 & 0 & 1 & 0 & 0 & 1 & \begin{tabular}{|l|}
1 \\
\end{tabular} & 1 & 1 & 1 & 1 \\
\hline 139B & 22 & 127 & 24 & 1 & 1 & 0 & 0 & 0 & \begin{tabular}{|l|}
0 \\
\end{tabular} & 0 & \begin{tabular}{|l|}
0 \\
\end{tabular} & 0 & \begin{tabular}{|l|}
0 \\
\end{tabular} & 0 & \begin{tabular}{|l|} 
\\
\end{tabular} & 1 & 1 & 0 & 11 & \begin{tabular}{|l|}
0 \\
\end{tabular} & 0 & 11 & 1 & 1 & 1 & 1 & 0 \\
\hline
\end{tabular}




\begin{tabular}{|c|c|c|c|c|c|c|c|c|c|c|c|c|c|c|c|c|c|c|c|c|c|c|c|c|c|c|c|}
\hline \multirow[b]{2}{*}{ Cycle } & \multirow[b]{2}{*}{ Timestep } & \multirow{2}{*}{$\begin{array}{l}\text { Cumulative } \\
\text { Timestep }\end{array}$} & \multirow{2}{*}{$\begin{array}{c}\text { Time } \\
\text { Interval } \\
(\mathrm{hrs})\end{array}$} & \multicolumn{6}{|c|}{$\begin{array}{c}\text { NW } \\
\text { Neck Shims }\end{array}$} & \multicolumn{6}{|c|}{$\begin{array}{c}\text { NE } \\
\text { Neck Shims }\end{array}$} & \multicolumn{6}{|c|}{$\begin{array}{c}\text { SW } \\
\text { Neck Shims }\end{array}$} & \multicolumn{6}{|c|}{$\begin{array}{c}\text { SE } \\
\text { Neck Shims }\end{array}$} \\
\hline & & & & 1 & 2 & 3 & 4 & 5 & 6 & 1 & \begin{tabular}{|l|}
2 \\
\end{tabular} & 3 & \begin{tabular}{|l|}
4 \\
\end{tabular} & \begin{tabular}{|l|}
5 \\
\end{tabular} & 6 & 1 & \begin{tabular}{|l|l|}
2 \\
\end{tabular} & 3 & \begin{tabular}{|l|}
4 \\
\end{tabular} & 5 & 6 & & 2 & \begin{tabular}{|l|}
3 \\
\end{tabular} & 4 & & 6 \\
\hline $139 \mathrm{~B}$ & 23 & 128 & 24 & 1 & 1 & 0 & 0 & 0 & 0 & 0 & 0 & 0 & 0 & 0 & 0 & 1 & 1 & 0 & 1 & 0 & 0 & 1 & 1 & 1 & 1 & 1 & 0 \\
\hline 139B & 24 & 129 & 24 & 1 & 1 & 0 & 0 & 0 & 0 & 0 & 0 & 0 & 0 & 0 & 0 & 1 & 1 & 0 & 1 & 0 & 0 & 1 & 1 & 1 & 1 & 1 & 0 \\
\hline 139B & 25 & 130 & 24 & 1 & 0 & 0 & 0 & 0 & 0 & 0 & 0 & 0 & 0 & 0 & 0 & 1 & 1 & 0 & 1 & 0 & 0 & 1 & 1 & 1 & 1 & 1 & 0 \\
\hline 139B & 26 & 131 & 24 & 1 & 0 & 0 & 0 & 0 & 0 & 0 & 0 & 0 & 0 & 0 & 0 & 1 & 1 & 0 & 1 & 0 & 0 & 1 & 1 & 1 & 1 & 1 & 0 \\
\hline 139B & 27 & 132 & 24 & 1 & 0 & 0 & 0 & 0 & 0 & 0 & 0 & 0 & 0 & 0 & 0 & 1 & 1 & 0 & 1 & 0 & 0 & 1 & 1 & 1 & 1 & 1 & 0 \\
\hline 139B & 28 & 133 & 24 & 1 & 0 & 0 & 0 & 0 & 0 & 0 & 0 & 0 & 0 & 0 & 0 & 1 & 1 & 0 & 1 & 0 & 0 & 1 & 1 & 1 & 1 & 1 & 0 \\
\hline 139B & 29 & 134 & 24 & 1 & 0 & 0 & 0 & 0 & 0 & 0 & 0 & 0 & 0 & 0 & 0 & 1 & 1 & 0 & 1 & 0 & 0 & 1 & 1 & 1 & 1 & 0 & 0 \\
\hline 139B & 30 & 135 & 24 & 1 & 0 & 0 & 0 & 0 & 0 & 0 & 0 & 0 & 0 & 0 & 0 & 1 & 1 & 0 & 1 & 0 & 0 & 1 & 1 & 1 & 1 & 0 & 0 \\
\hline 139B & 31 & 136 & 24 & 1 & 0 & 0 & 0 & 0 & 0 & 0 & 0 & 0 & 0 & 0 & 0 & 1 & 0 & 0 & 1 & 0 & 0 & 1 & 1 & 1 & 1 & 0 & 0 \\
\hline 139B & 32 & 137 & 24 & 1 & 0 & 0 & 0 & 0 & 0 & 0 & 0 & 0 & 0 & 0 & 0 & 1 & 0 & 0 & 1 & 0 & 0 & 1 & 1 & 1 & 1 & 0 & 0 \\
\hline 139B & 33 & 138 & 7 & 1 & 0 & 0 & 0 & 0 & 0 & 0 & 0 & 0 & 0 & 0 & 0 & 1 & 0 & 0 & 1 & 0 & 0 & 1 & 1 & 1 & 1 & 0 & 0 \\
\hline 139B & 34 & 139 & 1044 & 1 & 0 & 0 & 0 & 0 & 0 & 0 & 0 & 0 & 0 & 0 & 0 & 1 & 0 & 0 & 1 & 0 & 0 & 1 & 1 & 1 & 1 & 0 & 0 \\
\hline 139B & 35 & 140 & 13 & 1 & 0 & 0 & 0 & 0 & 0 & 0 & 0 & 0 & 0 & 0 & 0 & 1 & 0 & 0 & 1 & 0 & 0 & 1 & 1 & 1 & 1 & 0 & 0 \\
\hline 139B & 36 & 141 & 24 & 0 & 0 & 0 & 0 & 0 & 0 & 0 & 0 & 0 & 0 & 0 & 0 & 1 & 0 & 0 & 1 & 0 & 0 & 1 & 1 & 1 & 1 & 0 & 0 \\
\hline 139B & 37 & 142 & 24 & 0 & 0 & 0 & 0 & 0 & 0 & 0 & 0 & 0 & 0 & 0 & 0 & 0 & 0 & 0 & 1 & 0 & 0 & 1 & 1 & 1 & 1 & 0 & 0 \\
\hline 139B & 38 & 143 & 24 & 0 & 0 & 0 & 0 & 0 & 0 & 0 & 0 & 0 & 0 & 0 & 0 & 0 & 0 & 0 & 1 & 0 & 0 & 1 & 1 & 1 & 1 & 0 & 0 \\
\hline 139B & 39 & 144 & 24 & 0 & 0 & 0 & 0 & 0 & 0 & 0 & 0 & 0 & 0 & 0 & 0 & 0 & 0 & 0 & 1 & 0 & 0 & 1 & 1 & 1 & 1 & 0 & 0 \\
\hline 139B & 40 & 145 & 24 & 0 & 0 & 0 & 0 & 0 & 0 & 0 & 0 & 0 & 0 & 0 & 0 & 0 & 0 & 0 & 1 & 0 & 0 & 1 & 1 & 0 & 1 & 0 & 0 \\
\hline 139B & 41 & 146 & 24 & 0 & 0 & 0 & 0 & 0 & 0 & 0 & 0 & 0 & 0 & 0 & 0 & 0 & 0 & 0 & 1 & 0 & 0 & 1 & 1 & 0 & 1 & 0 & 0 \\
\hline 139B & 42 & 147 & 24 & 0 & 0 & 0 & 0 & 0 & 0 & 0 & 0 & 0 & 0 & 0 & 0 & 0 & 0 & 0 & 1 & 0 & 0 & 1 & 0 & 0 & 1 & 0 & 0 \\
\hline 139B & 43 & 148 & 24 & 0 & 0 & 0 & 0 & 0 & 0 & 0 & 0 & 0 & 0 & 0 & 0 & 0 & 0 & 0 & 1 & 0 & 0 & 1 & 0 & 0 & 1 & 0 & 0 \\
\hline 139B & 44 & 149 & 24 & 0 & 0 & 0 & 0 & 0 & 0 & 0 & 0 & 0 & 0 & 0 & 0 & 0 & 0 & 0 & 1 & 0 & 0 & 1 & 0 & 0 & 1 & 0 & 0 \\
\hline 139B & 45 & 150 & 24 & 0 & 0 & 0 & 0 & 0 & 0 & 0 & 0 & 0 & 0 & 0 & 0 & 0 & 0 & 0 & 1 & 0 & 0 & 0 & 0 & 0 & 1 & 0 & 0 \\
\hline 139B & 46 & 151 & 24 & 0 & 0 & 0 & 0 & 0 & 0 & 0 & 0 & 0 & 0 & 0 & 0 & 0 & 0 & 0 & 1 & 0 & 0 & 0 & 0 & 0 & 1 & 0 & 0 \\
\hline 139B & 47 & 1 & 24 & 0 & 0 & 0 & 0 & 0 & 0 & 0 & 0 & 0 & 0 & 0 & 0 & 0 & 0 & 0 & 1 & 0 & 0 & 0 & 0 & 0 & 1 & 0 & 0 \\
\hline 139B & 48 & 153 & 24 & 0 & 0 & 0 & 0 & 0 & 0 & 0 & 0 & 0 & 0 & 0 & 0 & 0 & 0 & 0 & 1 & 0 & 0 & 0 & 0 & 0 & 1 & 0 & 0 \\
\hline 139B & 49 & 154 & 24 & 0 & 0 & 0 & 0 & 0 & 0 & 0 & 0 & 0 & 0 & 0 & 0 & 0 & 0 & 0 & 1 & 0 & 0 & 0 & 0 & 0 & 1 & 0 & 0 \\
\hline 139B & 50 & 155 & 24 & 0 & 0 & 0 & 0 & 0 & 0 & 0 & 0 & 0 & 0 & 0 & 0 & 0 & 0 & 0 & 1 & 0 & 0 & 0 & 0 & 0 & 1 & 0 & 0 \\
\hline 139B & 51 & 156 & 24 & 0 & 0 & 0 & 0 & 0 & 0 & 0 & 0 & 0 & 0 & 0 & 0 & 0 & 0 & 0 & 1 & 0 & 0 & 0 & 0 & 0 & 1 & 0 & 0 \\
\hline 139B & 52 & 157 & 24 & 0 & 0 & 0 & 0 & 0 & 0 & 0 & 0 & 0 & 0 & 0 & 0 & 0 & 0 & 0 & 1 & 0 & 0 & 0 & 0 & 0 & 1 & 0 & 0 \\
\hline 139B & 53 & 158 & 24 & 0 & 0 & 0 & 0 & 0 & 0 & 0 & 0 & 0 & 0 & 0 & 0 & 0 & 0 & 0 & 1 & 0 & 0 & 0 & 0 & 0 & 1 & 0 & 0 \\
\hline 139B & 54 & 159 & 24 & 0 & 0 & 0 & 0 & 0 & 0 & 0 & 0 & 0 & 0 & 0 & 0 & 0 & 0 & 0 & 1 & 0 & 0 & 0 & 0 & 0 & 1 & 0 & 0 \\
\hline 139B & 55 & 160 & 21 & 0 & 0 & 0 & 0 & 0 & 0 & 0 & 0 & 0 & 0 & 0 & 0 & 0 & 0 & 0 & 1 & 0 & 0 & 0 & 0 & 0 & 1 & 0 & 0 \\
\hline $140 \mathrm{~A}$ & 1 & 161 & 8 & 1 & 1 & 1 & 1 & 1 & 1 & 1 & 1 & 1 & 1 & 1 & 1 & 1 & 1 & 1 & 1 & 1 & 1 & 1 & 1 & 1 & 1 & 1 & 1 \\
\hline $140 \mathrm{~A}$ & 2 & 162 & 24 & 1 & 1 & 1 & 1 & 1 & 0 & 0 & 1 & 0 & 0 & 0 & 0 & 1 & 1 & 1 & 1 & 1 & 0 & 1 & 1 & 1 & 1 & 1 & 1 \\
\hline $140 \mathrm{~A}$ & 3 & 163 & 24 & 1 & 1 & 1 & 1 & 1 & 0 & 0 & 0 & 0 & 0 & 0 & 0 & 1 & 1 & 1 & 1 & 1 & 0 & 1 & 1 & 1 & 1 & 1 & 1 \\
\hline $140 \mathrm{~A}$ & 4 & 164 & 24 & 1 & 1 & 1 & 1 & 1 & 0 & 0 & 0 & 0 & 0 & 0 & 0 & 1 & 1 & 1 & 1 & 1 & 0 & 1 & 1 & 1 & 1 & 1 & 1 \\
\hline $140 \mathrm{~A}$ & 5 & 165 & 24 & 1 & 1 & 1 & 1 & 1 & 0 & 0 & 0 & 0 & 0 & 0 & 0 & 1 & 1 & 1 & 1 & 1 & 0 & 1 & 1 & 1 & 1 & 1 & 1 \\
\hline $140 \mathrm{~A}$ & 6 & 166 & 24 & 1 & 1 & 1 & 1 & 1 & 0 & 0 & 0 & 0 & 0 & 0 & 0 & 1 & 1 & 1 & 1 & 0 & 0 & 1 & 1 & 1 & 1 & 1 & 1 \\
\hline $140 \mathrm{~A}$ & 7 & 167 & 24 & 1 & 1 & 1 & 1 & 1 & 0 & 0 & 0 & 0 & 0 & 0 & 0 & 1 & 1 & 1 & 1 & 0 & 0 & 1 & 1 & 1 & 1 & 1 & 1 \\
\hline $140 \mathrm{~A}$ & 8 & 168 & 24 & 1 & 1 & 1 & 1 & 1 & 0 & 0 & 0 & 0 & 0 & 0 & 0 & 1 & 1 & 1 & 1 & 0 & 0 & 1 & 1 & 1 & 1 & 1 & 1 \\
\hline $140 \mathrm{~A}$ & 9 & 169 & 24 & 1 & 1 & 1 & 1 & 1 & 0 & 0 & 0 & 0 & 0 & 0 & 0 & 1 & 1 & 1 & 1 & 0 & 0 & 1 & 1 & 1 & 1 & 1 & 1 \\
\hline $140 \mathrm{~A}$ & 10 & 170 & 24 & 1 & 1 & 1 & 1 & 1 & 0 & 0 & 0 & 0 & 0 & 0 & 0 & 1 & 1 & 1 & 1 & 0 & 0 & 1 & 1 & 1 & 1 & 1 & 1 \\
\hline $140 \mathrm{~A}$ & 11 & 171 & 24 & 1 & 1 & 1 & 1 & 1 & 0 & 0 & 0 & 0 & 0 & 0 & 0 & 1 & 1 & 0 & 1 & 0 & 0 & 1 & 1 & 1 & 1 & 1 & 0 \\
\hline $140 \mathrm{~A}$ & 12 & 172 & 24 & 1 & 1 & 1 & 1 & 1 & 0 & 0 & \begin{tabular}{|l|}
0 \\
\end{tabular} & 0 & \begin{tabular}{|l|}
0 \\
\end{tabular} & 0 & 0 & 1 & 1 & 0 & 1 & 0 & 0 & 1 & 1 & 1 & 1 & 1 & 0 \\
\hline
\end{tabular}




\begin{tabular}{|c|c|c|c|c|c|c|c|c|c|c|c|c|c|c|c|c|c|c|c|c|c|c|c|c|c|c|c|}
\hline \multirow[b]{2}{*}{ Cycle } & \multirow[b]{2}{*}{ Timestep } & \multirow{2}{*}{$\begin{array}{l}\text { Cumulative } \\
\text { Timestep }\end{array}$} & \multirow{2}{*}{$\begin{array}{c}\text { Time } \\
\text { Interval } \\
(\mathrm{hrs})\end{array}$} & \multicolumn{6}{|c|}{$\begin{array}{c}\text { NW } \\
\text { Neck Shims }\end{array}$} & \multicolumn{6}{|c|}{$\begin{array}{c}\text { NE } \\
\text { Neck Shims }\end{array}$} & \multicolumn{6}{|c|}{$\begin{array}{c}\text { SW } \\
\text { Neck Shims }\end{array}$} & \multicolumn{6}{|c|}{$\begin{array}{c}\text { SE } \\
\text { Neck Shims }\end{array}$} \\
\hline & & & & \begin{tabular}{|l|}
1 \\
\end{tabular} & 2 & \begin{tabular}{|l|}
3 \\
\end{tabular} & 4 & 5 & 6 & 1 & 2 & 3 & 4 & 5 & 6 & 1 & \begin{tabular}{|l|}
2 \\
\end{tabular} & 3 & 4 & 5 & 6 & & 2 & 3 & 4 & & 6 \\
\hline $140 \mathrm{~A}$ & 13 & 173 & 24 & 1 & 1 & 1 & 1 & 1 & 0 & 0 & 0 & 0 & 0 & 0 & 0 & 1 & 1 & 0 & 1 & 0 & 0 & 1 & 1 & 1 & 1 & 1 & 0 \\
\hline $140 \mathrm{~A}$ & 14 & 174 & 24 & 1 & 1 & 1 & 1 & 1 & 0 & 0 & 0 & 0 & 0 & 0 & 0 & 1 & 1 & 0 & 1 & 0 & 0 & 1 & 1 & 1 & 1 & 1 & 0 \\
\hline $140 \mathrm{~A}$ & 15 & 175 & 24 & 1 & 1 & 1 & 1 & 1 & 0 & 0 & 0 & 0 & 0 & 0 & 0 & 1 & 1 & 0 & 1 & 0 & 0 & 1 & 1 & 1 & 1 & 1 & 0 \\
\hline $140 \mathrm{~A}$ & 16 & 176 & 24 & 1 & 1 & 1 & 1 & 1 & 0 & 0 & 0 & 0 & 0 & 0 & 0 & 1 & 1 & 0 & 1 & 0 & 0 & 1 & 1 & 1 & 1 & 1 & 0 \\
\hline $140 \mathrm{~A}$ & 17 & 177 & 24 & 1 & 1 & 1 & 1 & 1 & 0 & 0 & 0 & 0 & 0 & 0 & 0 & 1 & 1 & 0 & 1 & 0 & 0 & 1 & 1 & 1 & 1 & 1 & 0 \\
\hline $140 \mathrm{~A}$ & 18 & 178 & 24 & 1 & 1 & 1 & 1 & 1 & 0 & 0 & 0 & 0 & 0 & 0 & 0 & 1 & 1 & 0 & 1 & 0 & 0 & 1 & 1 & 1 & 1 & 1 & 0 \\
\hline $140 \mathrm{~A}$ & 19 & 179 & 24 & 1 & 1 & 1 & 1 & 1 & 0 & 0 & 0 & 0 & 0 & 0 & 0 & 1 & 1 & 0 & 1 & 0 & 0 & 1 & 1 & 1 & 1 & 1 & 0 \\
\hline $140 \mathrm{~A}$ & 20 & 180 & 24 & 1 & 1 & 1 & 1 & 0 & 0 & 0 & 0 & 0 & 0 & 0 & 0 & 1 & 0 & 0 & 1 & 0 & 0 & 1 & 1 & $\begin{array}{ll}1 \\
\end{array}$ & 1 & 1 & 0 \\
\hline $140 \mathrm{~A}$ & 21 & 181 & 24 & 1 & 1 & 1 & 1 & 0 & 0 & 0 & 0 & 0 & 0 & 0 & 0 & 1 & 0 & 0 & 1 & 0 & 0 & 1 & 1 & 1 & 1 & 1 & 0 \\
\hline $140 \mathrm{~A}$ & 22 & 182 & 24 & 1 & 1 & 1 & 1 & 0 & 0 & 0 & 0 & 0 & 0 & 0 & 0 & 1 & 0 & 0 & 1 & 0 & 0 & 1 & 1 & 1 & 1 & 1 & 0 \\
\hline $140 \mathrm{~A}$ & 23 & 183 & 24 & 1 & 1 & 1 & 1 & 0 & 0 & 0 & 0 & 0 & 0 & 0 & 0 & 1 & 0 & 0 & 1 & 0 & 0 & 1 & 1 & 1 & 1 & 1 & 0 \\
\hline $140 \mathrm{~A}$ & 24 & 184 & 24 & 1 & 1 & 1 & 1 & 0 & 0 & 0 & 0 & 0 & 0 & 0 & 0 & 1 & 0 & 0 & 1 & 0 & 0 & 1 & 1 & 1 & 1 & 1 & 0 \\
\hline $140 \mathrm{~A}$ & 25 & 185 & 24 & 1 & 1 & 1 & 1 & 0 & 0 & 0 & 0 & 0 & 0 & 0 & 0 & 1 & 0 & 0 & 1 & 0 & 0 & 1 & 1 & 1 & 1 & 1 & 0 \\
\hline $140 \mathrm{~A}$ & 26 & 186 & 24 & 1 & 1 & 1 & 1 & 0 & 0 & 0 & 0 & 0 & 0 & 0 & 0 & 1 & 0 & 0 & 1 & 0 & 0 & 1 & 1 & 1 & 1 & 0 & 0 \\
\hline $140 \mathrm{~A}$ & 27 & 187 & 24 & 1 & 1 & 1 & 1 & 0 & 0 & 0 & 0 & 0 & 0 & 0 & 0 & 1 & 0 & 0 & 1 & 0 & 0 & 1 & 1 & 1 & 1 & 0 & 0 \\
\hline $140 \mathrm{~A}$ & 28 & 188 & 24 & 1 & 1 & 1 & 1 & 0 & 0 & 0 & 0 & 0 & 0 & 0 & 0 & 1 & 0 & 0 & 1 & 0 & 0 & 1 & 1 & 1 & 1 & 0 & 0 \\
\hline $140 \mathrm{~A}$ & 29 & 189 & 24 & 1 & 1 & 1 & 0 & 0 & 0 & 0 & 0 & 0 & 0 & 0 & 0 & 0 & 0 & 0 & 1 & 0 & 0 & 1 & 1 & 1 & 1 & 0 & 0 \\
\hline $140 \mathrm{~A}$ & 30 & 190 & 24 & 1 & 1 & 1 & 0 & 0 & 0 & 0 & 0 & 0 & 0 & 0 & 0 & 0 & 0 & 0 & 1 & 0 & 0 & 1 & 1 & 1 & 1 & 0 & 0 \\
\hline $140 \mathrm{~A}$ & 31 & 191 & 24 & 1 & 1 & 1 & 0 & 0 & 0 & 0 & 0 & 0 & 0 & 0 & 0 & 0 & 0 & 0 & 1 & 0 & 0 & 1 & 1 & 1 & 1 & 0 & 0 \\
\hline $140 \mathrm{~A}$ & 32 & 192 & 24 & 1 & 1 & 0 & 0 & 0 & 0 & 0 & 0 & 0 & 0 & 0 & 0 & 0 & 0 & 0 & 1 & 0 & 0 & 1 & 1 & 1 & 1 & 0 & 0 \\
\hline $140 \mathrm{~A}$ & 33 & 193 & 24 & 1 & 1 & 0 & 0 & 0 & 0 & 0 & 0 & 0 & 0 & 0 & 0 & 0 & 0 & 0 & 1 & 0 & 0 & 1 & 1 & 1 & 1 & 0 & 0 \\
\hline $140 \mathrm{~A}$ & 34 & 194 & 24 & 1 & 1 & 0 & 0 & 0 & 0 & 0 & 0 & 0 & 0 & 0 & 0 & 0 & 0 & 0 & 1 & 0 & 0 & 1 & 1 & 1 & 1 & 0 & 0 \\
\hline $140 \mathrm{~A}$ & 35 & 195 & 24 & 1 & 1 & 0 & 0 & 0 & 0 & 0 & 0 & 0 & 0 & 0 & 0 & 0 & 0 & 0 & 1 & 0 & 0 & 1 & 1 & 0 & 1 & 0 & 0 \\
\hline $140 \mathrm{~A}$ & 36 & 196 & 24 & 1 & 1 & 0 & 0 & 0 & 0 & 0 & 0 & 0 & 0 & 0 & 0 & 0 & 0 & 0 & 1 & 0 & 0 & 1 & 1 & 0 & 1 & 0 & 0 \\
\hline $140 \mathrm{~A}$ & 37 & 197 & 2 & 1 & 1 & 0 & 0 & 0 & 0 & 0 & 0 & 0 & 0 & 0 & 0 & 0 & 0 & 0 & 1 & 0 & 0 & 1 & 1 & 0 & 1 & 0 & 0 \\
\hline $140 \mathrm{~A}$ & 38 & 198 & 24 & 1 & 0 & 0 & 0 & 0 & 0 & 0 & 0 & 0 & 0 & 0 & 0 & 0 & 0 & 0 & 1 & 0 & 0 & 1 & 1 & 0 & 1 & 0 & 0 \\
\hline $140 \mathrm{~A}$ & 39 & 199 & 24 & 1 & 0 & 0 & 0 & 0 & 0 & 0 & 0 & 0 & 0 & 0 & 0 & 0 & 0 & 0 & 1 & 0 & 0 & 1 & 0 & 0 & 1 & 0 & 0 \\
\hline $140 \mathrm{~A}$ & 40 & 200 & 24 & 1 & 0 & 0 & 0 & 0 & 0 & 0 & 0 & 0 & 0 & 0 & 0 & 0 & 0 & 0 & 1 & 0 & 0 & 1 & 0 & 0 & 1 & 0 & 0 \\
\hline $140 \mathrm{~A}$ & 41 & 201 & 24 & 1 & 0 & 0 & 0 & 0 & 0 & 0 & 0 & 0 & 0 & 0 & 0 & 0 & 0 & 0 & 1 & 0 & 0 & 1 & 0 & 0 & 1 & 0 & 0 \\
\hline $140 \mathrm{~A}$ & 42 & 202 & 24 & 0 & 0 & 0 & 0 & 0 & 0 & 0 & 0 & 0 & 0 & 0 & 0 & 0 & 0 & 0 & 1 & 0 & 0 & 1 & 0 & 0 & 1 & 0 & 0 \\
\hline $140 \mathrm{~A}$ & 43 & 203 & 24 & 0 & 0 & 0 & 0 & 0 & 0 & 0 & 0 & 0 & 0 & 0 & 0 & 0 & 0 & 0 & 1 & 0 & 0 & 1 & 0 & 0 & 1 & 0 & 0 \\
\hline $140 \mathrm{~A}$ & 44 & 204 & 24 & 0 & 0 & 0 & 0 & 0 & 0 & 0 & 0 & 0 & 0 & 0 & 0 & 0 & 0 & 0 & 1 & 0 & 0 & 1 & 0 & 0 & 1 & 0 & 0 \\
\hline $140 \mathrm{~A}$ & 45 & 205 & 24 & 0 & 0 & 0 & 0 & 0 & 0 & 0 & 0 & 0 & 0 & 0 & 0 & 0 & 0 & 0 & 1 & 0 & 0 & 0 & 0 & 0 & 1 & 0 & 0 \\
\hline $140 \mathrm{~A}$ & 46 & 206 & 24 & 0 & 0 & 0 & 0 & 0 & 0 & 0 & 0 & 0 & 0 & 0 & 0 & 0 & 0 & 0 & 1 & 0 & 0 & 0 & 0 & 0 & 1 & 0 & 0 \\
\hline $140 \mathrm{~A}$ & 47 & 207 & 24 & 0 & 0 & 0 & 0 & 0 & 0 & 0 & 0 & 0 & 0 & 0 & 0 & 0 & 0 & 0 & 1 & 0 & 0 & 0 & 0 & 0 & 1 & 0 & 0 \\
\hline $140 \mathrm{~A}$ & 48 & 208 & 11 & 0 & 0 & 0 & 0 & 0 & 0 & 0 & 0 & 0 & 0 & 0 & 0 & 0 & 0 & 0 & 1 & 0 & 0 & 0 & 0 & 0 & 1 & 0 & 0 \\
\hline 140B & 1 & 209 & 8 & 1 & 1 & 1 & 1 & 1 & 1 & 1 & 1 & 1 & 1 & 1 & 1 & 1 & 1 & 1 & 1 & 1 & 1 & 1 & 1 & 1 & 1 & 1 & 1 \\
\hline 140B & 2 & 210 & 24 & 0 & 1 & 1 & 1 & 1 & 0 & 1 & 1 & 1 & 1 & 1 & 0 & 1 & 0 & 0 & 1 & 0 & 0 & 1 & 1 & 1 & 1 & 1 & 1 \\
\hline 140B & 3 & 211 & 24 & 0 & 1 & 1 & 1 & 1 & 0 & 0 & 1 & 1 & 0 & 0 & 0 & 0 & 0 & 0 & 1 & 0 & 0 & 1 & 1 & 1 & 1 & 1 & 0 \\
\hline 140B & 4 & 212 & 24 & 0 & 1 & 1 & 1 & 1 & 0 & 0 & 1 & 1 & 0 & 0 & 0 & 0 & 0 & 0 & 1 & 0 & 0 & 1 & 1 & 1 & 1 & 1 & 0 \\
\hline 140B & 5 & 213 & 24 & 0 & 1 & 1 & 1 & 1 & 0 & 0 & 1 & 1 & 0 & 0 & 0 & 0 & 0 & 0 & 1 & 0 & 0 & 1 & 1 & 1 & 1 & 1 & 0 \\
\hline 140B & 6 & 214 & 24 & 0 & 1 & 1 & 1 & 1 & 0 & 0 & 1 & 1 & 0 & 0 & 0 & 0 & 0 & 0 & 1 & 0 & 0 & 1 & 1 & 1 & 1 & 1 & 0 \\
\hline 140B & 7 & 215 & 24 & 0 & 1 & 1 & 1 & 1 & 0 & 0 & 1 & 1 & 0 & 0 & 0 & 0 & 0 & 0 & 1 & 0 & 0 & 1 & 1 & 1 & 1 & 1 & 0 \\
\hline 140B & 8 & 216 & 24 & 0 & 1 & 1 & 1 & 1 & 0 & 0 & 1 & 1 & 0 & 0 & 0 & 0 & 0 & 0 & 1 & 0 & 0 & 1 & 1 & 1 & 1 & 1 & 0 \\
\hline 140B & 9 & 217 & 24 & 0 & 1 & 1 & 1 & 0 & 0 & 0 & 1 & 1 & 0 & 0 & 0 & 0 & 0 & 0 & 1 & 0 & 0 & 1 & 1 & 1 & 1 & 0 & 0 \\
\hline
\end{tabular}




\begin{tabular}{|c|c|c|c|c|c|c|c|c|c|c|c|c|c|c|c|c|c|c|c|c|c|c|c|c|c|c|c|}
\hline \multirow[b]{2}{*}{ Cycle } & \multirow[b]{2}{*}{ Timestep } & \multirow{2}{*}{$\begin{array}{l}\text { Cumulative } \\
\text { Timestep }\end{array}$} & \multirow{2}{*}{$\begin{array}{c}\text { Time } \\
\text { Interval } \\
(\mathrm{hrs})\end{array}$} & \multicolumn{6}{|c|}{$\begin{array}{c}\text { NW } \\
\text { Neck Shims } \\
\end{array}$} & \multicolumn{6}{|c|}{$\begin{array}{c}\text { NE } \\
\text { Neck Shims }\end{array}$} & \multicolumn{6}{|c|}{$\begin{array}{c}\text { SW } \\
\text { Neck Shims }\end{array}$} & \multicolumn{6}{|c|}{$\begin{array}{c}\text { SE } \\
\text { Neck Shims }\end{array}$} \\
\hline & & & & \begin{tabular}{|l|}
1 \\
\end{tabular} & 2 & \begin{tabular}{|l|l|}
3 \\
\end{tabular} & 4 & 5 & 6 & 1 & \begin{tabular}{|l|}
2 \\
\end{tabular} & 3 & \begin{tabular}{|l|}
4 \\
\end{tabular} & \begin{tabular}{|l|}
5 \\
\end{tabular} & 6 & 1 & \begin{tabular}{|l|l|}
2 \\
\end{tabular} & 3 & \begin{tabular}{|l|}
4 \\
\end{tabular} & 5 & 6 & & 2 & \begin{tabular}{|l|}
3 \\
\end{tabular} & 4 & & 6 \\
\hline 140B & 10 & 218 & 24 & 0 & 1 & 1 & 0 & 0 & 0 & 0 & 1 & 1 & 0 & 0 & 0 & 0 & 0 & 0 & 1 & 0 & 0 & 1 & 1 & 1 & 1 & 0 & 0 \\
\hline 140B & 11 & 219 & 24 & 0 & 1 & 1 & 0 & 0 & 0 & 0 & 1 & 1 & 0 & 0 & 0 & 0 & 0 & 0 & 1 & 0 & 0 & 1 & 1 & 1 & 1 & 0 & 0 \\
\hline 140B & 12 & 220 & 24 & 0 & 1 & 1 & 0 & 0 & 0 & 0 & 1 & 1 & 0 & 0 & 0 & 0 & 0 & 0 & 1 & 0 & 0 & 1 & 1 & 1 & 1 & 0 & 0 \\
\hline 140B & 13 & 221 & 24 & 0 & 1 & 1 & 0 & 0 & 0 & 0 & 1 & 1 & 0 & 0 & 0 & 0 & 0 & 0 & 1 & 0 & 0 & 1 & 1 & 0 & 1 & 0 & 0 \\
\hline 140B & 14 & 222 & 24 & 0 & 1 & 1 & 0 & 0 & 0 & 0 & 1 & 1 & 0 & 0 & 0 & 0 & 0 & 0 & 1 & 0 & 0 & 1 & 1 & 0 & 1 & 0 & 0 \\
\hline 140B & 15 & 223 & 24 & 0 & 1 & 1 & 0 & 0 & 0 & 0 & 1 & 1 & 0 & 0 & 0 & 0 & 0 & 0 & 1 & 0 & 0 & 1 & 1 & 0 & 1 & 0 & 0 \\
\hline 140B & 16 & 224 & 24 & 0 & 1 & 1 & 0 & 0 & 0 & 0 & 1 & 1 & 0 & 0 & 0 & 0 & 0 & 0 & 1 & 0 & 0 & 1 & 1 & 0 & 1 & 0 & 0 \\
\hline 140B & 17 & 225 & 24 & 0 & 1 & 1 & 0 & 0 & 0 & 0 & 1 & 1 & 0 & 0 & 0 & 0 & 0 & 0 & 1 & 0 & 0 & 1 & 1 & 0 & 1 & 0 & 0 \\
\hline $140 \mathrm{~B}$ & 18 & 226 & 24 & 0 & 1 & 0 & 0 & 0 & 0 & 0 & 1 & 1 & 0 & 0 & 0 & 0 & 0 & 0 & 1 & 0 & 0 & 1 & 1 & 0 & 1 & 0 & 0 \\
\hline 140B & 19 & 227 & 24 & 0 & 1 & 0 & 0 & 0 & 0 & 0 & 1 & 1 & 0 & 0 & 0 & 0 & 0 & 0 & 1 & 0 & 0 & 1 & 1 & 0 & 1 & 0 & 0 \\
\hline 140B & 20 & 228 & 24 & 0 & 1 & 0 & 0 & 0 & 0 & 0 & 1 & 1 & 0 & 0 & 0 & 0 & 0 & 0 & 1 & 0 & 0 & 1 & 1 & 0 & 1 & 0 & 0 \\
\hline 140B & 21 & 229 & 24 & 0 & 1 & 0 & 0 & 0 & 0 & 0 & 1 & 1 & 0 & 0 & 0 & 0 & 0 & 0 & 1 & 0 & 0 & 1 & 1 & 0 & 1 & 0 & 0 \\
\hline 140B & 22 & 230 & 24 & 0 & 1 & 0 & 0 & 0 & 0 & 0 & 1 & 1 & 0 & 0 & 0 & 0 & 0 & 0 & 1 & 0 & 0 & 1 & 1 & 0 & 1 & 0 & 0 \\
\hline 140B & 23 & 231 & 24 & 0 & 1 & 0 & 0 & 0 & 0 & 0 & 1 & 1 & 0 & 0 & 0 & 0 & 0 & 0 & 1 & 0 & 0 & 1 & 1 & 0 & 1 & 0 & 0 \\
\hline 140B & 24 & 232 & 24 & 0 & 1 & 0 & 0 & 0 & 0 & 0 & 1 & 1 & 0 & 0 & 0 & 0 & 0 & 0 & 1 & 0 & 0 & 1 & 1 & 0 & 1 & 0 & 0 \\
\hline $140 \mathrm{~B}$ & 25 & 233 & 24 & 0 & 1 & 0 & 0 & 0 & 0 & 0 & 0 & 1 & 0 & 0 & 0 & 0 & 0 & 0 & 1 & 0 & 0 & 1 & 1 & 0 & 1 & 0 & 0 \\
\hline 140B & 26 & 234 & 19 & 0 & 1 & 0 & 0 & 0 & 0 & 0 & 0 & 0 & 0 & 0 & 0 & 0 & 0 & 0 & 1 & 0 & 0 & 1 & 1 & 0 & 1 & 0 & 0 \\
\hline 140B & 27 & 235 & 185 & 0 & 1 & 0 & 0 & 0 & 0 & 0 & 0 & 0 & 0 & 0 & 0 & 0 & 0 & 0 & 1 & 0 & 0 & 1 & 1 & 0 & 1 & 0 & 0 \\
\hline 140B & 28 & 236 & 11 & 0 & 1 & 0 & 0 & 0 & 0 & 0 & 0 & 0 & 0 & 0 & 0 & 0 & 0 & 0 & 1 & 0 & 0 & 1 & 1 & 0 & 1 & 0 & 0 \\
\hline 140B & 29 & 237 & 24 & 0 & 1 & 0 & 0 & 0 & 0 & 0 & 0 & 0 & 0 & 0 & 0 & 0 & 0 & 0 & 1 & 0 & 0 & 1 & 0 & 0 & 1 & 0 & 0 \\
\hline 140B & 30 & 238 & 24 & 0 & 1 & 0 & 0 & 0 & 0 & 0 & 0 & 0 & 0 & 0 & 0 & 0 & 0 & 0 & 1 & 0 & 0 & 1 & 0 & 0 & 1 & 0 & 0 \\
\hline 140B & 31 & 239 & 24 & 0 & 1 & 0 & 0 & 0 & 0 & 0 & 0 & 0 & 0 & 0 & 0 & 0 & 0 & 0 & 1 & 0 & 0 & 1 & 0 & 0 & 1 & 0 & 0 \\
\hline 140B & 32 & 240 & 24 & 0 & 0 & 0 & 0 & 0 & 0 & 0 & 0 & 0 & 0 & 0 & 0 & 0 & 0 & 0 & 1 & 0 & 0 & 1 & 0 & 0 & 1 & 0 & 0 \\
\hline 140B & 33 & 241 & 24 & 0 & 0 & 0 & 0 & 0 & 0 & 0 & 0 & 0 & 0 & 0 & 0 & 0 & 0 & 0 & 1 & 0 & 0 & 0 & 0 & 0 & 1 & 0 & 0 \\
\hline 140B & 34 & 2 & 24 & 0 & 0 & 0 & 0 & 0 & 0 & 0 & 0 & 0 & 0 & 0 & 0 & 0 & 0 & 0 & 1 & 0 & 0 & 0 & 0 & 0 & 1 & 0 & 0 \\
\hline 140B & 35 & 243 & 24 & 0 & 0 & 0 & 0 & 0 & 0 & 0 & 0 & 0 & 0 & 0 & 0 & 0 & 0 & 0 & 1 & 0 & 0 & 0 & 0 & 0 & 1 & 0 & 0 \\
\hline $140 \mathrm{~B}$ & 36 & 244 & 24 & 0 & 0 & 0 & 0 & 0 & 0 & 0 & 0 & 0 & 0 & 0 & 0 & 0 & 0 & 0 & 1 & 0 & 0 & 0 & 0 & 0 & 1 & 0 & 0 \\
\hline 140B & 37 & 245 & 13 & 0 & 0 & 0 & 0 & 0 & 0 & 0 & 0 & 0 & 0 & 0 & 0 & 0 & 0 & 0 & 1 & 0 & 0 & 0 & 0 & 0 & 1 & 0 & 0 \\
\hline $141 \mathrm{~A}$ & 1 & 246 & 8 & 1 & 1 & 1 & 1 & 1 & 1 & 1 & 1 & 1 & 1 & 1 & 1 & 1 & 1 & 1 & 1 & 1 & 1 & 1 & 1 & 1 & 1 & 1 & 1 \\
\hline $141 \mathrm{~A}$ & 2 & 247 & 24 & 1 & 1 & 1 & 1 & 1 & 0 & 1 & 1 & 0 & 0 & 0 & 0 & 1 & 1 & 0 & 1 & 0 & 0 & 1 & 1 & 1 & 1 & 1 & 0 \\
\hline $141 \mathrm{~A}$ & 3 & 248 & 24 & 1 & 1 & 1 & 1 & 1 & 0 & 1 & 0 & 0 & 0 & 0 & 0 & 1 & 0 & 0 & 1 & 0 & 0 & 1 & 1 & 1 & 1 & 1 & 0 \\
\hline $141 \mathrm{~A}$ & 4 & 249 & 24 & 1 & 1 & 1 & 1 & 1 & 1 & 1 & 0 & 0 & 0 & 0 & 0 & 1 & 0 & 0 & 1 & 0 & 0 & 1 & 1 & 1 & 1 & 1 & 0 \\
\hline $141 \mathrm{~A}$ & 5 & 250 & 24 & 1 & 1 & 1 & 1 & 1 & 1 & 1 & 0 & 0 & 0 & 0 & 0 & 1 & 0 & 0 & 1 & 0 & 0 & 1 & 1 & 1 & 1 & 1 & 0 \\
\hline $141 \mathrm{~A}$ & 6 & 251 & 24 & 1 & 1 & 1 & 1 & 1 & 1 & 1 & \begin{tabular}{|l|}
0 \\
\end{tabular} & 0 & 0 & 0 & 0 & 0 & 0 & 0 & 1 & 0 & 0 & 1 & 1 & 1 & 1 & 1 & 0 \\
\hline $141 \mathrm{~A}$ & 7 & 252 & 24 & 1 & 1 & 1 & 1 & 1 & 1 & 1 & 0 & 0 & 0 & 0 & 0 & 0 & 0 & 0 & 1 & 0 & 0 & 1 & 1 & 1 & 1 & 1 & 0 \\
\hline $141 \mathrm{~A}$ & 8 & 253 & 24 & 1 & 1 & 1 & 1 & 1 & 1 & 1 & 0 & 0 & 0 & 0 & 0 & 0 & 0 & 0 & 1 & 0 & 0 & 1 & 1 & 1 & 1 & 1 & 0 \\
\hline $141 \mathrm{~A}$ & 9 & 254 & 24 & 1 & 1 & 1 & 1 & 1 & 1 & 1 & 0 & 0 & 0 & 0 & 0 & 0 & 0 & 0 & 1 & 0 & 0 & 1 & 1 & 1 & 1 & 1 & 0 \\
\hline $141 \mathrm{~A}$ & 10 & 255 & 24 & 1 & 1 & 1 & 1 & 1 & 1 & 1 & 0 & 0 & 0 & 0 & 0 & 0 & 0 & 0 & 1 & 0 & 0 & 1 & 1 & 1 & 1 & 1 & 0 \\
\hline $141 \mathrm{~A}$ & 11 & 256 & 24 & 1 & 1 & 1 & 1 & 1 & 1 & 1 & 0 & 0 & 0 & 0 & 0 & 0 & 0 & 0 & 1 & 0 & 0 & 1 & 1 & 1 & 1 & 1 & 0 \\
\hline $141 \mathrm{~A}$ & 12 & 257 & 24 & 1 & 1 & 1 & 1 & 1 & 1 & 1 & 0 & 0 & 0 & 0 & 0 & 0 & 0 & 0 & 1 & 0 & 0 & 1 & 1 & 1 & 1 & 1 & 0 \\
\hline $141 \mathrm{~A}$ & 13 & 258 & 24 & 1 & 1 & 1 & 1 & 1 & 1 & 1 & 0 & 0 & 0 & 0 & 0 & 0 & 0 & 0 & 1 & 0 & 0 & 1 & 1 & 1 & 1 & 1 & 0 \\
\hline $141 \mathrm{~A}$ & 14 & 259 & 24 & 1 & 1 & 1 & 1 & 1 & 1 & 1 & 0 & 0 & 0 & 0 & 0 & 0 & 0 & 0 & 1 & 0 & 0 & 1 & 1 & 1 & 1 & 1 & 0 \\
\hline $141 \mathrm{~A}$ & 15 & 260 & 24 & 1 & 1 & 1 & 1 & 1 & 1 & 1 & 0 & 0 & 0 & 0 & 0 & 0 & 0 & 0 & 1 & 0 & 0 & 1 & 1 & 1 & 1 & 1 & 0 \\
\hline $141 \mathrm{~A}$ & 16 & 261 & 24 & 1 & 1 & 1 & 1 & 1 & 1 & 1 & 0 & 0 & 0 & 0 & 0 & 0 & 0 & 0 & 1 & 0 & 0 & 1 & 1 & 1 & 1 & 0 & 0 \\
\hline $141 \mathrm{~A}$ & 17 & 262 & 24 & 1 & 1 & 1 & 1 & 1 & 1 & 1 & \begin{tabular}{|l|}
0 \\
\end{tabular} & 0 & \begin{tabular}{|l|}
0 \\
\end{tabular} & 0 & 0 & 0 & 0 & 0 & 1 & 0 & 0 & 1 & 1 & 1 & 1 & 0 & 0 \\
\hline
\end{tabular}




\begin{tabular}{|c|c|c|c|c|c|c|c|c|c|c|c|c|c|c|c|c|c|c|c|c|c|c|c|c|c|c|c|}
\hline \multirow[b]{2}{*}{ Cycle } & \multirow[b]{2}{*}{ Timestep } & \multirow{2}{*}{$\begin{array}{l}\text { Cumulative } \\
\text { Timestep }\end{array}$} & \multirow{2}{*}{$\begin{array}{l}\text { Time } \\
\text { Interval } \\
(\mathrm{hrs})\end{array}$} & \multicolumn{6}{|c|}{$\begin{array}{c}\text { NW } \\
\text { Neck Shims } \\
\end{array}$} & \multicolumn{6}{|c|}{$\begin{array}{c}\text { NE } \\
\text { Neck Shims }\end{array}$} & \multicolumn{6}{|c|}{$\begin{array}{c}\text { SW } \\
\text { Neck Shims }\end{array}$} & \multicolumn{6}{|c|}{$\begin{array}{c}\text { SE } \\
\text { Neck Shims }\end{array}$} \\
\hline & & & & \begin{tabular}{|l|}
1 \\
\end{tabular} & \begin{tabular}{|l|}
2 \\
\end{tabular} & \begin{tabular}{|l|l|}
3 \\
\end{tabular} & 4 & 5 & 6 & 1 & \begin{tabular}{|l|}
2 \\
\end{tabular} & 3 & \begin{tabular}{|l|}
4 \\
\end{tabular} & \begin{tabular}{|l|}
5 \\
\end{tabular} & 6 & 1 & \begin{tabular}{|l|l|}
2 \\
\end{tabular} & 3 & \begin{tabular}{|l|}
4 \\
\end{tabular} & 5 & 6 & 1 & \begin{tabular}{|l|}
2 \\
\end{tabular} & 3 & 4 & & 6 \\
\hline $141 \mathrm{~A}$ & 18 & 263 & 24 & 1 & 1 & 1 & 1 & 1 & 1 & 1 & 0 & 0 & 0 & 0 & 0 & 0 & 0 & 0 & 1 & 0 & 0 & 1 & 1 & 1 & 1 & 0 & 0 \\
\hline $141 \mathrm{~A}$ & 19 & 264 & 24 & 1 & 1 & 1 & 1 & 1 & 0 & 0 & 0 & 0 & 0 & 0 & 0 & 0 & 0 & 0 & 1 & 0 & 0 & 1 & 1 & 1 & 1 & 0 & 0 \\
\hline $141 \mathrm{~A}$ & 20 & 265 & 24 & 1 & 1 & 1 & 1 & 0 & 0 & 0 & 0 & 0 & 0 & 0 & 0 & 0 & 0 & 0 & 1 & 0 & 0 & 1 & \begin{tabular}{|l|}
1 \\
\end{tabular} & 1 & 1 & 0 & 0 \\
\hline $141 \mathrm{~A}$ & 21 & 266 & 24 & 1 & 1 & 1 & 1 & 0 & 0 & 0 & 0 & 0 & 0 & 0 & 0 & 0 & 0 & 0 & 1 & 0 & 0 & 1 & 1 & 1 & 1 & 0 & 0 \\
\hline $141 \mathrm{~A}$ & 22 & 267 & 24 & 1 & 1 & 1 & 1 & 0 & 0 & 0 & 0 & 0 & 0 & 0 & 0 & 0 & 0 & 0 & 1 & 0 & 0 & 1 & \begin{tabular}{|l|}
1 \\
\end{tabular} & 1 & 1 & 0 & 0 \\
\hline $141 \mathrm{~A}$ & 23 & 268 & 24 & 1 & 1 & 1 & 1 & 0 & 0 & 0 & 0 & 0 & 0 & 0 & 0 & 0 & 0 & 0 & 1 & 0 & 0 & 1 & 1 & 1 & 1 & 0 & 0 \\
\hline $141 \mathrm{~A}$ & 24 & 269 & 24 & 1 & 1 & 1 & 1 & 0 & 0 & 0 & 0 & 0 & 0 & 0 & 0 & 0 & 0 & 0 & 1 & 0 & 0 & 1 & \begin{tabular}{|l|}
1 \\
\end{tabular} & 1 & 1 & 0 & 0 \\
\hline $141 \mathrm{~A}$ & 25 & 270 & 24 & 1 & 1 & 1 & 1 & 0 & 0 & 0 & 0 & 0 & 0 & 0 & 0 & 0 & 0 & 0 & 1 & 0 & 0 & 1 & \begin{tabular}{|l|}
1 \\
\end{tabular} & 1 & 1 & 0 & 0 \\
\hline $141 \mathrm{~A}$ & 26 & 271 & 24 & 1 & 1 & 1 & 1 & 0 & 0 & 0 & 0 & 0 & 0 & 0 & 0 & 0 & 0 & 0 & 1 & 0 & 0 & 1 & 1 & 1 & 1 & 0 & 0 \\
\hline $141 \mathrm{~A}$ & 27 & 272 & 24 & 1 & 1 & 1 & 1 & 0 & 0 & 0 & 0 & 0 & 0 & 0 & 0 & 0 & 0 & 0 & 1 & 0 & 0 & 1 & 1 & 1 & 1 & 0 & 0 \\
\hline $141 \mathrm{~A}$ & 28 & 273 & 24 & 1 & 1 & 0 & 0 & 0 & 0 & 0 & 0 & 0 & 0 & 0 & 0 & 0 & 0 & 0 & 1 & 0 & 0 & 1 & 1 & 1 & 1 & 0 & 0 \\
\hline $141 \mathrm{~A}$ & 29 & 274 & 24 & 1 & 1 & 0 & 0 & 0 & 0 & 0 & 0 & 0 & 0 & 0 & 0 & 0 & 0 & 0 & 1 & 0 & 0 & 1 & 1 & 1 & 1 & 0 & 0 \\
\hline $141 \mathrm{~A}$ & 30 & 275 & 24 & 1 & 0 & 0 & 0 & 0 & 0 & 0 & 0 & 0 & 0 & 0 & 0 & 0 & 0 & 0 & 1 & 0 & 0 & 1 & \begin{tabular}{|l|}
1 \\
\end{tabular} & 1 & 1 & 0 & 0 \\
\hline $141 \mathrm{~A}$ & 31 & 276 & 24 & 1 & 0 & 0 & 0 & 0 & 0 & 0 & 0 & 0 & 0 & 0 & 0 & 0 & 0 & 0 & 1 & 0 & 0 & 1 & \begin{tabular}{|l|}
1 \\
\end{tabular} & 1 & 1 & 0 & 0 \\
\hline $141 \mathrm{~A}$ & 32 & 277 & 24 & 1 & 0 & 0 & 0 & 0 & 0 & 0 & 0 & 0 & 0 & 0 & 0 & 0 & 0 & 0 & 1 & 0 & 0 & 1 & \begin{tabular}{|l|}
1 \\
\end{tabular} & 0 & 1 & 0 & 0 \\
\hline $141 \mathrm{~A}$ & 33 & 278 & 24 & 1 & 0 & 0 & 0 & 0 & 0 & 0 & 0 & 0 & 0 & 0 & 0 & 0 & 0 & 0 & 1 & 0 & 0 & 1 & 1 & 0 & 1 & 0 & 0 \\
\hline $141 \mathrm{~A}$ & 34 & 279 & 7 & 1 & 0 & 0 & 0 & 0 & 0 & 0 & 0 & 0 & 0 & 0 & 0 & 0 & 0 & 0 & 1 & 0 & 0 & 1 & \begin{tabular}{|l|}
1 \\
\end{tabular} & 0 & 1 & 0 & 0 \\
\hline $142 \mathrm{~A}$ & 1 & 280 & 8 & 1 & 1 & 1 & 1 & 1 & 1 & 1 & 1 & 1 & 1 & 1 & 1 & 1 & 1 & 1 & 1 & 1 & 1 & 1 & 1 & 1 & 1 & 1 & 1 \\
\hline $142 \mathrm{~A}$ & 2 & 281 & 24 & 1 & 1 & 1 & 1 & 1 & 0 & 1 & 1 & 1 & 1 & 0 & 0 & 1 & 1 & 1 & 1 & 1 & 1 & 1 & \begin{tabular}{|l|}
1 \\
\end{tabular} & 1 & 1 & 1 & 0 \\
\hline $142 \mathrm{~A}$ & 3 & 282 & 24 & 1 & 1 & 1 & 1 & 0 & 0 & 1 & 1 & 1 & 1 & 0 & 0 & 1 & 1 & 1 & 1 & 1 & 1 & 1 & 1 & 1 & 1 & 1 & 0 \\
\hline $142 \mathrm{~A}$ & 4 & 283 & 24 & 1 & 1 & 1 & 1 & 0 & 0 & 1 & 1 & 1 & 1 & 1 & 0 & 1 & 1 & 1 & 1 & 1 & 1 & 1 & 1 & 1 & 1 & 1 & 0 \\
\hline $142 \mathrm{~A}$ & 5 & 284 & 24 & 1 & 1 & 1 & 1 & 0 & 0 & 1 & 1 & 1 & 1 & 1 & 0 & 1 & 1 & 1 & 1 & 1 & 1 & 1 & \begin{tabular}{|l|}
1 \\
\end{tabular} & 1 & 1 & 1 & 0 \\
\hline $142 \mathrm{~A}$ & 6 & 285 & 24 & 1 & 1 & \begin{tabular}{|l|}
1 \\
\end{tabular} & 1 & 0 & 0 & 1 & 1 & 1 & 1 & 1 & 0 & 1 & \begin{tabular}{|l|}
1 \\
\end{tabular} & 1 & 1 & 1 & \begin{tabular}{|l|}
1 \\
\end{tabular} & 1 & \begin{tabular}{|l|}
1 \\
\end{tabular} & 1 & 1 & 1 & 0 \\
\hline $142 \mathrm{~A}$ & 7 & 286 & 25 & 1 & 1 & 1 & 1 & 0 & 0 & 1 & 1 & 1 & 1 & 1 & 0 & 1 & 1 & 1 & 1 & 1 & 0 & 1 & 1 & 1 & 1 & 1 & 0 \\
\hline $142 \mathrm{~A}$ & 8 & 287 & 24 & 1 & 1 & 1 & 1 & 0 & 0 & 1 & 1 & 1 & 1 & 1 & 0 & 1 & 1 & 1 & 1 & 1 & 0 & 1 & 1 & 1 & 1 & 1 & 0 \\
\hline $142 \mathrm{~A}$ & 9 & 288 & 24 & 1 & 1 & 1 & 1 & 0 & 0 & 1 & 1 & 1 & 1 & 1 & 0 & 1 & 1 & 1 & 1 & 1 & 0 & 1 & 1 & 1 & 1 & 1 & 0 \\
\hline $142 \mathrm{~A}$ & 10 & 289 & 24 & 1 & 1 & 1 & 1 & 0 & 0 & 1 & 1 & 1 & 1 & 1 & 0 & 1 & 1 & 1 & 1 & 1 & 0 & 1 & 1 & 1 & 1 & 1 & 0 \\
\hline $142 \mathrm{~A}$ & 11 & 290 & 24 & 1 & 1 & 1 & 1 & 0 & 0 & 1 & 1 & 1 & 1 & 0 & 0 & 1 & 1 & 1 & 1 & 1 & 0 & 1 & 1 & 1 & 1 & 1 & 0 \\
\hline $142 \mathrm{~A}$ & 12 & 291 & 24 & 1 & 1 & 1 & 1 & 0 & 0 & 1 & 1 & 1 & 1 & 0 & 0 & 1 & 1 & 1 & 1 & 1 & 0 & 1 & 1 & 1 & 1 & 1 & 0 \\
\hline $142 \mathrm{~A}$ & 13 & 292 & 24 & 1 & 1 & 1 & 1 & 0 & 0 & 1 & 1 & 1 & 1 & 0 & 0 & 1 & 1 & 1 & 1 & 1 & 0 & 1 & 1 & 1 & 1 & 1 & 0 \\
\hline $142 \mathrm{~A}$ & 14 & 293 & 24 & 1 & 1 & 1 & 1 & 0 & 0 & 1 & 1 & 1 & 1 & 0 & 0 & 1 & 1 & 1 & 1 & 1 & 0 & 1 & 1 & 1 & 1 & 1 & 0 \\
\hline $142 \mathrm{~A}$ & 15 & 294 & 24 & 1 & 1 & 1 & 1 & 0 & 0 & 1 & 1 & 1 & 1 & 0 & 0 & 1 & 1 & 1 & 1 & 0 & 0 & 1 & 1 & 1 & 1 & 1 & 0 \\
\hline $142 \mathrm{~A}$ & 16 & 295 & 24 & 1 & 1 & 1 & 1 & 0 & 0 & 1 & 1 & 1 & 0 & 0 & 0 & 1 & 1 & 1 & 1 & 0 & 0 & 1 & 1 & 1 & 1 & 1 & 0 \\
\hline $142 \mathrm{~A}$ & 17 & 296 & 24 & 1 & 1 & 1 & 1 & 0 & 0 & 1 & 1 & 1 & 0 & 0 & 0 & 1 & 1 & 0 & 1 & 0 & 0 & 1 & 1 & 1 & 1 & 1 & 0 \\
\hline $142 \mathrm{~A}$ & 18 & 297 & 24 & 1 & 1 & 1 & 1 & 0 & 0 & 1 & 1 & 1 & 0 & 0 & 0 & 1 & 1 & 0 & 1 & 0 & 0 & 1 & 1 & 1 & 1 & 1 & 0 \\
\hline $142 \mathrm{~A}$ & 19 & 298 & 24 & 1 & 1 & 1 & 1 & 0 & 0 & 1 & 1 & 1 & 0 & 0 & 0 & 1 & 1 & 0 & 1 & 0 & 0 & 1 & 1 & 1 & 1 & 1 & 0 \\
\hline $142 \mathrm{~A}$ & 20 & 299 & 24 & 1 & 1 & 1 & 1 & 0 & 0 & 1 & 1 & 0 & 0 & 0 & 0 & 1 & 1 & 0 & 1 & 0 & 0 & 1 & 1 & 1 & 1 & 1 & 0 \\
\hline $142 \mathrm{~A}$ & 21 & 300 & 24 & 1 & 1 & 1 & 1 & 0 & 0 & 1 & 1 & 0 & 0 & 0 & 0 & 1 & 1 & 0 & 1 & 0 & \begin{tabular}{|l|}
0 \\
\end{tabular} & 1 & 1 & 1 & 1 & 1 & 0 \\
\hline $142 \mathrm{~A}$ & 22 & 301 & 24 & 1 & 1 & 1 & 1 & 0 & 0 & 1 & 1 & 0 & 0 & 0 & 0 & 1 & 1 & 0 & 1 & 0 & 0 & 1 & 1 & 1 & 1 & 1 & 0 \\
\hline $142 \mathrm{~A}$ & 23 & 302 & 24 & 1 & 1 & 1 & 1 & 0 & 0 & 1 & 1 & 0 & 0 & 0 & 0 & 1 & 0 & 0 & 1 & 0 & 0 & 1 & 1 & 1 & 1 & 1 & 0 \\
\hline $142 \mathrm{~A}$ & 24 & 303 & 24 & 1 & 1 & 1 & 1 & 0 & 0 & 1 & 1 & 0 & 0 & 0 & 0 & 1 & 0 & 0 & 1 & 0 & 0 & 1 & 1 & 1 & 1 & 1 & 0 \\
\hline $142 \mathrm{~A}$ & 25 & 304 & 24 & 1 & 1 & 1 & 1 & 0 & 0 & 1 & 1 & 0 & 0 & 0 & 0 & 0 & 0 & 0 & 1 & 0 & 0 & 1 & 1 & 1 & 1 & 1 & 0 \\
\hline $142 \mathrm{~A}$ & 26 & 305 & 24 & 1 & 1 & 1 & 1 & 0 & 0 & 1 & 1 & 0 & 0 & 0 & 0 & 0 & 0 & 0 & 1 & 0 & 0 & 1 & \begin{tabular}{|l|}
1 \\
\end{tabular} & 1 & 1 & 1 & 0 \\
\hline $142 \mathrm{~A}$ & 27 & 306 & 24 & 1 & 1 & 1 & 1 & 0 & 0 & 1 & 1 & 0 & 0 & 0 & 0 & 0 & 0 & 0 & 1 & 0 & 0 & 1 & \begin{tabular}{|l|}
1 \\
\end{tabular} & 1 & 1 & 1 & 0 \\
\hline $142 \mathrm{~A}$ & 28 & 307 & 24 & 1 & 1 & 1 & 1 & 0 & \begin{tabular}{|l|}
0 \\
\end{tabular} & 1 & 1 & 0 & \begin{tabular}{|l|}
0 \\
\end{tabular} & 0 & 0 & 0 & 0 & 0 & 1 & 0 & 0 & 1 & 1 & 1 & 1 & 1 & 0 \\
\hline
\end{tabular}




\begin{tabular}{|c|c|c|c|c|c|c|c|c|c|c|c|c|c|c|c|c|c|c|c|c|c|c|c|c|c|c|c|}
\hline \multirow[b]{2}{*}{ Cycle } & \multirow[b]{2}{*}{ Timestep } & \multirow{2}{*}{$\begin{array}{l}\text { Cumulative } \\
\text { Timestep }\end{array}$} & \multirow{2}{*}{$\begin{array}{c}\text { Time } \\
\text { Interval } \\
(\mathrm{hrs})\end{array}$} & \multicolumn{6}{|c|}{$\begin{array}{c}\text { NW } \\
\text { Neck Shims } \\
\end{array}$} & \multicolumn{6}{|c|}{$\begin{array}{c}\text { NE } \\
\text { Neck Shims }\end{array}$} & \multicolumn{6}{|c|}{$\begin{array}{c}\text { SW } \\
\text { Neck Shims }\end{array}$} & \multicolumn{6}{|c|}{$\begin{array}{c}\text { SE } \\
\text { Neck Shims }\end{array}$} \\
\hline & & & & \begin{tabular}{|l|}
1 \\
\end{tabular} & 2 & \begin{tabular}{|l|}
3 \\
\end{tabular} & 4 & 5 & 6 & 1 & 2 & 3 & 4 & 5 & 6 & 1 & \begin{tabular}{|l|}
2 \\
\end{tabular} & 3 & 4 & 5 & 6 & & 2 & 3 & 4 & & 6 \\
\hline $142 \mathrm{~A}$ & 29 & 308 & 24 & 1 & 1 & 1 & 1 & 0 & 0 & 1 & 1 & 0 & 0 & 0 & 0 & 0 & 0 & 0 & 1 & 0 & 0 & 1 & 1 & 1 & 1 & 1 & 0 \\
\hline $142 \mathrm{~A}$ & 30 & 309 & 24 & 1 & 1 & 1 & 1 & 0 & 0 & 1 & 0 & 0 & 0 & 0 & 0 & 0 & 0 & 0 & 1 & 0 & 0 & 1 & 1 & 1 & 1 & 1 & 0 \\
\hline $142 \mathrm{~A}$ & 31 & 310 & 25 & 1 & 1 & 1 & 0 & 0 & 0 & 1 & 0 & 0 & 0 & 0 & 0 & 0 & 0 & 0 & 1 & 0 & 0 & 1 & 1 & 1 & 1 & 1 & 0 \\
\hline $142 \mathrm{~A}$ & 32 & 311 & 24 & 1 & 1 & 1 & 0 & 0 & 0 & 1 & 0 & 0 & 0 & 0 & 0 & 0 & 0 & 0 & 1 & 0 & 0 & 1 & 1 & 1 & 1 & 1 & 0 \\
\hline $142 \mathrm{~A}$ & 33 & 312 & 20 & 1 & 1 & 1 & 0 & 0 & 0 & 1 & 0 & 0 & 0 & 0 & 0 & 0 & 0 & 0 & 1 & 0 & 0 & 1 & 1 & 1 & 1 & 1 & 0 \\
\hline $142 \mathrm{~A}$ & 34 & 313 & 17 & 1 & 1 & 1 & 0 & 0 & 0 & 1 & 0 & 0 & 0 & 0 & 0 & 0 & 0 & 0 & 1 & 0 & 0 & 1 & 1 & 1 & 1 & 1 & 0 \\
\hline $142 \mathrm{~A}$ & 35 & 314 & 11 & 1 & 1 & 1 & 0 & 0 & 0 & 1 & 0 & 0 & 0 & 0 & 0 & 0 & 0 & 0 & 1 & 0 & 0 & 1 & 1 & 1 & 1 & 1 & 0 \\
\hline $142 \mathrm{~A}$ & 36 & 315 & 24 & 1 & 1 & 1 & 0 & 0 & 0 & 1 & 0 & 0 & 0 & 0 & 0 & 0 & 0 & 0 & 1 & 0 & 0 & 1 & 1 & 1 & 1 & 1 & 0 \\
\hline $142 \mathrm{~A}$ & 37 & 316 & 24 & 1 & 1 & 1 & 0 & 0 & 0 & 1 & 0 & 0 & 0 & 0 & 0 & 0 & 0 & 0 & 1 & 0 & 0 & 1 & 1 & 1 & 1 & 0 & 0 \\
\hline $142 \mathrm{~A}$ & 38 & 317 & 24 & 1 & 1 & 1 & 0 & 0 & 0 & 1 & 0 & 0 & 0 & 0 & 0 & 0 & 0 & 0 & 1 & 0 & 0 & 1 & 1 & 1 & 1 & 0 & 0 \\
\hline $142 \mathrm{~A}$ & 39 & 318 & 24 & 1 & 1 & 0 & 0 & 0 & 0 & 1 & 0 & 0 & 0 & 0 & 0 & 0 & 0 & 0 & 1 & 0 & 0 & 1 & 1 & 1 & 1 & 0 & 0 \\
\hline $142 \mathrm{~A}$ & 40 & 319 & 24 & 1 & 1 & 0 & 0 & 0 & 0 & 1 & 0 & 0 & 0 & 0 & 0 & 0 & 0 & 0 & 1 & 0 & 0 & 1 & 1 & 1 & 1 & 0 & 0 \\
\hline $142 \mathrm{~A}$ & 41 & 320 & 24 & 1 & 1 & 0 & 0 & 0 & 0 & 1 & 0 & 0 & 0 & 0 & 0 & 0 & 0 & 0 & 1 & 0 & 0 & 1 & 1 & 0 & 1 & 0 & 0 \\
\hline $142 \mathrm{~A}$ & 42 & 321 & 24 & 1 & 1 & 0 & 0 & 0 & 0 & 1 & 0 & 0 & 0 & 0 & 0 & 0 & 0 & 0 & 1 & 0 & 0 & 1 & 1 & 0 & 1 & 0 & 0 \\
\hline $142 \mathrm{~A}$ & 43 & 322 & 24 & 1 & 0 & 0 & 0 & 0 & 0 & 0 & 0 & 0 & 0 & 0 & 0 & 0 & 0 & 0 & 1 & 0 & 0 & 1 & 1 & 0 & 1 & 0 & 0 \\
\hline $142 \mathrm{~A}$ & 44 & 323 & 24 & 1 & 0 & 0 & 0 & 0 & 0 & 0 & 0 & 0 & 0 & 0 & 0 & 0 & 0 & 0 & 1 & 0 & 0 & 1 & 1 & 0 & 1 & 0 & 0 \\
\hline $142 \mathrm{~A}$ & 45 & 324 & 24 & 1 & 0 & 0 & 0 & 0 & 0 & 0 & 0 & 0 & 0 & 0 & 0 & 0 & 0 & 0 & 1 & 0 & 0 & 1 & 0 & 0 & 1 & 0 & 0 \\
\hline $142 \mathrm{~A}$ & 46 & 325 & 24 & 1 & 0 & 0 & 0 & 0 & 0 & 0 & 0 & 0 & 0 & 0 & 0 & 0 & 0 & 0 & 1 & 0 & 0 & 1 & 0 & 0 & 1 & 0 & 0 \\
\hline $142 \mathrm{~A}$ & 47 & 326 & 24 & 1 & 0 & 0 & 0 & 0 & 0 & 0 & 0 & 0 & 0 & 0 & 0 & 0 & 0 & 0 & 1 & 0 & 0 & 1 & 0 & 0 & 1 & 0 & 0 \\
\hline $142 \mathrm{~A}$ & 48 & 327 & 24 & 0 & 0 & 0 & 0 & 0 & 0 & 0 & 0 & 0 & 0 & 0 & 0 & 0 & 0 & 0 & 1 & 0 & 0 & 1 & 0 & 0 & 1 & 0 & 0 \\
\hline $142 \mathrm{~A}$ & 49 & 328 & 24 & 0 & 0 & 0 & 0 & 0 & 0 & 0 & 0 & 0 & 0 & 0 & 0 & 0 & 0 & 0 & 1 & 0 & 0 & 1 & 0 & 0 & 1 & 0 & 0 \\
\hline $142 \mathrm{~A}$ & 50 & 329 & 24 & 0 & 0 & 0 & 0 & 0 & 0 & 0 & 0 & 0 & 0 & 0 & 0 & 0 & 0 & 0 & 1 & 0 & 0 & 0 & 0 & 0 & 1 & 0 & 0 \\
\hline $142 \mathrm{~A}$ & 51 & 330 & 15 & 0 & 0 & 0 & 0 & 0 & 0 & 0 & 0 & 0 & 0 & 0 & 0 & 0 & 0 & 0 & 1 & 0 & 0 & 0 & 0 & 0 & 1 & 0 & 0 \\
\hline 142B & 1 & 331 & 8 & 1 & 1 & 1 & 1 & 1 & 1 & 1 & 1 & 1 & 1 & 1 & 1 & 1 & 1 & 1 & 1 & 1 & 1 & 1 & 1 & 1 & 1 & 1 & 1 \\
\hline 142B & 2 & 332 & 24 & 1 & 1 & 1 & 1 & 1 & 1 & 1 & 1 & 1 & 1 & 0 & 0 & 1 & 1 & 1 & 1 & 1 & 0 & 1 & 1 & 1 & 1 & 1 & 1 \\
\hline 142B & 3 & 333 & 24 & 1 & 1 & 1 & 1 & 1 & 1 & 1 & 1 & 1 & 1 & 0 & 0 & 1 & 1 & 1 & 1 & 1 & 0 & 1 & 1 & 1 & 1 & 1 & 1 \\
\hline 142B & 4 & 334 & 45 & 1 & 1 & 1 & 1 & 1 & 1 & 1 & 1 & 1 & 1 & 0 & 0 & 1 & 1 & 1 & 1 & 1 & 0 & 1 & 1 & 1 & 1 & 1 & 1 \\
\hline 142B & 5 & 335 & 13 & 1 & 1 & 1 & 1 & 1 & 1 & 1 & 1 & 1 & 1 & 0 & 0 & 1 & 1 & 1 & 1 & 1 & 0 & 1 & 1 & 1 & 1 & 1 & 1 \\
\hline 142B & 6 & 336 & 24 & 1 & 1 & 1 & 1 & 1 & 1 & 1 & 1 & 1 & 1 & 0 & 0 & 1 & 1 & 1 & 1 & 1 & 0 & 1 & 1 & 1 & 1 & 1 & 0 \\
\hline 142B & 7 & 337 & 24 & 1 & 1 & 1 & 1 & 1 & 1 & 1 & 1 & 1 & 1 & 0 & 0 & 1 & 1 & 1 & 1 & 1 & 0 & 1 & 1 & 1 & 1 & 1 & 0 \\
\hline 142B & 8 & 338 & 24 & 1 & 1 & 1 & 1 & 1 & 1 & 1 & 1 & 1 & 1 & 0 & 0 & 1 & 1 & 1 & 1 & 1 & 0 & 1 & 1 & 1 & 1 & 1 & 0 \\
\hline 142B & 9 & 339 & 24 & 1 & 1 & 1 & 1 & 1 & 1 & 1 & 1 & 1 & 1 & 0 & 0 & 1 & 1 & 1 & 1 & 1 & 0 & 1 & 1 & 1 & 1 & 0 & 0 \\
\hline 142B & 10 & 340 & 24 & 1 & 1 & 1 & 1 & 1 & 1 & 1 & 1 & 1 & 1 & 0 & 0 & 1 & 1 & 1 & 1 & 1 & 0 & 1 & 1 & 1 & 1 & 0 & 0 \\
\hline 142B & 11 & 341 & 24 & 1 & 1 & 1 & 1 & 1 & 1 & 1 & 1 & 1 & 1 & 0 & 0 & 1 & 1 & 1 & 1 & 1 & 0 & 1 & 1 & 1 & 1 & 0 & 0 \\
\hline 142B & 12 & 342 & 24 & 1 & 1 & 1 & 1 & 1 & 1 & 1 & 1 & 1 & 1 & 0 & 0 & 1 & 1 & 1 & 1 & 1 & 0 & 1 & 1 & 1 & 1 & 0 & 0 \\
\hline 142B & 13 & 343 & 24 & 1 & 1 & 1 & 1 & 1 & 1 & 1 & 1 & 1 & 1 & 0 & 0 & 1 & 1 & 1 & 1 & 1 & 0 & 1 & 1 & 1 & 1 & 0 & 0 \\
\hline 142B & 14 & 344 & 24 & 1 & 1 & 1 & 1 & 1 & 1 & 1 & 1 & 1 & 1 & 0 & 0 & 1 & 1 & 1 & 1 & 1 & 0 & 1 & 1 & 1 & 1 & 0 & 0 \\
\hline 142B & 15 & 345 & 24 & 1 & 1 & 1 & 1 & 1 & 0 & 1 & 1 & 1 & 1 & 0 & 0 & 1 & 1 & 1 & 1 & 0 & 0 & 1 & 1 & 0 & 1 & 0 & 0 \\
\hline 142B & 16 & 346 & 24 & 1 & 1 & 1 & 1 & 1 & 0 & 1 & 1 & 1 & 1 & 0 & 0 & 1 & 1 & 1 & 1 & 0 & 0 & 1 & 1 & 0 & 1 & 0 & 0 \\
\hline 142B & 17 & 347 & 24 & 1 & 1 & 1 & 1 & 0 & 0 & 1 & 1 & 1 & 1 & 0 & 0 & 1 & 1 & 1 & 1 & 0 & 0 & 1 & 1 & 0 & 1 & 0 & 0 \\
\hline 142B & 18 & 348 & 24 & 1 & 1 & 1 & 1 & 0 & 0 & 1 & 1 & 1 & 1 & 0 & 0 & 1 & 1 & 1 & 1 & 0 & 0 & 1 & 1 & 0 & 1 & 0 & 0 \\
\hline 142B & 19 & 349 & 24 & 1 & 1 & 1 & 1 & 0 & 0 & 1 & 1 & 1 & 1 & 0 & 0 & 1 & 1 & 1 & 1 & 0 & 0 & 1 & 1 & 0 & 1 & 0 & 0 \\
\hline 142B & 20 & 350 & 24 & 1 & 1 & 1 & 1 & 0 & 0 & 1 & 1 & 1 & 1 & 0 & 0 & 1 & 1 & 1 & 1 & 0 & 0 & 1 & 1 & 0 & 1 & 0 & 0 \\
\hline 142B & 21 & 351 & 24 & 1 & 1 & 1 & 1 & 0 & 0 & 1 & 1 & 1 & 1 & 0 & 0 & 1 & 1 & 1 & 1 & 0 & 0 & 1 & 1 & 0 & 1 & 0 & 0 \\
\hline 142B & 22 & 352 & 24 & 1 & 1 & 1 & 1 & 0 & 0 & 1 & 1 & 1 & 1 & 0 & 0 & 1 & 1 & 1 & 1 & 0 & 0 & 1 & 1 & \begin{tabular}{|l|}
0 \\
\end{tabular} & 1 & $\begin{array}{l}0 \\
\end{array}$ & 0 \\
\hline
\end{tabular}




\begin{tabular}{|c|c|c|c|c|c|c|c|c|c|c|c|c|c|c|c|c|c|c|c|c|c|c|c|c|c|c|c|}
\hline \multirow[b]{2}{*}{ Cycle } & \multirow[b]{2}{*}{ Timestep } & \multirow{2}{*}{$\begin{array}{l}\text { Cumulative } \\
\text { Timestep }\end{array}$} & \multirow{2}{*}{$\begin{array}{c}\text { Time } \\
\text { Interval } \\
(\mathrm{hrs})\end{array}$} & \multicolumn{6}{|c|}{$\begin{array}{c}\text { NW } \\
\text { Neck Shims }\end{array}$} & \multicolumn{6}{|c|}{$\begin{array}{c}\text { NE } \\
\text { Neck Shims }\end{array}$} & \multicolumn{6}{|c|}{$\begin{array}{c}\text { SW } \\
\text { Neck Shims }\end{array}$} & \multicolumn{6}{|c|}{$\begin{array}{c}\text { SE } \\
\text { Neck Shims }\end{array}$} \\
\hline & & & & 1 & 2 & 3 & 4 & 5 & 6 & 1 & 2 & 3 & 4 & 5 & 6 & & 2 & 3 & 4 & 5 & 6 & & 2 & 3 & 4 & & \\
\hline 142B & 23 & 353 & 24 & 1 & 1 & 1 & 1 & $\overline{0}$ & $\overline{0}$ & 1 & 1 & 1 & 0 & 0 & 0 & 1 & 1 & 1 & 1 & 0 & 0 & 1 & 1 & 0 & 1 & 0 & 0 \\
\hline 142B & 24 & 354 & 24 & 1 & 1 & 1 & 1 & 0 & 0 & 1 & 1 & 1 & 0 & 0 & 0 & 1 & 1 & 1 & 1 & 0 & 0 & 1 & 1 & 0 & 1 & 0 & 0 \\
\hline 142B & 25 & 355 & 24 & 1 & 1 & 1 & 0 & 0 & 0 & 1 & 1 & 1 & 0 & 0 & 0 & 1 & 1 & 1 & 1 & 0 & 0 & 1 & 1 & 0 & 1 & 0 & 0 \\
\hline 142B & 26 & 356 & 24 & 1 & 1 & 1 & 0 & 0 & 0 & 1 & 1 & 1 & 0 & 0 & 0 & 1 & 1 & 1 & 1 & 0 & 0 & 1 & 1 & 0 & 1 & 0 & 0 \\
\hline 142B & 27 & 357 & 24 & 1 & 1 & 1 & 0 & 0 & 0 & 1 & 1 & 0 & 0 & 0 & 0 & 1 & 1 & 1 & 1 & 0 & 0 & 1 & 1 & 0 & 1 & 0 & 0 \\
\hline 142B & 28 & 358 & 24 & 1 & 1 & 1 & 0 & 0 & 0 & 1 & 1 & 0 & 0 & 0 & 0 & 1 & 1 & 1 & 1 & 0 & 0 & 1 & 1 & 0 & 1 & 0 & 0 \\
\hline 142B & 29 & 359 & 24 & 1 & 1 & 1 & 0 & 0 & 0 & 1 & 1 & 0 & 0 & 0 & 0 & 1 & 1 & 1 & 1 & 0 & 0 & 1 & 1 & 0 & 1 & 0 & 0 \\
\hline 142B & 30 & 360 & 24 & 1 & 1 & 1 & 0 & 0 & 0 & 1 & 1 & 0 & 0 & 0 & 0 & 1 & 1 & 1 & 1 & 0 & 0 & 1 & 1 & 0 & 1 & 0 & 0 \\
\hline 142B & 31 & 361 & 24 & 1 & 1 & 1 & 0 & 0 & 0 & 1 & 1 & 0 & 0 & 0 & 0 & 1 & 1 & 1 & 1 & 0 & 0 & 1 & 1 & 0 & 1 & 0 & 0 \\
\hline 142B & 32 & 362 & 31 & 1 & 1 & 0 & 0 & 0 & 0 & 1 & 1 & 0 & 0 & 0 & 0 & 1 & 1 & 0 & 1 & 0 & 0 & 1 & 1 & 0 & 1 & 0 & 0 \\
\hline 142B & 33 & 363 & 46 & 1 & 1 & 0 & 0 & 0 & 0 & 1 & 1 & 0 & 0 & 0 & 0 & 1 & 1 & 0 & 1 & 0 & 0 & 1 & 1 & 0 & 1 & 0 & 0 \\
\hline 142B & 34 & 364 & 11 & 1 & 1 & 0 & 0 & 0 & 0 & 1 & 1 & 0 & 0 & 0 & 0 & 1 & 1 & 0 & 1 & 0 & 0 & 1 & 1 & 0 & 1 & 0 & 0 \\
\hline 142B & 35 & 365 & 24 & 1 & 1 & 0 & 0 & 0 & 0 & 1 & 1 & 0 & 0 & 0 & 0 & 1 & 1 & 0 & 1 & 0 & 0 & 1 & 1 & 0 & 1 & 0 & 0 \\
\hline 142B & 36 & 366 & 24 & 1 & 1 & 0 & 0 & 0 & 0 & 1 & 1 & 0 & 0 & 0 & 0 & 1 & 1 & 0 & 1 & 0 & 0 & 1 & 1 & 0 & 1 & 0 & 0 \\
\hline 142B & 37 & 367 & 24 & 1 & 1 & 0 & 0 & 0 & 0 & 1 & 1 & 0 & 0 & 0 & 0 & 1 & 1 & 0 & 1 & 0 & 0 & 1 & 1 & 0 & 1 & 0 & 0 \\
\hline 142B & 38 & 368 & 24 & 1 & 1 & 0 & 0 & 0 & 0 & 1 & 1 & 0 & 0 & 0 & 0 & 1 & 1 & 0 & 1 & 0 & 0 & 1 & 1 & 0 & 1 & 0 & 0 \\
\hline 142B & 39 & 369 & 24 & 1 & 1 & 0 & 0 & 0 & 0 & 1 & 1 & 0 & 0 & 0 & 0 & 1 & 1 & 0 & 1 & 0 & 0 & 1 & 1 & 0 & 1 & 0 & 0 \\
\hline 142B & 40 & 370 & 24 & 1 & 1 & 0 & 0 & 0 & 0 & 1 & 1 & 0 & 0 & 0 & 0 & 1 & 0 & 0 & 1 & 0 & 0 & 1 & 1 & 0 & 1 & 0 & 0 \\
\hline 142B & 41 & 371 & 24 & 1 & 1 & 0 & 0 & 0 & 0 & 1 & 1 & 0 & 0 & 0 & 0 & 1 & 0 & 0 & 1 & 0 & 0 & 1 & 1 & 0 & 1 & 0 & 0 \\
\hline 142B & 42 & 372 & 24 & 1 & 1 & 0 & 0 & 0 & 0 & 1 & 1 & 0 & 0 & 0 & 0 & 1 & 0 & 0 & 1 & 0 & 0 & 1 & 1 & 0 & 1 & 0 & 0 \\
\hline 142B & 43 & 373 & 24 & 1 & 1 & 0 & 0 & 0 & 0 & 1 & 1 & 0 & 0 & 0 & 0 & 1 & 0 & 0 & 1 & 0 & 0 & 1 & 1 & 0 & 1 & 0 & 0 \\
\hline 142B & 44 & 374 & 24 & 1 & 1 & 0 & 0 & 0 & 0 & 1 & 1 & 0 & 0 & 0 & 0 & 0 & 0 & 0 & 1 & 0 & 0 & 0 & 0 & 0 & 1 & 0 & 0 \\
\hline 142B & 45 & 375 & 24 & 1 & 1 & 0 & 0 & 0 & 0 & 1 & 1 & 0 & 0 & 0 & 0 & 0 & 0 & 0 & 1 & 0 & 0 & 0 & 0 & 0 & 1 & 0 & 0 \\
\hline 142B & 46 & 376 & 24 & 1 & 1 & 0 & 0 & 0 & 0 & 1 & 1 & 0 & 0 & 0 & 0 & 0 & 0 & 0 & 1 & 0 & 0 & 0 & 0 & 0 & 1 & 0 & 0 \\
\hline 142B & 47 & 377 & 24 & 1 & 1 & 0 & 0 & 0 & 0 & 1 & 1 & 0 & 0 & 0 & 0 & 0 & 0 & 0 & 1 & 0 & 0 & 0 & 0 & 0 & 1 & 0 & 0 \\
\hline 142B & 48 & 378 & 24 & 1 & 1 & 0 & 0 & 0 & 0 & 1 & 1 & 0 & 0 & 0 & 0 & 0 & 0 & 0 & 1 & 0 & 0 & 0 & 0 & 0 & 1 & 0 & 0 \\
\hline 142B & 49 & 379 & 24 & 1 & 1 & 0 & 0 & 0 & 0 & 1 & 1 & 0 & 0 & 0 & 0 & 0 & 0 & 0 & 1 & 0 & 0 & 0 & 0 & 0 & 1 & 0 & 0 \\
\hline 142B & 50 & 380 & 24 & 1 & 1 & 0 & 0 & 0 & 0 & 1 & 1 & 0 & 0 & 0 & 0 & 0 & 0 & 0 & 1 & 0 & 0 & 0 & 0 & 0 & 1 & 0 & 0 \\
\hline 142B & 51 & 381 & 24 & 1 & 1 & 0 & 0 & 0 & 0 & 1 & 0 & 0 & 0 & 0 & 0 & 0 & 0 & 0 & 1 & 0 & 0 & 0 & 0 & 0 & 1 & 0 & 0 \\
\hline 142B & 52 & 382 & 24 & 0 & 0 & 0 & 0 & 0 & 0 & 1 & 0 & 0 & 0 & 0 & 0 & 0 & 0 & 0 & 1 & 0 & 0 & 0 & 0 & 0 & 1 & 0 & 0 \\
\hline 142B & 53 & 383 & 24 & 0 & 0 & 0 & 0 & 0 & 0 & 0 & 0 & 0 & 0 & 0 & 0 & 0 & 0 & 0 & 1 & 0 & 0 & 0 & 0 & 0 & 1 & 0 & 0 \\
\hline 142B & 54 & 384 & 24 & 0 & 0 & 0 & 0 & 0 & 0 & 0 & 0 & 0 & 0 & 0 & 0 & 0 & 0 & 0 & 1 & 0 & 0 & 0 & 0 & 0 & 1 & 0 & 0 \\
\hline 142B & 55 & 385 & 24 & 0 & 0 & 0 & 0 & 0 & 0 & 0 & 0 & 0 & 0 & 0 & 0 & 0 & 0 & 0 & 1 & 0 & 0 & 0 & 0 & 0 & 1 & 0 & 0 \\
\hline 142B & 56 & 386 & 24 & 0 & 0 & 0 & 0 & 0 & 0 & 0 & 0 & 0 & 0 & 0 & 0 & 0 & 0 & 0 & 1 & 0 & 0 & 0 & 0 & 0 & 1 & 0 & 0 \\
\hline 142B & 57 & 387 & 21 & 0 & 0 & 0 & 0 & 0 & 0 & 0 & 0 & 0 & 0 & 0 & 0 & 0 & 0 & 0 & 1 & 0 & 0 & 0 & 0 & 0 & 1 & 0 & 0 \\
\hline $143 \mathrm{~A}$ & 1 & 388 & 8 & 1 & 1 & 1 & 1 & 1 & 1 & 1 & 1 & 1 & 1 & 1 & 1 & 1 & 1 & 1 & 1 & 1 & 1 & 1 & 1 & 1 & 1 & 1 & 1 \\
\hline $143 \mathrm{~A}$ & 2 & 389 & 24 & 1 & 1 & 1 & 1 & 1 & 1 & 1 & 1 & 1 & 1 & 1 & 0 & 1 & 1 & 1 & 1 & 1 & 1 & 1 & 1 & 1 & 1 & 1 & 1 \\
\hline $143 \mathrm{~A}$ & 3 & 390 & 24 & 1 & 1 & 1 & 1 & 1 & 1 & 1 & 1 & 1 & 0 & 0 & 0 & 1 & 1 & 1 & 1 & 1 & 1 & 1 & 1 & 1 & 1 & 1 & 1 \\
\hline $143 \mathrm{~A}$ & 4 & 391 & 24 & 1 & 1 & 1 & 1 & 1 & 1 & 1 & 1 & 1 & 0 & 0 & 0 & 1 & 1 & 1 & 1 & 1 & 0 & 1 & 1 & 1 & 1 & 1 & 1 \\
\hline $143 \mathrm{~A}$ & 5 & 392 & 24 & 1 & 1 & 1 & 1 & 1 & 1 & 1 & 1 & 1 & 0 & 0 & 0 & 1 & 1 & 1 & 1 & 1 & 0 & 1 & 1 & 1 & 1 & 1 & 1 \\
\hline $143 \mathrm{~A}$ & 6 & 393 & 24 & 1 & 1 & 1 & 1 & 1 & 1 & 1 & 1 & 1 & 0 & 0 & 0 & 1 & 1 & 1 & 1 & 1 & 0 & 1 & 1 & 1 & 1 & 1 & 1 \\
\hline $143 \mathrm{~A}$ & 7 & 394 & 24 & 1 & 1 & 1 & 1 & 1 & 1 & 1 & 1 & 1 & 0 & 0 & 0 & 1 & 1 & 1 & 1 & 1 & 0 & 1 & 1 & 1 & 1 & 1 & 4 \\
\hline $143 \mathrm{~A}$ & 8 & 395 & 24 & 1 & 1 & 1 & 1 & 1 & 1 & 1 & 1 & 1 & 0 & 0 & 0 & 1 & 1 & 1 & 1 & 1 & 0 & 1 & 1 & 1 & 1 & 1 & 1 \\
\hline $143 \mathrm{~A}$ & 9 & 396 & 24 & 1 & 1 & 1 & 1 & 1 & 1 & 1 & 1 & 0 & 0 & 0 & 0 & 1 & 1 & 1 & 1 & 1 & 0 & 1 & 1 & 1 & 1 & 1 & 1 \\
\hline $143 \mathrm{~A}$ & 10 & 397 & 24 & 1 & 1 & 1 & 1 & 1 & 1 & 1 & 1 & 0 & 0 & 0 & 0 & 1 & 1 & 1 & 1 & 1 & 0 & 1 & 1 & 1 & 1 & 1 & \\
\hline
\end{tabular}




\begin{tabular}{|c|c|c|c|c|c|c|c|c|c|c|c|c|c|c|c|c|c|c|c|c|c|c|c|c|c|c|c|}
\hline \multirow[b]{2}{*}{ Cycle } & \multirow[b]{2}{*}{ Timestep } & \multirow{2}{*}{$\begin{array}{l}\text { Cumulative } \\
\text { Timestep }\end{array}$} & \multirow{2}{*}{$\begin{array}{c}\text { Time } \\
\text { Interval } \\
(\mathrm{hrs})\end{array}$} & \multicolumn{6}{|c|}{$\begin{array}{c}\text { NW } \\
\text { Neck Shims }\end{array}$} & \multicolumn{6}{|c|}{$\begin{array}{c}\text { NE } \\
\text { Neck Shims }\end{array}$} & \multicolumn{6}{|c|}{$\begin{array}{c}\text { SW } \\
\text { Neck Shims }\end{array}$} & \multicolumn{6}{|c|}{$\begin{array}{c}\text { SE } \\
\text { Neck Shims }\end{array}$} \\
\hline & & & & \begin{tabular}{|l|}
1 \\
\end{tabular} & 2 & \begin{tabular}{|l|}
3 \\
\end{tabular} & 4 & 5 & 6 & 1 & 2 & 3 & 4 & 5 & 6 & & 2 & 3 & 4 & 5 & 6 & & 12 & 3 & 4 & & 6 \\
\hline $143 \mathrm{~A}$ & 11 & 398 & 24 & 1 & 1 & 1 & 1 & 1 & 1 & 1 & 1 & 0 & 0 & 0 & 0 & 1 & 1 & 1 & 1 & 1 & 0 & 1 & 1 & 1 & 1 & 1 & 1 \\
\hline $143 \mathrm{~A}$ & 12 & 399 & 24 & 1 & 1 & 1 & 1 & 1 & 1 & 1 & 1 & 0 & 0 & 0 & 0 & 1 & 1 & 1 & 1 & 1 & 0 & 1 & 1 & 1 & 1 & 1 & 1 \\
\hline $143 \mathrm{~A}$ & 13 & 400 & 24 & 1 & 1 & 1 & 1 & 1 & 1 & 1 & 1 & 0 & 0 & 0 & 0 & 1 & 1 & 1 & 1 & 1 & 0 & 1 & 1 & 1 & 1 & 1 & 1 \\
\hline $143 \mathrm{~A}$ & 14 & 401 & 24 & 1 & 1 & 1 & 1 & 1 & 1 & 1 & 1 & 0 & 0 & 0 & 0 & 1 & 1 & 1 & 1 & 1 & 0 & 1 & 1 & 1 & 1 & 1 & 1 \\
\hline $143 \mathrm{~A}$ & 15 & 402 & 24 & 1 & 1 & 1 & 1 & 1 & 1 & 1 & 1 & 0 & 0 & 0 & 0 & 1 & 1 & 1 & 1 & 1 & 0 & 1 & 1 & 1 & 1 & 1 & 1 \\
\hline $143 \mathrm{~A}$ & 16 & 403 & 24 & 1 & 1 & 1 & 1 & 1 & 1 & 1 & 1 & 0 & 0 & 0 & 0 & 1 & 1 & 1 & 1 & 1 & 0 & 1 & 1 & 1 & 1 & 1 & 1 \\
\hline $143 \mathrm{~A}$ & 17 & 404 & 24 & 1 & 1 & 1 & 1 & 1 & 1 & 1 & 1 & 0 & 0 & 0 & 0 & 1 & 1 & 1 & 1 & 1 & 0 & 1 & 1 & 1 & 1 & 1 & 1 \\
\hline $143 \mathrm{~A}$ & 18 & 405 & 24 & 1 & 1 & 1 & 1 & 1 & 1 & 1 & 0 & 0 & 0 & 0 & 0 & 1 & 1 & 1 & 1 & 1 & 0 & 1 & 1 & 1 & 1 & 1 & 1 \\
\hline $143 \mathrm{~A}$ & 19 & 406 & 24 & 1 & 1 & 1 & 1 & 1 & 1 & 1 & 0 & 0 & 0 & 0 & 0 & 1 & 1 & 1 & 1 & 1 & 0 & 1 & 1 & 1 & 1 & 1 & 1 \\
\hline $143 \mathrm{~A}$ & 20 & 407 & 24 & 1 & 1 & 1 & 1 & 1 & 1 & 1 & 0 & 0 & 0 & 0 & 0 & 1 & 1 & 1 & 1 & 1 & 0 & 1 & 1 & 1 & 1 & 1 & 1 \\
\hline $143 \mathrm{~A}$ & 21 & 408 & 24 & 1 & 1 & 1 & 1 & 1 & 1 & 1 & 0 & 0 & 0 & 0 & 0 & 1 & 1 & 1 & 1 & 1 & 0 & 1 & 1 & 1 & 1 & 1 & 1 \\
\hline $143 \mathrm{~A}$ & 22 & 409 & 24 & 1 & 1 & 1 & 1 & 1 & 1 & 1 & 0 & 0 & 0 & 0 & 0 & 1 & 1 & 1 & 1 & 1 & 0 & 1 & 1 & 1 & 1 & 1 & 1 \\
\hline $143 \mathrm{~A}$ & 23 & 410 & 24 & 1 & 1 & 1 & 1 & 1 & 1 & 0 & 0 & 0 & 0 & 0 & 0 & 1 & 1 & 1 & 1 & 1 & 0 & 1 & 1 & 1 & 1 & 1 & 1 \\
\hline $143 \mathrm{~A}$ & 24 & 411 & 15 & 1 & 1 & 1 & 1 & 1 & 1 & 0 & 0 & 0 & 0 & 0 & 0 & 1 & 1 & 1 & 1 & 1 & 0 & 1 & 1 & 1 & 1 & 1 & 1 \\
\hline $143 \mathrm{~A}$ & 25 & 412 & 449 & 1 & 1 & 1 & 1 & 1 & 1 & 0 & 0 & 0 & 0 & 0 & 0 & 1 & 1 & 1 & 1 & 1 & 0 & 1 & 1 & 1 & 1 & 1 & 1 \\
\hline $143 \mathrm{~A}$ & 26 & 413 & 24 & 1 & 1 & 1 & 1 & 1 & 1 & 0 & 0 & 0 & 0 & 0 & 0 & 1 & 1 & 1 & 1 & 1 & 0 & 1 & 1 & 1 & 1 & 1 & 1 \\
\hline $143 \mathrm{~A}$ & 27 & 414 & 24 & 1 & 1 & 1 & 1 & 1 & 1 & 0 & 0 & 0 & 0 & 0 & 0 & 1 & 1 & 1 & 1 & 0 & 0 & 1 & 1 & 1 & 1 & 1 & 1 \\
\hline $143 \mathrm{~A}$ & 28 & 415 & 24 & 1 & 1 & 1 & 1 & 1 & 1 & 0 & 0 & 0 & 0 & 0 & 0 & 1 & 1 & 1 & 1 & 0 & 0 & 1 & 1 & 1 & 1 & 1 & 1 \\
\hline $143 \mathrm{~A}$ & 29 & 416 & 24 & 1 & 1 & 1 & 1 & 1 & 1 & 0 & 0 & 0 & 0 & 0 & 0 & 1 & 1 & 1 & 1 & 0 & 0 & 1 & 1 & 1 & 1 & 1 & 1 \\
\hline $143 \mathrm{~A}$ & 30 & 417 & 24 & 1 & 1 & 1 & 1 & 1 & 1 & 0 & 0 & 0 & 0 & 0 & 0 & 1 & 1 & 1 & 1 & 0 & 0 & 1 & 1 & 1 & 1 & 1 & 1 \\
\hline $143 \mathrm{~A}$ & 31 & 418 & 24 & 1 & 1 & 1 & 1 & 1 & 1 & 0 & 0 & 0 & 0 & 0 & 0 & 1 & 1 & 0 & 1 & 0 & 0 & 1 & 1 & 1 & 1 & 1 & 1 \\
\hline $143 \mathrm{~A}$ & 32 & 419 & 24 & 1 & 1 & 1 & 1 & 1 & 1 & 0 & 0 & 0 & 0 & 0 & 0 & 1 & 1 & 0 & 1 & 0 & 0 & 1 & 1 & 1 & 1 & 1 & 1 \\
\hline $143 \mathrm{~A}$ & 33 & 420 & 24 & 1 & 1 & 1 & 1 & 1 & 1 & 0 & 0 & 0 & 0 & 0 & 0 & 1 & 0 & 0 & 1 & 0 & 0 & 1 & 1 & 1 & 1 & 1 & 1 \\
\hline $143 \mathrm{~A}$ & 34 & 421 & 24 & 1 & 1 & 1 & 1 & 1 & 1 & 0 & 0 & 0 & 0 & 0 & 0 & 1 & 0 & 0 & 1 & 0 & 0 & 1 & 1 & 1 & 1 & 1 & 0 \\
\hline $143 \mathrm{~A}$ & 35 & 422 & 24 & 1 & 1 & 1 & 1 & 1 & 1 & 0 & 0 & 0 & 0 & 0 & 0 & 1 & 0 & 0 & 1 & 0 & 0 & 1 & 1 & 1 & 1 & 1 & 0 \\
\hline $143 \mathrm{~A}$ & 36 & 423 & 24 & 1 & 1 & 1 & 1 & 1 & 1 & 0 & 0 & 0 & 0 & 0 & 0 & 1 & 0 & 0 & 1 & 0 & 0 & 1 & 1 & 1 & 1 & 1 & 0 \\
\hline $143 \mathrm{~A}$ & 37 & 424 & 24 & 1 & 1 & 1 & 1 & 1 & 1 & 0 & 0 & 0 & 0 & 0 & 0 & 0 & 0 & 0 & 1 & 0 & 0 & 1 & 1 & 1 & 1 & 1 & 0 \\
\hline $143 \mathrm{~A}$ & 38 & 425 & 24 & 1 & 1 & 1 & 1 & 1 & 1 & 0 & 0 & 0 & 0 & 0 & 0 & 0 & 0 & 0 & 1 & 0 & 0 & 1 & 1 & 1 & 1 & 1 & 0 \\
\hline $143 \mathrm{~A}$ & 39 & 426 & 24 & 1 & 1 & 1 & 1 & 1 & 1 & 0 & 0 & 0 & 0 & 0 & 0 & 0 & 0 & 0 & 1 & 0 & 0 & 1 & 1 & 1 & 1 & 0 & 0 \\
\hline $143 \mathrm{~A}$ & 40 & 427 & 24 & 1 & 1 & 1 & 1 & 1 & 1 & 0 & 0 & 0 & 0 & 0 & 0 & 0 & 0 & 0 & 1 & 0 & 0 & 1 & 1 & 1 & 1 & 0 & 0 \\
\hline $143 \mathrm{~A}$ & 41 & 428 & 28 & 1 & 1 & 1 & 1 & 1 & 1 & 0 & 0 & 0 & 0 & 0 & 0 & 0 & 0 & 0 & 1 & 0 & 0 & 1 & 1 & 1 & 1 & 0 & 0 \\
\hline $143 \mathrm{~A}$ & 42 & 429 & 112 & 1 & 1 & 1 & 1 & 1 & 1 & 0 & 0 & 0 & 0 & 0 & 0 & 0 & 0 & 0 & 1 & 0 & 0 & 1 & 1 & 1 & 1 & 0 & 0 \\
\hline $143 \mathrm{~A}$ & 43 & 430 & 8 & 1 & 1 & 1 & 1 & 1 & 1 & 0 & 0 & 0 & 0 & 0 & 0 & 0 & 0 & 0 & 1 & 0 & 0 & 1 & 1 & 1 & 1 & 0 & 0 \\
\hline $143 \mathrm{~A}$ & 44 & 431 & 24 & 1 & 1 & 1 & 1 & 1 & 0 & 0 & 0 & 0 & 0 & 0 & 0 & 0 & 0 & 0 & 1 & 0 & 0 & 1 & 1 & 0 & 1 & 0 & 0 \\
\hline $143 \mathrm{~A}$ & 45 & 432 & 24 & 1 & 1 & 1 & 1 & 1 & 0 & 0 & 0 & 0 & 0 & 0 & 0 & 0 & 0 & 0 & 1 & 0 & 0 & 1 & 1 & 0 & 1 & 0 & 0 \\
\hline $143 \mathrm{~A}$ & 46 & 433 & 24 & 1 & 1 & 1 & 1 & 1 & 0 & 0 & 0 & 0 & 0 & 0 & 0 & 0 & 0 & 0 & 1 & 0 & 0 & 1 & 0 & 0 & 1 & 0 & 0 \\
\hline $143 \mathrm{~A}$ & 47 & 434 & 24 & 1 & 1 & 1 & 1 & 1 & 0 & 0 & 0 & 0 & 0 & 0 & 0 & 0 & 0 & 0 & 1 & 0 & 0 & 1 & 0 & 0 & 1 & 0 & 0 \\
\hline $143 \mathrm{~A}$ & 48 & 435 & 24 & 1 & 1 & 1 & 1 & 1 & 0 & 0 & 0 & 0 & 0 & 0 & 0 & 0 & 0 & 0 & 1 & 0 & 0 & 1 & 0 & 0 & 1 & 0 & 0 \\
\hline $143 \mathrm{~A}$ & 49 & 436 & 24 & 1 & 1 & 1 & 1 & 1 & 0 & 0 & 0 & 0 & 0 & 0 & 0 & 0 & 0 & 0 & 1 & 0 & 0 & 0 & 0 & 0 & 1 & 0 & 0 \\
\hline $143 \mathrm{~A}$ & 50 & 437 & 24 & 1 & 1 & 1 & 1 & 1 & 0 & 0 & 0 & 0 & 0 & 0 & 0 & 0 & 0 & 0 & 1 & 0 & 0 & 0 & 0 & 0 & 1 & 0 & 0 \\
\hline $143 \mathrm{~A}$ & 51 & 438 & 24 & 1 & 1 & 1 & 1 & 0 & 0 & 0 & 0 & 0 & 0 & 0 & 0 & 0 & 0 & 0 & 1 & 0 & 0 & 0 & 0 & 0 & 1 & 0 & 0 \\
\hline $143 \mathrm{~A}$ & 52 & 439 & 24 & 1 & 1 & 1 & 0 & 0 & 0 & 0 & 0 & 0 & 0 & 0 & 0 & 0 & 0 & 0 & 1 & 0 & 0 & 0 & 0 & 0 & 1 & 0 & 0 \\
\hline $143 \mathrm{~A}$ & 53 & 440 & 22 & 1 & 1 & 0 & 0 & 0 & 0 & 0 & 0 & 0 & 0 & 0 & 0 & 0 & 0 & 0 & 1 & 0 & 0 & 0 & 0 & 0 & 1 & 0 & 0 \\
\hline 143B & 1 & 441 & 8 & 1 & 1 & 1 & 1 & 1 & 1 & 1 & 1 & 1 & 1 & 1 & 1 & 1 & 1 & 1 & 1 & 1 & 1 & 1 & 1 & 1 & 1 & 1 & 1 \\
\hline 143B & 2 & 442 & 24 & 1 & 1 & 1 & 1 & 1 & - & 1 & 1 & 1 & 1 & 1 & 1 & 1 & 1 & 1 & 1 & 1 & 1 & 1 & 1 & 1 & 1 & 1 & 1 \\
\hline
\end{tabular}




\begin{tabular}{|c|c|c|c|c|c|c|c|c|c|c|c|c|c|c|c|c|c|c|c|c|c|c|c|c|c|c|c|}
\hline \multirow[b]{2}{*}{ Cycle } & \multirow[b]{2}{*}{ Timestep } & \multirow{2}{*}{$\begin{array}{l}\text { Cumulative } \\
\text { Timestep }\end{array}$} & \multirow{2}{*}{$\begin{array}{c}\text { Time } \\
\text { Interval } \\
\text { (hrs) }\end{array}$} & \multicolumn{6}{|c|}{$\begin{array}{c}\text { NW } \\
\text { Neck Shims }\end{array}$} & \multicolumn{6}{|c|}{$\begin{array}{c}\text { NE } \\
\text { Neck Shims }\end{array}$} & \multicolumn{6}{|c|}{$\begin{array}{c}\text { SW } \\
\text { Neck Shims }\end{array}$} & \multicolumn{6}{|c|}{$\begin{array}{c}\text { SE } \\
\text { Neck Shims }\end{array}$} \\
\hline & & & & 1 & 2 & 3 & 4 & 5 & 6 & 1 & 2 & 3 & 4 & 5 & 6 & 1 & \begin{tabular}{|l|}
2 \\
\end{tabular} & 3 & \begin{tabular}{|l|}
4 \\
\end{tabular} & 5 & 6 & & 2 & 3 & \begin{tabular}{|l|}
4 \\
\end{tabular} & & 6 \\
\hline $143 B$ & 3 & 443 & 24 & 1 & 1 & 1 & 1 & 1 & 1 & 1 & 1 & 1 & 1 & 1 & 1 & 1 & 1 & 1 & 1 & 1 & 1 & 1 & 1 & 1 & 1 & 0 & 0 \\
\hline 143B & 4 & 444 & 24 & 1 & 1 & 1 & 1 & 1 & 1 & 1 & 1 & 1 & 1 & 1 & 1 & 1 & 1 & 1 & 1 & 1 & 1 & 1 & 1 & 1 & 1 & 0 & 0 \\
\hline 143B & 5 & 445 & 24 & 1 & 1 & 1 & 1 & 1 & 1 & 1 & 1 & 1 & 1 & 1 & 1 & 1 & 1 & 1 & 1 & 1 & 1 & 1 & 1 & 1 & 1 & 0 & 0 \\
\hline 143B & 6 & 446 & 24 & 1 & 1 & 1 & 1 & 1 & 1 & 1 & 1 & 1 & 1 & 1 & 1 & 1 & 1 & 1 & 1 & 1 & 0 & 1 & 1 & 1 & 1 & 0 & 0 \\
\hline 143B & 7 & 447 & 24 & 1 & 1 & 1 & 1 & 1 & 1 & 1 & 1 & $1 \mid$ & 1 & 1 & 1 & 1 & 1 & 1 & 1 & 1 & 0 & 1 & 1 & 1 & 1 & 0 & 0 \\
\hline 143B & 8 & 448 & 24 & 1 & 1 & 1 & 1 & 1 & 1 & 1 & 1 & 1 & 1 & 1 & 1 & 1 & 1 & 1 & 1 & 1 & 0 & 1 & 1 & 1 & 1 & 0 & 0 \\
\hline 143B & 9 & 449 & 24 & 1 & 1 & 1 & 1 & 1 & 1 & 1 & 1 & 1 & 1 & 1 & 1 & 1 & 1 & 1 & 1 & 0 & 0 & 1 & 1 & 1 & 1 & 0 & 0 \\
\hline 143B & 10 & 450 & 24 & 1 & 1 & 1 & 1 & 1 & 1 & 1 & 1 & 1 & 1 & 1 & 1 & 1 & 1 & 1 & 1 & 0 & 0 & 1 & 1 & 1 & 1 & 0 & 0 \\
\hline 143B & 11 & 451 & 24 & 1 & 1 & 1 & 1 & 1 & 1 & 1 & 1 & 1 & 1 & 1 & 1 & 1 & 1 & 1 & 1 & 0 & 0 & 1 & 1 & 1 & 1 & 0 & 0 \\
\hline 143B & 12 & 452 & 24 & 1 & 1 & 1 & 1 & 1 & 1 & 1 & 1 & 1 & 1 & 1 & 1 & 1 & 1 & 1 & 1 & 0 & 0 & 1 & 1 & 1 & 1 & 0 & 0 \\
\hline $143 \mathrm{~B}$ & 13 & 453 & 24 & 1 & 1 & 1 & 1 & 1 & 1 & 1 & 1 & 1 & 1 & 1 & 1 & 1 & 1 & 1 & 1 & 0 & 0 & 1 & 1 & 1 & 1 & 0 & 0 \\
\hline 143B & 14 & 454 & 24 & 1 & 1 & 1 & 1 & 1 & 1 & 1 & 1 & 1 & 1 & 1 & 0 & 1 & 1 & 1 & 1 & 0 & 0 & 1 & 1 & 1 & 1 & 0 & 0 \\
\hline 143B & 15 & 455 & 24 & 1 & 1 & 1 & 1 & 1 & 1 & 1 & 1 & 1 & 1 & 1 & 0 & 1 & 1 & 1 & 1 & 0 & 0 & 1 & 1 & 1 & 1 & 0 & 0 \\
\hline 143B & 16 & 456 & 24 & 1 & 1 & 1 & 1 & 1 & 1 & 1 & 1 & 1 & 1 & 1 & 0 & 1 & 1 & 0 & 1 & 0 & 0 & 1 & 1 & 1 & 1 & 0 & 0 \\
\hline 143B & 17 & 457 & 24 & 1 & 1 & 1 & 1 & 1 & 1 & 1 & 1 & 1 & 1 & 1 & 0 & 1 & 1 & 0 & 1 & 0 & 0 & 1 & 1 & 1 & 1 & 0 & 0 \\
\hline 143B & 18 & 458 & 24 & 1 & 1 & 1 & 1 & 1 & 1 & 1 & 1 & 1 & 1 & 1 & 0 & 1 & 1 & 0 & 1 & 0 & 0 & 1 & 1 & 1 & 1 & 0 & 0 \\
\hline 143B & 19 & 459 & 24 & 1 & 1 & 1 & 1 & 1 & 1 & 1 & 1 & 1 & 1 & 1 & 0 & 1 & 0 & 0 & 1 & 0 & 0 & 1 & 1 & 1 & 1 & 0 & 0 \\
\hline 143B & 20 & 460 & 24 & 1 & 1 & 1 & 1 & 1 & 1 & 1 & 1 & 1 & 1 & 1 & 0 & 1 & 0 & 0 & 1 & 0 & 0 & 1 & 1 & 1 & 1 & 0 & 0 \\
\hline 143B & 21 & 461 & 24 & 1 & 1 & 1 & 1 & 1 & 1 & 1 & 1 & 1 & 1 & 1 & 0 & 1 & 0 & 0 & 1 & 0 & 0 & 1 & 1 & 1 & 1 & 0 & 0 \\
\hline 143B & 22 & 462 & 24 & 1 & 1 & 1 & 1 & 1 & 1 & 1 & 1 & 1 & 1 & 0 & 0 & 1 & 0 & 0 & 1 & 0 & 0 & 1 & 1 & 1 & 1 & 0 & 0 \\
\hline 143B & 23 & 463 & 24 & 1 & 1 & 1 & 1 & 1 & 1 & 1 & 1 & 1 & 1 & 0 & 0 & 1 & 0 & 0 & 1 & 0 & 0 & 1 & 1 & 1 & 1 & 0 & 0 \\
\hline 143B & 24 & 464 & 24 & 1 & 1 & 1 & 1 & 1 & 1 & 1 & 1 & $1 \mid$ & 1 & 0 & 0 & 1 & 0 & 0 & 1 & 0 & 0 & 1 & 1 & 1 & 1 & 0 & 0 \\
\hline 143B & 25 & 465 & 24 & 1 & 1 & 1 & 1 & 1 & 1 & 1 & 1 & \begin{tabular}{|l|}
1 \\
\end{tabular} & 1 & 0 & 0 & 0 & 0 & 0 & 1 & 0 & 0 & 1 & 1 & 1 & 1 & 0 & 0 \\
\hline 143B & 26 & 466 & 24 & 1 & 1 & 1 & 1 & 1 & 1 & 1 & 1 & 1 & 1 & 0 & 0 & 0 & 0 & 0 & 1 & 0 & 0 & 1 & 1 & 1 & 1 & 0 & 0 \\
\hline 143B & 27 & 467 & 24 & 1 & 1 & 1 & 1 & 1 & 1 & 1 & 1 & 1 & 1 & 0 & 0 & 0 & 0 & 0 & 1 & 0 & 0 & 1 & 1 & 1 & 1 & 0 & 0 \\
\hline 143B & 28 & 468 & 24 & 1 & 1 & 1 & 1 & 1 & 1 & 1 & 1 & 1 & 0 & 0 & 0 & 0 & 0 & 0 & 1 & 0 & 0 & 1 & 1 & 1 & 1 & 0 & 0 \\
\hline 143B & 29 & 469 & 53 & 1 & 1 & 1 & 1 & 1 & 1 & 1 & 1 & 1 & 0 & 0 & 0 & 0 & 0 & 0 & 1 & 0 & 0 & 1 & 1 & 1 & 1 & 0 & 0 \\
\hline 143B & 30 & 470 & 24 & 1 & 1 & 1 & 1 & 1 & 1 & 1 & 1 & 1 & 0 & 0 & 0 & 0 & 0 & 0 & 1 & 0 & 0 & 1 & 1 & 1 & 1 & 0 & 0 \\
\hline $143 \mathrm{~B}$ & 31 & 471 & 24 & 1 & 1 & 1 & 1 & 1 & 1 & 1 & 1 & 1 & 0 & 0 & 0 & 0 & 0 & 0 & 1 & 0 & 0 & 1 & 1 & 1 & 1 & 0 & 0 \\
\hline 143B & 32 & 472 & 24 & 1 & 1 & 1 & 1 & 1 & 0 & 1 & 1 & 1 & 0 & 0 & 0 & 0 & 0 & 0 & 1 & 0 & 0 & 1 & 1 & 1 & 1 & 0 & 0 \\
\hline 143B & 33 & 473 & 24 & 1 & 1 & 1 & 1 & 1 & 0 & 1 & 1 & 1 & 0 & 0 & 0 & 0 & 0 & 0 & 1 & 0 & 0 & 1 & 1 & 1 & 1 & 0 & 0 \\
\hline 143B & 34 & 474 & 24 & 1 & 1 & 1 & 1 & 1 & 0 & 1 & 1 & \begin{tabular}{|l|}
1 \\
\end{tabular} & 0 & 0 & 0 & 0 & 0 & 0 & 1 & 0 & 0 & 1 & 1 & 1 & 1 & 0 & 0 \\
\hline 143B & 35 & 475 & 24 & 1 & 1 & 1 & 1 & 1 & 0 & 1 & 1 & 0 & 0 & 0 & 0 & 0 & 0 & 0 & 1 & 0 & 0 & 1 & 1 & 1 & 1 & 0 & 0 \\
\hline 143B & 36 & 476 & 24 & 1 & 1 & 1 & 1 & 1 & 0 & 1 & 1 & 0 & 0 & 0 & 0 & 0 & 0 & 0 & 1 & 0 & 0 & 1 & 1 & 1 & 1 & 0 & 0 \\
\hline 143B & 37 & 477 & 24 & 1 & 1 & 1 & 1 & 1 & 0 & 1 & 1 & 0 & 0 & 0 & 0 & 0 & 0 & 0 & 1 & 0 & 0 & 1 & 1 & 0 & 1 & 0 & 0 \\
\hline 143B & 38 & 478 & 24 & 1 & 1 & 1 & 1 & 1 & 0 & 1 & 1 & 0 & 0 & 0 & 0 & 0 & 0 & 0 & 1 & 0 & 0 & 1 & 1 & 0 & 1 & 0 & 0 \\
\hline 143B & 39 & 479 & 24 & 1 & 1 & 1 & 1 & 0 & 0 & 1 & 1 & 0 & 0 & 0 & 0 & 0 & 0 & 0 & 1 & 0 & 0 & 1 & 1 & 0 & 1 & 0 & 0 \\
\hline 143B & 40 & 480 & 24 & 1 & 1 & 1 & 1 & 0 & 0 & 1 & 1 & 0 & 0 & 0 & 0 & 0 & 0 & 0 & 1 & 0 & 0 & 1 & 0 & 0 & 1 & 0 & 0 \\
\hline 143B & 41 & 481 & 24 & 1 & 1 & 1 & 1 & 0 & 0 & 1 & 1 & 0 & 0 & 0 & 0 & 0 & 0 & 0 & 1 & 0 & 0 & 0 & 0 & 0 & 1 & 0 & 0 \\
\hline 143B & 42 & 482 & 24 & 1 & 1 & 1 & 1 & 0 & 0 & 1 & 1 & 0 & 0 & 0 & 0 & 0 & 0 & 0 & 1 & 0 & 0 & 0 & 0 & 0 & 1 & 0 & 0 \\
\hline 143B & 43 & 483 & 24 & 1 & 1 & 1 & 0 & 0 & 0 & 1 & 1 & 1 & 1 & 0 & 0 & 0 & 0 & 0 & 1 & 0 & 0 & 0 & 0 & 0 & 1 & 0 & 0 \\
\hline 143B & 44 & 484 & 24 & 1 & 1 & 1 & 0 & 0 & 0 & 1 & 1 & 1 & 1 & 0 & 0 & 0 & 0 & 0 & 1 & 0 & 0 & 0 & 0 & 0 & 1 & 0 & 0 \\
\hline 143B & 45 & 485 & 24 & 1 & 1 & 1 & 0 & 0 & 0 & 1 & 1 & \begin{tabular}{|l|}
1 \\
\end{tabular} & 0 & 0 & 0 & \begin{tabular}{|l|}
0 \\
\end{tabular} & 0 & 0 & 1 & 0 & 0 & 0 & 0 & 0 & 1 & 0 & 0 \\
\hline 143B & 46 & 486 & 24 & 1 & 1 & 0 & 0 & 0 & 0 & 1 & 1 & 1 & 0 & 0 & 0 & 0 & 0 & 0 & 1 & 0 & 0 & 0 & 0 & 0 & 1 & 0 & 0 \\
\hline 143B & 47 & 487 & 24 & 1 & 1 & 0 & 0 & 0 & 0 & 1 & 1 & 1 & 0 & 0 & 0 & 0 & 0 & 0 & 1 & 0 & 0 & 0 & 0 & 0 & 1 & 0 & 0 \\
\hline
\end{tabular}




\begin{tabular}{|c|c|c|c|c|c|c|c|c|c|c|c|c|c|c|c|c|c|c|c|c|c|c|c|c|c|c|c|}
\hline \multirow[b]{2}{*}{ Cycle } & \multirow[b]{2}{*}{ Timestep } & \multirow{2}{*}{$\begin{array}{l}\text { Cumulative } \\
\text { Timestep }\end{array}$} & \multirow{2}{*}{$\begin{array}{l}\text { Time } \\
\text { Interval } \\
(\mathrm{hrs})\end{array}$} & \multicolumn{6}{|c|}{$\begin{array}{c}\text { NW } \\
\text { Neck Shims } \\
\end{array}$} & \multicolumn{6}{|c|}{$\begin{array}{c}\text { NE } \\
\text { Neck Shims }\end{array}$} & \multicolumn{6}{|c|}{$\begin{array}{c}\text { SW } \\
\text { Neck Shims }\end{array}$} & \multicolumn{6}{|c|}{$\begin{array}{c}\text { SE } \\
\text { Neck Shims }\end{array}$} \\
\hline & & & & \begin{tabular}{|l|}
1 \\
\end{tabular} & 2 & \begin{tabular}{|l|l|}
3 \\
\end{tabular} & 4 & 5 & 6 & 1 & \begin{tabular}{|l|}
2 \\
\end{tabular} & 3 & \begin{tabular}{|l|}
4 \\
\end{tabular} & \begin{tabular}{|l|}
5 \\
\end{tabular} & 6 & 1 & \begin{tabular}{|l|}
2 \\
\end{tabular} & 3 & \begin{tabular}{|l|}
4 \\
\end{tabular} & 5 & 6 & 1 & 2 & \begin{tabular}{|l|}
3 \\
\end{tabular} & 4 & & 6 \\
\hline $143 B$ & 48 & 488 & 24 & 1 & 1 & 0 & 0 & 0 & 0 & 1 & 1 & 1 & 0 & 0 & 0 & 0 & 0 & 0 & 1 & 0 & 0 & $\overline{0}$ & 0 & 0 & 1 & 0 & 0 \\
\hline 143B & 49 & 489 & 24 & 1 & 0 & 0 & 0 & 0 & 0 & 1 & 1 & 1 & 0 & 0 & 0 & 0 & 0 & 0 & 1 & 0 & 0 & 0 & 0 & 0 & 1 & 0 & 0 \\
\hline 143B & 50 & 490 & 24 & 1 & 0 & 0 & 0 & 0 & 0 & 1 & 1 & 0 & 0 & 0 & 0 & 0 & 0 & 0 & 1 & 0 & 0 & 0 & 0 & 0 & 1 & 0 & 0 \\
\hline 143B & 51 & 491 & 24 & 1 & 0 & 0 & 0 & 0 & 0 & 1 & 1 & 0 & 0 & 0 & 0 & 0 & 0 & 0 & 1 & 0 & 0 & 0 & 0 & 0 & 1 & 0 & 0 \\
\hline 143B & 52 & 492 & 24 & 1 & 0 & 0 & 0 & 0 & 0 & 1 & 1 & 0 & 0 & 0 & 0 & 0 & 0 & 0 & 1 & 0 & 0 & 0 & 0 & 0 & 1 & 0 & 0 \\
\hline 143B & 53 & 493 & 24 & 0 & 0 & 0 & 0 & 0 & 0 & 1 & 1 & 0 & 0 & 0 & 0 & 0 & 0 & 0 & 1 & 0 & 0 & 0 & 0 & 0 & 1 & 0 & 0 \\
\hline 143B & 54 & 494 & 24 & 0 & 0 & 0 & 0 & 0 & 0 & 1 & 0 & 0 & 0 & 0 & 0 & 0 & 0 & 0 & 1 & 0 & 0 & 0 & 0 & 0 & 1 & 0 & 0 \\
\hline 143B & 55 & 495 & 24 & 0 & 0 & 0 & 0 & 0 & 0 & 1 & 0 & 0 & 0 & 0 & 0 & 0 & 0 & 0 & 1 & 0 & 0 & 0 & 0 & 0 & 1 & 0 & 0 \\
\hline 143B & 56 & 496 & 24 & 0 & 0 & 0 & 0 & 0 & 0 & 0 & 0 & 0 & 0 & 0 & 0 & 0 & 0 & 0 & 1 & 0 & 0 & 0 & 0 & 0 & 1 & 0 & 0 \\
\hline 143B & 57 & 497 & 24 & 0 & 0 & 0 & 0 & 0 & 0 & 0 & 0 & 0 & 0 & 0 & 0 & 0 & 0 & 0 & 1 & 0 & 0 & 0 & 0 & 0 & 1 & 0 & 0 \\
\hline $143 \mathrm{~B}$ & 58 & 498 & 24 & 0 & 0 & 0 & 0 & 0 & 0 & 0 & 0 & 0 & 0 & 0 & 0 & 0 & 0 & 0 & 1 & 0 & 0 & 0 & 0 & 0 & 1 & 0 & 0 \\
\hline 143B & 59 & 499 & 24 & 0 & 0 & 0 & 0 & 0 & 0 & 0 & 0 & 0 & 0 & 0 & 0 & 0 & 0 & 0 & 1 & 0 & 0 & 0 & 0 & 0 & 1 & 0 & 0 \\
\hline 143B & 60 & 500 & 9 & 0 & 0 & 0 & 0 & 0 & 0 & 0 & 0 & 0 & 0 & 0 & 0 & 0 & 0 & 0 & 1 & 0 & 0 & 0 & 0 & 0 & 1 & 0 & 0 \\
\hline $144 \mathrm{~A}$ & 1 & 501 & 8 & 1 & 1 & 1 & 1 & 1 & 1 & 1 & 1 & 1 & 1 & 1 & 1 & 1 & 1 & 1 & 1 & 1 & 1 & 1 & 1 & 1 & 1 & 1 & 1 \\
\hline $144 \mathrm{~A}$ & 2 & 502 & 24 & 1 & 1 & 1 & 1 & 1 & 1 & 1 & 1 & 1 & 1 & 1 & 1 & 1 & 1 & 1 & 1 & 1 & 0 & 1 & 1 & 1 & 1 & 1 & 0 \\
\hline $144 \mathrm{~A}$ & 3 & 503 & 24 & 1 & 1 & 1 & 1 & 1 & 1 & 1 & 1 & 1 & 1 & 1 & 1 & 1 & 1 & 1 & 1 & 1 & 0 & 1 & 1 & 1 & 1 & 0 & 0 \\
\hline $144 \mathrm{~A}$ & 4 & 504 & 24 & 1 & 1 & 1 & 1 & 1 & 1 & 1 & 1 & 1 & 1 & 1 & 1 & 1 & 1 & 1 & 1 & 1 & 0 & 1 & 1 & 1 & 1 & 0 & 0 \\
\hline $144 \mathrm{~A}$ & 5 & 505 & 24 & 1 & 1 & 1 & 1 & 1 & 1 & 1 & 1 & 1 & 1 & 1 & 1 & 1 & 1 & 1 & 1 & 1 & 0 & 1 & 1 & 1 & 1 & 0 & 0 \\
\hline $144 \mathrm{~A}$ & 6 & 506 & 24 & 1 & 1 & 1 & 1 & 1 & 1 & 1 & 1 & 1 & 1 & 1 & 1 & 1 & 1 & 1 & 1 & 1 & 0 & 1 & 1 & 1 & 1 & 0 & 0 \\
\hline $144 \mathrm{~A}$ & 7 & 507 & 24 & 1 & 1 & 1 & 1 & 1 & 1 & 1 & 1 & 1 & 1 & 1 & 1 & 1 & 1 & 1 & 1 & 1 & 0 & 1 & 1 & 0 & 1 & 0 & 0 \\
\hline $144 \mathrm{~A}$ & 8 & 508 & 24 & 1 & 1 & 1 & 1 & 1 & 1 & 1 & 1 & 1 & 1 & 1 & 1 & 1 & 1 & 1 & 1 & 1 & 0 & 1 & 1 & 0 & 1 & 0 & 0 \\
\hline $144 \mathrm{~A}$ & 9 & 509 & 24 & 1 & 1 & 1 & 1 & 1 & 1 & 1 & 1 & 1 & 1 & 1 & 1 & 1 & 1 & 1 & 1 & 1 & 0 & 1 & 1 & 0 & 1 & 0 & 0 \\
\hline $144 \mathrm{~A}$ & 10 & 510 & 24 & 1 & 1 & 1 & 1 & 1 & 1 & 1 & 1 & 1 & 1 & 1 & 1 & 1 & 1 & 1 & 1 & 1 & 0 & 1 & 1 & 0 & 1 & 0 & 0 \\
\hline $144 \mathrm{~A}$ & 11 & 511 & 24 & 1 & 1 & 1 & 1 & 1 & 1 & 1 & 1 & 1 & 1 & 1 & 1 & 1 & 1 & 1 & 1 & 0 & 0 & 1 & 1 & 0 & 1 & 0 & 0 \\
\hline $144 \mathrm{~A}$ & 12 & 51 & 24 & 1 & 1 & 1 & 1 & 1 & 1 & 1 & 1 & 1 & 1 & 1 & 0 & 1 & 1 & 1 & 1 & 0 & 0 & 1 & 1 & 0 & 1 & 0 & 0 \\
\hline $144 \mathrm{~A}$ & 13 & 513 & 24 & 1 & 1 & 1 & 1 & 1 & 1 & 1 & 1 & 1 & 1 & 1 & 0 & 1 & 1 & 1 & 1 & 0 & 0 & 1 & 1 & 0 & 1 & 0 & 0 \\
\hline $144 \mathrm{~A}$ & 14 & 514 & 24 & 1 & 1 & 1 & 1 & 1 & 1 & 1 & 1 & 1 & 1 & 0 & 0 & 1 & 1 & 1 & 1 & 0 & 0 & 1 & 1 & 0 & 1 & 0 & 0 \\
\hline $144 \mathrm{~A}$ & 15 & 515 & 24 & 1 & 1 & 1 & 1 & 1 & 1 & 1 & 1 & 1 & 1 & 0 & 0 & 1 & 1 & 1 & 1 & 0 & 0 & 1 & 1 & 0 & 1 & 0 & 0 \\
\hline $144 \mathrm{~A}$ & 16 & 516 & 24 & 1 & 1 & 1 & 1 & 1 & 1 & 1 & 1 & 1 & 1 & 0 & 0 & 1 & 1 & 1 & 1 & 0 & 0 & 1 & 1 & 0 & 1 & 0 & 0 \\
\hline $144 \mathrm{~A}$ & 17 & 517 & 24 & 1 & 1 & 1 & 1 & 1 & 1 & 1 & 1 & 1 & 1 & 0 & 0 & 1 & 1 & 1 & 1 & 0 & 0 & 1 & 1 & 0 & 1 & 0 & 0 \\
\hline $144 \mathrm{~A}$ & 18 & 518 & 24 & 1 & 1 & 1 & 1 & 1 & 1 & 1 & 1 & 1 & 1 & 0 & 0 & 1 & 1 & 1 & 1 & 0 & 0 & 1 & 1 & 0 & 1 & 0 & 0 \\
\hline $144 \mathrm{~A}$ & 19 & 519 & 24 & 1 & 1 & 1 & 1 & 1 & 1 & 1 & 1 & 1 & 1 & 0 & 0 & 1 & 1 & 1 & 1 & 0 & 0 & 1 & 1 & 0 & 1 & 0 & 0 \\
\hline $144 \mathrm{~A}$ & 20 & 520 & 24 & 1 & 1 & 1 & 1 & 1 & 1 & 1 & 1 & 1 & 1 & 0 & 0 & 1 & 1 & 1 & 1 & 0 & 0 & 1 & 1 & 0 & 1 & 0 & 0 \\
\hline $144 \mathrm{~A}$ & 21 & 521 & 24 & 1 & 1 & 1 & 1 & 1 & 1 & 1 & 1 & 1 & 0 & 0 & 0 & 1 & 1 & 1 & 1 & 0 & 0 & 1 & 1 & 0 & 1 & 0 & 0 \\
\hline $144 \mathrm{~A}$ & 22 & 522 & 24 & 1 & 1 & 1 & 1 & 1 & 1 & 1 & 1 & 1 & 0 & 0 & 0 & 1 & 1 & 1 & 1 & 0 & 0 & 1 & 1 & 0 & 1 & 0 & 0 \\
\hline $144 \mathrm{~A}$ & 23 & 523 & 24 & 1 & 1 & 1 & 1 & 1 & 4 & 1 & 1 & 1 & 0 & 0 & 0 & 1 & 1 & 0 & 1 & 0 & 0 & 1 & 1 & 0 & 1 & 0 & 0 \\
\hline $144 \mathrm{~A}$ & 24 & 524 & 24 & 1 & 1 & 1 & 1 & 1 & 1 & 1 & 1 & 1 & 0 & 0 & 0 & 1 & 1 & 0 & 1 & 0 & 0 & 1 & 1 & 0 & 1 & 0 & 0 \\
\hline $144 \mathrm{~A}$ & 25 & 525 & 24 & 1 & 1 & 1 & 1 & 1 & 1 & 1 & 1 & 1 & 0 & 0 & 0 & 1 & 1 & 0 & 1 & 0 & 0 & 1 & 1 & 0 & 1 & 0 & 0 \\
\hline $144 \mathrm{~A}$ & 26 & 526 & 24 & 1 & 1 & 1 & 1 & 1 & 4 & 1 & 1 & 1 & 0 & 0 & 0 & 1 & 1 & 0 & 1 & 0 & 0 & 1 & 0 & 0 & 1 & 0 & 0 \\
\hline $144 \mathrm{~A}$ & 27 & 527 & 24 & 1 & 1 & 1 & 1 & 1 & 1 & 1 & 1 & 1 & 0 & 0 & 0 & 1 & 1 & 0 & 1 & 0 & 0 & 1 & 0 & 0 & 1 & 0 & 0 \\
\hline $144 \mathrm{~A}$ & 28 & 528 & 24 & 1 & 1 & 1 & 1 & 1 & 1 & 1 & 1 & 1 & 0 & 0 & 0 & 1 & 1 & 0 & 1 & 0 & 0 & 1 & 0 & 0 & 1 & 0 & 0 \\
\hline $144 \mathrm{~A}$ & 29 & 529 & 24 & 1 & 1 & 1 & 1 & 1 & 1 & 1 & 1 & 1 & 0 & 0 & 0 & 1 & 1 & 0 & 1 & 0 & 0 & 1 & 0 & 0 & 1 & 0 & 0 \\
\hline $144 \mathrm{~A}$ & 30 & 530 & 24 & 1 & 1 & 1 & 1 & 1 & 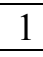 & 1 & 1 & 1 & 0 & 0 & 0 & 1 & 1 & 0 & 1 & 0 & 0 & 1 & 0 & 0 & 1 & 0 & 0 \\
\hline $144 \mathrm{~A}$ & 31 & 531 & 24 & 1 & 1 & 1 & 1 & 1 & 1 & 1 & 1 & 1 & 0 & 0 & 0 & 1 & 0 & 0 & 1 & 0 & 0 & 1 & 0 & 0 & 1 & 0 & 0 \\
\hline $144 \mathrm{~A}$ & 32 & 532 & 24 & 1 & 1 & 1 & 1 & 1 & 1 & 1 & 1 & 1 & \begin{tabular}{|l|}
0 \\
\end{tabular} & 0 & 0 & 1 & 0 & 0 & 1 & 0 & 0 & 1 & 0 & \begin{tabular}{|l|}
0 \\
\end{tabular} & 1 & 0 & 0 \\
\hline
\end{tabular}




\begin{tabular}{|c|c|c|c|c|c|c|c|c|c|c|c|c|c|c|c|c|c|c|c|c|c|c|c|c|c|c|c|}
\hline \multirow[b]{2}{*}{ Cycle } & \multirow[b]{2}{*}{ Timestep } & \multirow{2}{*}{$\begin{array}{l}\text { Cumulative } \\
\text { Timestep }\end{array}$} & \multirow{2}{*}{$\begin{array}{l}\text { Time } \\
\text { Interval } \\
(\mathrm{hrs})\end{array}$} & \multicolumn{6}{|c|}{$\begin{array}{c}\text { NW } \\
\text { Neck Shims } \\
\end{array}$} & \multicolumn{6}{|c|}{$\begin{array}{c}\text { NE } \\
\text { Neck Shims }\end{array}$} & \multicolumn{6}{|c|}{$\begin{array}{c}\text { SW } \\
\text { Neck Shims }\end{array}$} & \multicolumn{6}{|c|}{$\begin{array}{c}\text { SE } \\
\text { Neck Shims }\end{array}$} \\
\hline & & & & \begin{tabular}{|l|}
1 \\
\end{tabular} & \begin{tabular}{|l|}
2 \\
\end{tabular} & \begin{tabular}{|l|l|}
3 \\
\end{tabular} & 4 & 5 & 6 & \begin{tabular}{|l|l|}
1 & \\
\end{tabular} & 2 & 3 & 4 & 5 & 6 & 1 & \begin{tabular}{|l|l|}
2 \\
\end{tabular} & 3 & \begin{tabular}{|l|}
4 \\
\end{tabular} & \begin{tabular}{|l|}
5 \\
\end{tabular} & 6 & 1 & \begin{tabular}{|l|}
2 \\
\end{tabular} & & 4 & & 6 \\
\hline $144 \mathrm{~A}$ & 33 & 533 & 24 & 1 & 1 & 1 & 1 & 1 & 1 & \begin{tabular}{l|}
1 \\
\end{tabular} & 1 & 1 & 0 & 0 & 0 & 1 & 0 & 0 & 1 & 0 & 0 & 1 & \begin{tabular}{|l|}
0 \\
\end{tabular} & 0 & 1 & 0 & 0 \\
\hline $144 \mathrm{~A}$ & 34 & 534 & 24 & 1 & 1 & 1 & 1 & 1 & 1 & 1 & 1 & 0 & 0 & 0 & 0 & 1 & 0 & 0 & 1 & 0 & 0 & 1 & 0 & 0 & 1 & 0 & 0 \\
\hline $144 \mathrm{~A}$ & 35 & 535 & 24 & 1 & 1 & 1 & 1 & 1 & 1 & 1 & 1 & 0 & 0 & 0 & 0 & 1 & 0 & 0 & 1 & \begin{tabular}{|l|}
0 \\
\end{tabular} & 0 & 1 & \begin{tabular}{|l|}
0 \\
\end{tabular} & 0 & 1 & 0 & 0 \\
\hline $144 \mathrm{~A}$ & 36 & 536 & 24 & 1 & 1 & 1 & 1 & 1 & 1 & 1 & 1 & 0 & 0 & 0 & 0 & 1 & 0 & 0 & 1 & 0 & 0 & 1 & 0 & 0 & 1 & 0 & 0 \\
\hline $144 \mathrm{~A}$ & 37 & 537 & 24 & 1 & 1 & 1 & 1 & 1 & 1 & 1 & 1 & 0 & 0 & 0 & 0 & 1 & 0 & 0 & 1 & 0 & 0 & 1 & \begin{tabular}{|l|}
0 \\
\end{tabular} & 0 & 1 & 0 & 0 \\
\hline $144 \mathrm{~A}$ & 38 & 538 & 24 & 1 & 1 & 1 & 1 & 0 & 0 & \begin{tabular}{|l|}
1 \\
\end{tabular} & 1 & 0 & 0 & 0 & 0 & 1 & 0 & 0 & 1 & 0 & 0 & 1 & 0 & 0 & 1 & 0 & 0 \\
\hline $144 \mathrm{~A}$ & 39 & 539 & 24 & 1 & 1 & 1 & 1 & 0 & 0 & 1 & 1 & 0 & 0 & 0 & 0 & 0 & 0 & 0 & 1 & 0 & 0 & 1 & \begin{tabular}{|l|}
0 \\
\end{tabular} & 0 & 1 & 0 & 0 \\
\hline $144 \mathrm{~A}$ & 40 & 540 & 24 & 1 & 1 & 1 & 1 & 0 & 0 & \begin{tabular}{|l|}
1 \\
\end{tabular} & 1 & 0 & 0 & 0 & 0 & 0 & 0 & 0 & 1 & \begin{tabular}{|l|}
0 \\
\end{tabular} & 0 & 1 & \begin{tabular}{|l|}
0 \\
\end{tabular} & 0 & 1 & 0 & 0 \\
\hline $144 \mathrm{~A}$ & 41 & 541 & 24 & 1 & 1 & 1 & 1 & 0 & 0 & 1 & 1 & 0 & 0 & 0 & 0 & 0 & 0 & 0 & 1 & 0 & 0 & 1 & 0 & 0 & 1 & 0 & 0 \\
\hline $144 \mathrm{~A}$ & 42 & 542 & 24 & 1 & 1 & 1 & 1 & 0 & 0 & 1 & 1 & 0 & 0 & 0 & 0 & 0 & 0 & 0 & 1 & 0 & 0 & 1 & 0 & 0 & 1 & 0 & 0 \\
\hline $144 \mathrm{~A}$ & 43 & 543 & 24 & 1 & 1 & 1 & 1 & 0 & 0 & 1 & 1 & 0 & 0 & 0 & 0 & 0 & 0 & 0 & 1 & \begin{tabular}{|l|} 
\\
\end{tabular} & 0 & 1 & \begin{tabular}{|l|}
0 \\
\end{tabular} & 0 & 1 & 0 & 0 \\
\hline $144 \mathrm{~A}$ & 44 & 544 & 24 & 1 & 1 & 1 & 1 & 0 & 0 & 1 & 1 & 0 & 0 & 0 & 0 & 0 & 0 & 0 & 1 & 0 & 0 & 1 & 0 & 0 & 1 & 0 & 0 \\
\hline $144 \mathrm{~A}$ & 45 & 545 & 18 & 1 & 1 & 1 & 1 & 0 & 0 & 1 & 1 & 0 & 0 & 0 & 0 & 0 & 0 & 0 & 1 & 0 & 0 & 1 & \begin{tabular}{|l|}
0 \\
\end{tabular} & 0 & 1 & 0 & 0 \\
\hline 144B & 1 & 546 & 8 & 1 & 1 & 1 & 1 & 1 & 1 & 1 & 1 & 1 & 1 & 1 & 1 & 1 & 1 & 1 & 1 & 1 & 1 & 1 & \begin{tabular}{|l|}
1 \\
\end{tabular} & 1 & 1 & 1 & 1 \\
\hline 144B & 2 & 547 & 24 & 1 & 1 & 1 & 1 & 1 & 0 & 1 & 1 & 1 & 1 & 1 & 1 & 1 & 1 & 1 & 1 & 1 & 1 & 1 & \begin{tabular}{|l|}
1 \\
\end{tabular} & 1 & 1 & 1 & 1 \\
\hline 144B & 3 & 548 & 24 & 1 & 1 & 1 & 1 & 1 & 0 & 1 & 1 & 1 & 1 & 1 & 0 & 1 & 1 & 1 & 1 & 1 & 1 & 1 & 1 & 1 & 1 & 1 & 1 \\
\hline 144B & 4 & 549 & 24 & 1 & 1 & 1 & 1 & 1 & 0 & 1 & 1 & 1 & 1 & 1 & 0 & 1 & 1 & 1 & 1 & 1 & 0 & 1 & \begin{tabular}{|l|}
1 \\
\end{tabular} & 1 & 1 & 1 & 1 \\
\hline 144B & 5 & 550 & 24 & 1 & 1 & 1 & 1 & 1 & 0 & 1 & 1 & 1 & 1 & 1 & 0 & 1 & 1 & 1 & 1 & 1 & 0 & 1 & 1 & 1 & 1 & 1 & 1 \\
\hline 144B & 6 & 551 & 24 & 1 & 1 & 1 & 1 & 1 & 0 & 1 & 1 & 1 & 1 & 1 & 0 & 1 & 1 & 1 & 1 & 1 & 0 & 1 & \begin{tabular}{|l|}
1 \\
\end{tabular} & 1 & 1 & 1 & 1 \\
\hline 144B & 7 & 552 & 24 & 1 & 1 & 1 & 1 & 1 & 0 & 1 & 1 & 1 & 1 & 1 & 0 & 1 & 1 & 1 & 1 & 1 & 0 & 1 & 1 & 1 & 1 & 1 & 1 \\
\hline 144B & 8 & 553 & 24 & 1 & 1 & 1 & 1 & 1 & 0 & 1 & 1 & 1 & 1 & 1 & 0 & 1 & 1 & 1 & 1 & 1 & 0 & 1 & 1 & 1 & 1 & 1 & 1 \\
\hline 144B & 9 & 554 & 24 & 1 & 1 & 1 & 1 & 1 & 0 & 1 & 1 & 1 & 1 & 1 & 0 & 1 & 1 & 1 & 1 & 1 & 0 & 1 & \begin{tabular}{|l|}
1 \\
\end{tabular} & 1 & 1 & 1 & 0 \\
\hline 144B & 10 & 555 & 24 & 1 & 1 & 1 & 1 & 1 & 0 & 1 & 1 & 1 & 1 & 1 & 0 & 1 & 1 & 1 & 1 & 1 & 0 & 1 & \begin{tabular}{|l|}
1 \\
\end{tabular} & 1 & 1 & 1 & 0 \\
\hline 144B & 11 & 556 & 24 & 1 & 1 & 1 & 1 & 1 & 0 & 1 & 1 & 1 & 1 & 0 & 0 & 1 & 1 & 1 & 1 & 1 & 0 & 1 & 1 & 1 & 1 & 1 & 0 \\
\hline 144B & 12 & 557 & 24 & 1 & 1 & 1 & 1 & 1 & 0 & 1 & 1 & 1 & 0 & 0 & 0 & 1 & 1 & 1 & 1 & 1 & 0 & 1 & 1 & 1 & 1 & 1 & 0 \\
\hline 144B & 13 & 558 & 24 & 1 & 1 & 1 & 1 & 1 & 0 & 1 & 1 & 1 & 0 & 0 & 0 & 1 & 1 & 1 & 1 & 1 & 0 & 1 & 1 & 1 & 1 & 1 & 0 \\
\hline 144B & 14 & 559 & 24 & 1 & 1 & 1 & 1 & 1 & 0 & 1 & 1 & 1 & 0 & 0 & 0 & 1 & 1 & 1 & 1 & 1 & 0 & 1 & 1 & 1 & 1 & 1 & 0 \\
\hline 144B & 15 & 560 & 24 & 1 & 1 & 1 & 1 & 1 & 0 & 1 & 1 & 1 & 0 & 0 & 0 & 1 & 1 & 1 & 1 & 1 & 0 & 1 & 1 & 1 & 1 & 1 & 0 \\
\hline 144B & 16 & 561 & 24 & 1 & 1 & 1 & 1 & 1 & 0 & 1 & 1 & 1 & 0 & 0 & 0 & 1 & 1 & 1 & 1 & 1 & 0 & 1 & 1 & 1 & 1 & 1 & 0 \\
\hline 144B & 17 & 562 & 24 & 1 & 1 & 1 & 1 & 1 & 0 & 1 & 1 & 1 & 0 & 0 & 0 & 1 & 1 & 1 & 1 & 1 & 0 & 1 & 1 & 1 & 1 & 1 & 0 \\
\hline 144B & 18 & 563 & 24 & 1 & 1 & 1 & 1 & 1 & 0 & 1 & 1 & 1 & 0 & 0 & 0 & 1 & 1 & 1 & 1 & 1 & 0 & 1 & 1 & 1 & 1 & 1 & 0 \\
\hline 144B & 19 & 564 & 24 & 1 & 1 & 1 & 1 & 1 & 0 & 1 & 1 & 1 & 0 & 0 & 0 & 1 & 1 & 1 & 1 & 1 & 0 & 1 & 1 & 1 & 1 & 1 & 0 \\
\hline 144B & 20 & 565 & 24 & 1 & 1 & 1 & 1 & 1 & 0 & 1 & 1 & 1 & 0 & 0 & 0 & 1 & 1 & 1 & 1 & 1 & 0 & 1 & 1 & 1 & 1 & 1 & 0 \\
\hline 144B & 21 & 566 & 24 & 1 & 1 & 1 & 1 & 1 & 0 & \begin{tabular}{|l|}
1 \\
\end{tabular} & 1 & 1 & 0 & 0 & 0 & 1 & 1 & 1 & 1 & 1 & 0 & 1 & 1 & 1 & 1 & 1 & 0 \\
\hline 144B & 22 & 567 & 19 & 1 & 1 & 1 & 1 & 1 & 0 & 1 & 1 & 1 & 0 & 0 & 0 & 1 & 1 & 1 & 1 & 1 & 0 & 1 & 1 & 1 & 1 & 1 & 0 \\
\hline 144B & 23 & 568 & 66 & 1 & 1 & 1 & 1 & 1 & 0 & 1 & 1 & 1 & 0 & 0 & 0 & 1 & 1 & 1 & 1 & 1 & 0 & 1 & 1 & 1 & 1 & 1 & 0 \\
\hline 144B & 24 & 569 & 19 & 1 & 1 & 1 & 1 & 1 & 0 & 1 & 1 & 1 & 0 & 0 & 0 & 1 & 1 & 1 & 1 & 1 & 0 & 1 & 1 & 1 & 1 & 1 & 0 \\
\hline 144B & 25 & 570 & 24 & 1 & 1 & 1 & 1 & 1 & 0 & 1 & 1 & 1 & 0 & 0 & 0 & 1 & 1 & 1 & 1 & 1 & 0 & 1 & 1 & 1 & 1 & 1 & 0 \\
\hline 144B & 26 & 571 & 24 & 1 & 1 & 1 & 1 & 1 & 0 & 1 & 1 & 1 & 0 & 0 & 0 & 1 & 1 & 1 & 1 & 1 & 0 & 1 & 1 & 1 & 1 & 1 & 0 \\
\hline 144B & 27 & 572 & 24 & 1 & 1 & 1 & 1 & 0 & 0 & 1 & 1 & 1 & 0 & 0 & 0 & 1 & 1 & 1 & 1 & 1 & 0 & 1 & 1 & 1 & 1 & 1 & 0 \\
\hline 144B & 28 & 573 & 24 & 1 & 1 & 1 & 1 & 0 & 0 & 1 & 1 & 1 & 0 & 0 & 0 & 1 & 1 & 1 & 1 & 1 & 0 & 1 & 1 & 1 & 1 & 1 & 0 \\
\hline 144B & 29 & 574 & 24 & 1 & 1 & 1 & 1 & 0 & 0 & 1 & 1 & 1 & 0 & 0 & 0 & 1 & 1 & 1 & 1 & 1 & 0 & 1 & 1 & 1 & 1 & 1 & 0 \\
\hline 144B & 30 & 575 & 24 & 1 & 1 & 1 & 1 & 0 & 0 & 1 & 1 & 1 & 0 & 0 & 0 & 1 & 1 & 1 & 1 & 1 & 0 & 1 & \begin{tabular}{|l|}
1 \\
\end{tabular} & 1 & 1 & 1 & 0 \\
\hline 144B & 31 & 576 & 24 & 1 & 1 & 1 & 1 & 0 & 0 & 1 & 1 & 1 & 0 & 0 & 0 & 1 & 1 & 1 & 1 & 1 & 0 & 1 & \begin{tabular}{|l|}
1 \\
\end{tabular} & 1 & 1 & 0 & 0 \\
\hline 144B & 32 & 577 & 24 & 1 & 1 & 1 & 1 & 0 & 0 & $1 \mid$ & 1 & 1 & 0 & 0 & 0 & 1 & 1 & 1 & 1 & 11 & 0 & 1 & 1 & 1 & 1 & 0 & 0 \\
\hline
\end{tabular}




\begin{tabular}{|c|c|c|c|c|c|c|c|c|c|c|c|c|c|c|c|c|c|c|c|c|c|c|c|c|c|c|c|}
\hline \multirow[b]{2}{*}{ Cycle } & \multirow[b]{2}{*}{ Timestep } & \multirow{2}{*}{$\begin{array}{l}\text { Cumulative } \\
\text { Timestep }\end{array}$} & \multirow{2}{*}{$\begin{array}{c}\text { Time } \\
\text { Interval } \\
\text { (hrs) }\end{array}$} & \multicolumn{6}{|c|}{$\begin{array}{c}\text { NW } \\
\text { Neck Shims } \\
\end{array}$} & \multicolumn{6}{|c|}{$\begin{array}{c}\text { NE } \\
\text { Neck Shims }\end{array}$} & \multicolumn{6}{|c|}{$\begin{array}{c}\text { SW } \\
\text { Neck Shims } \\
\end{array}$} & \multicolumn{6}{|c|}{$\begin{array}{c}\text { SE } \\
\text { Neck Shims }\end{array}$} \\
\hline & & & & 1 & 2 & 3 & 4 & & 6 & 1 & 2 & 3 & 4 & 5 & 6 & 1 & \begin{tabular}{|l|}
2 \\
\end{tabular} & 3 & 4 & 5 & 6 & & 2 & 3 & \begin{tabular}{|l|l|}
4 & \\
\end{tabular} & & 6 \\
\hline 144B & 33 & 578 & 24 & 1 & 1 & 1 & 0 & 0 & 0 & 1 & 1 & 1 & 0 & 0 & 0 & 1 & 1 & 1 & \begin{tabular}{|l|}
1 \\
\end{tabular} & 1 & $\overline{0}$ & 1 & 1 & 1 & 1 & 0 & 0 \\
\hline 144B & 34 & 579 & 24 & 1 & 1 & 1 & 0 & 0 & 0 & 1 & 1 & 1 & 0 & 0 & 0 & 1 & 1 & 1 & 1 & 1 & 0 & 1 & 1 & 1 & 1 & 0 & 0 \\
\hline 144B & 35 & 580 & 24 & 1 & 1 & 1 & 0 & 0 & 0 & 1 & 1 & 1 & 0 & 0 & 0 & 1 & 1 & 1 & 1 & 1 & 0 & 1 & 1 & 1 & 1 & 0 & 0 \\
\hline 144B & 36 & 581 & 24 & 1 & 1 & 1 & 0 & 0 & 0 & 1 & 1 & 1 & 0 & 0 & 0 & 1 & 1 & 1 & 1 & 0 & 0 & 1 & 1 & 0 & 1 & 0 & 0 \\
\hline 144B & 37 & 582 & 24 & 1 & 1 & 1 & 0 & 0 & 0 & 1 & 1 & 1 & 0 & 0 & 0 & 1 & 1 & 1 & 1 & 0 & 0 & 1 & 1 & 0 & 1 & 0 & 0 \\
\hline 144B & 38 & 583 & 24 & 1 & 1 & 1 & 0 & 0 & 0 & 1 & 1 & 0 & 0 & 0 & 0 & 1 & 1 & 1 & 1 & 0 & 0 & 1 & 1 & 0 & 1 & 0 & 0 \\
\hline 144B & 39 & 584 & 24 & 1 & 1 & 1 & 0 & 0 & 0 & 1 & 1 & 0 & 0 & 0 & 0 & 1 & 1 & 1 & 1 & 0 & 0 & 1 & 1 & 0 & 1 & 0 & 0 \\
\hline 144B & 40 & 585 & 24 & 1 & 1 & 1 & 0 & 0 & 0 & 1 & 1 & 0 & 0 & 0 & 0 & 1 & 1 & 1 & $\begin{array}{ll}1 \\
\end{array}$ & 0 & 0 & 1 & 1 & 0 & 1 & 0 & 0 \\
\hline 144B & 41 & 586 & 24 & 1 & 1 & 0 & 0 & 0 & 0 & 1 & 1 & 0 & 0 & 0 & 0 & 1 & 1 & 1 & 1 & 0 & 0 & 1 & 1 & 0 & 1 & 0 & 0 \\
\hline 144B & 42 & 587 & 24 & 1 & 1 & 0 & 0 & 0 & 0 & 1 & 1 & 0 & 0 & 0 & 0 & 1 & 1 & 1 & 1 & 0 & 0 & 1 & 1 & 0 & 1 & 0 & 0 \\
\hline 144B & 43 & 588 & 24 & 1 & 1 & 0 & 0 & 0 & 0 & 1 & 1 & 0 & 0 & 0 & 0 & 1 & 1 & 1 & 1 & 0 & 0 & 1 & 1 & 0 & 1 & 0 & 0 \\
\hline 144B & 44 & 589 & 24 & 1 & 1 & 0 & 0 & 0 & 0 & 1 & 1 & 0 & 0 & 0 & 0 & 1 & 1 & 1 & 1 & 0 & 0 & 1 & 1 & 0 & 1 & 0 & 0 \\
\hline 144B & 45 & 590 & 24 & 1 & 1 & 0 & 0 & 0 & 0 & 1 & 1 & 0 & 0 & 0 & 0 & 1 & 1 & 1 & 1 & 0 & 0 & 1 & 0 & 0 & 1 & 0 & 0 \\
\hline 144B & 46 & 591 & 24 & 1 & 1 & 0 & 0 & 0 & 0 & 1 & 1 & 0 & 0 & 0 & 0 & 1 & 1 & 1 & 1 & 0 & 0 & 1 & 0 & 0 & 1 & 0 & 0 \\
\hline 144B & 47 & 592 & 24 & 1 & 1 & 0 & 0 & 0 & 0 & 1 & 1 & 0 & 0 & 0 & 0 & 1 & 1 & 1 & 1 & 0 & 0 & 1 & 0 & 0 & 1 & 0 & 0 \\
\hline 144B & 48 & 593 & 24 & 1 & 1 & 0 & 0 & 0 & 0 & 1 & 1 & 0 & 0 & 0 & 0 & 1 & 1 & 0 & 1 & 0 & 0 & 1 & 0 & 0 & 1 & 0 & 0 \\
\hline 144B & 49 & 594 & 24 & 1 & 0 & 0 & 0 & 0 & 0 & 1 & 1 & 0 & 0 & 0 & 0 & 1 & 1 & 0 & 1 & 0 & 0 & 1 & 0 & 0 & 1 & 0 & 0 \\
\hline 144B & 50 & 595 & 24 & 1 & 0 & 0 & 0 & 0 & 0 & 1 & 0 & 0 & 0 & 0 & 0 & 1 & 1 & 0 & 1 & 0 & 0 & 0 & 0 & 0 & 1 & 0 & 0 \\
\hline 144B & 51 & 596 & 24 & 1 & 0 & 0 & 0 & 0 & 0 & 1 & 0 & 0 & 0 & 0 & 0 & 1 & 1 & 0 & 1 & 0 & 0 & 0 & 0 & 0 & 1 & 0 & 0 \\
\hline 144B & 52 & 597 & 24 & 1 & 0 & 0 & 0 & 0 & 0 & 1 & 0 & 0 & 0 & 0 & 0 & 1 & 1 & 0 & 1 & 0 & 0 & 0 & 0 & 0 & 1 & 0 & 0 \\
\hline 144B & 53 & 598 & 24 & 1 & 0 & 0 & 0 & 0 & 0 & 1 & 0 & 0 & 0 & 0 & 0 & 1 & 0 & 0 & 1 & 0 & 0 & 0 & 0 & 0 & 1 & 0 & 0 \\
\hline 144B & 54 & 599 & 27 & 1 & 0 & 0 & 0 & 0 & 0 & 1 & 0 & 0 & 0 & 0 & 0 & 1 & 0 & 0 & 1 & 0 & 0 & 0 & 0 & 0 & 1 & 0 & 0 \\
\hline $145 \mathrm{~A}$ & 1 & 600 & 11 & 1 & 1 & 1 & 1 & 1 & 1 & 1 & 1 & 1 & 1 & 1 & 1 & 1 & 1 & 1 & 1 & 1 & 1 & 1 & 1 & 1 & 1 & 1 & 1 \\
\hline $145 \mathrm{~A}$ & 2 & 601 & 11 & 1 & 1 & 1 & 1 & 1 & 1 & 1 & 1 & 1 & 1 & 1 & 1 & 1 & 1 & 1 & 1 & 1 & 1 & 1 & 1 & 1 & 1 & 1 & 1 \\
\hline $145 \mathrm{~A}$ & 3 & 602 & 24 & 1 & 1 & 1 & 1 & 1 & 1 & 1 & 1 & 1 & 1 & 1 & 1 & 1 & 1 & 1 & 1 & 1 & 1 & 1 & 1 & 1 & 1 & 1 & 1 \\
\hline $145 \mathrm{~A}$ & 4 & 603 & 24 & 1 & 1 & 1 & 1 & 1 & 1 & 1 & 1 & 1 & 1 & 1 & 1 & 1 & 1 & 1 & 1 & 1 & 1 & 1 & 1 & 1 & 1 & 1 & 1 \\
\hline $145 \mathrm{~A}$ & 5 & 604 & 24 & 1 & 1 & 1 & 1 & 1 & 1 & 1 & 1 & 1 & 1 & 1 & 1 & 1 & 1 & 1 & 1 & 1 & 1 & 1 & 1 & 1 & 1 & 1 & 1 \\
\hline $145 \mathrm{~A}$ & 6 & 605 & 24 & 1 & 1 & 1 & 1 & 1 & 1 & 1 & 1 & 1 & 1 & 1 & 0 & 1 & 1 & 1 & 1 & 1 & 1 & 1 & 1 & 1 & 1 & 1 & 1 \\
\hline $145 \mathrm{~A}$ & 7 & 606 & 24 & 1 & 1 & 1 & 1 & 1 & 1 & 1 & 1 & 1 & 1 & 1 & 0 & 1 & 1 & 1 & 1 & 1 & 1 & 1 & 1 & 1 & 1 & 1 & 1 \\
\hline $145 \mathrm{~A}$ & 8 & 607 & 24 & 1 & 1 & 1 & 1 & 1 & 1 & 1 & 1 & 1 & 1 & 1 & 0 & 1 & 1 & 1 & 1 & 1 & 1 & 1 & 1 & 1 & 1 & 1 & 1 \\
\hline $145 \mathrm{~A}$ & 9 & 608 & 24 & 1 & 1 & 1 & 1 & 1 & 1 & 1 & 1 & 1 & 1 & 1 & 0 & 1 & 1 & 1 & 1 & 1 & 1 & 1 & 1 & 1 & 1 & 1 & 1 \\
\hline $145 \mathrm{~A}$ & 10 & 609 & 24 & 1 & 1 & 1 & 1 & 1 & 1 & 1 & 1 & 1 & 1 & 1 & 0 & 1 & 1 & 1 & 1 & 1 & 1 & 1 & 1 & 1 & 1 & 1 & 1 \\
\hline $145 \mathrm{~A}$ & 11 & 610 & 24 & 1 & 1 & 1 & 1 & 1 & 1 & 1 & 1 & 1 & 1 & 1 & 0 & 1 & 1 & 1 & 1 & 1 & 1 & 1 & 1 & 1 & 1 & 1 & 1 \\
\hline $145 \mathrm{~A}$ & 12 & 611 & 24 & 1 & 1 & 1 & 1 & 1 & 1 & 1 & 1 & 1 & 1 & 0 & 0 & 1 & 1 & 1 & 1 & 1 & 1 & 1 & 1 & 1 & 1 & 1 & 1 \\
\hline $145 \mathrm{~A}$ & 13 & 612 & 24 & 1 & 1 & 1 & 1 & 1 & 1 & 1 & 1 & 1 & 1 & 0 & 0 & 1 & 1 & 1 & 1 & 1 & 1 & 1 & 1 & 1 & 1 & 1 & 1 \\
\hline $145 \mathrm{~A}$ & 14 & 613 & 24 & 1 & 1 & 1 & 1 & 1 & 1 & 1 & 1 & 1 & 1 & 0 & 0 & 1 & 1 & 1 & 1 & 1 & 1 & 1 & 1 & 1 & 1 & 1 & 1 \\
\hline $145 \mathrm{~A}$ & 15 & 614 & 24 & 1 & 1 & 1 & 1 & 1 & 1 & 1 & 1 & 1 & 1 & 0 & 0 & 1 & 1 & 1 & 1 & 1 & 1 & 1 & 1 & 1 & 1 & 1 & 0 \\
\hline $145 \mathrm{~A}$ & 16 & 615 & 24 & 1 & 1 & 1 & 1 & 1 & 1 & 1 & 1 & 1 & 1 & 0 & 0 & 1 & 1 & 1 & 1 & 1 & 1 & 1 & 1 & 1 & 1 & 1 & 0 \\
\hline $145 \mathrm{~A}$ & 17 & 616 & 24 & 1 & 1 & 1 & 1 & 1 & 1 & 1 & 1 & 1 & 1 & 0 & 0 & 1 & 1 & 1 & 1 & 1 & 1 & 1 & 1 & 1 & 1 & 1 & 0 \\
\hline $145 \mathrm{~A}$ & 18 & 617 & 24 & 1 & 1 & 1 & 1 & 1 & 1 & 1 & 1 & 1 & 1 & 0 & 0 & 1 & 1 & 1 & 1 & 1 & 1 & 1 & 1 & 1 & 1 & 1 & 0 \\
\hline $145 \mathrm{~A}$ & 19 & 618 & 24 & 1 & 1 & 1 & 1 & 1 & 1 & 1 & 1 & 1 & 1 & 0 & 0 & 1 & 1 & 1 & 1 & 1 & 1 & 1 & 1 & 1 & 1 & 1 & 0 \\
\hline $145 \mathrm{~A}$ & 20 & 619 & 24 & 1 & 1 & 1 & 1 & 1 & 1 & 1 & 1 & 1 & 1 & 0 & 0 & 1 & 1 & 1 & 1 & 1 & 1 & 1 & 1 & 1 & 1 & 1 & 0 \\
\hline $145 \mathrm{~A}$ & 21 & 620 & 24 & 1 & 1 & 1 & 1 & 1 & 1 & 1 & 1 & 1 & 0 & 0 & 0 & 1 & 1 & 1 & 1 & 1 & 1 & 1 & 1 & 1 & 1 & 1 & 0 \\
\hline $145 \mathrm{~A}$ & 22 & 621 & 24 & 1 & 1 & 1 & 1 & 1 & 1 & 1 & 1 & 1 & 0 & 0 & 0 & 1 & 1 & 1 & 1 & 1 & 1 & 1 & 1 & 1 & 1 & 0 & 0 \\
\hline $145 \mathrm{~A}$ & 23 & 622 & 24 & 1 & 1 & 1 & 1 & 1 & 1 & 1 & 1 & 1 & 0 & 0 & 0 & 1 & 1 & 1 & 1 & 1 & 1 & 1 & 1 & 1 & 1 & 0 & 0 \\
\hline
\end{tabular}




\begin{tabular}{|c|c|c|c|c|c|c|c|c|c|c|c|c|c|c|c|c|c|c|c|c|c|c|c|c|c|c|c|}
\hline \multirow[b]{2}{*}{ Cycle } & \multirow[b]{2}{*}{ Timestep } & \multirow{2}{*}{$\begin{array}{l}\text { Cumulative } \\
\text { Timestep }\end{array}$} & \multirow{2}{*}{$\begin{array}{c}\text { Time } \\
\text { Interval } \\
(\text { hrs })\end{array}$} & \multicolumn{6}{|c|}{$\begin{array}{c}\text { NW } \\
\text { Neck Shims } \\
\end{array}$} & \multicolumn{6}{|c|}{$\begin{array}{c}\text { NE } \\
\text { Neck Shims } \\
\end{array}$} & \multicolumn{6}{|c|}{$\begin{array}{c}\text { SW } \\
\text { Neck Shims } \\
\end{array}$} & \multicolumn{6}{|c|}{$\begin{array}{c}\text { SE } \\
\text { Neck Shims }\end{array}$} \\
\hline & & & & 11 & \begin{tabular}{|l|}
2 \\
\end{tabular} & 3 & 4 & 5 & 6 & 1 & 2 & 3 & 4 & 5 & 6 & 1 & \begin{tabular}{|l|}
2 \\
\end{tabular} & 3 & \begin{tabular}{|l|l}
4 \\
\end{tabular} & 5 & 6 & 1 & \begin{tabular}{|l|}
2 \\
\end{tabular} & 3 & 4 & & 6 \\
\hline $145 \mathrm{~A}$ & 24 & 623 & 24 & 1 & 1 & 1 & 1 & 1 & 1 & 1 & 1 & 1 & 0 & 0 & 0 & 1 & 1 & 1 & 1 & 1 & 1 & 1 & 1 & 1 & 1 & 0 & 0 \\
\hline $145 \mathrm{~A}$ & 25 & 624 & 24 & 1 & 1 & 1 & 1 & 1 & 1 & 1 & 1 & 1 & 0 & 0 & 0 & 1 & 1 & 1 & 1 & 1 & 1 & 1 & 1 & 1 & 1 & 0 & 0 \\
\hline $145 \mathrm{~A}$ & 26 & 625 & 8 & 1 & 1 & 1 & 1 & 1 & 1 & 1 & 1 & 1 & 0 & 0 & 0 & 1 & 1 & 1 & 1 & 1 & 1 & 1 & 1 & 1 & 1 & 0 & 0 \\
\hline $145 \mathrm{~A}$ & 27 & 626 & 43 & 1 & 1 & 1 & 1 & 1 & 1 & 1 & 1 & 1 & 0 & 0 & 0 & 1 & 1 & 1 & 1 & 1 & 1 & 1 & 1 & 1 & 1 & 0 & 0 \\
\hline $145 \mathrm{~A}$ & 28 & 627 & 12 & 1 & 1 & 1 & 1 & 1 & 1 & 1 & 1 & 1 & 0 & 0 & 0 & 1 & 1 & 1 & 1 & 1 & 1 & 1 & 1 & 1 & 1 & 0 & 0 \\
\hline $145 \mathrm{~A}$ & 29 & 628 & 10 & 1 & 1 & 1 & 1 & 1 & 1 & 1 & 1 & 1 & 0 & 0 & 0 & 1 & 1 & 1 & 1 & 1 & 1 & 1 & 1 & 1 & 1 & 0 & 0 \\
\hline $145 \mathrm{~A}$ & 30 & 629 & 24 & 1 & 1 & 1 & 1 & 1 & 1 & 1 & 1 & 1 & 0 & 0 & 0 & 1 & 1 & 1 & 1 & 1 & 1 & 1 & 1 & 1 & 1 & 0 & 0 \\
\hline $145 \mathrm{~A}$ & 31 & 630 & 24 & 1 & 1 & 1 & 1 & 1 & 0 & 1 & 1 & 1 & 0 & 0 & 0 & 1 & 1 & 1 & 1 & 1 & 1 & 1 & 1 & 1 & 1 & 0 & 0 \\
\hline $145 \mathrm{~A}$ & 32 & 631 & 24 & 1 & 1 & 1 & 1 & 1 & 0 & 1 & 1 & 1 & 0 & 0 & 0 & 1 & 1 & 1 & 1 & 1 & 1 & 1 & 1 & 1 & 1 & 0 & 0 \\
\hline $145 \mathrm{~A}$ & 33 & 632 & 24 & 1 & 1 & 1 & 1 & 1 & 0 & 1 & 1 & 1 & 0 & 0 & 0 & 1 & 1 & 1 & 1 & 1 & 1 & 1 & 1 & 1 & 1 & 0 & 0 \\
\hline $145 \mathrm{~A}$ & 34 & 633 & 24 & 1 & 1 & 1 & 1 & 1 & 0 & 1 & 11 & 1 & 0 & 0 & 0 & 1 & 1 & 1 & 1 & 1 & 0 & 1 & 1 & 1 & 1 & 0 & 0 \\
\hline $145 \mathrm{~A}$ & 35 & 634 & 25 & 1 & 1 & 1 & 1 & 1 & 0 & 1 & 11 & 1 & 0 & 0 & 0 & 1 & 1 & 1 & 1 & 1 & 0 & 1 & 1 & 1 & 1 & 0 & 0 \\
\hline $145 \mathrm{~A}$ & 36 & 635 & 60 & 1 & 1 & 1 & 1 & 1 & 0 & 1 & 11 & 1 & 0 & 0 & 0 & \begin{tabular}{|l|}
1 \\
\end{tabular} & 1 & 1 & 1 & 1 & 0 & 1 & 1 & 1 & 1 & 0 & 0 \\
\hline $145 \mathrm{~A}$ & 37 & 63 & 11 & 1 & 1 & 1 & 1 & 1 & 0 & 1 & 11 & 1 & 0 & 0 & 0 & \begin{tabular}{|l|}
1 \\
\end{tabular} & 1 & 1 & 1 & 1 & 0 & 1 & 1 & 1 & 1 & 0 & 0 \\
\hline $145 \mathrm{~A}$ & 38 & 637 & 24 & 1 & 1 & 1 & 1 & 1 & 0 & 1 & 1 & 0 & 0 & 0 & 0 & 1 & 1 & 1 & 1 & 1 & 0 & 1 & 1 & 1 & 1 & 0 & 0 \\
\hline $145 \mathrm{~A}$ & 39 & 638 & 18 & 1 & 1 & 1 & 1 & 1 & 0 & 1 & 11 & 0 & 0 & 0 & 0 & 1 & 1 & 1 & 1 & 1 & 0 & 1 & 1 & 1 & 1 & 0 & 0 \\
\hline $145 \mathrm{~A}$ & 40 & 639 & 58 & 1 & 1 & 1 & 1 & 1 & 0 & 1 & 11 & 0 & 0 & 0 & 0 & 1 & 1 & 1 & 1 & 1 & 0 & 1 & 1 & 1 & 1 & 0 & 0 \\
\hline $145 \mathrm{~A}$ & 41 & 640 & 8 & 1 & 1 & 1 & 1 & 1 & 0 & 1 & 1 & 0 & 0 & 0 & 0 & 1 & 1 & 1 & 1 & 1 & 0 & 1 & 1 & 1 & 1 & 0 & 0 \\
\hline $145 \mathrm{~A}$ & 42 & 641 & 12 & 1 & 1 & 1 & 1 & 1 & 0 & 1 & 11 & 0 & 0 & 0 & 0 & 1 & 1 & 1 & 1 & 1 & 0 & 1 & 1 & 1 & 1 & 0 & 0 \\
\hline $145 \mathrm{~A}$ & 43 & 642 & 24 & 1 & 1 & 1 & 1 & 1 & 0 & 1 & 11 & 0 & 0 & 0 & 0 & 1 & 1 & 1 & 1 & 1 & 0 & 1 & 1 & 1 & 1 & 0 & 0 \\
\hline $145 \mathrm{~A}$ & 44 & 6 & 24 & 1 & 1 & 1 & 1 & 1 & 0 & 1 & 11 & 0 & 0 & 0 & 0 & 1 & 1 & 1 & 1 & 1 & 0 & 1 & 1 & 1 & 1 & 0 & 0 \\
\hline $145 \mathrm{~A}$ & 45 & 644 & 24 & 1 & 1 & 1 & 1 & 1 & 0 & 1 & 11 & 0 & 0 & 0 & 0 & 1 & 1 & 1 & 1 & 1 & 0 & 1 & 1 & 1 & 1 & 0 & 0 \\
\hline $145 \mathrm{~A}$ & 46 & 645 & 24 & 1 & 1 & 1 & 1 & 0 & 0 & 1 & 1 & 0 & 0 & 0 & 0 & 1 & 1 & 1 & 1 & 1 & 0 & 1 & 1 & 1 & 1 & 0 & 0 \\
\hline $145 \mathrm{~A}$ & 47 & 646 & 24 & 1 & 1 & 1 & 1 & 0 & 0 & 1 & 10 & 0 & 0 & 0 & 0 & 1 & 1 & 1 & 1 & 1 & 0 & 1 & 1 & 1 & 1 & 0 & 0 \\
\hline $145 \mathrm{~A}$ & 48 & 647 & 24 & 1 & 1 & 1 & 1 & 0 & 0 & 1 & 100 & 0 & 0 & 0 & 0 & 1 & 1 & 1 & 1 & 1 & 0 & 1 & 1 & 1 & 1 & 0 & 0 \\
\hline $145 \mathrm{~A}$ & 49 & 648 & 24 & 1 & 1 & 1 & 1 & 0 & 0 & 1 & 10 & 0 & 0 & 0 & 0 & 1 & 1 & 1 & 1 & 0 & 0 & 1 & 1 & 1 & 1 & 0 & 0 \\
\hline $145 \mathrm{~A}$ & 50 & 649 & 24 & 1 & 1 & 1 & 1 & 0 & 0 & 1 & 10 & 0 & 0 & 0 & 0 & 1 & 1 & 1 & 1 & 0 & 0 & 1 & 1 & 1 & 1 & 0 & 0 \\
\hline $145 \mathrm{~A}$ & 51 & 650 & 24 & 1 & 1 & 1 & 1 & 0 & 0 & 0 & \begin{tabular}{|l|l}
0 \\
\end{tabular} & 0 & 0 & 0 & 0 & 1 & 1 & 1 & 1 & 0 & 0 & 1 & 1 & 1 & 1 & 0 & 0 \\
\hline $145 \mathrm{~A}$ & 52 & 651 & 24 & 1 & 1 & 1 & 1 & 0 & 0 & 0 & \begin{tabular}{|l|l}
0 \\
\end{tabular} & 0 & 0 & 0 & 0 & 1 & 1 & 1 & 1 & 0 & 0 & 1 & 1 & 1 & 1 & 0 & 0 \\
\hline $145 \mathrm{~A}$ & 53 & 652 & 24 & 1 & 1 & 1 & 1 & 0 & 0 & 0 & 0 & 0 & 0 & 0 & 0 & 1 & 1 & 0 & 1 & 0 & 0 & 1 & 1 & 1 & 1 & 0 & 0 \\
\hline $145 \mathrm{~A}$ & 54 & 653 & 24 & 1 & 1 & 1 & 1 & 0 & 0 & 0 & \begin{tabular}{|l|l|}
0 & 0 \\
\end{tabular} & 0 & 0 & 0 & 0 & 1 & 1 & 0 & 1 & 0 & 0 & 1 & 1 & 0 & 1 & 0 & 0 \\
\hline $145 \mathrm{~A}$ & 55 & 654 & 24 & 1 & 1 & 1 & 1 & 0 & 0 & 0 & \begin{tabular}{|l|l}
0 \\
\end{tabular} & 0 & 0 & 0 & 0 & 1 & 1 & 0 & 1 & 0 & 0 & 1 & 1 & 0 & 1 & 0 & 0 \\
\hline $145 \mathrm{~A}$ & 56 & 655 & 24 & 1 & 1 & 1 & 1 & 0 & 0 & 0 & \begin{tabular}{|l|l}
0 \\
\end{tabular} & 0 & 0 & 0 & 0 & 1 & 1 & 0 & 1 & 0 & 0 & 1 & 1 & 0 & 1 & 0 & 0 \\
\hline $145 \mathrm{~A}$ & 57 & 656 & 24 & 1 & 1 & 1 & 1 & 0 & 0 & 0 & 0 & 0 & 0 & 0 & 0 & 1 & 1 & 0 & 1 & 0 & 0 & 1 & 0 & 0 & 1 & 0 & 0 \\
\hline $145 \mathrm{~A}$ & 58 & 657 & 24 & 1 & 1 & 1 & 1 & 0 & 0 & 0 & 0 & 0 & 0 & 0 & 0 & 1 & 1 & 0 & 1 & 0 & 0 & 1 & 0 & 0 & 1 & 0 & 0 \\
\hline $145 \mathrm{~A}$ & 59 & 658 & 24 & 1 & 1 & 1 & 1 & 0 & 0 & 0 & 0 & 0 & 0 & 0 & 0 & 1 & 1 & 0 & 1 & 0 & 0 & 1 & 0 & 0 & 1 & 0 & 0 \\
\hline $145 \mathrm{~A}$ & 60 & 659 & 24 & 1 & 1 & 1 & 1 & 0 & 0 & 0 & 0 & 0 & 0 & 0 & 0 & 1 & 0 & 0 & 1 & 0 & 0 & 1 & 0 & 0 & 1 & 0 & 0 \\
\hline $145 \mathrm{~A}$ & 61 & 660 & 24 & 1 & 1 & 1 & 0 & 0 & 0 & 0 & \begin{tabular}{|l|l}
0 & 0 \\
\end{tabular} & 0 & 0 & 0 & 0 & 1 & 0 & 0 & 1 & 0 & 0 & 1 & 0 & 0 & 1 & 0 & 0 \\
\hline $145 \mathrm{~A}$ & 62 & 661 & 24 & 1 & 1 & 1 & 0 & $\underline{0}$ & 0 & 0 & 0 & 0 & 0 & 0 & 0 & 1 & 0 & 0 & 1 & 0 & 0 & 1 & 0 & 0 & 1 & 0 & 0 \\
\hline $145 \mathrm{~A}$ & 63 & 662 & 29 & 1 & 1 & 1 & 0 & 0 & 0 & 0 & \begin{tabular}{|l|l}
0 \\
\end{tabular} & 0 & 0 & 0 & 0 & 1 & 0 & 0 & 1 & 0 & 0 & & \begin{tabular}{l|l|}
0 \\
\end{tabular} & 0 & & 0 & 4 \\
\hline
\end{tabular}

$0=$ neck shim withdrawn

$1=$ neck shim inserted 


\section{Appendix D}

\section{MCNP Quarter-Core ATR Model Input File Listing}

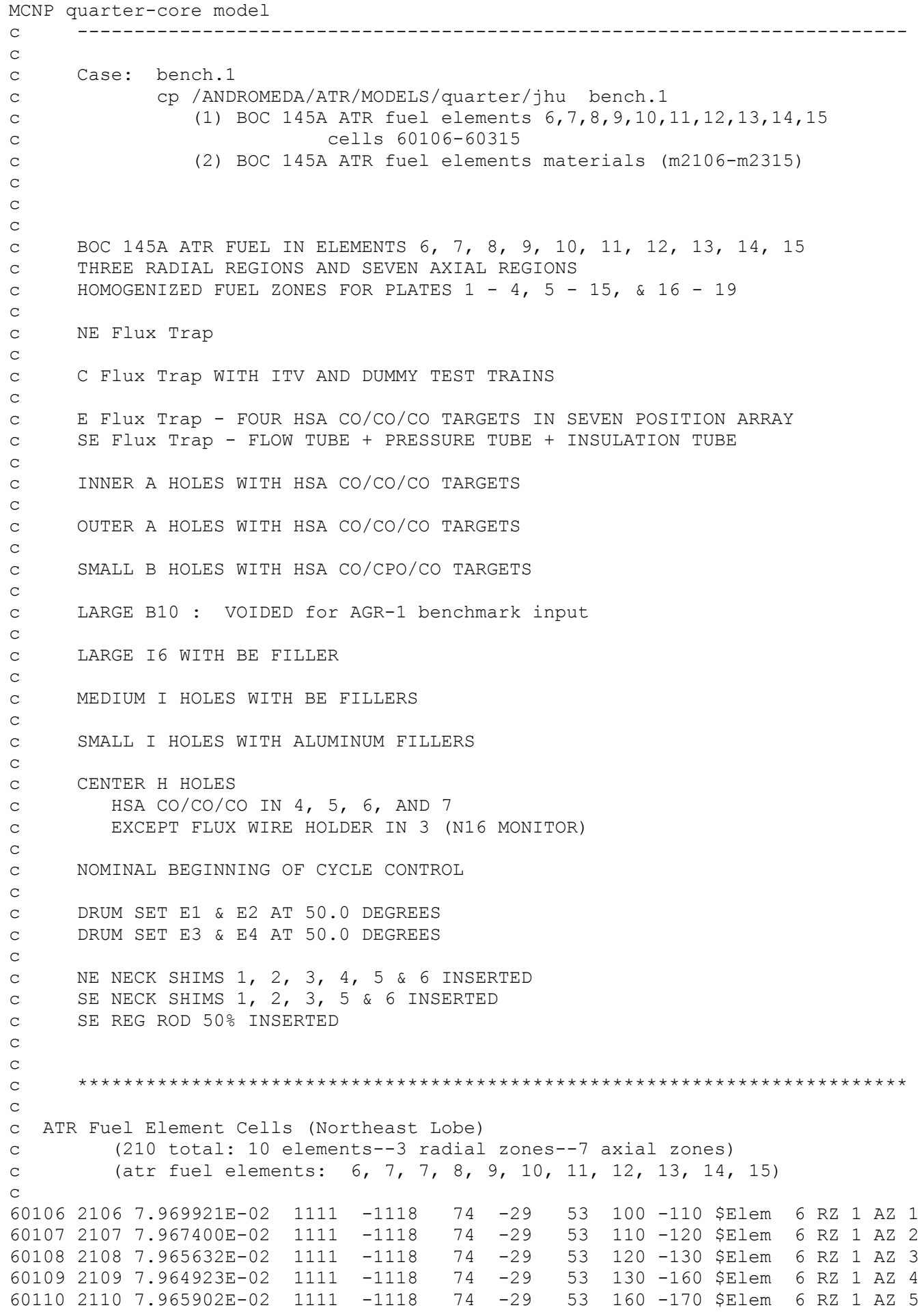




\begin{tabular}{|c|c|c|c|c|c|c|c|c|c|c|c|c|c|c|}
\hline 1 & 2111 & & 111 & -1118 & 74 & -29 & 53 & 170 & -180 & lem & 6 & RZ & AZ & 6 \\
\hline 112 & 2112 & $7.971317 \mathrm{E}-02$ & 1111 & -1118 & 74 & -29 & 53 & 180 & -200 & $\$ E l e m$ & 6 & RZ & AZ & \\
\hline 13 & 2113 & $8.290653 \mathrm{E}-02$ & 19 & -1120 & 74 & -29 & 53 & 100 & -110 & $\$ E l e m$ & 6 & RZ & AZ & \\
\hline 14 & 114 & $.288057 \mathrm{E}-02$ & 119 & -1120 & 74 & -29 & 53 & 110 & -120 & $\$$ Elem & 1 & $\mathrm{RZ}$ & AZ & \\
\hline 15 & 115 & $8.286028 \mathrm{E}-02$ & 119 & -1120 & 74 & -29 & 53 & 120 & -130 & $\$$ Elem & 61 & RZ & AZ & \\
\hline 0116 & 2116 & $8.285143 E-02$ & 119 & -1120 & 74 & -29 & 53 & 130 & -160 & $\$ E l e m$ & 1 & RZ & AZ & \\
\hline 117 & 2117 & $8.286359 \mathrm{E}-02$ & 119 & -1120 & 74 & -29 & 53 & 160 & -170 & $\$ \mathrm{Elem}$ & 6 & RZ & AZ & \\
\hline 118 & 118 & $8.288571 \mathrm{E}-02$ & 19 & -1120 & 74 & -29 & 53 & 170 & -180 & $\$ E l e m$ & 0 & RZ & AZ & \\
\hline 19 & 119 & $291521 \mathrm{E}-02$ & & -1120 & 74 & -29 & 53 & 180 & -200 & $\$$ Elem & 61 & RZ & AZ & \\
\hline 120 & 120 & $7.864934 \mathrm{E}-02$ & 21 & -1128 & 74 & -29 & 53 & 100 & -110 & $\$$ Elem & 61 & RZ & AZ & \\
\hline 0121 & 2121 & $7.862680 \mathrm{E}-02$ & 121 & -1128 & 74 & -29 & 53 & 110 & -120 & $\$ E l e m$ & 1 & RZ & AZ & \\
\hline 0122 & 2122 & $7.861021 \mathrm{E}-02$ & 121 & -1128 & 74 & -29 & 53 & 120 & -130 & $\$ E l e m$ & 6 & RZ & AZ & \\
\hline 123 & 2123 & $7.860361 \mathrm{E}-02$ & 121 & -1128 & 74 & -29 & 53 & 130 & -160 & $\$ E l e m$ & 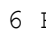 & RZ & $\mathrm{AZ}$ & \\
\hline 124 & 24 & $7.861274 \mathrm{E}-02$ & 21 & -1128 & 74 & -29 & 53 & 160 & -170 & $\$ E l e m$ & 61 & RZ & AZ & \\
\hline 125 & 2125 & $7.863101 \mathrm{E}-02$ & 21 & -1128 & 74 & -29 & 53 & 170 & -180 & $\$ E l e m$ & 1 & RZ 3 & $\mathrm{AZ}$ & \\
\hline 0126 & 2126 & $7.865541 \mathrm{E}-02$ & 121 & -1128 & 74 & -29 & 53 & 180 & -200 & $\$ \mathrm{Elem}$ & 61 & RZ 3 & AZ & \\
\hline 0127 & 2127 & $7.975810 \mathrm{E}-02$ & 111 & -1118 & 17 & -72 & -30 & 100 & -110 & $\$ E l e m$ & 7 & RZ & AZ & \\
\hline 28 & 2128 & $7.975810 \mathrm{E}-02$ & 111 & -1118 & 17 & -72 & -30 & 110 & -120 & $\$$ Elem & 1 & RZ & AZ & \\
\hline 29 & 129 & $7.975810 \mathrm{E}-02$ & 111 & -1118 & 17 & -72 & -30 & 120 & -130 & $\$ E l e m$ & 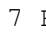 & RZ & AZ & \\
\hline 30 & 2130 & $7.975810 \mathrm{E}-02$ & 11 & -1118 & 17 & -72 & -30 & 130 & -160 & $\$$ Elem & 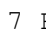 & RZ & $\mathrm{AZ}$ & \\
\hline 131 & 2131 & $7.975810 \mathrm{E}-02$ & 111 & -1118 & 17 & -72 & -30 & 160 & -170 & $\$ E l e m$ & 71 & RZ & AZ & \\
\hline 0132 & 2132 & $7.975810 \mathrm{E}-02$ & 111 & -1118 & 17 & -72 & -30 & 170 & -180 & $\$$ Elem & 7 & RZ & AZ & \\
\hline 133 & 2133 & $.0 E-02$ & 111 & -1118 & 17 & -72 & -30 & 180 & -200 & $\$ E l e m$ & 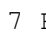 & RZ & AZ & \\
\hline 134 & 2134 & $8.294907 \mathrm{E}-02$ & 119 & -1120 & 17 & -72 & -30 & 100 & -110 & $\$ E l e m$ & 1 & RZ & AZ & \\
\hline 135 & 135 & $8.294907 \mathrm{E}-02$ & 119 & -1120 & 17 & -72 & -30 & 110 & -120 & $\$$ Elem & 1 & RZ 2 & $\mathrm{AZ}$ & \\
\hline 36 & 136 & 8.294907. & 19 & -1120 & 17 & -72 & -30 & 120 & -130 & $\$ E l e m$ & s & RZ 2 & $2 \mathrm{AZ}$ & \\
\hline 0137 & 2137 & $8.294907 \mathrm{E}-02$ & 119 & -1120 & 17 & -72 & -30 & 130 & -160 & $\$ E l e m$ & 71 & RZ & AZ & \\
\hline 138 & 2138 & 8.294 & 119 & -1120 & 17 & -72 & -30 & 160 & -170 & $\$ E l e m$ & 71 & RZ & AZ & \\
\hline 139 & 2139 & $7 E-02$ & 119 & -1120 & 17 & -72 & -30 & 170 & -180 & $\$ E l e m$ & t & RZ 2 & AZ & \\
\hline 140 & 140 & $8.294907 \mathrm{E}-02$ & 119 & -1120 & 17 & -72 & -30 & 180 & -200 & $\$$ Elem & 1 & RZ 2 & $\mathrm{AZ}$ & \\
\hline 41 & 41 & 7.858601 & 121 & -1128 & 17 & -72 & -30 & 100 & -110 & $\$ E l e m$ & 71 & RZ & AZ & \\
\hline 0142 & 2142 & $7.858601 \mathrm{E}-02$ & 121 & -1128 & 17 & -72 & -30 & 110 & -120 & $\$ E l e m$ & 71 & RZ & AZ & \\
\hline 43 & 2143 & 7.85 & 121 & -1128 & 17 & -72 & -30 & 120 & -130 & $\$ E l e m$ & 71 & RZ 3 & $3 \mathrm{AZ}$ & \\
\hline 44 & 2144 & 7.8 & 121 & -1128 & 17 & -72 & -30 & 130 & -160 & $\$$ Elem & t & RZ 3 & $3 \mathrm{AZ}$ & \\
\hline 145 & 2145 & $7.858601 \mathrm{E}-02$ & 121 & -1128 & 17 & -72 & -30 & 160 & -170 & $\$$ Elem & 71 & RZ 3 & $\mathrm{AZ}$ & \\
\hline 50146 & 146 & $7.858601 \mathrm{E}-02$ & 121 & -1128 & 17 & -72 & -30 & 170 & -180 & $\$$ Elem & 71 & RZ 3 & $\mathrm{Az}$ & \\
\hline 60147 & 2147 & $7.858601 \mathrm{E}-02$ & 1121 & -1128 & 17 & -72 & -30 & 180 & -200 & $\$ E l e m$ & 71 & RZ 3 & $3 \mathrm{AZ}$ & \\
\hline 50148 & 2148 & 7.97 & 1111 & -1118 & 54 & -15 & -73 & 100 & -110 & $\$$ Elem & 81 & RZ & $\mathrm{AZ}$ & \\
\hline 49 & 2149 & 7. & 11 & -1 & 54 & -15 & -73 & 110 & -120 & $\$ E l e m$ & 81 & RZ 1 & $1 \mathrm{AZ}$ & \\
\hline 150 & 2150 & $7.973803 \mathrm{E}-02$ & 111 & -1118 & 54 & -15 & -73 & 120 & -130 & $\$$ Elem & $81-x-1$ & RZ & AZ & \\
\hline 151 & 2151 & $7.973803 \mathrm{E}-02$ & 111 & -1118 & 54 & -15 & -73 & 130 & -160 & $\$$ Elem & $81-r a$ & RZ 1 & AZ & \\
\hline 0152 & 2152 & $7.973803 \mathrm{E}-02$ & 1111 & -1118 & 54 & -15 & -73 & 160 & -170 & $\$ E l e m$ & 81 & RZ & AZ & \\
\hline 0153 & 2153 & 7.9 & 1111 & -1118 & 54 & -15 & -73 & 170 & -180 & $\$ E l e m$ & 8 & RZ & AZ & \\
\hline 54 & 2154 & 7. & 11 & -1 & 54 & -15 & -73 & 180 & -200 & $\$ E l e m$ & $81-x-1$ & RZ 1 & $1 \mathrm{AZ}$ & \\
\hline 155 & 2155 & 8.294 & 119 & -1120 & 54 & -15 & -73 & 100 & -110 & \$Elem & 81 & RZ 2 & AZ & \\
\hline 156 & 2156 & $8.294866 \mathrm{E}-02$ & 119 & -1120 & 54 & -15 & -73 & 110 & -120 & $\$$ Elem & $81-r a$ & RZ 2 & AZ & \\
\hline 60157 & 2157 & $8.294866 \mathrm{E}-02$ & 1119 & -1120 & 54 & -15 & -73 & 120 & -130 & $\$ E l e m$ & 81 & RZ & AZ & \\
\hline 60158 & 2158 & 8. & 1119 & -1120 & 54 & -15 & -73 & 130 & -160 & $\$ E l e m$ & 8 & RZ 2 & $2 \mathrm{AZ}$ & 4 \\
\hline 59 & 2159 & 8. & 9 & -1 & 54 & -15 & -73 & 160 & -170 & em & $81-x-1$ & RZ 2 & $2 \mathrm{AZ}$ & \\
\hline 160 & 2160 & 8.29 & 119 & -1120 & 54 & -15 & -73 & 170 & -180 & \$Elem & 81 & RZ 2 & $2 \mathrm{AZ}$ & \\
\hline 161 & 2161 & 8.29 & 119 & -1120 & 54 & -15 & -73 & 180 & -200 & $\$$ Elem & $81-r a$ & RZ 2 & $\mathrm{AZ}$ & \\
\hline 60162 & 2162 & $7.868379 \mathrm{E}-02$ & 121 & -1128 & 54 & -15 & -73 & 100 & -110 & $\$ E l e m$ & 81 & RZ & AZ & \\
\hline 60163 & 2163 & 7.868379 & 1121 & -1128 & 54 & -15 & -73 & 110 & -120 & $\$$ Elem & $81-r a$ & RZ 3 & $3 \mathrm{AZ}$ & \\
\hline 164 & 2164 & 7.86 & 1121 & -1128 & 54 & -15 & -73 & 120 & -130 & $\$$ Elem & $81-x-1$ & RZ 3 & $3 \mathrm{AZ}$ & \\
\hline 65 & 2165 & 7.8 & 21 & -1128 & 54 & -15 & -73 & 130 & -160 & $\$$ Elem & 81 & RZ & Az & \\
\hline 66 & 2166 & 02 & 21 & 28 & 54 & -15 & -73 & 160 & -170 & $\$ \mathrm{Elem}$ & $81-r a$ & RZ & $\mathrm{Az}$ & \\
\hline 60167 & 2167 & $7.868379 \mathrm{E}-02$ & 1121 & -1128 & 54 & -15 & -73 & 170 & -180 & $\$ E l e m$ & 81 & RZ 3 & $3 \mathrm{Az}$ & \\
\hline 60168 & 2168 & $7.868379 \mathrm{E}-02$ & 1121 & -1128 & 54 & -15 & -73 & 180 & -200 & $\$$ Elem & 81 & RZ 3 & $3 \mathrm{Az}$ & \\
\hline 60169 & 2169 & 7.9738031 & 1821 & -1828 & 14 & -52 & 27 & 100 & -110 & $\$ E l e m$ & 91 & RZ & $\mathrm{Az}$ & \\
\hline 50170 & 2170 & -02 & 821 & -1828 & 14 & -52 & 27 & 110 & -120 & \$Elem & 91 & RZ & $A z$ & \\
\hline 60171 & 2171 & 7. & 821 & -1828 & 14 & -52 & 27 & 120 & -130 & $\$$ Elem & 91 & RZ & Az & \\
\hline 60172 & 2172 & 797 & 821 & -1828 & 14 & -52 & 27 & 130 & -160 & $\$ E l e m$ & 91 & RZ & $\mathrm{Az}$ & \\
\hline 60173 & 2173 & $7.973803 \mathrm{E}-02$ & 1821 & -1828 & 14 & -52 & 27 & 160 & -170 & $\$$ Elem & 91 & RZ 1 & $1 \mathrm{Az}$ & 5 \\
\hline 60174 & 2174 & $7.973803 \mathrm{E}-02$ & 1821 & -1828 & 14 & -52 & 27 & 170 & -180 & $\$ E l e m$ & 91 & RZ & Az & \\
\hline 60175 & 2175 & 7.973 & 1821 & -1828 & 14 & -52 & 27 & 180 & -200 & $\$ E l e m$ & 91 & RZ & Az & \\
\hline 60176 & 2176 & $8.294866 \mathrm{E}-02$ & 1829 & -1830 & 14 & -52 & 27 & 100 & -110 & $\$$ Elem & 9 & RZ & $\pi$ & \\
\hline & & 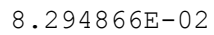 & 1829 & 830 & 14 & -52 & 27 & 110 & -120 & $\$$ Elem & 9 & RZ 2 & $\mathrm{Az}$ & \\
\hline 60178 & 2178 & $8.294866 \mathrm{E}-02$ & 1829 & -1830 & 14 & -52 & 27 & 120 & -130 & $\$ E l e m$ & 91 & RZ 2 & Az & \\
\hline 60179 & 2179 & $8.294866 \mathrm{E}-02$ & 1829 & -1830 & 14 & -52 & 27 & 130 & -160 & $\$ E l e m$ & 9 & RZ 2 & Az & \\
\hline 60180 & 2180 & $366 \mathrm{E}-02$ & 1829 & -1830 & 14 & -52 & 27 & 160 & -170 & $\$$ Elem & 9 & RZ & $A 2$ & \\
\hline 60181 & & $8.294866 \mathrm{E}-02$ & 829 & -1830 & 14 & -52 & 27 & 170 & -180 & $\$ E l e m$ & 9 & RZ & $A 2$ & \\
\hline
\end{tabular}




\begin{tabular}{|c|c|c|c|c|c|c|c|c|c|c|c|c|c|c|}
\hline 182 & 182 & 2 & 29 & -1830 & 14 & -52 & 27 & 180 & -200 & $1 \mathrm{em}$ & 9 & RZ & A & $\mathrm{Az}$ \\
\hline 183 & 2183 & $.868379 \mathrm{E}-02$ & 1831 & -1838 & 14 & -52 & 27 & 100 & -110 & $\$ E l e m$ & 9 & B7 & 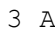 & $\Delta$ \\
\hline 84 & 2184 & $.868379 \mathrm{E}-02$ & 831 & -1838 & 14 & -52 & 27 & 110 & -120 & $\$ E l e m$ & 9 & RZ & A & $A:$ \\
\hline 85 & 185 & $868379 \mathrm{E}-02$ & 831 & -1838 & 14 & -52 & 27 & 120 & -130 & $\$$ Elem & 9 & $\mathrm{RZ}$ & $3 \mathrm{~A}$ & $\mathrm{AZ}$ \\
\hline 86 & 86 & $.868379 \mathrm{E}-02$ & 831 & -1838 & 14 & -52 & 27 & 130 & -160 & $\$$ Elem & 9 & RZ & $3 \mathrm{~A}$ & $\mathrm{Az}$ \\
\hline 187 & 2187 & $7.868379 \mathrm{E}-02$ & 1831 & -1838 & 14 & -52 & 27 & 160 & -170 & $\$ E l e m$ & 9 & RZ & A & AZ \\
\hline 188 & 2188 & $7.868379 \mathrm{E}-02$ & 831 & -1838 & 14 & -52 & 27 & 170 & -180 & $\$ \mathrm{Elem}$ & 9 & RZ & 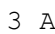 & 7 \\
\hline 189 & 189 & $7.868379 \mathrm{E}-02$ & 831 & -1838 & 14 & & 27 & 180 & -200 & $\$ E l e m$ & 9 & $\mathrm{RZ}$ & A & $\mathrm{AZ}$ \\
\hline 90 & 190 & $.967879 \mathrm{E}-02$ & 821 & -1828 & 71 & -12 & -53 & 100 & -110 & $\$$ Elem & 10 & RZ & A & $\mathrm{Az}$ \\
\hline 191 & 191 & $7.964312 \mathrm{E}-02$ & 821 & -1828 & 71 & -12 & -53 & 110 & -120 & $\$$ Elem & 10 & RZ & & \\
\hline 192 & 2192 & $7.961814 \mathrm{E}-02$ & 821 & -1828 & 71 & -12 & -53 & 120 & -130 & $\$ E l e m$ & 10 & RZ & & Az \\
\hline 193 & 2193 & $7.960810 \mathrm{E}-02$ & 821 & -1828 & 71 & -12 & -53 & 130 & -160 & $\$ E l e m$ & 10 & RZ & & Az \\
\hline 194 & 2194 & $7.962194 \mathrm{E}-02$ & 821 & -1828 & 71 & -12 & -53 & 160 & -170 & $\$ E l e m$ & 10 & RZ & & $\mathrm{Az}$ \\
\hline 95 & 95 & $7.965042 \mathrm{E}-02$ & 321 & -1828 & 71 & -12 & -53 & 70 & -180 & $\$ E l e m$ & 10 & RZ & A & Az \\
\hline 96 & 2196 & $7.969851 \mathrm{E}-02$ & 821 & -1828 & 71 & -12 & -53 & 180 & -200 & $\$ E l e m$ & 10 & RZ & $1 \mathrm{~A}$ & $\mathrm{Az}$ \\
\hline 197 & 2197 & $8.288951 \mathrm{E}-02$ & 829 & -1830 & 71 & -12 & -53 & 100 & -110 & $\$ \mathrm{Elem}$ & 10 & RZ & 2 & Az \\
\hline 198 & 198 & $74 E-02$ & 829 & -1830 & 71 & -12 & -53 & 110 & -120 & $\$ E l e m$ & 10 & RZ & $2 \mathrm{~A}$ & $\mathrm{Az}$ \\
\hline 99 & 2199 & $8.282410 \mathrm{E}-02$ & 829 & -1830 & 71 & -12 & -53 & 120 & -130 & $\$$ Elem & 10 & RZ & A & $\mathrm{Az}$ \\
\hline 200 & 200 & $8.281159 \mathrm{E}-02$ & 829 & -1830 & 71 & -12 & -53 & 130 & -160 & $\$ E l e m$ & 10 & RZ & A & Az \\
\hline 01 & 2201 & $8.282881 \mathrm{E}-02$ & 829 & -1830 & 71 & -12 & -53 & 160 & -170 & $\$$ Elem & 10 & RZ & $2 A$ & $\mathrm{AZ}$ \\
\hline 202 & 2202 & $8.286008 \mathrm{E}-02$ & 829 & -1830 & 71 & -12 & -53 & 170 & -180 & $\$ E l e m$ & 10 & RZ & 2 & Az \\
\hline 203 & 2203 & $8.290178 \mathrm{E}-02$ & 1829 & -1830 & 71 & -12 & -53 & 180 & -200 & $\$$ Elem & 10 & RZ & $2 A$ & $\mathrm{Az}$ \\
\hline 204 & 2204 & $7.851893 \mathrm{E}-02$ & 831 & -1838 & 71 & -12 & -53 & 100 & -110 & $\$ E l e m$ & 10 & RZ & A & Az \\
\hline 05 & 2205 & $7.848707 \mathrm{E}-02$ & 831 & -1838 & 71 & -12 & -53 & 110 & -120 & $\$ E l e m$ & 10 & RZ & $3 \mathrm{~A}$ & $\mathrm{Az}$ \\
\hline 206 & 06 & $7.846358 \mathrm{E}-02$ & 831 & -1838 & 71 & -12 & -53 & 120 & -130 & $\$$ Elem & 10 & RZ & $3 \mathrm{~A}$ & Az \\
\hline 207 & 207 & $7.845429 \mathrm{E}-02$ & 831 & -1838 & 71 & -12 & -53 & 130 & -160 & $\$ E l e m$ & 10 & RZ & $3 \mathrm{~A}$ & Az \\
\hline 208 & 2208 & $7.846721 \mathrm{E}-02$ & 831 & -1838 & 71 & -12 & -53 & 160 & -170 & $\$$ Elem & 10 & RZ & $3 \mathrm{~A}$ & $\mathrm{AZ}$ \\
\hline 209 & 2209 & 7.8493 & 831 & -1838 & 71 & -12 & -53 & 170 & -180 & $\$ E l e m$ & 10 & RZ & $3 \mathrm{~A}$ & Az \\
\hline 210 & 2210 & $7.852753 \mathrm{E}-02$ & 831 & -1838 & 71 & -12 & -53 & 180 & -200 & $\$ E l e m$ & 10 & RZ & $3 \mathrm{~A}$ & AZ \\
\hline 211 & 211 & $7.969562 \mathrm{E}-02$ & 821 & -1828 & 28 & -69 & -13 & 100 & -110 & $\$$ Elem & 11 & $\mathrm{RZ}$ & & $\mathrm{Az}$ \\
\hline 212 & 212 & $7.966863 \mathrm{E}$ & 821 & -1828 & 28 & -69 & -13 & 110 & -120 & $\$ E l e m$ & 11 & RZ & A & $A Z$ \\
\hline 213 & 2213 & $7.964972 \mathrm{E}-02$ & 821 & -1828 & 28 & -69 & -13 & 120 & -130 & $\$ E l e m$ & 11 & RZ & $1 \mathrm{~A}$ & $\mathrm{AZ}$ \\
\hline 214 & 2214 & 7.964 & 821 & -1828 & 28 & -69 & -13 & 130 & -160 & $\$ E l e m$ & 11 & RZ & A & $\mathrm{AZ}$ \\
\hline 15 & 2215 & 7.9 & 821 & -1828 & 28 & -69 & -13 & 160 & -170 & $\$$ Elem & 11 & RZ & $1 \mathrm{~A}$ & $\mathrm{AZ}$ \\
\hline 216 & 2216 & $7.967413 \mathrm{E}-02$ & 821 & -1828 & 28 & -69 & -13 & 170 & -180 & $\$ E l e m$ & 11 & RZ & $1 \mathrm{~A}$ & $\mathrm{AZ}$ \\
\hline 217 & 2217 & $7.971054 \mathrm{E}-02$ & 821 & -1828 & 28 & -69 & -13 & 180 & -200 & $\$$ Elem & 11 & RZ & $1 \mathrm{~A}$ & A \\
\hline 218 & 2218 & $8.290397 \mathrm{E}-02$ & 1829 & -1830 & 28 & -69 & -13 & 100 & -110 & $\$ E l e m$ & 11 & RZ & $2 A$ & AZ \\
\hline 219 & 2219 & & 1829 & -1830 & 28 & -69 & -13 & 110 & -120 & $\$ E l e m$ & 11 & RZ & $2 A$ & AZ \\
\hline 20 & 2220 & 8. & 29 & -1 & 28 & -69 & -13 & 120 & -130 & $\$ E l e m$ & 11 & RZ & $2 \mathrm{~A}$ & AZ \\
\hline 221 & 2221 & 8.2844 & 829 & -1830 & 28 & -69 & -13 & 130 & -160 & \$Elem & 11 & RZ & $2 \mathrm{~A}$ & AZ \\
\hline 222 & 2222 & $8.285798 \mathrm{E}-02$ & 829 & -1830 & 28 & -69 & -13 & 160 & -170 & $\$$ Elem & 11 & RZ & $2 \mathrm{~A}$ & $\mathrm{Az}$ \\
\hline 223 & 2223 & $8.288167 \mathrm{E}-02$ & 1829 & -1830 & 28 & -69 & -13 & 170 & -180 & $\$ E l e m$ & 11 & RZ & $2 \mathrm{~A}$ & Az \\
\hline 224 & 2224 & & 1829 & -1830 & 28 & -69 & -13 & 180 & -200 & $\$ E l e m$ & 11 & RZ & $2 \mathrm{~A}$ & Az \\
\hline 25 & 2225 & $7.8 .8-3$. & 31 & -1 & 28 & -69 & -13 & 100 & -110 & $\$ E l e m$ & 11 & RZ & $3 \mathrm{~A}$ & $\mathrm{Az}$ \\
\hline 226 & 2226 & 7.85 & 331 & -1838 & 28 & -69 & -13 & 110 & -120 & \$Elem & 11 & RZ & $3 \mathrm{~A}$ & AZ \\
\hline 227 & 2227 & 7.849169 & 831 & -1838 & 28 & -69 & -13 & 20 & -130 & $\$$ Elem & 11 & RZ & $3 A$ & Az \\
\hline 228 & 2228 & $7.848465 \mathrm{E}-02$ & 1831 & -1838 & 28 & -69 & -13 & 130 & -160 & $\$ E l e m$ & 11 & RZ & $3 \mathrm{~A}$ & A \\
\hline 229 & 2229 & & 1 & - & 28 & -69 & -13 & 50 & -170 & $\$ E l e m$ & 11 & RZ & $3 \mathrm{~A}$ & AZ \\
\hline 30 & 2230 & 78 & 831 & -1 & 28 & -69 & -13 & 170 & -180 & em & 11 & RZ & $3 \mathrm{~A}$ & $\mathrm{AZ}$ \\
\hline 31 & 2231 & 7.8 & 831 & -1838 & 28 & -69 & -13 & 180 & -200 & \$Elem & 11 & RZ & $3 \mathrm{~A}$ & AZ \\
\hline 232 & 2232 & 7.975780 & 821 & -1828 & -52 & -26 & -70 & 100 & -110 & $\$$ Elem & 12 & $\mathrm{RZ}$ & $1 \mathrm{~A}$ & Az \\
\hline 60233 & 2233 & $7.975780 \mathrm{E}-02$ & 1821 & -1828 & 52 & -26 & -70 & 110 & -120 & $\$ E l e m$ & 12 & RZ & $1 \mathrm{~A}$ & AZ \\
\hline 60234 & 2234 & 7.97578 & 1821 & -1828 & -52 & -26 & -70 & 120 & -130 & $\$$ Elem & 12 & RZ & $1 \mathrm{~A}$ & A \\
\hline 235 & 2235 & 7.97 & 21 & $-1 \varepsilon$ & -52 & -26 & -70 & 130 & -160 & $\$$ Elem & 12 & RZ & $1 \mathrm{~A}$ & $\mathrm{Az}$ \\
\hline & 36 & 7.9 & 21 & $-1 \varepsilon$ & -52 & -26 & -70 & 60 & -170 & $\$ E l e m$ & 12 & RZ & $1 \mathrm{~A}$ & AZ \\
\hline 237 & 2237 & 7.97 & 321 & -1828 & -52 & -26 & -70 & 70 & -180 & $\$ \mathrm{Elem}$ & 12 & $\mathrm{RZ}$ & & \\
\hline 60238 & 2238 & $7.975780 \mathrm{E}-02$ & 1821 & -1828 & -52 & -26 & -70 & 180 & -200 & $\$ E l e m$ & 12 & RZ & A & Az \\
\hline 60239 & 2239 & $8.294866 \mathrm{E}-02$ & 1829 & -1830 & -52 & -26 & -70 & 100 & -110 & $\$$ Elem & 12 & RZ & $2 A$ & A \\
\hline 60240 & 2240 & 8.2948 & 1829 & -1830 & -52 & -26 & -70 & 110 & -120 & $\$ E l e m$ & 12 & RZ & $2 \mathrm{~A}$ & Az \\
\hline 60241 & 2241 & -02 & 829 & -1830 & -52 & -26 & -70 & 120 & -130 & $\$ E l e m$ & 12 & RZ & A & $\mathrm{AZ}$ \\
\hline 242 & 2242 & & 829 & -1830 & -52 & 26 & -70 & 130 & -160 & $\$$ Elem & 12 & RZ & & $\mathrm{A}$ \\
\hline 60243 & 2243 & 82 & 1829 & -1830 & -52 & 6 & -70 & 160 & -170 & $\$ E l e m$ & 12 & RZ & 27 & Az \\
\hline 60244 & 2244 & $8.294866 \mathrm{E}-02$ & 1829 & -1830 & -52 & -26 & -70 & 170 & -180 & $\$ E l e m$ & 12 & RZ & 27 & $\mathrm{AZ}$ \\
\hline 60245 & 2245 & $8.294866 \mathrm{E}-02$ & 1829 & -1830 & -52 & -26 & -70 & 180 & -200 & $\$ E l e m$ & 12 & RZ & $2 F$ & AZ \\
\hline 60246 & 2246 & $7.87 \mathrm{C}$ & 1831 & -1838 & -52 & -26 & -70 & 100 & -110 & $\$ E l e m$ & 12 & RZ & F & Az \\
\hline 60247 & 2247 & $7.870346 \mathrm{E}-02$ & 1831 & -1838 & -52 & 2r & -70 & 110 & -120 & $\$$ Elem & 12 & RZ & & \\
\hline & & ת ת & & 1838 & -52 & & -70 & 120 & -130 & $\$$ Elem & 12 & RZ & 3 & \\
\hline 60249 & 2249 & $7.870346 \mathrm{E}-02$ & 1831 & -1838 & -52 & -26 & -70 & 130 & -160 & $\$ E l e m$ & 12 & RZ & $3 t$ & A \\
\hline 60250 & 2250 & $7.870346 \mathrm{E}-02$ & 1831 & -1838 & -52 & -26 & -70 & 160 & -170 & $\$ E l e m$ & 12 & RZ & J & A \\
\hline & 2251 & 7.87 & 831 & -1838 & -52 & -26 & -70 & 170 & -180 & $\$$ Elem & 12 & RZ & 3 & A \\
\hline 204 & 52 & $7.870346 \mathrm{E}-02$ & 831 & -1838 & -52 & -26 & -70 & 180 & -200 & $\$ E l e m$ & 12 & RZ & & \\
\hline
\end{tabular}




\begin{tabular}{|c|c|c|c|c|c|c|c|c|c|c|c|c|c|c|}
\hline 253 & 253 & -02 & 1721 & -1728 & 25 & 54 & 10 & 100 & -110 & $\$ E l e m$ & 13 & RZ & $1 A$ & AZ \\
\hline 54 & 254 & $.975780 \mathrm{E}-02$ & 1721 & -1728 & 25 & 54 & 10 & 110 & -120 & $\$ E l e m$ & 13 & RZ & $1 \mathrm{~F}$ & AZ \\
\hline & 255 & $.975780 \mathrm{E}-02$ & 1721 & 1728 & 25 & 54 & 10 & 120 & -130 & $\$$ Elem & 13 & RZ & 1 & Az \\
\hline 56 & 256 & $.975780 \mathrm{E}-02$ & 1721 & -1728 & 5 & t & 10 & 130 & -160 & $\$ E l e m$ & 13 & RZ & 1 & $\mathrm{AZ}$ \\
\hline 7 & 57 & $75780 \mathrm{E}-02$ & 121 & & D & 52 & 10 & 160 & -170 & $\$ E l e m$ & 13 & RZ & 1 & $\mathrm{z}$ \\
\hline & 58 & $.975780 \mathrm{E}-02$ & 721 & 1728 & 25 & 54 & 10 & 70 & -180 & Elem & 13 & RZ & 2 & $\mathrm{AZ}$ \\
\hline & 59 & $.975780 \mathrm{E}-02$ & 721 & 1728 & 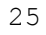 & 52 & 10 & 180 & -200 & $\$$ Elem & 13 & RZ & & AZ \\
\hline & 60 & $.294866 \mathrm{E}-02$ & 729 & -1730 & 25 & 54 & 10 & 100 & -110 & $\$ E l e m$ & 13 & RZ & 2 & AZ \\
\hline 261 & 261 & $8.294866 \mathrm{E}-02$ & 1729 & -1730 & 25 & 54 & 10 & 110 & -120 & $\$$ Elem & 13 & RZ & 2 & $\mathrm{AZ}$ \\
\hline 26 & 262 & $866 \mathrm{E}-02$ & 729 & & 25 & & 10 & 120 & & $\$ E l e m$ & 13 & RZ & & $\pi$ \\
\hline 3 & 203 & $294866 \mathrm{E}-02$ & 129 & & 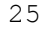 & 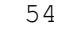 & 10 & 130 & -160 & $\$$ Elem & 13 & RZ & ${ }^{5}$ & 14 \\
\hline & 64 & $.294866 \mathrm{E}-02$ & 729 & 1730 & 5 & 54 & 10 & 160 & -170 & Elem & 13 & RZ & & Az \\
\hline & 265 & $8.294866 \mathrm{E}-02$ & 729 & -1730 & 25 & 54 & 10 & 170 & -180 & $\$$ Elem & 13 & RZ & & AZ \\
\hline 6 & 266 & $.294866 \mathrm{E}-02$ & 729 & -1730 & 25 & 52 & 10 & 180 & -200 & $\$$ Elem & 13 & RZ & & AZ \\
\hline 267 & 267 & -02 & 731 & & 5 & 5 & 10 & 100 & -110 & $\$ E l e m$ & 13 & RZ & & 7 \\
\hline 8 & 268 & -02 & 131 & -1 & 25 & 54 & 10 & 110 & -120 & $\$$ Elem & 13 & RZ & 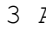 & A2 \\
\hline & 69 & 02 & 731 & 至 & 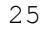 & 5 & 10 & 120 & -130 & $\$$ Elem & 13 & RZ & $F$ & $\mathrm{AZ}$ \\
\hline & 270 & $.870346 \mathrm{E}-02$ & 731 & -1738 & 25 & 54 & 10 & 130 & -160 & $\$$ Elem & 13 & RZ & t & AZ \\
\hline 271 & 271 & $7.870346 \mathrm{E}-02$ & 731 & -173 & 25 & 52 & 10 & 160 & -170 & $\$$ Elem & 13 & RZ & & AZ \\
\hline 272 & 272 & 02 & 731 & & 5 & & 10 & 170 & -180 & $\$$ Elem & 13 & RZ & & AZ \\
\hline 273 & 273 & 02 & 731 & -17 & 25 & 5 & 10 & 180 & -200 & $\$$ Elem & 13 & RZ & t & Az \\
\hline 74 & 274 & $80 \mathrm{E}-02$ & 1721 & -1 & 68 & -23 & 53 & 100 & -110 & $\$$ Elem & 14 & RZ & $1 F$ & $\mathrm{AZ}$ \\
\hline 0275 & 275 & $780 \mathrm{E}-02$ & 1721 & -172 & 8 & & 53 & 110 & -120 & $\$$ Elem & 14 & RZ & & AZ \\
\hline 76 & 276 & -02 & 721 & -1 & & & 53 & 120 & -130 & $\$ E l e m$ & 14 & RZ & 2 & AZ \\
\hline & 277 & & 721 & & 8 & 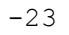 & 53 & 130 & -160 & $\$ E l e m$ & 14 & RZ & 1 & AZ \\
\hline 278 & 278 & 02 & 1721 & -1 & 68 & 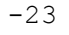 & 53 & 60 & -170 & $\$$ Elem & 14 & RZ & $t$ & Az \\
\hline 60279 & 279 & -02 & 1721 & -1 & 68 & -23 & 53 & 170 & -180 & $\$$ Elem & 14 & RZ & t & AZ \\
\hline 60280 & 280 & 7.97 & 1721 & -172 & 68 & 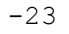 & 53 & 180 & -200 & $\$ E l e m$ & 14 & RZ & $F$ & AZ \\
\hline 60281 & 281 & 8.2 & 729 & & & 2 & 53 & 100 & -110 & $\$ E l e m$ & 14 & RZ & $2 F$ & AZ \\
\hline & 282 & & 1729 & & 8 & -2 & 3 & 110 & & em & 14 & RZ & 2 & AZ \\
\hline 83 & 283 & 02 & 1729 & & 8 & $-2-2$ & 53 & 120 & -130 & $\$ E l e m$ & 14 & RZ & 27 & Az \\
\hline 60284 & 284 & -02 & 1729 & -1 & 68 & -23 & 53 & 130 & -160 & $\$$ Elem & 14 & RZ & 27 & AZ \\
\hline 60285 & 285 & $866 \mathrm{E}-02$ & 1729 & -17 & 68 & -23 & 53 & 160 & -170 & $\$$ Elem & 14 & RZ & $t$ & AZ \\
\hline 60286 & 286 & -02 & 1729 & -1 & 68 & -2 & 53 & 170 & -180 & $\$ E l e m$ & 14 & RZ & $t$ & AZ \\
\hline & 287 & & 1729 & & 8 & -2 & 53 & 30 & & em & 14 & RZ & 25 & AZ \\
\hline 6 & 288 & 02 & 1731 & & 58 & -23 & 3 & 0 & -110 & 1 em & 14 & RZ & 37 & AZ \\
\hline 89 & 289 & 02 & 1731 & -1 & 58 & -2 & 53 & 110 & -120 & \$Elem & 14 & RZ & $3 z$ & Az \\
\hline 60290 & 290 & -02 & 1731 & -1 & 68 & -23 & 53 & 120 & -130 & $\$ E l e m$ & 14 & RZ & t & AZ \\
\hline 60291 & 291 & -02 & 1731 & -1 & 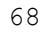 & -23 & 53 & 130 & -160 & $\$ E l e m$ & 14 & RZ & $3 z$ & AZ \\
\hline 60292 & 292 & 02 & 1731 & -1 & 68 & -23 & 53 & 160 & -170 & 1 em & 14 & RZ & $3 t$ & Az \\
\hline & 3 & & 1731 & & 8 & -23 & 53 & 10 & & $m$ & 14 & RZ & $t$ & AZ \\
\hline & 294 & 2 & 17 & & 8 & - & 53 & 30 & 0 & em & 14 & RZ & $3 z$ & Az \\
\hline 95 & 295 & 02 & 1721 & -1 & 11 & -66 & 24 & 100 & -110 & $\$ E l e m$ & 15 & RZ & 17 & Az \\
\hline 60296 & 96 & -02 & 1721 & -17 & 11 & 6 & -24 & 110 & -120 & $\$ E l e m$ & 15 & RZ & F & AZ \\
\hline 60297 & 297 & 02 & 1721 & -1 & 5 & -66 & -24 & 120 & -130 & $\$ E l e m$ & 15 & RZ & 1 & $\mathrm{AZ}$ \\
\hline & 8 & & & & 1 & -66 & -24 & 30 & & $m$ & 15 & RZ & 2 & A2 \\
\hline & 299 & 2 & 17 & & 11 & & -24 & 50 & & em & 15 & RZ & 1 & Az \\
\hline & 300 & 22 & 1721 & -1 & 1 & -66 & -24 & 170 & -180 & $\$ E l e m$ & 15 & RZ & 12 & $\mathrm{AZ}$ \\
\hline 60301 & 301 & $780 \mathrm{E}$ & 1721 & -17 & 11 & -66 & -24 & 180 & -200 & $\$ E l e m$ & 15 & RZ & E & AZ \\
\hline 02 & 302 & 02 & 1729 & -17 & 11 & -66 & -24 & 100 & -110 & $\$$ Elem & 15 & RZ & 27 & Az \\
\hline & 303 & 02 & 1729 & -1 & 11 & -66 & -24 & 110 & 20 & $\$$ Elem & 15 & RZ & $F$ & $\mathrm{AZ}$ \\
\hline & 304 & 02 & 1729 & & 11 & 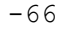 & -24 & 120 & & $\$ E l e m$ & 15 & RZ & t & Az \\
\hline & 305 & -02 & 1729 & & 11 & 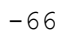 & -24 & 130 & -160 & $\$ E l e m$ & 15 & RZ & $2 z$ & $\mathrm{Az}$ \\
\hline & 306 & $8.294866 \mathrm{E}-02$ & 1729 & -1 & 11 & -66 & -24 & 160 & -170 & $\$ E l e m$ & 15 & RZ & $2 z$ & AZ \\
\hline 07 & 307 & 22 & 1729 & -1 & 11 & $-\epsilon$ & -24 & 170 & -180 & $\$$ Elem & 15 & RZ & E & AZ \\
\hline & 2308 & $866 \mathrm{E}$ & 1729 & -17 & 11 & -66 & -24 & 180 & -200 & $\$$ Elem & 15 & RZ & 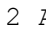 & AZ \\
\hline & 2309 & $46 \mathrm{E}-02$ & 1731 & & 11 & -6 & -24 & 100 & -110 & $\$ E l e m$ & 15 & RZ & & Az \\
\hline 60310 & 310 & $6 \mathrm{E}-02$ & 1731 & & 11 & -6 & -24 & 110 & -120 & $\$ E l e m$ & 15 & RZ & & AZ \\
\hline & 11 & $46 \mathrm{~F}-02$ & 1731 & -1738 & 11 & -6 & -24 & 120 & -130 & $\$ E l e m$ & 15 & RZ & & AZ \\
\hline & $B 12$ & $.870346 \mathrm{E}-02$ & 1731 & -1 & 11 & -6 & -24 & 130 & -160 & $\$$ Elem & 15 & RZ & & AZ \\
\hline & & .8703461 & 1731 & & 11 & -6 & -24 & 160 & 70 & $\$ E l e m$ & 15 & RZ & & Az \\
\hline & & & 173 & & 11 & -6 & -24 & 170 & & $\$ E l e m$ & 13 & RZ & & AZ \\
\hline & & $7.870346 \mathrm{E}-02$ & 1731 & -1738 & 11 & -66 & -24 & 180 & -200 & \$Elem & I & RZ & & \\
\hline
\end{tabular}

c

c

c

NE LOBE

$\begin{array}{lllll}60901 & 029 & 7.21241-2 & 1111 & -1128\end{array}$ $60902 \quad 0101.00276-1 \quad 1118-1119$ $60903 \quad 0101.00276-1 \quad 1120-1121$ $\begin{array}{lllll}60904 & 029 & 7.21241-2 & 1111 & -1128\end{array}$

$\begin{array}{llllllll}53 & -54 & -73 & 100 & -200 & \$ \# 8 & \text { side plate } \\ 54 & -15 & -73 & 100 & -200 & \$ \# 8 & \text { H2O } & 4 \text { \& } 5 \\ 54 & -15 & -73 & 100 & -200 & \$ \# 8 & \text { H2O } 15 \text { \& } 16 \\ 15 & -17 & -30 & 100 & -200 & \$ \# 8,7 \text { side plate }\end{array}$ 


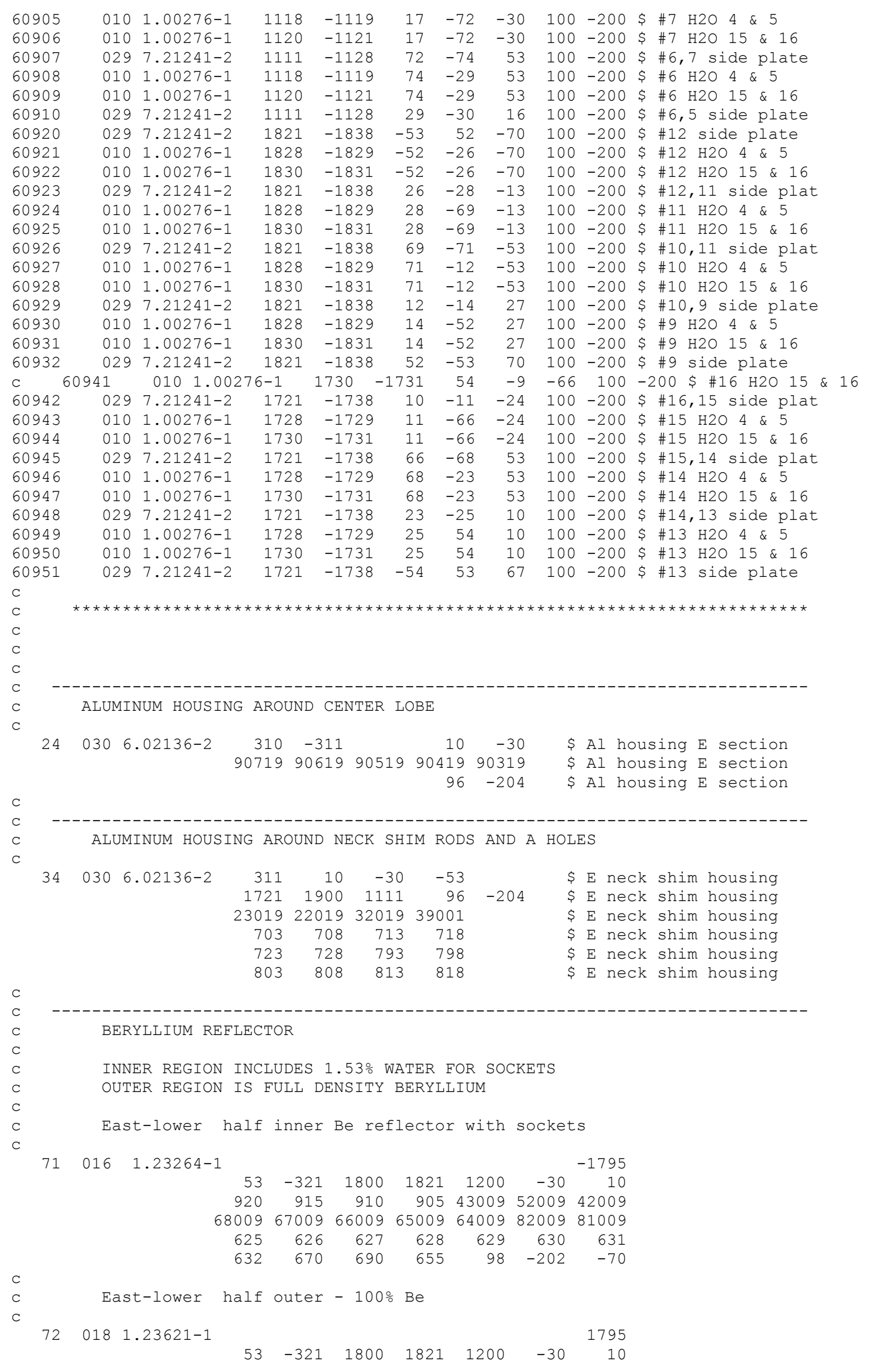


$\mathrm{C}$

C

C

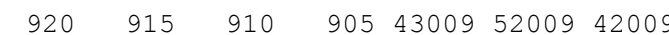

68009670096600965009640098200981009

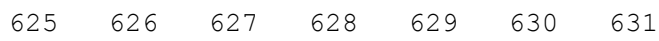

$\begin{array}{lllllll}632 & 670 & 690 & 655 & 98 & -202 & -70\end{array}$

$73016 \quad 1.23264-1$

East-upper half inner Be reflector with sockets

$\begin{array}{rrrrrrrr} & 53 & -321 & 1800 & 1821 & 1200 & -30 & 10\end{array}$

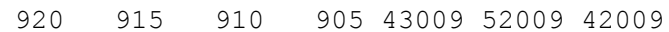

68009670096600965009640098200981009

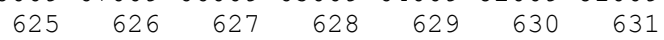

$\begin{array}{lllllll}632 & 670 & 690 & 655 & 98 & -202 & 70\end{array}$

$740181.23621-1$

East-upper half outer - $100 \% \mathrm{Be}$

$\begin{array}{rrrrrrrr}53 & -321 & 1800 & 1821 & 1200 & -30 & 10\end{array}$

$\begin{array}{lllllll}920 & 915 & 910 & 905 & 43009 & 52009 & 42009\end{array}$

68009670096600965009640098200981009

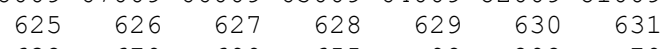

C

$\begin{array}{lllllll}632 & 670 & 690 & 655 & 98 & -202 & 70\end{array}$

C

REGIONS OUTSIDE BE REFLECTOR

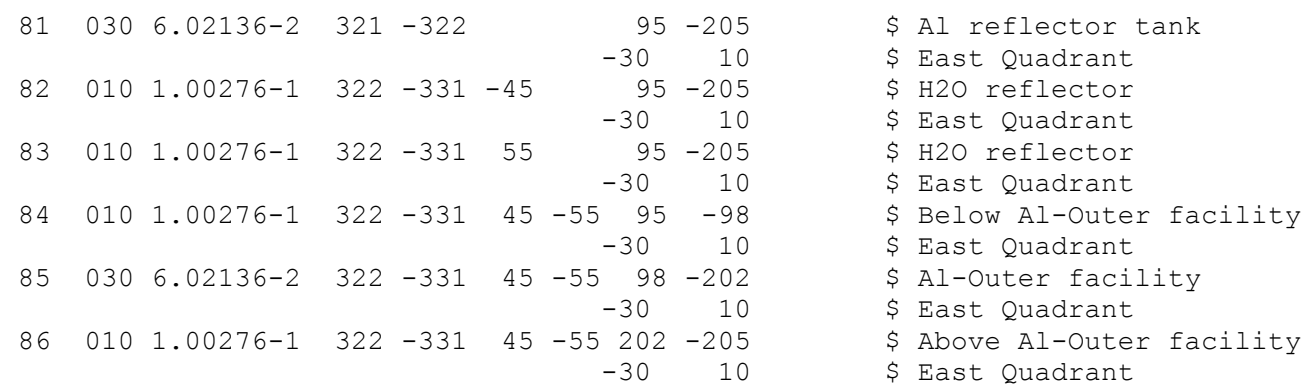

C

C

WATER AND ALUMINUM BELOW FUEL MEAT (1.905 CM)

$\begin{array}{lllllllll}101 & 031 & 8.4631-2 & 1111 & -1200 & 53 & 1821 & 99 & -100\end{array}$ Elem 8,7,6,5

$\begin{array}{llll}-30 & 10 & \text { \$ East Quadrant }\end{array}$

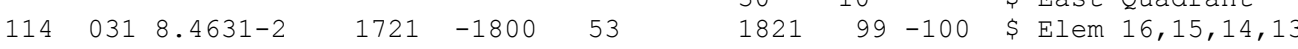

$-30 \quad 10 \quad$ \$ East Quadrant

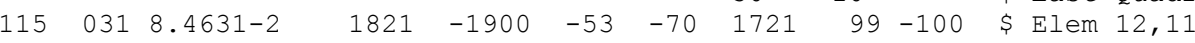

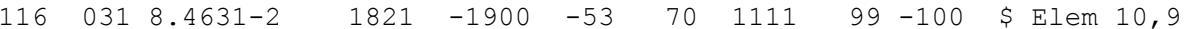

$\mathrm{C}$

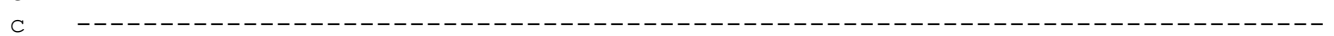

C WATER AND ALUMinUM BELOW FUEL PLATES

$121033 \quad 9.3665-2 \quad 1111-1200 \quad 53 \quad 1821 \quad 97 \quad-99 \quad \$ \quad E l e m \quad 8,7,6,5$

$\begin{array}{llll}-30 & 10 & \text { \$ East Quadrant }\end{array}$

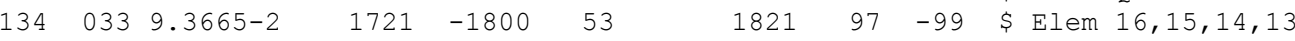

$\begin{array}{llllllllll} & 033 & 9.3665-2 & 1821 & -1900 & -53 & -70 & -30 & 10 & 97\end{array}$

$\begin{array}{lllllllllll}136 & 033 & 9.3665-2 & 1821 & -1900 & -53 & 70 & 1111 & 97 & -99 & \text { Elem } 10,9\end{array}$

C

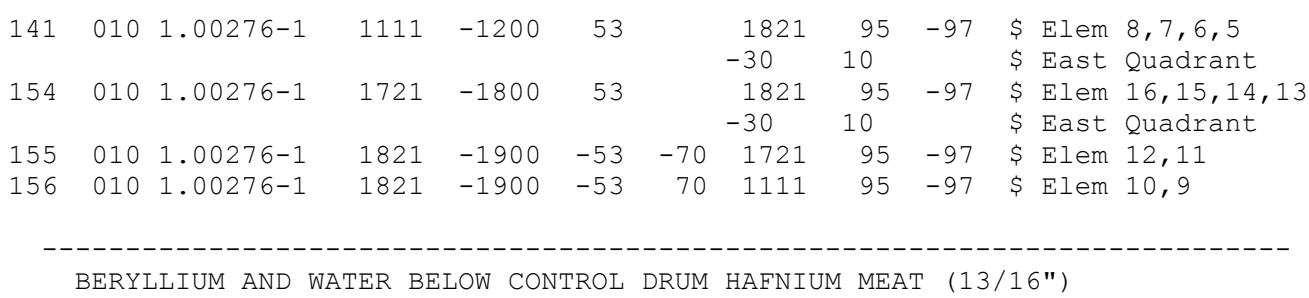


C

$\begin{array}{llllll}173 & 19 & 1.21987-1 & -920 & 102-101 & \text { E4 } \\ 174 & 19 & 1.21987-1 & -915 & 102-101 & \text { E3 } \\ 175 & 19 & 1.21987-1 & -910 & 102-101 & \text { E2 } \\ 176 & 19 & 1.21987-1 & -905 & 102-101 & \text { E1 }\end{array}$

$\mathrm{C}$

$\mathrm{C}$

C

STAINLESS STEEL, ALUMINUM AND WATER BELOW BERYLLIUM DRUMS (2")

$\begin{array}{lllllll}193 & 22 & 8.7091-2 & -920 & 98-102 & \text { E4 } \\ 194 & 22 & 8.7091-2 & -915 & 98-102 & \text { E3 } \\ 195 & 22 & 8.7091-2 & -910 & 98-102 & \text { E2 } \\ 196 & 22 & 8.7091-2 & -905 & 98-102 & \text { \$ } 1\end{array}$

$\mathrm{C}$

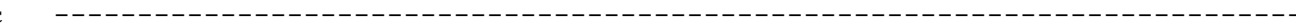

WATER AND ALUMINUM ABOVE FUEL MEAT

$2010318.4631-2 \quad 1111-1200 \quad 53 \quad 1821 \quad 200-201 \quad \$ \quad E l e m 8,7,6,5$

$\begin{array}{lll}-30 & 10 \quad \text { East Quadrant }\end{array}$

$2140318.4631-2 \quad 1721 \quad-1800 \quad 53 \quad 1821 \quad 200-201 \quad$ Elem 16,15,14,13

$\begin{array}{lllllll}-53 & -70 & 1721 & 200 & -201 \quad \$ \text { Elem 12,11 }\end{array}$

$\begin{array}{lllllllllll}216 & 031 & 8.4631-2 & 1821 & -1900 & -53 & 70 & 1111 & 200 & -201 & \text { Elem } 10,9\end{array}$

C

C

WATER AND ALUMINUM ABOVE FUEL PLATES

$\begin{array}{lllllllll}221 & 032 & 9.2652-2 & 1111 & -1200 & 53 & 1821 & 201 & -203\end{array} \quad$ Elem 8,7,6,5

$\begin{array}{llll}-30 & 10 \quad \$ \text { East Quadrant }\end{array}$

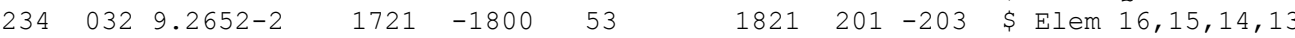

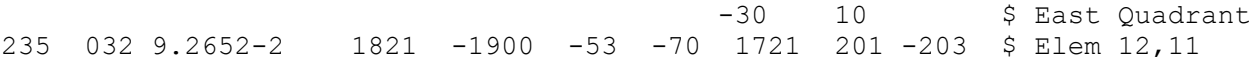

C

$\begin{array}{llllllllll} & 032 & 9.2652-2 & 1821 & -1900 & -53 & 70 & 1111 & 201 & -203\end{array}$ Elem 10,9

WATER ABOVE FUEL ELEMENT

$2410101.00276-1 \quad 1111-1200 \quad 53 \quad 1821 \quad 203-205 \quad \$ \quad E l e m \quad 8,7,6,5$

$\begin{array}{llll}-30 & 10 & -30 & \text { Sast Quadrant }\end{array}$

$2540101.00276-1 \quad 1721-1800 \quad 53 \quad 1821 \quad 203-205 \quad \$ \quad E l e m \quad 16,15,14,13$

$255 \quad 0101.00276-1 \quad 1821 \quad-1900 \quad-53 \quad-70 \quad 1721 \quad 203-205 \quad \$ \quad E l e m \quad 12,11$

$256 \quad 0101.00276-1 \quad 1821 \quad-1900 \quad-53 \quad 70 \quad 1111 \quad 203-205 \quad \$ \quad E l e m \quad 10,9$

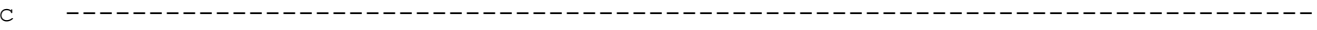

$\begin{array}{llllll}273 & 21 & 9.46985-2 & -920 & 200 & -202\end{array} \mathrm{E4}$

$27421 \quad 9.46985-2 \quad-915 \quad 200-202 \quad$ E3

$\begin{array}{llllll}275 & 21 & 9.46985-2 & -910 & 200 & -202\end{array}$

$\begin{array}{lllll}276 & 21 & 9.46985-2 & -905 & 200-202\end{array}$

C

SS, AL AND WATER ABOVE CONTROL DRUMS

$\begin{array}{llllllll}293 & 23 & 7.6442-2 & -920 & -67 & 202 & -208 & \text { \$ }\end{array}$

$29423 \quad 7.6442-2 \quad-915 \quad 202-208 \quad$ E3

$29523 \quad 7.6442-2 \quad-910 \quad 202-208 \quad \$ \quad E 2$

$\begin{array}{lllllll}296 & 23 & 7.6442-2 & -905 & 73 & 202 & -208\end{array}$

$\mathrm{C}$

C Stainless STEEL, ALUMinum, AND WATER BELOW CONTROL DRUMS

C

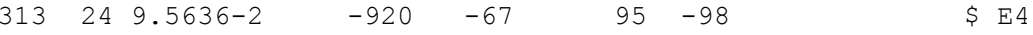

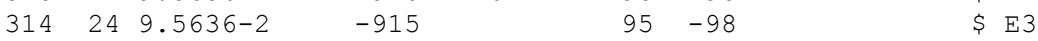

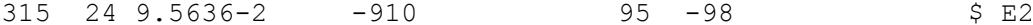

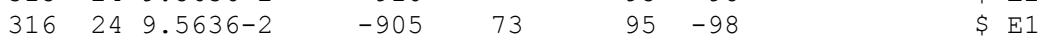

C

ALUMINUM AND WATER BELOW BERYLLIUM REFLECTOR 


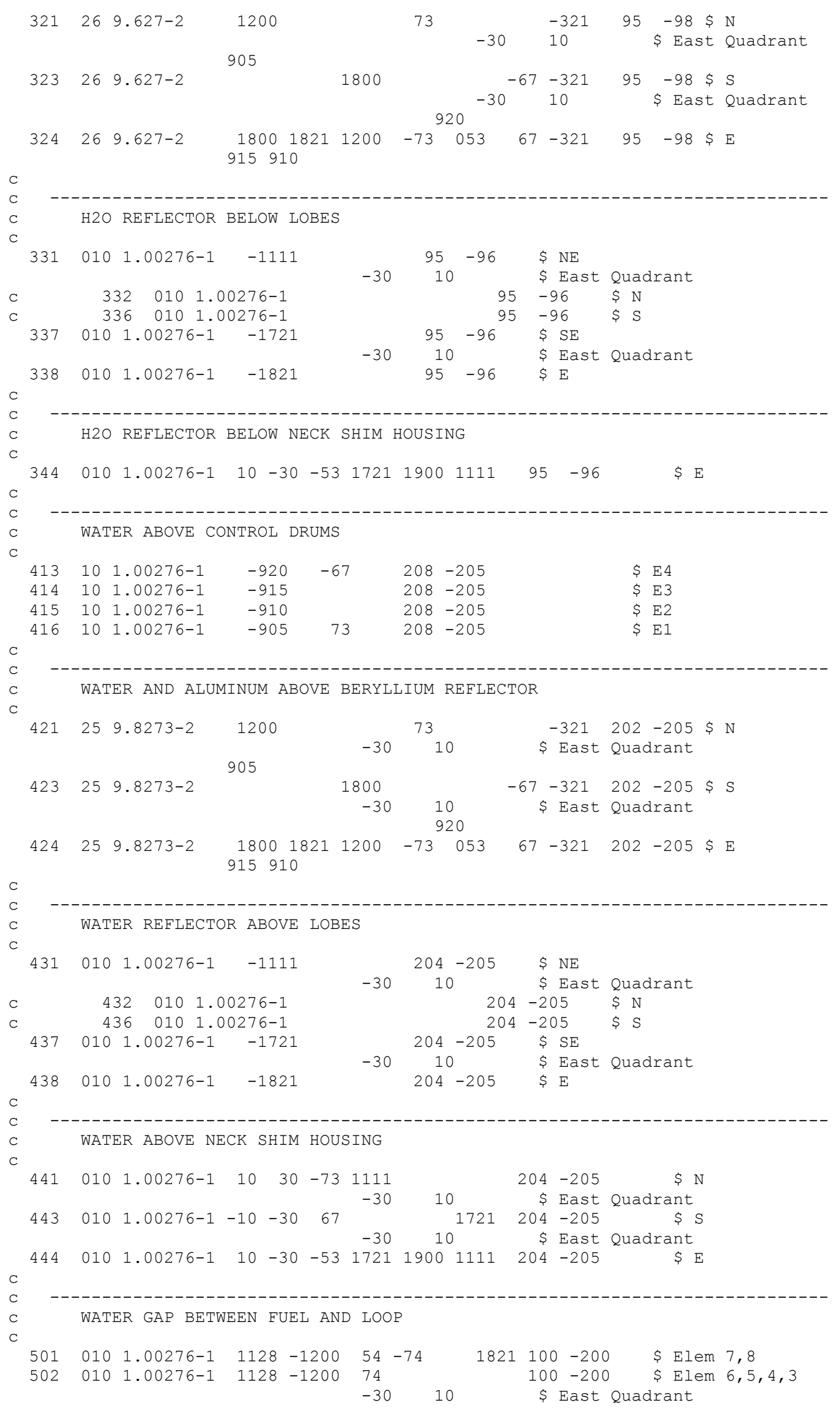




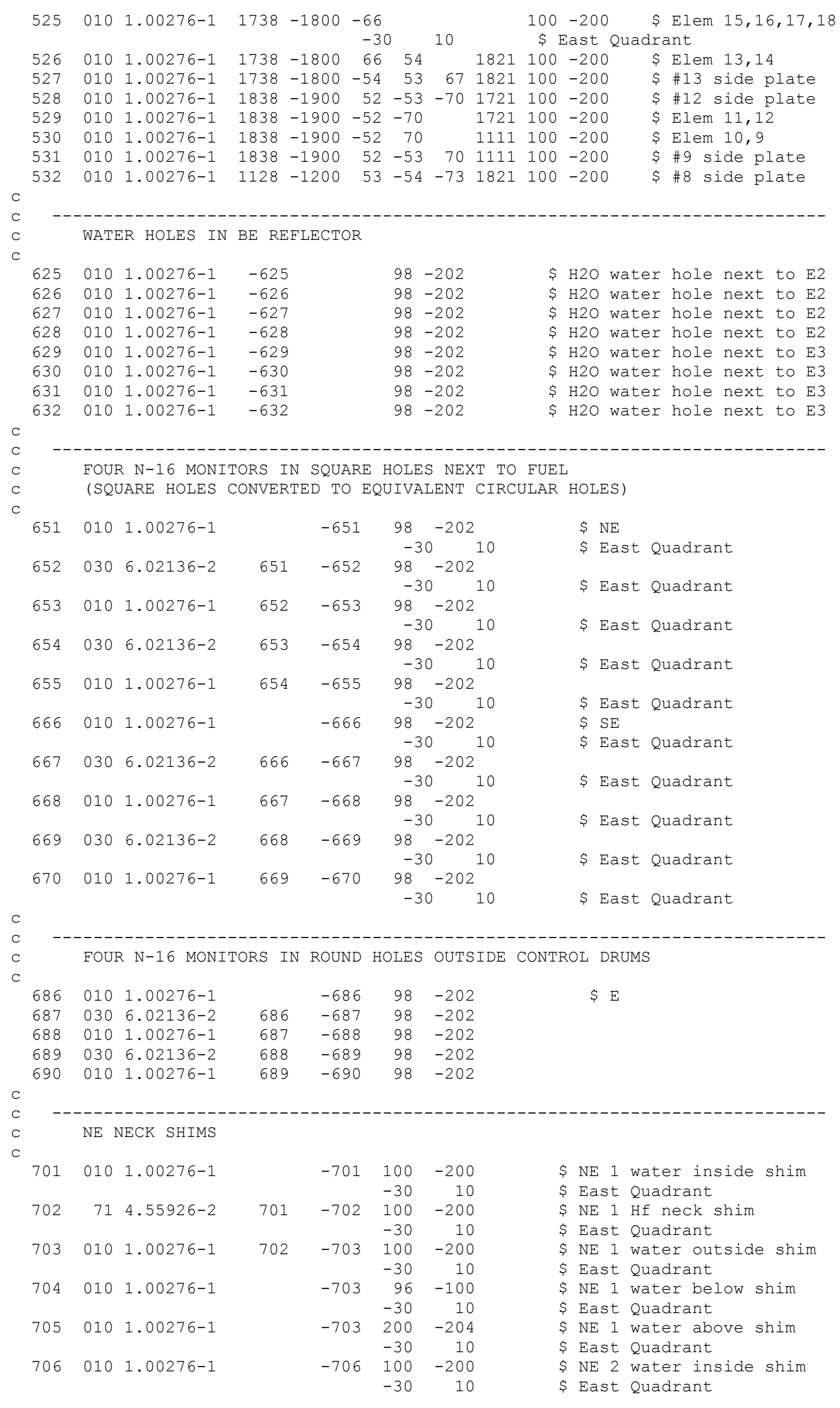




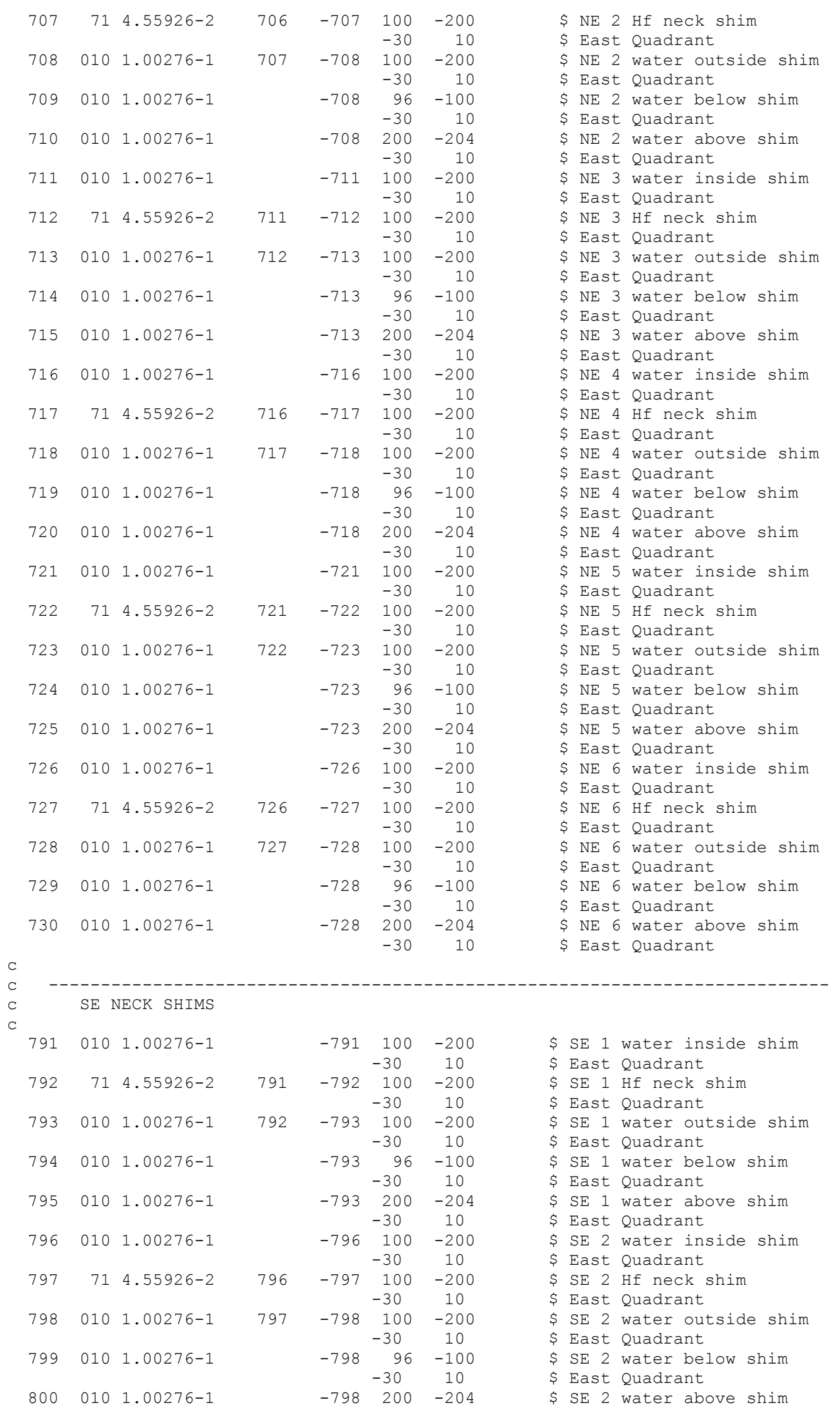




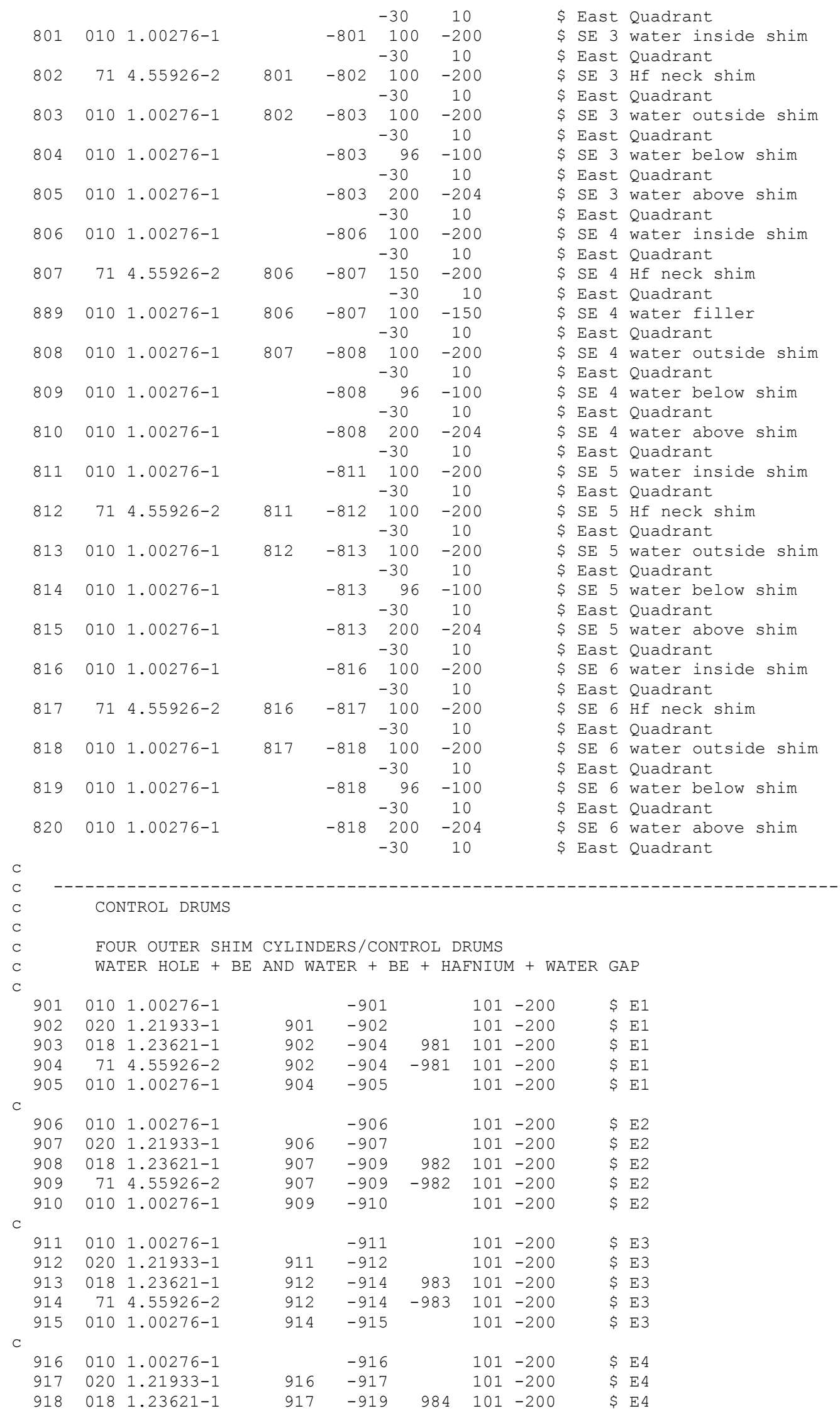




$\begin{array}{rrrrrrrrr}919 & 71 & 4.55926-2 & 917 & -919 & -984 & 101 & -200 & \text { \$ } E 4 \\ 920 & 010 & 1.00276-1 & 919 & -920 & & 101 & -200 & \text { \$ E4 }\end{array}$

C

C

C

C Flux Trap WITH ITV AND THREE DUMMY TEST TRAINS

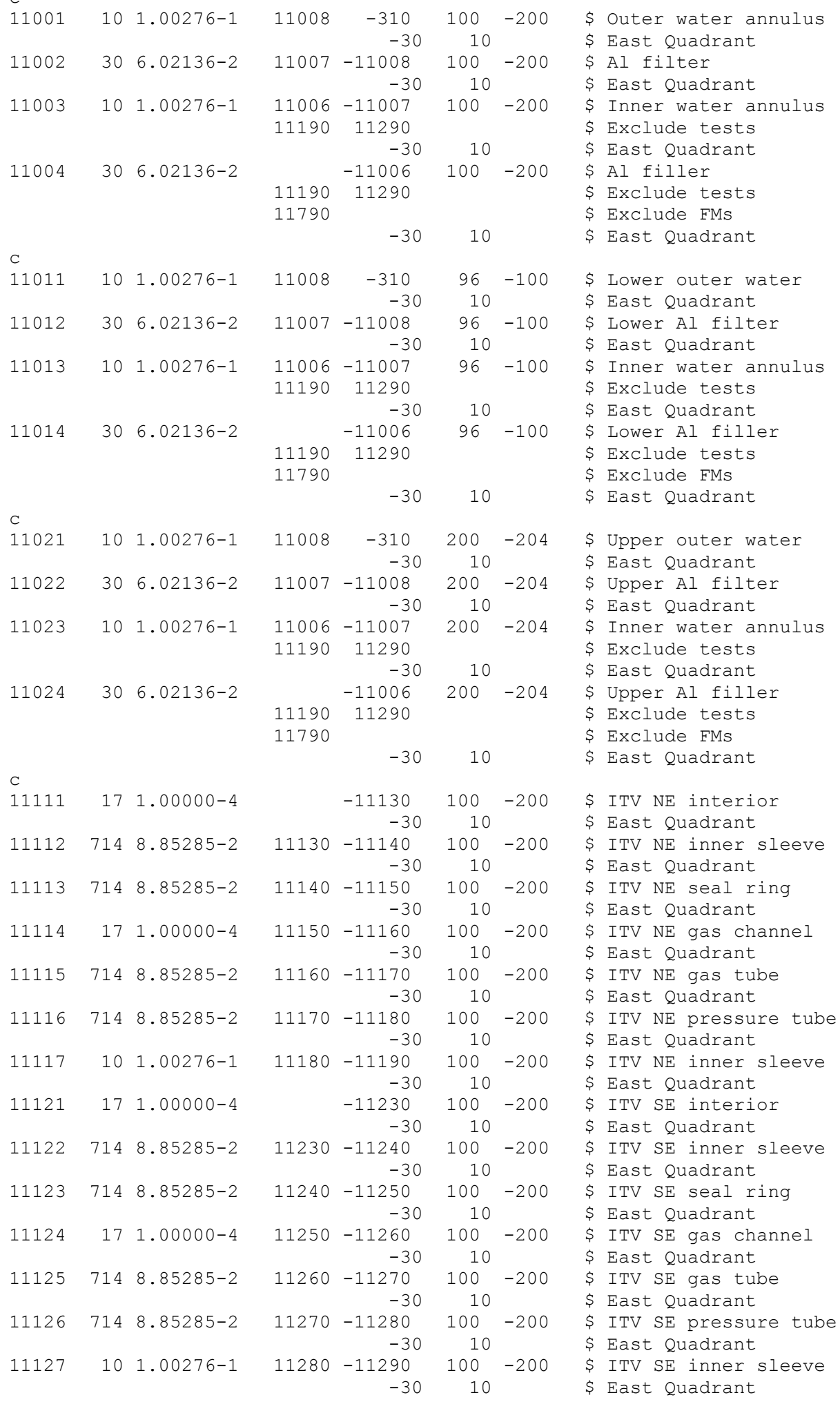




\begin{tabular}{|c|c|c|c|c|c|c|c|}
\hline 11177 & 10 & $1.00276-1$ & & -11770 & 100 & -200 & \$ ITV E FM interior \\
\hline 11178 & 30 & $6.02136-2$ & 11770 & -11780 & 100 & -200 & \$ ITV E FM holder \\
\hline 11179 & 10 & $1.00276-1$ & 11780 & -11790 & 100 & -200 & \$ ITV E FM water \\
\hline \multicolumn{8}{|l|}{ C } \\
\hline \multirow[t]{2}{*}{11211} & 17 & $1.00000-4$ & & -11130 & 96 & -100 & \$ ITV NE interior \\
\hline & & & & -30 & 10 & & \$ East Quadrant \\
\hline \multirow{2}{*}{11212} & 714 & $8.85285-2$ & 11130 & -11140 & 96 & -100 & \$ ITV NE inner sleeve \\
\hline & & & & -30 & 10 & & \$ East Quadrant \\
\hline \multirow{2}{*}{11213} & 714 & $8.85285-2$ & 11140 & -11150 & 96 & -100 & \$ ITV NE seal ring \\
\hline & & & & -30 & 10 & & \$ East Quadrant \\
\hline \multirow{2}{*}{11214} & 17 & $1.00000-4$ & 11150 & -11160 & 96 & -100 & \$ ITV NE gas channel \\
\hline & & & & -30 & 10 & & \$ East Quadrant \\
\hline \multirow{2}{*}{11215} & 714 & $8.85285-2$ & 11160 & -11170 & 96 & -100 & \$ ITV NE gas tube \\
\hline & & & & -30 & 10 & & \$ East Quadrant \\
\hline \multirow{2}{*}{11216} & 714 & $8.85285-2$ & 11170 & -11180 & 96 & -100 & \$ ITV NE pressure tube \\
\hline & & & & -30 & 10 & & \$ East Quadrant \\
\hline \multirow{2}{*}{11217} & 10 & $1.00276-1$ & 11180 & -11190 & 96 & -100 & \$ ITV NE inner sleeve \\
\hline & & & & -30 & 10 & & \$ East Quadrant \\
\hline \multirow{2}{*}{11221} & 17 & $1.00000-4$ & & -11230 & 96 & -100 & \$ ITV SE interior \\
\hline & & & & -30 & 10 & & \$ East Quadrant \\
\hline \multirow{2}{*}{11222} & 714 & $8.85285-2$ & 11230 & -11240 & 96 & -100 & \$ ITV SE inner sleeve \\
\hline & & & & -30 & 10 & & \$ East Quadrant \\
\hline 11223 & 714 & $8.85285-2$ & 11240 & -11250 & 96 & -100 & \$ ITV SE seal ring \\
\hline & & & & -30 & 10 & & \$ East Quadrant \\
\hline 11224 & 17 & $1.00000-4$ & 11250 & -11260 & 96 & -100 & \$ ITV SE gas channel \\
\hline & & & & -30 & 10 & & \$ East Quadrant \\
\hline 11225 & 714 & $8.85285-2$ & 11260 & -11270 & 96 & -100 & \$ ITV SE gas tube \\
\hline & & & & -30 & 10 & & \$ East Quadrant \\
\hline 11226 & 714 & $8.85285-2$ & 11270 & -11280 & 96 & -100 & \$ ITV SE pressure tube \\
\hline & & & & -30 & 10 & & \$ East Quadrant \\
\hline 11227 & 10 & $1.00276-1$ & 11280 & -11290 & 96 & -100 & \$ ITV SE inner sleeve \\
\hline & & & & -30 & 10 & & \$ East Quadrant \\
\hline 11277 & 10 & $1.00276-1$ & & -11770 & 96 & -100 & \$ ITV E FM interior \\
\hline 11278 & 30 & $6.02136-2$ & 11770 & -11780 & 96 & -100 & \$ ITV E FM holder \\
\hline 11279 & 10 & $1.00276-1$ & 11780 & -11790 & 96 & -100 & \$ ITV E FM water \\
\hline C & & & & & & & \\
\hline 11311 & 17 & $1.00000-4$ & & -11130 & 200 & -204 & \$ ITV NE interior \\
\hline & & & & -30 & 10 & & \$ East Quadrant \\
\hline 11312 & 714 & $8.85285-2$ & 11130 & -11140 & 200 & -204 & \$ ITV NE inner sleeve \\
\hline & & & & -30 & 10 & & \$ East Quadrant \\
\hline 11313 & 714 & $8.85285-2$ & 11140 & -11150 & 200 & -204 & \$ ITV NE seal ring \\
\hline & & & & -30 & 10 & & \$ East Quadrant \\
\hline 11314 & 17 & $1.00000-4$ & 11150 & -11160 & 200 & -204 & \$ ITV NE gas channel \\
\hline & & & & -30 & 10 & & \$ East Quadrant \\
\hline 11315 & 714 & $8.85285-2$ & 11160 & -11170 & 200 & -204 & \$ ITV NE gas tube \\
\hline & & & & -30 & 10 & & \$ East Quadrant \\
\hline 11316 & 714 & $8.85285-2$ & 11170 & -11180 & 200 & -204 & \$ ITV NE pressure tube \\
\hline & & & & -30 & 10 & & \$ East Quadrant \\
\hline 11317 & 10 & $1.00276-1$ & 11180 & -11190 & 200 & -204 & \$ ITV NE inner sleeve \\
\hline & & & & -30 & 10 & & \$ East Quadrant \\
\hline 11321 & 17 & $1.00000-4$ & & -11230 & 200 & -204 & \$ ITV SE interior \\
\hline & & & & -30 & 10 & & \$ East Quadrant \\
\hline 11322 & 714 & $8.85285-2$ & 11230 & -11240 & 200 & -204 & \$ ITV SE inner sleeve \\
\hline & & & & -30 & 10 & & \$ East Quadrant \\
\hline 11323 & 714 & $8.85285-2$ & 11240 & -11250 & 200 & -204 & \$ ITV SE seal ring \\
\hline & & & & -30 & 10 & & \$ East Quadrant \\
\hline 11324 & 17 & $1.00000-4$ & 11250 & -11260 & 200 & -204 & \$ ITV SE gas channel \\
\hline & & & & -30 & 10 & & \$ East Quadrant \\
\hline 11325 & 714 & $8.85285-2$ & 11260 & -11270 & 200 & -204 & \$ ITV SE gas tube \\
\hline & & & & -30 & 10 & & \$ East Quadrant \\
\hline 11326 & 714 & $8.85285-2$ & 11270 & -11280 & 200 & -204 & \$ ITV SE pressure tube \\
\hline & & & & -30 & 10 & & \$ East Quadrant \\
\hline 11327 & 10 & $1.00276-1$ & 11280 & -11290 & 200 & -204 & \$ ITV SE inner sleeve \\
\hline & & & & -30 & 10 & & \$ East Quadrant \\
\hline 11377 & 10 & $1.00276-1$ & & -11770 & 200 & -204 & \$ ITV E FM interior \\
\hline 11378 & 30 & $6.02136-2$ & 11770 & -11780 & 200 & -204 & \$ ITV E FM holder \\
\hline 11379 & 10 & $1.00276-1$ & 11780 & -11790 & 200 & -204 & \$ ITV E FM water \\
\hline C & & & & & & & \\
\hline C & & & & & & & \\
\hline & & & & & & & \\
\hline & & & & & & & \\
\hline
\end{tabular}




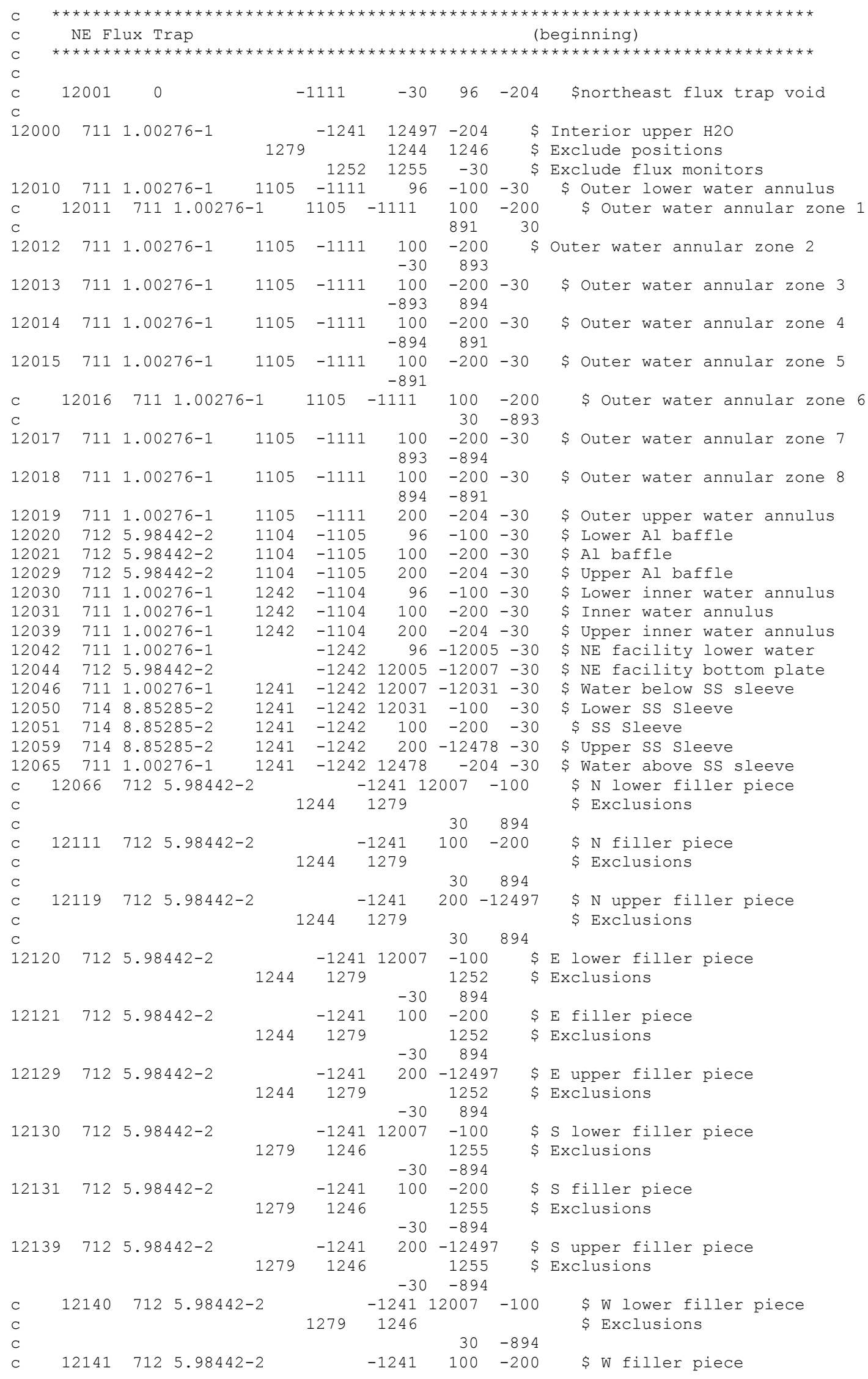




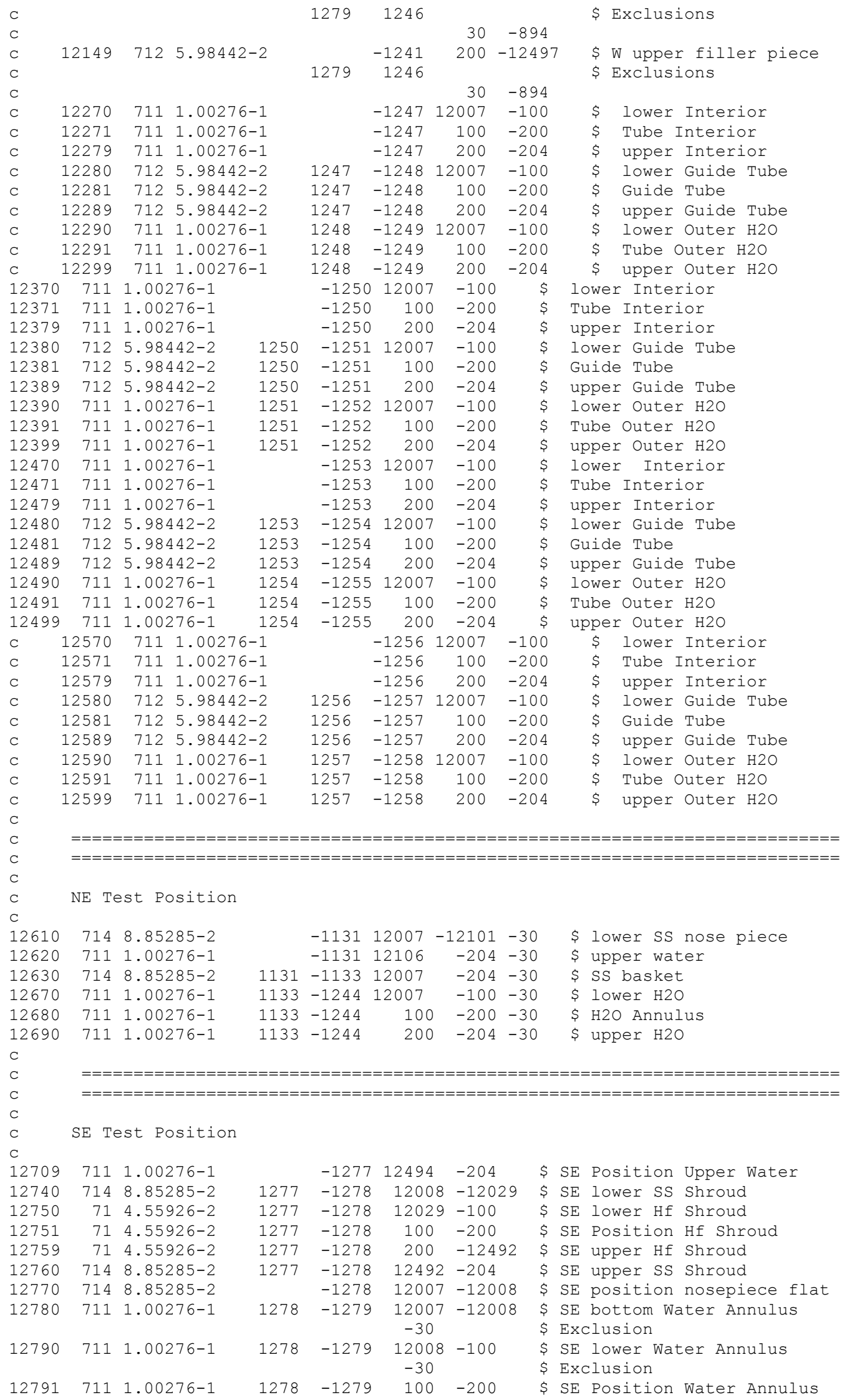




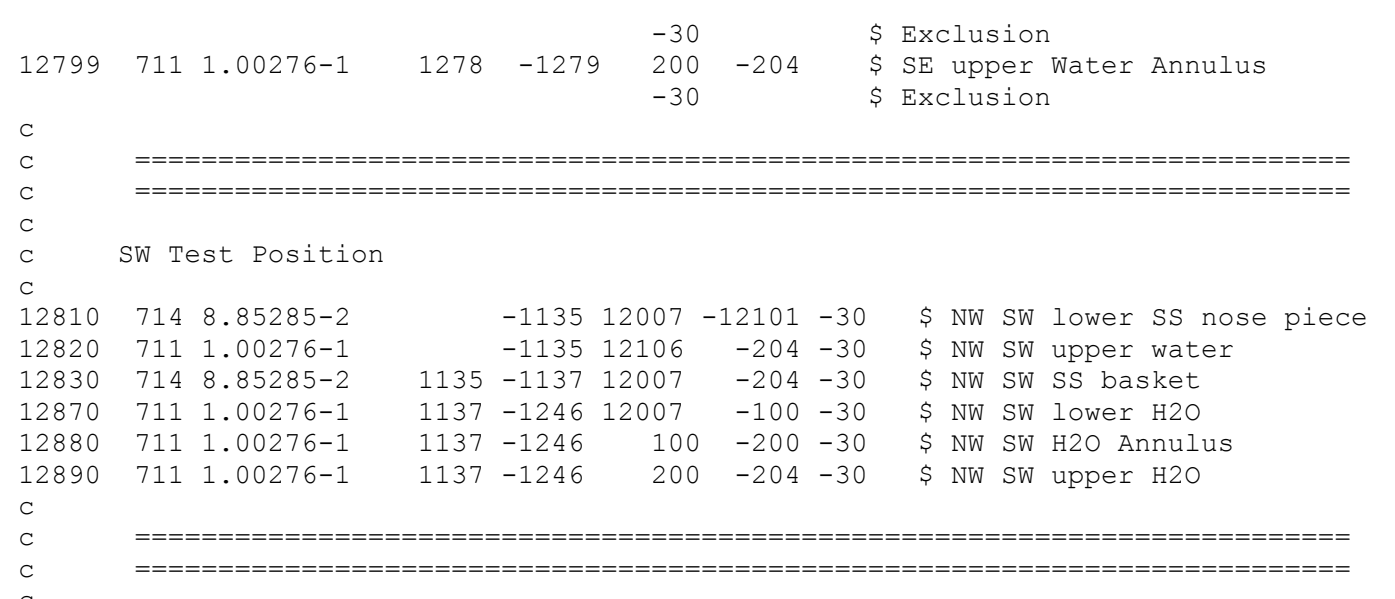

NW Test Position

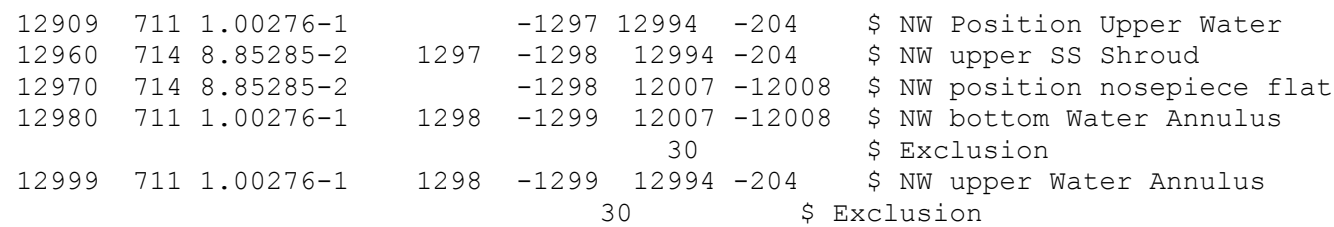

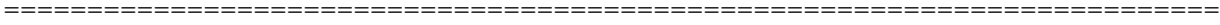

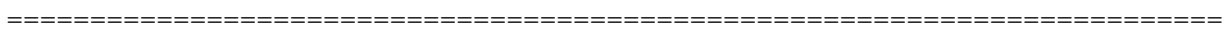

SE Test Position

$120017148.85285-2 \quad-127712008 \quad-2211 \quad$ SE Position lower steel

$120027111.00276-1 \quad-1277 \quad 2221-12494 \quad$ \$ SE Position upper water

$121017327.48430-2 \quad-1277 \quad 2211 \quad-2212 \quad$ \$ SE Test Level 1

$121027327.98326-2 \quad-1277 \quad 2212 \quad-2213 \quad$ \$ SE Test Level 2

$121037327.48430-2 \quad-1277 \quad 2213 \quad-2214 \quad$ \$ SE Test Level 3

$121047327.48430-2 \quad-1277 \quad 2214 \quad-2215 \quad$ \$ SE Test Level 4

$121057327.48430-2 \quad-1277 \quad 2215 \quad-2216 \quad$ SE Test Level 5

$121067327.48430-2 \quad-1277 \quad 2216 \quad-2217 \quad$ \$ SE Test Level 6

$12107 \quad 7327.48430-2 \quad-1277 \quad 2217 \quad-2218 \quad$ \$ SE Test Level 7

$121087327.48430-2 \quad-1277 \quad 2218 \quad-2219 \quad$ \$ SE Test Level 8

$121097327.48430-2 \quad-1277 \quad 2219-2220 \quad$ \$ SE Test Level 9

$121107327.48430-2 \quad-12772220-222 \quad$ \$ SE Test Level 10

C

C

C

(

C

C $\quad 12501714 \quad 8.85285-2$

C $\quad 12502 \quad 711 \quad 1.00276-1$

C $\quad 125107327.48430-2$

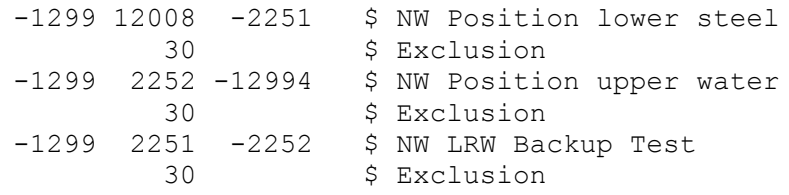

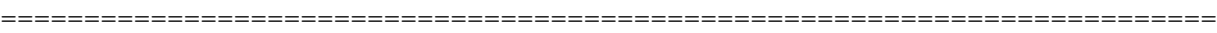

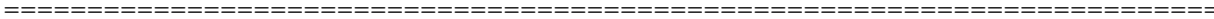

NE Test Position 


\begin{tabular}{|c|c|c|c|c|c|c|}
\hline \multicolumn{7}{|l|}{$C$} \\
\hline 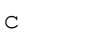 & \multicolumn{6}{|l|}{ SW Test Position } \\
\hline \\
\hline 12801 & $75107.32218-2$ & & -113512101 & -12102 & -30 & \$ SW Test Tier 1 \\
\hline 12802 & $75107.32218-2$ & & -113512102 & -12103 & -30 & \$SW Test Tier 2 \\
\hline 12803 & $75107.32218-2$ & & -113512103 & -12104 & -30 & \$SW Test Tier 3 \\
\hline 12804 & $75107.32218-2$ & & $-1135 \quad 12104$ & -12105 & -30 & \$SW Test Tier 4 \\
\hline \multirow{2}{*}{12805} & $75107.32218-2$ & & -113512105 & -12106 & -30 & \$ SW Test Tier 5 \\
\hline \multirow{2}{*}{\multicolumn{7}{|c|}{ 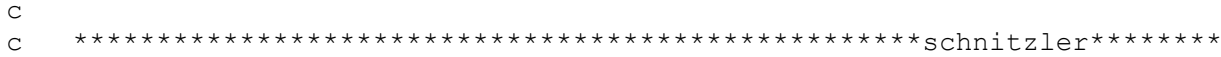 }} \\
\hline & & & & & & \\
\hline 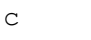 & \multirow{2}{*}{\multicolumn{3}{|c|}{ 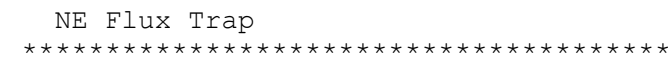 }} & (end) & & \\
\hline \multicolumn{4}{|c|}{ 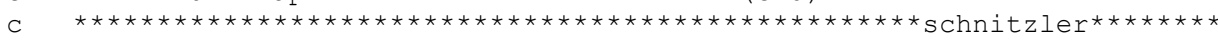 } & & & \\
\hline & & & & & & \\
\hline & & & & & & \\
\hline & & & & & & \\
\hline & & & & & & \\
\hline & E Flux Trap WI & ITH SEVEN & N POSITION TARC & GET ARRA & & \\
\hline & Four HSA C & $\mathrm{CO} / \mathrm{CO} / \mathrm{CO}$ & Targets In Pos & sitions & 2,4, & , 6 , and 7 \\
\hline & Three Alum & minum Fil & Ilers In Positi & ions 1 , & 3, an & and 5 \\
\hline & & & & & & \\
\hline 13050 & $101.00276-1$ & & -1881 & -204 & $\$ E$ & Interior water \\
\hline & & 13109 & 1320913309 & & $\$ E$ & interior water \\
\hline & & 13409 & 1350913609 & & $\$ E$ & Interior water \\
\hline & & 13709 & & & $\$ E$ & Interior water \\
\hline 13051 & $306.02136-2$ & 1881 & -1882 & -204 & $\$ E$ & Housing \\
\hline 13052 & $101.00276-1$ & 1882 & -1883 & -204 & $\$ E$ & Water \\
\hline 13053 & $306.02136-2$ & 1883 & -1884 & -204 & $\$ E$ & Envelope Tube \\
\hline 13054 & $101.00276-1$ & 1884 & -1807 & -204 & $\$ E$ & Water \\
\hline 13055 & $306.02136-2$ & 1807 & -1808 & -204 & $\$ E$ & Al SR guide tube \\
\hline 13056 & $101.00276-1$ & 1808 & -1809 & -204 & $\$ \mathrm{E}$ & Water \\
\hline 13057 & $306.02136-2$ & 1809 & -1811 & -185 & $\$ E$ & Al SR follower \\
\hline 13058 & $101.00276-1$ & 1811 & -1812 & -204 & $\$ E$ & Water \\
\hline 13059 & $30 \quad 6.02136-2$ & 1812 & -1813 & -204 & $\$ \mathrm{E}$ & Al baffle \\
\hline 13060 & $101.00276-1$ & 1813 & -1821 & -204 & $\$ E$ & Water \\
\hline 13061 & $306.02136-2$ & 1809 & -1810 & -204 & $\$ E$ & $\mathrm{Al}$ \\
\hline 13062 & $714.55926-2$ & 1810 & -1811 & -204 & $\$ E$ & Hf safety rod \\
\hline C & & & & & & \\
\hline 13101 & $101.00276-1$ & & -13104 & -401 & $\$ \mathrm{E} 1$ & i1 lower water \\
\hline 13102 & $101.00276-1$ & & -13104 & -204 & \$ E1 & 1 upper water \\
\hline 13104 & $306.02136-2$ & & -13104 & -416 & $\$ E 1$ & 1 Al filler \\
\hline 13105 & $101.00276-1$ & 13104 & -13105 & -204 & $\$ \mathrm{E} 1$ & $1 \mathrm{H} 20$ annulus \\
\hline c & & & & & & \\
\hline 13194 & $306.02136-2$ & 13105 & -13106 & -204 & $\$ E 1$ & 1 Al basket \\
\hline 13196 & $101.00276-1$ & 13106 & -13108 & -204 & \$ E1 & $1 \mathrm{H} 20$ annulus \\
\hline 13198 & $306.02136-2$ & 13108 & -13109 & -204 & $\$ E 1$ & 1 Al guide tube \\
\hline C & & & & & & \\
\hline 13251 & $101.00276-1$ & & -13246 & -100 & $\$ \mathrm{E} 2$ & 2 lower water \\
\hline 13252 & $101.00276-1$ & & -13246 & -204 & $\$ \mathrm{E} 2$ & 22 upper water \\
\hline 13253 & $101.00276-1$ & & -13241 & -200 & $\$ \mathrm{E} 2$ & 2 interior water \\
\hline 13254 & $306.02136-2$ & (13242 & -13245 & $-421)$ & $\$ E 2$ & $2 \mathrm{Al}$ end caps \\
\hline & & $:(13242$ & -13245 & $-426)$ & $\$ \mathrm{E} 2$ & $2 \mathrm{Al}$ end caps \\
\hline & & : (13242 & -13245 & $-432)$ & $\$ \mathrm{E} 2$ & 2 Al end caps \\
\hline & & : (13242 & -13245 & $-200)$ & $\$ E 2$ & $2 \mathrm{Al}$ end caps \\
\hline 13255 & $306.02136-2$ & 13241 & -13242 & -200 & $\$ \mathrm{E} 2$ & 2 Al holder \\
\hline 13256 & $306.02136-2$ & 13245 & -13246 & -200 & $\$ \mathrm{E} 2$ & 2 Al housing \\
\hline 13257 & $101.00276-1$ & 13246 & -13205 & -204 & $\$ \mathrm{E} 2$ & $2 \mathrm{H} 2 \mathrm{O}$ annulus \\
\hline 13260 & $445.60276-2$ & 13242 & -13245 & -424 & $\$ E 2$ & 2 lower HSA Co \\
\hline 13270 & $445.60276-2$ & 13242 & -13245 & -430 & $\$ E 2$ & 2 middle HSA Co \\
\hline 13280 & $445.60276-2$ & 13242 & -13245 & -435 & $\$ \mathrm{E} 2$ & 2 upper HSA Co \\
\hline c & & & & & & \\
\hline 13294 & $306.02136-2$ & 13205 & -13206 & -204 & $\$ E 2$ & 2 Al basket \\
\hline 13296 & $101.00276-1$ & 13206 & -13208 & -204 & $\$ E 2$ & $2 \mathrm{H} 2 \mathrm{O}$ annulus \\
\hline 13298 & $306.02136-2$ & 13208 & -13209 & -204 & $\$ \mathrm{E} 2$ & 2 Al guide tube \\
\hline . & & & & & & \\
\hline 13301 & $101.00276-1$ & & -13304 & -401 & $\$ \mathrm{E} 3$ & 3 lower water \\
\hline 13302 & $101.00276-1$ & & -13304 & -204 & $\$$ E3 & 3 upper water \\
\hline 13304 & $306.02136-2$ & & -13304 & -416 & $\$$ E3 & 3 Al filler \\
\hline 13305 & $101.00276-1$ & 13304 & -13305 & -204 & $\$ E 3$ & $3 \mathrm{H} 20$ annulus \\
\hline
\end{tabular}




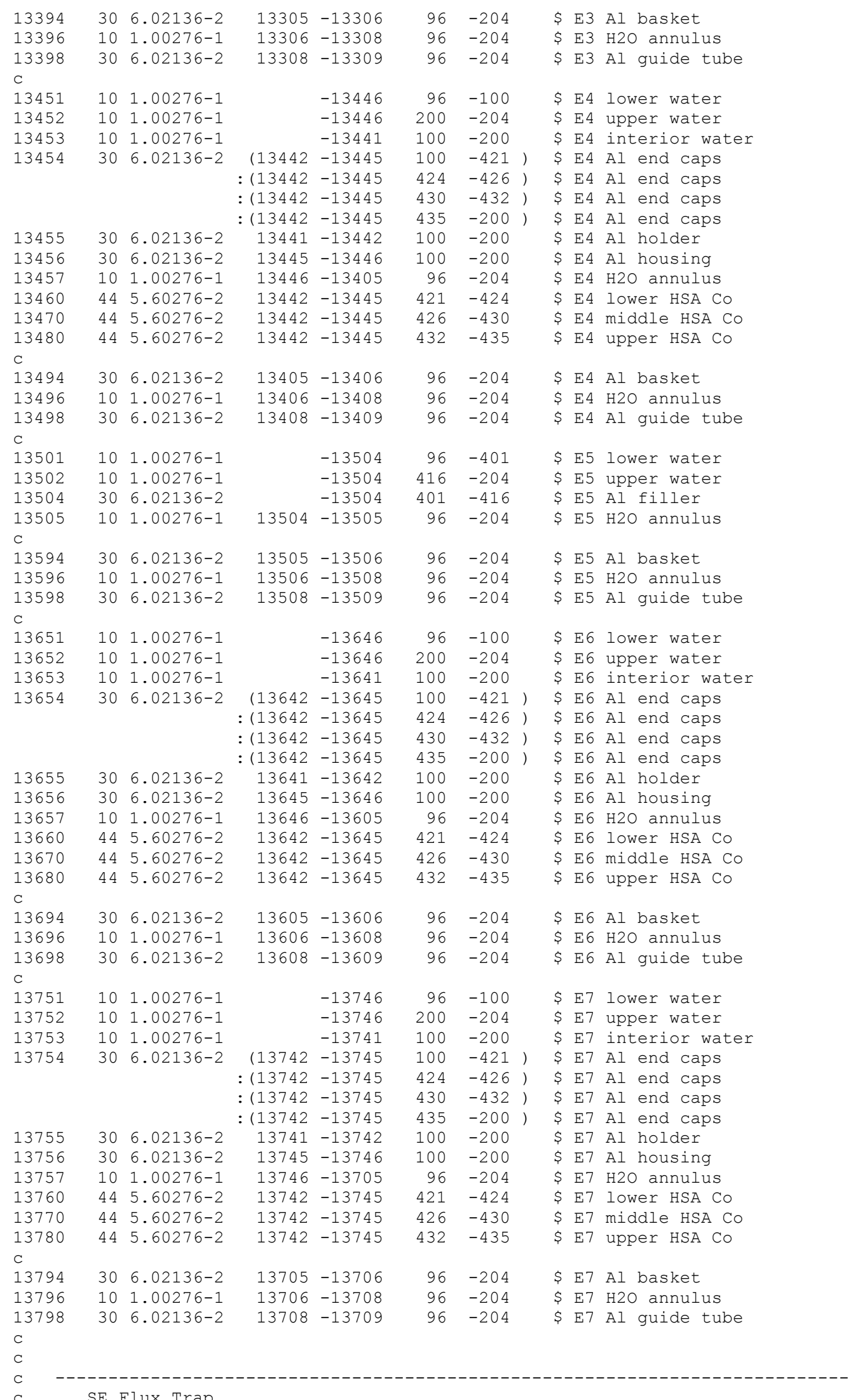

SE Flux Trap 


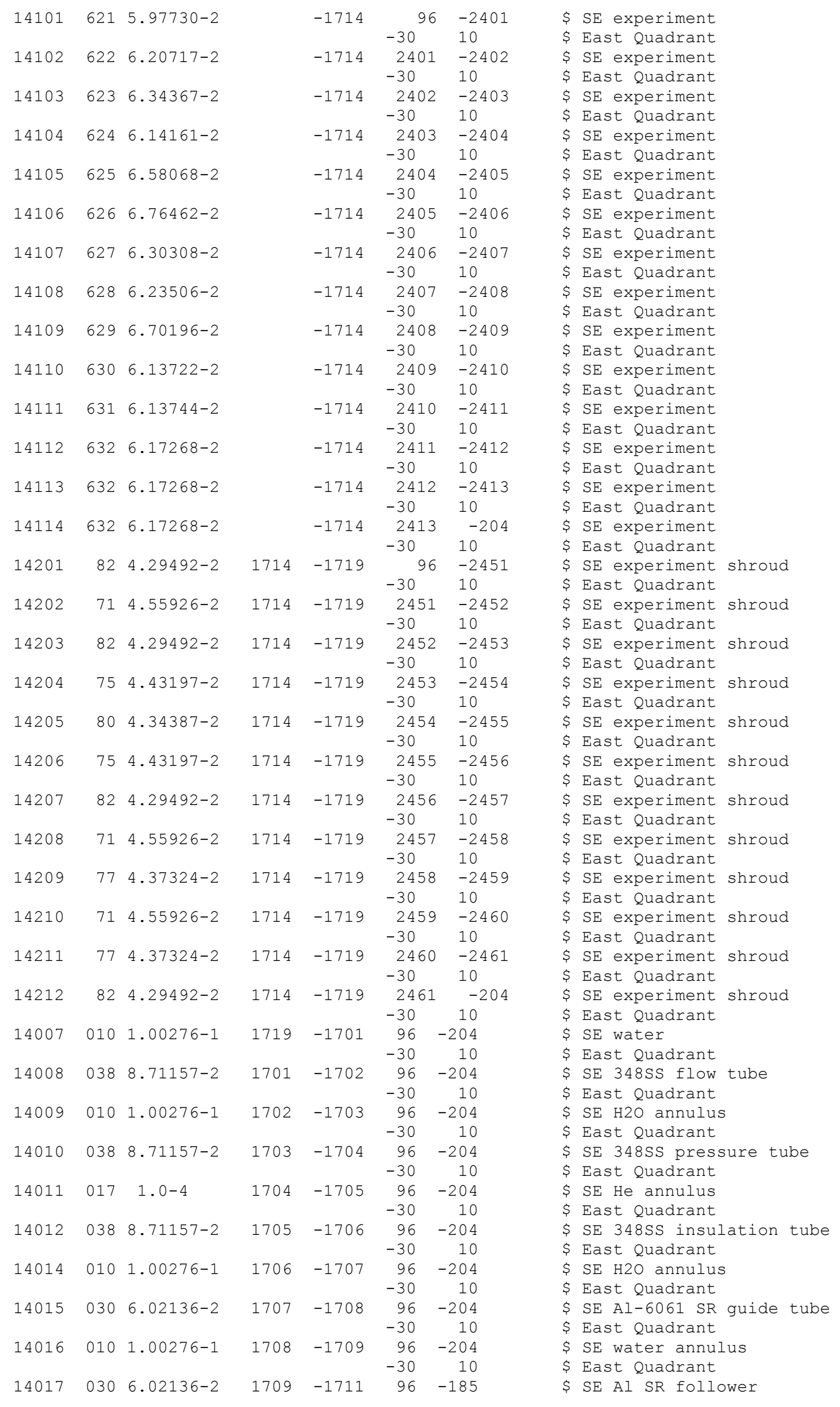




\begin{tabular}{|c|c|c|c|c|c|c|c|c|}
\hline & & & & & -30 & 10 & $\$$ & East Quadrant \\
\hline 14031 & 010 & $1.00276-1$ & 1711 & -1712 & $\begin{array}{r}96 \\
-30\end{array}$ & $\begin{array}{r}-204 \\
10\end{array}$ & $\begin{array}{l}\$ \\
\$\end{array}$ & $\begin{array}{l}\text { SE water annulus } \\
\text { East Quadrant }\end{array}$ \\
\hline 14032 & 030 & $6.02136-2$ & 1712 & -1713 & $\begin{array}{r}96 \\
-30\end{array}$ & $\begin{array}{r}-204 \\
10\end{array}$ & $\begin{array}{l}\$ \\
\$\end{array}$ & $\begin{array}{l}\text { SE Al baffle } \\
\text { East Quadrant }\end{array}$ \\
\hline 14033 & 010 & $1.00276-1$ & 1713 & -1721 & $\begin{array}{r}96 \\
-30\end{array}$ & $\begin{array}{r}-204 \\
10\end{array}$ & $\begin{array}{l}\$ \\
\$\end{array}$ & $\begin{array}{l}\text { SE water annulus } \\
\text { East Quadrant }\end{array}$ \\
\hline 14018 & 030 & $6.02136-2$ & 1709 & -1710 & $\begin{array}{l}185 \\
-30\end{array}$ & $\begin{array}{r}-204 \\
10\end{array}$ & $\begin{array}{l}\$ \\
\$\end{array}$ & $\begin{array}{l}\text { SE Al } \\
\text { East Quadrant }\end{array}$ \\
\hline 4019 & 71 & $4.55926-2$ & 1710 & -1711 & $\begin{array}{l}185 \\
-30\end{array}$ & $\begin{array}{r}-204 \\
10\end{array}$ & $\begin{array}{l}\$ \\
\$\end{array}$ & $\begin{array}{l}\text { SE Hf Safety Rod } \\
\text { East Quadrant }\end{array}$ \\
\hline
\end{tabular}

C

c

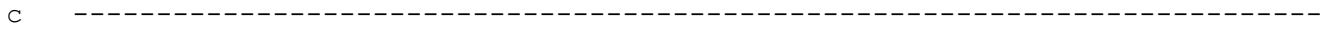

C

C

$22001 \quad 101.00276-1 \quad-22011 \quad 100-200 \quad \$$ A2 H2O interior

$22002 \quad 101.00276-1 \quad-22014 \quad 96-100 \quad \$$ A2 lower water

$22003 \quad 101.00276-1 \quad-22014 \quad 200-204 \quad \$$ A2 upper water

$2200530 \quad 6.02136-2 \quad 22011-22012 \quad 100 \quad-200 \quad \$$ A2 Al holder

$22010 \quad 445.60276-2 \quad 22012-22013 \quad 421 \quad-424 \quad$ \$ A2 lower HSA Co

$22020 \quad 445.60276-2 \quad 22012-22013 \quad 426 \quad-430 \quad \$$ A2 middle HSA Co

$22030 \quad 445.60276-2 \quad 22012-22013 \quad 432-435$ \$ A2 upper HSA Co

$22040306.02136-2(22012-22013 \quad 100-421)$ \$ A2 A1 caps

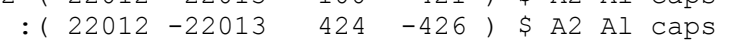

:( $\left.\begin{array}{llll}22012 & -22013 & 430 & -432\end{array}\right)$ \$ A2 Al caps

$22060 \quad:\left(\begin{array}{llll}22012 & -22013 \quad 435 & -200\end{array}\right)$ \$ A2 A1 caps

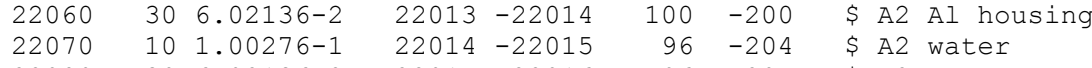

$22080 \quad 30 \quad 6.02136-2 \quad 22015-22016 \quad 96-204 \quad$ \$ A2 Al basket

$22090 \quad 101.00276-1 \quad 22016-22019 \quad 96 \quad-204 \quad \$$ A2 water

$23001 \quad 101.00276-1 \quad-23011 \quad 100-200 \quad \$$ A3 H2O interior

$23002 \quad 101.00276-1 \quad-23014 \quad 96-100 \quad \$$ A3 lower water

$23003 \quad 101.00276-1 \quad-23014 \quad 200-204 \quad \$$ A3 upper water

$2300530 \quad 6.02136-2 \quad 23011-23012 \quad 100 \quad-200 \quad \$$ A3 Al holder

$23010 \quad 445.60276-2 \quad 23012-23013 \quad 421 \quad-424 \quad$ \$ A3 lower HSA Co

$23020 \quad 445.60276-2 \quad 23012-23013 \quad 426$-430 \$A3 middle HSA Co

$2303044 \quad 5.60276-2 \quad 23012-23013 \quad 432-435$ \$ A3 upper HSA CO

$23040306.02136-2(23012-23013 \quad 100-421)$ \$ A3 A1 caps

$:\left(\begin{array}{llll}23012 & -23013 & 424 & -426\end{array}\right)$ \$ A3 Al caps

:( $\left.\begin{array}{llll}23012 & -23013 & 430 & -432\end{array}\right)$ \$ A3 Al caps

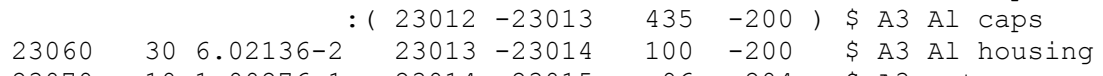

$23070 \quad 101.00276-1 \quad 23014-23015 \quad 96 \quad-204 \quad$ \$ A3 water

$23080 \quad 30 \quad 6.02136-2 \quad 23015-23016 \quad 96-204 \quad$ \$ A3 Al basket

$23090 \quad 101.00276-1 \quad 23016-23019 \quad 96 \quad-204 \quad$ \$ A3 water

C
C
C




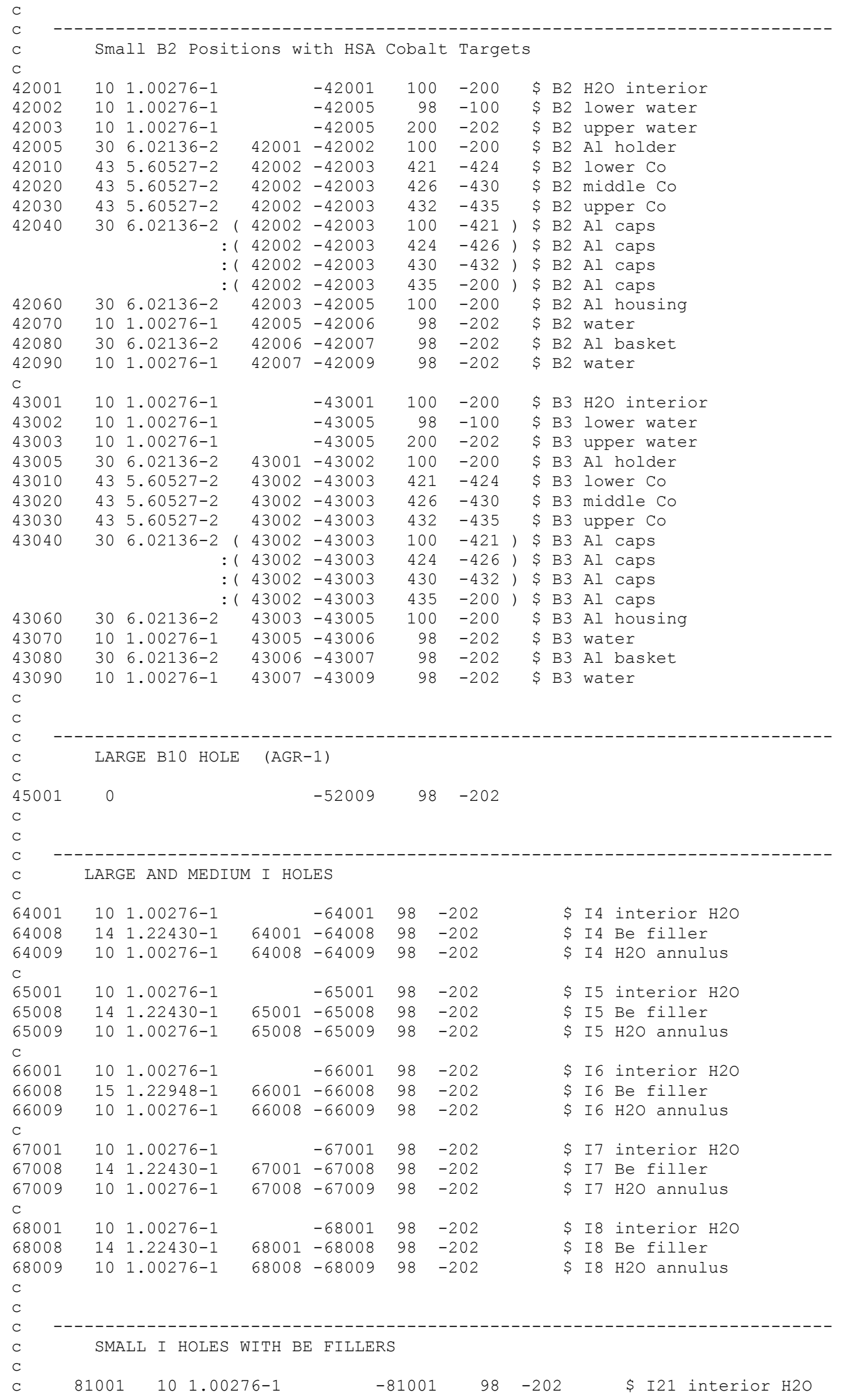




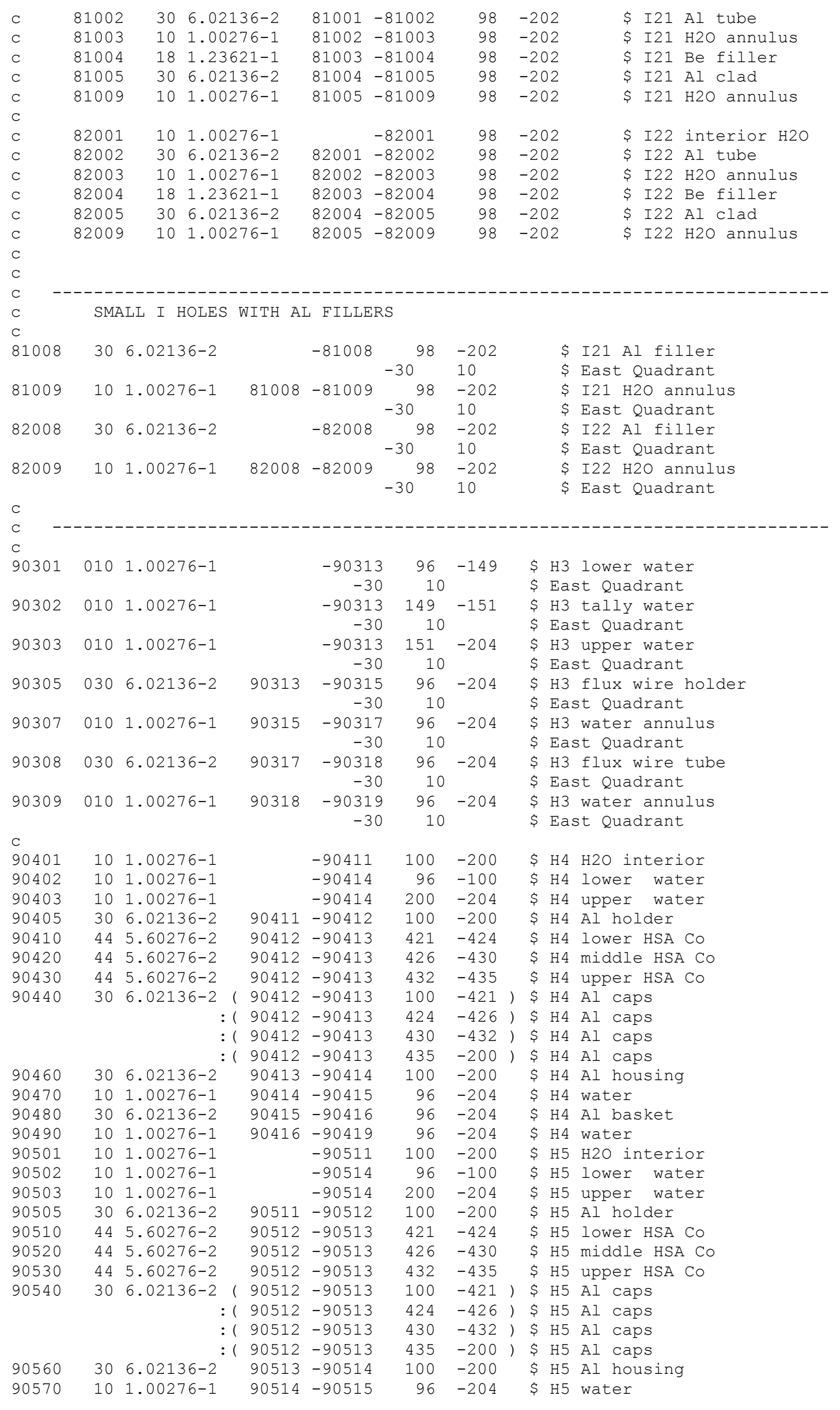




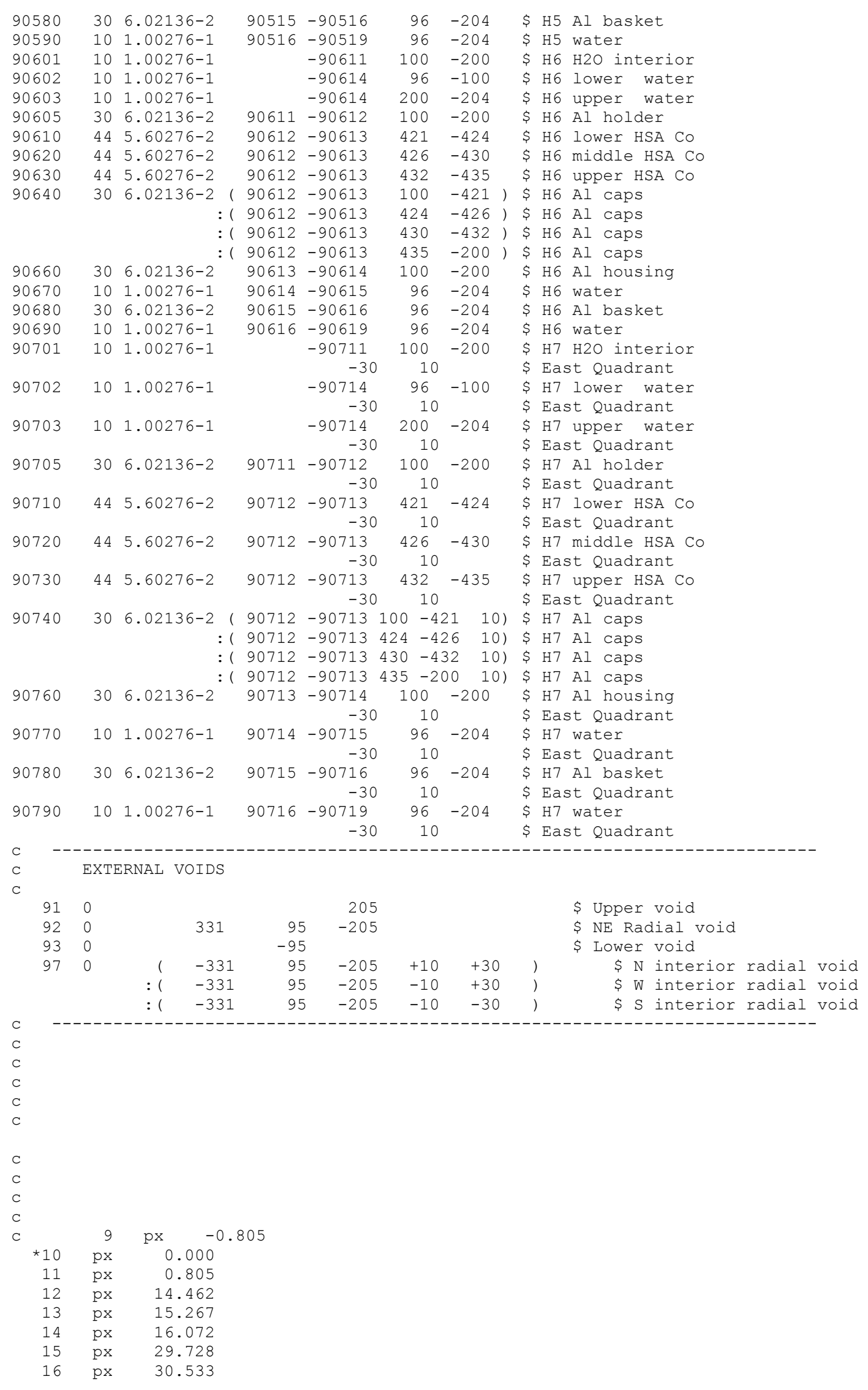




\begin{tabular}{|c|c|c|c|c|c|}
\hline 17 & $\mathrm{px}$ & \multicolumn{4}{|c|}{31.338} \\
\hline 23 & py & \multicolumn{4}{|c|}{-31.338} \\
\hline 24 & py & \multicolumn{4}{|c|}{-30.533} \\
\hline 25 & py & \multicolumn{4}{|c|}{-29.728} \\
\hline 26 & py & \multicolumn{4}{|c|}{-16.072} \\
\hline 27 & py & \multicolumn{4}{|c|}{-15.267} \\
\hline 28 & py & \multicolumn{4}{|c|}{-14.462} \\
\hline 29 & py & \multicolumn{4}{|c|}{-0.805} \\
\hline$\star 30$ & py & \multicolumn{4}{|c|}{0.000} \\
\hline & 31 & \multicolumn{2}{|c|}{ py } & \multicolumn{2}{|c|}{0.805} \\
\hline & & & & & \\
\hline 45 & $\mathrm{p}$ & 1 & -1 & 0.0 & -42.65 \\
\hline 52 & $\mathrm{p}$ & 1 & -1 & 0.0 & 29.39 \\
\hline 53 & $\mathrm{p}$ & 1 & -1 & 0.0 & 30.53 \\
\hline 54 & $\mathrm{p}$ & 1 & -1 & 0.0 & 31.67 \\
\hline 55 & $\mathrm{p}$ & 1 & -1 & 0.0 & 42.65 \\
\hline 66 & $\mathrm{p}$ & 1 & 1 & 0.0 & -31.67 \\
\hline 67 & $\mathrm{p}$ & 1 & 1 & 0.0 & -30.53 \\
\hline 68 & $\mathrm{p}$ & 1 & 1 & 0.0 & -29.39 \\
\hline 69 & $\mathrm{p}$ & 1 & 1 & 0.0 & -1.13 \\
\hline 70 & $\mathrm{p}$ & 1 & 1 & 0.0 & 0.0 \\
\hline 71 & $\mathrm{p}$ & 1 & 1 & 0.0 & 1.13 \\
\hline 72 & $\mathrm{p}$ & 1 & 1 & 0.0 & 29.39 \\
\hline 73 & $\mathrm{p}$ & 1 & 1 & 0.0 & 30.53 \\
\hline 74 & $\mathrm{p}$ & 1 & 1 & 0.0 & 31.67 \\
\hline
\end{tabular}

C

pz $\quad-64.540$

pz $\quad-41.540$

pz $\quad-20.955$

pz $\quad-2.540$

pz $\quad-1.905$

pz $\quad 0.000$

pz $\quad 5.080$

pz $\quad 3.0163$

15.240

30.480

45.720

$\begin{array}{ll}\mathrm{pz} & 45.720 \\ \mathrm{pz} & 57.150\end{array}$

60.960

64.770

76.200

91.440

106.680

$\begin{array}{ll}\mathrm{pz} & 114.30 \\ \mathrm{pz} & 121.920\end{array}$

pz 123.825

pz 127.0000

pz 147.320

pz $\quad 166.0000$

pz $\quad 187.0000$

208 pz 137.1600

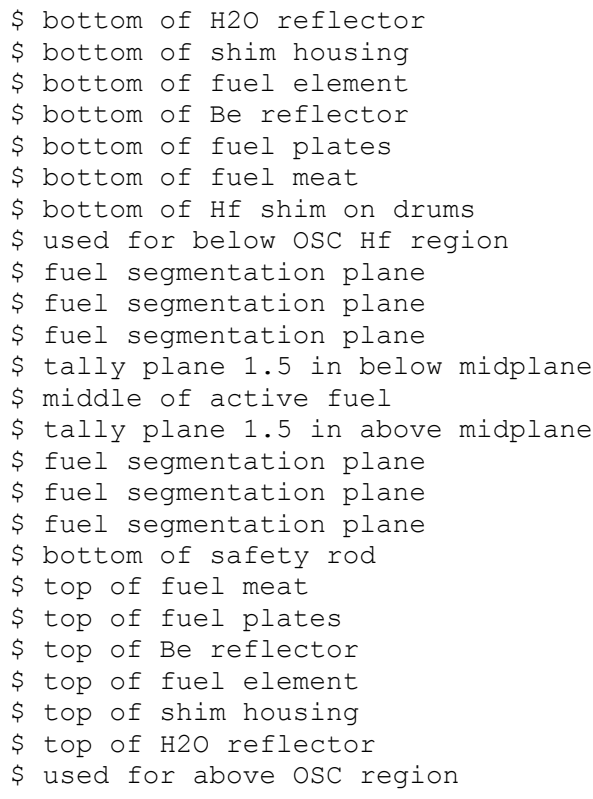

cylinders cetered at origin

$\mathrm{Cz} \quad 4.09702$

Cz 6.587

64.294

Cz $\quad 68.580$

CZ 100.00

C

C

C

target definition planes

$401 \mathrm{pz} \quad-2.05740$

$416 \mathrm{pz} \quad 123.97740$

\$ bottom of flux trap target stack

C

C

$421 \mathrm{pz} \quad 1.27000$

C $\quad 422$ pz 13.97000

$\begin{array}{llll}\text { C } & 423 & \text { pz } & 26.67000\end{array}$

$424 \mathrm{pz} \quad 39.37000$

$\$$ i.r of $\mathrm{H}$ holes housing

$\$$ o.r. of $\mathrm{H}$ holes housing

\$i.r of Al reflector tank

\$ o.r of Al reflector tank

$\$$ o.r of water reflector

\$ bottom of HSA lower target

\$ HSA target segmentation surface

\$ HSA target segmentation surface

\$ top of HSA lower target 


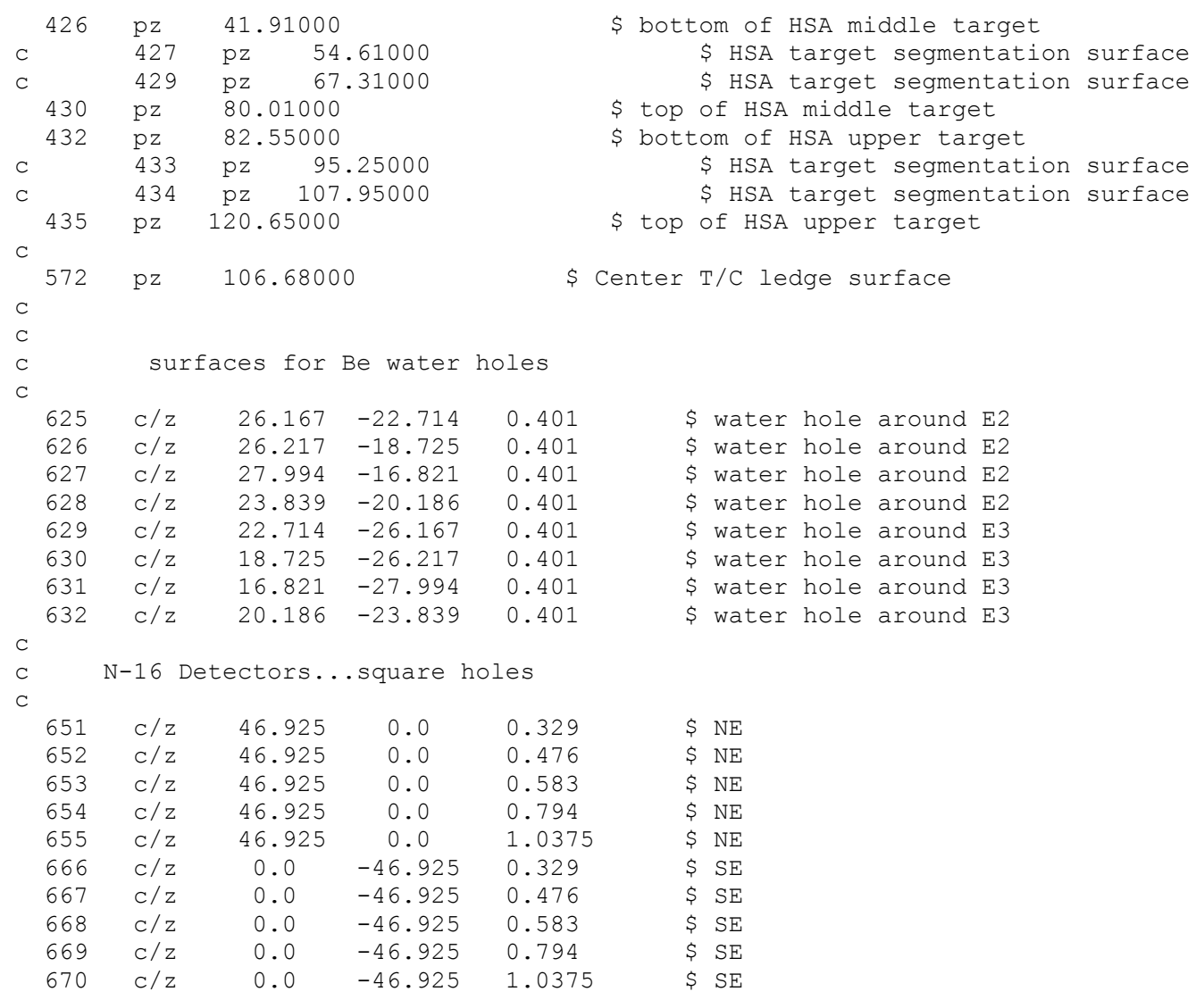

c N-16 Detectors...outside drums (round holes)

c

$\begin{array}{llllll}686 & \mathrm{C} / \mathrm{z} & 34.123 & -34.123 & 0.329 & \text { \$ } \mathrm{E}\end{array}$

$\begin{array}{llllll}687 & \mathrm{C} / \mathrm{z} & 34.123 & -34.123 & 0.476 \quad \text { \$ }\end{array}$

$\begin{array}{llllll}688 & c / z & 34.123 & -34.123 & 0.583 & \$ \mathrm{E}\end{array}$

$\begin{array}{llllll}689 \mathrm{c} / \mathrm{z} & 34.123 & -34.123 & 0.794 & \text { \$ } \mathrm{E}\end{array}$

C

SHIM RODS

$\begin{array}{llllll}701 & c / z & 9.474 & 0.0 & 0.239 & \text { \$ NE } 1\end{array}$

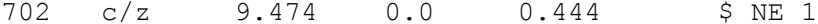

$\begin{array}{lllll}703 & \mathrm{c} / \mathrm{z} & 9.474 & 0.0 & 0.752\end{array}$

$\begin{array}{lllll}706 & c / z & 11.405 & 0.0 & 0.239\end{array}$

$\begin{array}{lllll}707 & c / z & 11.405 & 0.0 & 0.444\end{array}$

$\begin{array}{lllll}708 & c / z & 11.405 & 0.0 & 0.752\end{array}$

$\begin{array}{lllll}711 \mathrm{C} / \mathrm{z} & 13.335 & 0.0 & 0.239 & \text { \$ NE } 3\end{array}$

$\begin{array}{lllll}712 \mathrm{c} / \mathrm{z} & 13.335 & 0.0 & 0.444 & \text { \$ NE } 3\end{array}$

$\begin{array}{lllll}716 \mathrm{c} / \mathrm{z} & 15.265 & 0.0 & 0.239 & \text { \$ NE } 4\end{array}$

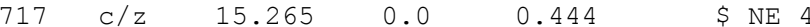

$\begin{array}{lllll}718 \mathrm{c} / \mathrm{z} & 15.265 & 0.0 & 0.752 & \text { \$ NE } 4\end{array}$

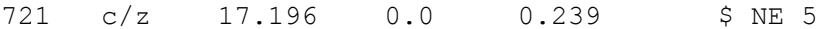

$\begin{array}{llllll}722 & c / z & 17.196 & 0.0 & 0.444 & \text { \$ NE } 5\end{array}$

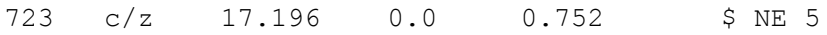

$\begin{array}{lllll}726 & c / z & 19.126 & 0.0 & 0.239\end{array}$

$\begin{array}{lllll}727 & c / z & 19.126 & 0.0 & 0.444\end{array}$

C

$\mathrm{c} / \mathrm{z}=19.126$

$0.0 \quad 0.752$

$\$ N E 6$

$\begin{array}{llllll}791 & c / z & 0.0 & -9.474 & 0.239 & \text { S SE } 1\end{array}$

$\begin{array}{lllll}792 \mathrm{C} / \mathrm{z} & 0.0 & -9.474 & 0.444 & \text { \$ SE } 1\end{array}$

$\begin{array}{llllll}793 & c / z & 0.0 & -9.474 & 0.752 & \text { \$ SE } 1\end{array}$

$\begin{array}{lllll}796 \mathrm{C} / \mathrm{z} & 0.0 & -11.405 & 0.239 & \text { \$ SE } 2\end{array}$

$\begin{array}{lllll}797 \mathrm{C} / \mathrm{z} & 0.0 & -11.405 & 0.444 & \text { \$ SE } 2\end{array}$

$\begin{array}{lllll}798 & \mathrm{C} / \mathrm{z} & 0.0 & -11.405 & 0.752\end{array}$ 


\begin{tabular}{|c|c|c|c|c|c|}
\hline 801 & $\mathrm{c} / \mathrm{z}$ & 0.0 & -13.335 & 0.239 & $\$ \mathrm{SE} 3$ \\
\hline 802 & $\mathrm{c} / \mathrm{z}$ & 0.0 & -13.335 & 0.444 & \$SE 3 \\
\hline 803 & $\mathrm{C} / \mathrm{z}$ & 0.0 & -13.335 & 0.752 & $\$ \mathrm{SE} 3$ \\
\hline 806 & $\mathrm{c} / \mathrm{z}$ & 0.0 & -15.265 & 0.239 & $\$ S E$ \\
\hline 807 & $\mathrm{c} / \mathrm{z}$ & 0.0 & -15.265 & 0.444 & $\$ S E$ \\
\hline 808 & $\mathrm{C} / \mathrm{z}$ & 0.0 & -15.265 & 0.752 & \$SE 4 \\
\hline 811 & $\mathrm{c} / \mathrm{z}$ & 0.0 & -17.196 & 0.239 & \$SE 5 \\
\hline 812 & $\mathrm{c} / \mathrm{z}$ & 0.0 & -17.196 & 0.444 & $\$ S E$ \\
\hline 813 & $\mathrm{c} / \mathrm{z}$ & 0.0 & -17.196 & 0.752 & $\$ S E$ \\
\hline 816 & $\mathrm{C} / \mathrm{z}$ & 0.0 & -19.126 & 0.239 & $\$ S E$ \\
\hline 817 & $\mathrm{C} / \mathrm{z}$ & 0.0 & -19.126 & 0.444 & $\$ \mathrm{SE} 6$ \\
\hline 818 & $\mathrm{C} / \mathrm{z}$ & 0.0 & -19.126 & 0.752 & $\$ S E$ \\
\hline
\end{tabular}

C

C

CONTROL DRUMS

\begin{tabular}{|c|c|c|c|c|c|}
\hline 901 & $\mathrm{C} / \mathrm{z}$ & 52.596 & -10.157 & 1.429 & $\$$ \\
\hline 902 & $\mathrm{C} / \mathrm{z}$ & 52.596 & -10.157 & 8.560 & $\$$ \\
\hline 904 & $\mathrm{C} / \mathrm{z}$ & 52.596 & -10.157 & 9.195 & $\$$ \\
\hline 905 & $\mathrm{C} / \mathrm{z}$ & 52.596 & -10.157 & 9.525 & $\$$ \\
\hline 906 & $C / z$ & 37.610 & -23.228 & 1.429 & $\$$ \\
\hline 907 & $\mathrm{C} / \mathrm{z}$ & 37.610 & -23.228 & 8.560 & $\$$ \\
\hline 909 & $\mathrm{C} / \mathrm{z}$ & 37.610 & $-23 \cdot 228$ & 9.195 & $\$$ \\
\hline 910 & $\mathrm{C} / \mathrm{z}$ & 37.610 & -23.228 & 9.525 & $\$$ \\
\hline 11 & $C / z$ & 23.228 & -37.610 & 1.429 & $\$$ \\
\hline 912 & $c / z$ & 23.228 & -37.610 & 8.560 & $\$$ \\
\hline 14 & $C / z$ & 23.228 & -37.610 & 9.195 & $\$$ \\
\hline 915 & $c / z$ & 23.228 & -37.610 & 9.525 & $\$$ \\
\hline 16 & $C / z$ & 10.157 & -52.596 & 1.429 & $\$$ \\
\hline 17 & $\mathrm{C} / \mathrm{z}$ & 10.157 & -52.596 & 8.560 & $\$$ \\
\hline & $c / z$ & 10.157 & -52.596 & 9.195 & $\$$ \\
\hline & $\mathrm{C} / \mathrm{z}$ & 10.157 & -52.596 & 9.525 & $\$$ \\
\hline
\end{tabular}

C

CONTROL DRUM POSITION SURFACES

Nominal Beginning of Cycle Positions

$\begin{array}{llllllllll}\mathrm{C} / \mathrm{z} & 44.2816 & -14.0837 & 9.195 & & \text { \$ DRUM E1 } & \text { AT } & 50 & \text { DEGREES } \\ \mathrm{C} / \mathrm{z} & 28.6141 & -25.1311 & 9.195 & & \text { \$ DRUM E2 } & \text { AT } & 50 & \text { DEGREES } \\ \mathrm{C} / \mathrm{z} & 25.1311 & -28.6141 & 9.195 & & \text { \$ DRUM E3 AT } & 50 & \text { DEGREES } \\ \mathrm{C} / \mathrm{z} & 14.0837 & -44.2816 & 9.195 & & \text { \$ DRUM E4 } & \text { AT } & 50 & \text { DEGREES }\end{array}$

\begin{tabular}{|c|c|c|c|c|c|c|c|c|c|c|}
\hline 981 & $\mathrm{c} / \mathrm{z}$ & 44.2436 & -6.3119 & 9.195 & $\$$ & DRUM & E1 & $\mathrm{AT}$ & 0 & DEGREES \\
\hline 982 & $\mathrm{c} / \mathrm{z}$ & 30.3697 & -17.5600 & 9.195 & $\$$ & DRUM & $\mathrm{E} 2$ & $\mathrm{AT}$ & 0 & DEGREES \\
\hline 983 & $\mathrm{C} / \mathrm{z}$ & 17.5600 & -30.3697 & 9.195 & $\$$ & DRUM & E3 & $\mathrm{AT}$ & 0 & DEGREES \\
\hline 984 & $\mathrm{C} / \mathrm{z}$ & 6.3119 & -44.2436 & 9.195 & $\$$ & DRUM & E4 & $\mathrm{AT}$ & 0 & DEGREES \\
\hline 981 & $\mathrm{C} / \mathrm{z}$ & 43.4011 & -10.2020 & 9.195 & $\$$ & DRUM & E1 & $\mathrm{AT}$ & 25 & DEGREES \\
\hline 982 & $\mathrm{C} / \mathrm{z}$ & 28.6527 & -21.1509 & 9.195 & $\$$ & DRUM & $\mathrm{E} 2$ & $\mathrm{AT}$ & 25 & DEGREES \\
\hline 983 & $\mathrm{C} / \mathrm{z}$ & 21.1509 & -28.6527 & 9.195 & $\$$ & DRUM & E3 & $\mathrm{AT}$ & 25 & DEGREES \\
\hline 984 & $\mathrm{C} / \mathrm{z}$ & 10.2020 & -43.4011 & 9.195 & $\$$ & DRUM & E4 & $\mathrm{AT}$ & 25 & DEGREES \\
\hline 981 & $\mathrm{C} / \mathrm{z}$ & 43.7261 & -12.5803 & 9.195 & $\$$ & DRUM & E1 & $\mathrm{AT}$ & 40 & DEGREES \\
\hline 982 & $\mathrm{C} / \mathrm{z}$ & 28.4203 & -23.5400 & 9.195 & $\$$ & DRUM & $\mathrm{E} 2$ & $\mathrm{AT}$ & 40 & DEGREES \\
\hline 983 & $\mathrm{C} / \mathrm{z}$ & 23.5400 & -28.4203 & 9.195 & $\$$ & DRUM & E3 & $\mathrm{AT}$ & 40 & DEGREES \\
\hline 984 & $\mathrm{C} / \mathrm{z}$ & 12.5803 & -43.7261 & 9.195 & $\$$ & DRUM & E4 & $\mathrm{AT}$ & 40 & DEGREES \\
\hline 981 & $\mathrm{C} / \mathrm{z}$ & 44.2816 & -14.0837 & 9.195 & $\$$ & DRUM & E1 & $\mathrm{AT}$ & 50 & DEGREES \\
\hline 982 & $\mathrm{C} / \mathrm{z}$ & 28.6141 & -25.1311 & 9.195 & $\$$ & DRUM & E2 & $\mathrm{AT}$ & 50 & DEGREES \\
\hline 983 & $\mathrm{C} / \mathrm{z}$ & 25.1311 & -28.6141 & 9.195 & $\$$ & DRUM & E3 & $\mathrm{AT}$ & 50 & DEGREES \\
\hline 984 & $\mathrm{C} / \mathrm{z}$ & 14.0837 & -44.2816 & 9.195 & $\$$ & DRUM & E4 & $\mathrm{AT}$ & 50 & DEGREES \\
\hline 981 & $\mathrm{c} / \mathrm{z}$ & 45.0898 & -15.4678 & 9.195 & $\$$ & DRUM & E1 & $\mathrm{AT}$ & 60 & DEGREES \\
\hline 982 & $\mathrm{C} / \mathrm{z}$ & 29.0812 & -26.6643 & 9.195 & $\$$ & DRUM & E2 & $\mathrm{AT}$ & 60 & DEGREES \\
\hline 983 & $\mathrm{C} / \mathrm{z}$ & 26.6643 & -29.0812 & 9.195 & $\$$ & DRUM & E3 & $\mathrm{AT}$ & 60 & DEGREES \\
\hline 984 & $\mathrm{C} / \mathrm{z}$ & 15.4678 & -45.0898 & 9.195 & $\$$ & DRUM & $\mathrm{E} 4$ & $\mathrm{AT}$ & 60 & DEGREES \\
\hline
\end{tabular}




\begin{tabular}{|c|c|c|c|c|c|c|c|c|c|c|c|}
\hline C & 981 & $c / z$ & 45.5812 & -16.1018 & 9.195 & $\$$ & DRUM & E1 & $\mathrm{AT}$ & 65 I & DEGREES \\
\hline C & 982 & $c / z$ & 29.4132 & -27.3945 & 9.195 & $\$$ & DRUM & E2 & $\mathrm{AT}$ & 65 I & DEGREES \\
\hline C & 983 & $c / z$ & 27.3945 & -29.4132 & 9.195 & $\$$ & DRUM & E3 & $\mathrm{AT}$ & 65 & DEGREES \\
\hline C & 984 & $c / z$ & 16.1018 & -45.5812 & 9.195 & $\$$ & DRUM & E4 & $\mathrm{AT}$ & 65 & DEGREES \\
\hline \multicolumn{12}{|l|}{ C } \\
\hline C & 981 & $c / z$ & 46.7201 & -17.2296 & 9.195 & $\$$ & DRUM & E1 & $\mathrm{AT}$ & 75 & DEGREES \\
\hline C & 982 & $c / z$ & 30.2612 & -28.7546 & 9.195 & $\$$ & DRUM & E2 & $\mathrm{AT}$ & 75 & DEGREES \\
\hline C & 983 & $c / z$ & 28.7546 & -30.2612 & 9.195 & $\$$ & DRUM & E3 & $\mathrm{AT}$ & 75 & DEGREES \\
\hline C & 984 & $c / z$ & 17.2296 & -46.7201 & 9.195 & $\$$ & DRUM & E4 & $\mathrm{AT}$ & 75 & DEGREES \\
\hline \multicolumn{12}{|c|}{ C } \\
\hline C & 981 & $c / z$ & 47.3589 & -17.7148 & 9.195 & $\$$ & DRUM & E1 & $\mathrm{AT}$ & 80 & DEGREES \\
\hline C & 982 & $c / z$ & 30.7708 & -29.3741 & 9.195 & $\$$ & DRUM & E2 & $\mathrm{AT}$ & 80 & DEGREES \\
\hline C & 983 & $c / z$ & 29.3741 & -30.7708 & 9.195 & $\$$ & DRUM & E3 & $\mathrm{AT}$ & 80 & DEGREES \\
\hline C & 984 & $c / z$ & 17.7148 & -47.3589 & 9.195 & $\$$ & DRUM & E4 & $\mathrm{AT}$ & 80 I & DEGREES \\
\hline \multicolumn{12}{|c|}{ C } \\
\hline C & 981 & $c / z$ & 48.0375 & -18.1425 & 9.195 & $\$$ & DRUM & E1 & $\mathrm{AT}$ & 85 & DEGREES \\
\hline C & 982 & $c / z$ & 31.3325 & -29.9467 & 9.195 & $\$$ & DRUM & E2 & $\mathrm{AT}$ & 85 & DEGREES \\
\hline C & 983 & $c / z$ & 29.9467 & -31.3325 & 9.195 & $\$$ & DRUM & E3 & $\mathrm{AT}$ & 85 & DEGREES \\
\hline C & 984 & $c / z$ & 18.1425 & -48.0375 & 9.195 & $\$$ & DRUM & E4 & $\mathrm{AT}$ & 85 & DEGREES \\
\hline \multicolumn{12}{|c|}{ C } \\
\hline C & 981 & $c / z$ & 50.2597 & -19.0502 & 9.195 & $\$$ & DRUM & E1 & $\mathrm{AT}$ & 100 & DEGREES \\
\hline C & 982 & $c / z$ & 33.2854 & -31.3425 & 9.195 & $\$$ & DRUM & E2 & $\mathrm{AT}$ & 100 & DEGREES \\
\hline C & 983 & $c / z$ & 31.3425 & -33.2854 & 9.195 & $\$$ & DRUM & E3 & $\mathrm{AT}$ & 100 & DEGREES \\
\hline C & 984 & $c / z$ & 19.0502 & -50.2597 & 9.195 & $\$$ & DRUM & E4 & $\mathrm{AT}$ & 100 & DEGREES \\
\hline \multicolumn{12}{|l|}{ C } \\
\hline C & 981 & $c / z$ & 53.4422 & -19.3130 & 9.195 & $\$$ & DRUM & E1 & $\mathrm{AT}$ & 120 & DEGREES \\
\hline C & 982 & $c / z$ & 36.3215 & -32.3323 & 9.195 & $\$$ & DRUM & E2 & $\mathrm{AT}$ & 120 & DEGREES \\
\hline C & 983 & $c / z$ & 32.3323 & -36.3215 & 9.195 & $\$$ & DRUM & E3 & $\mathrm{AT}$ & 120 & DEGREES \\
\hline C & 984 & $c / z$ & 19.3130 & -53.4422 & 9.195 & $\$$ & DRUM & E4 & $\mathrm{AT}$ & 120 & DEGREES \\
\hline \multicolumn{12}{|c|}{ C } \\
\hline C & 981 & $c / z$ & 54.2370 & -19.2044 & 9.195 & $\$$ & DRUM & E1 & $\mathrm{AT}$ & 125 & DEGREES \\
\hline C & 982 & $c / z$ & 37.1199 & -32.4099 & 9.195 & $\$$ & DRUM & E2 & $\mathrm{AT}$ & 125 & DEGREES \\
\hline C & 983 & $c / z$ & 32.4099 & -37.1199 & 9.195 & $\$$ & DRUM & E3 & $\mathrm{AT}$ & 125 & DEGREES \\
\hline C & 984 & $c / z$ & 19.2044 & -54.2370 & 9.195 & $\$$ & DRUM & E4 & $\mathrm{AT}$ & 125 & DEGREES \\
\hline \multicolumn{12}{|c|}{$\mathrm{C}$} \\
\hline C & 981 & $c / z$ & 57.9068 & -17.6632 & 9.195 & $\$$ & DRUM & E1 & $\mathrm{AT}$ & 150 & DEGREES \\
\hline C & 982 & $c / z$ & 41.0463 & -31.7568 & 9.195 & $\$$ & DRUM & E2 & $\mathrm{AT}$ & 150 & DEGREES \\
\hline C & 983 & $c / z$ & 31.7568 & -41.0463 & 9.195 & $\$$ & DRUM & E3 & $\mathrm{AT}$ & 150 & DEGREES \\
\hline C & 984 & $c / z$ & 17.6632 & -57.9068 & 9.195 & $\$$ & DRUM & E4 & $\mathrm{AT}$ & 150 & DEGREES \\
\hline
\end{tabular}

LOBE AND FUEL REGIONS

NE lobe

$1104 \mathrm{C} / \mathrm{z} \quad 30.533 \quad 0.0 \quad 6.82625 \quad$ \$ water

\begin{tabular}{|c|c|c|c|c|c|c|}
\hline 1111 & 2 & إ. & . & 7.050 & $?$ & Euel plate-inner \\
\hline & 1112 & $\mathrm{c} / \mathrm{z}$ & 30.533 & 0.0 & 7.861 & \$1st fuel plate-outer \\
\hline $\mathrm{C}$ & 1113 & $\mathrm{C} / \mathrm{z}$ & 30.533 & 0.0 & 8.059 & \$2nd fuel plate-inner \\
\hline & 1114 & $\mathrm{C} / \mathrm{z}$ & 30.533 & 0.0 & 8.186 & \$2nd fuel plate-outer \\
\hline & 1115 & $\mathrm{C} / \mathrm{z}$ & 30.533 & 0.0 & 8.385 & \$ 3rd fuel plate-inner \\
\hline & 1116 & $\mathrm{c} / \mathrm{z}$ & 30.533 & 0.0 & 8.512 & \$3rd fuel plate-outer \\
\hline & 1117 & $\mathrm{c} / \mathrm{z}$ & 30.533 & 0.0 & 8.710 & \$ 4th fuel plate-inner \\
\hline 1118 & $c / z$ & 30.533 & 0.0 & 8.837 & $\$$ & 4th fuel plate-outer \\
\hline 1119 & $c / z$ & 30.533 & 0.0 & 9.0348 & $\$$ & 5th fuel plate-inner \\
\hline 1120 & $\mathrm{C} / \mathrm{z}$ & 30.533 & 0.0 & 12.413 & $\$$ & 15th fuel plate-outer \\
\hline 1121 & $\mathrm{c} / \mathrm{z}$ & 30.533 & 0.0 & 12.611 & \$ & 16thfuel plate-inner \\
\hline$c$ & 1122 & $\mathrm{c} / \mathrm{z}$ & 30.533 & 0.0 & 12.738 & \$16thfuel plate-outer \\
\hline & 1123 & $\mathrm{C} / \mathrm{z}$ & 30.533 & 0.0 & 12.936 & \$17thfuel plate-inner \\
\hline & 1124 & $\mathrm{c} / \mathrm{z}$ & 30.533 & 0.0 & 13.063 & \$17thfuel plate-outer \\
\hline & 1125 & $\mathrm{c} / \mathrm{z}$ & 30.533 & 0.0 & 13.261 & \$18thfuel plate-inner \\
\hline & 1126 & $\mathrm{c} / \mathrm{z}$ & 30.533 & 0.0 & 13.388 & \$18thfuel plate-outer \\
\hline F & 1127 & $\mathrm{C} / \mathrm{z}$ & 30.533 & 0.0 & 13.586 & \$19thfuel plate-inner \\
\hline 128 & $\mathrm{c} / \mathrm{z}$ & 30.533 & 0.0 & 13.84 & $\$$ & 19thfuel plate-outer \\
\hline 200 & $\mathrm{c} / \mathrm{z}$ & 30.533 & 0.0 & 14.133 & $\$$ & o.r. of $\mathrm{NE}$ loop \\
\hline & $\mathrm{c} / \mathrm{z}$ & 29.862 & 2.455 & 26.353 & $\$$ & Be with sawcuts \\
\hline & 1195 & $\mathrm{c} / \mathrm{z}$ & 29.862 & -2.455 & 26.353 & $\$$ Be with sawcuts \\
\hline
\end{tabular}




\begin{tabular}{|c|c|c|c|c|c|c|}
\hline \\
\hline \\
\hline C & 1718 & $\mathrm{C} / \mathrm{z}$ & 0.0 & -30.533 & 0.114 & \$ water \\
\hline C & 1717 & $\mathrm{C} / \mathrm{z}$ & 0.0 & -30.533 & 0.238 & \$ Al flux wire tube \\
\hline C & 1715 & $\mathrm{C} / \mathrm{z}$ & 0.0 & -30.533 & 0.311 & \$ water \\
\hline C & 1716 & $\mathrm{C} / \mathrm{z}$ & 0.0 & -30.533 & 0.476 & \$ SS304 flux wire holder \\
\hline 1714 & $\mathrm{C} / \mathrm{z}$ & 0.0 & -30.533 & $3 \quad 2.04470$ & $0 \quad \$$ & shroud inner radius \\
\hline 1719 & $\mathrm{C} / \mathrm{z}$ & 0.0 & -30.533 & 2.17170 & $\$$ & shroud outer radius \\
\hline 1701 & $\mathrm{C} / \mathrm{z}$ & 0.0 & -30.533 & 2.216 & $\$$ & water \\
\hline 1702 & $\mathrm{C} / \mathrm{z}$ & 0.0 & -30.533 & 2.381 & $\$$ & flow tube \\
\hline 1703 & $\mathrm{C} / \mathrm{z}$ & 0.0 & -30.533 & 2.696 & $\$$ & water \\
\hline 1704 & $c / z$ & 0.0 & -30.533 & 3.213 & $\$$ & pressure tube \\
\hline 1705 & $\mathrm{c} / \mathrm{z}$ & 0.0 & -30.533 & 3.341 & $\$$ & He gap \\
\hline 1706 & $\mathrm{c} / \mathrm{z}$ & 0.0 & -30.533 & 3.651 & $\$$ & insulation tube \\
\hline 1707 & $\mathrm{c} / \mathrm{z}$ & 0.0 & -30.533 & 4.128 & $\$$ & water \\
\hline 1708 & $\mathrm{C} / \mathrm{z}$ & 0.0 & -30.533 & 4.763 & $\$$ & Safety Rod Guide Tube \\
\hline 1709 & $\mathrm{c} / \mathrm{z}$ & 0.0 & -30.533 & 4.920 & $\$$ & water \\
\hline 1710 & $\mathrm{c} / \mathrm{z}$ & 0.0 & -30.533 & 5.791 & $\$$ & inner radius of $\mathrm{Hf}$ safety rod \\
\hline 1711 & $\mathrm{C} / \mathrm{z}$ & 0.0 & -30.533 & 6.426 & $\$$ & o.r of $\mathrm{Hf}$ \\
\hline 1712 & $\mathrm{c} / \mathrm{z}$ & 0.0 & -30.533 & 6.826 & $\$$ & water \\
\hline 1713 & $c / z$ & 0.0 & -30.533 & 7.461 & $\$$ & Al baffle \\
\hline \multicolumn{7}{|c|}{ C } \\
\hline 1721 & $c / z$ & 0.0 & -30.533 & 7.658 & $\$$ & 1st fuel plate-inner \\
\hline C & 1722 & $\mathrm{C} / \mathrm{z}$ & 0.0 & -30.533 & 7.861 & \$ 1st fuel plate-outer \\
\hline C & 1723 & $\mathrm{c} / \mathrm{z}$ & 0.0 & -30.533 & 8.059 & \$ 2nd fuel plate-inner \\
\hline C & 1724 & $\mathrm{C} / \mathrm{z}$ & 0.0 & -30.533 & 8.186 & $\$ 2$ nd fuel plate-outer \\
\hline C & 1725 & $\mathrm{C} / \mathrm{z}$ & 0.0 & -30.533 & 8.385 & \$ 3rd fuel plate-inner \\
\hline C & 1726 & $\mathrm{C} / \mathrm{z}$ & 0.0 & -30.533 & 8.512 & \$ 3rd fuel plate-outer \\
\hline C & 1727 & $\mathrm{C} / \mathrm{z}$ & 0.0 & -30.533 & 8.710 & \$ 4 th fuel plate-inner \\
\hline 1728 & $\mathrm{c} / \mathrm{z}$ & 0.0 & -30.533 & 8.837 & $\$$ & 4th fuel plate-outer \\
\hline 1729 & $\mathrm{c} / \mathrm{z}$ & 0.0 & -30.533 & 9.0348 & $\$$ & 5th fuel plate-inner \\
\hline 1730 & $\mathrm{c} / \mathrm{z}$ & 0.0 & -30.533 & 12.413 & $\$$ & 15thfuel plate-outer \\
\hline 1731 & $\mathrm{c} / \mathrm{z}$ & 0.0 & -30.533 & 12.611 & $\$$ & 16thfuel plate-inner \\
\hline C & 1732 & $\mathrm{c} / \mathrm{z}$ & 0.0 & -30.533 & 12.738 & \$16thfuel plate-outer \\
\hline C & 1733 & $\mathrm{c} / \mathrm{z}$ & 0.0 & -30.533 & 12.936 & \$17thfuel plate-inner \\
\hline C & 1734 & $\mathrm{c} / \mathrm{z}$ & 0.0 & -30.533 & 13.063 & \$17thfuel plate-outer \\
\hline C & 1735 & $\mathrm{C} / \mathrm{z}$ & 0.0 & -30.533 & 13.261 & \$18thfuel plate-inner \\
\hline C & 1736 & $\mathrm{c} / \mathrm{z}$ & 0.0 & -30.533 & 13.388 & \$18thfuel plate-outer \\
\hline C & 1737 & $\mathrm{C} / \mathrm{z}$ & 0.0 & -30.533 & 13.586 & \$19thfuel plate-inner \\
\hline 1738 & $\mathrm{c} / \mathrm{z}$ & 0.0 & -30.533 & $3 \quad 13.84$ & $\$$ & 19thfuel plate-outer \\
\hline 1800 & $\mathrm{c} / \mathrm{z}$ & 0.0 & -30.533 & 14.133 & $\$$ & o.r. of SE loop \\
\hline C & 1796 & $\mathrm{C} / \mathrm{z}$ & 2.455 & -29.862 & 26.353 & $\$$ Be with sockets \\
\hline 1795 & $\mathrm{c} / \mathrm{z}$ & -2.455 & -29.862 & 26.353 & $\$$ & Be with sockets \\
\hline \multicolumn{7}{|l|}{ C } \\
\hline C & E loop & & & & & \\
\hline \multicolumn{7}{|l|}{ C } \\
\hline 1807 & $\mathrm{c} / \mathrm{z}$ & 15.267 & -15.267 & 4.128 & $\$$ & water \\
\hline 1808 & $\mathrm{c} / \mathrm{z}$ & 15.267 & -15.267 & 4.763 & $\$$ & Safety Rod Guide Tube \\
\hline 1809 & $\mathrm{C} / \mathrm{z}$ & 15.267 & -15.267 & 4.920 & $\$$ & water \\
\hline 1810 & $\mathrm{C} / \mathrm{z}$ & 15.267 & -15.267 & 5.791 & $\$$ & inner radius of Hf safety rod \\
\hline 1811 & $\mathrm{C} / \mathrm{z}$ & 15.267 & -15.267 & 6.426 & $\$$ & o.r. of $\mathrm{Hf}$ \\
\hline 1812 & $\mathrm{C} / \mathrm{z}$ & 15.267 & -15.267 & 6.826 & $\$$ & water \\
\hline 1813 & $\mathrm{C} / \mathrm{z}$ & 15.267 & -15.267 & 7.461 & $\$$ & Al baffle \\
\hline 1881 & $\mathrm{C} / \mathrm{z}$ & 15.267 & -15.267 & 2.85750 & $\$$ & E flux trap interior $\mathrm{H} 20$ \\
\hline 1882 & $\mathrm{C} / \mathrm{z}$ & 15.267 & -15.267 & 3.17500 & $\$$ & E flux trap housing \\
\hline 1883 & $\mathrm{c} / \mathrm{z}$ & 15.267 & -15.267 & 3.33248 & $\$$ & E water \\
\hline 1884 & $c / z$ & 15.267 & -15.267 & 3.65125 & $\$$ & E envelope tube \\
\hline \multicolumn{7}{|l|}{$\mathrm{C}$} \\
\hline 1821 & $c / z$ & 15.267 & -15.267 & 7.658 & & \$1st fuel plate-inner \\
\hline C & 1822 & $\mathrm{c} / \mathrm{z}$ & 15.267 & -15.267 & 7.861 & \$ 1st fuel plate-outer \\
\hline C & 1823 & $\mathrm{C} / \mathrm{z}$ & 15.267 & -15.267 & 8.059 & \$ 2nd fuel plate-inner \\
\hline C & 1824 & $\mathrm{C} / \mathrm{z}$ & 15.267 & -15.267 & 8.186 & \$2nd fuel plate-outer \\
\hline C & 1825 & $\mathrm{c} / \mathrm{z}$ & 15.267 & -15.267 & 8.385 & \$ 3rd fuel plate-inner \\
\hline C & 1826 & $\mathrm{C} / \mathrm{z}$ & 15.267 & -15.267 & 8.512 & \$ 3rd fuel plate-outer \\
\hline C & 1827 & $\mathrm{c} / \mathrm{z}$ & 15.267 & -15.267 & 8.710 & \$ 4th fuel plate-inner \\
\hline 1828 & $\mathrm{c} / \mathrm{z}$ & 15.267 & -15.267 & 8.837 & & \$ 4 th fuel plate-outer \\
\hline 1829 & $c / z$ & 15.267 & -15.267 & 9.0348 & & \$ 5th fuel plate-inner \\
\hline 1830 & $\mathrm{C} / \mathrm{z}$ & 15.267 & -15.267 & 12.413 & & \$15thfuel plate-outer \\
\hline 1831 & $\mathrm{c} / \mathrm{z}$ & 15.267 & -15.267 & 12.611 & & \$16thfuel plate-inner \\
\hline C & 1832 & $\mathrm{C} / \mathrm{z}$ & 15.267 & -15.267 & 12.738 & \$16thfuel plate-outer \\
\hline C & 1833 & $\mathrm{C} / \mathrm{z}$ & 15.267 & -15.267 & 12.936 & \$17thfuel plate-inner \\
\hline
\end{tabular}




\begin{tabular}{|c|c|c|c|c|c|c|}
\hline C & 1834 & $c / z$ & 5.267 & 13.0 & & \$17thfuel plate-outer \\
\hline C & 1835 & $c / z$ & 5.267 & 13.2 & & \$ 18thfuel plate-inner \\
\hline C & 1836 & $c / z$ & 5.267 & $13 \cdot 3$ & & \$18thfuel plate-outer \\
\hline C & 1837 & $c / z$ & 5.267 & 13.5 & & \$19thfuel plate-inner \\
\hline 1838 & $c / z$ & 15.267 & $-15.267 \quad 13$ & 84 & $\$$ & 19thfuel plate-outer \\
\hline 1900 & $c / z$ & 15.267 & -15.267 & 181 & $\$$ & E loop outer radius \\
\hline C & & & & & & \\
\hline 2461 & $\mathrm{pz}$ & 122.55500 & & & & \\
\hline 2460 & $\mathrm{pz}$ & 118.11000 & & & & \\
\hline 2459 & $\mathrm{pz}$ & 104.77500 & & & & \\
\hline 2458 & $\mathrm{pz}$ & 91.44000 & & & & \\
\hline 2457 & $\mathrm{pz}$ & 87.63000 & & & & \\
\hline 2456 & $\mathrm{pz}$ & 69.21500 & & & & \\
\hline 2455 & $\mathrm{pz}$ & 64.77000 & & & & \\
\hline 2454 & $\mathrm{pz}$ & 55.88000 & & & & \\
\hline 2453 & $\mathrm{pz}$ & 51.43500 & & & & \\
\hline 2452 & $\mathrm{pz}$ & 20.32000 & & & & \\
\hline 2451 & $\mathrm{pz}$ & -1.90500 & & & & \\
\hline C & & & & & & \\
\hline 2413 & $\mathrm{pz}$ & 156.84500 & & & & \\
\hline 2412 & $\mathrm{pz}$ & 139.06500 & & & & \\
\hline 2411 & $\mathrm{pz}$ & 121.28500 & & & & \\
\hline 2410 & $\mathrm{pz}$ & 103.50500 & & & & \\
\hline 2409 & $\mathrm{pz}$ & 85.72500 & & & & \\
\hline 2408 & $\mathrm{pz}$ & 67.94500 & & & & \\
\hline 2407 & $\mathrm{pz}$ & 50.16500 & & & & \\
\hline 2406 & $\mathrm{pz}$ & 32.38500 & & & & \\
\hline 2405 & $\mathrm{pz}$ & 27.94000 & & & & \\
\hline 2404 & $\mathrm{pz}$ & 19.05000 & & & & \\
\hline 2403 & $\mathrm{pz}$ & 1.27000 & & & & \\
\hline 2402 & $\mathrm{pz}$ & -16.51000 & & & & \\
\hline 2401 & $\mathrm{pz}$ & -34.29000 & & & & \\
\hline C & & & & & & \\
\hline C & & & & & & \\
\hline C & C Flux & Trap & & & & \\
\hline C & & & & & & \\
\hline 11006 & $\mathrm{Cz}$ & 3.62966 & & & $\$$ & ITV center filler outer radius \\
\hline 11007 & $\mathrm{Cz}$ & 3.73880 & & & $\$$ & ITV filter inner radius \\
\hline 11008 & $\mathrm{Cz}$ & 3.98780 & & & $\$$ & ITV filter outer radius \\
\hline 11130 & $c / z$ & 1.98730 & 0.53249 & 0.64770 & $\$$ & ITV NE interior void radius \\
\hline 11140 & $c / z$ & 1.98730 & 0.53249 & 0.95250 & $\$$ & ITV NE seal ring inner radius \\
\hline 11150 & $c / z$ & 1.98730 & 0.53249 & 1.23190 & $\$$ & ITV NE seal ring outer radius \\
\hline 11160 & $c / z$ & 1.98730 & 0.53249 & 1.27000 & $\$$ & ITV NE gas tube inner radius \\
\hline 11170 & $c / z$ & 1.98730 & 0.53249 & 1.39700 & $\$$ & ITV NE gas tube outer radius \\
\hline 11180 & $c / z$ & 1.98730 & 0.53249 & 1.55956 & $\$$ & ITV NE pressure tube outer radius \\
\hline 11190 & $c / z$ & 1.98730 & 0.53249 & 1.66878 & $\$$ & ITV NE test annulus outer radius \\
\hline 11230 & $c / z$ & -0.53249 & -1.98730 & 0.64770 & $\$$ & ITV SE interior void radius \\
\hline 11240 & $c / z$ & -0.53249 & -1.98730 & 0.95250 & $\$$ & ITV SE seal ring inner radius \\
\hline 11250 & $\mathrm{c} / \mathrm{z}$ & -0.53249 & -1.98730 & 1.23190 & $\$$ & ITV SE seal ring outer radius \\
\hline 11260 & $c / z$ & -0.53249 & -1.98730 & 1.27000 & $\$$ & ITV SE gas tube inner radius \\
\hline 11270 & $c / z$ & -0.53249 & -1.98730 & 1.39700 & $\$$ & ITV SE gas tube outer radius \\
\hline 11280 & $c / z$ & -0.53249 & -1.98730 & 1.55956 & $\$$ & ITV SE pressure tube outer radius \\
\hline 11290 & $c / z$ & -0.53249 & -1.98730 & 1.66878 & $\$$ & ITV SE test annulus outer radius \\
\hline 11770 & $c / z$ & 1.97566 & -1.97566 & 0.11430 & $\$$ & ITV E FM sheath inner radius \\
\hline 11780 & $c / z$ & 1.97566 & -1.97566 & 0.23876 & $\$$ & ITV E FM sheath outer radius \\
\hline 11790 & $c / z$ & 1.97566 & -1.97566 & 0.31750 & $\$$ & ITV E FM position outer radius \\
\hline C & & & & & & \\
\hline $\mathrm{C}$ & & & & & & \\
\hline C & 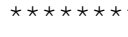 & $\star \star \star \star * \star * \star * \star * \star *$ & $\approx * \star \star \star \star * \star * \star * *$ & $\approx \star \star \star \star \star * ⿻ 一 𠃋 十$ & 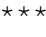 & 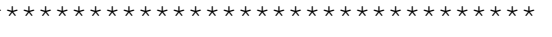 \\
\hline C & & & & & & \\
\hline C & & NE Flux & Trap & & & (beginning) \\
\hline C & & & & & & \\
\hline C & $\star \star \star \star \star * * *$ & $\star \star \star \star \star \star * \star * \star *$ & 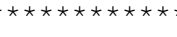 & $\star \star \star \star \star \star * \star * *$ & & 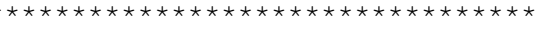 \\
\hline C & & & & & & \\
\hline C & & & & & & \\
\hline C & $\mathrm{NE}$ Expe & riment Axia & l Levels & & & \\
\hline C & & & & & & \\
\hline 2221 & $\mathrm{pz}$ & 129.84480 & & & & \\
\hline 2220 & $\mathrm{pz}$ & 104.47020 & & & & \\
\hline 2219 & $\mathrm{pz}$ & 102.53980 & & & & \\
\hline 2218 & $\mathrm{pz}$ & 77.16520 & & & & \\
\hline
\end{tabular}




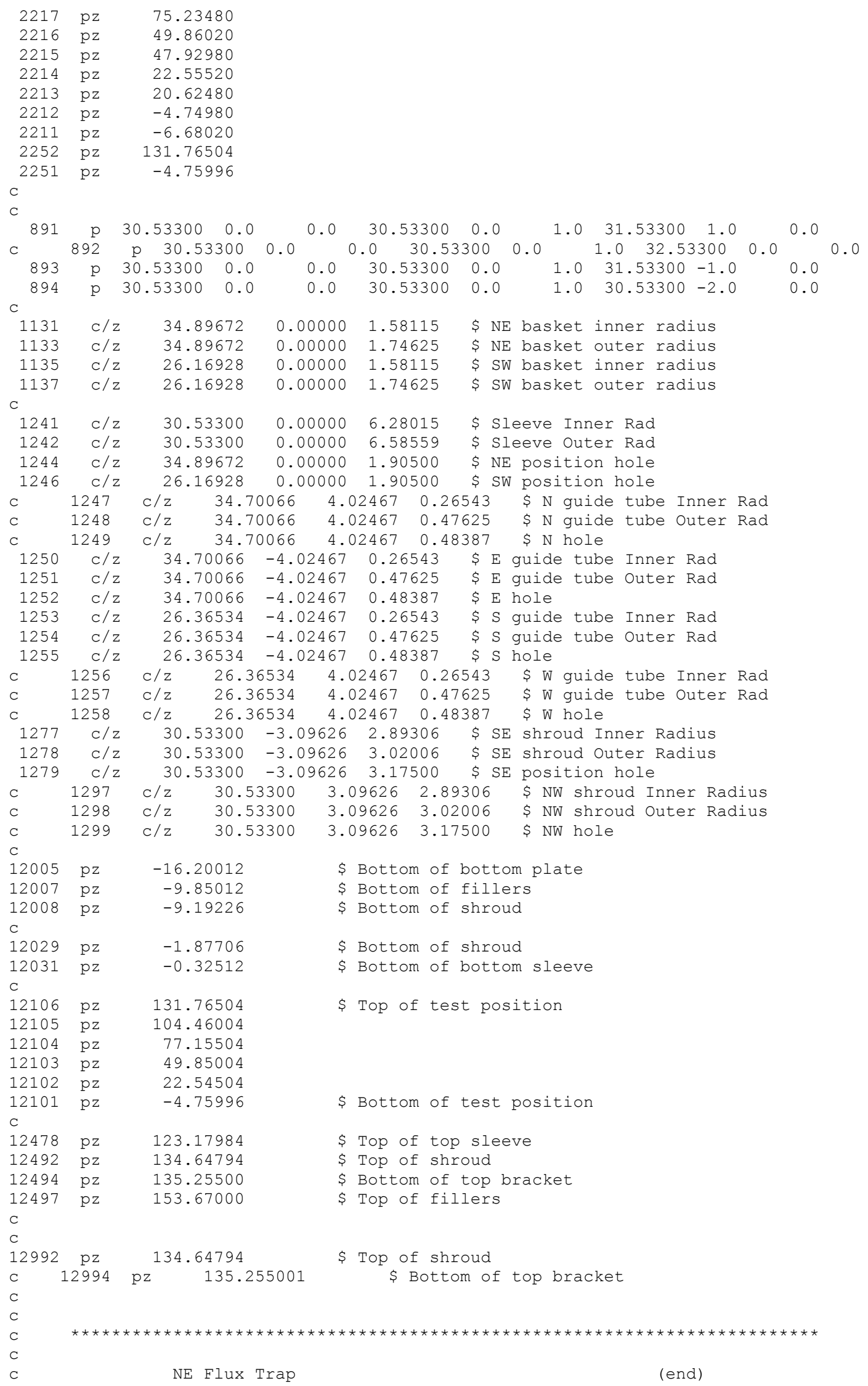




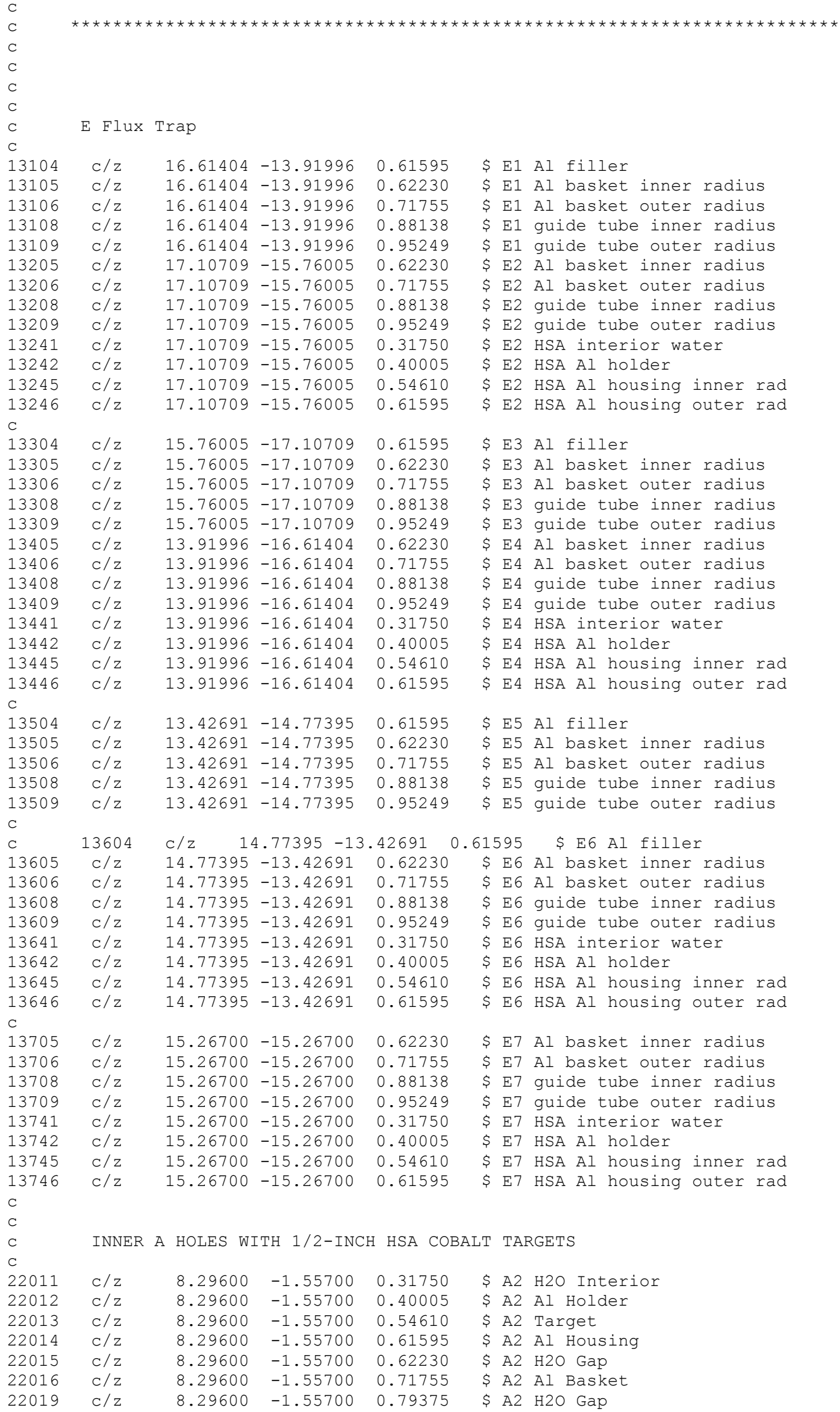




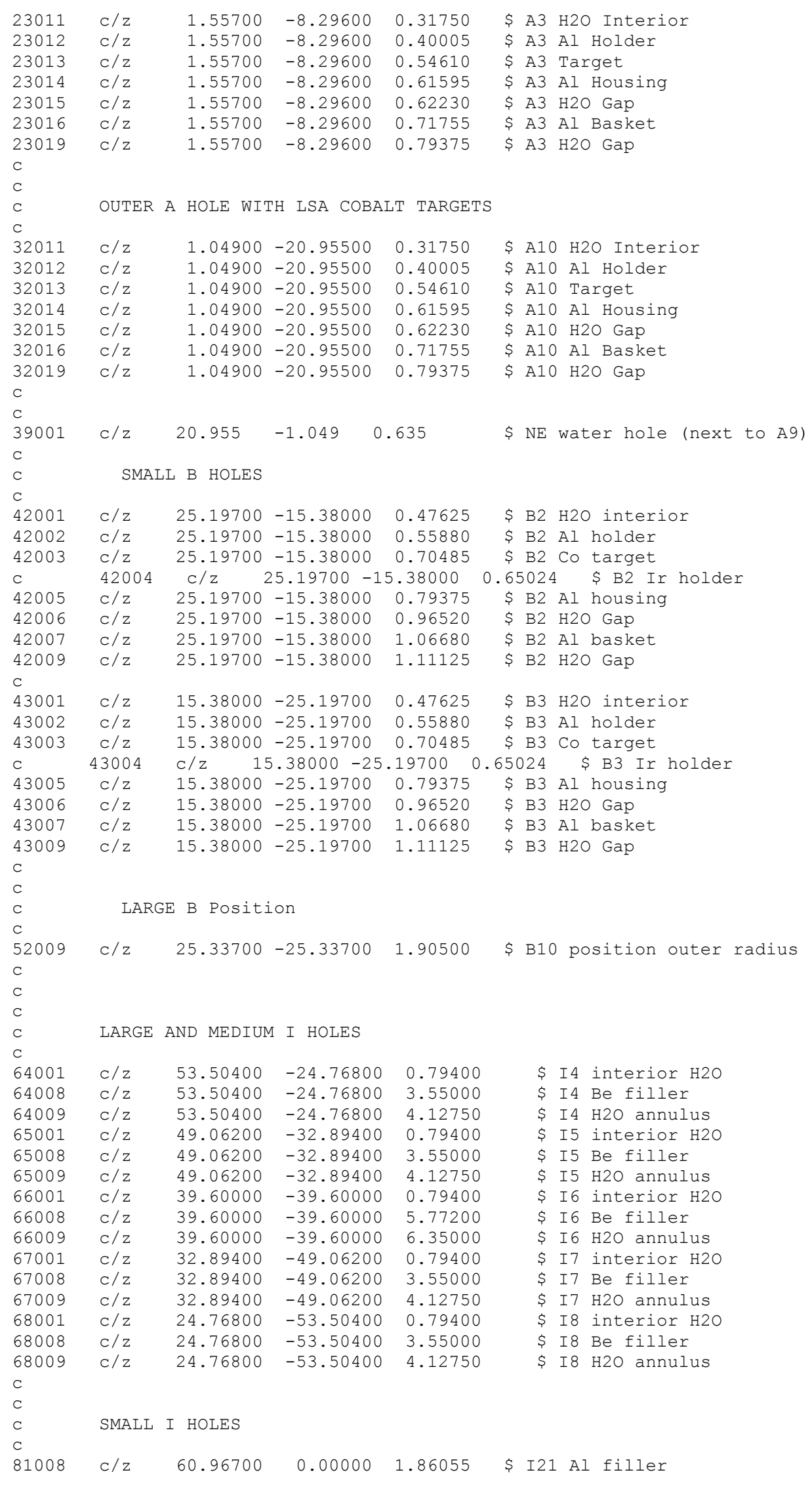




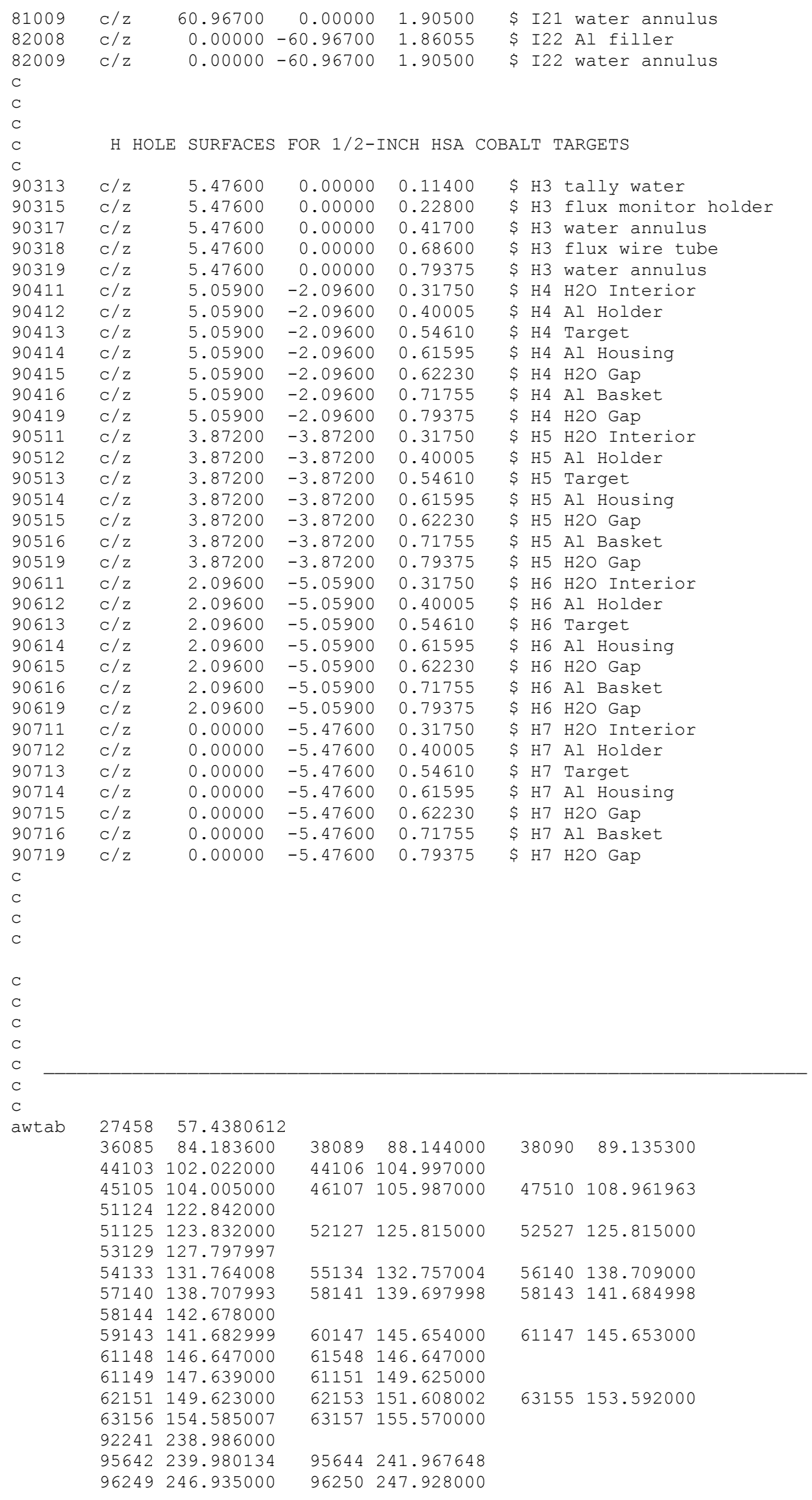




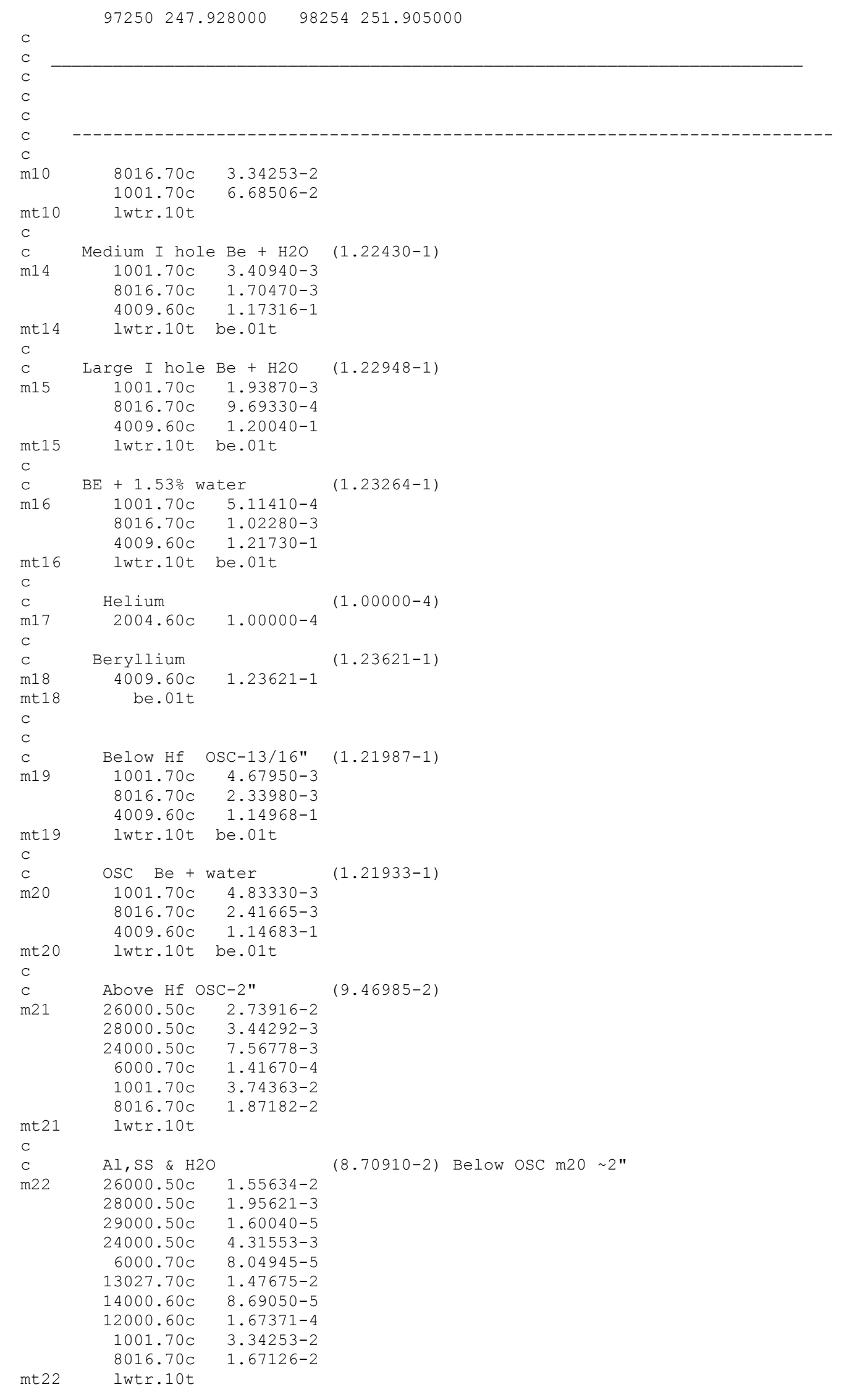




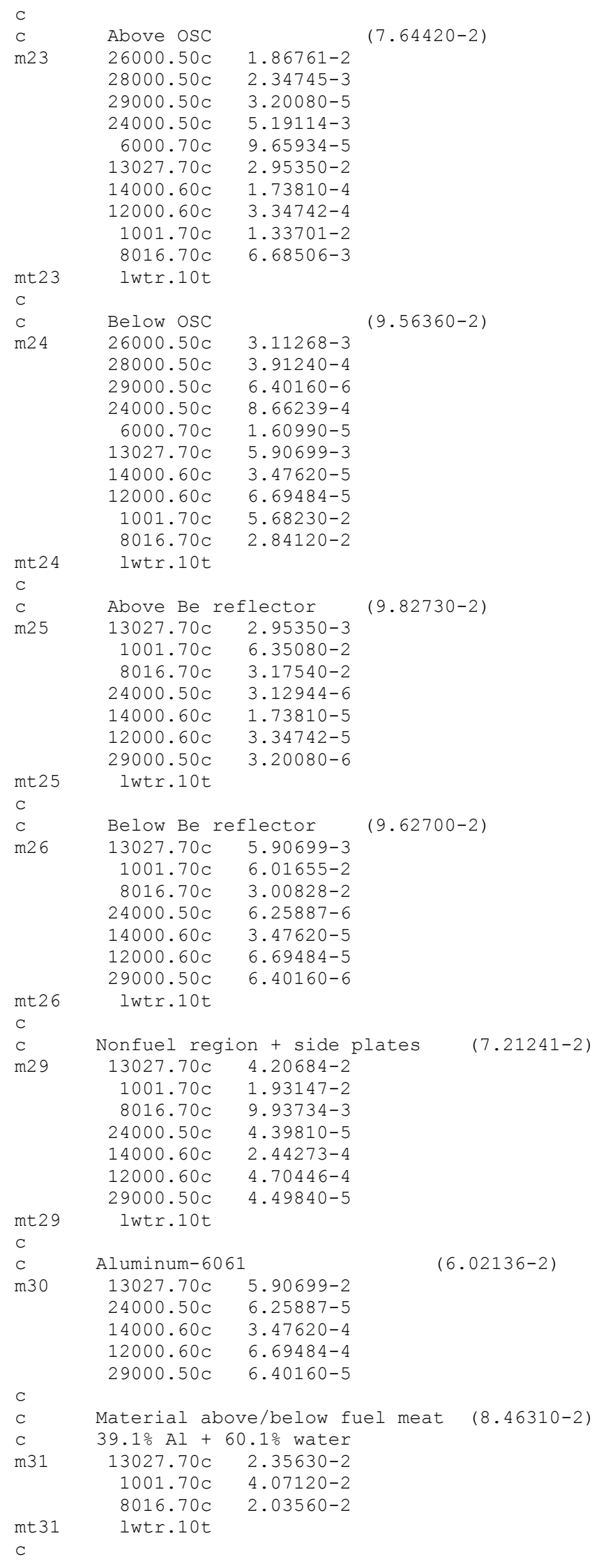




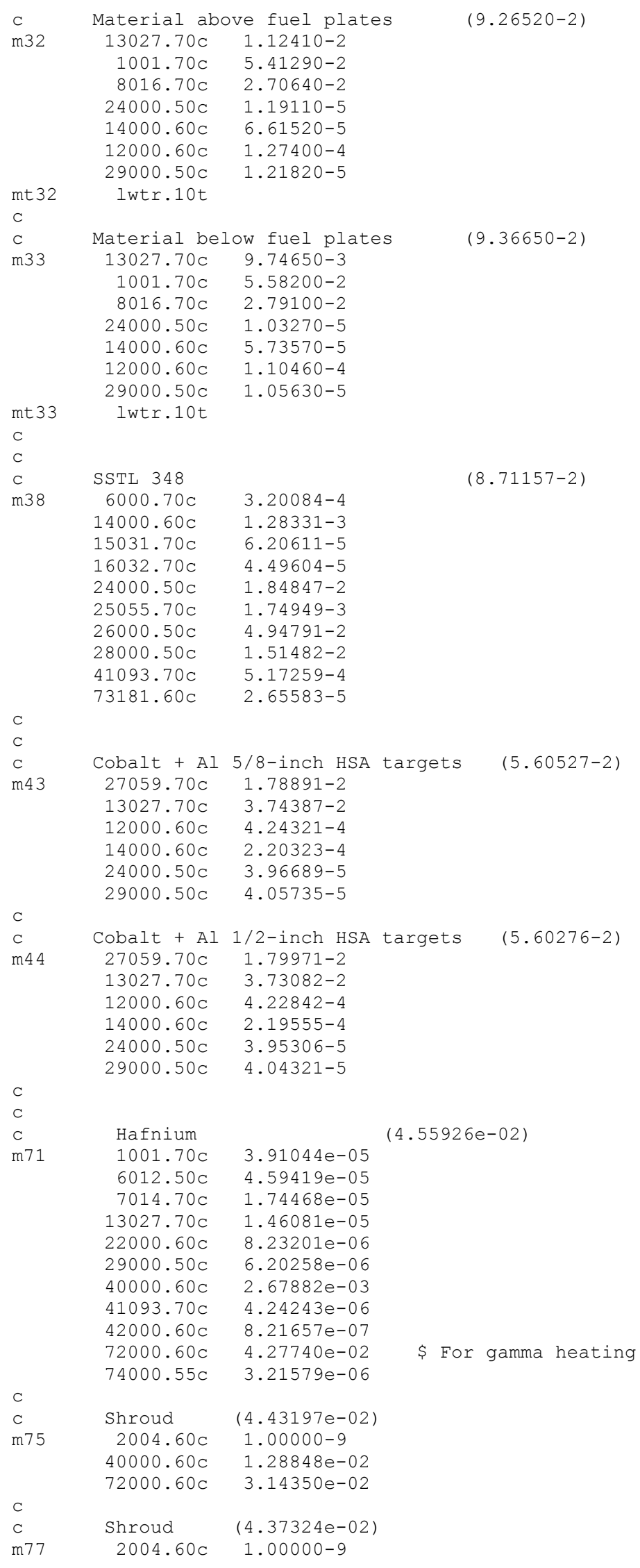




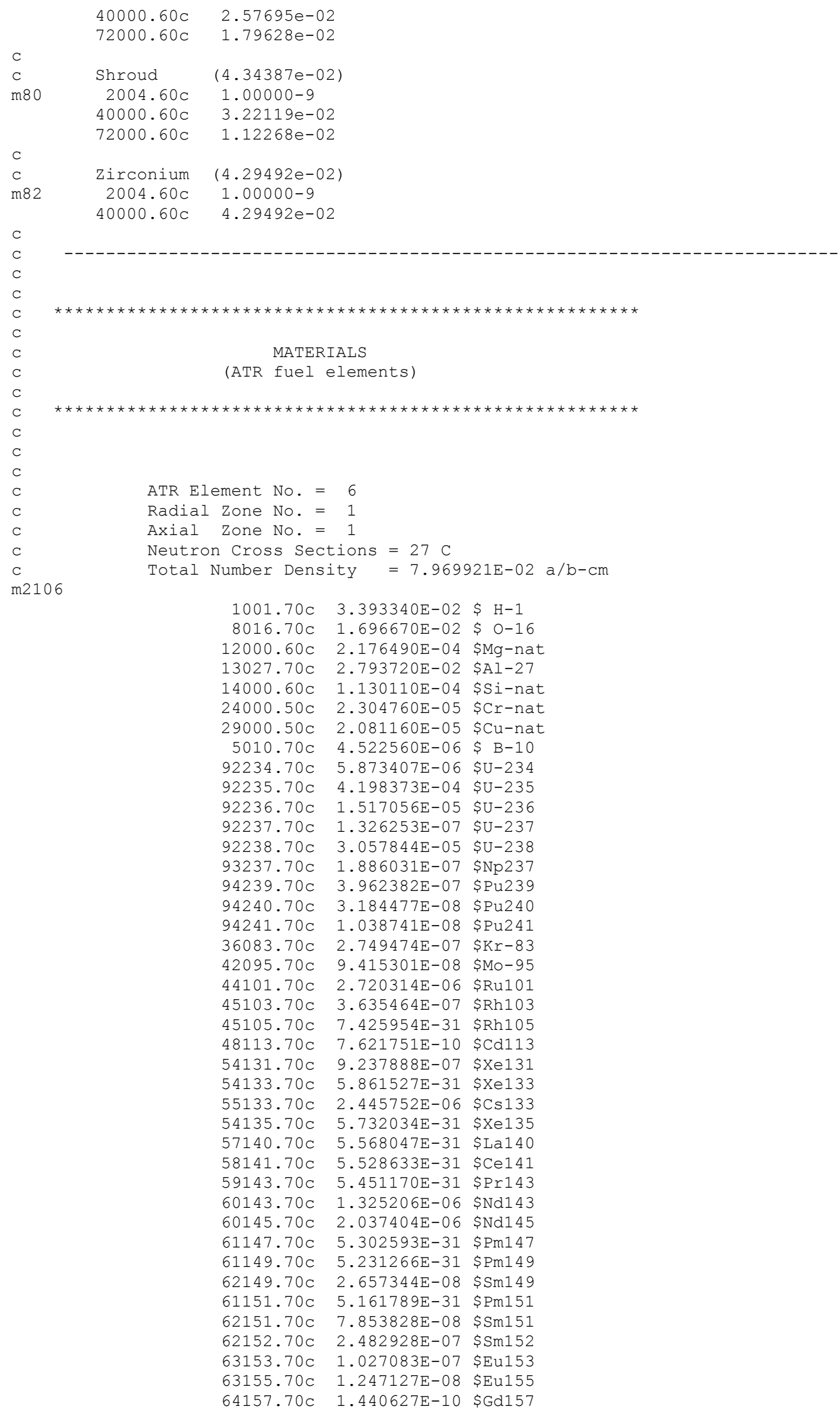




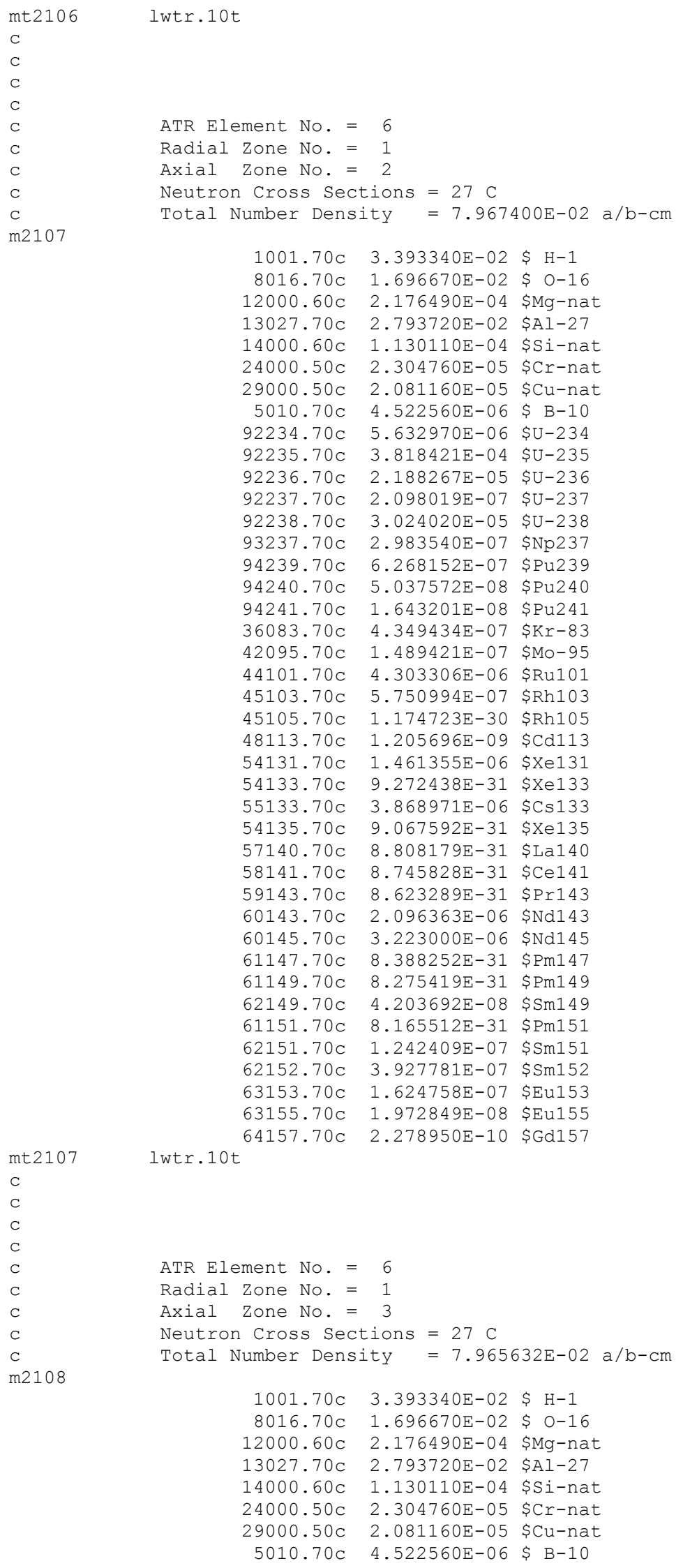




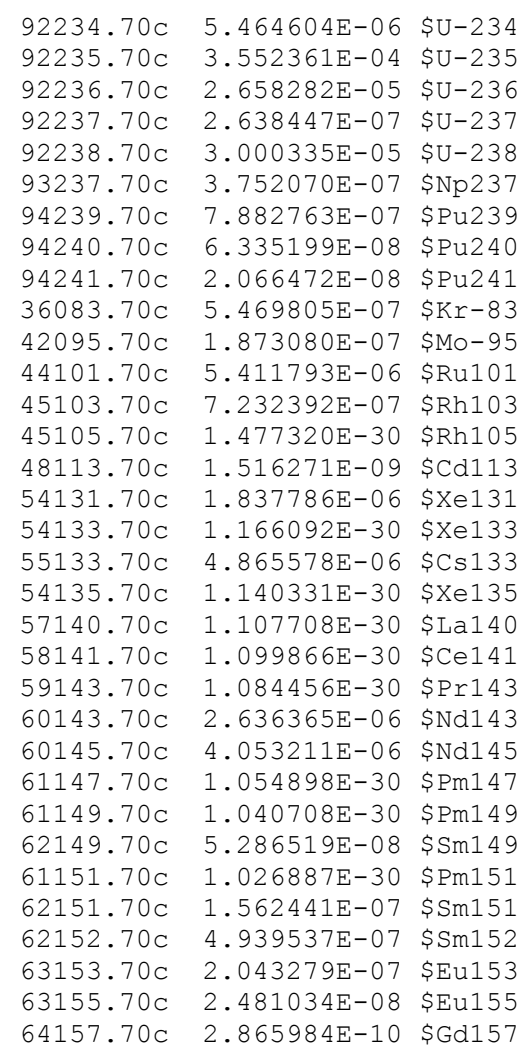

mt2108 lwtr.10t

C

C

$\mathrm{C}$

C

C ATR Element No. = 6

C Radial Zone No. = 1

C Axial Zone No. = 4

C Neutron Cross Sections $=27 \mathrm{C}$

Total Number Density $=7.964923 \mathrm{E}-02 \mathrm{a} / \mathrm{b}-\mathrm{cm}$

m2109

\begin{tabular}{|c|c|c|}
\hline $1001.70 \mathrm{C}$ & $3.393340 \mathrm{E}-02$ & $\$ \mathrm{H}-1$ \\
\hline $8016.70 \mathrm{c}$ & $1.696670 \mathrm{E}-02$ & $\$ 0-16$ \\
\hline $12000.60 \mathrm{C}$ & $2.176490 \mathrm{E}-04$ & $\$ M g-n a t$ \\
\hline $13027.70 \mathrm{C}$ & $2.793720 \mathrm{E}-02$ & $\$ A l-27$ \\
\hline $14000.60 \mathrm{C}$ & $1.130110 \mathrm{E}-04$ & \$Si-nat \\
\hline $24000.50 \mathrm{c}$ & $2.304760 \mathrm{E}-05$ & \$Cr-nat \\
\hline $29000.50 \mathrm{C}$ & $2.081160 \mathrm{E}-05$ & $\$ \mathrm{Cu}-\mathrm{nat}$ \\
\hline $5010.70 \mathrm{c}$ & $4.522561 \mathrm{E}-06$ & $\$ B-10$ \\
\hline $92234.70 \mathrm{C}$ & $5.396837 \mathrm{E}-06$ & $\$ U-234$ \\
\hline $92235.70 \mathrm{C}$ & $3.445273 E-04$ & $\$ U-235$ \\
\hline $92236.70 \mathrm{C}$ & $2.847462 \mathrm{E}-05$ & $\$ U-236$ \\
\hline $92237.70 \mathrm{C}$ & $2.855968 \mathrm{E}-07$ & $\$ U-237$ \\
\hline $92238.70 \mathrm{C}$ & $2.990802 \mathrm{E}-05$ & $\$ U-238$ \\
\hline $93237.70 \mathrm{C}$ & $4.061401 E-07$ & $\$ N p 237$ \\
\hline $94239.70 \mathrm{C}$ & $8.532641 E-07$ & \$Pu239 \\
\hline $94240.70 \mathrm{C}$ & $6.857490 \mathrm{E}-08$ & $\$ \mathrm{Pu} 240$ \\
\hline $94241.70 \mathrm{C}$ & $2.236838 \mathrm{E}-08$ & \$Pu241 \\
\hline $36083.70 \mathrm{C}$ & $5.920750 \mathrm{E}-07$ & $\$ K r-83$ \\
\hline $42095.70 \mathrm{C}$ & $2.027502 \mathrm{E}-07$ & $\$ M o-95$ \\
\hline $44101.70 \mathrm{C}$ & $5.857956 \mathrm{E}-06$ & \$Ru101 \\
\hline $45103.70 \mathrm{C}$ & $7.828650 \mathrm{E}-07$ & $\$ R h 103$ \\
\hline $45105.70 \mathrm{C}$ & $1.599114 \mathrm{E}-30$ & \$Rh105 \\
\hline $48113.70 \mathrm{C}$ & $1.641277 \mathrm{E}-09$ & $\$ C d 113$ \\
\hline $54131.70 \mathrm{C}$ & $1.989298 \mathrm{E}-06$ & $\$ x e 131$ \\
\hline $3.70 \mathrm{c}$ & $1.262228 \mathrm{E}-30$ & $\$ x e 133$ \\
\hline $55133.70 \mathrm{C}$ & $5.266710 \mathrm{E}-06$ & $\$ C s 133$ \\
\hline $54135.70 \mathrm{C}$ & $1.234343 E-30$ & $\$ x e 135$ \\
\hline
\end{tabular}


$57140.70 \mathrm{C} \quad 1.199030 \mathrm{E}-30 \quad$ Sa140

$58141.70 \mathrm{C} \quad 1.190542 \mathrm{E}-30 \$ \mathrm{Ce} 141$

$59143.70 \mathrm{C} \quad 1.173861 \mathrm{E}-30 \quad \$ \operatorname{Pr} 143$

$60143.70 \mathrm{C} \quad 2.853714 \mathrm{E}-06 \$ \mathrm{Nd} 143$

$60145.70 \mathrm{C} \quad 4.387370 \mathrm{E}-06 \$ \mathrm{Nd145}$

$61147.70 \mathrm{C} \quad 1.141867 \mathrm{E}-30 \quad \$ \mathrm{Pm} 147$

$61149.70 \mathrm{C} \quad 1.126507 \mathrm{E}-30 \$ \operatorname{Pm} 149$

$62149.70 \mathrm{C} \quad 5.722355 \mathrm{E}-08 \quad \$ \mathrm{Sm} 149$

$61151.70 \mathrm{C} \quad 1.111546 \mathrm{E}-30$ \$Pm151

$62151.70 \mathrm{C} \quad 1.691252 \mathrm{E}-07$ \$Sm151

$62152.70 \mathrm{C} \quad 5.346767 \mathrm{E}-07 \quad \$$ Sm152

$63153.70 \mathrm{C} \quad 2.211732 \mathrm{E}-07 \quad \$ \mathrm{Eu} 153$

$63155.70 \mathrm{C} \quad 2.685577 \mathrm{E}-08 \quad$ \$Eu155

$\begin{array}{lll}64157.70 \mathrm{C} \quad 3.102264 \mathrm{E}-10 \quad \$ G d 157 & \end{array}$

C

C

C

C

m2110

mt 2110

lwtr.10t
ATR Element No. $=6$

Radial Zone No. $=1$

Axial Zone No. = 5

Neutron Cross Sections $=27 \mathrm{C}$

Total Number Density $=7.965902 \mathrm{E}-02 \mathrm{a} / \mathrm{b}-\mathrm{cm}$

$1001.70 \mathrm{C} \quad 3.393340 \mathrm{E}-02$ \$ $\mathrm{H}-1$

$8016.70 \mathrm{C} \quad 1.696670 \mathrm{E}-02 \$ 0-16$

$12000.60 \mathrm{C} \quad 2.176490 \mathrm{E}-04$ \$Mg-nat

$13027.70 \mathrm{C} \quad 2.793720 \mathrm{E}-02$ \$Al-27

$14000.60 \mathrm{C} \quad 1.130110 \mathrm{E}-04$ \$Si-nat

$24000.50 \mathrm{C} \quad 2.304760 \mathrm{E}-05$ \$Cr-nat

$29000.50 \mathrm{C} \quad 2.081160 \mathrm{E}-05$ \$Cu-nat

$5010.70 \mathrm{C} \quad 4.522560 \mathrm{E}-06$ \$ B-10

$92234.70 \mathrm{C} \quad 5.490160 \mathrm{E}-06$ \$U-234

$92235.70 \mathrm{C} \quad 3.592745 \mathrm{E}-04$ \$U-235

$92236.70 \mathrm{C} \quad 2.586940 \mathrm{E}-05$ \$U-236

$92237.70 \mathrm{C} \quad 2.556417 \mathrm{E}-07 \quad \$ \mathrm{U}-237$

$92238.70 \mathrm{C} \quad 3.003930 \mathrm{E}-05$ \$U-238

$93237.70 \mathrm{C} \quad 3.635416 \mathrm{E}-07$ \$Np237

$94239.70 \mathrm{C} \quad 7.637686 \mathrm{E}-07$ \$Pu239

$94240.70 \mathrm{C} \quad 6.138235 \mathrm{E}-08$ \$Pu240

$94241.70 \mathrm{C} \quad 2.002225 \mathrm{E}-08$ \$Pu241

$36083.70 \mathrm{C} \quad 5.299746 \mathrm{E}-07 \quad \$ \mathrm{Kr}-83$

$42095.70 \mathrm{C} \quad 1.814845 \mathrm{E}-07$ \$Mo-95

$44101.70 \mathrm{C} \quad 5.243538 \mathrm{E}-06$ \$Ru101

$45103.70 \mathrm{C} \quad 7.007535 \mathrm{E}-07$ \$Rh103

$45105.70 \mathrm{C} \quad 1.431389 \mathrm{E}-30$ \$Rh105

$48113.70 \mathrm{C} \quad 1.469130 \mathrm{E}-09$ \$Cd113

$54131.70 \mathrm{C} \quad 1.780648 \mathrm{E}-06$ \$Xe131

$54133.70 \mathrm{C} \quad 1.129838 \mathrm{E}-30$ \$Xe133

$55133.70 \mathrm{C} \quad 4.714306 \mathrm{E}-06 \$ \mathrm{Cs} 133$

$54135.70 \mathrm{C} \quad 1.104878 \mathrm{E}-30$ \$Xe135

$57140.70 \mathrm{C} \quad 1.073269 \mathrm{E}-30$ \$La140

$58141.70 \mathrm{C} \quad 1.065671 \mathrm{E}-30 \quad \$ \mathrm{Ce} 141$

$59143.70 \mathrm{C} \quad 1.050740 \mathrm{E}-30 \quad \$ \operatorname{Pr} 143$

$60143.70 \mathrm{C} \quad 2.554399 \mathrm{E}-06 \$ \mathrm{Nd} 143$

$60145.70 \mathrm{C} \quad 3.927196 \mathrm{E}-06$ \$Nd145

$61147.70 \mathrm{C} \quad 1.022101 \mathrm{E}-30$ \$Pm147

$61149.70 \mathrm{C} \quad 1.008352 \mathrm{E}-30$ \$Pm149

$62149.70 \mathrm{C} \quad 5.122159 \mathrm{E}-08$ \$Sm149

$61151.70 \mathrm{C} \quad 9.949601 \mathrm{E}-31$ \$Pm151

$62151.70 \mathrm{C} \quad 1.513864 \mathrm{E}-07$ \$Sm151

$62152.70 \mathrm{C} \quad 4.785965 \mathrm{E}-07$ \$Sm152

$63153.70 \mathrm{C} \quad 1.979753 \mathrm{E}-07$ \$Eu153

$63155.70 \mathrm{C} \quad 2.403898 \mathrm{E}-08$ \$Eu155

$64157.70 \mathrm{C} \quad 2.776880 \mathrm{E}-10$ \$Gd157

$\mathrm{C}$

C

C 


\begin{tabular}{|c|c|c|c|}
\hline C & \multicolumn{3}{|c|}{ ATR Element No. $=6$} \\
\hline C & Radial Zone No. = & 1 & \\
\hline C & Zone No. = & 6 & \\
\hline C & \multicolumn{3}{|c|}{ Neutron Cross Sections $=27 \mathrm{C}$} \\
\hline C & Total Number Dens: & $=7.967913 \mathrm{E}-02$ & $\mathrm{a} / \mathrm{b}-\mathrm{cm}$ \\
\hline \multicolumn{4}{|l|}{ m2111 } \\
\hline & $1001.70 \mathrm{C}$ & $3.393340 \mathrm{E}-02 \$ \mathrm{H}-1$ & \\
\hline & $8016.70 \mathrm{C}$ & $1.696670 \mathrm{E}-02 \$ 0-16$ & \\
\hline & $12000.60 \mathrm{c}$ & $2.176490 \mathrm{E}-04$ \$Mg-nat & \\
\hline & $13027.70 \mathrm{C}$ & $2.793720 \mathrm{E}-02$ \$AI-27 & \\
\hline & $14000.60 \mathrm{c}$ & $1.130110 \mathrm{E}-04$ \$Si-nat & \\
\hline & $24000.50 \mathrm{c}$ & $2.304760 \mathrm{E}-05$ \$Cr-nat & \\
\hline & $29000.50 \mathrm{C}$ & $2.081160 \mathrm{E}-05$ \$Cu-nat & \\
\hline & $5010.70 \mathrm{c}$ & $4.522560 \mathrm{E}-06 \$ \mathrm{~B}-10$ & \\
\hline & $92234.70 \mathrm{C}$ & $5.682128 E-06 \$ U-234$ & \\
\hline & $92235.70 \mathrm{C}$ & $3.896104 \mathrm{E}-04 \quad \$ U-235$ & \\
\hline & $92236.70 \mathrm{C}$ & $2.051036 \mathrm{E}-05 \$ \mathrm{U}-236$ & \\
\hline & $92237.70 \mathrm{C}$ & $1.940229 \mathrm{E}-07 \quad \$ U-237$ & \\
\hline & $92238.70 \mathrm{C}$ & $3.030936 \mathrm{E}-05 \$ \mathrm{U}-238$ & \\
\hline & $93237.70 \mathrm{C}$ & $2.759150 \mathrm{E}-07 \quad \$ \mathrm{~Np} 237$ & \\
\hline & $94239.70 \mathrm{C}$ & $5.796729 \mathrm{E}-07$ \$Pu239 & \\
\hline & $94240.70 \mathrm{C}$ & $4.658700 \mathrm{E}-08$ \$Pu 240 & \\
\hline & $94241.70 \mathrm{C}$ & $1.519617 \mathrm{E}-08$ \$Pu241 & \\
\hline & $36083.70 \mathrm{C}$ & $4.022317 E-07 \$ K r-83$ & \\
\hline & $42095.70 \mathrm{c}$ & $1.377402 \mathrm{E}-07$ \$Mo-95 & \\
\hline & $44101.70 \mathrm{c}$ & $3.979657 \mathrm{E}-06$ \$Ru101 & \\
\hline & $45103.70 \mathrm{c}$ & $5.318466 \mathrm{E}-07$ \$Rh103 & \\
\hline & $45105.70 \mathrm{C}$ & $1.086373 \mathrm{E}-30 \$ \mathrm{Rh} 105$ & \\
\hline & $48113.70 \mathrm{C}$ & $1.115017 \mathrm{E}-09 \$ \mathrm{Cd} 113$ & \\
\hline & $54131.70 \mathrm{c}$ & $1.351448 E-06 \$ \operatorname{Xe131}$ & \\
\hline & $54133.70 \mathrm{c}$ & $8.575064 \mathrm{E}-31$ \$Xe133 & \\
\hline & $55133.70 \mathrm{C}$ & $3.577989 \mathrm{E}-06 \$ \mathrm{Cs} 133$ & \\
\hline & $54135.70 \mathrm{C}$ & $8.385625 \mathrm{E}-31 \$ \mathrm{Xe1} 35$ & \\
\hline & $57140.70 \mathrm{C}$ & $8.145722 \mathrm{E}-31$ \$La140 & \\
\hline & $58141.70 \mathrm{C}$ & $8.088060 \mathrm{E}-31$ \$Ce141 & \\
\hline & $59143.70 \mathrm{C}$ & $7.974737 \mathrm{E}-31 \quad \$ \operatorname{Pr} 143$ & \\
\hline & $60143.70 \mathrm{c}$ & $1.938697 \mathrm{E}-06 \$ \mathrm{Nd1} 43$ & \\
\hline & $60145.70 \mathrm{C}$ & $2.980600 \mathrm{E}-06 \$ \mathrm{Nd1} 45$ & \\
\hline & $61147.70 \mathrm{C}$ & $7.757377 \mathrm{E}-31 \quad \$ \operatorname{Pm} 147$ & \\
\hline & $61149.70 \mathrm{C}$ & $7.653031 E-31 \$ \operatorname{Pm} 149$ & \\
\hline & $62149.70 \mathrm{c}$ & $3.887535 E-08 \$ \operatorname{Sm} 149$ & \\
\hline & $61151.70 \mathrm{C}$ & $7.551389 \mathrm{E}-31 \quad \$ \operatorname{Pm} 151$ & \\
\hline & $62151.70 \mathrm{c}$ & $1.148968 \mathrm{E}-07$ \$Sm151 & \\
\hline & $62152.70 \mathrm{C}$ & $3.632376 \mathrm{E}-07 \$ \operatorname{Sm} 152$ & \\
\hline & $63153.70 \mathrm{c}$ & $1.502561 \mathrm{E}-07$ \$Eu153 & \\
\hline & $63155.70 \mathrm{C}$ & $1.824472 E-08$ \$Eu155 & \\
\hline & $64157.70 \mathrm{C}$ & $2.107552 \mathrm{E}-10$ \$Gd157 & \\
\hline mt2111 & \multicolumn{3}{|l|}{ lwtr.10t } \\
\hline \multicolumn{4}{|l|}{ C } \\
\hline \multicolumn{4}{|l|}{ C } \\
\hline \multicolumn{4}{|l|}{ C } \\
\hline \multicolumn{4}{|l|}{ C } \\
\hline C & ATR Element No. = & 6 & \\
\hline C & Radial Zone No. = & 1 & \\
\hline C & Zone No. = & 7 & \\
\hline C & Neutron Cross Sect & tions $=27 \mathrm{C}$ & \\
\hline C & Total Number Dens: & $=7.971317 \mathrm{E}-02$ & $\mathrm{a} / \mathrm{b}-\mathrm{cm}$ \\
\hline \multicolumn{4}{|l|}{ m2112 } \\
\hline & $1001.70 \mathrm{C}$ & $3.393340 \mathrm{E}-02$ \$ $\mathrm{H}-1$ & \\
\hline & $8016.70 \mathrm{C}$ & $1.696670 \mathrm{E}-02 \$ 0-16$ & \\
\hline & $12000.60 \mathrm{c}$ & $2.176490 \mathrm{E}-04$ \$Mg-nat & \\
\hline & $13027.70 \mathrm{C}$ & $2.793720 \mathrm{E}-02 \quad \$ \mathrm{Al}-27$ & \\
\hline & $14000.60 \mathrm{C}$ & 1.130110E-04 \$Si-nat & \\
\hline & $24000.50 \mathrm{C}$ & $2.304760 \mathrm{E}-05$ \$Cr-nat & \\
\hline & $29000.50 \mathrm{C}$ & $2.081160 \mathrm{E}-05 \$ \mathrm{Cu}-\mathrm{nat}$ & \\
\hline & $5010.70 \mathrm{C}$ & $4.522560 E-06 \$ B-10$ & \\
\hline & $92234.70 \mathrm{C}$ & $6.006495 \mathrm{E}-06 \$ \mathrm{U}-234$ & \\
\hline & $92235.70 \mathrm{C}$ & $4.408686 \mathrm{E}-04 \$ \mathrm{~S}-235$ & \\
\hline & $92236.70 \mathrm{C}$ & $1.145522 \mathrm{E}-05 \$ \mathrm{U}-236$ & \\
\hline & $92237.70 \mathrm{C}$ & $8.990592 \mathrm{E}-08$ \$U-237 & \\
\hline & $92238.70 \mathrm{c}$ & $3.076566 \mathrm{E}-05 \$ \mathrm{U}-238$ & \\
\hline
\end{tabular}




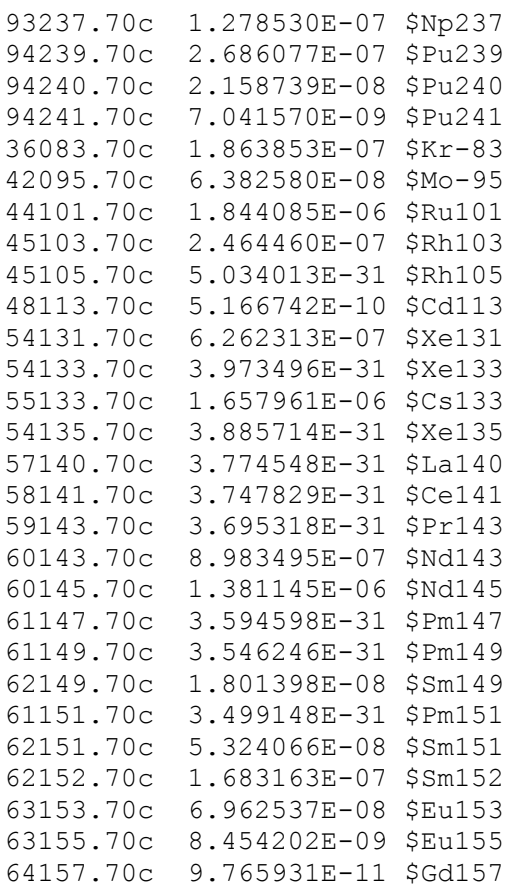
mt2112 lwtr.10t

C

$\mathrm{C}$

C ATR Element No. = 6

C Radial Zone No. $=2$

C Axial Zone No. = 1

C Neutron Cross Sections $=27 \mathrm{C}$

Total Number Density $=8.290653 \mathrm{E}-02 \mathrm{a} / \mathrm{b}-\mathrm{cm}$

m2113

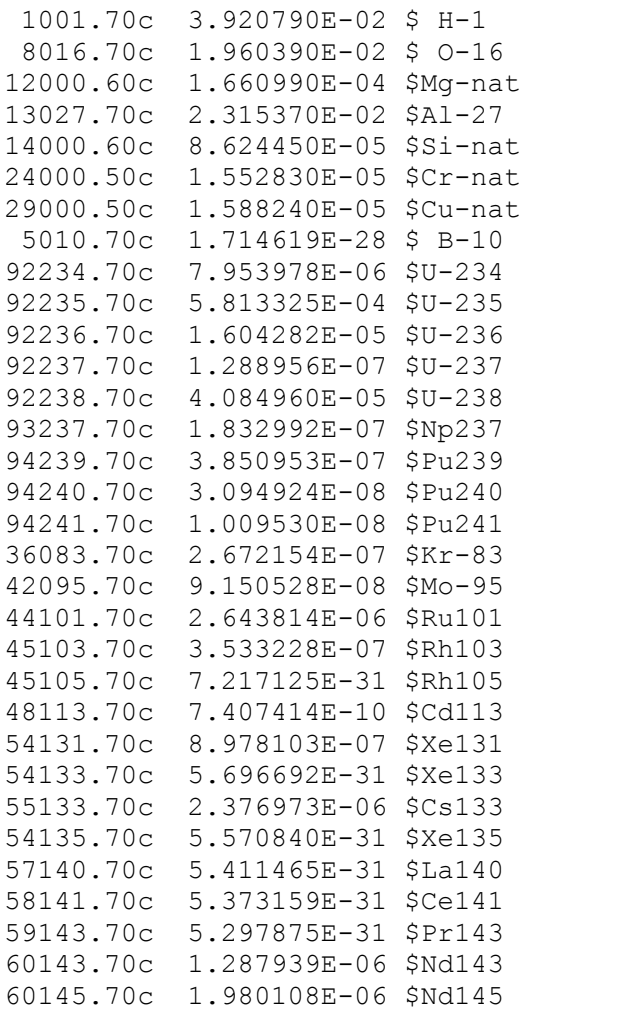




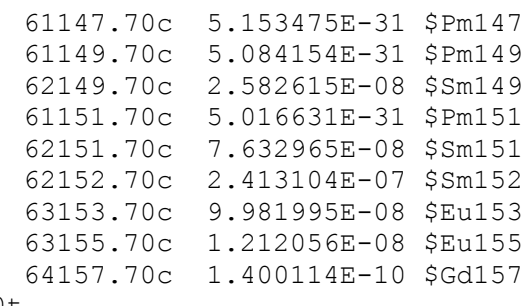

mt2113
C
C
C
C
C
C
C
C
C
m2114 lwtr.10t

$64157.70 \mathrm{C} \quad 1.400114 \mathrm{E}-10 \quad \$ \mathrm{Gd} 157$

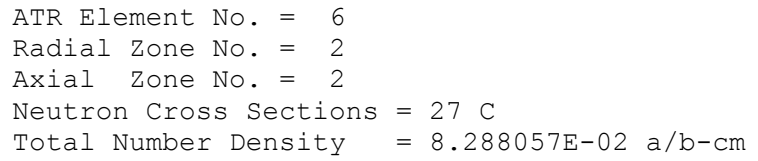




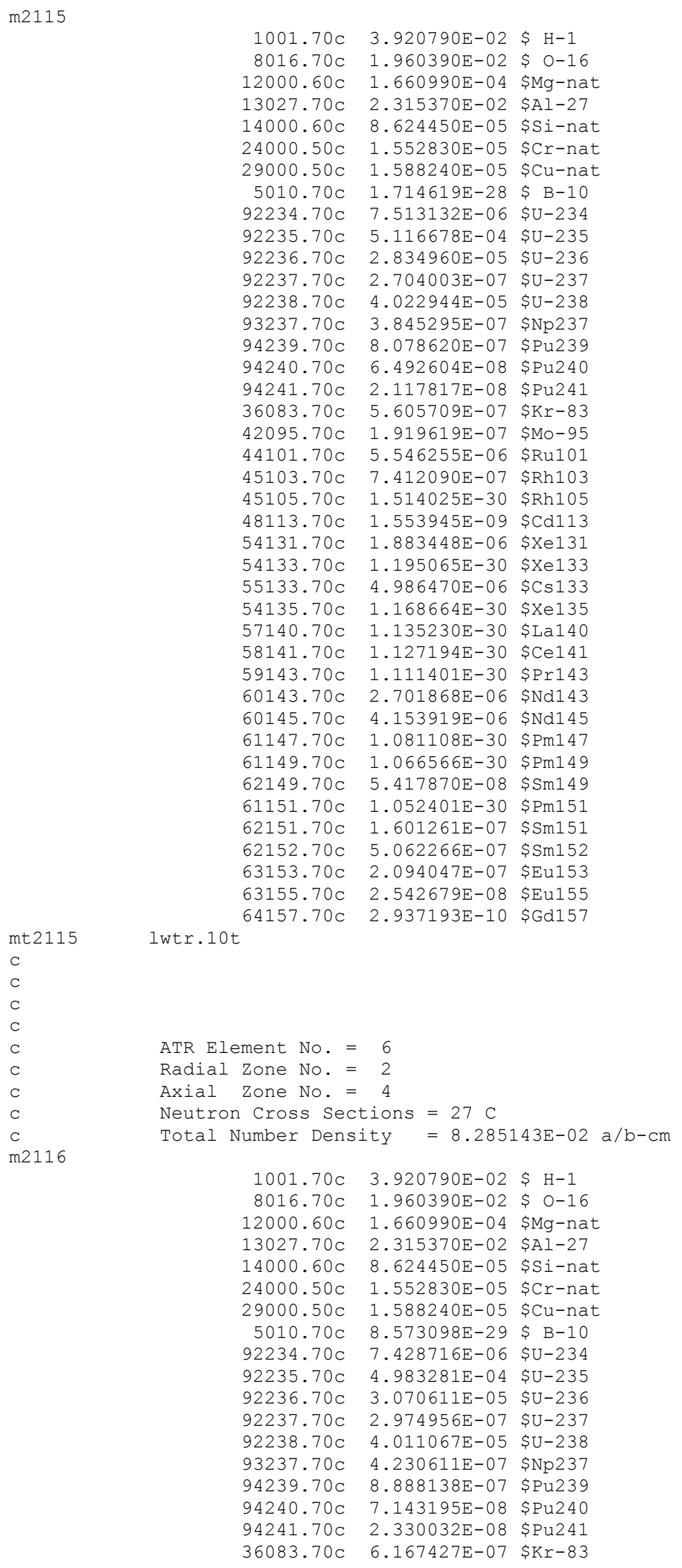




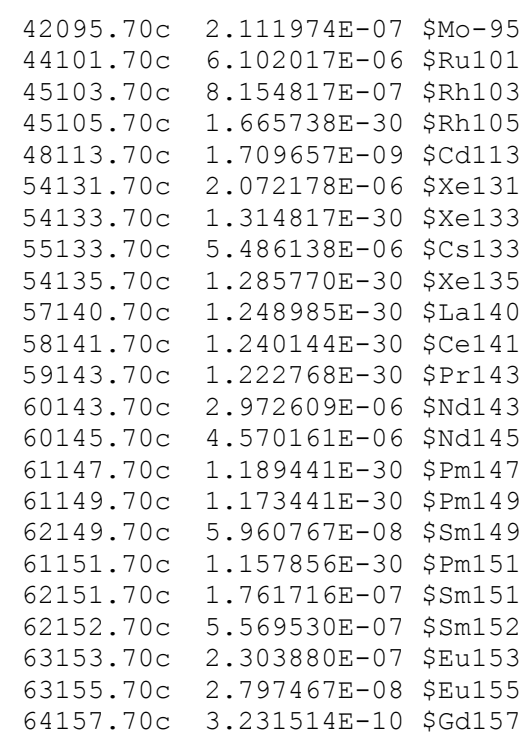

mt2116 lwtr.10t

$\mathrm{c}$

C

C

C

C

C

C

m2117

ATR Element No. $=6$

Radial Zone No. = 2

Axial Zone No. = 5

Neutron Cross Sections $=27 \mathrm{C}$

Total Number Density $=8.286359 \mathrm{E}-02 \mathrm{a} / \mathrm{b}-\mathrm{cm}$

$1001.70 \mathrm{C} \quad 3.920790 \mathrm{E}-02$ \$ $\mathrm{H}-1$

$8016.70 \mathrm{C} \quad 1.960390 \mathrm{E}-02$ \$ O-16

$12000.60 \mathrm{C} \quad 1.660990 \mathrm{E}-04$ \$Mg-nat

$13027.70 \mathrm{C} \quad 2.315370 \mathrm{E}-02$ \$Al-27

$14000.60 \mathrm{C} \quad 8.624450 \mathrm{E}-05$ \$Si-nat

$24000.50 \mathrm{C} \quad 1.552830 \mathrm{E}-05$ \$Cr-nat

$29000.50 \mathrm{C} \quad 1.588240 \mathrm{E}-05$ \$Cu-nat

$5010.70 \mathrm{C} \quad 1.714619 \mathrm{E}-28$ \$ B-10

$92234.70 \mathrm{C} \quad 7.544610 \mathrm{E}-06 \quad \$ \mathrm{U}-234$

$92235.70 \mathrm{C} \quad 5.166420 \mathrm{E}-04 \quad \$ \mathrm{U}-235$

$92236.70 \mathrm{C} \quad 2.747086 \mathrm{E}-05 \mathrm{\$ U}-236$

$92237.70 \mathrm{C} \quad 2.602965 \mathrm{E}-07 \quad \$ \mathrm{U}-237$

$92238.70 \mathrm{C} \quad 4.027372 \mathrm{E}-05 \quad \$ \mathrm{U}-238$

$93237.70 \mathrm{C} \quad 3.701611 \mathrm{E}-07 \quad \$ \mathrm{~Np} 237$

$94239.70 \mathrm{C} \quad 7.776754 \mathrm{E}-07$ \$Pu239

$94240.70 \mathrm{C} \quad 6.250002 \mathrm{E}-08$ \$Pu240

$94241.70 \mathrm{C} \quad 2.038682 \mathrm{E}-08$ \$Pu241

$36083.70 \mathrm{C} \quad 5.396245 \mathrm{E}-07$ \$Kr-83

$42095.70 \mathrm{C} \quad 1.847891 \mathrm{E}-07$ \$Mo-95

$44101.70 \mathrm{C} \quad 5.339014 \mathrm{E}-06$ \$Ru101

45103.70C 7.135129E-07 \$Rh103

$45105.70 \mathrm{C} \quad 1.457452 \mathrm{E}-30$ \$Rh105

$48113.70 \mathrm{C} \quad 1.495880 \mathrm{E}-09$ \$Cd113

$54131.70 \mathrm{C} \quad 1.813071 \mathrm{E}-06$ \$Xe131

$54133.70 \mathrm{C} \quad 1.150411 \mathrm{E}-30$ \$Xe133

$55133.70 \mathrm{C} \quad 4.800145 \mathrm{E}-06 \$ \mathrm{Cs} 133$

$54135.70 \mathrm{C} \quad 1.124996 \mathrm{E}-30$ \$Xe135

$57140.70 \mathrm{C} \quad 1.092811 \mathrm{E}-30$ \$La140

$58141.70 \mathrm{C} \quad 1.085075 \mathrm{E}-30$ \$Ce141

$59143.70 \mathrm{C} \quad 1.069872 \mathrm{E}-30 \quad \$ \mathrm{Pr} 143$

$60143.70 \mathrm{C} \quad 2.600910 \mathrm{E}-06 \$ \mathrm{Nd1} 43$

$60145.70 \mathrm{C} \quad 3.998703 \mathrm{E}-06$ \$Nd145

$61147.70 \mathrm{C} \quad 1.040712 \mathrm{E}-30 \quad \$ \mathrm{Pm} 147$

$61149.70 \mathrm{C} \quad 1.026713 \mathrm{E}-30 \quad \$ \mathrm{Pm} 149$

$62149.70 \mathrm{C} \quad 5.215425 \mathrm{E}-08$ \$Sm149

$61151.70 \mathrm{C} \quad 1.013077 \mathrm{E}-30$ \$Pm151

$62151.70 \mathrm{C} \quad 1.541429 \mathrm{E}-07$ \$Sm151 
$62152.70 \mathrm{C} \quad 4.873110 \mathrm{E}-07$ \$Sm152

$63153.70 \mathrm{C} \quad 2.015801 \mathrm{E}-07$ \$Eu153

$63155.70 \mathrm{C} \quad 2.447669 \mathrm{E}-08 \quad \$ \mathrm{Eu} 155$

mt2117 lwtr.10t

$64157.70 \mathrm{C} \quad 2.827442 \mathrm{E}-10 \quad \$ \mathrm{Gd} 157$

$\mathrm{C}$

C ATR Element No. = 6

C Radial Zone No. = 2

C Axial Zone No. = 6

C Neutron Cross Sections $=27 \mathrm{C}$

c Total Number Density $=8.288571 \mathrm{E}-02 \mathrm{a} / \mathrm{b}-\mathrm{cm}$

m2118

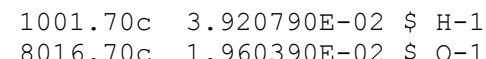

$12000.60 \mathrm{C} \quad 1.660990 \mathrm{E}-04$ \$Mg-nat

$13027.70 \mathrm{C} \quad 2.315370 \mathrm{E}-02$ \$Al-27

$14000.60 \mathrm{C} \quad 8.624450 \mathrm{E}-05$ \$Si-nat

$24000.50 \mathrm{C} \quad 1.552830 \mathrm{E}-05$ \$Cr-nat

$29000.50 \mathrm{C} \quad 1.588240 \mathrm{E}-05$ \$Cu-nat

$5010.70 \mathrm{C} \quad 1.714619 \mathrm{E}-28$ \$ B -10

$92234.70 \mathrm{C} \quad 7.755540 \mathrm{E}-06$ \$U-234

$92235.70 \mathrm{C} \quad 5.499743 \mathrm{E}-04 \quad \$ \mathrm{U}-235$

$92236.70 \mathrm{C} \quad 2.158247 \mathrm{E}-05 \quad \$ \mathrm{U}-236$

$92237.70 \mathrm{C} \quad 1.925910 \mathrm{E}-07 \quad \$ \mathrm{U}-237$

$92238.70 \mathrm{C} \quad 4.057045 \mathrm{E}-05 \quad \mathrm{SU}-238$

$93237.70 \mathrm{C} \quad 2.738789 \mathrm{E}-07$ \$Np237

$94239.70 \mathrm{C} \quad 5.753952 \mathrm{E}-07$ \$Pu239

$94240.70 \mathrm{C} \quad 4.624321 \mathrm{E}-08$ \$Pu240

$94241.70 \mathrm{C} \quad 1.508403 \mathrm{E}-08$ \$Pu241

$36083.70 \mathrm{C} \quad 3.992634 \mathrm{E}-07 \quad \$ \mathrm{Kr}-83$

$42095.70 \mathrm{C} \quad 1.367238 \mathrm{E}-07$ \$Mo-95

$44101.70 \mathrm{C} \quad 3.950289 \mathrm{E}-06$ \$Ru101

$45103.70 \mathrm{C} \quad 5.279219 \mathrm{E}-07$ \$Rh103

$45105.70 \mathrm{C} \quad 1.078356 \mathrm{E}-30$ \$Rh105

$48113.70 \mathrm{C} \quad 1.106788 \mathrm{E}-09 \$ \mathrm{Cd} 113$

$54131.70 \mathrm{c} \quad 1.341475 \mathrm{E}-06$ \$Xe131

$54133.70 \mathrm{C} \quad 8.511786 \mathrm{E}-31$ \$Xe133

$55133.70 \mathrm{C} \quad 3.551585 \mathrm{E}-06$ \$Cs133

$54135.70 \mathrm{C} \quad 8.323743 \mathrm{E}-31$ \$Xe135

$57140.70 \mathrm{C} \quad 8.085611 \mathrm{E}-31$ \$La140

$58141.70 \mathrm{C} \quad 8.028375 \mathrm{E}-31$ \$Ce141

$59143.70 \mathrm{C} \quad 7.915889 \mathrm{E}-31 \quad \mathrm{P} r 143$

$60143.70 \mathrm{C} \quad 1.924390 \mathrm{E}-06 \$ \mathrm{Nd} 143$

$60145.70 \mathrm{C} \quad 2.958605 \mathrm{E}-06 \$ \mathrm{Nd} 145$

$61147.70 \mathrm{C} \quad 7.700132 \mathrm{E}-31 \$ \mathrm{Pm} 147$

$61149.70 \mathrm{C} \quad 7.596555 \mathrm{E}-31 \$ \mathrm{Pm} 149$

$62149.70 \mathrm{C} \quad 3.858847 \mathrm{E}-08 \quad \$ \mathrm{Sm} 149$

$61151.70 \mathrm{C} \quad 7.495665 \mathrm{E}-31$ \$Pm151

$62151.70 \mathrm{C} \quad 1.140489 \mathrm{E}-07$ \$Sm151

$62152.70 \mathrm{C} \quad 3.605571 \mathrm{E}-07$ \$Sm152

$63153.70 \mathrm{C} \quad 1.491473 \mathrm{E}-07$ \$ Eu153

$63155.70 \mathrm{C} \quad 1.811008 \mathrm{E}-08$ \$Eu155

mt2118 lwtr.10t

c

C

C

$\mathrm{C}$

C

$\mathrm{C}$

C

$64157.70 \mathrm{C} \quad 2.091999 \mathrm{E}-10 \quad \$ \mathrm{Gd} 157$

m2119

ATR Element No. $=6$

Radial Zone No. = 2

Axial Zone No. = 7

Neutron Cross Sections $=27 \mathrm{C}$

Total Number Density $=8.291521 \mathrm{E}-02 \mathrm{a} / \mathrm{b}-\mathrm{cm}$

$1001.70 \mathrm{C} \quad 3.920790 \mathrm{E}-02$ \$ $\mathrm{H}-1$

$8016.70 \mathrm{C} \quad 1.960390 \mathrm{E}-02$ \$ O-16

$12000.60 \mathrm{C} \quad 1.660990 \mathrm{E}-04$ \$Mg-nat

$13027.70 \mathrm{C} \quad 2.315370 \mathrm{E}-02$ \$Al-27 


\begin{tabular}{|c|c|c|}
\hline $14000.60 \mathrm{C}$ & $8.624450 \mathrm{E}-05$ & \$Si-nat \\
\hline $24000.50 \mathrm{c}$ & $1.552830 \mathrm{E}-05$ & $\$ \mathrm{Cr}-\mathrm{nat}$ \\
\hline $9000.50 \mathrm{c}$ & $1.588240 \mathrm{E}-05$ & $\$ \mathrm{Cu}$-nat \\
\hline $5010.70 \mathrm{c}$ & $1.714619 \mathrm{E}-28$ & $\$ B-10$ \\
\hline $2234.70 \mathrm{C}$ & $8.036629 \mathrm{E}-06$ & $\$ U-234$ \\
\hline $92235.70 \mathrm{C}$ & $5.943936 \mathrm{E}-04$ & $\$ U-235$ \\
\hline $92236.70 \mathrm{c}$ & $1.373548 \mathrm{E}-05$ & $\$ U-236$ \\
\hline $92237.70 \mathrm{C}$ & $1.023656 \mathrm{E}-07$ & $\$ U-237$ \\
\hline $2238.70 c$ & $4.096588 \mathrm{E}-05$ & $\$ U-238$ \\
\hline $237.70 \mathrm{c}$ & $1.455716 \mathrm{E}-07$ & \$Np237 \\
\hline $4239.70 \mathrm{C}$ & $3.058329 \mathrm{E}-07$ & \$Pu239 \\
\hline $94240.70 \mathrm{c}$ & $2.457910 \mathrm{E}-08$ & \$Pu240 \\
\hline $94241.70 \mathrm{C}$ & $8.017431 \mathrm{E}-09$ & \$Pu2 41 \\
\hline $36083.70 \mathrm{c}$ & $2.122157 \mathrm{E}-07$ & $\$ K r-83$ \\
\hline $42095.70 \mathrm{C}$ & $7.267115 \mathrm{E}-08$ & \$Mo-95 \\
\hline $44101.70 \mathrm{C}$ & $2.099650 \mathrm{E}-06$ & \$Ru101 \\
\hline $45103.70 \mathrm{C}$ & $2.806000 \mathrm{E}-07$ & $\$ R h 103$ \\
\hline $45105.70 \mathrm{c}$ & $5.731656 \mathrm{E}-31$ & \$Rh105 \\
\hline $48113.70 \mathrm{C}$ & $5.882779 \mathrm{E}-10$ & $\$ \mathrm{Cd} 113$ \\
\hline $54131.70 \mathrm{C}$ & $7.130180 \mathrm{E}-07$ & \$Xe131 \\
\hline $54133.70 \mathrm{C}$ & $4.524167 \mathrm{E}-31$ & $\$ \mathrm{Xe133}$ \\
\hline $55133.70 \mathrm{C}$ & $1.887731 \mathrm{E}-06$ & $\$ C s 133$ \\
\hline $54135.70 \mathrm{C}$ & $4.424219 \mathrm{E}-31$ & $\$ \mathrm{Xe135}$ \\
\hline $57140.70 \mathrm{C}$ & $4.297647 \mathrm{E}-31$ & \$La140 \\
\hline $58141.70 \mathrm{C}$ & $4.267226 \mathrm{E}-31$ & $\$ C e 141$ \\
\hline $59143.70 \mathrm{C}$ & $4.207437 \mathrm{E}-31$ & $\$ \operatorname{Pr} 143$ \\
\hline $60143.70 \mathrm{c}$ & $1.022848 \mathrm{E}-06$ & $\$ N d 143$ \\
\hline $60145.70 \mathrm{c}$ & $1.572552 \mathrm{E}-06$ & $\$ N d 145$ \\
\hline $61147.70 \mathrm{c}$ & $4.092758 \mathrm{E}-31$ & \$Pm1 47 \\
\hline $61149.70 \mathrm{C}$ & $4.037706 \mathrm{E}-31$ & \$Pm149 \\
\hline $62149.70 \mathrm{c}$ & $2.051046 \mathrm{E}-08$ & $\$$ Sm149 \\
\hline $61151.70 \mathrm{C}$ & $3.984080 \mathrm{E}-31$ & \$Pm151 \\
\hline $62151.70 \mathrm{C}$ & $6.061905 \mathrm{E}-08$ & \$Sm151 \\
\hline $62152.70 \mathrm{c}$ & $1.916426 \mathrm{E}-07$ & \$Sm152 \\
\hline $63153.70 \mathrm{c}$ & $7.927446 \mathrm{E}-08$ & \$Eu153 \\
\hline $63155.70 \mathrm{C}$ & $9.625834 \mathrm{E}-09$ & \$Eu155 \\
\hline 64157.7 & $1.111935 \mathrm{E}-10$ & \$Gd157 \\
\hline
\end{tabular}

mt2119 lwtr.10t

C

$\mathrm{C}$

C ATR Element No. = 6

C Radial Zone No. = 3

C Axial Zone No. = 1

C Neutron Cross Sections $=27 \mathrm{C}$

C Total Number Density $=7.864934 \mathrm{E}-02 \mathrm{a} / \mathrm{b}-\mathrm{cm}$

m2120

$\begin{array}{rll}1001.70 \mathrm{C} & 3.212840 \mathrm{E}-02 & \text { \$ }-1 \\ 8016.70 \mathrm{C} & 1.606420 \mathrm{E}-02 & \text { \$ }-16 \\ 12000.60 \mathrm{C} & 2.399430 \mathrm{E}-04 & \text { \$Mg-nat } \\ 13027.70 \mathrm{C} & 2.958200 \mathrm{E}-02 & \text { \$Al-27 } \\ 14000.60 \mathrm{C} & 1.245870 \mathrm{E}-04 & \text { \$Si-nat } \\ 24000.50 \mathrm{C} & 2.243180 \mathrm{E}-05 & \text { \$Cr-nat } \\ 29000.50 \mathrm{C} & 2.294330 \mathrm{E}-05 & \text { \$Cu-nat } \\ 5010.70 \mathrm{C} & 4.498556 \mathrm{E}-06 & \text { \$ }-10 \\ 92234.70 \mathrm{C} & 5.593492 \mathrm{E}-06 & \text { \$U-234 } \\ 92235.70 \mathrm{C} & 4.016172 \mathrm{E}-04 & \text { \$U-235 } \\ 92236.70 \mathrm{C} & 1.381726 \mathrm{E}-05 & \text { \$U-236 } \\ 92237.70 \mathrm{C} & 1.192045 \mathrm{E}-07 & \text { \$U-237 } \\ 92238.70 \mathrm{C} & 2.904261 \mathrm{E}-05 & \text { \$U-238 } \\ 93237.70 \mathrm{C} & 1.695178 \mathrm{E}-07 & \text { \$Np237 } \\ 94239.70 \mathrm{C} & 3.561417 \mathrm{E}-07 & \text { \$Pu239 } \\ 94240.70 \mathrm{C} & 2.862230 \mathrm{E}-08 & \text { \$Pu240 } \\ 94241.70 \mathrm{C} & 9.336279 \mathrm{E}-09 & \text { \$Pu241 } \\ 36083.70 \mathrm{C} & 2.471246 \mathrm{E}-07 & \text { \$Kr-83 } \\ 42095.70 \mathrm{C} & 8.462538 \mathrm{E}-08 & \text { \$Mo-95 } \\ 44101.70 \mathrm{C} & 2.445037 \mathrm{E}-06 & \text { \$Ru101 } \\ 45103.70 \mathrm{C} & 3.267580 \mathrm{E}-07 & \text { \$Rh103 } \\ 45105.70 \mathrm{C} & 6.674500 \mathrm{E}-31 & \text { \$Rh105 } \\ 48113.70 \mathrm{C} & 6.850482 \mathrm{E}-10 & \text { \$Cd113 }\end{array}$




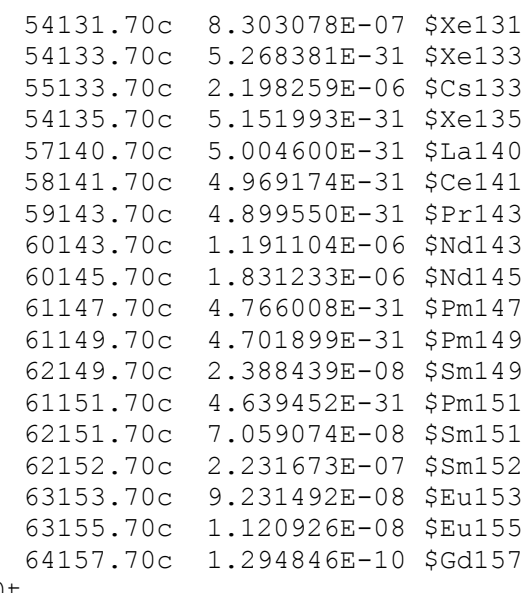

$\begin{array}{ll}\text { mt2120 lwtr.10t } \\ \text { C } \\ \text { C } \\ \text { C } \\ \text { C } \\ \text { ATR Element No. }=6 \\ \text { C } & \text { Radial Zone No. }=3 \\ \text { C } & \text { Axial Zone No. }=2 \\ \text { C } & \text { Neutron Cross Sections }=27 \mathrm{C} \\ \text { C } & \text { Total Number Density }=7.862680 \mathrm{E}-02 \mathrm{a} / \mathrm{b}-\mathrm{Cm}\end{array}$

$1001.70 \mathrm{C} \quad 3.212840 \mathrm{E}-02 \$ \mathrm{H}-1$

$8016.70 \mathrm{c} \quad 1.606420 \mathrm{E}-02 \$ 0-16$

$12000.60 \mathrm{C} \quad 2.399430 \mathrm{E}-04$ \$Mg-nat $13027.70 \mathrm{C} \quad 2.958200 \mathrm{E}-02 \quad \$ \mathrm{Al}-27$

$14000.60 \mathrm{C} \quad 1.245870 \mathrm{E}-04$ \$Si-nat $24000.50 \mathrm{C} \quad 2.243180 \mathrm{E}-05$ \$Cr-nat $29000.50 \mathrm{C} \quad 2.294330 \mathrm{E}-05$ \$Cu-nat $5010.70 \mathrm{C} \quad 4.498556 \mathrm{E}-06$ \$ B-10 $92234.70 \mathrm{C} \quad 5.378763 \mathrm{E}-06 \quad \$ \mathrm{U}-234$ $92235.70 \mathrm{C} \quad 3.676846 \mathrm{E}-04 \quad \$ \mathrm{U}-235$ $92236.70 \mathrm{C} \quad 1.981170 \mathrm{E}-05 \quad \$ \mathrm{U}-236$ $92237.70 \mathrm{C} \quad 1.881292 \mathrm{E}-07 \quad \$ \mathrm{U}-237$ $92238.70 \mathrm{C} \quad 2.874054 \mathrm{E}-05 \quad \$ \mathrm{U}-238$ $93237.70 \mathrm{C} \quad 2.675338 \mathrm{E}-07 \quad \$ \mathrm{~Np} 237$ $94239.70 \mathrm{C} \quad 5.620647 \mathrm{E}-07$ \$Pu239 $94240.70 \mathrm{C} \quad 4.517187 \mathrm{E}-08$ \$Pu240 $94241.70 \mathrm{C} \quad 1.473457 \mathrm{E}-08$ \$Pu241 $36083.70 \mathrm{C} \quad 3.900134 \mathrm{E}-07 \quad \$ \mathrm{Kr}-83$ $42095.70 \mathrm{C} \quad 1.335562 \mathrm{E}-07$ \$MO-95 $44101.70 \mathrm{C} \quad 3.858770 \mathrm{E}-06$ \$Ru101 $45103.70 \mathrm{C} \quad 5.156912 \mathrm{E}-07$ \$Rh103 $45105.70 \mathrm{C} \quad 1.053373 \mathrm{E}-30$ \$Rh105 $48113.70 \mathrm{C} \quad 1.081147 \mathrm{E}-09$ \$Cd113 $54131.70 \mathrm{C} \quad 1.310396 \mathrm{E}-06$ \$Xe131 $54133.70 \mathrm{C} \quad 8.314588 \mathrm{E}-31$ \$Xe133 $55133.70 \mathrm{C} \quad 3.469303 \mathrm{E}-06 \quad \$ \mathrm{Cs} 133$ $54135.70 \mathrm{C} \quad 8.130903 \mathrm{E}-31$ \$Xe135 $57140.70 \mathrm{C} \quad 7.898288 \mathrm{E}-31$ \$La140 $58141.70 \mathrm{C} \quad 7.842376 \mathrm{E}-31$ \$Ce141 $59143.70 \mathrm{C} \quad 7.732496 \mathrm{E}-31 \quad \$ \operatorname{Pr} 143$ $60143.70 \mathrm{C} \quad 1.879807 \mathrm{E}-06$ \$Nd143 $60145.70 \mathrm{C} \quad 2.890061 \mathrm{E}-06$ \$Nd145 $61147.70 \mathrm{C} \quad 7.521739 \mathrm{E}-31$ \$Pm147 $61149.70 \mathrm{C} \quad 7.420562 \mathrm{E}-31$ \$Pm149 $62149.70 \mathrm{C} \quad 3.769447 \mathrm{E}-08 \quad \$ \mathrm{Sm} 149$ $61151.70 \mathrm{C} \quad 7.322008 \mathrm{E}-31$ \$Pm151 $62151.70 \mathrm{C} \quad 1.114067 \mathrm{E}-07$ \$Sm151 $62152.70 \mathrm{C} \quad 3.522038 \mathrm{E}-07$ \$Sm152 $63153.70 \mathrm{C} \quad 1.456919 \mathrm{E}-07 \quad \$ \mathrm{Eu} 153$ $63155.70 \mathrm{C} \quad 1.769052 \mathrm{E}-08$ \$Eu155 $64157.70 \mathrm{C} \quad 2.043533 \mathrm{E}-10$ \$Gd157 
C
ATR Element No. $=6$

Radial Zone No. $=3$

Axial Zone No. $=3$

Neutron Cross Sections $=27 \mathrm{C}$

Total Number Density $=7.861021 \mathrm{E}-02 \mathrm{a} / \mathrm{b}-\mathrm{cm}$

\begin{abstract}
$1001.70 \mathrm{C} \quad 3.212840 \mathrm{E}-02$ \$ $\mathrm{H}-1$


$92235.70 \mathrm{C} \quad 3.327364 \mathrm{E}-04 \quad \$ \mathrm{U}-235$

$92236.70 \mathrm{C} \quad 2.598572 \mathrm{E}-05 \quad \$ \mathrm{U}-236$

$92237.70 \mathrm{C} \quad 2.591186 \mathrm{E}-07 \quad \$ \mathrm{U}-237$

$92238.70 \mathrm{C} \quad 2.842948 \mathrm{E}-05$ \$U-238

$93237.70 \mathrm{C} \quad 3.684862 \mathrm{E}-07$ \$Np237

$94239.70 \mathrm{C} \quad 7.741566 \mathrm{E}-07$ \$Pu239

$94240.70 \mathrm{C} \quad 6.221721 \mathrm{E}-08$ \$Pu240

$94241.70 \mathrm{C} \quad 2.029457 \mathrm{E}-08$ \$Pu241

$36083.70 \mathrm{C} \quad 5.371828 \mathrm{E}-07$ \$Kr-83

$42095.70 \mathrm{C} \quad 1.839529 \mathrm{E}-07$ \$Mo-95

$44101.70 \mathrm{C} \quad 5.314855 \mathrm{E}-06$ \$Ru101

$45103.70 \mathrm{C} \quad 7.102844 \mathrm{E}-07$ \$Rh103

$45105.70 \mathrm{C} \quad 1.450857 \mathrm{E}-30$ \$Rh105

$48113.70 \mathrm{C} \quad 1.489111 \mathrm{E}-09$ \$Cd113

$54131.70 \mathrm{C} \quad 1.804867 \mathrm{E}-06$ \$Xe131

$54133.70 \mathrm{C} \quad 1.145205 \mathrm{E}-30 \quad \$ \mathrm{Xe} 133$

$55133.70 \mathrm{C} \quad 4.778425 \mathrm{E}-06$ \$Cs133

$54135.70 \mathrm{C} \quad 1.119905 \mathrm{E}-30$ \$Xe135

$57140.70 \mathrm{C} \quad 1.087866 \mathrm{E}-30$ \$La140

$58141.70 \mathrm{C} \quad 1.080165 \mathrm{E}-30$ \$Ce141

$59143.70 \mathrm{C} \quad 1.065031 \mathrm{E}-30 \quad \$ \operatorname{Pr} 143$

$60143.70 \mathrm{C} \quad 2.589141 \mathrm{E}-06 \$ \mathrm{Nd1} 43$

$60145.70 \mathrm{C} \quad 3.980609 \mathrm{E}-06 \$ \mathrm{Nd1} 45$

$61147.70 \mathrm{C} \quad 1.036002 \mathrm{E}-30 \quad \$ \mathrm{Pm} 147$

$61149.70 \mathrm{C} \quad 1.022067 \mathrm{E}-30 \$ \mathrm{Pm} 149$

$62149.70 \mathrm{C} \quad 5.191826 \mathrm{E}-08 \quad \$ \mathrm{Sm} 149$

$61151.70 \mathrm{C} \quad 1.008493 \mathrm{E}-30$ \$Pm151

$62151.70 \mathrm{C} \quad 1.534454 \mathrm{E}-07$ \$Sm151

$62152.70 \mathrm{C} \quad 4.851059 \mathrm{E}-07$ \$Sm152

$63153.70 \mathrm{C} \quad 2.006679 \mathrm{E}-07$ \$Eu153

$63155.70 \mathrm{C} \quad 2.436593 \mathrm{E}-08$ \$Eu155

mt2123 lwtr.10t

$64157.70 \mathrm{C} \quad 2.814648 \mathrm{E}-10$ \$Gd157

C

C

$\mathrm{C}$

ATR Element No. $=6$

Radial Zone No. $=3$

Axial Zone No. = 5

Neutron Cross Sections $=27 \mathrm{C}$

Total Number Density $=7.861274 \mathrm{E}-02 \mathrm{a} / \mathrm{b}-\mathrm{cm}$

$1001.70 \mathrm{C} \quad 3.212840 \mathrm{E}-02$ \$ $\mathrm{H}-1$

$8016.70 \mathrm{C} \quad 1.606420 \mathrm{E}-02$ \$ O-16

$12000.60 \mathrm{c} \quad 2.399430 \mathrm{E}-04$ \$Mg-nat

$13027.70 \mathrm{C} \quad 2.958200 \mathrm{E}-02$ \$Al-27

$14000.60 \mathrm{C} \quad 1.245870 \mathrm{E}-04$ \$Si-nat

$24000.50 \mathrm{C} \quad 2.243180 \mathrm{E}-05$ \$Cr-nat

$29000.50 \mathrm{C} \quad 2.294330 \mathrm{E}-05$ \$Cu-nat

$5010.70 \mathrm{C} \quad 4.498556 \mathrm{E}-06 \$ \mathrm{~B}-10$

$92234.70 \mathrm{C} \quad 5.244710 \mathrm{E}-06$ \$U-234

$92235.70 \mathrm{C} \quad 3.465008 \mathrm{E}-04 \quad \mathrm{SU}-235$

$92236.70 \mathrm{C} \quad 2.355397 \mathrm{E}-05 \quad \$ \mathrm{U}-236$

$92237.70 \mathrm{C} \quad 2.311582 \mathrm{E}-07 \quad \$ \mathrm{U}-237$

$92238.70 \mathrm{C} \quad 2.855196 \mathrm{E}-05 \quad \$ \mathrm{U}-238$

$93237.70 \mathrm{C} \quad 3.287243 \mathrm{E}-07$ \$Np237

$94239.70 \mathrm{C} \quad 6.906205 \mathrm{E}-07$ \$Pu239

$94240.70 \mathrm{C} \quad 5.550361 \mathrm{E}-08$ \$Pu240

$94241.70 \mathrm{C} \quad 1.810467 \mathrm{E}-08$ \$Pu241

$36083.70 \mathrm{C} \quad 4.792175 \mathrm{E}-07$ \$Kr-83

$42095.70 \mathrm{C} \quad 1.641033 \mathrm{E}-07$ \$Mo-95

$44101.70 \mathrm{C} \quad 4.741351 \mathrm{E}-06$ \$Ru101

$45103.70 \mathrm{C} \quad 6.336405 \mathrm{E}-07$ \$Rh103

$45105.70 \mathrm{C} \quad 1.294301 \mathrm{E}-30$ \$Rh105

$48113.70 \mathrm{C} \quad 1.328427 \mathrm{E}-09$ \$Cd113

$54131.70 \mathrm{C} \quad 1.610111 \mathrm{E}-06$ \$Xe131

$54133.70 \mathrm{C} \quad 1.021631 \mathrm{E}-30$ \$xe133

$55133.70 \mathrm{C} \quad 4.262805 \mathrm{E}-06 \$ \mathrm{Cs} 133$

$54135.70 \mathrm{C} \quad 9.990608 \mathrm{E}-31$ \$Xe135

$57140.70 \mathrm{C} \quad 9.704788 \mathrm{E}-31$ \$La140 


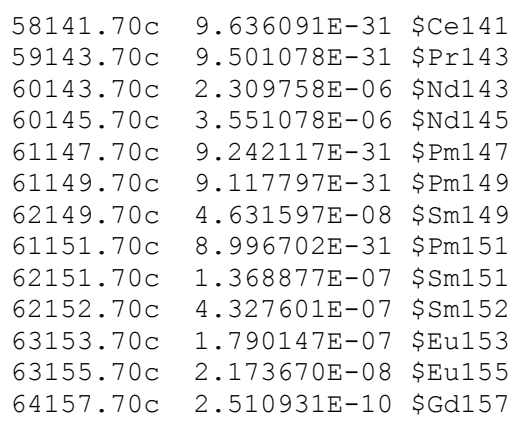

mt2124 lwtr.10t

c

C

$\mathrm{C}$

ATR Element No. = 6

C Radial Zone No. = 3

Axial Zone No. $=6$

C Neutron Cross Sections $=27 \mathrm{C}$

C Total Number Density $=7.863101 \mathrm{E}-02 \mathrm{a} / \mathrm{b}-\mathrm{cm}$

m2125

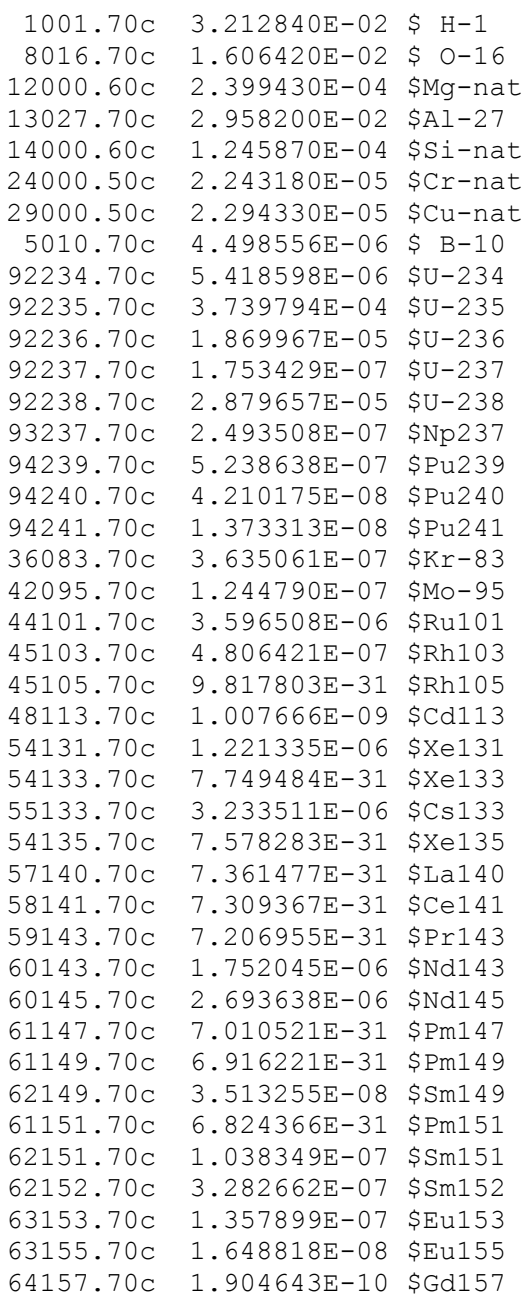




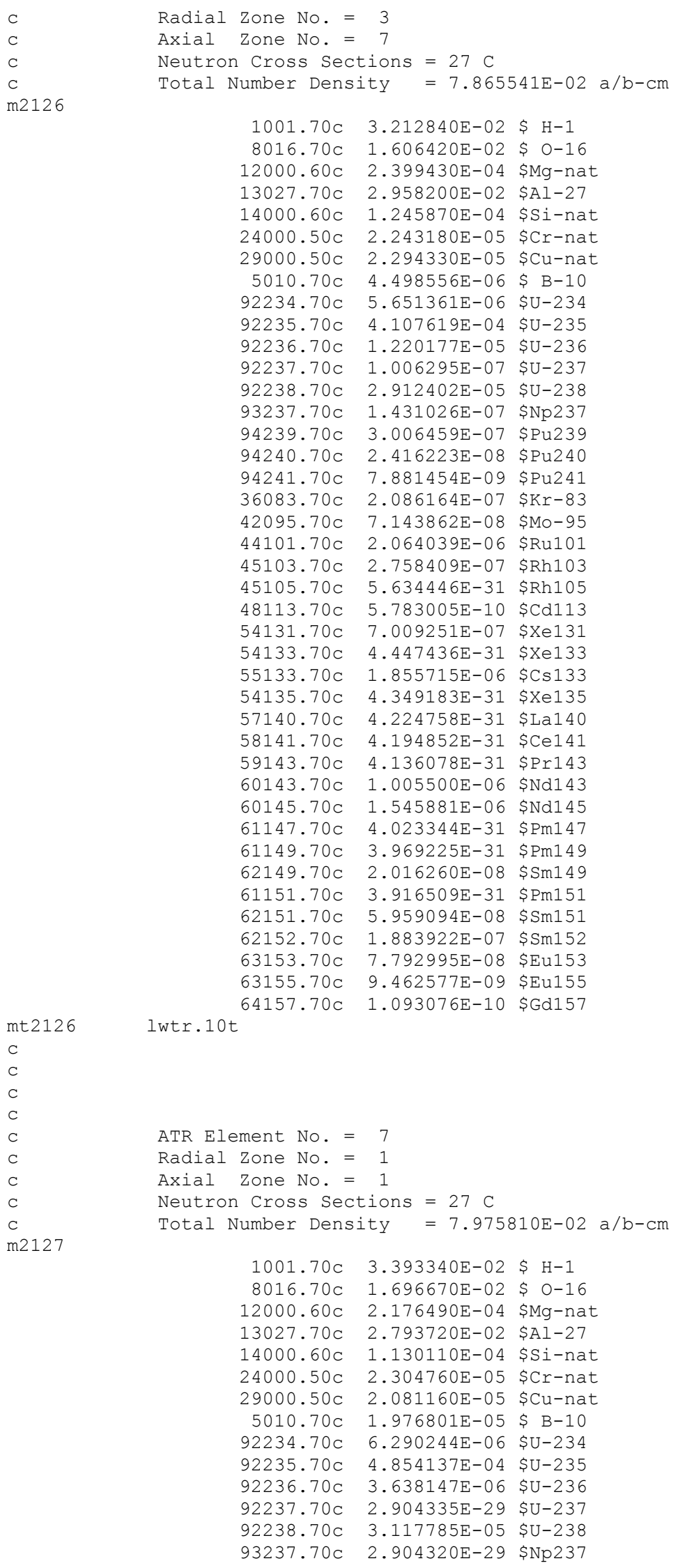




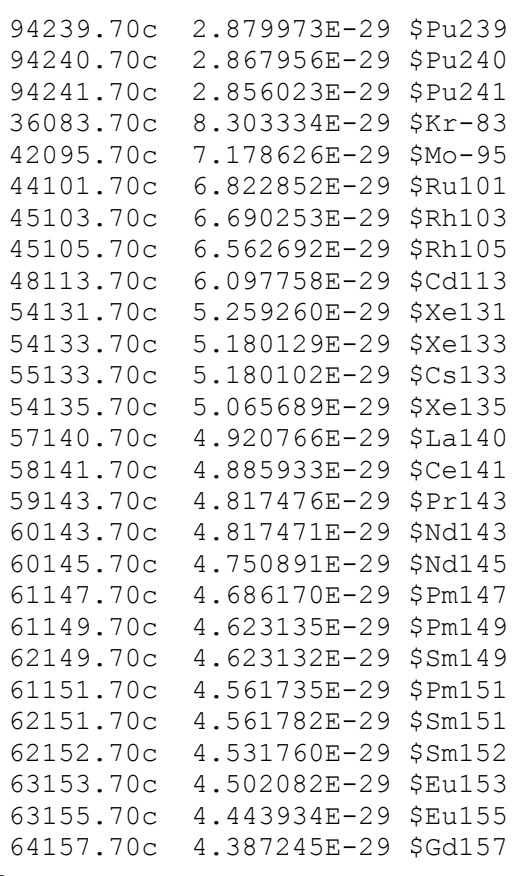

mt2127 lwtr.10t

c

C

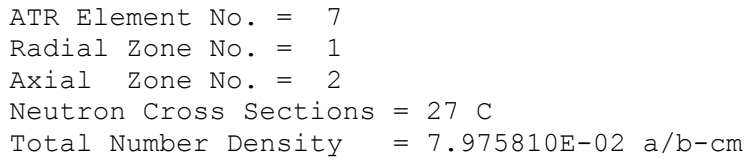

\begin{tabular}{|c|c|c|}
\hline $1001.70 \mathrm{c}$ & $3.393340 \mathrm{E}-02$ & $\$ \mathrm{H}-1$ \\
\hline $8016.70 \mathrm{C}$ & $1.696670 \mathrm{E}-02$ & $\$ 0-16$ \\
\hline $2000.60 \mathrm{c}$ & $2.176490 \mathrm{E}-04$ & $\$ M g-n a t$ \\
\hline $027.70 \mathrm{C}$ & $2.793720 \mathrm{E}-02$ & $\$ A 1-27$ \\
\hline $000.60 \mathrm{C}$ & $1.130110 \mathrm{E}-04$ & \$Si-nat \\
\hline $4000.50 \mathrm{c}$ & $2.304760 \mathrm{E}-05$ & \$Cr-nat \\
\hline $000.50 \mathrm{C}$ & $2.081160 \mathrm{E}-05$ & $\$ \mathrm{Cu}-\mathrm{nat}$ \\
\hline$C$ & $1.976801 \mathrm{E}-05$ & $\$ B-10$ \\
\hline $.70 \mathrm{C}$ & $6.290244 E-06$ & $\$ U-234$ \\
\hline $70 \mathrm{c}$ & $4.854137 E-04$ & $\$ U-235$ \\
\hline $.70 \mathrm{C}$ & $3.638147 E-06$ & $\$ U-236$ \\
\hline $.70 \mathrm{C}$ & $2.904335 E-29$ & $\$ U-237$ \\
\hline $.70 \mathrm{c}$ & $3.117785 E-05$ & $\$ U-238$ \\
\hline $.70 \mathrm{C}$ & $2.904320 \mathrm{E}-29$ & $\$ N p 237$ \\
\hline$C$ & $9973 E-29$ & $\$ P u 239$ \\
\hline $.70 \mathrm{C}$ & $2.867956 \mathrm{E}-29$ & $\$ \mathrm{Pu} 240$ \\
\hline$c$ & $23 E-29$ & \$Pu241 \\
\hline $.70 \mathrm{C}$ & $8.303334 E-29$ & $\$ K r-83$ \\
\hline$c$ & $7.178626 \mathrm{E}-29$ & $\$ M o-95$ \\
\hline $.70 \mathrm{C}$ & $2 E-29$ & \$Ru101 \\
\hline & $6.690253 E-29$ & $\$ R h 103$ \\
\hline $.70 \mathrm{C}$ & $2 E-29$ & \$Rh105 \\
\hline & $58 E-29$ & $\$ \mathrm{Cd} 113$ \\
\hline .7 & $0 E-29$ & $\$ x e 131$ \\
\hline $.70 \mathrm{C}$ & $5.180129 \mathrm{E}-29$ & $\$ x e 133$ \\
\hline $.70 \mathrm{C}$ & $2 E-29$ & $\$ C$ s 133 \\
\hline $.70 \mathrm{C}$ & $5.065689 \mathrm{E}-29$ & \$Xe135 \\
\hline $.70 \mathrm{C}$ & $5 E-29$ & \$La140 \\
\hline .70 & $4.885933 E-29$ & $\$ C e 141$ \\
\hline 59 & $76 E-29$ & $\$ \operatorname{Pr} 143$ \\
\hline & $471 E-29$ & $\$ N d 143$ \\
\hline 601 & $4.750891 \mathrm{E}-29$ & $\$ N d 145$ \\
\hline 611 & $4.686170 \mathrm{E}-29$ & $\$ P m 147$ \\
\hline
\end{tabular}




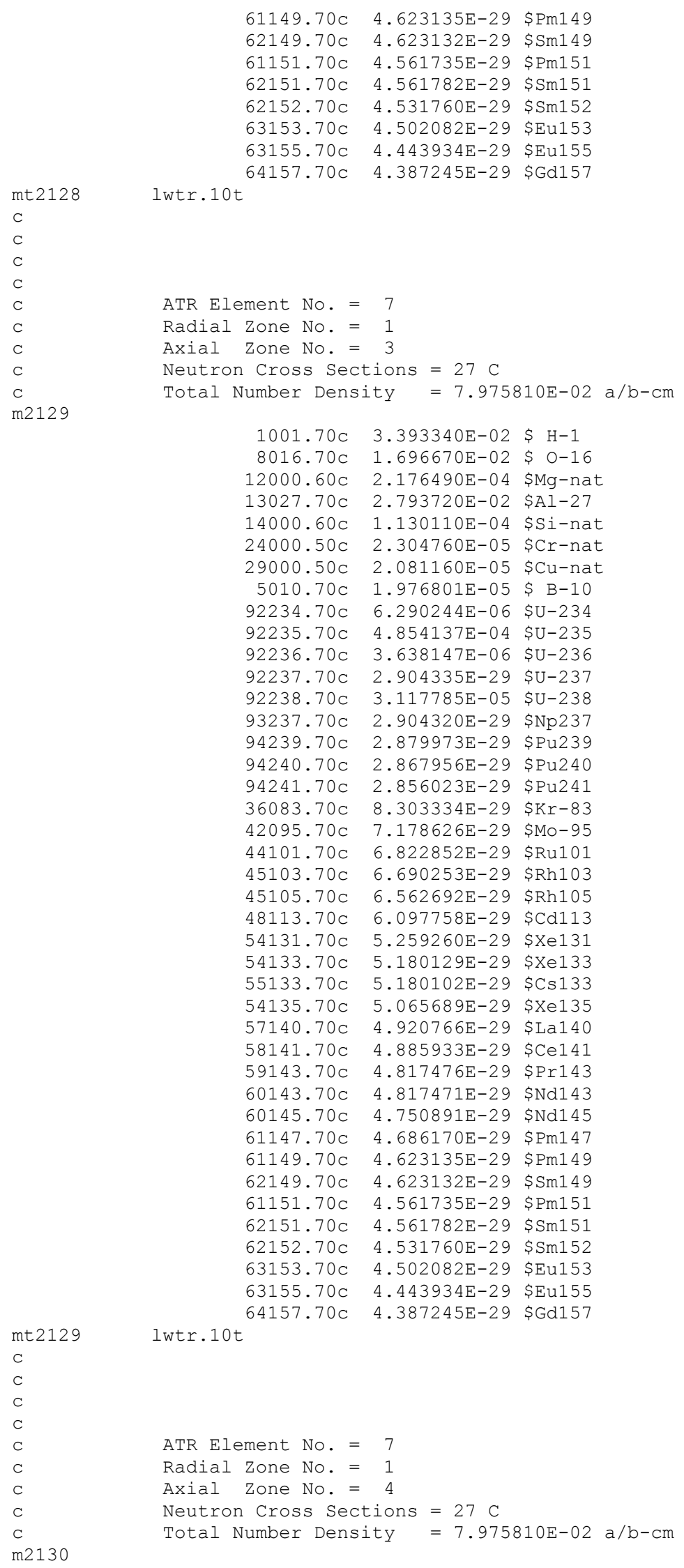




\begin{tabular}{|c|c|c|}
\hline $1001.70 \mathrm{C}$ & $3.393340 \mathrm{E}-02$ & $\$ \mathrm{H}-1$ \\
\hline $8016.70 \mathrm{c}$ & $1.696670 \mathrm{E}-02$ & $\$ 0-16$ \\
\hline $12000.60 \mathrm{c}$ & $2.176490 \mathrm{E}-04$ & \$Mg-nat \\
\hline $13027.70 \mathrm{C}$ & $2.793720 \mathrm{E}-02$ & $\$ A l-27$ \\
\hline $14000.60 \mathrm{c}$ & $1.130110 \mathrm{E}-04$ & \$Si-nat \\
\hline $24000.50 \mathrm{C}$ & $2.304760 \mathrm{E}-05$ & $\$ \mathrm{Cr}-\mathrm{nat}$ \\
\hline $29000.50 \mathrm{C}$ & $2.081160 \mathrm{E}-05$ & $\$ \mathrm{Cu}-\mathrm{nat}$ \\
\hline 5010. & $1.976802 \mathrm{E}-05$ & $\$ B-10$ \\
\hline 2234.70 & $6.290264 \mathrm{E}-06$ & $\$ \mathrm{U}-234$ \\
\hline $92235.70 \mathrm{C}$ & $4.854152 \mathrm{E}-04$ & $\$ U-235$ \\
\hline $92236.70 \mathrm{C}$ & $3.638159 \mathrm{E}-06$ & $\$ U-236$ \\
\hline $92237.70 \mathrm{C}$ & $1.452167 \mathrm{E}-29$ & $\$ \mathrm{U}-237$ \\
\hline $92238.70 \mathrm{C}$ & $3.117796 \mathrm{E}-05$ & $\$ U-238$ \\
\hline $93237.70 \mathrm{C}$ & $1.452160 \mathrm{E}-29$ & $\$ N p 237$ \\
\hline $94239.70 \mathrm{C}$ & $1.439987 \mathrm{E}-29$ & \$Pu239 \\
\hline $94240.70 \mathrm{C}$ & $1.433978 \mathrm{E}-29$ & \$Pu240 \\
\hline $94241.70 \mathrm{C}$ & $1.428012 \mathrm{E}-29$ & \$Pu 241 \\
\hline $36083.70 \mathrm{c}$ & $4.151667 \mathrm{E}-29$ & $\$ K r-83$ \\
\hline $42095.70 \mathrm{C}$ & $3.589313 E-29$ & $\$ M o-95$ \\
\hline $44101.70 \mathrm{C}$ & $3.411426 \mathrm{E}-29$ & \$Ru101 \\
\hline $45103.70 \mathrm{C}$ & $3.345127 \mathrm{E}-29$ & \$Rh103 \\
\hline $45105.70 \mathrm{C}$ & $3.281347 \mathrm{E}-29$ & \$Rh105 \\
\hline $48113.70 \mathrm{C}$ & $3.048880 \mathrm{E}-29$ & $\$ \mathrm{Cd} 113$ \\
\hline $54131.70 \mathrm{C}$ & $2.629630 \mathrm{E}-29$ & $\$ \mathrm{Xe131}$ \\
\hline 54133.700 & $2.590065 \mathrm{E}-29$ & $\$ \mathrm{Xe133}$ \\
\hline $55133.70 c$ & $2.590051 \mathrm{E}-29$ & $\$ \mathrm{Cs} 133$ \\
\hline $54135.70 \mathrm{C}$ & $2.532845 \mathrm{E}-29$ & $\$$ Xe135 \\
\hline $57140.70 \mathrm{C}$ & $2.460383 E-29$ & \$La140 \\
\hline $58141.70 \mathrm{C}$ & $2.442967 \mathrm{E}-29$ & $\$ C e 141$ \\
\hline 59143.700 & $2.408738 \mathrm{E}-29$ & $\$ \operatorname{Pr} 143$ \\
\hline 60143.70 & $2.408736 \mathrm{E}-29$ & $\$ N d 143$ \\
\hline 60145.70 & $2.375446 \mathrm{E}-29$ & $\$ N d 145$ \\
\hline $61147.70 \mathrm{c}$ & $2.343085 E-29$ & $\$ \operatorname{Pm} 147$ \\
\hline $61149.70 \mathrm{c}$ & $2.311568 \mathrm{E}-29$ & $\$ \operatorname{Pm} 149$ \\
\hline $62149.70 c$ & $2.311566 \mathrm{E}-29$ & $\$ \operatorname{Sm} 149$ \\
\hline 61151.70 & $2.280868 \mathrm{E}-29$ & \$Pm151 \\
\hline 62151.70 & $2.280891 \mathrm{E}-29$ & \$Sm151 \\
\hline $62152.70 \mathrm{C}$ & $2.265881 \mathrm{E}-29$ & $\$$ Sm152 \\
\hline $63153.70 \mathrm{c}$ & $2.251041 \mathrm{E}-29$ & \$Eu153 \\
\hline 63155.7 & $2.221967 \mathrm{E}-29$ & \$Eu155 \\
\hline 64157.7 & $2.193623 \mathrm{E}-29$ & \$Gd157 \\
\hline
\end{tabular}

mt2130
C
$C$
$C$
$C$
$C$
$C$
$C$
$C$
$C$
m2131

lwtr.10t

$63155.70 \mathrm{C} \quad 2.221967 \mathrm{E}-29 \quad \$ \mathrm{Eu} 155$

ATR Element No. $=7$

Radial Zone No. $=1$

Axial Zone No. = 5

Neutron Cross Sections $=27 \mathrm{C}$

Total Number Density $=7.975810 \mathrm{E}-02 \mathrm{a} / \mathrm{b}-\mathrm{cm}$

\begin{tabular}{|c|c|c|}
\hline & 22 & T \\
\hline & $1.696670 \mathrm{E}-02$ & $0-16$ \\
\hline & $2.176490 \mathrm{E}-04$ & $x_{0}$ \\
\hline & $20 E-02$ & \\
\hline & $1.130110 \mathrm{E}-04$ & $-n$ \\
\hline & $4760 E-05$ & $\$ \mathrm{Cr}-\mathrm{n}$ \\
\hline & 2.0 & $\$ \mathrm{Cu}-\mathrm{n}$ \\
\hline & 1.9 & $\$ B-10$ \\
\hline & 6.2 & $\$ \mathrm{U}-234$ \\
\hline & 4.854 & $\$ \mathrm{U}-2$ \\
\hline & & $\$ U-236$ \\
\hline & & \\
\hline & 3. & $\$ \mathrm{U}-$ \\
\hline & $20 E-29$ & $\$ \mathrm{~Np} 2$ \\
\hline & $2.879973 \mathrm{E}-29$ & \$Pu23 \\
\hline & $2.867956 \mathrm{E}-29$ & \$Pu240 \\
\hline & $2.856023 \mathrm{E}-29$ & \$Pu241 \\
\hline & $8.303334 \mathrm{E}-29$ & $\$ K r-8$ \\
\hline & $71786265-2$ & $90-9$ \\
\hline
\end{tabular}




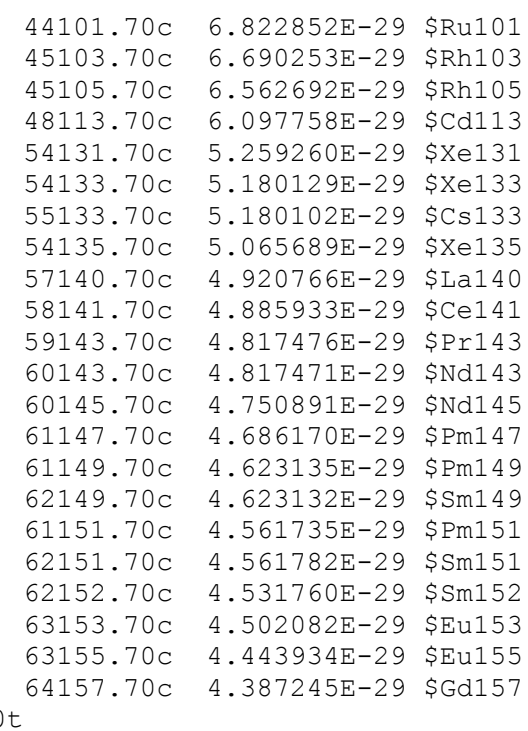

mt213 13
C
C
C
C
C
C
C
C
m2132 lwtr.10t

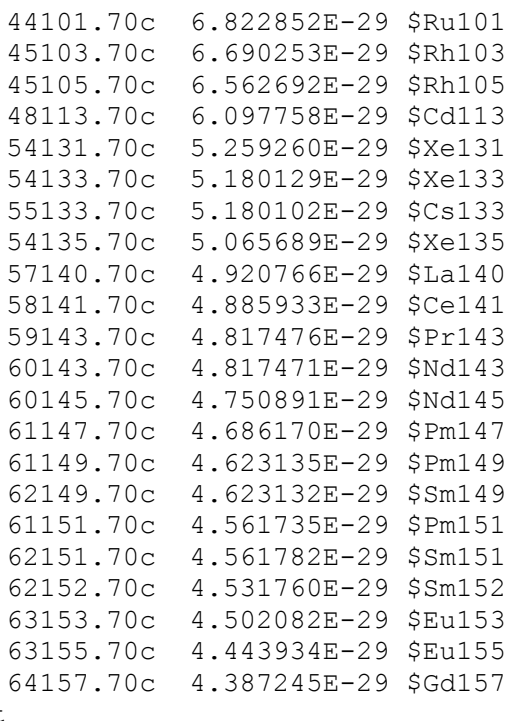

ATR Element No. $=7$

Radial Zone No. $=1$

Axial Zone No. $=6$

Neutron Cross Sections $=27 \mathrm{C}$

Total Number Density $=7.975810 \mathrm{E}-02 \mathrm{a} / \mathrm{b}-\mathrm{cm}$

$1001.70 \mathrm{C} \quad 3.393340 \mathrm{E}-02 \$ \mathrm{H}-1$

$8016.70 \mathrm{C} \quad 1.696670 \mathrm{E}-02$ \$ $0-16$

$12000.60 \mathrm{C} \quad 2.176490 \mathrm{E}-04$ \$Mg-nat

$13027.70 \mathrm{C} \quad 2.793720 \mathrm{E}-02$ \$Al-27

$14000.60 \mathrm{C} \quad 1.130110 \mathrm{E}-04$ \$Si-nat

$24000.50 \mathrm{C} \quad 2.304760 \mathrm{E}-05$ \$Cr-nat

$29000.50 \mathrm{C} \quad 2.081160 \mathrm{E}-05$ \$Cu-nat

$5010.70 \mathrm{C} \quad 1.976801 \mathrm{E}-05$ \$ -10

$92234.70 \mathrm{C} \quad 6.290244 \mathrm{E}-06$ \$U-234

$92235.70 \mathrm{C} \quad 4.854137 \mathrm{E}-04 \quad \$ \mathrm{U}-235$

$92236.70 \mathrm{C} \quad 3.638147 \mathrm{E}-06 \quad \$ \mathrm{U}-236$

$92237.70 \mathrm{C} \quad 2.904335 \mathrm{E}-29$ \$U-237

$92238.70 \mathrm{C} \quad 3.117785 \mathrm{E}-05$ \$U-238

$93237.70 \mathrm{C} \quad 2.904320 \mathrm{E}-29 \quad \$ \mathrm{~Np} 237$

$94239.70 \mathrm{C} \quad 2.879973 \mathrm{E}-29$ \$Pu239

$94240.70 \mathrm{C} \quad 2.867956 \mathrm{E}-29$ \$Pu240

$94241.70 \mathrm{C} \quad 2.856023 \mathrm{E}-29$ \$Pu241

$36083.70 \mathrm{C} \quad 8.303334 \mathrm{E}-29 \quad \$ \mathrm{Kr}-83$

$42095.70 \mathrm{C} \quad 7.178626 \mathrm{E}-29$ \$Mo-95

$44101.70 \mathrm{C} \quad 6.822852 \mathrm{E}-29$ \$Ru101

$45103.70 \mathrm{C} \quad 6.690253 \mathrm{E}-29$ \$Rh103

$45105.70 \mathrm{C} \quad 6.562692 \mathrm{E}-29$ \$Rh105

$48113.70 \mathrm{C} \quad 6.097758 \mathrm{E}-29 \$ \mathrm{Cd} 113$

$54131.70 \mathrm{C} \quad 5.259260 \mathrm{E}-29$ \$Xe131

$54133.70 \mathrm{C} \quad 5.180129 \mathrm{E}-29$ \$Xe133

$55133.70 \mathrm{C} \quad 5.180102 \mathrm{E}-29$ \$Cs133

$54135.70 \mathrm{C} \quad 5.065689 \mathrm{E}-29 \$ \mathrm{Xe} 135$

$57140.70 \mathrm{C} \quad 4.920766 \mathrm{E}-29$ \$La140

$58141.70 \mathrm{C} \quad 4.885933 \mathrm{E}-29 \$ \mathrm{Ce} 141$

$59143.70 \mathrm{C} \quad 4.817476 \mathrm{E}-29 \$ \mathrm{Pr} 143$

$60143.70 \mathrm{C} \quad 4.817471 \mathrm{E}-29 \$ \mathrm{Nd} 143$

$60145.70 \mathrm{C} \quad 4.750891 \mathrm{E}-29 \$ \mathrm{Nd} 145$

$61147.70 \mathrm{C} \quad 4.686170 \mathrm{E}-29 \$ \mathrm{Pm} 147$

$61149.70 \mathrm{C} \quad 4.623135 \mathrm{E}-29 \$ \mathrm{Pm} 149$

$62149.70 \mathrm{C} \quad 4.623132 \mathrm{E}-29 \quad \$ \mathrm{Sm} 149$

$61151.70 \mathrm{C} \quad 4.561735 \mathrm{E}-29 \$ \mathrm{Pm} 151$

$62151.70 \mathrm{C} \quad 4.561782 \mathrm{E}-29$ \$Sm151

$62152.70 \mathrm{C} \quad 4.531760 \mathrm{E}-29$ \$Sm152 
$63153.70 \mathrm{C} \quad 4.502082 \mathrm{E}-29 \$ \mathrm{Eu} 153$

$63155.70 \mathrm{C} \quad 4.443934 \mathrm{E}-29$ \$Eu155

$64157.70 \mathrm{C} \quad 4.387245 \mathrm{E}-29 \$ \mathrm{Gd} 157$

mt2132
C
C
c
c
c
c
C
C
C
m2133

lwtr.10t

ATR Element No. $=7$

Radial Zone No. $=1$

Axial Zone No. $=7$

Neutron Cross Sections $=27 \mathrm{C}$

Total Number Density $=7.975810 \mathrm{E}-02 \mathrm{a} / \mathrm{b}-\mathrm{cm}$

\begin{abstract}
$1001.70 \mathrm{C} \quad 3.393340 \mathrm{E}-02$ \$ $\mathrm{H}-1$
$8016.70 \mathrm{C} \quad 1.696670 \mathrm{E}-02 \$ 0-16$

$12000.60 \mathrm{C} \quad 2.176490 \mathrm{E}-04$ \$Mg-nat

$13027.70 \mathrm{C} \quad 2.793720 \mathrm{E}-02$ \$Al-27

$14000.60 \mathrm{C} \quad 1.130110 \mathrm{E}-04$ \$Si-nat

$24000.50 \mathrm{C} \quad 2.304760 \mathrm{E}-05$ \$Cr-nat

$29000.50 \mathrm{C} \quad 2.081160 \mathrm{E}-05$ \$Cu-nat

$5010.70 \mathrm{C} \quad 1.976801 \mathrm{E}-05$ \$ B-10

$92234.70 \mathrm{C} \quad 6.290244 \mathrm{E}-06 \quad \$ \mathrm{U}-234$

$92235.70 \mathrm{C} \quad 4.854137 \mathrm{E}-04 \quad \$ \mathrm{U}-235$

$92236.70 \mathrm{C} \quad 3.638147 \mathrm{E}-06 \$ \mathrm{U}-236$

$92237.70 \mathrm{C} \quad 2.904335 \mathrm{E}-29$ \$U-237

$92238.70 \mathrm{C} \quad 3.117785 \mathrm{E}-05$ \$U-238

$93237.70 \mathrm{C} \quad 2.904320 \mathrm{E}-29 \quad \$ N p 237$

$94239.70 \mathrm{C} \quad 2.879973 \mathrm{E}-29$ \$Pu239

$94240.70 \mathrm{C} \quad 2.867956 \mathrm{E}-29$ \$Pu240

$94241.70 \mathrm{C} \quad 2.856023 \mathrm{E}-29$ \$Pu241

$36083.70 \mathrm{C} \quad 8.303334 \mathrm{E}-29 \$ \mathrm{Kr}-83$

$42095.70 \mathrm{C} \quad 7.178626 \mathrm{E}-29$ \$Mo-95

$44101.70 \mathrm{C} \quad 6.822852 \mathrm{E}-29$ \$Ru101

$45103.70 \mathrm{C} \quad 6.690253 \mathrm{E}-29$ \$Rh103

$45105.70 \mathrm{C} \quad 6.562692 \mathrm{E}-29$ \$Rh105

$48113.70 \mathrm{C} \quad 6.097758 \mathrm{E}-29$ \$Cd113

$54131.70 \mathrm{C} \quad 5.259260 \mathrm{E}-29$ \$Xe131

$54133.70 \mathrm{C} \quad 5.180129 \mathrm{E}-29$ \$Xe133

$55133.70 \mathrm{C} \quad 5.180102 \mathrm{E}-29 \$ \mathrm{Cs} 133$

$54135.70 \mathrm{C} \quad 5.065689 \mathrm{E}-29$ \$Xe135

$57140.70 \mathrm{C} \quad 4.920766 \mathrm{E}-29$ \$La140

$58141.70 \mathrm{C} \quad 4.885933 \mathrm{E}-29 \$ \mathrm{Ce} 141$

$59143.70 \mathrm{C} \quad 4.817476 \mathrm{E}-29 \quad \$ \operatorname{Pr} 143$

$60143.70 \mathrm{C} \quad 4.817471 \mathrm{E}-29 \$ \mathrm{Nd143}$

$60145.70 \mathrm{C} \quad 4.750891 \mathrm{E}-29 \$ \mathrm{Nd} 145$

$61147.70 \mathrm{C} \quad 4.686170 \mathrm{E}-29 \$ \mathrm{Pm} 147$

$61149.70 \mathrm{C} \quad 4.623135 \mathrm{E}-29 \$ \mathrm{Pm} 149$

$62149.70 \mathrm{C} \quad 4.623132 \mathrm{E}-29 \$ \mathrm{Sm} 149$

$61151.70 \mathrm{C} \quad 4.561735 \mathrm{E}-29 \$ \mathrm{Pm} 151$

$62151.70 \mathrm{C} \quad 4.561782 \mathrm{E}-29 \$ \mathrm{Sm} 151$

$62152.70 \mathrm{C} \quad 4.531760 \mathrm{E}-29 \$ \mathrm{Sm} 152$

$63153.70 \mathrm{C} \quad 4.502082 \mathrm{E}-29 \$ \mathrm{Eu} 153$

$63155.70 \mathrm{C} \quad 4.443934 \mathrm{E}-29$ \$Eu155

$64157.70 \mathrm{C} \quad 4.387245 \mathrm{E}-29$ \$Gd157
\end{abstract}

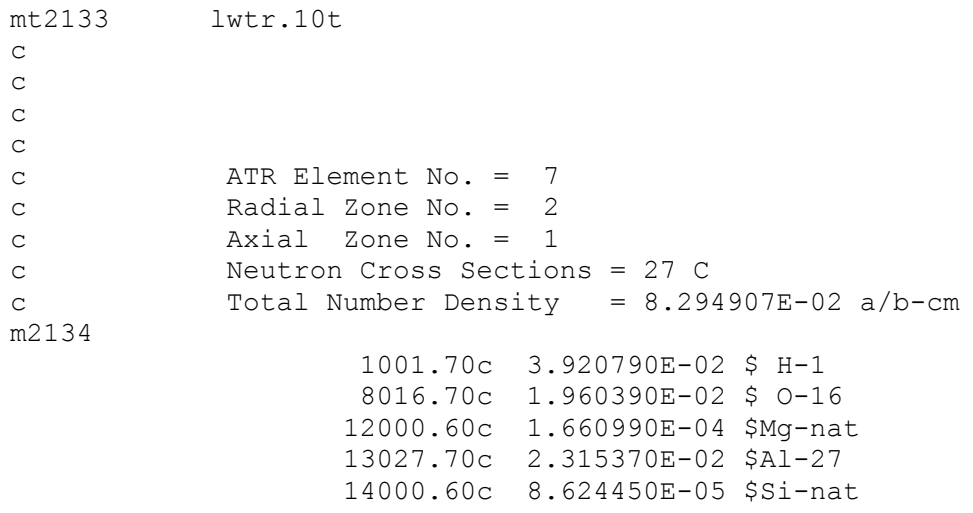

lwtr. 10 t 


\begin{tabular}{|c|c|c|c|}
\hline \\
\hline \multicolumn{4}{|c|}{$1.552830 \mathrm{E}-05$ \$Cr-nat } \\
\hline & $29000.50 \mathrm{c}$ & $1.588240 \mathrm{E}-05$ & \$Cu-nat \\
\hline & $5010.70 c$ & $1.714619 \mathrm{E}-28$ & $\$ B-10$ \\
\hline & $92234.70 c$ & $8.360426 \mathrm{E}-06$ & $\$ U-234$ \\
\hline & $92235.70 \mathrm{c}$ & $6.451682 \mathrm{E}-04$ & $\$ \mathrm{U}-235$ \\
\hline & $92236.70 \mathrm{C}$ & $4.835498 \mathrm{E}-06$ & $\$ U-236$ \\
\hline & $92237.70 \mathrm{C}$ & $7.242605 \mathrm{E}-30$ & $\$ \mathrm{U}-237$ \\
\hline & $92238.70 \mathrm{c}$ & $4.143880 \mathrm{E}-05$ & $\$ \mathrm{U}-238$ \\
\hline & $93237.70 \mathrm{c}$ & $7.242569 \mathrm{E}-30$ & \$Np237 \\
\hline & $94239.70 \mathrm{c}$ & $7.181854 \mathrm{E}-30$ & \$Pu239 \\
\hline & $94240.70 \mathrm{C}$ & $7.151887 \mathrm{E}-30$ & \$Pu240 \\
\hline & $94241.70 \mathrm{c}$ & $7.122128 \mathrm{E}-30$ & \$Pu241 \\
\hline & $36083.70 \mathrm{c}$ & $2.070621 \mathrm{E}-29$ & $\$ \mathrm{Kr}-83$ \\
\hline & $42095.70 \mathrm{c}$ & $1.790150 \mathrm{E}-29$ & $\$ M o-95$ \\
\hline & $44101.70 \mathrm{c}$ & $1.701430 \mathrm{E}-29$ & \$Ru101 \\
\hline & $45103.70 \mathrm{C}$ & $1.668363 \mathrm{E}-29$ & \$Rh103 \\
\hline & $45105.70 \mathrm{c}$ & $1.636554 \mathrm{E}-29$ & $\$ R h 105$ \\
\hline & $48113.70 \mathrm{c}$ & $1.520612 \mathrm{E}-29$ & $\$ \mathrm{Cd113}$ \\
\hline & $54131.70 \mathrm{c}$ & $1.311513 \mathrm{E}-29$ & $\$ \mathrm{Xe131}$ \\
\hline & $54133.70 \mathrm{c}$ & $1.291780 \mathrm{E}-29$ & $\$$ Xe133 \\
\hline & $55133.70 \mathrm{C}$ & $1.291774 \mathrm{E}-29$ & $\$ \mathrm{Cs} 133$ \\
\hline & $54135.70 \mathrm{C}$ & $1.263242 \mathrm{E}-29$ & $\$ \mathrm{Xe1} 35$ \\
\hline & $57140.70 \mathrm{c}$ & $1.227103 \mathrm{E}-29$ & $\$ \mathrm{La} 140$ \\
\hline & $58141.70 \mathrm{C}$ & $1.218416 \mathrm{E}-29$ & $\$ \mathrm{Ce} 141$ \\
\hline & $59143.70 \mathrm{c}$ & $1.201345 \mathrm{E}-29$ & $\$ \operatorname{Pr} 143$ \\
\hline & $60143.70 \mathrm{c}$ & $1.201344 \mathrm{E}-29$ & $\$ N d 143$ \\
\hline & $60145.70 \mathrm{c}$ & $1.184740 \mathrm{E}-29$ & \$Nd14 5 \\
\hline & $61147.70 \mathrm{c}$ & $1.168601 \mathrm{E}-29$ & $\$ \operatorname{Pm} 147$ \\
\hline & $61149.70 \mathrm{c}$ & $1.152882 \mathrm{E}-29$ & $\$ \operatorname{Pm} 149$ \\
\hline & $62149.70 \mathrm{c}$ & $1.152881 \mathrm{E}-29$ & $\$$ Sm1 49 \\
\hline & $61151.70 \mathrm{C}$ & $1.137570 \mathrm{E}-29$ & $\$ \operatorname{Pm} 151$ \\
\hline & $62151.70 \mathrm{c}$ & $1.137582 \mathrm{E}-29$ & $\$ \operatorname{Sm151}$ \\
\hline & $62152.70 \mathrm{c}$ & $1.130095 \mathrm{E}-29$ & \$Sm152 \\
\hline & $63153.70 \mathrm{c}$ & $1.122694 \mathrm{E}-29$ & \$Eu153 \\
\hline & $63155.70 \mathrm{c}$ & $1.108194 \mathrm{E}-29$ & \$Eu155 \\
\hline & $64157.70 \mathrm{c}$ & $1.094057 \mathrm{E}-29$ & \$Gd157 \\
\hline lwtr. 10 & & & \\
\hline ATR El & ement No. $=$ & 7 & \\
\hline Radial & Zone No. = & 2 & \\
\hline Axial & Zone No. = & 2 & \\
\hline Neutro & n Cross Sect & tions $=27 \mathrm{C}$ & \\
\hline & Number Densi & $=8.294$ & $907 \mathrm{E}-02 \mathrm{a} / \mathrm{b}-\mathrm{cm}$ \\
\hline & $1001.70 \mathrm{C}$ & $3.920790 \mathrm{E}-02$ & $\$ \mathrm{H}-1$ \\
\hline & $8016.70 \mathrm{c}$ & $1.960390 \mathrm{E}-02$ & $\$ 0-16$ \\
\hline & $12000.60 \mathrm{c}$ & $1.660990 \mathrm{E}-04$ & $\$ M g-n a t$ \\
\hline & $13027.70 \mathrm{c}$ & $2.315370 \mathrm{E}-02$ & $\$ A l-27$ \\
\hline & $14000.60 \mathrm{C}$ & $8.624450 \mathrm{E}-05$ & \$Si-nat \\
\hline & $24000.50 \mathrm{c}$ & $1.552830 \mathrm{E}-05$ & \$Cr-nat \\
\hline & $29000.50 \mathrm{c}$ & $1.588240 \mathrm{E}-05$ & $\$ \mathrm{Cu}-$ nat \\
\hline & $5010.70 \mathrm{c}$ & $1.714619 \mathrm{E}-28$ & $\$ B-10$ \\
\hline & $92234.70 \mathrm{c}$ & $8.360426 \mathrm{E}-06$ & $\$ \mathrm{U}-234$ \\
\hline & $92235.70 \mathrm{C}$ & $6.451682 \mathrm{E}-04$ & $\$ U-235$ \\
\hline & $92236.70 \mathrm{c}$ & $4.835498 \mathrm{E}-06$ & $\$ U-236$ \\
\hline & $92237.70 \mathrm{c}$ & $7.242605 \mathrm{E}-30$ & $\$ \mathrm{U}-237$ \\
\hline & $92238.70 \mathrm{c}$ & $4.143880 \mathrm{E}-05$ & $\$ U-238$ \\
\hline & $93237.70 \mathrm{C}$ & $7.242569 \mathrm{E}-30$ & $\$ \mathrm{~Np} 237$ \\
\hline & $94239.70 \mathrm{C}$ & $7.181854 \mathrm{E}-30$ & \$Pu239 \\
\hline & $94240.70 \mathrm{c}$ & $7.151887 \mathrm{E}-30$ & \$Pu240 \\
\hline & $94241.70 \mathrm{c}$ & $7.122128 \mathrm{E}-30$ & \$Pu241 \\
\hline & $36083.70 \mathrm{c}$ & $2.070621 E-29$ & $\$ K r-83$ \\
\hline & $42095.70 \mathrm{c}$ & $1.790150 \mathrm{E}-29$ & $\$ M o-95$ \\
\hline & $44101.70 \mathrm{C}$ & $1.701430 \mathrm{E}-29$ & \$Ru101 \\
\hline & $45103.70 \mathrm{C}$ & $1.668363 \mathrm{E}-29$ & \$Rh103 \\
\hline & $45105.70 \mathrm{c}$ & $1.636554 \mathrm{E}-29$ & \$Rh105 \\
\hline & $48113.70 \mathrm{c}$ & $1.520612 \mathrm{E}-29$ & $\$ C d 113$ \\
\hline & $54131.70 \mathrm{C}$ & $1.311513 \mathrm{E}-29$ & $\$ \mathrm{Xe131}$ \\
\hline
\end{tabular}

mt2134 lwtr.10t

c

C

C

c

C

C

C

m2135 


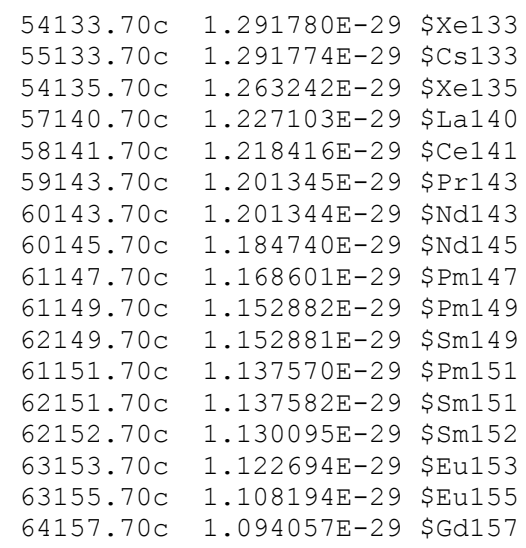
mt2135 lwtr.10t

$\mathrm{C}$

C

C

C

C ATR Element No. = 7

C Radial Zone No. $=2$

C Axial Zone No. = 3

C Neutron Cross Sections $=27 \mathrm{C}$

C Total Number Density $=8.294907 \mathrm{E}-02 \mathrm{a} / \mathrm{b}-\mathrm{cm}$

m2136

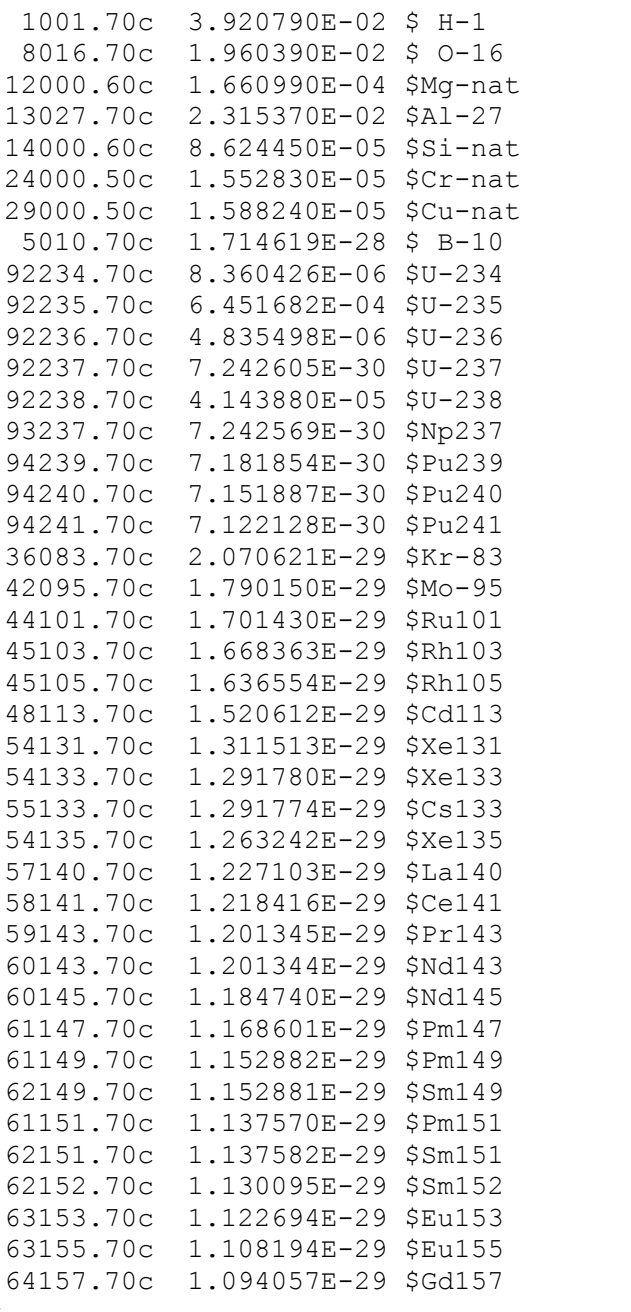

mt2136 lwtr.10t

C 
ATR Element No. $=7$

Radial Zone No. $=2$

Axial Zone No. $=4$

Neutron Cross Sections $=27 \mathrm{C}$

Total Number Density $=8.294907 \mathrm{E}-02 \mathrm{a} / \mathrm{b}-\mathrm{cm}$

lwtr.10t

\begin{tabular}{|c|c|c|}
\hline 1001 & $3.920790 \mathrm{E}-02$ & $\$ \mathrm{H}-1$ \\
\hline $8016.70 \mathrm{c}$ & $1.960390 \mathrm{E}-02$ & $\$ 0-16$ \\
\hline $000.60 \mathrm{c}$ & $1.660990 \mathrm{E}-04$ & $\$ M g-n a t$ \\
\hline $7.70 \mathrm{c}$ & $2.315370 \mathrm{E}-02$ & $\$ A I-27$ \\
\hline $0.60 \mathrm{C}$ & $8.624450 \mathrm{E}-05$ & \$Si-nat \\
\hline & $1.552830 \mathrm{E}-05$ & $\$ C r-n a t$ \\
\hline $.50 \mathrm{c}$ & $1.588240 \mathrm{E}-05$ & $\$ \mathrm{Cu}-\mathrm{nat}$ \\
\hline & $8.573098 E-29$ & $\$ B-10$ \\
\hline $.70 \mathrm{C}$ & $8.360423 E-06$ & $\$ U-234$ \\
\hline 7 & $6.451679 \mathrm{E}-04$ & $\$ U-235$ \\
\hline $.70 \mathrm{C}$ & $4.835496 \mathrm{E}-06$ & $\$ U-236$ \\
\hline $.70 \mathrm{c}$ & $3.621303 E-30$ & $\$ U-237$ \\
\hline $.70 \mathrm{C}$ & $4.143878 \mathrm{E}-05$ & $\$ U-238$ \\
\hline $.70 \mathrm{C}$ & $3.621285 E-30$ & $\$ N p 237$ \\
\hline $.70 \mathrm{C}$ & $3.590928 \mathrm{E}-30$ & $\$ P u 239$ \\
\hline $.70 \mathrm{c}$ & $3.575944 \mathrm{E}-30$ & $\$ \mathrm{Pu} 240$ \\
\hline $.70 \mathrm{C}$ & $3.561065 \mathrm{E}-30$ & $\$ \mathrm{Pu} 241$ \\
\hline $.70 \mathrm{c}$ & $311 E-29$ & $\$ K r-83$ \\
\hline $.70 \mathrm{C}$ & 8.9507 & $\$ M o-95$ \\
\hline & & \$Ru101 \\
\hline $\mathrm{OC}_{\mathrm{C}}$ & $3 E-30$ & \$Rh103 \\
\hline & & $\$ R h 105$ \\
\hline $0 \mathrm{c}$ & $E-30$ & $\$ \mathrm{Cd} 113$ \\
\hline & & $\$ x e 131$ \\
\hline $70 \mathrm{c}$ & $3 E-30$ & $\$ x e 133$ \\
\hline $.70 \mathrm{C}$ & $6.458870 \mathrm{E}-30$ & $\$ \mathrm{Cs} 133$ \\
\hline $.70 \mathrm{C}$ & $3 E-30$ & $\$ x e 135$ \\
\hline & 6.13551 & $\$ \mathrm{La} 140$ \\
\hline C & $2 E-30$ & $\$ \mathrm{Ce} 141$ \\
\hline $.70 \mathrm{C}$ & $6.006725 \mathrm{E}-30$ & $\$ \operatorname{Pr} 143$ \\
\hline & $E-30$ & $\$ N d 143$ \\
\hline $.70 \mathrm{C}$ & $3 E-30$ & $\$ N d 145$ \\
\hline .7 & $E-30$ & $\$ P m 147$ \\
\hline $.70 \mathrm{C}$ & 5.7644 & $\$ P m 149$ \\
\hline .7 & $5 E-30$ & $\$ S m 149$ \\
\hline & $5.687851 \mathrm{E}-30$ & $\$ P m 151$ \\
\hline .7 & $5.687911 \mathrm{E}-30$ & $\$ S m 151$ \\
\hline & $5.650478 \mathrm{E}-30$ & $\$ S m 152$ \\
\hline & $5.613472 \mathrm{E}-30$ & $\$ E u 153$ \\
\hline & $969 E-30$ & \$Eu155 \\
\hline & 5.4 & $\$$ Gd1 57 \\
\hline
\end{tabular}
$64157.70 \mathrm{C} 5.470287 \mathrm{E}-30$ SGd 57

ATR Element No. $=7$

Radial Zone No. $=2$

Axial Zone No. = 5

Neutron Cross Sections $=27 \mathrm{C}$

Total Number Density $=8.294907 \mathrm{E}-02 \mathrm{a} / \mathrm{b}-\mathrm{cm}$

1001.70C 3.920790E-02 \$ H-1

$8016.70 \mathrm{C} \quad 1.960390 \mathrm{E}-02$ \$ O-16

$12000.60 \mathrm{C} \quad 1.660990 \mathrm{E}-04$ \$Mg-nat

$13027.70 \mathrm{C} \quad 2.315370 \mathrm{E}-02$ \$Al-27

$14000.60 \mathrm{C} \quad 8.624450 \mathrm{E}-05$ \$Si-nat

$24000.50 \mathrm{C} \quad 1.552830 \mathrm{E}-05$ \$Cr-nat

$29000.50 \mathrm{C} \quad 1.588240 \mathrm{E}-05$ \$Cu-nat

$5010.70 \mathrm{C} \quad 1.714619 \mathrm{E}-28$ \$ B-10

$92234.70 \mathrm{C} \quad 8.360426 \mathrm{E}-06$ \$U-234

$92235.70 \mathrm{C} \quad 6.451682 \mathrm{E}-04 \quad \$ \mathrm{U}-235$ 
$92236.70 \mathrm{C} \quad 4.835498 \mathrm{E}-06 \quad \$ \mathrm{U}-236$

$92237.70 \mathrm{C} \quad 7.242605 \mathrm{E}-30 \quad \$ \mathrm{U}-237$

$92238.70 \mathrm{C} \quad 4.143880 \mathrm{E}-05$ \$U-238

$93237.70 \mathrm{C} \quad 7.242569 \mathrm{E}-30 \quad \$ \mathrm{~Np} 237$

$94239.70 \mathrm{C} \quad 7.181854 \mathrm{E}-30$ \$Pu239

$94240.70 \mathrm{C} \quad 7.151887 \mathrm{E}-30$ \$Pu240

$94241.70 \mathrm{C} \quad 7.122128 \mathrm{E}-30$ \$Pu241

$36083.70 \mathrm{C} \quad 2.070621 \mathrm{E}-29$ \$Kr-83

$42095.70 \mathrm{C} \quad 1.790150 \mathrm{E}-29$ \$Mo-95

$44101.70 \mathrm{C} \quad 1.701430 \mathrm{E}-29$ \$Ru101

$45103.70 \mathrm{C} \quad 1.668363 \mathrm{E}-29$ \$Rh103

$45105.70 \mathrm{C} \quad 1.636554 \mathrm{E}-29$ \$Rh105

$48113.70 \mathrm{C} \quad 1.520612 \mathrm{E}-29$ \$Cd113

$54131.70 \mathrm{C} \quad 1.311513 \mathrm{E}-29$ \$Xe131

$54133.70 \mathrm{C} \quad 1.291780 \mathrm{E}-29$ \$Xe133

$55133.70 \mathrm{C} \quad 1.291774 \mathrm{E}-29$ \$Cs133

$54135.70 \mathrm{C} \quad 1.263242 \mathrm{E}-29$ \$Xe135

$57140.70 \mathrm{C} \quad 1.227103 \mathrm{E}-29$ \$La140

$58141.70 \mathrm{C} \quad 1.218416 \mathrm{E}-29$ \$Ce141

$59143.70 \mathrm{C} \quad 1.201345 \mathrm{E}-29 \quad \$ \operatorname{Pr} 143$

$60143.70 \mathrm{C} \quad 1.201344 \mathrm{E}-29 \$ \mathrm{Nd} 143$

$60145.70 \mathrm{C} \quad 1.184740 \mathrm{E}-29$ \$Nd145

$61147.70 \mathrm{C} \quad 1.168601 \mathrm{E}-29$ \$Pm147

$61149.70 \mathrm{C} \quad 1.152882 \mathrm{E}-29 \$ \mathrm{Pm} 149$

$62149.70 \mathrm{C} \quad 1.152881 \mathrm{E}-29 \$ \mathrm{Sm} 149$

$61151.70 \mathrm{C} \quad 1.137570 \mathrm{E}-29$ \$Pm151

$62151.70 \mathrm{C} \quad 1.137582 \mathrm{E}-29$ \$Sm151

$62152.70 \mathrm{C} \quad 1.130095 \mathrm{E}-29 \quad \$ \mathrm{Sm} 152$

$63153.70 \mathrm{C} \quad 1.122694 \mathrm{E}-29$ \$Eu153

$63155.70 \mathrm{C} \quad 1.108194 \mathrm{E}-29$ \$Eu155

mt2138 lwtr.10t

$64157.70 \mathrm{C} \quad 1.094057 \mathrm{E}-29$ \$Gd157

c

C

C

m2139

ATR Element No. $=7$
Radial Zone No. $=2$
Axial Zone No. $=6$
Neutron Cross Sections $=27 \mathrm{C}$
Total Number Density $=8.294907 \mathrm{E}-02 \mathrm{a} / \mathrm{b}-\mathrm{cm}$

$1001.70 \mathrm{C} \quad 3.920790 \mathrm{E}-02$ \$ H-1

$8016.70 \mathrm{C} \quad 1.960390 \mathrm{E}-02$ \$ O-16

$12000.60 \mathrm{C} \quad 1.660990 \mathrm{E}-04$ \$Mg-nat

$13027.70 \mathrm{C} \quad 2.315370 \mathrm{E}-02$ \$Al-27

$14000.60 \mathrm{C} \quad 8.624450 \mathrm{E}-05$ \$Si-nat

$24000.50 \mathrm{C} \quad 1.552830 \mathrm{E}-05$ SCr-nat

$29000.50 \mathrm{C} \quad 1.588240 \mathrm{E}-05$ \$Cu-nat

$5010.70 \mathrm{C} \quad 1.714619 \mathrm{E}-28$ \$ B-10

$92234.70 \mathrm{C} \quad 8.360426 \mathrm{E}-06 \quad \$ \mathrm{U}-234$

$92235.70 \mathrm{C} \quad 6.451682 \mathrm{E}-04 \quad \$ \mathrm{U}-235$

$92236.70 \mathrm{C} \quad 4.835498 \mathrm{E}-06$ \$U-236

$92237.70 \mathrm{C} \quad 7.242605 \mathrm{E}-30 \quad \$ \mathrm{U}-237$

$92238.70 \mathrm{C} \quad 4.143880 \mathrm{E}-05 \mathrm{SU}-238$

$93237.70 \mathrm{C} \quad 7.242569 \mathrm{E}-30 \quad \$ \mathrm{~Np} 237$

$94239.70 \mathrm{C} \quad 7.181854 \mathrm{E}-30 \quad \$ \mathrm{Pu} 239$

$94240.70 \mathrm{C} \quad 7.151887 \mathrm{E}-30$ \$Pu240

$94241.70 \mathrm{C} \quad 7.122128 \mathrm{E}-30$ \$Pu2 41

$36083.70 \mathrm{C} \quad 2.070621 \mathrm{E}-29$ \$Kr-83

$42095.70 \mathrm{C} \quad 1.790150 \mathrm{E}-29$ \$Mo-95

$44101.70 \mathrm{C} \quad 1.701430 \mathrm{E}-29$ \$Ru101

$45103.70 \mathrm{C} \quad 1.668363 \mathrm{E}-29$ \$Rh103

$45105.70 \mathrm{C} \quad 1.636554 \mathrm{E}-29$ \$Rh105

$48113.70 \mathrm{C} \quad 1.520612 \mathrm{E}-29$ \$Cd113

$54131.70 \mathrm{C} \quad 1.311513 \mathrm{E}-29$ \$Xe131

$54133.70 \mathrm{C} \quad 1.291780 \mathrm{E}-29$ \$Xe133

$55133.70 \mathrm{C} \quad 1.291774 \mathrm{E}-29 \quad \$ \mathrm{Cs} 133$

$54135.70 \mathrm{C} \quad 1.263242 \mathrm{E}-29$ \$Xe135

$57140.70 \mathrm{C} \quad 1.227103 \mathrm{E}-29$ \$La140

$58141.70 \mathrm{C} \quad 1.218416 \mathrm{E}-29$ \$Ce141 
$59143.70 \mathrm{C} \quad 1.201345 \mathrm{E}-29 \quad \$ \operatorname{Pr} 143$

$60143.70 \mathrm{C} \quad 1.201344 \mathrm{E}-29 \$ \mathrm{Nd} 143$

$60145.70 \mathrm{C} \quad 1.184740 \mathrm{E}-29$ \$Nd145

$61147.70 \mathrm{C} \quad 1.168601 \mathrm{E}-29 \$ \mathrm{Pm} 147$

$61149.70 \mathrm{C} \quad 1.152882 \mathrm{E}-29 \$ \mathrm{Pm} 149$

$62149.70 \mathrm{C} \quad 1.152881 \mathrm{E}-29 \quad \$ \mathrm{Sm} 149$

$61151.70 \mathrm{C} \quad 1.137570 \mathrm{E}-29$ \$Pm151

$62151.70 \mathrm{C} \quad 1.137582 \mathrm{E}-29$ \$Sm151

$62152.70 \mathrm{C} \quad 1.130095 \mathrm{E}-29 \$ \mathrm{Sm} 152$

$63153.70 \mathrm{C} \quad 1.122694 \mathrm{E}-29$ \$Eu153

$63155.70 \mathrm{C} \quad 1.108194 \mathrm{E}-29$ \$Eu155

$64157.70 \mathrm{C} \quad 1.094057 \mathrm{E}-29$ \$Gd157

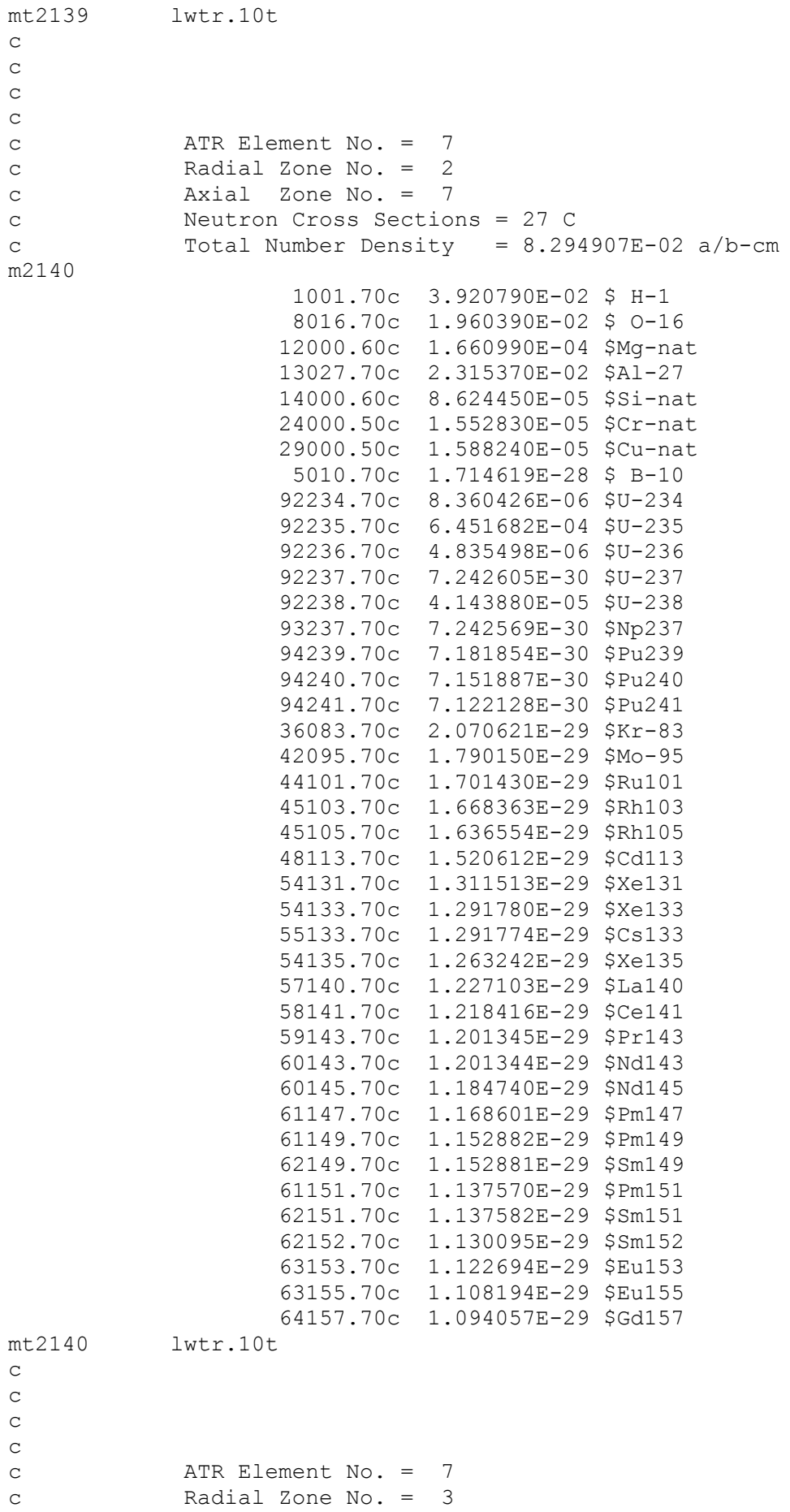


C Axial Zone No. = 1

C Neutron Cross Sections $=27 \mathrm{C}$

C Total Number Density $=7.858601 \mathrm{E}-02 \mathrm{a} / \mathrm{b}-\mathrm{cm}$

m2141

$\begin{array}{llll}1001.70 \mathrm{C} & 3.212840 \mathrm{E}-02 & \$ \mathrm{H}-1 \\ 8016.70 \mathrm{C} & 1.606420 \mathrm{E}-02 & \$ \mathrm{O}-1\end{array}$

$12000.60 \mathrm{C} \quad 2.399430 \mathrm{E}-04$ \$Mg-nat

$13027.70 \mathrm{C} \quad 2.958200 \mathrm{E}-02$ \$Al-27

$14000.60 \mathrm{C} \quad 1.245870 \mathrm{E}-04$ \$Si-nat

$24000.50 \mathrm{C} \quad 2.243180 \mathrm{E}-05$ \$Cr-nat

$29000.50 \mathrm{C} \quad 2.294330 \mathrm{E}-05$ \$Cu-nat

$5010.70 \mathrm{C} \quad 1.312339 \mathrm{E}-05$ \$ B -10

$92234.70 \mathrm{C} \quad 4.639810 \mathrm{E}-06$ \$U-234

$92235.70 \mathrm{C} \quad 3.580509 \mathrm{E}-04 \quad \$ \mathrm{U}-235$

$92236.70 \mathrm{C} \quad 2.683571 \mathrm{E}-06 \mathrm{SU}-236$

$92237.70 \mathrm{C} \quad 1.545203 \mathrm{E}-29$ \$U-237

$92238.70 \mathrm{C} \quad 2.299741 \mathrm{E}-05$ \$U-238

$93237.70 \mathrm{C} \quad 1.545195 \mathrm{E}-29 \$ \mathrm{~Np} 237$

$94239.70 \mathrm{C} \quad 1.532242 \mathrm{E}-29$ \$Pu239

$94240.70 \mathrm{C} \quad 1.525848 \mathrm{E}-29$ \$Pu240

$94241.70 \mathrm{C} \quad 1.519499 \mathrm{E}-29$ \$Pu241

$36083.70 \mathrm{C} \quad 4.417651 \mathrm{E}-29$ \$Kr-83

$42095.70 \mathrm{C} \quad 3.819269 \mathrm{E}-29$ \$Mo-95

$44101.70 \mathrm{C} \quad 3.629985 \mathrm{E}-29$ \$Ru101

$45103.70 \mathrm{C} \quad 3.559438 \mathrm{E}-29$ \$Rh103

$45105.70 \mathrm{C} \quad 3.491572 \mathrm{E}-29$ \$Rh105

$48113.70 \mathrm{C} \quad 3.244211 \mathrm{E}-29$ \$Cd113

$54131.70 \mathrm{C} \quad 2.798102 \mathrm{E}-29$ \$Xe131

$54133.70 \mathrm{C} \quad 2.756002 \mathrm{E}-29 \$ \mathrm{Xe133}$

$55133.70 \mathrm{C} \quad 2.755987 \mathrm{E}-29$ S 2133

$54135.70 \mathrm{C} \quad 2.695116 \mathrm{E}-29$ \$Xe135

$57140.70 \mathrm{C} \quad 2.618012 \mathrm{E}-29$ \$La140

$58141.70 \mathrm{C} \quad 2.599480 \mathrm{E}-29 \quad \$ \mathrm{Ce} 141$

$59143.70 \mathrm{C} \quad 2.563058 \mathrm{E}-29 \$ \mathrm{Pr} 143$

$60143.70 \mathrm{C} \quad 2.563055 \mathrm{E}-29$ \$Nd143

$60145.70 \mathrm{C} \quad 2.527633 \mathrm{E}-29 \$ \mathrm{Nd1} 45$

$61147.70 \mathrm{C} \quad 2.493199 \mathrm{E}-29 \quad \$ \mathrm{Pm} 147$

$61149.70 \mathrm{C} \quad 2.459663 \mathrm{E}-29 \$ \mathrm{Pm} 149$

$62149.70 \mathrm{C} \quad 2.459661 \mathrm{E}-29 \$ \mathrm{Sm} 149$

$61151.70 \mathrm{C} \quad 2.426995 \mathrm{E}-29 \$ \mathrm{Pm} 151$

$62151.70 \mathrm{C} \quad 2.427021 \mathrm{E}-29$ \$Sm151

$62152.70 \mathrm{C} \quad 2.411048 \mathrm{E}-29$ \$Sm152

$63153.70 \mathrm{C} \quad 2.395258 \mathrm{E}-29$ \$Eu153

$63155.70 \mathrm{C} \quad 2.364321 \mathrm{E}-29$ \$Eu155

mt2141 lwtr.10t

c

C

$\mathrm{C}$

$\mathrm{C}$

C

C

C

C

$64157.70 \mathrm{C} \quad 2.334161 \mathrm{E}-29 \$ \mathrm{Gd} 157$

m2142

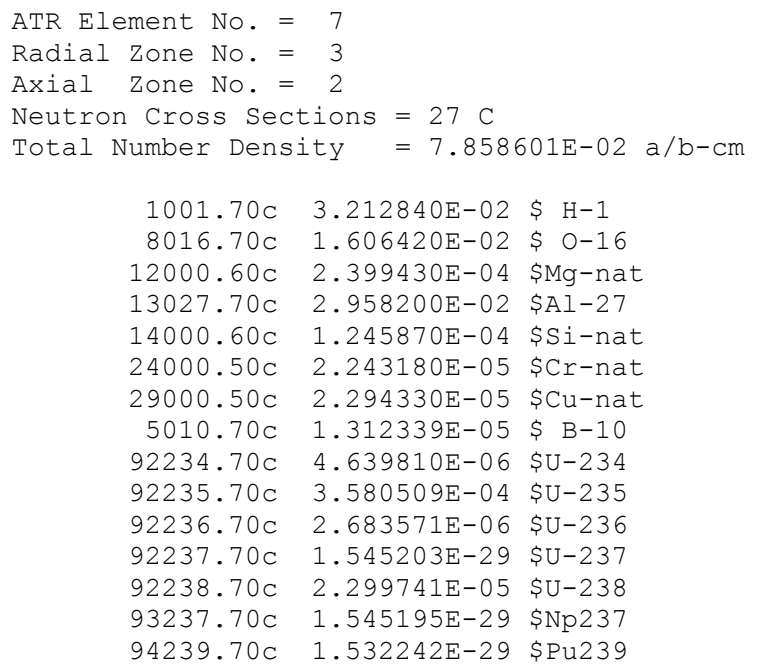




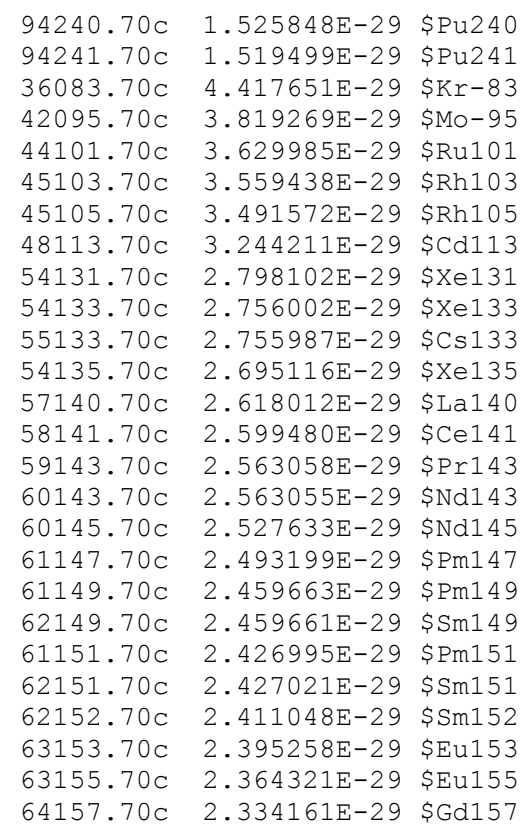

mt2142 lwtr.10t

$\mathrm{C}$

$\mathrm{C}$

$\mathrm{C}$

C ATR Element No. $=7$

C Radial Zone No. = 3

C Axial Zone No. = 3

C Neutron Cross Sections $=27 \mathrm{C}$

C Total Number Density $=7.858601 \mathrm{E}-02 \mathrm{a} / \mathrm{b}-\mathrm{cm}$

$\mathrm{m} 2143$

\begin{tabular}{|c|c|c|}
\hline $1001.70 \mathrm{c}$ & $3.212840 \mathrm{E}-02$ & $\$ \mathrm{H}-1$ \\
\hline $8016.70 \mathrm{c}$ & $1.606420 \mathrm{E}-02$ & $\$ 0-16$ \\
\hline $12000.60 \mathrm{c}$ & $2.399430 \mathrm{E}-04$ & $\$ M g-n a t$ \\
\hline $13027.70 \mathrm{C}$ & $2.958200 \mathrm{E}-02$ & $\$ A I-27$ \\
\hline $14000.60 \mathrm{c}$ & $1.245870 \mathrm{E}-04$ & \$Si-nat \\
\hline $24000.50 \mathrm{c}$ & $2.243180 \mathrm{E}-05$ & \$Cr-nat \\
\hline $29000.50 \mathrm{C}$ & $2.294330 \mathrm{E}-05$ & \$Cu-nat \\
\hline $5010.70 \mathrm{C}$ & $1.312339 \mathrm{E}-05$ & $\$ B-10$ \\
\hline $92234.70 \mathrm{C}$ & $4.639810 \mathrm{E}-06$ & $\$ U-234$ \\
\hline $92235.70 \mathrm{C}$ & $3.580509 \mathrm{E}-04$ & $\$ U-235$ \\
\hline $92236.70 \mathrm{C}$ & $2.683571 \mathrm{E}-06$ & $\$ U-236$ \\
\hline $92237.70 \mathrm{C}$ & $1.545203 E-29$ & $\$ U-237$ \\
\hline $92238.70 \mathrm{C}$ & $2.299741 \mathrm{E}-05$ & $\$ U-238$ \\
\hline $93237.70 \mathrm{C}$ & $1.545195 \mathrm{E}-29$ & $\$ N p 237$ \\
\hline $94239.70 \mathrm{C}$ & $1.532242 \mathrm{E}-29$ & $\$ P u 239$ \\
\hline $94240.70 \mathrm{C}$ & $1.525848 \mathrm{E}-29$ & $\$ P u 240$ \\
\hline $94241.70 \mathrm{C}$ & $1.519499 \mathrm{E}-29$ & $\$ P u 241$ \\
\hline $36083.70 \mathrm{c}$ & $4.417651 \mathrm{E}-29$ & $\$ K r-83$ \\
\hline $42095.70 \mathrm{C}$ & $3.819269 \mathrm{E}-29$ & $\$ M o-95$ \\
\hline $44101.70 \mathrm{C}$ & $3.629985 E-29$ & \$Ru101 \\
\hline $45103.70 \mathrm{C}$ & $3.559438 E-29$ & \$Rh103 \\
\hline $45105.70 \mathrm{C}$ & $3.491572 \mathrm{E}-29$ & \$Rh105 \\
\hline $48113.70 \mathrm{C}$ & $3.244211 E-29$ & $\$ \mathrm{Cd} 113$ \\
\hline $54131.70 \mathrm{C}$ & $2.798102 \mathrm{E}-29$ & \$xe131 \\
\hline $54133.70 \mathrm{C}$ & $2.756002 \mathrm{E}-29$ & $\$ x e 133$ \\
\hline $55133.70 \mathrm{C}$ & $2.755987 E-29$ & $\$ C s 133$ \\
\hline $54135.70 \mathrm{C}$ & $2.695116 \mathrm{E}-29$ & $\$ x e 135$ \\
\hline $57140.70 \mathrm{C}$ & $2.618012 \mathrm{E}-29$ & \$La1 40 \\
\hline $58141.70 \mathrm{C}$ & $2.599480 E-29$ & $\$ \mathrm{Ce} 141$ \\
\hline $59143.70 \mathrm{C}$ & $2.563058 \mathrm{E}-29$ & $\$ \operatorname{Pr} 143$ \\
\hline $60143.70 \mathrm{c}$ & $2.563055 E-29$ & $\$ N d 143$ \\
\hline $60145.70 \mathrm{c}$ & $2.527633 E-29$ & $\$ N d 145$ \\
\hline $61147.70 \mathrm{c}$ & $2.493199 \mathrm{E}-29$ & $\$ P m 147$ \\
\hline $61149.70 c$ & $2.459663 \mathrm{E}-29$ & $\$ P m 149$ \\
\hline
\end{tabular}


$62149.70 \mathrm{C} \quad 2.459661 \mathrm{E}-29 \$ \mathrm{Sm} 149$

$61151.70 \mathrm{C} \quad 2.426995 \mathrm{E}-29$ \$Pm151

$62151.70 \mathrm{C} \quad 2.427021 \mathrm{E}-29 \$ \mathrm{Sm} 151$

$62152.70 \mathrm{C} \quad 2.411048 \mathrm{E}-29 \$ \mathrm{Sm} 152$

$63153.70 \mathrm{C} \quad 2.395258 \mathrm{E}-29$ \$Eu153

$63155.70 \mathrm{C} \quad 2.364321 \mathrm{E}-29$ \$Eu155

mt2143 lwtr.10t $64157.70 \mathrm{C} 2.334161 \mathrm{E}-29$ \$Gd157

c

C

C

C

C

C

C

m2144

ATR Element No. $=7$

Radial Zone No. $=3$

Axial Zone No. = 4

Neutron Cross Sections $=27 \mathrm{C}$

Total Number Density $=7.858601 \mathrm{E}-02 \mathrm{a} / \mathrm{b}-\mathrm{cm}$

\begin{tabular}{|c|c|c|}
\hline $1001.70 \mathrm{C}$ & $3.212840 \mathrm{E}-02$ & \\
\hline $8016.70 \mathrm{C}$ & $1.606420 \mathrm{E}-02$ & $\$ 0-16$ \\
\hline $2000.60 \mathrm{c}$ & $2.399430 E-04$ & $\$ M g-n a t$ \\
\hline $27.70 \mathrm{C}$ & $2.958200 \mathrm{E}-02$ & $\$ A 1-27$ \\
\hline $000.60 \mathrm{C}$ & $1.245870 \mathrm{E}-04$ & $\$ S i-n a t$ \\
\hline 4000.50 & $2.243180 \mathrm{E}-05$ & $\$ C r-n a t$ \\
\hline 9000.50 & $2.294330 \mathrm{E}-05$ & $\$ \mathrm{Cu}-\mathrm{nat}$ \\
\hline 10.70 & $1.312339 \mathrm{E}-05$ & $\$ B-10$ \\
\hline 34.70 & $4.639812 E-06$ & $\$ U-234$ \\
\hline & $3.580510 E-04$ & $\$ \mathrm{U}-235$ \\
\hline $5.70 \mathrm{C}$ & $2.683571 \mathrm{E}-06$ & $\$ U-236$ \\
\hline & $7.726017 E-30$ & $\$ \mathrm{U}-237$ \\
\hline 3 & $2.299742 E-05$ & $\$ U-238$ \\
\hline & $7.725979 \mathrm{E}-30$ & \$Np237 \\
\hline . & $7.661212 \mathrm{E}-30$ & \$Pu239 \\
\hline & $7.629244 \mathrm{E}-30$ & \$Pu240 \\
\hline $.10 \mathrm{C}$ & $7.597500 \mathrm{E}-30$ & $\$ \mathrm{Pu} 241$ \\
\hline & $2.208826 E-29$ & $\$ K r-83$ \\
\hline $.70 \mathrm{C}$ & $1.909635 \mathrm{E}-29$ & $\$ \mathrm{Mo}-95$ \\
\hline 4 & $1.814993 E-29$ & \$Ru101 \\
\hline $.70 \mathrm{C}$ & $1.779720 \mathrm{E}-29$ & \$Rh103 \\
\hline C & $1.745786 \mathrm{E}-29$ & \$Rh105 \\
\hline $3.70 \mathrm{c}$ & $1.622106 \mathrm{E}-29$ & $\$ C d 113$ \\
\hline $70 \mathrm{c}$ & $1.399051 E-29$ & $\$ x e 131$ \\
\hline $.70 \mathrm{C}$ & $1.378001 \mathrm{E}-29$ & $\$ x e 133$ \\
\hline $\mathrm{C}$ & $1.377994 \mathrm{E}-29$ & $\$ \mathrm{Cs} 133$ \\
\hline .70 & $58 E-29$ & $\$ x e 135$ \\
\hline 57 & $06 E-29$ & \$La140 \\
\hline 58 & $40 E-29$ & $\$ \mathrm{Ce} 141$ \\
\hline & $-529 E-29$ & \$Pr143 \\
\hline 60 & $28 E-29$ & $\$ N d 143$ \\
\hline & $7 E-29$ & $\$ N d 145$ \\
\hline 61 & $00 E-29$ & $\$ \operatorname{Pm} 147$ \\
\hline & $32 E-29$ & $\$ P m 149$ \\
\hline 62 & $831 E-29$ & $\$ \operatorname{Sm} 149$ \\
\hline 61 & $98 E-29$ & \$Pm151 \\
\hline 62 & $511 E-29$ & $\$ S m 151$ \\
\hline & $24 E-29$ & $\$ S m 152$ \\
\hline 63 & $29 E-29$ & \$Eu153 \\
\hline & $E-29$ & \$Eu155 \\
\hline & 1 & \$Gd15 \\
\hline
\end{tabular}

mt2144

C

$\mathrm{C}$

C

C

C

$\mathrm{C}$

C

$\mathrm{C}$

C

m2145 lwtr.10t

ATR Element No. $=7$

Radial Zone No. = 3

Axial Zone No. = 5

Neutron Cross Sections $=27 \mathrm{C}$

Total Number Density $=7.858601 \mathrm{E}-02 \mathrm{a} / \mathrm{b}-\mathrm{cm}$

$1001.70 \mathrm{C} \quad 3.212840 \mathrm{E}-02 \$ \mathrm{H}-1$ 


\begin{tabular}{|c|c|c|c|}
\hline \\
\hline & $8016.70 \mathrm{C}$ & $1.606420 \mathrm{E}-02$ & $\$ 0-16$ \\
\hline & $12000.60 \mathrm{c}$ & $2.399430 \mathrm{E}-04$ & $\$ M g-n a t$ \\
\hline & $13027.70 \mathrm{c}$ & $2.958200 \mathrm{E}-02$ & $\$ A 1-27$ \\
\hline & $14000.60 \mathrm{c}$ & $1.245870 \mathrm{E}-04$ & \$Si-nat \\
\hline & $24000.50 \mathrm{c}$ & $2.243180 \mathrm{E}-05$ & $\$ C r-n a t$ \\
\hline & $29000.50 \mathrm{c}$ & $2.294330 \mathrm{E}-05$ & $\$ \mathrm{Cu}-$ nat \\
\hline & $5010.70 \mathrm{C}$ & $1.312339 \mathrm{E}-05$ & $\$ B-10$ \\
\hline & $92234.70 \mathrm{c}$ & $4.639810 \mathrm{E}-06$ & $\$ U-234$ \\
\hline & $92235.70 \mathrm{c}$ & $3.580509 \mathrm{E}-04$ & $\$ \mathrm{U}-235$ \\
\hline & $92236.70 \mathrm{c}$ & $2.683571 \mathrm{E}-06$ & $\$ U-236$ \\
\hline & $92237.70 \mathrm{c}$ & $1.545203 \mathrm{E}-29$ & $\$ \mathrm{U}-237$ \\
\hline & $92238.70 \mathrm{c}$ & $2.299741 E-05$ & $\$ \mathrm{U}-238$ \\
\hline & $93237.70 \mathrm{c}$ & $1.545195 \mathrm{E}-29$ & $\$ N p 237$ \\
\hline & $94239.70 \mathrm{c}$ & $1.532242 \mathrm{E}-29$ & \$Pu239 \\
\hline & $94240.70 \mathrm{C}$ & $1.525848 \mathrm{E}-29$ & \$Pu240 \\
\hline & $94241.70 \mathrm{c}$ & $1.519499 \mathrm{E}-29$ & \$Pu241 \\
\hline & $36083.70 \mathrm{c}$ & $4.417651 \mathrm{E}-29$ & $\$ \mathrm{Kr}-83$ \\
\hline & $42095.70 \mathrm{c}$ & $3.819269 \mathrm{E}-29$ & $\$ M o-95$ \\
\hline & $44101.70 \mathrm{c}$ & $3.629985 E-29$ & \$Ru101 \\
\hline & $45103.70 \mathrm{C}$ & $3.559438 \mathrm{E}-29$ & \$Rh103 \\
\hline & $45105.70 \mathrm{c}$ & $3.491572 \mathrm{E}-29$ & \$Rh105 \\
\hline & $48113.70 \mathrm{c}$ & $3.244211 \mathrm{E}-29$ & $\$ \mathrm{Cd} 113$ \\
\hline & $54131.70 \mathrm{C}$ & $2.798102 \mathrm{E}-29$ & $\$ \mathrm{Xe131}$ \\
\hline & $54133.70 \mathrm{c}$ & $2.756002 \mathrm{E}-29$ & $\$ x e 133$ \\
\hline & $55133.70 \mathrm{C}$ & $2.755987 \mathrm{E}-29$ & $\$ \mathrm{Cs} 133$ \\
\hline & $54135.70 \mathrm{c}$ & $2.695116 \mathrm{E}-29$ & $\$ \mathrm{Xe135}$ \\
\hline & $57140.70 \mathrm{c}$ & $2.618012 \mathrm{E}-29$ & $\$ \mathrm{La} 140$ \\
\hline & $58141.70 \mathrm{C}$ & $2.599480 \mathrm{E}-29$ & $\$ C e 141$ \\
\hline & $59143.70 \mathrm{c}$ & $2.563058 \mathrm{E}-29$ & $\$ \operatorname{Pr} 143$ \\
\hline & $60143.70 \mathrm{c}$ & $2.563055 E-29$ & $\$ N d 143$ \\
\hline & $60145.70 \mathrm{C}$ & $2.527633 E-29$ & $\$ N d 145$ \\
\hline & $61147.70 \mathrm{c}$ & $2.493199 \mathrm{E}-29$ & $\$ \operatorname{Pm} 147$ \\
\hline & $61149.70 \mathrm{C}$ & $2.459663 E-29$ & $\$ \operatorname{Pm} 149$ \\
\hline & $62149.70 \mathrm{c}$ & $2.459661 \mathrm{E}-29$ & $\$ \operatorname{Sm} 149$ \\
\hline & $61151.70 \mathrm{C}$ & $2.426995 E-29$ & \$Pm151 \\
\hline & $62151.70 \mathrm{c}$ & $2.427021 E-29$ & $\$$ Sm151 \\
\hline & $62152.70 \mathrm{c}$ & $2.411048 \mathrm{E}-29$ & $\$$ Sm152 \\
\hline & $63153.70 \mathrm{c}$ & $2.395258 \mathrm{E}-29$ & $\$$ Eu153 \\
\hline & $63155.70 \mathrm{c}$ & $2.364321 \mathrm{E}-29$ & \$Eu155 \\
\hline & $64157.70 \mathrm{C}$ & $2.334161 E-29$ & \$Gd157 \\
\hline lwtr. 10 & & & \\
\hline ATR El & ement No. = & 7 & \\
\hline Radial & Zone No. = & 3 & \\
\hline Axial & Zone No. = & 6 & \\
\hline Neutro & n Cross Sect & tions $=27 \mathrm{C}$ & \\
\hline & Number Densi & $=7.8586$ & $601 \mathrm{E}-02 \mathrm{a} / \mathrm{b}-\mathrm{cm}$ \\
\hline & $1001.70 \mathrm{C}$ & $3.212840 \mathrm{E}-02$ & $\$ \mathrm{H}-1$ \\
\hline & $8016.70 \mathrm{c}$ & $1.606420 \mathrm{E}-02$ & $\$ 0-16$ \\
\hline & $12000.60 \mathrm{c}$ & $2.399430 \mathrm{E}-04$ & $\$ M g-n a t$ \\
\hline & $13027.70 \mathrm{C}$ & $2.958200 \mathrm{E}-02$ & $\$ A I-27$ \\
\hline & $14000.60 \mathrm{c}$ & $1.245870 \mathrm{E}-04$ & \$Si-nat \\
\hline & $24000.50 c$ & $2.243180 \mathrm{E}-05$ & \$Cr-nat \\
\hline & $29000.50 c$ & $2.294330 \mathrm{E}-05$ & $\$ \mathrm{Cu}-$ nat \\
\hline & $5010.70 \mathrm{c}$ & $1.312339 \mathrm{E}-05$ & $\$ B-10$ \\
\hline & $92234.70 \mathrm{c}$ & $4.639810 \mathrm{E}-06$ & $\$ \mathrm{U}-234$ \\
\hline & $92235.70 \mathrm{c}$ & $3.580509 \mathrm{E}-04$ & $\$ \mathrm{U}-235$ \\
\hline & $92236.70 \mathrm{c}$ & $2.683571 E-06$ & $\$ U-236$ \\
\hline & $92237.70 \mathrm{c}$ & $1.545203 \mathrm{E}-29$ & $\$ \mathrm{U}-237$ \\
\hline & $92238.70 \mathrm{C}$ & $2.299741 \mathrm{E}-05$ & $\$ U-238$ \\
\hline & $93237.70 \mathrm{c}$ & $1.545195 \mathrm{E}-29$ & $\$ N p 237$ \\
\hline & $94239.70 \mathrm{c}$ & $1.532242 \mathrm{E}-29$ & \$Pu239 \\
\hline & $94240.70 \mathrm{C}$ & $1.525848 \mathrm{E}-29$ & $\$ \mathrm{Pu} 240$ \\
\hline & $94241.70 \mathrm{C}$ & $1.519499 \mathrm{E}-29$ & \$Pu241 \\
\hline & $36083.70 \mathrm{C}$ & $4.417651 \mathrm{E}-29$ & $\$ \mathrm{Kr}-83$ \\
\hline & $42095.70 \mathrm{c}$ & $3.819269 \mathrm{E}-29$ & $\$ M o-95$ \\
\hline & $44101.70 \mathrm{c}$ & $3.629985 E-29$ & \$Ru101 \\
\hline
\end{tabular}

c

c

C

C

C

C

C

m2146 
$45103.70 \mathrm{C} \quad 3.559438 \mathrm{E}-29 \$ \mathrm{Rh} 103$

$45105.70 \mathrm{C} \quad 3.491572 \mathrm{E}-29$ \$Rh105

$48113.70 \mathrm{C} \quad 3.244211 \mathrm{E}-29$ \$Cd113

$54131.70 \mathrm{C} \quad 2.798102 \mathrm{E}-29$ \$Xe131

$54133.70 \mathrm{C} \quad 2.756002 \mathrm{E}-29$ \$Xe133

$55133.70 \mathrm{C} \quad 2.755987 \mathrm{E}-29 \quad \$ \mathrm{Cs} 133$

$54135.70 \mathrm{C} \quad 2.695116 \mathrm{E}-29 \$ \mathrm{Xe} 135$

$57140.70 \mathrm{C} \quad 2.618012 \mathrm{E}-29$ \$La140

$58141.70 \mathrm{C} \quad 2.599480 \mathrm{E}-29$ \$Ce141

$59143.70 \mathrm{C} \quad 2.563058 \mathrm{E}-29 \$ \mathrm{Pr} 143$

$60143.70 \mathrm{C} \quad 2.563055 \mathrm{E}-29$ \$Nd143

$60145.70 \mathrm{C} \quad 2.527633 \mathrm{E}-29 \$ \mathrm{Nd1} 45$

$61147.70 \mathrm{C} \quad 2.493199 \mathrm{E}-29 \$ \mathrm{Pm} 147$

$61149.70 \mathrm{C} \quad 2.459663 \mathrm{E}-29 \quad \$ \mathrm{Pm} 149$

$62149.70 \mathrm{C} \quad 2.459661 \mathrm{E}-29$ \$Sm149

$61151.70 \mathrm{C} \quad 2.426995 \mathrm{E}-29 \$ \mathrm{Pm} 151$

$62151.70 \mathrm{C} \quad 2.427021 \mathrm{E}-29$ \$Sm151

$62152.70 \mathrm{C} \quad 2.411048 \mathrm{E}-29$ \$Sm152

$63153.70 \mathrm{C} \quad 2.395258 \mathrm{E}-29 \quad \$ \mathrm{Eu} 153$

$63155.70 \mathrm{C} \quad 2.364321 \mathrm{E}-29$ \$Eu155

mt2146 lwtr.10t

$64157.70 \mathrm{C} \quad 2.334161 \mathrm{E}-29 \$ \mathrm{Gd} 157$

C

$\mathrm{C}$

C

$\mathrm{C}$

m2147

ATR Element No. $=7$

Radial Zone No. $=3$

Axial Zone No. $=7$

Neutron Cross Sections $=27 \mathrm{C}$

Total Number Density $=7.858601 \mathrm{E}-02 \mathrm{a} / \mathrm{b}-\mathrm{cm}$

\begin{tabular}{|c|c|c|}
\hline 001. & $3.212840 \mathrm{E}-02$ & $\$ \mathrm{H}-1$ \\
\hline $8016.70 \mathrm{C}$ & $1.606420 \mathrm{E}-02$ & $\$ 0-16$ \\
\hline $2000.60 \mathrm{c}$ & $2.399430 \mathrm{E}-04$ & $\$ M g-n a t$ \\
\hline $3027.70 \mathrm{c}$ & $2.958200 \mathrm{E}-02$ & $\$ A I-27$ \\
\hline $4000.60 \mathrm{c}$ & $1.245870 \mathrm{E}-04$ & \$Si-nat \\
\hline $00.50 \mathrm{C}$ & $2.243180 \mathrm{E}-05$ & \$Cr-nat \\
\hline $9000.50 \mathrm{c}$ & $2.294330 \mathrm{E}-05$ & $\$ \mathrm{Cu}-\mathrm{nat}$ \\
\hline 10 תח 10 & $1.312339 \mathrm{E}-05$ & $\$ B-10$ \\
\hline $4.70 \mathrm{C}$ & $4.639810 \mathrm{E}-06$ & $\$ U-234$ \\
\hline $.70 \mathrm{C}$ & $3.580509 \mathrm{E}-04$ & $\$ U-235$ \\
\hline $.70 \mathrm{C}$ & $2.683571 \mathrm{E}-06$ & $\$ U-236$ \\
\hline $.70 \mathrm{C}$ & $1.545203 E-29$ & $\$ U-237$ \\
\hline $.70 \mathrm{C}$ & $2.299741 E-05$ & $\$ U-238$ \\
\hline $.70 \mathrm{C}$ & $1.545195 \mathrm{E}-29$ & $\$ N p 237$ \\
\hline $.70 \mathrm{C}$ & $1.532242 \mathrm{E}-29$ & \$Pu239 \\
\hline $0.70 \mathrm{c}$ & $3 E-29$ & \$Pu240 \\
\hline $.70 \mathrm{C}$ & 1.519 & \$Pu241 \\
\hline & $4.417651 E-29$ & $\$ K r-83$ \\
\hline $.70 \mathrm{C}$ & $9 E-29$ & $\$ M \circ-95$ \\
\hline & & \$Ru101 \\
\hline $70 \mathrm{C}$ & $8 E-29$ & $\$ R h 103$ \\
\hline & & \$Rh105 \\
\hline $.70 \mathrm{c}$ & 3.2442 & $\$ \mathrm{Cd} 113$ \\
\hline & $2.798102 \mathrm{E}-29$ & $\$ x e 131$ \\
\hline $.70 \mathrm{C}$ & $2 E-29$ & $\$ x e 133$ \\
\hline $.70 \mathrm{C}$ & $2.755987 \mathrm{E}-29$ & $\$ C$ s 133 \\
\hline $\mathrm{C}$ & $6 \mathrm{E}-29$ & $\$ x e 135$ \\
\hline & $E-29$ & \$La140 \\
\hline C & $O E-29$ & $\$ \mathrm{Ce} 141$ \\
\hline $.70 \mathrm{C}$ & $2.563058 \mathrm{E}-29$ & $\$ \operatorname{Pr} 143$ \\
\hline $.70 \mathrm{C}$ & $5 E-29$ & $\$ N d 143$ \\
\hline $.70 \mathrm{C}$ & $2.527633 E-29$ & $\$ N d 145$ \\
\hline $.70 \mathrm{C}$ & $2.493199 \mathrm{E}-29$ & $\$ \operatorname{Pm} 147$ \\
\hline $.70 \mathrm{C}$ & $2.459663 \mathrm{E}-29$ & \$Pm1 49 \\
\hline $.70 \mathrm{C}$ & $2.459661 \mathrm{E}-29$ & $\$ \operatorname{Sm} 149$ \\
\hline .70 & $2.426995 \mathrm{E}-29$ & $\$ P m 151$ \\
\hline 62 & $2.427021 \mathrm{E}-29$ & $\$ S m 151$ \\
\hline & $3 E-29$ & \$Sm152 \\
\hline .7 & $2.395258 \mathrm{E}-29$ & $\$$ Eu153 \\
\hline
\end{tabular}




\begin{tabular}{|c|c|c|c|}
\hline & $\begin{array}{l}63155.70 \mathrm{C} \\
64157.70 \mathrm{C}\end{array}$ & $\begin{array}{l}2.364321 \mathrm{E}-29 \\
2.334161 \mathrm{E}-29\end{array}$ & $\begin{array}{l}\$ \text { Eu155 } \\
\$ \text { Gd157 }\end{array}$ \\
\hline mt 2147 & \multicolumn{3}{|c|}{ lwtr.10t } \\
\hline \multicolumn{4}{|l|}{ c } \\
\hline \multicolumn{4}{|l|}{ c } \\
\hline \multirow{2}{*}{\multicolumn{4}{|c|}{$\begin{array}{l}\mathrm{C} \\
\mathrm{C}\end{array}$}} \\
\hline & & & \\
\hline $\mathrm{c}$ & ATR Element No. = & 8 & \\
\hline $\mathrm{c}$ & Radial Zone No. = & 1 & \\
\hline c & Axial Zone No. = & 1 & \\
\hline c & \multicolumn{3}{|c|}{ Neutron Cross Sections $=27 \mathrm{C}$} \\
\hline \multirow{2}{*}{\multicolumn{4}{|c|}{$=7.973803 \mathrm{E}-02 \mathrm{a} / \mathrm{b}-\mathrm{cm}$}} \\
\hline & & & \\
\hline & $1001.70 \mathrm{c}$ & $3.393340 \mathrm{E}-02$ & $\$ \mathrm{H}-1$ \\
\hline & $8016.70 \mathrm{c}$ & $1.696670 \mathrm{E}-02$ & $\$ 0-16$ \\
\hline & $12000.60 \mathrm{c}$ & $2.176490 \mathrm{E}-04$ & $\$ M g-n a t$ \\
\hline & $13027.70 \mathrm{c}$ & $2.793720 \mathrm{E}-02$ & $\$ A l-27$ \\
\hline & $14000.60 \mathrm{C}$ & $1.130110 \mathrm{E}-04$ & $\$$ Si-nat \\
\hline & $24000.50 \mathrm{c}$ & $2.304760 \mathrm{E}-05$ & $\$ \mathrm{Cr}$-nat \\
\hline & $29000.50 \mathrm{c}$ & $2.081160 \mathrm{E}-05$ & $\$ \mathrm{Cu}-$ nat \\
\hline & $5010.70 \mathrm{C}$ & $6.875743 \mathrm{E}-28$ & $\$ B-10$ \\
\hline & $92234.70 \mathrm{C}$ & $6.286590 \mathrm{E}-06$ & $\$ U-234$ \\
\hline & $92235.70 \mathrm{C}$ & $4.851306 \mathrm{E}-04$ & $\$ U-235$ \\
\hline & $92236.70 \mathrm{c}$ & $3.636026 \mathrm{E}-06$ & $\$ U-236$ \\
\hline & $92237.70 \mathrm{c}$ & $2.904335 \mathrm{E}-29$ & $\$ \mathrm{U}-237$ \\
\hline & $92238.70 \mathrm{c}$ & $3.115969 \mathrm{E}-05$ & $\$ \mathrm{U}-238$ \\
\hline & $93237.70 \mathrm{c}$ & $2.904320 \mathrm{E}-29$ & $\$ \mathrm{~Np} 237$ \\
\hline & $94239.70 \mathrm{C}$ & $2.879973 \mathrm{E}-29$ & \$Pu239 \\
\hline & $94240.70 \mathrm{c}$ & $2.867956 \mathrm{E}-29$ & $\$ P u 240$ \\
\hline & $94241.70 \mathrm{c}$ & $2.856023 \mathrm{E}-29$ & \$Pu241 \\
\hline & $36083.70 \mathrm{c}$ & $8.303334 \mathrm{E}-29$ & $\$ \mathrm{Kr}-83$ \\
\hline & $42095.70 \mathrm{c}$ & $7.178626 \mathrm{E}-29$ & $\$ M o-95$ \\
\hline & $44101.70 \mathrm{C}$ & $6.822852 \mathrm{E}-29$ & \$Ru101 \\
\hline & $45103.70 \mathrm{c}$ & $6.690253 \mathrm{E}-29$ & \$Rh103 \\
\hline & $45105.70 \mathrm{C}$ & $6.562692 \mathrm{E}-29$ & \$Rh105 \\
\hline & $48113.70 \mathrm{c}$ & $6.097758 \mathrm{E}-29$ & $\$ C d 113$ \\
\hline & $54131.70 \mathrm{c}$ & $5.259260 \mathrm{E}-29$ & $\$ \mathrm{Xe131}$ \\
\hline & $54133.70 \mathrm{c}$ & $5.180129 \mathrm{E}-29$ & $\$$ Xe133 \\
\hline & $55133.70 \mathrm{C}$ & $5.180102 \mathrm{E}-29$ & $\$ \mathrm{Cs} 133$ \\
\hline & $54135.70 \mathrm{c}$ & $5.065689 \mathrm{E}-29$ & $\$$ Xe135 \\
\hline & $57140.70 \mathrm{c}$ & $4.920766 \mathrm{E}-29$ & $\$ L a 140$ \\
\hline & $58141.70 \mathrm{C}$ & $4.885933 E-29$ & $\$ \mathrm{Ce} 141$ \\
\hline & $59143.70 \mathrm{c}$ & $4.817476 \mathrm{E}-29$ & $\$ \operatorname{Pr} 143$ \\
\hline & $60143.70 \mathrm{c}$ & $4.817471 \mathrm{E}-29$ & $\$ N d 143$ \\
\hline & $60145.70 \mathrm{C}$ & $4.750891 \mathrm{E}-29$ & $\$ N d 145$ \\
\hline & $61147.70 \mathrm{c}$ & $4.686170 \mathrm{E}-29$ & $\$ \operatorname{Pm} 147$ \\
\hline & $61149.70 \mathrm{C}$ & $4.623135 E-29$ & $\$ \operatorname{Pm} 149$ \\
\hline & $62149.70 \mathrm{c}$ & $4.623132 \mathrm{E}-29$ & $\$ \operatorname{Sm} 149$ \\
\hline & $61151.70 \mathrm{c}$ & $4.561735 \mathrm{E}-29$ & $\$ \operatorname{Pm} 151$ \\
\hline & $62151.70 \mathrm{C}$ & $4.561782 \mathrm{E}-29$ & $\$$ Sm1 51 \\
\hline & $62152.70 \mathrm{c}$ & $4.531760 \mathrm{E}-29$ & $\$ \operatorname{Sm} 152$ \\
\hline & $63153.70 \mathrm{C}$ & $4.502082 \mathrm{E}-29$ & \$Eu153 \\
\hline & $63155.70 \mathrm{C}$ & $4.443934 \mathrm{E}-29$ & \$Eu155 \\
\hline & $64157.70 \mathrm{c}$ & $4.387245 \mathrm{E}-29$ & \$Gd157 \\
\hline mt2148 & \multicolumn{3}{|l|}{ lwtr.10t } \\
\hline \multicolumn{4}{|l|}{$\begin{array}{l}\mathrm{C} \\
\mathrm{C}\end{array}$} \\
\hline \multirow{2}{*}{\multicolumn{4}{|c|}{$\begin{array}{l}\mathrm{C} \\
\mathrm{c}\end{array}$}} \\
\hline & & & \\
\hline \multicolumn{4}{|l|}{ c } \\
\hline C & \multicolumn{3}{|c|}{ ATR Element No. $=8$} \\
\hline c & \multicolumn{3}{|c|}{ Radial Zone No. $=1$} \\
\hline $\mathrm{c}$ & \multirow{2}{*}{\multicolumn{3}{|c|}{$\begin{array}{l}\text { Axial Zone No. }=2 \\
\text { Neutron Cross Sections }=27 \mathrm{C}\end{array}$}} \\
\hline C & & & \\
\hline$c$ & Total Number Densi & $=7.9738$ & 803E-02 a/b-cm \\
\hline & $1001.70 \mathrm{C}$ & $3.393340 \mathrm{E}-02$ & $\$ \mathrm{H}-1$ \\
\hline & $8016.70 c$ & $1.696670 \mathrm{E}-02$ & $\$ 0-16$ \\
\hline & $12000.60 \mathrm{c}$ & $2.176490 \mathrm{E}-04$ & $\$ M g-n a t$ \\
\hline & $13027.70 c$ & $2.793720 \mathrm{E}-02$ & $\$ A l-27$ \\
\hline & $14000.60 \mathrm{C}$ & $1.130110 \mathrm{E}-04$ & \$Si-nat \\
\hline & $24000.50 c$ & $2.304760 \mathrm{E}-05$ & $\$ C r-n a t$ \\
\hline
\end{tabular}




$$
\begin{aligned}
& 29000.50 \mathrm{C} \quad 2.081160 \mathrm{E}-05 \text { \$Cu-nat } \\
& 5010.70 \mathrm{C} \quad 6.875743 \mathrm{E}-28 \text { \$ B }-10 \\
& 92234.70 \mathrm{C} \quad 6.286590 \mathrm{E}-06 \quad \$ \mathrm{U}-234 \\
& 92235.70 \mathrm{C} \quad 4.851306 \mathrm{E}-04 \quad \$ \mathrm{U}-235 \\
& 92236.70 \mathrm{C} \quad 3.636026 \mathrm{E}-06 \quad \$ \mathrm{U}-236 \\
& 92237.70 \mathrm{C} \quad 2.904335 \mathrm{E}-29 \quad \$ \mathrm{U}-237 \\
& 92238.70 \mathrm{C} \quad 3.115969 \mathrm{E}-05 \quad \$ \mathrm{U}-238 \\
& 93237.70 \mathrm{C} \quad 2.904320 \mathrm{E}-29 \quad \$ \mathrm{~Np} 237 \\
& 94239.70 \mathrm{C} \quad 2.879973 \mathrm{E}-29 \text { \$Pu239 } \\
& 94240.70 \mathrm{C} \quad 2.867956 \mathrm{E}-29 \text { \$Pu240 } \\
& 94241.70 \mathrm{C} \quad 2.856023 \mathrm{E}-29 \text { \$Pu241 } \\
& 36083.70 \mathrm{C} \quad 8.303334 \mathrm{E}-29 \quad \$ \mathrm{Kr}-83 \\
& 42095.70 \mathrm{C} \quad 7.178626 \mathrm{E}-29 \text { \$Mo-95 } \\
& 44101.70 \mathrm{C} \quad 6.822852 \mathrm{E}-29 \text { \$Ru101 } \\
& 45103.70 \mathrm{C} \quad 6.690253 \mathrm{E}-29 \text { \$Rh103 } \\
& 45105.70 \mathrm{C} \quad 6.562692 \mathrm{E}-29 \text { \$Rh105 } \\
& 48113.70 \mathrm{C} \quad 6.097758 \mathrm{E}-29 \text { \$Cd113 } \\
& 54131.70 \mathrm{C} \quad 5.259260 \mathrm{E}-29 \text { \$Xe131 } \\
& 54133.70 \mathrm{c} \quad 5.180129 \mathrm{E}-29 \text { \$Xe133 } \\
& 55133.70 \mathrm{C} \quad 5.180102 \mathrm{E}-29 \text { \$Cs133 } \\
& 54135.70 \mathrm{C} \quad 5.065689 \mathrm{E}-29 \text { Xe135 } \\
& 57140.70 \mathrm{C} \quad 4.920766 \mathrm{E}-29 \text { \$La140 } \\
& 58141.70 \mathrm{C} \quad 4.885933 \mathrm{E}-29 \text { \$Ce141 } \\
& 59143.70 \mathrm{C} \quad 4.817476 \mathrm{E}-29 \quad \$ \operatorname{Pr} 143 \\
& 60143.70 \mathrm{C} \quad 4.817471 \mathrm{E}-29 \$ \mathrm{Nd} 143 \\
& 60145.70 \mathrm{C} \quad 4.750891 \mathrm{E}-29 \$ \mathrm{Nd} 145 \\
& 61147.70 \mathrm{C} \quad 4.686170 \mathrm{E}-29 \$ \mathrm{Pm} 147 \\
& 61149.70 \mathrm{C} \quad 4.623135 \mathrm{E}-29 \quad \$ \mathrm{Pm} 149 \\
& 62149.70 \mathrm{C} \quad 4.623132 \mathrm{E}-29 \$ \mathrm{Sm} 149 \\
& 61151.70 \mathrm{C} \quad 4.561735 \mathrm{E}-29 \text { \$Pm151 } \\
& 62151.70 \mathrm{C} \quad 4.561782 \mathrm{E}-29 \text { \$Sm151 } \\
& 62152.70 \mathrm{C} \quad 4.531760 \mathrm{E}-29 \text { \$Sm152 } \\
& 63153.70 \mathrm{C} \quad 4.502082 \mathrm{E}-29 \text { \$Eu153 } \\
& 63155.70 \mathrm{C} \quad 4.443934 \mathrm{E}-29 \text { \$Eu155 } \\
& 64157.70 \mathrm{C} \quad 4.387245 \mathrm{E}-29 \text { \$Gd157 }
\end{aligned}
$$

\begin{tabular}{|c|c|c|}
\hline $1001.70 \mathrm{C}$ & $3.393340 \mathrm{E}-02$ & $\$ \mathrm{H}-1$ \\
\hline $8016.70 \mathrm{C}$ & $1.696670 \mathrm{E}-02$ & $\$ 0-16$ \\
\hline $12000.60 \mathrm{c}$ & $2.176490 \mathrm{E}-04$ & \$Mg-nat \\
\hline $13027.70 \mathrm{C}$ & $2.793720 \mathrm{E}-02$ & $\$ A l-27$ \\
\hline $14000.60 \mathrm{c}$ & $1.130110 \mathrm{E}-04$ & \$Si-nat \\
\hline $24000.50 \mathrm{C}$ & $2.304760 \mathrm{E}-05$ & $\$ C r-n a t$ \\
\hline $29000.50 \mathrm{C}$ & $2.081160 \mathrm{E}-05$ & \$Cu-nat \\
\hline $5010.70 \mathrm{C}$ & $6.875743 E-28$ & $\$ B-10$ \\
\hline $92234.70 \mathrm{C}$ & $6.286590 \mathrm{E}-06$ & $\$ U-234$ \\
\hline $92235.70 \mathrm{C}$ & $4.851306 \mathrm{E}-04$ & $\$ U-235$ \\
\hline $92236.70 \mathrm{C}$ & $3.636026 \mathrm{E}-06$ & $\$ \mathrm{U}-236$ \\
\hline $92237.70 \mathrm{C}$ & $2.904335 E-29$ & $\$ U-237$ \\
\hline $92238.70 \mathrm{C}$ & $3.115969 \mathrm{E}-05$ & $\$ U-238$ \\
\hline $93237.70 \mathrm{C}$ & $2.904320 E-29$ & \$Np237 \\
\hline $94239.70 \mathrm{C}$ & $2.879973 E-29$ & $\$ P u 239$ \\
\hline $94240.70 \mathrm{C}$ & $2.867956 \mathrm{E}-29$ & \$Pu240 \\
\hline $94241.70 \mathrm{C}$ & $2.856023 E-29$ & \$Pu241 \\
\hline $36083.70 \mathrm{c}$ & $8.303334 \mathrm{E}-29$ & $\$ K r-83$ \\
\hline $42095.70 \mathrm{C}$ & $7.178626 \mathrm{E}-29$ & $\$ \mathrm{Mo}-95$ \\
\hline $44101.70 \mathrm{C}$ & $6.822852 \mathrm{E}-29$ & \$Ru101 \\
\hline $45103.70 \mathrm{C}$ & $6.690253 E-29$ & \$Rh103 \\
\hline $45105.70 \mathrm{C}$ & $6.562692 \mathrm{E}-29$ & \$Rh105 \\
\hline $48113.70 \mathrm{C}$ & $6.097758 E-29$ & $\$ \mathrm{Cd} 113$ \\
\hline $54131.70 \mathrm{C}$ & $5.259260 \mathrm{E}-29$ & $\$ x e 131$ \\
\hline $54133.70 \mathrm{C}$ & $5.180129 \mathrm{E}-29$ & $\$ \mathrm{Xe133}$ \\
\hline
\end{tabular}

mt2149 lwtr.10t

C

C

ATR Element No. $=8$

C Radial Zone No. $=1$

C Axial Zone No. = 3

C Neutron Cross Sections $=27 \mathrm{C}$

C Total Number Density $=7.973803 \mathrm{E}-02 \mathrm{a} / \mathrm{b}-\mathrm{cm}$

m2150 
$55133.70 \mathrm{C} \quad 5.180102 \mathrm{E}-29 \quad \$ \mathrm{Cs} 133$

$54135.70 \mathrm{C} \quad 5.065689 \mathrm{E}-29$ \$Xe135

$57140.70 \mathrm{C} \quad 4.920766 \mathrm{E}-29$ \$La140

$58141.70 \mathrm{C} \quad 4.885933 \mathrm{E}-29$ \$Ce141

$59143.70 \mathrm{C} \quad 4.817476 \mathrm{E}-29 \$ \operatorname{Pr} 143$

$60143.70 \mathrm{C} \quad 4.817471 \mathrm{E}-29 \$ \mathrm{Nd} 143$

$60145.70 \mathrm{C} \quad 4.750891 \mathrm{E}-29 \$ \mathrm{Nd} 145$

$61147.70 \mathrm{C} \quad 4.686170 \mathrm{E}-29$ \$Pm147

$61149.70 \mathrm{C} \quad 4.623135 \mathrm{E}-29 \$ \mathrm{Pm} 149$

$62149.70 \mathrm{C} \quad 4.623132 \mathrm{E}-29$ \$Sm149

$61151.70 \mathrm{c} \quad 4.561735 \mathrm{E}-29$ \$Pm151

$62151.70 \mathrm{C} \quad 4.561782 \mathrm{E}-29$ \$Sm151

$62152.70 \mathrm{C} \quad 4.531760 \mathrm{E}-29 \$ \mathrm{Sm} 152$

$63153.70 \mathrm{C} \quad 4.502082 \mathrm{E}-29 \$ \mathrm{Eu} 153$

$63155.70 \mathrm{C} \quad 4.443934 \mathrm{E}-29$ \$Eu155

$64157.70 \mathrm{C} \quad 4.387245 \mathrm{E}-29$ \$Gd157

mt2150 lwtr.10t
$\mathrm{C}$

m2151

mt2151

C

C

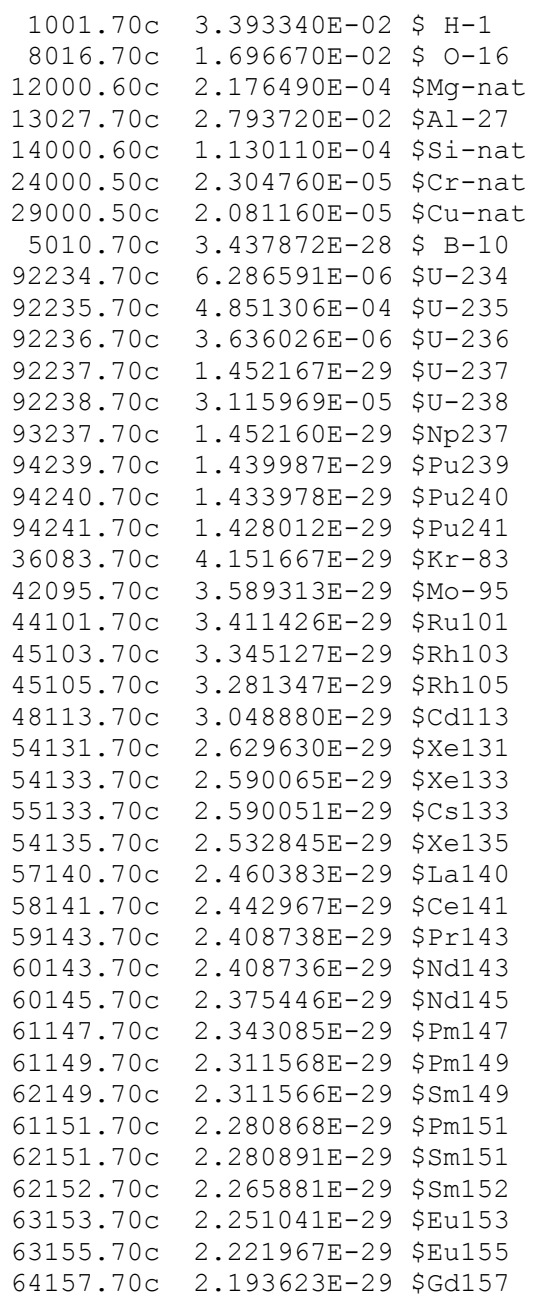


C

C

mt2152
C
C
C
C
C
C
C
C
C
m2153

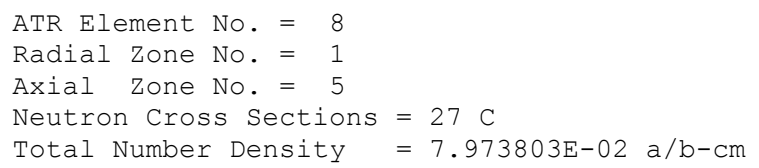




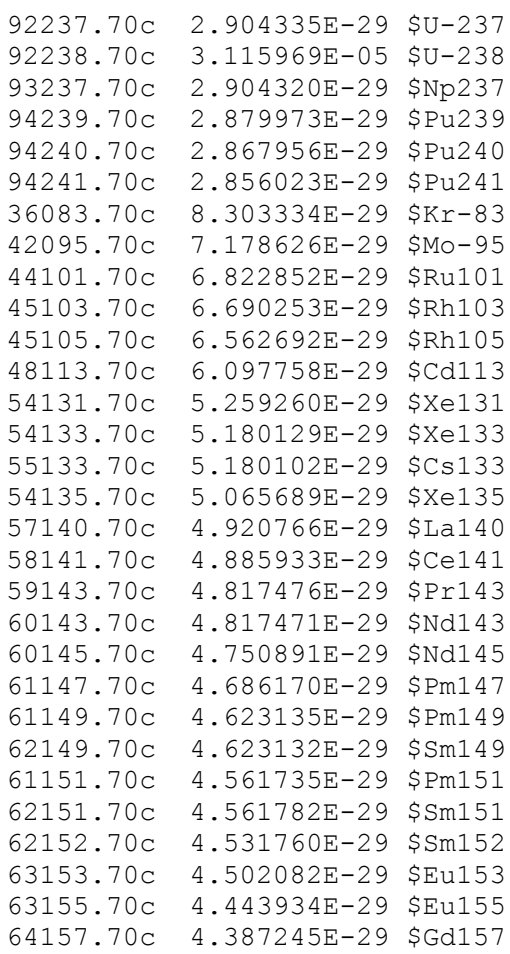

mt2153 lwtr.10t

C

C

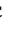

m2154

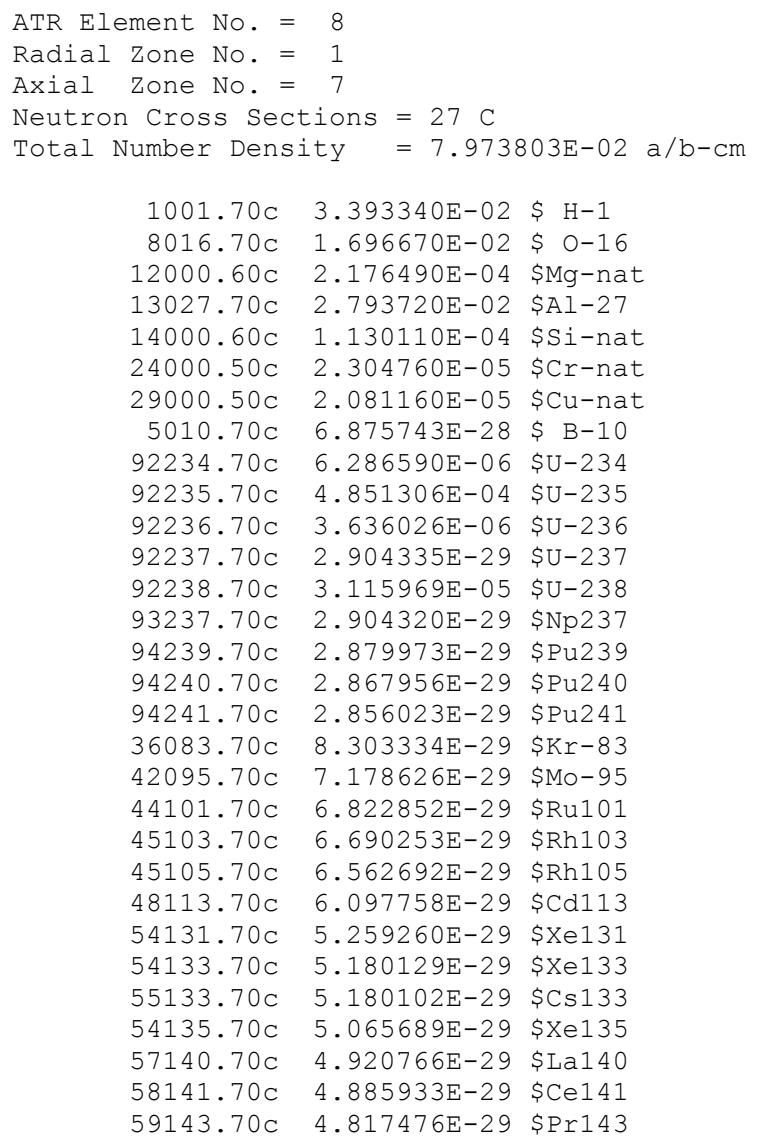




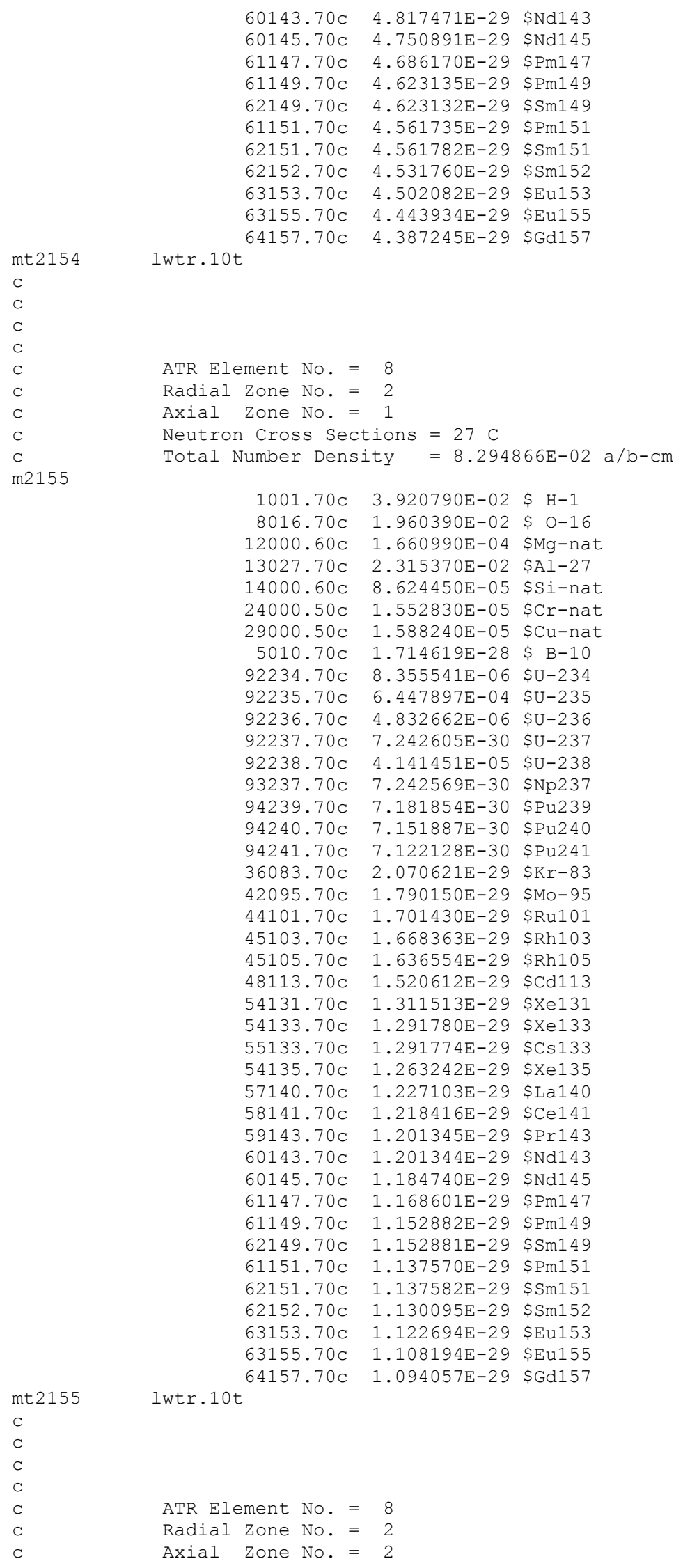




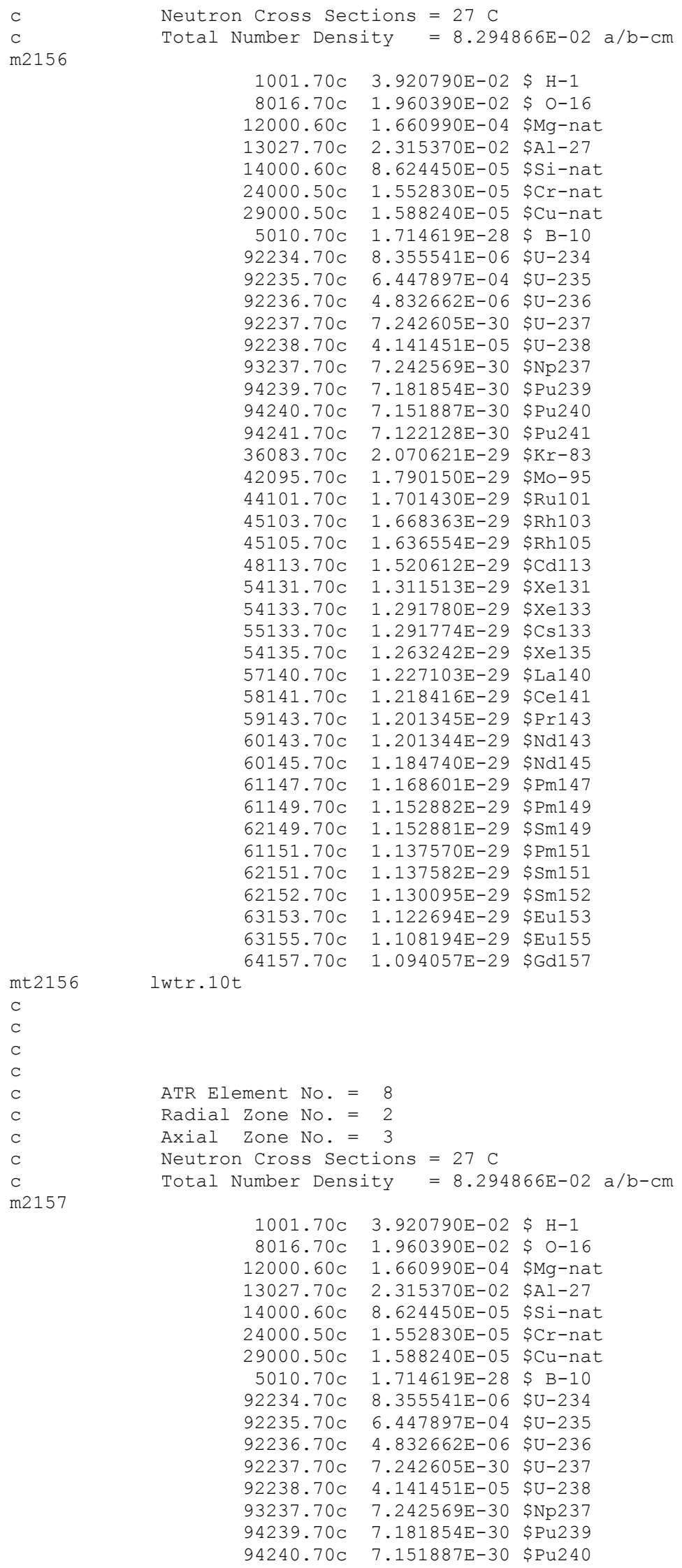




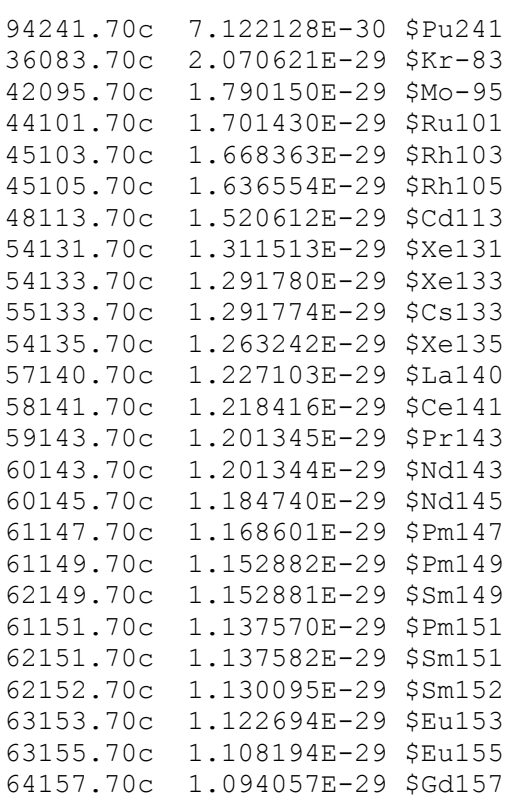

mt2157 lwtr.10t

c

C

C

C

C

m2158

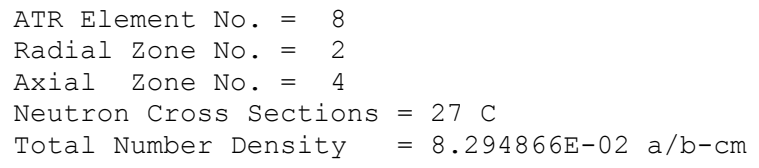
$1001.70 \mathrm{C} \quad 3.920790 \mathrm{E}-02 \$ \mathrm{H}-1$
$8016.70 \mathrm{C} \quad 1.960390 \mathrm{E}-02 \$ 0-16$
$12000.60 \mathrm{c} \quad 1.660990 \mathrm{E}-04$ \$Mg-nat
$13027.70 \mathrm{C} \quad 2.315370 \mathrm{E}-02$ \$Al-27
$14000.60 \mathrm{C} \quad 8.624450 \mathrm{E}-05$ \$Si-nat
$24000.50 \mathrm{C} \quad 1.552830 \mathrm{E}-05$ \$Cr-nat
$29000.50 \mathrm{C} \quad 1.588240 \mathrm{E}-05$ \$Cu-nat
$5010.70 \mathrm{C} \quad 8.573098 \mathrm{E}-29$ \$ B-10
$92234.70 \mathrm{C} \quad 8.355538 \mathrm{E}-06 \mathrm{\$ U}-234$
$92235.70 \mathrm{C} \quad 6.447894 \mathrm{E}-04 \quad \$ \mathrm{U}-235$
$92236.70 \mathrm{C} \quad 4.832660 \mathrm{E}-06 \quad \$ \mathrm{U}-236$
$92237.70 \mathrm{C} \quad 3.621303 \mathrm{E}-30 \quad \mathrm{SU}-237$
$92238.70 \mathrm{C} \quad 4.141449 \mathrm{E}-05 \quad \$ \mathrm{U}-238$
$93237.70 \mathrm{C} \quad 3.621285 \mathrm{E}-30 \quad \$ \mathrm{~Np} 237$
$94239.70 \mathrm{C} \quad 3.590928 \mathrm{E}-30$ \$Pu239
$94240.70 \mathrm{C} \quad 3.575944 \mathrm{E}-30$ \$Pu240
$94241.70 \mathrm{C} \quad 3.561065 \mathrm{E}-30$ \$Pu 241
$36083.70 \mathrm{C} \quad 1.035311 \mathrm{E}-29 \quad \$ \mathrm{Kr}-83$
$42095.70 \mathrm{C} \quad 8.950752 \mathrm{E}-30$ \$Mo-95
$44101.70 \mathrm{C} \quad 8.507152 \mathrm{E}-30$ \$Ru101
$45103.70 \mathrm{C} \quad 8.341818 \mathrm{E}-30 \quad \$ R h 103$
$45105.70 \mathrm{C} \quad 8.182769 \mathrm{E}-30$ \$Rh105
$48113.70 \mathrm{C} \quad 7.603060 \mathrm{E}-30 \quad \$ \mathrm{Cd} 113$
$54131.70 \mathrm{c} \quad 6.557568 \mathrm{E}-30 \quad \$ \mathrm{Xe131}$
$54133.70 \mathrm{c} \quad 6.458903 \mathrm{E}-30 \quad \$ \mathrm{Xe} 133$
$55133.70 \mathrm{C} \quad 6.458870 \mathrm{E}-30 \quad \$ \mathrm{Cs} 133$
$54135.70 \mathrm{C} \quad 6.316213 \mathrm{E}-30 \quad \$ \mathrm{Xe135}$
$57140.70 \mathrm{C} \quad 6.135514 \mathrm{E}-30$ \$La140
$58141.70 \mathrm{C} \quad 6.092082 \mathrm{E}-30 \$ \mathrm{Ce} 141$
$59143.70 \mathrm{c} \quad 6.006725 \mathrm{E}-30 \quad \$ \operatorname{Pr} 143$
$60143.70 \mathrm{C} \quad 6.006719 \mathrm{E}-30 \$ \mathrm{Nd14} 3$
$60145.70 \mathrm{C} \quad 5.923703 \mathrm{E}-30 \quad \$ \mathrm{Nd} 145$
$61147.70 \mathrm{C} \quad 5.843005 \mathrm{E}-30 \quad \$ \mathrm{Pm} 147$
$61149.70 \mathrm{C} \quad 5.764410 \mathrm{E}-30 \quad \$ \mathrm{Pm} 149$
$62149.70 \mathrm{C} \quad 5.764405 \mathrm{E}-30 \quad \$ \mathrm{Sm} 149$ 
$61151.70 \mathrm{C} \quad 5.687851 \mathrm{E}-30 \$ \mathrm{Pm} 151$

$62151.70 \mathrm{C} \quad 5.687911 \mathrm{E}-30 \quad \$ \mathrm{Sm} 151$

$62152.70 \mathrm{C} \quad 5.650478 \mathrm{E}-30 \quad \$ \mathrm{Sm} 152$

$63153.70 \mathrm{C} \quad 5.613472 \mathrm{E}-30 \quad \$ \mathrm{Eu} 153$

$63155.70 \mathrm{C} \quad 5.540969 \mathrm{E}-30$ \$Eu155

$64157.70 \mathrm{C} \quad 5.470287 \mathrm{E}-30 \quad \$ \mathrm{Gd} 157$

mt2158 lwtr.10t

$\mathrm{C}$

$\mathrm{C}$

$\mathrm{C}$

C ATR Element No. = 8

C Radial Zone No. $=2$

C Axial Zone No. = 5

C Neutron Cross Sections $=27 \mathrm{C}$

C Total Number Density $=8.294866 \mathrm{E}-02 \mathrm{a} / \mathrm{b}-\mathrm{cm}$

m2159

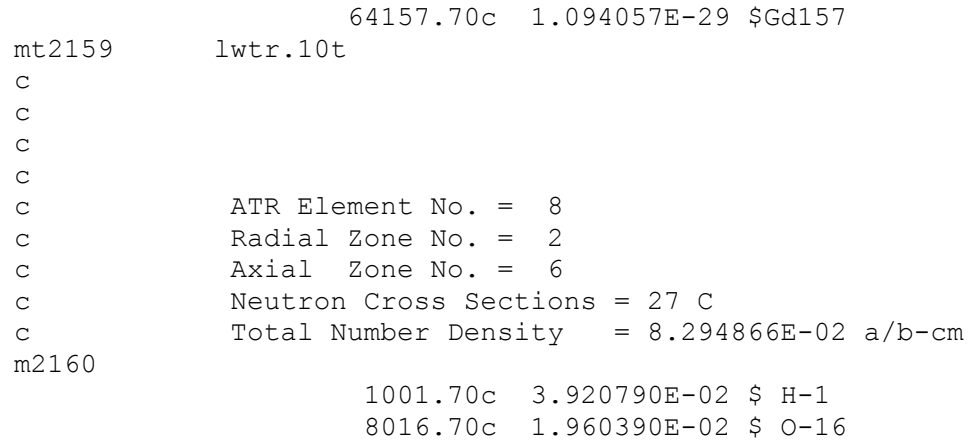




\begin{tabular}{|c|c|c|}
\hline $2000.60 \mathrm{C}$ & $1.660990 \mathrm{E}-04$ & $\$ M g-n a t$ \\
\hline $3027.70 \mathrm{C}$ & $2.315370 \mathrm{E}-02$ & $\$ A l-27$ \\
\hline $1000.60 \mathrm{c}$ & $8.624450 \mathrm{E}-05$ & \$Si-nat \\
\hline $000.50 \mathrm{c}$ & $1.552830 \mathrm{E}-05$ & $\$ C r-n a t$ \\
\hline $000.50 \mathrm{c}$ & $1.588240 \mathrm{E}-05$ & \$Cu-nat \\
\hline $5010.70 \mathrm{c}$ & $1.714619 \mathrm{E}-28$ & $\$ B-10$ \\
\hline $92234.70 \mathrm{C}$ & $8.355541 \mathrm{E}-06$ & $\$ U-234$ \\
\hline $92235.70 \mathrm{c}$ & $6.447897 \mathrm{E}-04$ & $\$ U-235$ \\
\hline $236.70 \mathrm{c}$ & $4.832662 \mathrm{E}-06$ & $\$ U-236$ \\
\hline $237.70 \mathrm{C}$ & $7.242605 \mathrm{E}-30$ & $\$ U-237$ \\
\hline $238.70 \mathrm{C}$ & $4.141451 \mathrm{E}-05$ & $\$ U-238$ \\
\hline $93237.70 \mathrm{C}$ & $7.242569 \mathrm{E}-30$ & \$Np237 \\
\hline $94239.70 \mathrm{c}$ & $7.181854 \mathrm{E}-30$ & \$Pu239 \\
\hline $4240.70 \mathrm{C}$ & $7.151887 \mathrm{E}-30$ & \$Pu2 40 \\
\hline $4241.70 \mathrm{C}$ & $7.122128 \mathrm{E}-30$ & \$Pu241 \\
\hline $36083.70 \mathrm{c}$ & $2.070621 \mathrm{E}-29$ & $\$ K r-83$ \\
\hline $42095.70 \mathrm{c}$ & $1.790150 \mathrm{E}-29$ & \$Mo-95 \\
\hline $44101.70 \mathrm{c}$ & $1.701430 \mathrm{E}-29$ & \$Ru101 \\
\hline $45103.70 \mathrm{C}$ & $1.668363 \mathrm{E}-29$ & \$Rh103 \\
\hline $45105.70 \mathrm{C}$ & $1.636554 \mathrm{E}-29$ & \$Rh105 \\
\hline $48113.70 \mathrm{c}$ & $1.520612 \mathrm{E}-29$ & $\$ \mathrm{Cd}$ 113 \\
\hline $54131.70 \mathrm{c}$ & $1.311513 \mathrm{E}-29$ & $\$ \mathrm{Xe131}$ \\
\hline $54133.70 \mathrm{c}$ & $1.291780 \mathrm{E}-29$ & $\$ \mathrm{Xe133}$ \\
\hline $55133.70 \mathrm{c}$ & $1.291774 \mathrm{E}-29$ & $\$ C s 133$ \\
\hline $54135.70 \mathrm{C}$ & $1.263242 \mathrm{E}-29$ & \$Xe135 \\
\hline $57140.70 \mathrm{C}$ & $1.227103 \mathrm{E}-29$ & \$La140 \\
\hline $58141.70 \mathrm{C}$ & $1.218416 \mathrm{E}-29$ & $\$ C e 141$ \\
\hline $59143.70 \mathrm{c}$ & $1.201345 \mathrm{E}-29$ & $\$ \operatorname{Pr} 143$ \\
\hline $60143.70 \mathrm{c}$ & $1.201344 \mathrm{E}-29$ & \$Nd143 \\
\hline $60145.70 \mathrm{c}$ & $1.184740 \mathrm{E}-29$ & \$Nd145 \\
\hline $61147.70 \mathrm{C}$ & $1.168601 \mathrm{E}-29$ & $\$ \operatorname{Pm} 147$ \\
\hline $61149.70 \mathrm{c}$ & $1.152882 \mathrm{E}-29$ & $\$ \operatorname{Pm} 149$ \\
\hline $62149.70 \mathrm{c}$ & $1.152881 \mathrm{E}-29$ & $\$$ Sm149 \\
\hline $61151.70 \mathrm{c}$ & $1.137570 \mathrm{E}-29$ & \$Pm151 \\
\hline $62151.70 \mathrm{C}$ & $1.137582 \mathrm{E}-29$ & $\$$ Sm151 \\
\hline $62152.70 \mathrm{c}$ & $1.130095 \mathrm{E}-29$ & $\$$ Sm152 \\
\hline $63153.70 \mathrm{c}$ & $1.122694 \mathrm{E}-29$ & \$Eu153 \\
\hline $3155.70 \mathrm{c}$ & $1.108194 \mathrm{E}-29$ & $\$$ Eu15 \\
\hline & $57 E-29$ & \$Gd157 \\
\hline
\end{tabular}

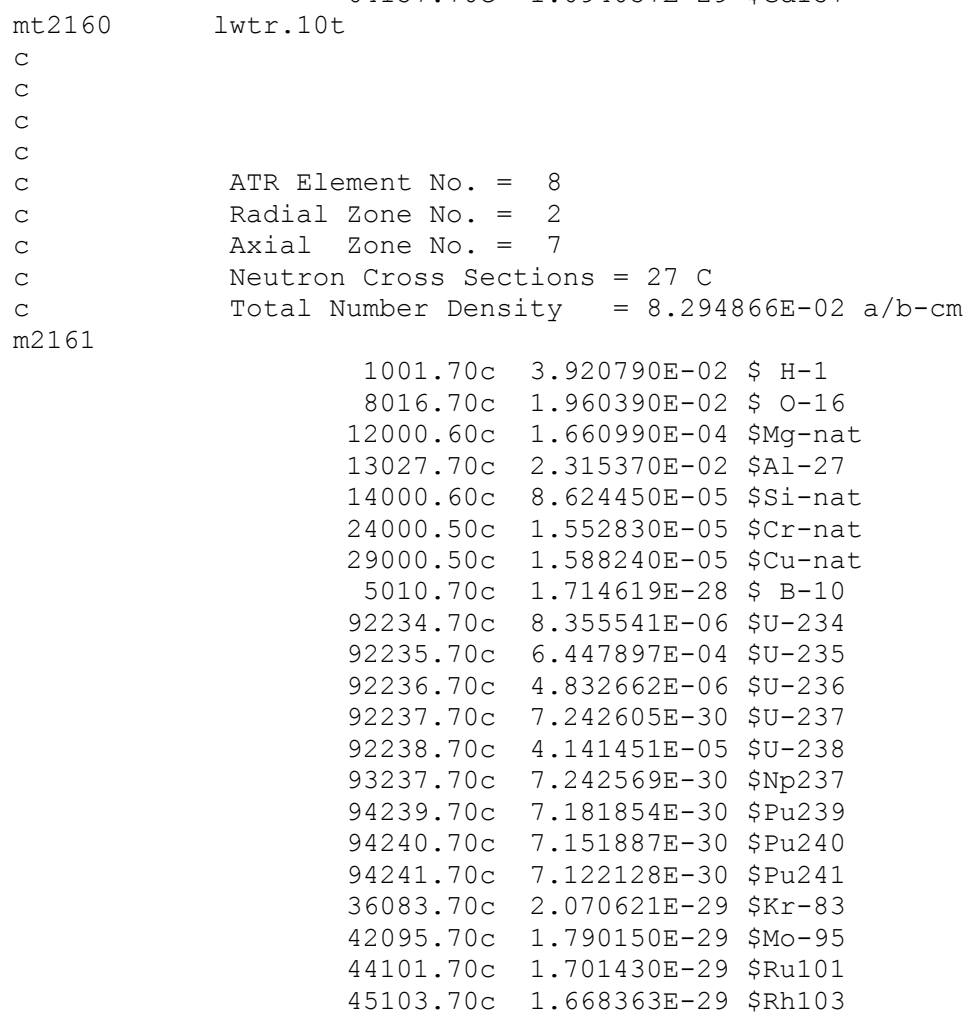




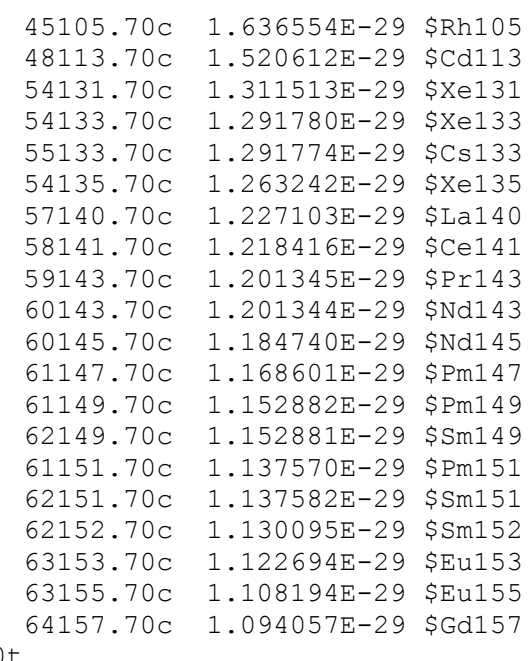

$\begin{array}{ll}\text { mt2161 lwtr.10t } \\ \mathrm{C} \\ \mathrm{C} \\ \mathrm{C} \\ \mathrm{C} & \text { ATR Element No. }=8 \\ \mathrm{C} & \text { Radial Zone No. }=3 \\ \mathrm{C} & \text { Axial Zone No. }=1 \\ \mathrm{C} & \text { Neutron Cross Sections }=27 \mathrm{C} \\ \mathrm{C} & \text { Total Number Density }=7.868379 \mathrm{E}-02 \mathrm{a} / \mathrm{b}-\mathrm{cm} \\ \mathrm{C} & \end{array}$

\begin{tabular}{|c|c|c|}
\hline $1001.70 \mathrm{c}$ & $3.212840 \mathrm{E}-02$ & $\$ \mathrm{H}-1$ \\
\hline $8016.70 \mathrm{c}$ & $1.606420 \mathrm{E}-02$ & $\$ 0-16$ \\
\hline $2000.60 \mathrm{c}$ & $2.399430 \mathrm{E}-04$ & $\$ M g-n a t$ \\
\hline $3027.70 \mathrm{c}$ & $2.958200 \mathrm{E}-02$ & $\$ A l-27$ \\
\hline $000.60 \mathrm{c}$ & $1.245870 \mathrm{E}-04$ & \$Si-nat \\
\hline $000.50 \mathrm{C}$ & $2.243180 \mathrm{E}-05$ & \$Cr-nat \\
\hline & $2.294330 \mathrm{E}-05$ & $\$ \mathrm{Cu}-$ nat \\
\hline $.70 \mathrm{C}$ & $3.658125 \mathrm{E}-28$ & $\$ B-10$ \\
\hline $4.70 \mathrm{C}$ & $5.964864 \mathrm{E}-06$ & $\$ U-234$ \\
\hline $.70 \mathrm{C}$ & $4.603032 E-04$ & $\$ U-235$ \\
\hline $.70 \mathrm{C}$ & $3.449947 E-06$ & $\$ U-236$ \\
\hline $.70 \mathrm{C}$ & $1.545203 E-29$ & $\$ U-237$ \\
\hline $.70 \mathrm{c}$ & $2.956504 \mathrm{E}-05$ & $\$ U-238$ \\
\hline $.70 \mathrm{C}$ & $1.545195 \mathrm{E}-29$ & $\$ N p 237$ \\
\hline $.70 \mathrm{C}$ & $1.532242 E-29$ & $\$ P u 239$ \\
\hline $.70 \mathrm{C}$ & $1.525848 \mathrm{E}-29$ & $\$ P u 240$ \\
\hline $.70 \mathrm{C}$ & $1.519499 \mathrm{E}-29$ & $\$ P u 241$ \\
\hline $.70 \mathrm{C}$ & $4.417651 E-29$ & $\$ K r-83$ \\
\hline $.70 \mathrm{c}$ & $3.819269 \mathrm{E}-29$ & $\$ M o-95$ \\
\hline $.70 \mathrm{C}$ & $3.629985 E-29$ & \$Ru101 \\
\hline $.70 \mathrm{C}$ & $38 E-29$ & $\$ R h 103$ \\
\hline $.70 \mathrm{C}$ & 3.4915 & \$Rh105 \\
\hline & $1 E-29$ & $\$ \mathrm{Cd} 113$ \\
\hline $.70 \mathrm{C}$ & $2.798102 E-29$ & \$Xe131 \\
\hline & $2 E-29$ & $\$ x e 133$ \\
\hline $.70 \mathrm{C}$ & $2.755987 E-29$ & $\$ \mathrm{Cs} 133$ \\
\hline & $2.695116 \mathrm{E}-29$ & $\$ x e 135$ \\
\hline $.70 \mathrm{C}$ & $2.618012 \mathrm{E}-29$ & $\$ \mathrm{La} 140$ \\
\hline & $480 E-29$ & $\$ C e 141$ \\
\hline $.70 \mathrm{C}$ & $58 E-29$ & $\$ \operatorname{Pr} 143$ \\
\hline & $2.563055 \mathrm{E}-29$ & $\$ N d 143$ \\
\hline $\mathrm{C}$ & $33 E-29$ & $\$ N d 145$ \\
\hline 61 & $2.493199 \mathrm{E}-29$ & $\$ \operatorname{Pm} 147$ \\
\hline $.70 \mathrm{C}$ & $2.459663 E-29$ & $\$ P m 149$ \\
\hline $.70 \mathrm{C}$ & $2.459661 \mathrm{E}-29$ & $\$ S m 149$ \\
\hline $.70 \mathrm{C}$ & $2.426995 E-29$ & $\$ P m 151$ \\
\hline $.70 \mathrm{C}$ & $2.427021 E-29$ & $\$ S m 151$ \\
\hline $.70 \mathrm{C}$ & 2. $411048 E-29$ & \$Sm152 \\
\hline & $5258 E-29$ & \$Eu153 \\
\hline 631 & $2.364321 E-29$ & \$Eu155 \\
\hline
\end{tabular}


$64157.70 \mathrm{C} \quad 2.334161 \mathrm{E}-29 \$ \mathrm{Gd} 157$

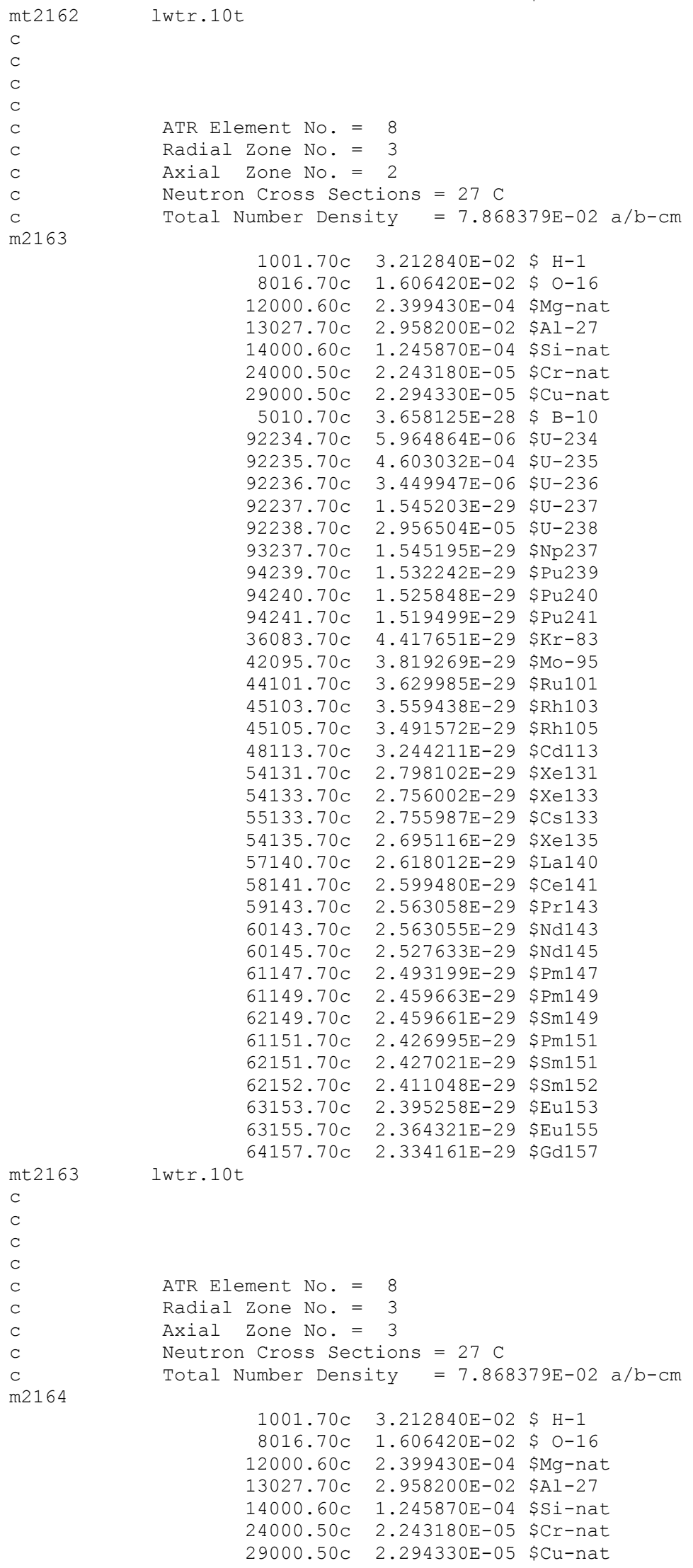




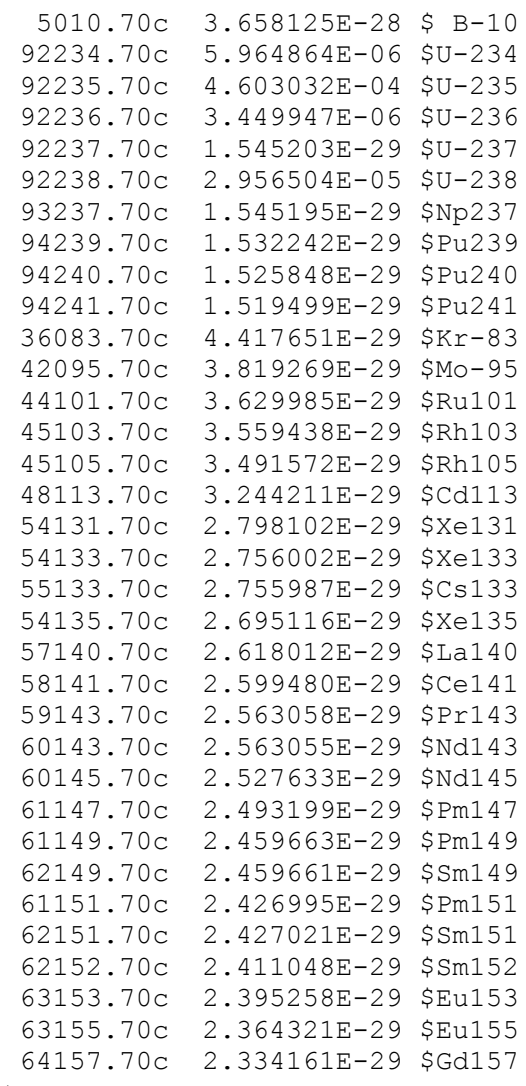

mt2164
C
C
C
C
C
C
C
C
C
m2165

lwtr.10t

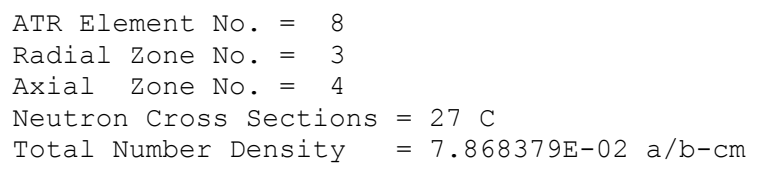

\begin{tabular}{|c|c|c|}
\hline $1001.70 \mathrm{C}$ & $3.212840 \mathrm{E}-02$ & $\$ \mathrm{H}-1$ \\
\hline $8016.70 \mathrm{c}$ & $1.606420 \mathrm{E}-02$ & $\$ 0-16$ \\
\hline $2000.60 \mathrm{c}$ & $2.399430 \mathrm{E}-04$ & $\$ M g-n a t$ \\
\hline $7.70 \mathrm{C}$ & $2.958200 \mathrm{E}-02$ & $\$ A l-27$ \\
\hline $00.60 \mathrm{c}$ & $1.245870 \mathrm{E}-04$ & \$Si-nat \\
\hline & $2.243180 E-05$ & $\$ C r-n a t$ \\
\hline $.50 \mathrm{C}$ & $2.294330 \mathrm{E}-05$ & $\$ \mathrm{Cu}-\mathrm{nat}$ \\
\hline & $1.829063 E-28$ & $\$ B-10$ \\
\hline 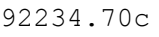 & $5.964876 \mathrm{E}-06$ & $\$ U-234$ \\
\hline & $4.603042 E-04$ & $\$ U-235$ \\
\hline$\cdot$ & $3.449954 \mathrm{E}-06$ & $\$ U-236$ \\
\hline & $7.726017 \mathrm{E}-30$ & $\$ U-237$ \\
\hline$\cdot$ & $2.956510 \mathrm{E}-05$ & $\$ U-238$ \\
\hline 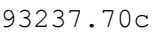 & 7.7259 & $\$ N p 237$ \\
\hline$c$ & $7.661212 \mathrm{E}-30$ & \$Pu239 \\
\hline C & $E-30$ & $\$ P u 240$ \\
\hline $.70 \mathrm{C}$ & $500 E-30$ & \$Pu241 \\
\hline 7 & $5 E-29$ & $\$ K r-83$ \\
\hline 70 & $E-29$ & $\$ M \circ-95$ \\
\hline & $E-29$ & \$Ru101 \\
\hline 70 & $E-29$ & $\$ R h 103$ \\
\hline & $5 E-29$ & $\$ R h 105$ \\
\hline 48 & $06 E-29$ & $\$ C d 113$ \\
\hline & $1 E-29$ & $\$ x e 131$ \\
\hline 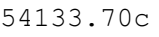 & $1 E-29$ & \$xe133 \\
\hline & $4 E-2$ & $\$ C s 133$ \\
\hline
\end{tabular}


$54135.70 \mathrm{C} \quad 1.347558 \mathrm{E}-29$ \$Xe135

$57140.70 \mathrm{C} \quad 1.309006 \mathrm{E}-29$ \$La140

$58141.70 \mathrm{C} \quad 1.299740 \mathrm{E}-29$ \$Ce141

$59143.70 \mathrm{C} \quad 1.281529 \mathrm{E}-29 \$ \operatorname{Pr} 143$

$60143.70 \mathrm{C} \quad 1.281528 \mathrm{E}-29 \$ \mathrm{Nd} 143$

$60145.70 \mathrm{C} \quad 1.263817 \mathrm{E}-29 \$ \mathrm{Nd} 145$

$61147.70 \mathrm{C} \quad 1.246600 \mathrm{E}-29 \$ \operatorname{Pm} 147$

$61149.70 \mathrm{C} \quad 1.229832 \mathrm{E}-29 \$ \mathrm{Pm} 149$

$62149.70 \mathrm{C} \quad 1.229831 \mathrm{E}-29$ \$Sm149

$61151.70 \mathrm{C} \quad 1.213498 \mathrm{E}-29 \$ \mathrm{Pm} 151$

$62151.70 \mathrm{C} \quad 1.213511 \mathrm{E}-29$ \$Sm151

$62152.70 \mathrm{C} \quad 1.205524 \mathrm{E}-29$ \$Sm152

$63153.70 \mathrm{C} \quad 1.197629 \mathrm{E}-29$ \$Eu153

$63155.70 \mathrm{C} \quad 1.182161 \mathrm{E}-29$ \$Eu155

mt2165 lwtr.10t

$64157.70 \mathrm{C} \quad 1.167081 \mathrm{E}-29$ \$Gd157

C

$\mathrm{C}$

$\mathrm{C}$

C ATR Element No. = 8

C Radial Zone No. = 3

C Axial Zone No. = 5

C Neutron Cross Sections $=27 \mathrm{C}$

C Total Number Density $=7.868379 \mathrm{E}-02 \mathrm{a} / \mathrm{b}-\mathrm{cm}$

m2166

$\begin{array}{lll}1001.70 \mathrm{C} & 3.212840 \mathrm{E}-02 & \$ \mathrm{H}-1 \\ 8016.70 \mathrm{C} & 1.606420 \mathrm{E}-02 & \$ \mathrm{O}-1\end{array}$

$12000.60 \mathrm{C} \quad 2.399430 \mathrm{E}-04 \$ \mathrm{Mg}-\mathrm{nat}$

$13027.70 \mathrm{C} \quad 2.958200 \mathrm{E}-02$ \$Al-27

$14000.60 \mathrm{C} \quad 1.245870 \mathrm{E}-04$ Si-nat

$24000.50 \mathrm{C} \quad 2.243180 \mathrm{E}-05$ \$Cr-nat

$29000.50 \mathrm{C} \quad 2.294330 \mathrm{E}-05$ \$Cu-nat

$5010.70 \mathrm{C} \quad 3.658125 \mathrm{E}-28$ \$ B -10

$92234.70 \mathrm{C} \quad 5.964864 \mathrm{E}-06 \quad \$ \mathrm{U}-234$

$92235.70 \mathrm{C} \quad 4.603032 \mathrm{E}-04$ \$U-235

$92236.70 \mathrm{C} \quad 3.449947 \mathrm{E}-06 \quad \$ \mathrm{U}-236$

$92237.70 \mathrm{C} \quad 1.545203 \mathrm{E}-29$ \$U-237

$92238.70 \mathrm{C} \quad 2.956504 \mathrm{E}-05 \quad \$ \mathrm{U}-238$

$93237.70 \mathrm{C} \quad 1.545195 \mathrm{E}-29 \$ \mathrm{~Np} 237$

$94239.70 \mathrm{C} \quad 1.532242 \mathrm{E}-29$ \$Pu239

$94240.70 \mathrm{C} \quad 1.525848 \mathrm{E}-29$ \$Pu240

$94241.70 \mathrm{C} \quad 1.519499 \mathrm{E}-29$ \$Pu241

$36083.70 \mathrm{C} \quad 4.417651 \mathrm{E}-29$ \$Kr-83

$42095.70 \mathrm{C} \quad 3.819269 \mathrm{E}-29$ \$Mo-95

$44101.70 \mathrm{C} \quad 3.629985 \mathrm{E}-29$ \$Ru101

$45103.70 \mathrm{C} \quad 3.559438 \mathrm{E}-29$ \$Rh103

$45105.70 \mathrm{C} \quad 3.491572 \mathrm{E}-29$ \$Rh105

$48113.70 \mathrm{C} \quad 3.244211 \mathrm{E}-29$ \$Cd113

$54131.70 \mathrm{C} \quad 2.798102 \mathrm{E}-29$ \$Xe131

$54133.70 \mathrm{C} \quad 2.756002 \mathrm{E}-29$ \$Xe133

$55133.70 \mathrm{C} \quad 2.755987 \mathrm{E}-29 \$ \mathrm{Cs} 133$

$54135.70 \mathrm{C} \quad 2.695116 \mathrm{E}-29$ \$Xe135

$57140.70 \mathrm{C} \quad 2.618012 \mathrm{E}-29$ \$La140

$58141.70 \mathrm{C} \quad 2.599480 \mathrm{E}-29$ \$Ce141

$59143.70 \mathrm{C} \quad 2.563058 \mathrm{E}-29 \quad \$ \mathrm{Pr} 143$

$60143.70 \mathrm{C} \quad 2.563055 \mathrm{E}-29 \$ \mathrm{Nd} 143$

$60145.70 \mathrm{C} \quad 2.527633 \mathrm{E}-29$ \$Nd145

$61147.70 \mathrm{C} \quad 2.493199 \mathrm{E}-29 \$ \mathrm{Pm} 147$

$61149.70 \mathrm{C} \quad 2.459663 \mathrm{E}-29 \$ \mathrm{Pm} 149$

$62149.70 \mathrm{C} \quad 2.459661 \mathrm{E}-29$ \$Sm149

$61151.70 \mathrm{C} \quad 2.426995 \mathrm{E}-29$ \$Pm151

$62151.70 \mathrm{C} \quad 2.427021 \mathrm{E}-29$ \$Sm151

$62152.70 \mathrm{C} \quad 2.411048 \mathrm{E}-29$ \$Sm152

$63153.70 \mathrm{C} \quad 2.395258 \mathrm{E}-29 \quad \$ \mathrm{Eu} 153$

$63155.70 \mathrm{C} \quad 2.364321 \mathrm{E}-29$ \$Eu155

mt2166 lwtr.10t

c

C

C 
C

C ATR Element No. $=8$

C Radial Zone No. = 3

C Axial Zone No. = 6

c Neutron Cross Sections $=27 \mathrm{C}$

m2167

mt 2167

$\mathrm{C}$

C

C

C

C

C

C

m2168

lwtr.10t

ATR Element No. $=8$

Radial Zone No. $=3$

Axial Zone No. = 7

Neutron Cross Sections $=27 \mathrm{C}$

Total Number Density $=7.868379 \mathrm{E}-02 \mathrm{a} / \mathrm{b}-\mathrm{cm}$

\begin{tabular}{|c|c|c|}
\hline & & \\
\hline & $1.606420 \mathrm{E}-02$ & $0-1$ \\
\hline & -04 & Mg-nat \\
\hline & $0 E-02$ & -2 \\
\hline & $1.245870 \mathrm{E}-04$ & $\sigma_{-}$ \\
\hline & $180 E-05$ & L \\
\hline & & $\mathrm{Cu}-\mathrm{na}$ \\
\hline & & ת \\
\hline 4 . & $5.964864 \mathrm{E}-06$ & $\$ U-234$ \\
\hline$\Gamma$ & $4.603032 \mathrm{E}-04$ & $\$ U-235$ \\
\hline & $3.449947 \mathrm{E}-06$ & $\$ U-$ \\
\hline & & $\$ U-2$ \\
\hline & & $\$ \mathrm{U}-2$ \\
\hline & 1.5 & \$Np237 \\
\hline & $32242 E-29$ & $\$ \mathrm{Pu} 2$ \\
\hline & & \\
\hline & 1.5 & $\$ \mathrm{Pu}$ \\
\hline & 4.4 & $\$ \mathrm{Kr}-$ \\
\hline & $3.819269 \mathrm{E}-29$ & \$Mo-95 \\
\hline & & \$Ru \\
\hline & & \\
\hline & 3.4 & $\$ R h$ \\
\hline & 3.2 & $\$ \mathrm{Cd}$ \\
\hline & $102 \mathrm{E}-29$ & $\$ \mathrm{Xe131}$ \\
\hline & 2.7 & $\$ \mathrm{Xe133}$ \\
\hline & & \\
\hline & & \\
\hline & 2.6 & $\$ \mathrm{La}$ \\
\hline & 2.5 & $\$ \mathrm{Ce} 1$ \\
\hline & $2.563058 \mathrm{E}-29$ & $\$ \operatorname{Pr} 143$ \\
\hline & $2.563055 \mathrm{E}-29$ & \$Nd143 \\
\hline & $633 E-29$ & Jd1 45 \\
\hline & 2.4 & $\$ P m$ \\
\hline & 2.45 & $\$ \operatorname{Pm} 149$ \\
\hline 19 & $2.459661 \mathrm{E}-29$ & \$Sm149 \\
\hline 51 & $2.426995 \mathrm{E}-29$ & \$Pm151 \\
\hline$\theta$ & $2.427021 \mathrm{E}-29$ & $\$$ Sm151 \\
\hline & $2.411048 \mathrm{E}-29$ & \$Sm1 \\
\hline & $2.395258 \mathrm{E}-29$ & \$Eu153 \\
\hline & $2.364321 \mathrm{E}-29$ & \$Eu 1 \\
\hline & & \\
\hline
\end{tabular}

Total Number Density $=7.868379 \mathrm{E}-02 \mathrm{a} / \mathrm{b}-\mathrm{cm}$

$1001.70 \mathrm{C} \quad 3.212840 \mathrm{E}-02 \$ \mathrm{H}-1$

$8016.70 \mathrm{C} \quad 1.606420 \mathrm{E}-02 \$ 0-16$

$12000.60 \mathrm{C} \quad 2.399430 \mathrm{E}-04$ \$Mg-nat

$13027.70 \mathrm{C} \quad 2.958200 \mathrm{E}-02$ \$Al-27

$14000.60 \mathrm{C} \quad 1.245870 \mathrm{E}-04$ \$Si-nat

$24000.50 \mathrm{C} \quad 2.243180 \mathrm{E}-05$ \$Cr-nat

$29000.50 \mathrm{C} \quad 2.294330 \mathrm{E}-05$ \$Cu-nat

$5010.70 \mathrm{C} \quad 3.658125 \mathrm{E}-28$ \$ B-10

$92234.70 \mathrm{C} \quad 5.964864 \mathrm{E}-06 \quad \$ \mathrm{U}-234$

$92235.70 \mathrm{C} \quad 4.603032 \mathrm{E}-04 \quad \$ \mathrm{U}-235$

$92236.70 \mathrm{C} \quad 3.449947 \mathrm{E}-06 \quad \$ \mathrm{U}-236$

$92237.70 \mathrm{C} \quad 1.545203 \mathrm{E}-29$ \$U-237 


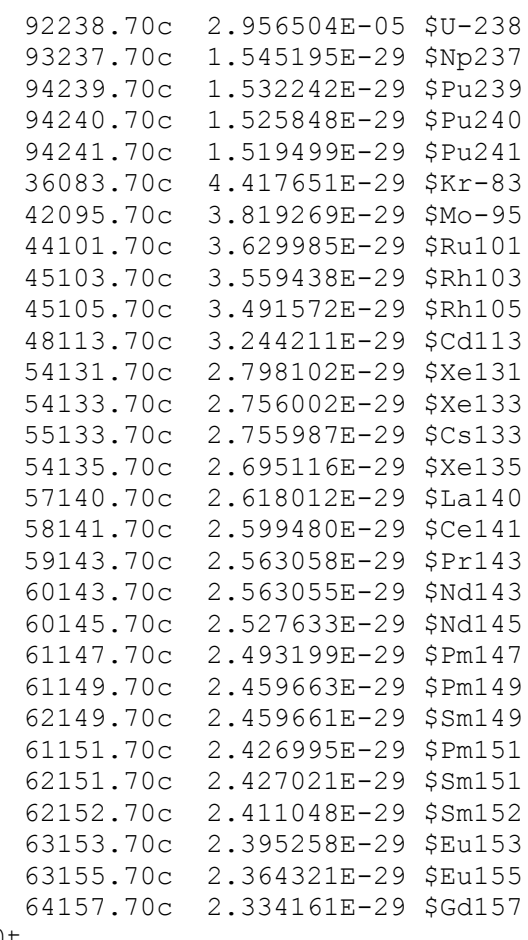

mt2168 lwtr.10t
C

\begin{tabular}{|c|c|c|}
\hline $1001.70 \mathrm{c}$ & $3.393340 \mathrm{E}-02$ & $\$ \mathrm{H}-1$ \\
\hline $8016.70 \mathrm{c}$ & $1.696670 \mathrm{E}-02$ & $\$ 0-16$ \\
\hline $12000.60 \mathrm{c}$ & $2.176490 \mathrm{E}-04$ & $\$ M g-n a t$ \\
\hline $13027.70 \mathrm{C}$ & $2.793720 \mathrm{E}-02$ & $\$ A l-27$ \\
\hline $14000.60 \mathrm{c}$ & $1.130110 \mathrm{E}-04$ & \$Si-nat \\
\hline $24000.50 \mathrm{c}$ & $2.304760 \mathrm{E}-05$ & $\$ C r-n a t$ \\
\hline $29000.50 \mathrm{c}$ & $2.081160 \mathrm{E}-05$ & $\$ \mathrm{Cu}-$ nat \\
\hline $5010.70 \mathrm{c}$ & $6.875743 \mathrm{E}-28$ & $\$ B-10$ \\
\hline $92234.70 \mathrm{C}$ & $6.286590 \mathrm{E}-06$ & $\$ \mathrm{U}-234$ \\
\hline $92235.70 \mathrm{c}$ & $4.851306 \mathrm{E}-04$ & $\$ U-235$ \\
\hline $92236.70 \mathrm{c}$ & $3.636026 \mathrm{E}-06$ & $\$ U-236$ \\
\hline $92237.70 \mathrm{C}$ & $2.904335 \mathrm{E}-29$ & $\$ U-237$ \\
\hline $92238.70 \mathrm{C}$ & $3.115969 \mathrm{E}-05$ & $\$ U-238$ \\
\hline $93237.70 \mathrm{C}$ & $2.904320 \mathrm{E}-29$ & $\$ N p 237$ \\
\hline $94239.70 \mathrm{C}$ & $2.879973 E-29$ & \$Pu239 \\
\hline $94240.70 \mathrm{C}$ & $2.867956 \mathrm{E}-29$ & \$Pu2 40 \\
\hline $94241.70 \mathrm{C}$ & $2.856023 E-29$ & \$Pu241 \\
\hline $36083.70 \mathrm{c}$ & $8.303334 \mathrm{E}-29$ & $\$ K r-83$ \\
\hline $42095.70 \mathrm{c}$ & $7.178626 \mathrm{E}-29$ & $\$ M \circ-95$ \\
\hline $44101.70 \mathrm{C}$ & $6.822852 \mathrm{E}-29$ & \$Ru101 \\
\hline $45103.70 \mathrm{C}$ & $6.690253 \mathrm{E}-29$ & \$Rh103 \\
\hline $45105.70 \mathrm{C}$ & $6.562692 \mathrm{E}-29$ & \$Rh105 \\
\hline $48113.70 \mathrm{C}$ & $6.097758 \mathrm{E}-29$ & $\$ \mathrm{Cd} 113$ \\
\hline $54131.70 \mathrm{C}$ & $5.259260 \mathrm{E}-29$ & $\$ x e 131$ \\
\hline $54133.70 \mathrm{C}$ & $5.180129 \mathrm{E}-29$ & $\$ x e 133$ \\
\hline $55133.70 \mathrm{c}$ & $5.180102 \mathrm{E}-29$ & $\$ C$ S133 \\
\hline $54135.70 \mathrm{C}$ & $5.065689 \mathrm{E}-29$ & $\$ \mathrm{Xe1} 35$ \\
\hline $57140.70 \mathrm{C}$ & $4.920766 \mathrm{E}-29$ & \$La140 \\
\hline $58141.70 \mathrm{C}$ & $4.885933 E-29$ & $\$ C e 141$ \\
\hline $59143.70 \mathrm{C}$ & $4.817476 \mathrm{E}-29$ & $\$ \operatorname{Pr} 143$ \\
\hline $60143.70 \mathrm{c}$ & $4.817471 E-29$ & $\$ N d 143$ \\
\hline
\end{tabular}




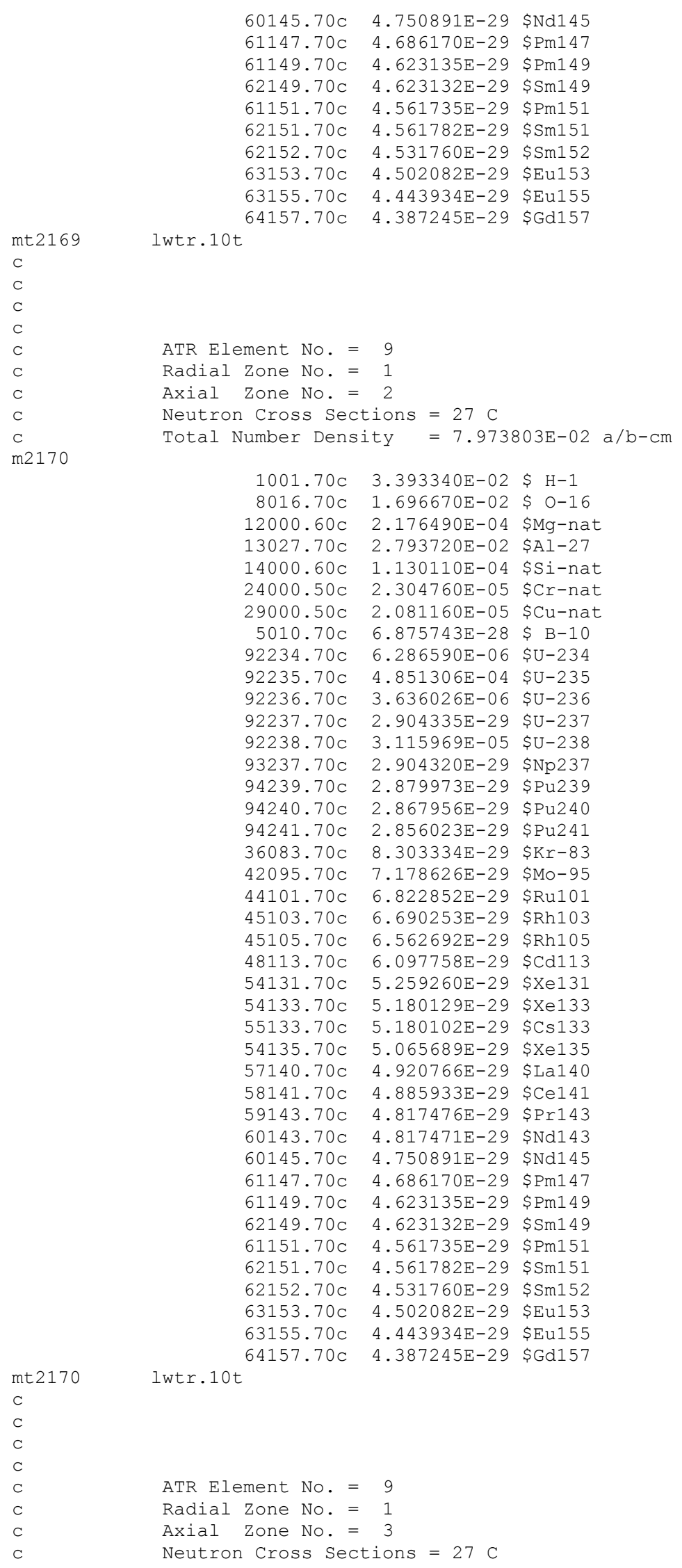


C 2171

mt2171
C
C
C
C
C
C
C
C
C
m2172
Total Number Density $=7.973803 \mathrm{E}-02 \mathrm{a} / \mathrm{b}-\mathrm{cm}$ 


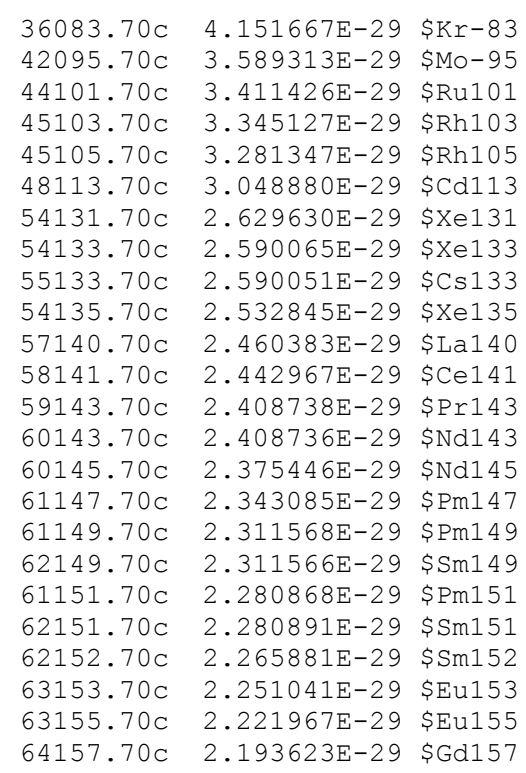

mt2172 lwtr.10t

C

C

C

ATR Element No = 9

C Radial Zone No. = 1

C Axial Zone No. = 5

C Neutron Cross Sections $=27 \mathrm{C}$

c Total Number Density $=7.973803 \mathrm{E}-02 \mathrm{a} / \mathrm{b}-\mathrm{cm}$

m2173

\begin{tabular}{|c|c|c|}
\hline $1001.70 \mathrm{C}$ & $3.393340 \mathrm{E}-02$ & $\$ \mathrm{H}-1$ \\
\hline $8016.70 \mathrm{C}$ & $1.696670 \mathrm{E}-02$ & $\$ 0-16$ \\
\hline $12000.60 \mathrm{c}$ & $2.176490 \mathrm{E}-04$ & $\$ M g-n a t$ \\
\hline $13027.70 \mathrm{c}$ & $2.793720 \mathrm{E}-02$ & $\$ A I-27$ \\
\hline $14000.60 \mathrm{c}$ & $1.130110 \mathrm{E}-04$ & \$Si-nat \\
\hline $24000.50 \mathrm{c}$ & $2.304760 \mathrm{E}-05$ & $\$ C r-n a t$ \\
\hline $29000.50 \mathrm{c}$ & $2.081160 \mathrm{E}-05$ & $\$ \mathrm{Cu}-\mathrm{nat}$ \\
\hline $5010.70 \mathrm{C}$ & $6.875743 E-28$ & $\$ B-10$ \\
\hline $92234.70 \mathrm{C}$ & $6.286590 \mathrm{E}-06$ & $\$ U-234$ \\
\hline $92235.70 \mathrm{c}$ & $4.851306 \mathrm{E}-04$ & $\$ U-235$ \\
\hline $92236.70 \mathrm{c}$ & $3.636026 \mathrm{E}-06$ & $\$ U-236$ \\
\hline $92237.70 \mathrm{C}$ & $2.904335 E-29$ & $\$ U-237$ \\
\hline $92238.70 \mathrm{c}$ & $3.115969 \mathrm{E}-05$ & $\$ U-238$ \\
\hline $93237.70 \mathrm{C}$ & $2.904320 E-29$ & $\$ \mathrm{~Np} 237$ \\
\hline $94239.70 \mathrm{C}$ & $2.879973 E-29$ & \$Pu239 \\
\hline $94240.70 \mathrm{C}$ & $2.867956 \mathrm{E}-29$ & $\$ \mathrm{Pu} 240$ \\
\hline $94241.70 \mathrm{C}$ & $2.856023 E-29$ & \$Pu241 \\
\hline $36083.70 \mathrm{c}$ & $8.303334 \mathrm{E}-29$ & $\$ K r-83$ \\
\hline $42095.70 \mathrm{c}$ & $7.178626 \mathrm{E}-29$ & $\$ M o-95$ \\
\hline $44101.70 \mathrm{C}$ & $6.822852 E-29$ & \$Ru101 \\
\hline $45103.70 \mathrm{c}$ & $6.690253 \mathrm{E}-29$ & $\$ R h 103$ \\
\hline $45105.70 \mathrm{C}$ & $6.562692 \mathrm{E}-29$ & $\$ R h 105$ \\
\hline $48113.70 \mathrm{c}$ & $6.097758 \mathrm{E}-29$ & $\$ \mathrm{Cd} 113$ \\
\hline $54131.70 \mathrm{C}$ & $5.259260 \mathrm{E}-29$ & \$Xe131 \\
\hline $54133.70 \mathrm{c}$ & $5.180129 \mathrm{E}-29$ & \$xe133 \\
\hline $55133.70 \mathrm{C}$ & $5.180102 E-29$ & $\$ \mathrm{Cs} 133$ \\
\hline $54135.70 \mathrm{C}$ & $5.065689 E-29$ & $\$ x e 135$ \\
\hline $57140.70 \mathrm{c}$ & $4.920766 \mathrm{E}-29$ & \$La140 \\
\hline $58141.70 \mathrm{C}$ & $4.885933 E-29$ & $\$ \mathrm{Ce} 141$ \\
\hline $59143.70 \mathrm{c}$ & $4.817476 E-29$ & $\$ \operatorname{Pr} 143$ \\
\hline $60143.70 \mathrm{c}$ & $4.817471 E-29$ & $\$ N d 143$ \\
\hline $60145.70 \mathrm{c}$ & $4.750891 E-29$ & $\$ N d 145$ \\
\hline $61147.70 \mathrm{c}$ & $4.686170 E-29$ & $\$ P m 147$ \\
\hline $61149.70 \mathrm{c}$ & $4.623135 E-29$ & $\$ P m 149$ \\
\hline $62149.70 \mathrm{c}$ & $4.623132 E-29$ & $\$ S m 149$ \\
\hline $61151.70 \mathrm{c}$ & $4.561735 \mathrm{E}-29$ & $\$ P m 151$ \\
\hline
\end{tabular}


$62151.70 \mathrm{C} \quad 4.561782 \mathrm{E}-29 \$ \mathrm{Sm} 151$

$62152.70 \mathrm{C} \quad 4.531760 \mathrm{E}-29$ \$Sm152

$63153.70 \mathrm{C} \quad 4.502082 \mathrm{E}-29 \$ \mathrm{Eu} 153$

$63155.70 \mathrm{C} \quad 4.443934 \mathrm{E}-29$ \$Eu155

mt2173 lwtr.10t $64157.70 \mathrm{c} \quad 4.387245 \mathrm{E}-29$ \$Gd157

C

C

C

C

C

C

C

C

m2174

ATR Element No. $=9$

Radial Zone No. $=1$

Axial Zone No. = 6

Neutron Cross Sections $=27 \mathrm{C}$

Total Number Density $=7.973803 \mathrm{E}-02 \mathrm{a} / \mathrm{b}-\mathrm{cm}$

\begin{abstract}
$1001.70 \mathrm{C} \quad 3.393340 \mathrm{E}-02$ \$ $\mathrm{H}-1$
$8016.70 \mathrm{C} \quad 1.696670 \mathrm{E}-02$ \$ O-16

$12000.60 \mathrm{C} \quad 2.176490 \mathrm{E}-04$ \$Mg-nat

$13027.70 \mathrm{C} \quad 2.793720 \mathrm{E}-02$ \$Al-27

$14000.60 \mathrm{C} \quad 1.130110 \mathrm{E}-04$ \$Si-nat

$24000.50 \mathrm{C} \quad 2.304760 \mathrm{E}-05$ \$Cr-nat

$29000.50 \mathrm{C} \quad 2.081160 \mathrm{E}-05$ \$Cu-nat

$5010.70 \mathrm{C} \quad 6.875743 \mathrm{E}-28$ \$ B-10

$92234.70 \mathrm{C} \quad 6.286590 \mathrm{E}-06 \quad \$ \mathrm{U}-234$

$92235.70 \mathrm{C} \quad 4.851306 \mathrm{E}-04 \quad \$ \mathrm{U}-235$

$92236.70 \mathrm{C} \quad 3.636026 \mathrm{E}-06$ \$U-236

$92237.70 \mathrm{C} \quad 2.904335 \mathrm{E}-29$ \$U-237

$92238.70 \mathrm{C} \quad 3.115969 \mathrm{E}-05$ \$U-238

$93237.70 \mathrm{C} \quad 2.904320 \mathrm{E}-29$ \$Np237

$94239.70 \mathrm{C} \quad 2.879973 \mathrm{E}-29$ \$Pu239

$94240.70 \mathrm{C} \quad 2.867956 \mathrm{E}-29$ \$Pu240

$94241.70 \mathrm{C} \quad 2.856023 \mathrm{E}-29$ \$Pu241

$36083.70 \mathrm{C} \quad 8.303334 \mathrm{E}-29 \quad \$ \mathrm{Kr}-83$

$42095.70 \mathrm{C} \quad 7.178626 \mathrm{E}-29$ \$Mo-95

$44101.70 \mathrm{C} \quad 6.822852 \mathrm{E}-29$ \$Ru101

$45103.70 \mathrm{C} \quad 6.690253 \mathrm{E}-29$ \$Rh103

$45105.70 \mathrm{C} \quad 6.562692 \mathrm{E}-29$ \$Rh105

$48113.70 \mathrm{C} \quad 6.097758 \mathrm{E}-29 \$ \mathrm{Cd} 113$

$54131.70 \mathrm{C} \quad 5.259260 \mathrm{E}-29 \$ \mathrm{Xe131}$

$54133.70 \mathrm{C} \quad 5.180129 \mathrm{E}-29 \$ \mathrm{Xe133}$

$55133.70 \mathrm{C} \quad 5.180102 \mathrm{E}-29 \$ \mathrm{Cs} 133$

$54135.70 \mathrm{C} \quad 5.065689 \mathrm{E}-29$ \$Xe135

$57140.70 \mathrm{C} \quad 4.920766 \mathrm{E}-29$ \$La140

$58141.70 \mathrm{C} \quad 4.885933 \mathrm{E}-29$ \$Ce141

$59143.70 \mathrm{C} \quad 4.817476 \mathrm{E}-29$ \$Pr143

$60143.70 \mathrm{C} \quad 4.817471 \mathrm{E}-29 \$ \mathrm{Nd} 143$

$60145.70 \mathrm{C} \quad 4.750891 \mathrm{E}-29 \quad \$ N d 145$

$61147.70 \mathrm{C} \quad 4.686170 \mathrm{E}-29 \$ \mathrm{Pm} 147$

$61149.70 \mathrm{C} \quad 4.623135 \mathrm{E}-29 \$ \mathrm{Pm} 149$

$62149.70 \mathrm{C} \quad 4.623132 \mathrm{E}-29 \$ \mathrm{Sm} 149$

$61151.70 \mathrm{C} \quad 4.561735 \mathrm{E}-29 \$ \mathrm{Pm} 151$

$62151.70 \mathrm{C} \quad 4.561782 \mathrm{E}-29 \$ \mathrm{Sm} 151$

$62152.70 \mathrm{C} \quad 4.531760 \mathrm{E}-29 \$ \mathrm{Sm} 152$

$63153.70 \mathrm{C} \quad 4.502082 \mathrm{E}-29$ \$Eu153

$63155.70 \mathrm{C} \quad 4.443934 \mathrm{E}-29$ \$Eu155
\end{abstract}

$64157.70 \mathrm{C} \quad 4.387245 \mathrm{E}-29$ \$Gd157

mt2174 lwtr.10t

C

C

$\mathrm{C}$

C ATR Element No. = 9

C Radial Zone No. = 1

C Axial Zone No. = 7

C Neutron Cross Sections $=27 \mathrm{C}$

c Total Number Density $=7.973803 \mathrm{E}-02 \mathrm{a} / \mathrm{b}-\mathrm{cm}$

m2175 


\begin{tabular}{|c|c|c|}
\hline $13027.70 \mathrm{C}$ & $2.793720 \mathrm{E}-02$ & $\$ A l-27$ \\
\hline $14000.60 \mathrm{c}$ & $1.130110 \mathrm{E}-04$ & \$Si-nat \\
\hline $24000.50 \mathrm{c}$ & $2.304760 \mathrm{E}-05$ & $\$ C r-n a t$ \\
\hline $9000.50 \mathrm{c}$ & $2.081160 \mathrm{E}-05$ & $\$ \mathrm{Cu}-$ nat \\
\hline $5010.70 \mathrm{c}$ & $6.875743 \mathrm{E}-28$ & $\$ B-10$ \\
\hline $92234.70 \mathrm{C}$ & $6.286590 \mathrm{E}-06$ & $\$ \mathrm{U}-234$ \\
\hline $92235.70 \mathrm{C}$ & $4.851306 \mathrm{E}-04$ & $\$ U-235$ \\
\hline $92236.70 \mathrm{C}$ & $3.636026 \mathrm{E}-06$ & $\$ U-236$ \\
\hline $92237.70 \mathrm{c}$ & $2.904335 \mathrm{E}-29$ & $\$ \mathrm{U}-237$ \\
\hline $238.70 c$ & $3.115969 \mathrm{E}-05$ & $\$ \mathrm{U}-238$ \\
\hline $93237.70 \mathrm{C}$ & $2.904320 E-29$ & \$Np237 \\
\hline $94239.70 \mathrm{c}$ & $2.879973 E-29$ & \$Pu239 \\
\hline $94240.70 \mathrm{c}$ & $2.867956 \mathrm{E}-29$ & \$Pu240 \\
\hline $94241.70 \mathrm{C}$ & $2.856023 E-29$ & \$Pu241 \\
\hline $36083.70 \mathrm{c}$ & $8.303334 E-29$ & $\$ \mathrm{Kr}-83$ \\
\hline $42095.70 \mathrm{c}$ & $7.178626 \mathrm{E}-29$ & $\$ M O-95$ \\
\hline $44101.70 \mathrm{c}$ & $6.822852 \mathrm{E}-29$ & \$Ru101 \\
\hline $45103.70 \mathrm{c}$ & $6.690253 \mathrm{E}-29$ & $\$ R h 103$ \\
\hline $45105.70 \mathrm{c}$ & $6.562692 \mathrm{E}-29$ & \$Rh105 \\
\hline $48113.70 \mathrm{C}$ & $6.097758 \mathrm{E}-29$ & $\$ C d 113$ \\
\hline $54131.70 \mathrm{C}$ & $5.259260 \mathrm{E}-29$ & $\$ \mathrm{Xe131}$ \\
\hline $54133.70 \mathrm{C}$ & $5.180129 \mathrm{E}-29$ & $\$$ Xe133 \\
\hline $55133.70 \mathrm{c}$ & $5.180102 \mathrm{E}-29$ & $\$ \mathrm{Cs} 133$ \\
\hline $54135.70 \mathrm{c}$ & $5.065689 \mathrm{E}-29$ & $\$ \mathrm{Xe135}$ \\
\hline $57140.70 \mathrm{C}$ & $4.920766 \mathrm{E}-29$ & $\$ L a 140$ \\
\hline $58141.70 \mathrm{C}$ & $4.885933 E-29$ & $\$ C e 141$ \\
\hline $59143.70 \mathrm{C}$ & $4.817476 \mathrm{E}-29$ & $\$ \operatorname{Pr} 143$ \\
\hline $60143.70 \mathrm{c}$ & $4.817471 \mathrm{E}-29$ & \$Nd143 \\
\hline $60145.70 \mathrm{c}$ & $4.750891 \mathrm{E}-29$ & \$Nd14 5 \\
\hline $61147.70 \mathrm{c}$ & $4.686170 \mathrm{E}-29$ & $\$ \operatorname{Pm} 147$ \\
\hline $61149.70 \mathrm{c}$ & $4.623135 E-29$ & $\$ \operatorname{Pm} 149$ \\
\hline $62149.70 \mathrm{C}$ & $4.623132 E-29$ & $\$ \operatorname{Sm} 149$ \\
\hline $61151.70 \mathrm{C}$ & $4.561735 E-29$ & $\$ \operatorname{Pm} 151$ \\
\hline $62151.70 \mathrm{C}$ & $4.561782 \mathrm{E}-29$ & $\$$ Sm151 \\
\hline $62152.70 \mathrm{c}$ & $4.531760 \mathrm{E}-29$ & \$Sm152 \\
\hline $63153.70 \mathrm{c}$ & $4.502082 E-29$ & \$Eu153 \\
\hline $63155.70 \mathrm{C}$ & $4.443934 E-29$ & $\$$ Eu155 \\
\hline $4157.70 \mathrm{C}$ & $4.387245 E-29$ & \$Gd157 \\
\hline
\end{tabular}

$\begin{array}{ll}\mathrm{mt} 2175 & \text { lwtr.10t } \\ \mathrm{C} & \\ \mathrm{C} & \\ \mathrm{C} & \text { ATR Element No. }=9 \\ \mathrm{C} & \text { Radial Zone No. }=2 \\ \mathrm{C} & \text { Axial Zone No. }=1 \\ \mathrm{C} & \text { Neutron Cross Sections }=27 \mathrm{C} \\ \mathrm{C} & \text { Total Number Density }=8.294866 \mathrm{E}-02 \mathrm{a} / \mathrm{b}-\mathrm{cm} \\ \mathrm{C} & \end{array}$

\begin{tabular}{|c|c|c|}
\hline & 2 & $\$ \mathrm{H}-1$ \\
\hline 80 & $1.960390 \mathrm{E}-02$ & $0-16$ \\
\hline & $60990=-04$ & $\mathrm{Mg}-\mathrm{n}$ \\
\hline & & \\
\hline & & \\
\hline & 1. & \\
\hline & & \\
\hline & & B \\
\hline & & $\$ \mathrm{U}-$ \\
\hline & 6. & \$U- \\
\hline & 4.8 & \$U-236 \\
\hline & & $\$ U_{-}$ \\
\hline & & \\
\hline & & $\$ \mathrm{~Np}$ \\
\hline & & $\$ P r$ \\
\hline & 7 . & $\$ \mathrm{Pu}$ \\
\hline & 7.1 & \$Pu241 \\
\hline & 2.0 & $\$ \mathrm{Kr}-83$ \\
\hline & $E-29$ & $\$ M \circ-95$ \\
\hline & & $\$ \mathrm{Ru}$ \\
\hline & & \\
\hline & $1.636554 \mathrm{E}-$ & 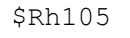 \\
\hline
\end{tabular}




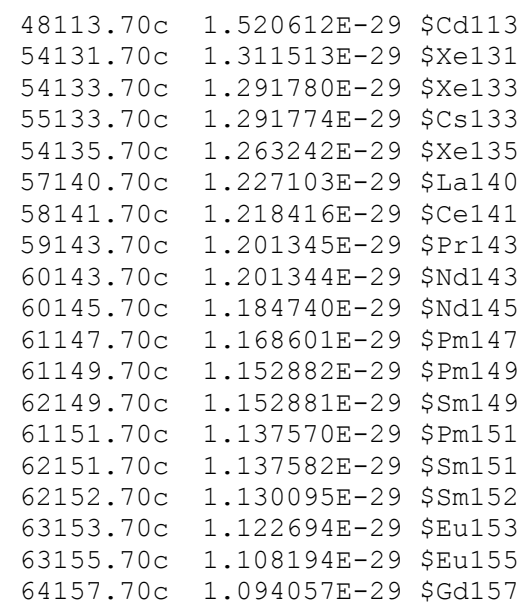
mt2176 lwtr.10t

C

$\mathrm{C}$

C

C

C

$\mathrm{C}$

C

C

m2177

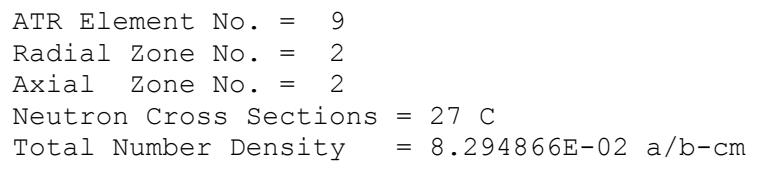




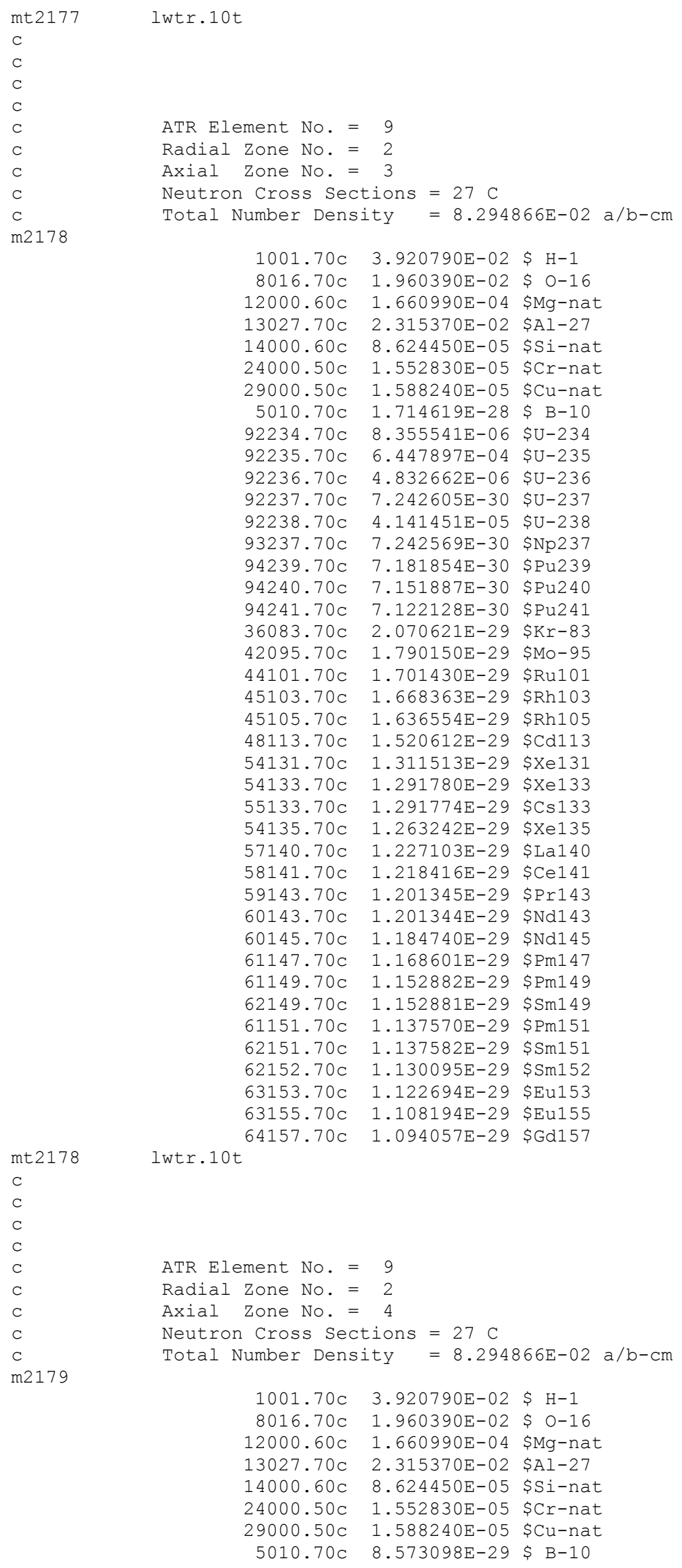




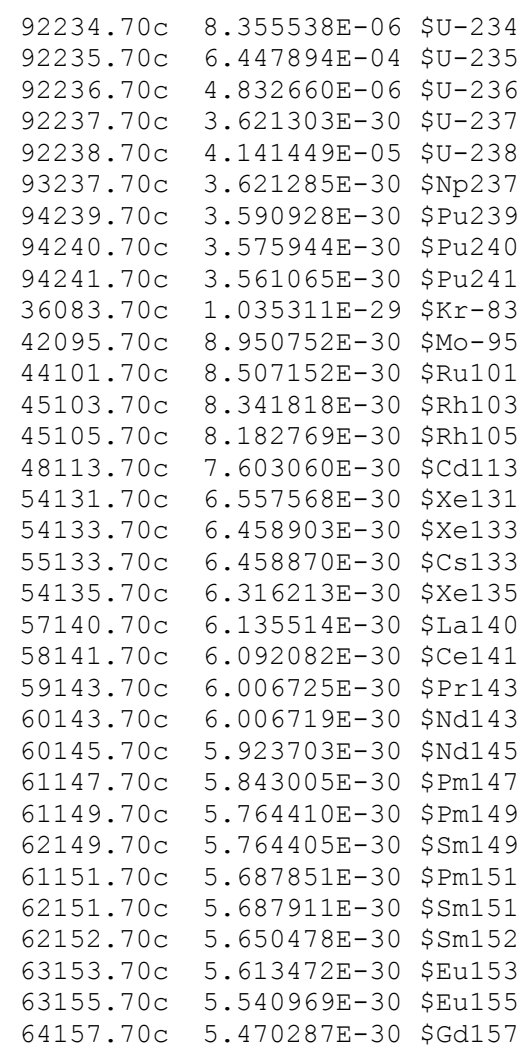

mt2179 lwtr.10t

c

C

$\mathrm{C}$

C

C ATR Element No. = 9

C Radial Zone No. $=2$

C Axial Zone No. = 5

C Neutron Cross Sections $=27 \mathrm{C}$

Total Number Density $=8.294866 \mathrm{E}-02 \mathrm{a} / \mathrm{b}-\mathrm{cm}$

m2180

\begin{tabular}{|c|c|c|}
\hline $1001.70 \mathrm{C}$ & $3.920790 \mathrm{E}-02$ & $\$ \mathrm{H}-1$ \\
\hline $8016.70 \mathrm{c}$ & $1.960390 \mathrm{E}-02$ & $\$ 0-16$ \\
\hline $12000.60 \mathrm{C}$ & $1.660990 \mathrm{E}-04$ & $\$ M g-n a t$ \\
\hline $13027.70 \mathrm{C}$ & $2.315370 \mathrm{E}-02$ & $\$ A l-27$ \\
\hline $14000.60 \mathrm{C}$ & $8.624450 \mathrm{E}-05$ & \$Si-nat \\
\hline $24000.50 \mathrm{c}$ & $1.552830 \mathrm{E}-05$ & \$Cr-nat \\
\hline $29000.50 \mathrm{C}$ & $1.588240 \mathrm{E}-05$ & $\$ \mathrm{Cu}-\mathrm{nat}$ \\
\hline $5010.70 \mathrm{c}$ & $1.714619 \mathrm{E}-28$ & $\$ B-10$ \\
\hline $92234.70 \mathrm{C}$ & $8.355541 \mathrm{E}-06$ & $\$ U-234$ \\
\hline $92235.70 \mathrm{C}$ & $6.447897 \mathrm{E}-04$ & $\$ U-235$ \\
\hline $92236.70 \mathrm{C}$ & $4.832662 \mathrm{E}-06$ & $\$ U-236$ \\
\hline $92237.70 \mathrm{C}$ & $7.242605 E-30$ & $\$ U-237$ \\
\hline $92238.70 \mathrm{C}$ & $4.141451 E-05$ & $\$ U-238$ \\
\hline $93237.70 \mathrm{C}$ & $7.242569 \mathrm{E}-30$ & $\$ N p 237$ \\
\hline $94239.70 \mathrm{C}$ & $7.181854 \mathrm{E}-30$ & $\$ P u 239$ \\
\hline $94240.70 \mathrm{C}$ & $7.151887 \mathrm{E}-30$ & $\$ \mathrm{Pu} 240$ \\
\hline $94241.70 \mathrm{C}$ & $7.122128 \mathrm{E}-30$ & \$Pu241 \\
\hline $36083.70 \mathrm{C}$ & $2.070621 E-29$ & $\$ K r-83$ \\
\hline $42095.70 \mathrm{C}$ & $1.790150 \mathrm{E}-29$ & $\$ M o-95$ \\
\hline $44101.70 \mathrm{C}$ & $1.701430 E-29$ & \$Ru101 \\
\hline $45103.70 \mathrm{C}$ & $1.668363 \mathrm{E}-29$ & $\$ R h 103$ \\
\hline $45105.70 \mathrm{C}$ & $1.636554 \mathrm{E}-29$ & \$Rh105 \\
\hline $48113.70 \mathrm{C}$ & $1.520612 \mathrm{E}-29$ & $\$ C d 113$ \\
\hline $54131.70 \mathrm{C}$ & 1.311513E-29 & $\$ x e 131$ \\
\hline $3.70 \mathrm{c}$ & $1.291780 \mathrm{E}-29$ & $\$ x e 133$ \\
\hline $55133.70 \mathrm{C}$ & $1.291774 \mathrm{E}-29$ & $\$ C s 133$ \\
\hline $54135.70 \mathrm{C}$ & $1.263242 E-29$ & $\$ x e 135$ \\
\hline
\end{tabular}


$57140.70 \mathrm{C} \quad 1.227103 \mathrm{E}-29$ \$La140

$58141.70 \mathrm{C} \quad 1.218416 \mathrm{E}-29$ \$Ce141

$59143.70 \mathrm{C} \quad 1.201345 \mathrm{E}-29$ \$Pr143

$60143.70 \mathrm{C} \quad 1.201344 \mathrm{E}-29 \$ \mathrm{Nd143}$

$60145.70 \mathrm{C} \quad 1.184740 \mathrm{E}-29 \$ \mathrm{Nd} 145$

$61147.70 \mathrm{C} \quad 1.168601 \mathrm{E}-29 \$ \mathrm{Pm} 147$

$61149.70 \mathrm{C} \quad 1.152882 \mathrm{E}-29 \$ \mathrm{Pm} 149$

$62149.70 \mathrm{C} \quad 1.152881 \mathrm{E}-29 \$ \mathrm{Sm} 149$

$61151.70 \mathrm{C} \quad 1.137570 \mathrm{E}-29$ \$Pm151

$62151.70 \mathrm{C} \quad 1.137582 \mathrm{E}-29$ \$Sm151

$62152.70 \mathrm{C} \quad 1.130095 \mathrm{E}-29 \$ \mathrm{Sm} 152$

$63153.70 \mathrm{C} \quad 1.122694 \mathrm{E}-29$ \$Eu153

$63155.70 \mathrm{C} \quad 1.108194 \mathrm{E}-29$ \$Eu155

64157.70 C $1.094057 \mathrm{E}-29$ \$Gd157

C

C

C

C

m2181

mt2181

lwtr.10t
ATR Element No. $=9$

Radial Zone No. $=2$

Axial Zone No. $=6$

Neutron Cross Sections $=27 \mathrm{C}$

Total Number Density $=8.294866 \mathrm{E}-02 \mathrm{a} / \mathrm{b}-\mathrm{cm}$

$1001.70 \mathrm{C} \quad 3.920790 \mathrm{E}-02$ \$ $\mathrm{H}-1$

$8016.70 \mathrm{C} \quad 1.960390 \mathrm{E}-02 \$ 0-16$

$12000.60 \mathrm{C} \quad 1.660990 \mathrm{E}-04$ \$Mg-nat

$13027.70 \mathrm{C} \quad 2.315370 \mathrm{E}-02$ \$Al-27

$14000.60 \mathrm{C} \quad 8.624450 \mathrm{E}-05$ \$Si-nat

$24000.50 \mathrm{C} \quad 1.552830 \mathrm{E}-05$ \$Cr-nat

$29000.50 \mathrm{C} \quad 1.588240 \mathrm{E}-05$ \$Cu-nat

$5010.70 \mathrm{C} \quad 1.714619 \mathrm{E}-28$ \$ B-10

$92234.70 \mathrm{C} \quad 8.355541 \mathrm{E}-06$ \$U-234

$92235.70 \mathrm{C} \quad 6.447897 \mathrm{E}-04 \quad \$ \mathrm{U}-235$

$92236.70 \mathrm{C} \quad 4.832662 \mathrm{E}-06$ \$U-236

$92237.70 \mathrm{C} \quad 7.242605 \mathrm{E}-30 \quad \$ \mathrm{U}-237$

$92238.70 \mathrm{C} \quad 4.141451 \mathrm{E}-05$ \$U-238

$93237.70 \mathrm{C} \quad 7.242569 \mathrm{E}-30 \quad \$ \mathrm{~Np} 237$

$94239.70 \mathrm{C} \quad 7.181854 \mathrm{E}-30$ \$Pu239

$94240.70 \mathrm{C} \quad 7.151887 \mathrm{E}-30$ \$Pu 240

$94241.70 \mathrm{C} \quad 7.122128 \mathrm{E}-30$ \$Pu241

$36083.70 \mathrm{C} \quad 2.070621 \mathrm{E}-29$ \$Kr-83

$42095.70 \mathrm{C} \quad 1.790150 \mathrm{E}-29$ \$Mo-95

$44101.70 \mathrm{C} \quad 1.701430 \mathrm{E}-29$ \$Ru101

$45103.70 \mathrm{C} \quad 1.668363 \mathrm{E}-29$ \$Rh103

$45105.70 \mathrm{C} \quad 1.636554 \mathrm{E}-29$ \$Rh105

$48113.70 \mathrm{C} \quad 1.520612 \mathrm{E}-29 \$ \mathrm{Cd} 113$

$54131.70 \mathrm{C} \quad 1.311513 \mathrm{E}-29$ \$Xe131

$54133.70 \mathrm{C} \quad 1.291780 \mathrm{E}-29$ \$Xe133

$55133.70 \mathrm{C} \quad 1.291774 \mathrm{E}-29 \$ \mathrm{Cs} 133$

$54135.70 \mathrm{C} \quad 1.263242 \mathrm{E}-29$ \$Xe135

$57140.70 \mathrm{C} \quad 1.227103 \mathrm{E}-29$ La140

$58141.70 \mathrm{C} \quad 1.218416 \mathrm{E}-29$ \$Ce141

$59143.70 \mathrm{C} \quad 1.201345 \mathrm{E}-29 \$ \operatorname{Pr} 143$

$60143.70 \mathrm{C} \quad 1.201344 \mathrm{E}-29 \$ \mathrm{Nd} 143$

$60145.70 \mathrm{C} \quad 1.184740 \mathrm{E}-29$ \$Nd145

$61147.70 \mathrm{C} \quad 1.168601 \mathrm{E}-29 \$ \mathrm{Pm} 147$

$61149.70 \mathrm{C} \quad 1.152882 \mathrm{E}-29$ \$Pm149

$62149.70 \mathrm{C} \quad 1.152881 \mathrm{E}-29 \$ \mathrm{Sm} 149$

$61151.70 \mathrm{C} \quad 1.137570 \mathrm{E}-29$ \$Pm151

$62151.70 \mathrm{C} \quad 1.137582 \mathrm{E}-29$ \$Sm151

$62152.70 \mathrm{C} \quad 1.130095 \mathrm{E}-29$ \$Sm152

$63153.70 \mathrm{C} \quad 1.122694 \mathrm{E}-29$ \$Eu153

$63155.70 \mathrm{C} \quad 1.108194 \mathrm{E}-29$ \$Eu155

$64157.70 \mathrm{C} \quad 1.094057 \mathrm{E}-29$ \$Gd157

C

C

C 


\begin{tabular}{|c|c|c|c|}
\hline C & \multicolumn{3}{|c|}{ ATR Element No. $=9$} \\
\hline C & Radial Zone No. = & 2 & \\
\hline C & Zone No. = & 7 & \\
\hline C & \multicolumn{3}{|c|}{ Neutron Cross Sections $=27 \mathrm{C}$} \\
\hline C & Total Number Dens: & $=8.294866 \mathrm{E}-02$ & $\mathrm{a} / \mathrm{b}-\mathrm{cm}$ \\
\hline \multicolumn{4}{|l|}{ m2182 } \\
\hline & $1001.70 \mathrm{C}$ & $3.920790 \mathrm{E}-02 \$ \mathrm{H}-1$ & \\
\hline & $8016.70 \mathrm{C}$ & $1.960390 \mathrm{E}-02 \$ 0-16$ & \\
\hline & $12000.60 \mathrm{c}$ & $1.660990 \mathrm{E}-04$ \$Mg-nat & \\
\hline & $13027.70 \mathrm{C}$ & $2.315370 \mathrm{E}-02$ \$AI-27 & \\
\hline & $14000.60 \mathrm{c}$ & $8.624450 \mathrm{E}-05$ \$Si-nat & \\
\hline & $24000.50 \mathrm{c}$ & $1.552830 \mathrm{E}-05$ \$Cr-nat & \\
\hline & $29000.50 \mathrm{C}$ & $1.588240 \mathrm{E}-05$ \$Cu-nat & \\
\hline & $5010.70 \mathrm{c}$ & $1.714619 \mathrm{E}-28$ \$ $\mathrm{B}-10$ & \\
\hline & $92234.70 \mathrm{c}$ & $8.355541 E-06 \$ U-234$ & \\
\hline & $92235.70 \mathrm{C}$ & $6.447897 \mathrm{E}-04 \quad \$ U-235$ & \\
\hline & $92236.70 \mathrm{C}$ & $4.832662 E-06 \$ U-236$ & \\
\hline & $92237.70 \mathrm{C}$ & $7.242605 \mathrm{E}-30 \quad \$ \mathrm{U}-237$ & \\
\hline & $92238.70 \mathrm{c}$ & $4.141451 \mathrm{E}-05$ \$U-238 & \\
\hline & $93237.70 \mathrm{C}$ & $7.242569 \mathrm{E}-30 \quad \$ \mathrm{~Np} 237$ & \\
\hline & $94239.70 \mathrm{C}$ & $7.181854 \mathrm{E}-30$ \$Pu239 & \\
\hline & $94240.70 \mathrm{C}$ & $7.151887 \mathrm{E}-30 \$ \mathrm{Pu} 240$ & \\
\hline & $94241.70 \mathrm{C}$ & $7.122128 \mathrm{E}-30$ \$Pu241 & \\
\hline & $36083.70 \mathrm{c}$ & $2.070621 E-29 \$ K r-83$ & \\
\hline & $42095.70 \mathrm{c}$ & $1.790150 \mathrm{E}-29$ \$Mo-95 & \\
\hline & $44101.70 \mathrm{c}$ & $1.701430 \mathrm{E}-29 \$ \mathrm{Ru} 01$ & \\
\hline & $45103.70 \mathrm{c}$ & $1.668363 \mathrm{E}-29 \$ \operatorname{Rh} 103$ & \\
\hline & $45105.70 \mathrm{c}$ & $1.636554 \mathrm{E}-29 \$ \mathrm{Rh} 105$ & \\
\hline & $48113.70 \mathrm{c}$ & $1.520612 \mathrm{E}-29 \$ \mathrm{Cd} 113$ & \\
\hline & $54131.70 \mathrm{c}$ & $1.311513 E-29 \$ \mathrm{Xe131}$ & \\
\hline & $54133.70 \mathrm{c}$ & $1.291780 \mathrm{E}-29 \$ \mathrm{Xe133}$ & \\
\hline & $55133.70 \mathrm{C}$ & $1.291774 \mathrm{E}-29 \$ \mathrm{Cs} 133$ & \\
\hline & $54135.70 \mathrm{C}$ & $1.263242 \mathrm{E}-29 \$ \mathrm{Xe} 135$ & \\
\hline & $57140.70 \mathrm{C}$ & 1.227103E-29\$La140 & \\
\hline & $58141.70 \mathrm{C}$ & $1.218416 \mathrm{E}-29 \$ \mathrm{Ce} 141$ & \\
\hline & $59143.70 \mathrm{C}$ & $1.201345 E-29 \$ \operatorname{Pr} 143$ & \\
\hline & $60143.70 \mathrm{c}$ & $1.201344 \mathrm{E}-29 \$ \mathrm{Nd1} 43$ & \\
\hline & $60145.70 \mathrm{C}$ & $1.184740 \mathrm{E}-29 \$ \mathrm{Nd} 145$ & \\
\hline & $61147.70 \mathrm{C}$ & $1.168601 \mathrm{E}-29 \$ \operatorname{Pm} 147$ & \\
\hline & $61149.70 \mathrm{c}$ & $1.152882 \mathrm{E}-29 \$ \operatorname{Pm} 149$ & \\
\hline & $62149.70 \mathrm{c}$ & $1.152881 \mathrm{E}-29 \$ \mathrm{Sm} 149$ & \\
\hline & $61151.70 \mathrm{C}$ & $1.137570 \mathrm{E}-29 \$ \operatorname{Pm} 151$ & \\
\hline & $62151.70 \mathrm{c}$ & $1.137582 \mathrm{E}-29 \$ \mathrm{Sm} 151$ & \\
\hline & $62152.70 \mathrm{C}$ & $1.130095 \mathrm{E}-29 \$ \mathrm{Sm} 152$ & \\
\hline & $63153.70 \mathrm{c}$ & $1.122694 \mathrm{E}-29$ \$Eu153 & \\
\hline & $63155.70 \mathrm{C}$ & $1.108194 \mathrm{E}-29$ \$Eu155 & \\
\hline & $64157.70 \mathrm{C}$ & $1.094057 \mathrm{E}-29 \$ \mathrm{Gd1} 57$ & \\
\hline mt2182 & \multicolumn{3}{|l|}{ lwtr.10t } \\
\hline \multicolumn{4}{|c|}{$\mathrm{C}$} \\
\hline \multicolumn{4}{|l|}{ C } \\
\hline \multicolumn{4}{|c|}{ C } \\
\hline \multicolumn{4}{|l|}{ C } \\
\hline C & ATR Element No. = & 9 & \\
\hline C & Radial Zone No. = & 3 & \\
\hline C & Zone No. = & 1 & \\
\hline C & Neutron Cross Sect & tions $=27 \mathrm{C}$ & \\
\hline C & Total Number Dens: & ity $=7.868379 \mathrm{E}-02$ & $\mathrm{a} / \mathrm{b}-\mathrm{cm}$ \\
\hline \multicolumn{4}{|l|}{ m2183 } \\
\hline & $1001.70 \mathrm{C}$ & $3.212840 \mathrm{E}-02 \$ \mathrm{H}-1$ & \\
\hline & $8016.70 \mathrm{C}$ & $1.606420 \mathrm{E}-02 \$ 0-16$ & \\
\hline & $12000.60 \mathrm{c}$ & 2.399430E-04 \$Mg-nat & \\
\hline & $13027.70 \mathrm{C}$ & $2.958200 \mathrm{E}-02$ \$Al-27 & \\
\hline & $14000.60 \mathrm{C}$ & 1.245870E-04 \$Si-nat & \\
\hline & $24000.50 \mathrm{C}$ & $2.243180 \mathrm{E}-05$ \$Cr-nat & \\
\hline & $29000.50 \mathrm{C}$ & 2.294330E-05 \$Cu-nat & \\
\hline & $5010.70 \mathrm{C}$ & $3.658125 E-28 \$ B-10$ & \\
\hline & $92234.70 \mathrm{C}$ & $5.964864 \mathrm{E}-06 \quad \$ U-234$ & \\
\hline & $92235.70 \mathrm{C}$ & $4.603032 E-04 \$ U-235$ & \\
\hline & $92236.70 \mathrm{C}$ & $3.449947 \mathrm{E}-06 \$ \mathrm{U}-236$ & \\
\hline & $92237.70 \mathrm{C}$ & $1.545203 E-29 \$ U-237$ & \\
\hline & $92238.70 \mathrm{c}$ & $2.956504 \mathrm{E}-05 \quad \$ U-238$ & \\
\hline
\end{tabular}




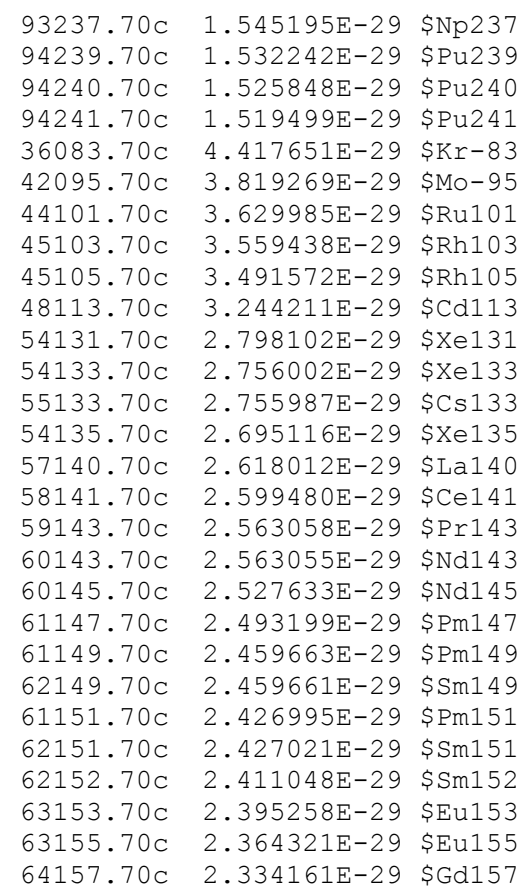
mt2183 lwtr.10t

$\mathrm{C}$

C

$\mathrm{C}$

C ATR Element No. = 9

Radial Zone No. = 3

C Axial Zone No. = 2

C Neutron Cross Sections $=27 \mathrm{C}$

c Total Number Density $=7.868379 \mathrm{E}-02 \mathrm{a} / \mathrm{b}-\mathrm{cm}$

m2184

\begin{tabular}{|c|c|c|}
\hline $1001.70 \mathrm{C}$ & $3.212840 \mathrm{E}-02$ & \$ $\mathrm{H}-1$ \\
\hline $8016.70 \mathrm{C}$ & $1.606420 \mathrm{E}-02$ & $\$ 0-16$ \\
\hline $12000.60 \mathrm{c}$ & $2.399430 \mathrm{E}-04$ & $\$ M g-n a t$ \\
\hline $13027.70 \mathrm{c}$ & $2.958200 \mathrm{E}-02$ & $\$ A 1-27$ \\
\hline $14000.60 \mathrm{c}$ & $1.245870 \mathrm{E}-04$ & \$Si-nat \\
\hline $24000.50 \mathrm{c}$ & $2.243180 \mathrm{E}-05$ & \$Cr-nat \\
\hline $29000.50 \mathrm{c}$ & $2.294330 \mathrm{E}-05$ & \$Cu-nat \\
\hline $5010.70 \mathrm{C}$ & $3.658125 \mathrm{E}-28$ & $\$ B-10$ \\
\hline $92234.70 \mathrm{c}$ & $5.964864 \mathrm{E}-06$ & $\$ \mathrm{U}-234$ \\
\hline $92235.70 \mathrm{C}$ & $4.603032 E-04$ & $\$ U-235$ \\
\hline $92236.70 \mathrm{c}$ & $3.449947 \mathrm{E}-06$ & $\$ U-236$ \\
\hline $92237.70 \mathrm{c}$ & $1.545203 \mathrm{E}-29$ & $\$ U-237$ \\
\hline $92238.70 \mathrm{c}$ & $2.956504 \mathrm{E}-05$ & $\$ U-238$ \\
\hline $93237.70 \mathrm{C}$ & $1.545195 \mathrm{E}-29$ & $\$ N p 237$ \\
\hline $94239.70 \mathrm{c}$ & $1.532242 \mathrm{E}-29$ & $\$ p u 239$ \\
\hline $94240.70 \mathrm{c}$ & $1.525848 \mathrm{E}-29$ & $\$ \mathrm{Pu} 240$ \\
\hline $94241.70 \mathrm{c}$ & $1.519499 \mathrm{E}-29$ & \$Pu241 \\
\hline $36083.70 \mathrm{c}$ & $4.417651 \mathrm{E}-29$ & $\$ K r-83$ \\
\hline $42095.70 \mathrm{c}$ & $3.819269 \mathrm{E}-29$ & $\$ M o-95$ \\
\hline $44101.70 \mathrm{c}$ & $3.629985 E-29$ & \$Ru101 \\
\hline $45103.70 \mathrm{c}$ & $3.559438 E-29$ & \$Rh103 \\
\hline $45105.70 \mathrm{c}$ & $3.491572 \mathrm{E}-29$ & \$Rh105 \\
\hline $48113.70 \mathrm{c}$ & $3.244211 E-29$ & $\$ \mathrm{Cd} 113$ \\
\hline $54131.70 \mathrm{c}$ & $2.798102 E-29$ & $\$ x e 131$ \\
\hline $54133.70 \mathrm{c}$ & $2.756002 \mathrm{E}-29$ & $\$ x e 133$ \\
\hline $55133.70 \mathrm{c}$ & $2.755987 \mathrm{E}-29$ & $\$ C$ S 133 \\
\hline $54135.70 \mathrm{c}$ & $2.695116 \mathrm{E}-29$ & $\$ \mathrm{Xe1} 35$ \\
\hline $57140.70 \mathrm{C}$ & $2.618012 \mathrm{E}-29$ & \$La140 \\
\hline $58141.70 \mathrm{c}$ & $2.599480 \mathrm{E}-29$ & $\$ \mathrm{Ce} 141$ \\
\hline $59143.70 \mathrm{c}$ & $2.563058 \mathrm{E}-29$ & $\$ \operatorname{Pr} 143$ \\
\hline $60143.70 \mathrm{c}$ & $2.563055 E-29$ & $\$ \mathrm{Nd} 143$ \\
\hline $60145.70 \mathrm{c}$ & $2.527633 E-29$ & $\$ N d 145$ \\
\hline
\end{tabular}




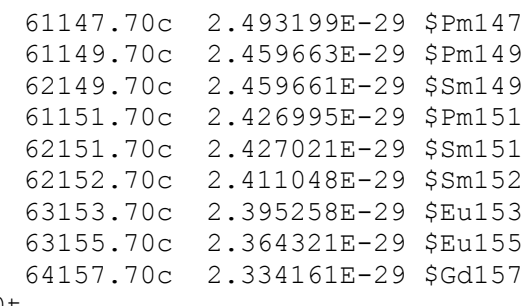

mt2184
$C$
$C$
$C$
$C$
$C$
$C$
$C$
$C$
$C$
m2185 lwtr.10t

$64157.70 \mathrm{C} \quad 2.334161 \mathrm{E}-29 \$ \mathrm{Gd} 157$

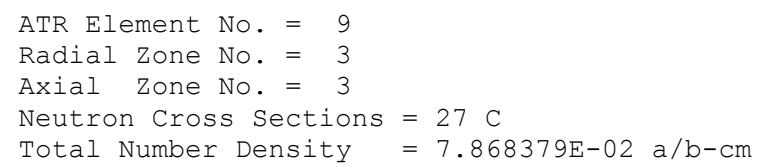

ATR Element No. = 9

Radial Zone No. $=3$

Axial Zone No. = 4

Neutron Cross Sections $=27 \mathrm{C}$

Total Number Density $=7.868379 \mathrm{E}-02 \mathrm{a} / \mathrm{b}-\mathrm{cm}$ 


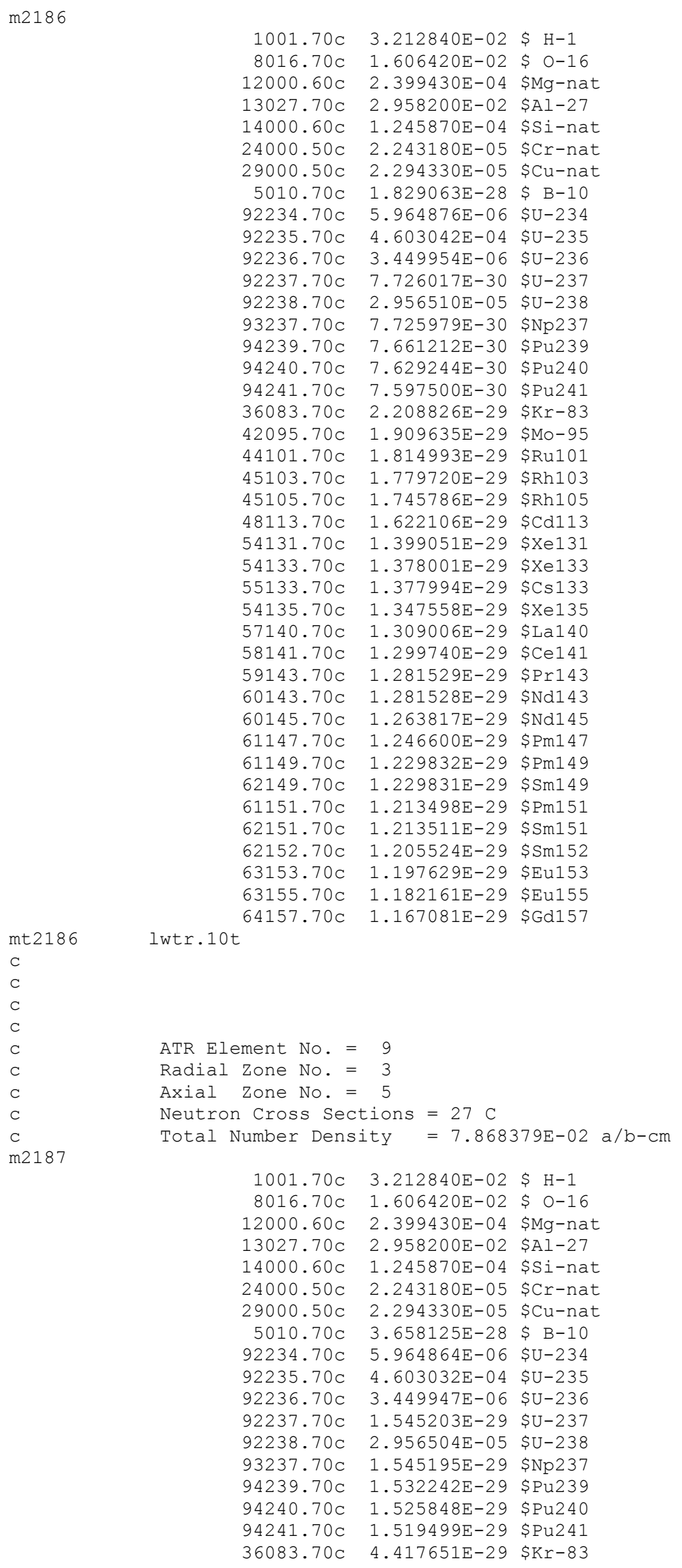




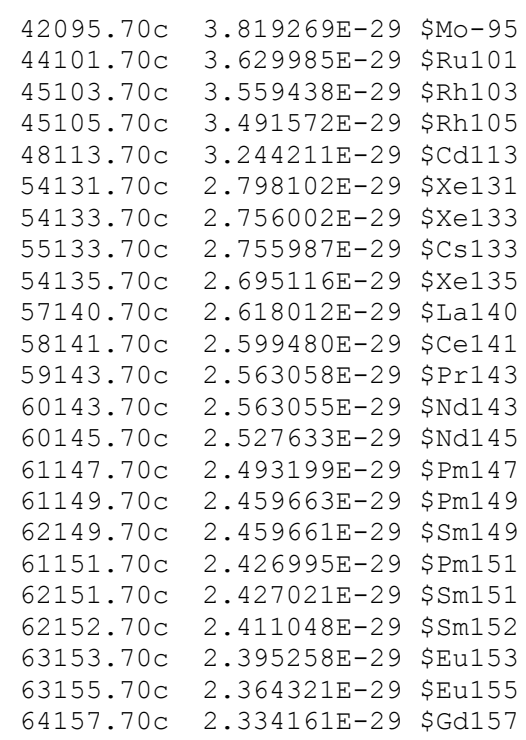

mt2187 lwtr.10t

c

C

C

C

C

C

C

m2188

ATR Element No. = 9

Radial Zone No. $=3$

Axial Zone No. $=6$

Neutron Cross Sections $=27 \mathrm{C}$

Total Number Density $=7.868379 \mathrm{E}-02 \mathrm{a} / \mathrm{b}-\mathrm{cm}$

\begin{abstract}
$1001.70 \mathrm{C} \quad 3.212840 \mathrm{E}-02$ \$ $\mathrm{H}-1$
$8016.70 \mathrm{C} \quad 1.606420 \mathrm{E}-02$ \$ O-16

$12000.60 \mathrm{C} \quad 2.399430 \mathrm{E}-04$ \$Mg-nat

$13027.70 \mathrm{C} \quad 2.958200 \mathrm{E}-02$ \$Al-27

$14000.60 \mathrm{C} \quad 1.245870 \mathrm{E}-04$ \$Si-nat

$24000.50 \mathrm{C} \quad 2.243180 \mathrm{E}-05$ \$Cr-nat

$29000.50 \mathrm{C} \quad 2.294330 \mathrm{E}-05$ \$Cu-nat

$5010.70 \mathrm{C} \quad 3.658125 \mathrm{E}-28$ \$ B 10

$92234.70 \mathrm{C} \quad 5.964864 \mathrm{E}-06 \quad \$ \mathrm{U}-234$

$92235.70 \mathrm{C} \quad 4.603032 \mathrm{E}-04 \quad \$ \mathrm{U}-235$

$92236.70 \mathrm{C} \quad 3.449947 \mathrm{E}-06$ \$U-236

$92237.70 \mathrm{C} \quad 1.545203 \mathrm{E}-29$ \$U-237

$92238.70 \mathrm{C} \quad 2.956504 \mathrm{E}-05 \quad \$ \mathrm{U}-238$

$93237.70 \mathrm{C} \quad 1.545195 \mathrm{E}-29 \quad \$ \mathrm{~Np} 237$

$94239.70 \mathrm{C} \quad 1.532242 \mathrm{E}-29$ \$Pu239

$94240.70 \mathrm{C} \quad 1.525848 \mathrm{E}-29$ \$Pu240

$94241.70 \mathrm{C} \quad 1.519499 \mathrm{E}-29$ \$Pu241

$36083.70 \mathrm{C} \quad 4.417651 \mathrm{E}-29$ \$Kr-83

$42095.70 \mathrm{C} \quad 3.819269 \mathrm{E}-29$ \$Mo-95

$44101.70 \mathrm{C} \quad 3.629985 \mathrm{E}-29$ \$Ru101

$45103.70 \mathrm{C} \quad 3.559438 \mathrm{E}-29$ \$Rh103

$45105.70 \mathrm{C} \quad 3.491572 \mathrm{E}-29 \$ \mathrm{Rh} 105$

$48113.70 \mathrm{C} \quad 3.244211 \mathrm{E}-29$ \$Cd113

$54131.70 \mathrm{C} \quad 2.798102 \mathrm{E}-29$ \$Xe131

$54133.70 \mathrm{C} \quad 2.756002 \mathrm{E}-29$ \$Xe133

$55133.70 \mathrm{C} \quad 2.755987 \mathrm{E}-29 \$ \mathrm{Cs} 133$

$54135.70 \mathrm{C} \quad 2.695116 \mathrm{E}-29$ \$Xe135

$57140.70 \mathrm{C} \quad 2.618012 \mathrm{E}-29$ \$La140

$58141.70 \mathrm{C} \quad 2.599480 \mathrm{E}-29$ \$Ce141

$59143.70 \mathrm{C} \quad 2.563058 \mathrm{E}-29 \$ \operatorname{Pr} 143$

$60143.70 \mathrm{C} \quad 2.563055 \mathrm{E}-29 \$ \mathrm{Nd1} 43$

$60145.70 \mathrm{C} \quad 2.527633 \mathrm{E}-29$ \$Nd145

$61147.70 \mathrm{C} \quad 2.493199 \mathrm{E}-29 \$ \mathrm{Pm} 147$

$61149.70 \mathrm{C} \quad 2.459663 \mathrm{E}-29 \quad \$ \mathrm{Pm} 149$

$62149.70 \mathrm{C} \quad 2.459661 \mathrm{E}-29$ \$Sm149

$61151.70 \mathrm{C} \quad 2.426995 \mathrm{E}-29$ \$Pm151

$62151.70 \mathrm{C} \quad 2.427021 \mathrm{E}-29$ \$Sm151
\end{abstract}


$62152.70 \mathrm{C} \quad 2.411048 \mathrm{E}-29 \$ \mathrm{Sm} 152$

$63153.70 \mathrm{C} \quad 2.395258 \mathrm{E}-29$ Su153

$63155.70 \mathrm{C} \quad 2.364321 \mathrm{E}-29 \quad \$ \mathrm{Eu} 155$

mt2188 lwtr.10t

$64157.70 \mathrm{C} \quad 2.334161 \mathrm{E}-29$ \$Gd157

$\mathrm{C}$

ATR Element No. = 9

C Radial Zone No. $=3$

C Axial Zone No. = 7

C Neutron Cross Sections $=27 \mathrm{C}$

c Total Number Density $=7.868379 \mathrm{E}-02 \mathrm{a} / \mathrm{b}-\mathrm{cm}$

m2189

$\begin{array}{llll}1001.70 \mathrm{C} & 3.212840 \mathrm{E}-02 & \$ \mathrm{H}-1 \\ 8016.70 \mathrm{C} & 1.606420 \mathrm{E}-02 & \$ & \mathrm{O}-1\end{array}$

$12000.60 \mathrm{C} \quad 2.399430 \mathrm{E}-04$ \$Mg-nat

$13027.70 \mathrm{C} \quad 2.958200 \mathrm{E}-02$ \$Al-27

$14000.60 \mathrm{C} \quad 1.245870 \mathrm{E}-04$ \$Si-nat

$24000.50 \mathrm{C} \quad 2.243180 \mathrm{E}-05$ \$Cr-nat

$29000.50 \mathrm{C} \quad 2.294330 \mathrm{E}-05$ \$Cu-nat

$5010.70 \mathrm{C} \quad 3.658125 \mathrm{E}-28$ \$ B -10

$92234.70 \mathrm{C} \quad 5.964864 \mathrm{E}-06 \quad \$ \mathrm{U}-234$

$92235.70 \mathrm{C} \quad 4.603032 \mathrm{E}-04 \quad \$ \mathrm{U}-235$

$92236.70 \mathrm{C} \quad 3.449947 \mathrm{E}-06 \quad \$ \mathrm{U}-236$

$92237.70 \mathrm{C} \quad 1.545203 \mathrm{E}-29$ \$U-237

$92238.70 \mathrm{C} \quad 2.956504 \mathrm{E}-05 \quad \mathrm{SU}-238$

$93237.70 \mathrm{C} \quad 1.545195 \mathrm{E}-29 \$ \mathrm{~Np} 237$

$94239.70 \mathrm{C} \quad 1.532242 \mathrm{E}-29$ \$Pu239

$94240.70 \mathrm{C} \quad 1.525848 \mathrm{E}-29$ \$Pu240

$94241.70 \mathrm{C} \quad 1.519499 \mathrm{E}-29$ \$Pu241

$36083.70 \mathrm{C} \quad 4.417651 \mathrm{E}-29$ \$Kr-83

$42095.70 \mathrm{C} \quad 3.819269 \mathrm{E}-29$ \$Mo-95

$44101.70 \mathrm{C} \quad 3.629985 \mathrm{E}-29$ \$Ru101

$45103.70 \mathrm{C} \quad 3.559438 \mathrm{E}-29$ \$Rh103

$45105.70 \mathrm{C} \quad 3.491572 \mathrm{E}-29$ \$Rh105

$48113.70 \mathrm{C} \quad 3.244211 \mathrm{E}-29 \$ \mathrm{Cd} 113$

$54131.70 \mathrm{C} \quad 2.798102 \mathrm{E}-29$ \$Xe131

$54133.70 \mathrm{C} \quad 2.756002 \mathrm{E}-29$ \$Xe133

$55133.70 \mathrm{C} \quad 2.755987 \mathrm{E}-29 \$ \mathrm{Cs} 133$

$54135.70 \mathrm{c} \quad 2.695116 \mathrm{E}-29$ \$Xe135

$57140.70 \mathrm{C} \quad 2.618012 \mathrm{E}-29$ La140

$58141.70 \mathrm{C} \quad 2.599480 \mathrm{E}-29 \$ \mathrm{Ce} 141$

$59143.70 \mathrm{C} \quad 2.563058 \mathrm{E}-29 \$ \mathrm{Pr} 143$

$60143.70 \mathrm{C} \quad 2.563055 \mathrm{E}-29 \$ \mathrm{Nd} 143$

$60145.70 \mathrm{C} \quad 2.527633 \mathrm{E}-29 \$ \mathrm{Nd} 145$

$61147.70 \mathrm{C} \quad 2.493199 \mathrm{E}-29$ \$Pm147

$61149.70 \mathrm{C} \quad 2.459663 \mathrm{E}-29$ \$Pm1 49

$62149.70 \mathrm{C} \quad 2.459661 \mathrm{E}-29$ \$Sm149

$61151.70 \mathrm{C} \quad 2.426995 \mathrm{E}-29 \$ \mathrm{Pm} 151$

$62151.70 \mathrm{C} \quad 2.427021 \mathrm{E}-29$ \$Sm151

$62152.70 \mathrm{C} \quad 2.411048 \mathrm{E}-29 \$ \mathrm{Sm} 152$

$63153.70 \mathrm{C} \quad 2.395258 \mathrm{E}-29$ \$Eu153

$63155.70 \mathrm{C} \quad 2.364321 \mathrm{E}-29$ \$Eu155

mt2189 lwtr.10t

c

C

C

$\mathrm{C}$

C

$\mathrm{C}$

C

m2190

ATR Element No. $=10$

Radial Zone No. = 1

Axial Zone No. = 1

Neutron Cross Sections $=27 \mathrm{C}$

Total Number Density $=7.967879 \mathrm{E}-02 \mathrm{a} / \mathrm{b}-\mathrm{cm}$

$1001.70 \mathrm{C} \quad 3.393340 \mathrm{E}-02 \$ \mathrm{H}-1$

$8016.70 \mathrm{C} \quad 1.696670 \mathrm{E}-02$ \$ O-16

$12000.60 \mathrm{C} \quad 2.176490 \mathrm{E}-04$ \$Mg-nat

$13027.70 \mathrm{C} \quad 2.793720 \mathrm{E}-02$ \$Al-27 


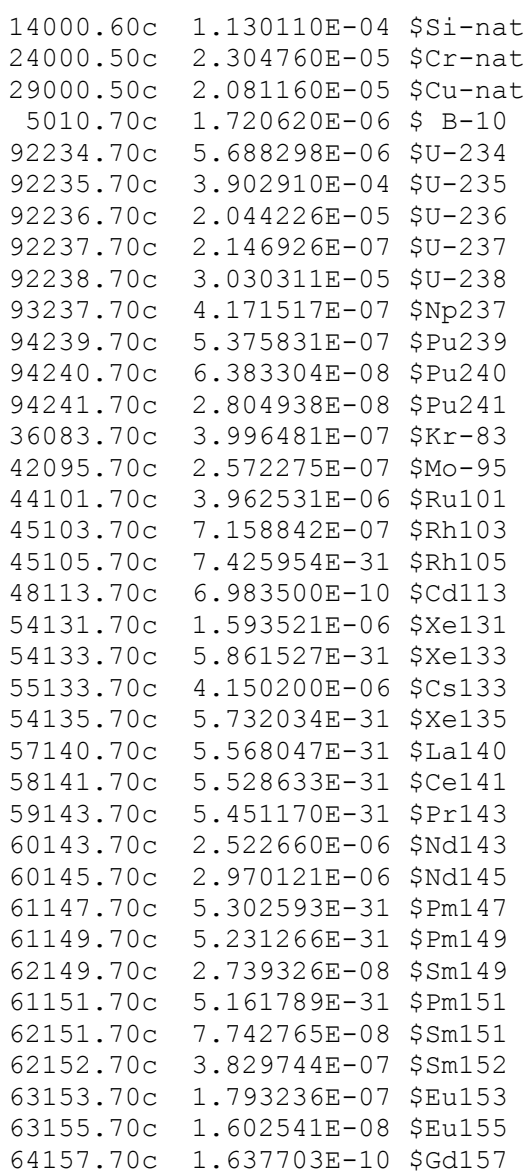

mt2190 lwtr.10t

$\mathrm{C}$

$\mathrm{C}$

C ATR Element No. = 10

C Radial Zone No. $=1$

C Axial Zone No. = 2

C Neutron Cross Sections $=27 \mathrm{C}$

c Total Number Density $=7.964312 \mathrm{E}-02 \mathrm{a} / \mathrm{b}-\mathrm{cm}$

m2191

$\begin{array}{rll}1001.70 \mathrm{C} & 3.393340 \mathrm{E}-02 & \text { \$ }-1 \\ 8016.70 \mathrm{C} & 1.696670 \mathrm{E}-02 & \text { \$ }-16 \\ 12000.60 \mathrm{C} & 2.176490 \mathrm{E}-04 & \text { \$Mg-nat } \\ 13027.70 \mathrm{C} & 2.793720 \mathrm{E}-02 & \text { \$Al-27 } \\ 14000.60 \mathrm{C} & 1.130110 \mathrm{E}-04 & \text { \$Si-nat } \\ 24000.50 \mathrm{C} & 2.304760 \mathrm{E}-05 & \text { \$Cr-nat } \\ 29000.50 \mathrm{C} & 2.081160 \mathrm{E}-05 & \text { \$Cu-nat } \\ 5010.70 \mathrm{C} & 1.720620 \mathrm{E}-06 & \text { \$ }-10 \\ 92234.70 \mathrm{C} & 5.338016 \mathrm{E}-06 & \text { \$U-234 } \\ 92235.70 \mathrm{C} & 3.349376 \mathrm{E}-04 & \text { \$U-235 } \\ 92236.70 \mathrm{C} & 3.022082 \mathrm{E}-05 & \text { \$U-236 } \\ 92237.70 \mathrm{C} & 3.396254 \mathrm{E}-07 & \text { \$U-237 } \\ 92238.70 \mathrm{C} & 2.979408 \mathrm{E}-05 & \text { \$U-238 } \\ 93237.70 \mathrm{C} & 6.598986 \mathrm{E}-07 & \text { \$Np237 } \\ 94239.70 \mathrm{C} & 8.504109 \mathrm{E}-07 & \text { \$Pu239 } \\ 94240.70 \mathrm{C} & 1.009784 \mathrm{E}-07 & \text { \$Pu240 } \\ 94241.70 \mathrm{C} & 4.437174 \mathrm{E}-08 & \text { \$Pu241 } \\ 36083.70 \mathrm{C} & 6.322094 \mathrm{E}-07 & \text { \$Kr-83 } \\ 42095.70 \mathrm{C} & 4.069121 \mathrm{E}-07 & \text { \$Mo-95 } \\ 44101.70 \mathrm{C} & 6.268387 \mathrm{E}-06 & \text { \$Ru101 } \\ 45103.70 \mathrm{C} & 1.132468 \mathrm{E}-06 & \text { \$Rh103 } \\ 45105.70 \mathrm{C} & 1.174723 \mathrm{E}-30 & \text { \$Rh105 } \\ 48113.70 \mathrm{C} & 1.104730 \mathrm{E}-09 & \text { \$Cd113 }\end{array}$




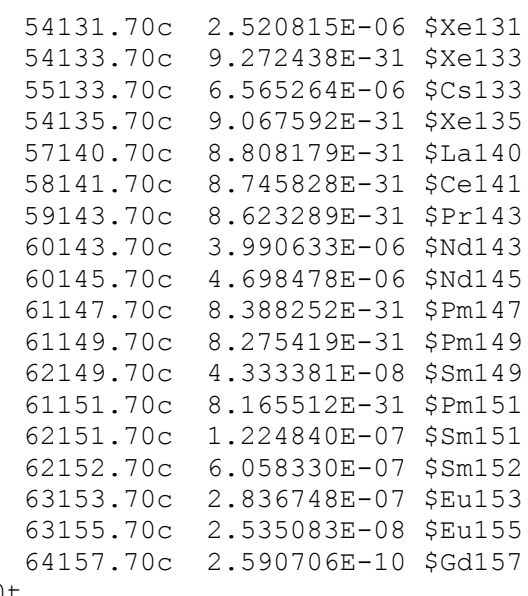

mt2191
C
C
C
C
C
C
C
C
C
m2192

lwtr.10t

$54131.70 \mathrm{C} \quad 2.520815 \mathrm{E}-06$ \$Xe131

$\begin{array}{ll}54133.70 \mathrm{C} & 9.272438 \mathrm{E}-31 \text { \$Xe133 }\end{array}$

$57140.70 \mathrm{C} \quad 8.808179 \mathrm{E}-31$ \$La140

$58141.70 \mathrm{C} \quad 8.745828 \mathrm{E}-31$ \$Ce141

$59143.70 \mathrm{C} \quad 8.623289 \mathrm{E}-31 \quad \$ \operatorname{Pr} 143$

$3.990633 \mathrm{E}-06$ \$Nd143

$60145.70 \mathrm{C} \quad 4.698478 \mathrm{E}-06$ \$Nd145

$8.388252 \mathrm{E}-31$ \$Pm147

$8.275419 \mathrm{E}-31$ \$Pm149

$62151.70 \mathrm{C} \quad 1.224840 \mathrm{E}-07$ \$Sm151

$62152.70 \mathrm{C} \quad 6.058330 \mathrm{E}-07$ \$Sm152

$64157.70 \mathrm{C} \quad 2.590706 \mathrm{E}-10 \quad \$ \mathrm{Gd} 157$

ATR Element No. = 10

Radial Zone No. = 1

Axial Zone No. $=3$

Neutron Cross Sections $=27 \mathrm{C}$

Total Number Density $=7.961814 \mathrm{E}-02 \mathrm{a} / \mathrm{b}-\mathrm{cm}$

\begin{abstract}
$1001.70 \mathrm{C} \quad 3.393340 \mathrm{E}-02 \$ \mathrm{H}-1$
$8016.70 \mathrm{C} \quad 1.696670 \mathrm{E}-02$ \$ O-16

$12000.60 \mathrm{C} \quad 2.176490 \mathrm{E}-04$ \$Mg-nat

$13027.70 \mathrm{C} \quad 2.793720 \mathrm{E}-02$ \$Al-27

$14000.60 \mathrm{C} \quad 1.130110 \mathrm{E}-04$ \$Si-nat

$24000.50 \mathrm{C} \quad 2.304760 \mathrm{E}-05$ \$Cr-nat

$29000.50 \mathrm{C} \quad 2.081160 \mathrm{E}-05$ \$Cu-nat

$5010.70 \mathrm{C} \quad 1.720620 \mathrm{E}-06$ \$ -10

$92234.70 \mathrm{C} \quad 5.092732 \mathrm{E}-06 \quad \$ \mathrm{U}-234$

$92235.70 \mathrm{C} \quad 2.961765 \mathrm{E}-04 \quad \$ \mathrm{U}-235$

$92236.70 \mathrm{C} \quad 3.706825 \mathrm{E}-05$ \$U-236

$92237.70 \mathrm{C} \quad 4.271094 \mathrm{E}-07$ \$U-237

$92238.70 \mathrm{C} \quad 2.943763 \mathrm{E}-05$ \$U-238

$93237.70 \mathrm{C} \quad 8.298817 \mathrm{E}-07 \quad \$ \mathrm{~Np} 237$

$94239.70 \mathrm{C} \quad 1.069468 \mathrm{E}-06$ \$Pu239

$94240.70 \mathrm{C} \quad 1.269895 \mathrm{E}-07$ \$Pu240

$94241.70 \mathrm{C} \quad 5.580144 \mathrm{E}-08$ \$Pu241

$36083.70 \mathrm{C} \quad 7.950601 \mathrm{E}-07$ \$Kr-83

$42095.70 \mathrm{C} \quad 5.117284 \mathrm{E}-07$ \$Mo-95

$44101.70 \mathrm{C} \quad 7.883059 \mathrm{E}-06$ \$Ru101

$45103.70 \mathrm{C} \quad 1.424180 \mathrm{E}-06$ \$Rh103

$45105.70 \mathrm{C} \quad 1.477320 \mathrm{E}-30$ \$Rh105

$48113.70 \mathrm{C} \quad 1.389298 \mathrm{E}-09 \$ \mathrm{Cd} 113$

$54131.70 \mathrm{C} \quad 3.170151 \mathrm{E}-06$ \$Xe131

$54133.70 \mathrm{C} \quad 1.166092 \mathrm{E}-30$ \$Xe133

$55133.70 \mathrm{C} \quad 8.256409 \mathrm{E}-06$ \$Cs133

$54135.70 \mathrm{C} \quad 1.140331 \mathrm{E}-30$ \$Xe135

$57140.70 \mathrm{C} \quad 1.107708 \mathrm{E}-30$ \$La140

$58141.70 \mathrm{C} \quad 1.099866 \mathrm{E}-30 \quad \$ \mathrm{Ce} 141$

$59143.70 \mathrm{C} \quad 1.084456 \mathrm{E}-30 \quad \$ \operatorname{Pr} 143$

$60143.70 \mathrm{C} \quad 5.018580 \mathrm{E}-06 \$ \mathrm{Nd} 143$

$60145.70 \mathrm{C} \quad 5.908758 \mathrm{E}-06 \$ \mathrm{Nd1} 45$

$61147.70 \mathrm{C} \quad 1.054898 \mathrm{E}-30 \quad \$ \mathrm{Pm} 147$

$61149.70 \mathrm{C} \quad 1.040708 \mathrm{E}-30 \$ \mathrm{Pm} 149$

$62149.70 \mathrm{C} \quad 5.449616 \mathrm{E}-08$ \$Sm149

$61151.70 \mathrm{C} \quad 1.026887 \mathrm{E}-30$ \$Pm151

$62151.70 \mathrm{C} \quad 1.540346 \mathrm{E}-07$ \$Sm151

$62152.70 \mathrm{C} \quad 7.618893 \mathrm{E}-07$ \$Sm152

$63153.70 \mathrm{C} \quad 3.567465 \mathrm{E}-07$ \$Eu153

$63155.70 \mathrm{C} \quad 3.188094 \mathrm{E}-08$ \$Eu155

$64157.70 \mathrm{C} \quad 3.258046 \mathrm{E}-10 \quad \$ \mathrm{Gd} 157$
\end{abstract}

mt2192 lwtr.10t 


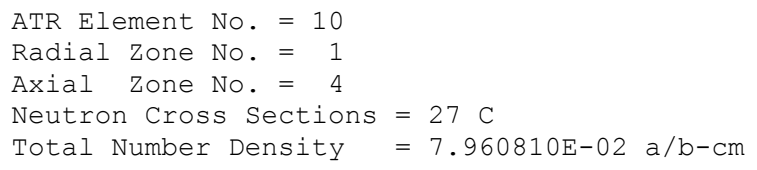




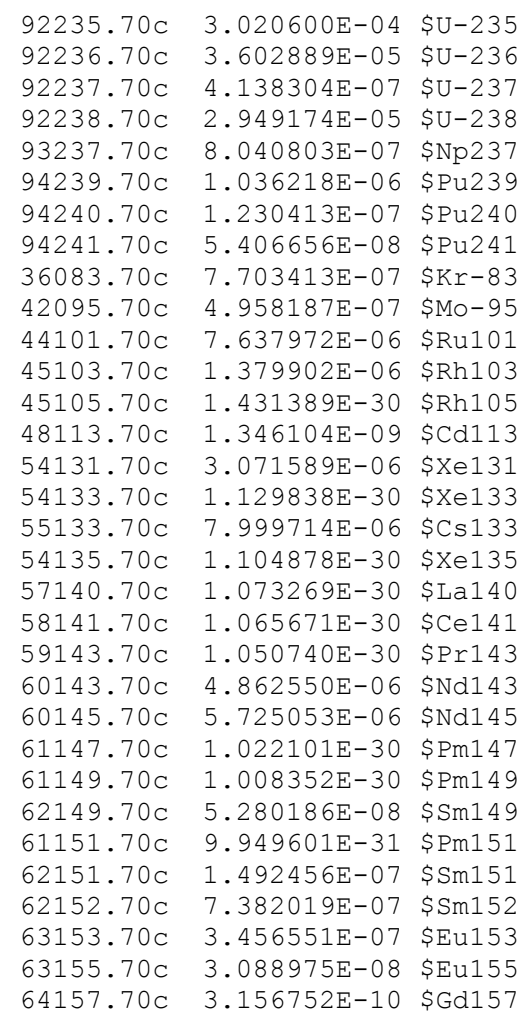

mt2194 lwtr.10t

C

C

C

C

C

C

C

C
ATR Element No. $=10$

Radial Zone No. $=1$

Axial Zone No. = 6

Neutron Cross Sections $=27 \mathrm{C}$

Total Number Density $=7.965042 \mathrm{E}-02 \mathrm{a} / \mathrm{b}-\mathrm{cm}$

$1001.70 \mathrm{C} \quad 3.393340 \mathrm{E}-02 \$ \mathrm{H}-1$

$8016.70 \mathrm{C} \quad 1.696670 \mathrm{E}-02 \mathrm{~S} 0-16$

$12000.60 \mathrm{C} \quad 2.176490 \mathrm{E}-04$ \$Mg-nat

$13027.70 \mathrm{C} \quad 2.793720 \mathrm{E}-02$ \$Al-27

$14000.60 \mathrm{C} \quad 1.130110 \mathrm{E}-04$ \$Si-nat

$24000.50 \mathrm{C} \quad 2.304760 \mathrm{E}-05$ \$Cr-nat

$29000.50 \mathrm{C} \quad 2.081160 \mathrm{E}-05$ \$Cu-nat

$5010.70 \mathrm{C} \quad 1.720620 \mathrm{E}-06$ \$ B -10

$92234.70 \mathrm{C} \quad 5.409632 \mathrm{E}-06 \quad \$ \mathrm{U}-234$

$92235.70 \mathrm{C} \quad 3.462548 \mathrm{E}-04 \quad \mathrm{SU}-235$

$92236.70 \mathrm{C} \quad 2.822156 \mathrm{E}-05 \mathrm{\$ U}-236$

$92237.70 \mathrm{C} \quad 3.140824 \mathrm{E}-07$ \$U-237

$92238.70 \mathrm{C} \quad 2.989815 \mathrm{E}-05$ \$U-238

$93237.70 \mathrm{C} \quad 6.102681 \mathrm{E}-07$ \$Np237

$94239.70 \mathrm{C} \quad 7.864521 \mathrm{E}-07$ \$Pu239

$94240.70 \mathrm{C} \quad 9.338393 \mathrm{E}-08$ \$Pu240

$94241.70 \mathrm{C} \quad 4.103458 \mathrm{E}-08$ \$Pu241

$36083.70 \mathrm{C} \quad 5.846614 \mathrm{E}-07$ \$Kr-83

$42095.70 \mathrm{C} \quad 3.763085 \mathrm{E}-07$ \$Mo-95

$44101.70 \mathrm{C} \quad 5.796946 \mathrm{E}-06$ \$Ru101

$45103.70 \mathrm{C} \quad 1.047296 \mathrm{E}-06$ \$Rh103

$45105.70 \mathrm{C} \quad 1.086373 \mathrm{E}-30$ \$Rh105

$48113.70 \mathrm{C} \quad 1.021644 \mathrm{E}-09$ \$Cd113

$54131.70 \mathrm{C} \quad 2.331226 \mathrm{E}-06$ \$Xe131

$54133.70 \mathrm{C} \quad 8.575064 \mathrm{E}-31$ \$Xe133

$55133.70 \mathrm{C} \quad 6.071496 \mathrm{E}-06 \$ \mathrm{Cs} 133$

$54135.70 \mathrm{C} \quad 8.385625 \mathrm{E}-31$ \$Xe135

$57140.70 \mathrm{C} \quad 8.145722 \mathrm{E}-31$ \$La140 


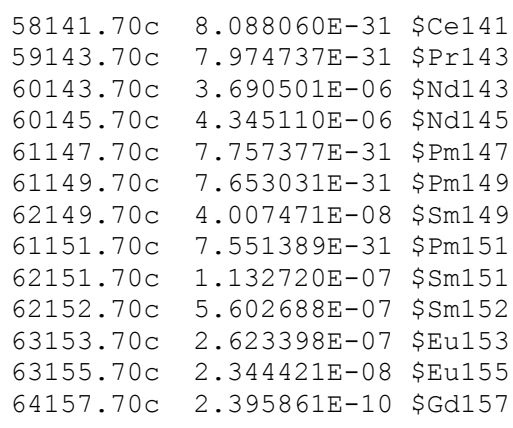

mt2195 lwtr.10t

c

C

$\mathrm{C}$

ATR Element No. $=10$

C Radial Zone No. = 1

Axial Zone No. = 7

C Neutron Cross Sections $=27 \mathrm{C}$

c Total Number Density $=7.969851 \mathrm{E}-02 \mathrm{a} / \mathrm{b}-\mathrm{cm}$

m2196

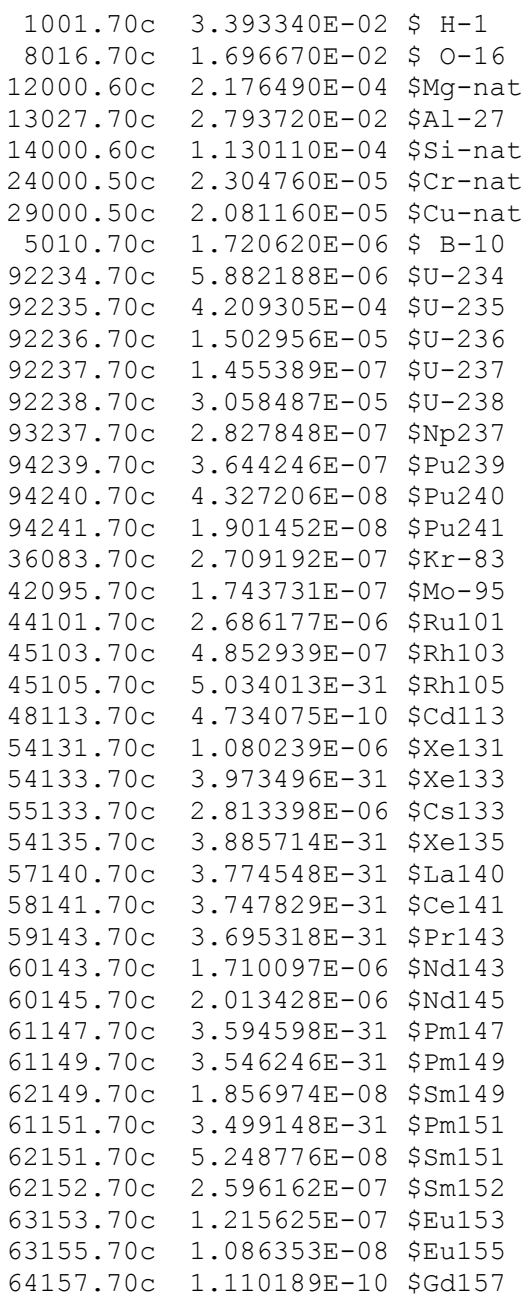




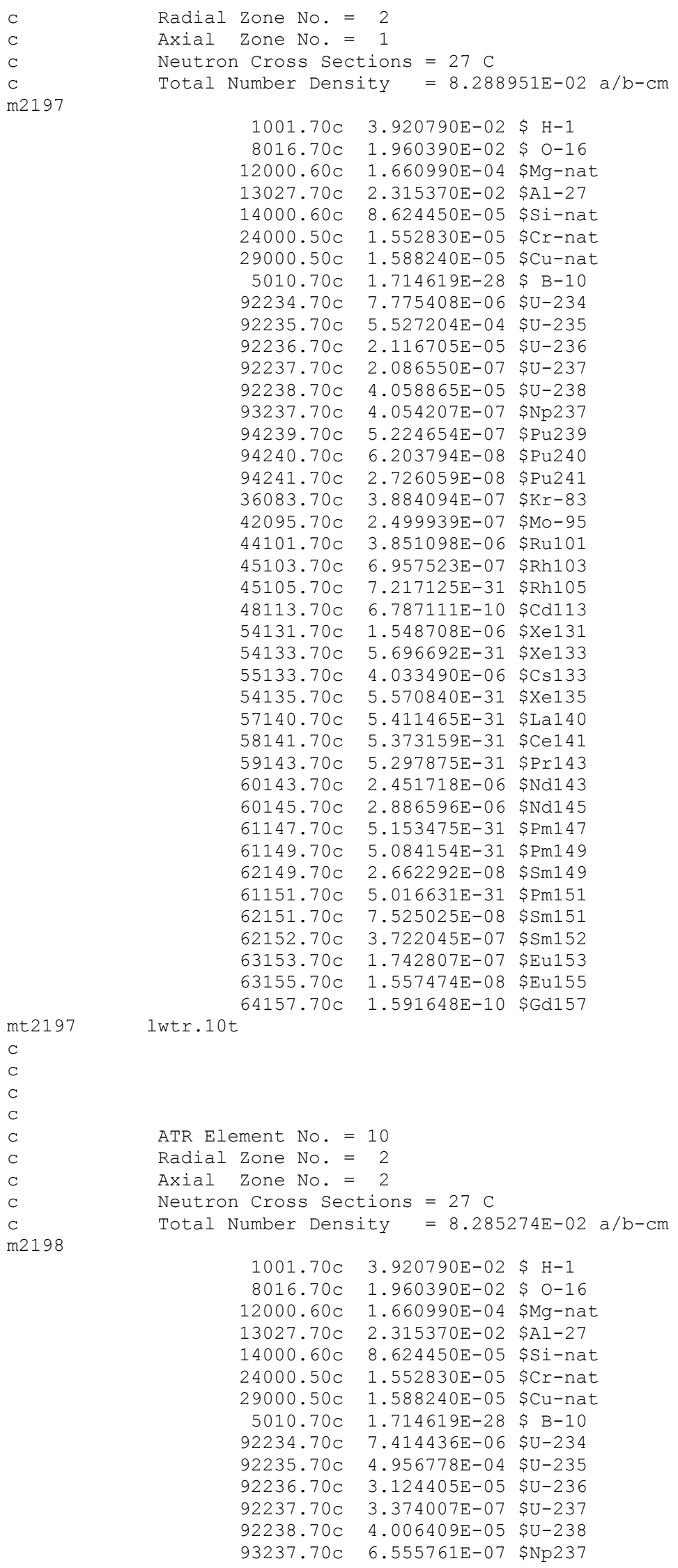


$94239.70 \mathrm{C} \quad 8.448404 \mathrm{E}-07$ \$Pu239

$94240.70 \mathrm{C} \quad 1.003170 \mathrm{E}-07$ \$Pu240

$94241.70 \mathrm{C} \quad 4.408109 \mathrm{E}-08$ \$Pu241

$36083.70 \mathrm{C} \quad 6.280683 \mathrm{E}-07$ \$Kr-83

$42095.70 \mathrm{C} \quad 4.042467 \mathrm{E}-07$ \$Mo-95

$44101.70 \mathrm{C} \quad 6.227328 \mathrm{E}-06$ \$Ru101

$45103.70 \mathrm{C} \quad 1.125050 \mathrm{E}-06$ \$Rh103

$45105.70 \mathrm{C} \quad 1.167028 \mathrm{E}-30$ \$Rh105

$48113.70 \mathrm{C} \quad 1.097494 \mathrm{E}-09 \$ \mathrm{Cd} 113$

$54131.70 \mathrm{C} \quad 2.504303 \mathrm{E}-06$ \$Xe131

$54133.70 \mathrm{C} \quad 9.211702 \mathrm{E}-31$ \$Xe133

$55133.70 \mathrm{C} \quad 6.522260 \mathrm{E}-06 \quad \$ \mathrm{Cs} 133$

$54135.70 \mathrm{C} \quad 9.008198 \mathrm{E}-31$ \$Xe135

$57140.70 \mathrm{C} \quad 8.750483 \mathrm{E}-31$ \$La140

$58141.70 \mathrm{C} \quad 8.688541 \mathrm{E}-31$ \$Ce141

$59143.70 \mathrm{C} \quad 8.566805 \mathrm{E}-31$ \$Pr143

$60143.70 \mathrm{C} \quad 3.964493 \mathrm{E}-06 \$ \mathrm{Nd} 143$

$60145.70 \mathrm{C} \quad 4.667702 \mathrm{E}-06 \$ \mathrm{Nd} 145$

$61147.70 \mathrm{C} \quad 8.333307 \mathrm{E}-31 \$ \mathrm{Pm} 147$

$61149.70 \mathrm{C} \quad 8.221213 \mathrm{E}-31$ \$Pm1 49

$62149.70 \mathrm{C} \quad 4.304997 \mathrm{E}-08 \quad \$ \mathrm{Sm} 149$

$61151.70 \mathrm{C} \quad 8.112026 \mathrm{E}-31$ \$Pm151

$62151.70 \mathrm{C} \quad 1.216817 \mathrm{E}-07$ \$Sm151

$62152.70 \mathrm{C} \quad 6.018647 \mathrm{E}-07$ \$Sm152

$63153.70 \mathrm{C} \quad 2.818166 \mathrm{E}-07$ \$Eu153

$63155.70 \mathrm{C} \quad 2.518478 \mathrm{E}-08$ \$Eu155

mt2198 lwtr.10t

c

$\mathrm{C}$

ATR Element No. $=10$

C Radial Zone No. = 2

C Axial Zone No. = 3

C Neutron Cross Sections $=27 \mathrm{C}$

Total Number Density $=8.282410 \mathrm{E}-02 \mathrm{a} / \mathrm{b}-\mathrm{cm}$

m2199

\begin{tabular}{|c|c|c|}
\hline $1001.70 \mathrm{c}$ & $3.920790 \mathrm{E}-02$ & $\$ \mathrm{H}-1$ \\
\hline $8016.70 \mathrm{c}$ & $1.960390 \mathrm{E}-02$ & $\$ 0-16$ \\
\hline $12000.60 \mathrm{c}$ & $1.660990 \mathrm{E}-04$ & $\$ M g-n a t$ \\
\hline $13027.70 \mathrm{C}$ & $2.315370 \mathrm{E}-02$ & $\$ A 1-27$ \\
\hline $14000.60 \mathrm{c}$ & $8.624450 \mathrm{E}-05$ & \$Si-nat \\
\hline $24000.50 \mathrm{c}$ & $1.552830 \mathrm{E}-05$ & $\$ C r-n a t$ \\
\hline $9000.50 \mathrm{c}$ & $1.588240 \mathrm{E}-05$ & $\$ \mathrm{Cu}-$ nat \\
\hline $010.70 \mathrm{c}$ & $1.714619 \mathrm{E}-28$ & $\$ B-10$ \\
\hline $234.70 \mathrm{C}$ & $7.133161 \mathrm{E}-06$ & $\$ U-234$ \\
\hline $35.70 \mathrm{C}$ & $4.512292 \mathrm{E}-04$ & $\$ U-235$ \\
\hline $6.70 \mathrm{c}$ & $3.909621 \mathrm{E}-05$ & $\$ U-236$ \\
\hline $37.70 \mathrm{C}$ & $4.377214 \mathrm{E}-07$ & $\$ U-237$ \\
\hline $8.70 \mathrm{c}$ & $3.965534 \mathrm{E}-05$ & $\$ U-238$ \\
\hline $7.70 \mathrm{C}$ & $8.505012 \mathrm{E}-07$ & $\$ N p 237$ \\
\hline $.70 \mathrm{C}$ & $1.096040 \mathrm{E}-06$ & \$Pu239 \\
\hline $70 \mathrm{c}$ & $1.301447 \mathrm{E}-07$ & $\$ \mathrm{Pu} 240$ \\
\hline $.70 \mathrm{C}$ & $5.718790 \mathrm{E}-08$ & \$Pu241 \\
\hline $.70 \mathrm{c}$ & $8.148143 \mathrm{E}-07$ & $\$ K r-83$ \\
\hline $.70 \mathrm{C}$ & $5.244430 E-07$ & $\$ M \circ-95$ \\
\hline $.70 \mathrm{C}$ & $8.078923 \mathrm{E}-06$ & \$Ru101 \\
\hline $.70 \mathrm{C}$ & $1.459566 \mathrm{E}-06$ & \$Rh103 \\
\hline $.70 \mathrm{C}$ & $1.514025 \mathrm{E}-30$ & \$Rh105 \\
\hline $3.70 \mathrm{c}$ & 1. $423816 \mathrm{E}-09$ & $\$ \mathrm{Cd} 113$ \\
\hline $.70 \mathrm{C}$ & $3.248917 \mathrm{E}-06$ & $\$ x e 131$ \\
\hline $.70 \mathrm{C}$ & $1.195065 \mathrm{E}-30$ & $\$ \mathrm{Xe1} 33$ \\
\hline $.70 \mathrm{C}$ & $8.461549 \mathrm{E}-06$ & $\$ C$ S 133 \\
\hline $.70 \mathrm{C}$ & $1.168664 \mathrm{E}-30$ & \$Xe135 \\
\hline $.70 \mathrm{C}$ & $1.135230 \mathrm{E}-30$ & \$La140 \\
\hline $.70 \mathrm{c}$ & $1.127194 \mathrm{E}-30$ & $\$ \mathrm{Ce} 141$ \\
\hline 59 & $1.111401 \mathrm{E}-30$ & $\$ \operatorname{Pr} 143$ \\
\hline & $5.143272 \mathrm{E}-06$ & $\$ N d 143$ \\
\hline & $569 E-06$ & $\$ N d 145$ \\
\hline 611 & $1.081108 \mathrm{E}-30$ & \$Pm1 47 \\
\hline
\end{tabular}


$61149.70 \mathrm{C} \quad 1.066566 \mathrm{E}-30 \$ \mathrm{Pm} 149$

$62149.70 \mathrm{C} \quad 5.585018 \mathrm{E}-08 \quad \$ \mathrm{Sm} 149$

$61151.70 \mathrm{C} \quad 1.052401 \mathrm{E}-30$ \$Pm151

$62151.70 \mathrm{C} \quad 1.578618 \mathrm{E}-07 \quad \$ \mathrm{Sm} 151$

$62152.70 \mathrm{C} \quad 7.808194 \mathrm{E}-07 \$ \mathrm{Sm} 152$

$63153.70 \mathrm{C} \quad 3.656103 \mathrm{E}-07 \quad \$ \mathrm{Eu} 153$

$63155.70 \mathrm{C} \quad 3.267306 \mathrm{E}-08$ \$Eu155

mt2199 lwtr.10t

$64157.70 \mathrm{C} \quad 3.338996 \mathrm{E}-10 \quad \$ \mathrm{Gd} 157$

C

C ATR Element No. = 10

C Radial Zone No. = 2

C Axial Zone No. = 4

C Neutron Cross Sections $=27 \mathrm{C}$

C Total Number Density $=8.281159 \mathrm{E}-02 \mathrm{a} / \mathrm{b}-\mathrm{cm}$

m2200

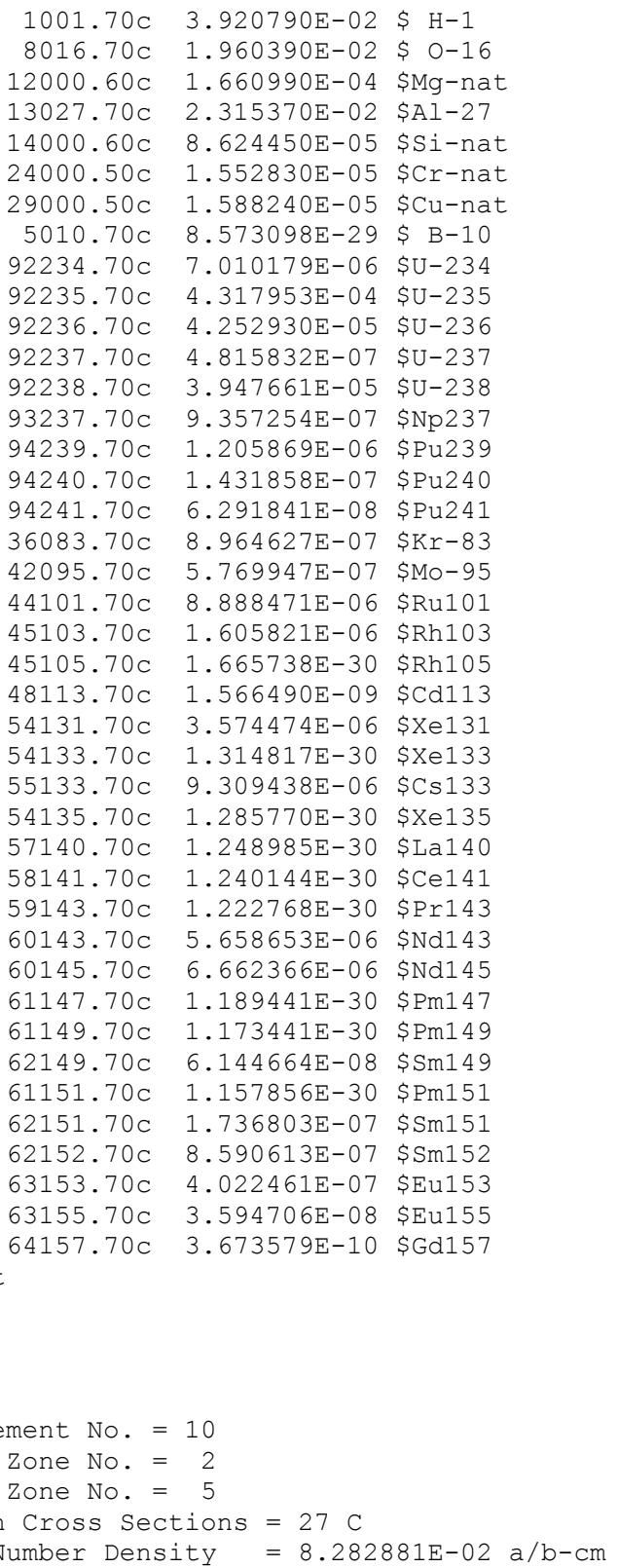

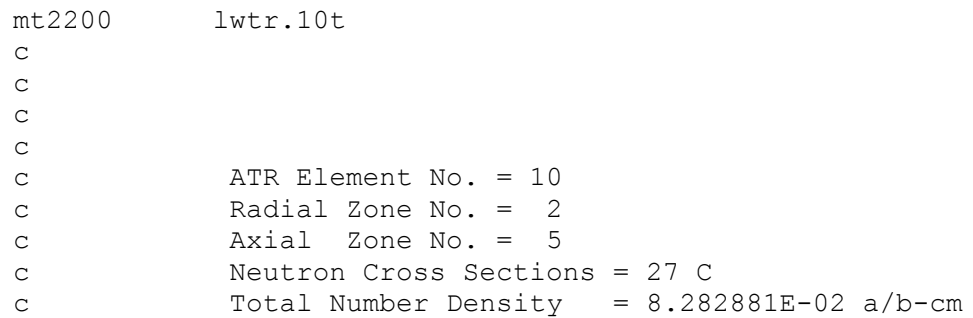




\begin{tabular}{|c|c|c|}
\hline $1001.70 \mathrm{C}$ & $3.920790 \mathrm{E}-02$ & $\$ H-1$ \\
\hline $8016.70 \mathrm{c}$ & $1.960390 \mathrm{E}-02$ & $\$ 0-16$ \\
\hline $12000.60 \mathrm{c}$ & $1.660990 \mathrm{E}-04$ & \$Mg-nat \\
\hline $13027.70 \mathrm{C}$ & $2.315370 \mathrm{E}-02$ & $\$ A l-27$ \\
\hline $4000.60 \mathrm{c}$ & $8.624450 \mathrm{E}-05$ & \$Si-nat \\
\hline $24000.50 \mathrm{C}$ & $1.552830 \mathrm{E}-05$ & $\$ \mathrm{Cr}-\mathrm{nat}$ \\
\hline $29000.50 \mathrm{C}$ & $1.588240 \mathrm{E}-05$ & $\$ \mathrm{Cu}-\mathrm{nat}$ \\
\hline $5010.70 \mathrm{C}$ & $1.714619 \mathrm{E}-28$ & $\$ B-10$ \\
\hline $2234.70 \mathrm{c}$ & $7.179019 \mathrm{E}-06$ & $\$ \mathrm{U}-234$ \\
\hline $92235.70 \mathrm{C}$ & $4.584759 \mathrm{E}-04$ & $\$ U-235$ \\
\hline $92236.70 \mathrm{C}$ & $3.781602 \mathrm{E}-05$ & $\$ U-236$ \\
\hline $92237.70 \mathrm{C}$ & $4.213655 \mathrm{E}-07$ & $\$ U-237$ \\
\hline $92238.70 \mathrm{C}$ & $3.972198 \mathrm{E}-05$ & $\$ U-238$ \\
\hline $93237.70 \mathrm{C}$ & $8.187213 \mathrm{E}-07$ & $\$ N p 237$ \\
\hline $94239.70 \mathrm{C}$ & $1.055086 \mathrm{E}-06$ & \$Pu239 \\
\hline $94240.70 \mathrm{C}$ & $1.252817 \mathrm{E}-07$ & \$Pu240 \\
\hline $94241.70 \mathrm{C}$ & $5.505101 \mathrm{E}-08$ & \$Pu 241 \\
\hline $36083.70 \mathrm{c}$ & $7.843680 \mathrm{E}-07$ & $\$ K r-83$ \\
\hline $42095.70 \mathrm{C}$ & $5.048466 \mathrm{E}-07$ & $\$ M o-95$ \\
\hline $44101.70 \mathrm{C}$ & $7.777046 \mathrm{E}-06$ & \$Ru101 \\
\hline $45103.70 \mathrm{C}$ & $1.405028 \mathrm{E}-06$ & \$Rh103 \\
\hline $45105.70 \mathrm{C}$ & $1.457452 \mathrm{E}-30$ & \$Rh105 \\
\hline $48113.70 \mathrm{C}$ & $1.370614 \mathrm{E}-09$ & $\$ \mathrm{Cd} 113$ \\
\hline $54131.70 \mathrm{C}$ & $3.127518 \mathrm{E}-06$ & $\$ \mathrm{Xe131}$ \\
\hline $54133.70 \mathrm{C}$ & $1.150411 \mathrm{E}-30$ & $\$ \mathrm{Xe133}$ \\
\hline $55133.70 \mathrm{c}$ & $8.145375 \mathrm{E}-06$ & $\$ \mathrm{Cs} 133$ \\
\hline $54135.70 \mathrm{C}$ & $1.124996 \mathrm{E}-30$ & $\$$ Xe135 \\
\hline $57140.70 \mathrm{C}$ & $1.092811 \mathrm{E}-30$ & \$La140 \\
\hline $58141.70 \mathrm{C}$ & $1.085075 \mathrm{E}-30$ & $\$ C e 141$ \\
\hline $59143.70 \mathrm{C}$ & $1.069872 \mathrm{E}-30$ & $\$ \operatorname{Pr} 143$ \\
\hline $60143.70 c$ & $4.951089 \mathrm{E}-06$ & $\$ N d 143$ \\
\hline $60145.70 \mathrm{c}$ & $5.829296 \mathrm{E}-06$ & $\$ N d 145$ \\
\hline $61147.70 \mathrm{c}$ & $1.040712 \mathrm{E}-30$ & $\$ \operatorname{Pm} 147$ \\
\hline $61149.70 \mathrm{c}$ & $1.026713 \mathrm{E}-30$ & $\$ \operatorname{Pm} 149$ \\
\hline $62149.70 \mathrm{c}$ & $5.376328 \mathrm{E}-08$ & $\$ \operatorname{Sm} 149$ \\
\hline $61151.70 c$ & $1.013077 \mathrm{E}-30$ & \$Pm151 \\
\hline $62151.70 \mathrm{C}$ & $1.519631 \mathrm{E}-07$ & \$Sm151 \\
\hline $62152.70 \mathrm{C}$ & $7.516433 \mathrm{E}-07$ & $\$$ Sm152 \\
\hline $63153.70 \mathrm{c}$ & $3.519488 \mathrm{E}-07$ & \$Eu153 \\
\hline 63155.70 & $3.145220 \mathrm{E}-08$ & \$Eu155 \\
\hline 64157.7 & $3.214231 \mathrm{E}-10$ & \$Gd157 \\
\hline
\end{tabular}

mt2201
$C$
$C$
$C$
$C$
$C$
$C$
$C$
$C$
$C$
m2202




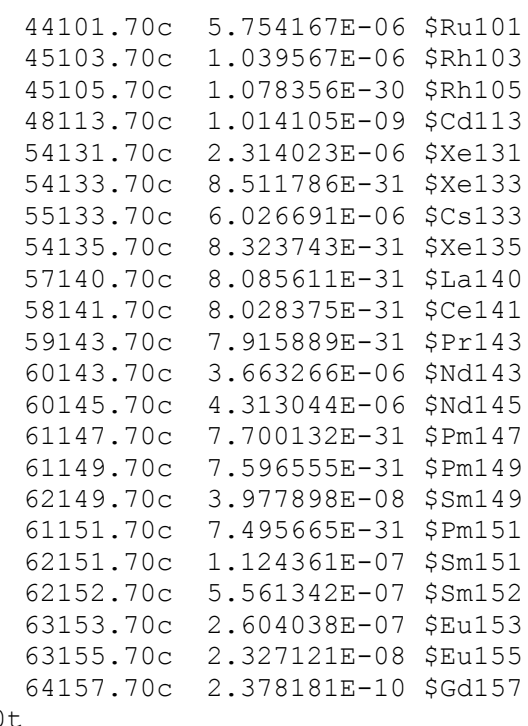

mt2202
C
C
C
C
C
C
C
C
C
m2203 lwtr.10t

$44101.70 \mathrm{C} \quad 5.754167 \mathrm{E}-06$ \$Ru101

$45103.70 \mathrm{C} \quad 1.039567 \mathrm{E}-06$ \$Rh103

$54131.70 \mathrm{C} \quad 2.314023 \mathrm{E}-06$ \$Xe131

$54133.70 \mathrm{C} \quad 8.511786 \mathrm{E}-31$ \$Xe133

$55133.70 \mathrm{C} \quad 6.026691 \mathrm{E}-06 \$ \mathrm{Cs} 133$

54135.70 $8.323743 \mathrm{E}-31$ \$Xe135

$57140.70 \mathrm{C} \quad 8.085611 \mathrm{E}-31$ \$La140

$8.028375 \mathrm{E}-31$ \$Ce141

$7.915889 \mathrm{E}-31 \$ \operatorname{Pr} 143$

$61147.70 \mathrm{C} \quad 7.700132 \mathrm{E}-31 \$ \mathrm{Pm} 147$

$61149.70 \mathrm{C} \quad 7.596555 \mathrm{E}-31$ \$Pm149

$62149.70 \mathrm{C} \quad 3.977898 \mathrm{E}-08$ \$Sm149

$61151.70 \mathrm{C} \quad 7.495665 \mathrm{E}-31$ \$Pm151

$62151.70 \mathrm{C} \quad 1.124361 \mathrm{E}-07$ \$Sm151

$5.561342 \mathrm{E}-07 \quad \$ \operatorname{Sm} 152$

$63155.70 \mathrm{C} 2.327121 \mathrm{E}-08$ \$Eu155

$64157.70 \mathrm{C} \quad 2.378181 \mathrm{E}-10$ \$Gd157

ATR Element No. = 10

Radial Zone No. $=2$

Axial Zone No. $=7$

Neutron Cross Sections $=27 \mathrm{C}$

Total Number Density $=8.290178 \mathrm{E}-02 \mathrm{a} / \mathrm{b}-\mathrm{cm}$

1001.70C 3.920790E-02 \$ H-1

$8016.70 \mathrm{C} \quad 1.960390 \mathrm{E}-02$ \$ $0-16$

$12000.60 \mathrm{C} \quad 1.660990 \mathrm{E}-04$ \$Mg-nat

$13027.70 \mathrm{C} \quad 2.315370 \mathrm{E}-02$ \$Al-27

$14000.60 \mathrm{C} \quad 8.624450 \mathrm{E}-05$ \$Si-nat

$24000.50 \mathrm{C} \quad 1.552830 \mathrm{E}-05$ \$Cr-nat

$29000.50 \mathrm{C} \quad 1.588240 \mathrm{E}-05$ \$Cu-nat

$5010.70 \mathrm{C} \quad 1.714619 \mathrm{E}-28$ \$ B 10

$92234.70 \mathrm{C} \quad 7.895820 \mathrm{E}-06$ \$U-234

$92235.70 \mathrm{C} \quad 5.717485 \mathrm{E}-04 \quad \$ \mathrm{U}-235$

$92236.70 \mathrm{C} \quad 1.780560 \mathrm{E}-05$ \$U-236

$92237.70 \mathrm{C} \quad 1.657085 \mathrm{E}-07$ \$U-237

$92238.70 \mathrm{C} \quad 4.076363 \mathrm{E}-05$ \$U-238

$93237.70 \mathrm{C} \quad 3.219748 \mathrm{E}-07 \quad \$ \mathrm{~Np} 237$

$94239.70 \mathrm{C} \quad 4.149287 \mathrm{E}-07$ \$Pu239

$94240.70 \mathrm{C} \quad 4.926895 \mathrm{E}-08$ \$Pu240

$94241.70 \mathrm{C} \quad 2.164966 \mathrm{E}-08$ \$Pu241

$36083.70 \mathrm{C} \quad 3.084648 \mathrm{E}-07$ \$Kr-83

$42095.70 \mathrm{C} \quad 1.985387 \mathrm{E}-07$ \$Mo-95

$44101.70 \mathrm{C} \quad 3.058443 \mathrm{E}-06$ \$Ru101

$45103.70 \mathrm{C} \quad 5.525487 \mathrm{E}-07$ \$Rh103

$45105.70 \mathrm{C} \quad 5.731656 \mathrm{E}-31$ \$Rh105

$48113.70 \mathrm{C} \quad 5.390151 \mathrm{E}-10 \quad \$ \mathrm{Cd} 113$

$54131.70 \mathrm{C} \quad 1.229945 \mathrm{E}-06$ \$Xe131

$54133.70 \mathrm{C} \quad 4.524167 \mathrm{E}-31$ \$Xe133

$55133.70 \mathrm{C} \quad 3.203295 \mathrm{E}-06$ \$Cs133

$54135.70 \mathrm{C} \quad 4.424219 \mathrm{E}-31$ \$Xe135

$57140.70 \mathrm{C} \quad 4.297647 \mathrm{E}-31$ \$La140

$58141.70 \mathrm{C} \quad 4.267226 \mathrm{E}-31$ \$Ce141

$59143.70 \mathrm{C} \quad 4.207437 \mathrm{E}-31$ \$Pr143

$60143.70 \mathrm{C} \quad 1.947092 \mathrm{E}-06 \$ \mathrm{Nd} 143$

$60145.70 \mathrm{C} \quad 2.292461 \mathrm{E}-06 \$ \mathrm{Nd1} 45$

$61147.70 \mathrm{C} \quad 4.092758 \mathrm{E}-31 \$ \mathrm{Pm} 147$

$61149.70 \mathrm{C} \quad 4.037706 \mathrm{E}-31 \$ \mathrm{Pm} 149$

$62149.70 \mathrm{C} \quad 2.114324 \mathrm{E}-08 \quad \$ \mathrm{Sm} 149$

$61151.70 \mathrm{C} \quad 3.984080 \mathrm{E}-31$ \$Pm151

$62151.70 \mathrm{C} \quad 5.976183 \mathrm{E}-08$ \$Sm151

$62152.70 \mathrm{C} \quad 2.955953 \mathrm{E}-07$ \$Sm152 
63153.70C 1.384093E-07 \$Eu153

$63155.70 \mathrm{C} \quad 1.236906 \mathrm{E}-08$ \$Eu155

mt2203 lwtr.10t

C

C

C

C

C

C

$\mathrm{C}$

ATR Element No. $=10$

Radial Zone No. $=3$

Axial Zone No. = 1

Neutron Cross Sections $=27 \mathrm{C}$

Total Number Density $=7.851893 \mathrm{E}-02 \mathrm{a} / \mathrm{b}-\mathrm{cm}$

\begin{tabular}{|c|c|c|}
\hline & & \\
\hline & $1.606420 \mathrm{E}-02$ & $0-16$ \\
\hline & $2.399430 \mathrm{E}-04$ & $\$ M g-n a$ \\
\hline & $2.958200 \mathrm{E}-02$ & $\$ A I-27$ \\
\hline & $870 E-04$ & $\$ \mathrm{Si}-\mathrm{n}$ \\
\hline & & \\
\hline & $30 E-05$ & $\$ \mathrm{Cu}-\mathrm{n}$ \\
\hline & $68 E-06$ & $\$ B-10$ \\
\hline 34 . & $4.098777 \mathrm{E}-06$ & $\$ U-234$ \\
\hline & & \\
\hline & $722 \mathrm{E}-05$ & $\$ \mathrm{U}-$ \\
\hline & 1.9 & $3 U-a_{1}$ \\
\hline 8. & $2.221118 \mathrm{E}-05$ & $\$ U-2$ \\
\hline & $8 E-07$ & $\$ N p$ \\
\hline & & $\$ \mathrm{Pu}$ \\
\hline & 5. & $\$ \mathrm{Pu}$ \\
\hline & $98 E-08$ & \$Pu2 \\
\hline & $2065 \mathrm{E}-07$ & $\$ \mathrm{Kr}-$ \\
\hline & 2.3 & \$Mo \\
\hline & & \\
\hline & 07 & $\$ R h$ \\
\hline & 6.6 & $\$ R h$ \\
\hline & $817 \mathrm{E}-10$ & $\$ C d 11$ \\
\hline & $1.432268 \mathrm{E}-06$ & $\$ \mathrm{Xe} 1$ \\
\hline & 5.2 & $\$ \mathrm{Xe}$ \\
\hline & & \\
\hline & & $\$ \mathrm{Xe}$ \\
\hline & $00 \mathrm{E}-31$ & \$La1 \\
\hline & $4.969174 \mathrm{E}-31$ & $\$ \mathrm{Ce} 1$ \\
\hline & 4.8 & $\$ \operatorname{Pr} 1$ \\
\hline & 2.2 & $\$ \mathrm{Nd1} 43$ \\
\hline & 2.6 & $\$ \mathrm{Nd}]$ \\
\hline & $08 E-31$ & $\$ \mathrm{Pm} 1$ \\
\hline & $4.701899 \mathrm{E}-31$ & $\$ P m 1$ \\
\hline , & $125 \mathrm{E}-08$ & $\$ \mathrm{Sm} 1$ \\
\hline & $4.639452 \mathrm{E}-31$ & $\$ \operatorname{Pm} 151$ \\
\hline & $251 E-08$ & $\$ \mathrm{Sm} 1$ \\
\hline & $3.442201 \mathrm{E}-07$ & $\$$ Sm1 \\
\hline & $1.611773 \mathrm{E}-07$ & \$Eu 1 \\
\hline & $1.440375 \mathrm{E}$ & Eul \\
\hline & & \\
\hline
\end{tabular}

mt2204 lwtr.10t

$\mathrm{C}$

C

C

C

c

C

C

C

m2205

ATR Element No. $=10$

Radial Zone No. $=3$

Axial Zone No. $=2$

Neutron Cross Sections $=27 \mathrm{C}$

Total Number Density $=7.848707 \mathrm{E}-02 \mathrm{a} / \mathrm{b}-\mathrm{cm}$

$1001.70 \mathrm{C} \quad 3.212840 \mathrm{E}-02 \$ \mathrm{H}-1$

$8016.70 \mathrm{C} \quad 1.606420 \mathrm{E}-02 \$ 0-16$

$12000.60 \mathrm{C} \quad 2.399430 \mathrm{E}-04$ \$Mg-nat

$13027.70 \mathrm{C} \quad 2.958200 \mathrm{E}-02$ \$Al-27

$14000.60 \mathrm{C} \quad 1.245870 \mathrm{E}-04$ \$Si-nat 


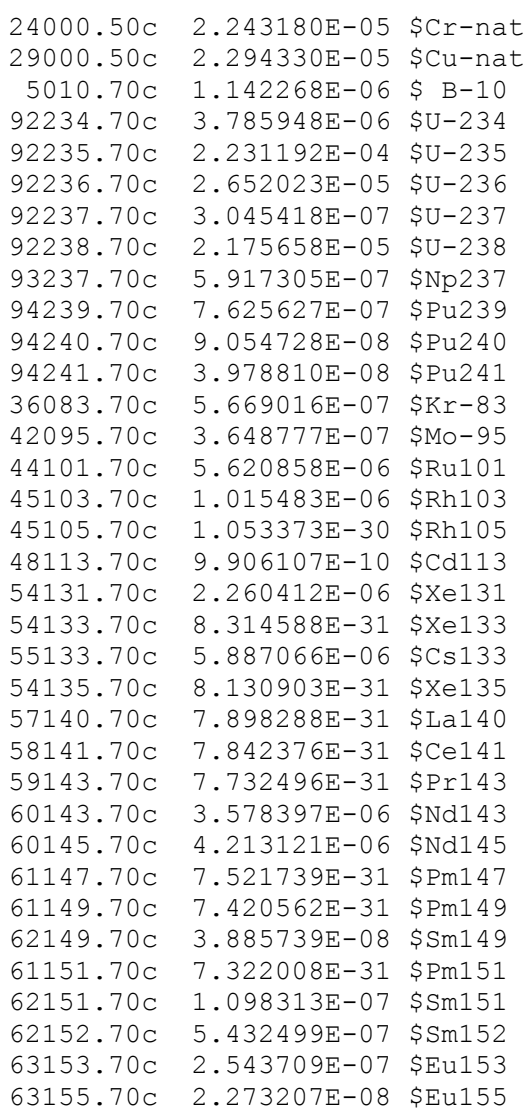

lwtr.10t $64157.70 \mathrm{c} \quad 2.323084 \mathrm{E}-10 \quad \$ \mathrm{Gd15} 7$

c

C

C

C

C

C

C

m2206

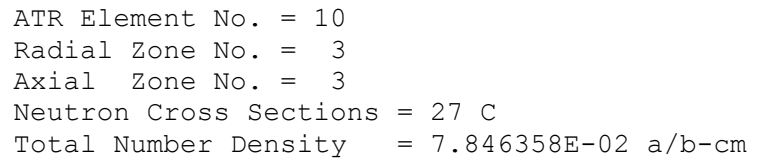

$\begin{array}{rll}1001.70 \mathrm{C} & 3.212840 \mathrm{E}-02 & \text { \$ H-1 } \\ 8016.70 \mathrm{C} & 1.606420 \mathrm{E}-02 & \text { \$ O-16 } \\ 12000.60 \mathrm{C} & 2.399430 \mathrm{E}-04 & \text { \$Mg-nat } \\ 13027.70 \mathrm{C} & 2.958200 \mathrm{E}-02 & \text { \$Al-27 } \\ 14000.60 \mathrm{C} & 1.245870 \mathrm{E}-04 & \text { \$Si-nat } \\ 24000.50 \mathrm{C} & 2.243180 \mathrm{E}-05 & \text { \$Cr-nat } \\ 29000.50 \mathrm{C} & 2.294330 \mathrm{E}-05 & \text { \$Cu-nat } \\ 5010.70 \mathrm{C} & 1.142268 \mathrm{E}-06 & \text { \$ B-10 } \\ 92234.70 \mathrm{C} & 3.555024 \mathrm{E}-06 & \text { \$U-234 } \\ 92235.70 \mathrm{C} & 1.866272 \mathrm{E}-04 & \text { \$U-235 } \\ 92236.70 \mathrm{C} & 3.296680 \mathrm{E}-05 & \text { \$U-236 } \\ 92237.70 \mathrm{C} & 3.869043 \mathrm{E}-07 & \text { \$U-237 } \\ 92238.70 \mathrm{C} & 2.142100 \mathrm{E}-05 & \text { \$U-238 } \\ 93237.70 \mathrm{C} & 7.517625 \mathrm{E}-07 & \text { \$Np237 } \\ 94239.70 \mathrm{C} & 9.687959 \mathrm{E}-07 & \text { \$Pu239 } \\ 94240.70 \mathrm{C} & 1.150356 \mathrm{E}-07 & \text { \$Pu240 } \\ 94241.70 \mathrm{C} & 5.054870 \mathrm{E}-08 & \text { \$Pu241 } \\ 36083.70 \mathrm{C} & 7.202187 \mathrm{E}-07 & \text { \$Kr-83 } \\ 42095.70 \mathrm{C} & 4.635580 \mathrm{E}-07 & \text { \$Mo-95 } \\ 44101.70 \mathrm{C} & 7.141005 \mathrm{E}-06 & \text { \$Ru101 } \\ 45103.70 \mathrm{C} & 1.290118 \mathrm{E}-06 & \text { \$Rh103 } \\ 45105.70 \mathrm{C} & 1.338255 \mathrm{E}-30 & \text { \$Rh105 } \\ 48113.70 \mathrm{C} & 1.258519 \mathrm{E}-09 & \text { \$Cd113 } \\ 54131.70 \mathrm{C} & 2.871735 \mathrm{E}-06 & \text { \$Xe131 }\end{array}$




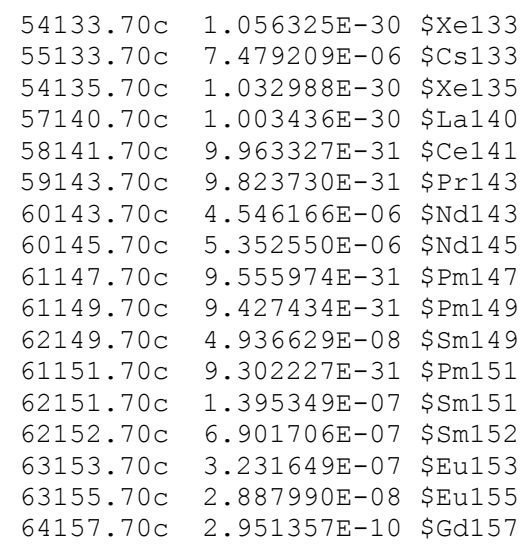

mt2206 lwtr.10t

c

$\mathrm{C}$

C

C

C ATR Element No. = 10

C Radial Zone No. = 3

C Axial Zone No. = 4

C Neutron Cross Sections $=27 \mathrm{C}$

C Total Number Density $=7.845429 \mathrm{E}-02 \mathrm{a} / \mathrm{b}-\mathrm{cm}$

m2207

mt2207 - lwtr.10t

\begin{abstract}
$1001.70 \mathrm{C} \quad 3.212840 \mathrm{E}-02$ \$ $\mathrm{H}-1$
$8016.70 \mathrm{C} \quad 1.606420 \mathrm{E}-02 \$ 0-16$

$12000.60 \mathrm{C} \quad 2.399430 \mathrm{E}-04$ \$Mg-nat

$13027.70 \mathrm{C} \quad 2.958200 \mathrm{E}-02$ \$Al-27

$14000.60 \mathrm{C} \quad 1.245870 \mathrm{E}-04$ \$Si-nat

$24000.50 \mathrm{C} \quad 2.243180 \mathrm{E}-05$ \$Cr-nat

$29000.50 \mathrm{C} \quad 2.294330 \mathrm{E}-05$ \$Cu-nat

$5010.70 \mathrm{C} \quad 1.142268 \mathrm{E}-06$ \$ B -10

$92234.70 \mathrm{C} \quad 3.463750 \mathrm{E}-06 \quad \$ \mathrm{U}-234$

$92235.70 \mathrm{C} \quad 1.722036 \mathrm{E}-04 \quad \$ \mathrm{U}-235$

$92236.70 \mathrm{C} \quad 3.551487 \mathrm{E}-05 \quad \$ \mathrm{U}-236$

$92237.70 \mathrm{C} \quad 4.194588 \mathrm{E}-07$ \$U-237

$92238.70 \mathrm{C} \quad 2.128837 \mathrm{E}-05 \quad \$ \mathrm{U}-238$

$93237.70 \mathrm{C} \quad 8.150166 \mathrm{E}-07$ \$Np237

$94239.70 \mathrm{C} \quad 1.050311 \mathrm{E}-06$ \$Pu239

$94240.70 \mathrm{C} \quad 1.247148 \mathrm{E}-07$ \$Pu240

$94241.70 \mathrm{C} \quad 5.480192 \mathrm{E}-08$ \$Pu241

$36083.70 \mathrm{C} \quad 7.808187 \mathrm{E}-07 \quad \$ \mathrm{Kr}-83$

$42095.70 \mathrm{C} \quad 5.025622 \mathrm{E}-07$ \$Mo-95

$44101.70 \mathrm{C} \quad 7.741855 \mathrm{E}-06$ \$Ru101

$45103.70 \mathrm{C} \quad 1.398670 \mathrm{E}-06$ \$Rh103

$45105.70 \mathrm{C} \quad 1.450857 \mathrm{E}-30$ \$Rh105

$48113.70 \mathrm{C} \quad 1.364412 \mathrm{E}-09 \$ \mathrm{Cd} 113$

$54131.70 \mathrm{C} \quad 3.113366 \mathrm{E}-06$ \$Xe131

$54133.70 \mathrm{C} \quad 1.145205 \mathrm{E}-30$ \$e133

$55133.70 \mathrm{C} \quad 8.108518 \mathrm{E}-06 \$ \mathrm{Cs} 133$

$54135.70 \mathrm{C} \quad 1.119905 \mathrm{E}-30$ \$Xe135

$57140.70 \mathrm{C} \quad 1.087866 \mathrm{E}-30$ \$La140

$58141.70 \mathrm{C} \quad 1.080165 \mathrm{E}-30$ \$Ce141

$59143.70 \mathrm{C} \quad 1.065031 \mathrm{E}-30$ \$Pr143

$60143.70 \mathrm{C} \quad 4.928685 \mathrm{E}-06 \$ \mathrm{Nd1} 43$

$60145.70 \mathrm{C} \quad 5.802919 \mathrm{E}-06 \$ \mathrm{Nd} 145$

$61147.70 \mathrm{C} \quad 1.036002 \mathrm{E}-30$ \$Pm147

$61149.70 \mathrm{C} \quad 1.022067 \mathrm{E}-30 \$ \mathrm{Pm} 149$

$62149.70 \mathrm{C} \quad 5.352000 \mathrm{E}-08$ \$Sm149

$61151.70 \mathrm{C} \quad 1.008493 \mathrm{E}-30$ \$Pm151

$62151.70 \mathrm{C} \quad 1.512755 \mathrm{E}-07$ \$Sm151

$62152.70 \mathrm{C} \quad 7.482421 \mathrm{E}-07$ \$Sm152

$63153.70 \mathrm{C} \quad 3.503563 \mathrm{E}-07$ \$Eu153

$63155.70 \mathrm{C} \quad 3.130988 \mathrm{E}-08$ \$Eu155

$64157.70 \mathrm{C} \quad 3.199687 \mathrm{E}-10$ \$Gd157
\end{abstract}

c 
C ATR Element No. = 10

lwtr.10t

ATR Element No. = 10

Radial Zone No. $=3$

Axial Zone No. = 6
Radial Zone No. $=3$

Axial Zone No. = 5

Neutron Cross Sections $=27 \mathrm{C}$

Total Number Density $=7.846721 \mathrm{E}-02 \mathrm{a} / \mathrm{b}-\mathrm{cm}$

\begin{tabular}{|c|c|c|}
\hline 1001 & $3.212840 \mathrm{E}-02$ & $\$ \mathrm{H}-1$ \\
\hline $8016.70 \mathrm{c}$ & $1.606420 \mathrm{E}-02$ & $\$ 0-16$ \\
\hline $000.60 \mathrm{c}$ & $2.399430 \mathrm{E}-04$ & $\$ M g-n a t$ \\
\hline $7.70 \mathrm{c}$ & $2.958200 \mathrm{E}-02$ & $\$ A I-27$ \\
\hline $0.60 \mathrm{c}$ & $1.245870 \mathrm{E}-04$ & \$Si-nat \\
\hline & $2.243180 \mathrm{E}-05$ & $\$ C r-n a t$ \\
\hline $.50 \mathrm{c}$ & $2.294330 \mathrm{E}-05$ & $\$ \mathrm{Cu}-\mathrm{nat}$ \\
\hline & $1.142268 \mathrm{E}-06$ & $\$ B-10$ \\
\hline $.70 \mathrm{C}$ & $3.590653 E-06$ & $\$ U-234$ \\
\hline- & $1.922575 \mathrm{E}-04$ & $\$ U-235$ \\
\hline $.70 \mathrm{C}$ & $3.197217 \mathrm{E}-05$ & $\$ U-236$ \\
\hline $.70 \mathrm{c}$ & $3.741968 \mathrm{E}-07$ & $\$ U-237$ \\
\hline $.70 \mathrm{C}$ & $2.147278 \mathrm{E}-05$ & $\$ U-238$ \\
\hline $.70 \mathrm{C}$ & $7.270715 \mathrm{E}-07$ & $\$ N p 237$ \\
\hline $.70 \mathrm{C}$ & $9.369766 \mathrm{E}-07$ & $\$ P u 239$ \\
\hline $.70 \mathrm{c}$ & $1.112573 \mathrm{E}-07$ & $\$ \mathrm{Pu} 240$ \\
\hline $.70 \mathrm{C}$ & $4.888847 \mathrm{E}-08$ & $\$ \mathrm{Pu} 241$ \\
\hline $.70 \mathrm{c}$ & $6.965638 \mathrm{E}-07$ & $\$ K r-83$ \\
\hline $.70 \mathrm{C}$ & $4.483328 \mathrm{E}-07$ & $\$ M o-95$ \\
\hline & 6 & \$Ru101 \\
\hline $\mathrm{OC}_{\mathrm{C}}$ & $5 E-06$ & \$Rh103 \\
\hline & 1 . & $\$ R h 105$ \\
\hline $0 \mathrm{c}$ & $E-09$ & $\$ \mathrm{Cd} 113$ \\
\hline & & $\$ x e 131$ \\
\hline $.70 \mathrm{C}$ & $L E-30$ & $\$ x e 133$ \\
\hline .7 & 7.2335 & $\$ \mathrm{Cs} 133$ \\
\hline $.70 \mathrm{C}$ & $3 E-31$ & $\$ x e 135$ \\
\hline & $9.704788 \mathrm{E}-31$ & $\$ \mathrm{La} 140$ \\
\hline C & $E-31$ & $\$ \mathrm{Ce} 141$ \\
\hline- & 9.5010 & $\$ \operatorname{Pr} 143$ \\
\hline & $E-06$ & $\$ N d 143$ \\
\hline $.70 \mathrm{C}$ & 5.176 & $\$ N d 145$ \\
\hline .7 & $7 E-31$ & $\$ P m 147$ \\
\hline .7 & $7 E-31$ & $\$ P m 149$ \\
\hline .7 & $9 E-08$ & $\$ S m 149$ \\
\hline & 8.9967 & $\$ P m 151$ \\
\hline $.70 \mathrm{C}$ & $1.349520 \mathrm{E}-07$ & $\$ S m 151$ \\
\hline & $6.675024 \mathrm{E}-07$ & $\$ S m 152$ \\
\hline & $3.125508 \mathrm{E}-07$ & $\$ E u 153$ \\
\hline & $3136 E-08$ & \$Eu155 \\
\hline & $2.854422 \mathrm{E}-1$ & \$Gd157 \\
\hline
\end{tabular}

$64157.70 \mathrm{C} \quad 2.854422 \mathrm{E}-10 \quad \$ \mathrm{Gd} 157$

Neutron Cross Sections $=27 \mathrm{C}$

Total Number Density $=7.849300 \mathrm{E}-02 \mathrm{a} / \mathrm{b}-\mathrm{cm}$

$1001.70 \mathrm{C} \quad 3.212840 \mathrm{E}-02 \$ \mathrm{H}-1$

$8016.70 \mathrm{C} \quad 1.606420 \mathrm{E}-02$ \$ O-16

$12000.60 \mathrm{C} \quad 2.399430 \mathrm{E}-04 \mathrm{\$ Mg}-\mathrm{nat}$

$13027.70 \mathrm{C} \quad 2.958200 \mathrm{E}-02$ \$Al-27

$14000.60 \mathrm{C} \quad 1.245870 \mathrm{E}-04 \mathrm{SSi-nat}$

$24000.50 \mathrm{C} \quad 2.243180 \mathrm{E}-05$ \$Cr-nat

$29000.50 \mathrm{C} \quad 2.294330 \mathrm{E}-05$ \$Cu-nat

$5010.70 \mathrm{C} \quad 1.142268 \mathrm{E}-06$ \$ B -10

$92234.70 \mathrm{C} \quad 3.843982 \mathrm{E}-06$ \$U-234

$92235.70 \mathrm{C} \quad 2.322898 \mathrm{E}-04$ \$U-235 


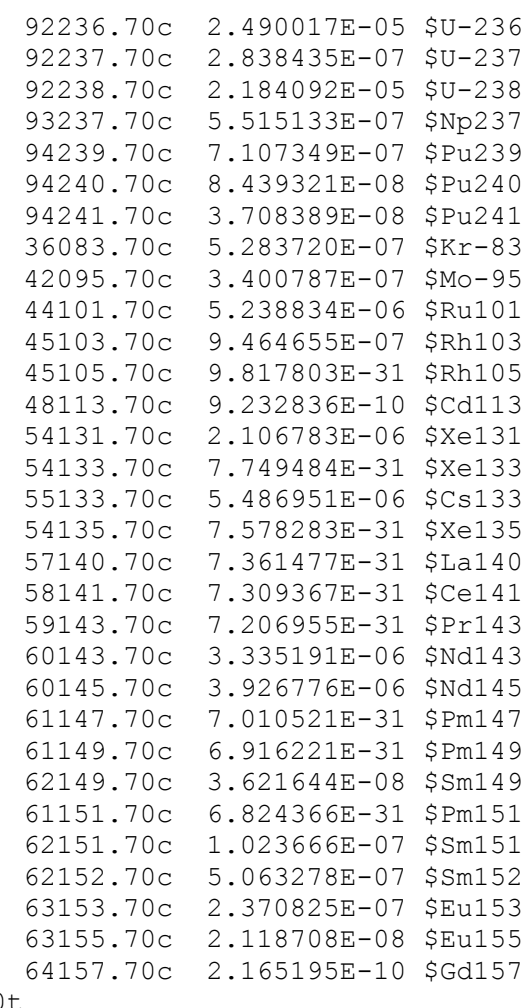

$\begin{array}{ll}\text { mt2209 lwtr.10t } \\ \mathrm{C} \\ \mathrm{C} \\ \mathrm{C} \\ \mathrm{C} & \text { ATR Element No. }=10 \\ \mathrm{C} & \text { Radial Zone No. }=3 \\ \mathrm{C} & \text { Axial Zone No. }=7 \\ \mathrm{C} & \text { Neutron Cross Sections }=27 \mathrm{C} \\ \mathrm{C} & \text { Total Number Density }=7.852753 \mathrm{E}-02 \mathrm{a} / \mathrm{b}-\mathrm{cm} \\ \mathrm{C} & \end{array}$ 
$59143.70 \mathrm{C} \quad 4.136078 \mathrm{E}-31 \quad \$ \operatorname{Pr} 143$

$60143.70 \mathrm{C} \quad 1.914068 \mathrm{E}-06$ \$Nd143

$60145.70 \mathrm{C} \quad 2.253580 \mathrm{E}-06$ \$Nd145

$61147.70 \mathrm{C} \quad 4.023344 \mathrm{E}-31$ \$Pm147

$61149.70 \mathrm{C} \quad 3.969225 \mathrm{E}-31$ \$Pm149

$62149.70 \mathrm{C} \quad 2.078465 \mathrm{E}-08 \quad \$ \mathrm{Sm} 149$

$61151.70 \mathrm{C} \quad 3.916509 \mathrm{E}-31$ \$Pm151

$62151.70 \mathrm{C} \quad 5.874825 \mathrm{E}-08 \quad \$ \mathrm{Sm} 151$

$62152.70 \mathrm{C} \quad 2.905819 \mathrm{E}-07$ \$Sm152

$63153.70 \mathrm{C} \quad 1.360619 \mathrm{E}-07$ \$Eu153

$63155.70 \mathrm{C} \quad 1.215928 \mathrm{E}-08$ \$Eu155

mt2210 lwtr.10t

$64157.70 \mathrm{C} \quad 1.242607 \mathrm{E}-10$ \$Gd157

C

C

C

C

$\mathrm{C}$

m2211

mt2211

C

C

C

C

c
ATR Element No. $=11$

Radial Zone No. = 1

Axial Zone No. = 1

Neutron Cross Sections $=27 \mathrm{C}$

Total Number Density $=7.969562 \mathrm{E}-02 \mathrm{a} / \mathrm{b}-\mathrm{cm}$

$1001.70 \mathrm{C} \quad 3.393340 \mathrm{E}-02$ \$ $\mathrm{H}-1$

$8016.70 \mathrm{C} \quad 1.696670 \mathrm{E}-02$ \$ O-16

$12000.60 \mathrm{C} \quad 2.176490 \mathrm{E}-04$ \$Mg-nat

$13027.70 \mathrm{C} \quad 2.793720 \mathrm{E}-02$ \$Al-27

$14000.60 \mathrm{C} \quad 1.130110 \mathrm{E}-04$ \$Si-nat

$24000.50 \mathrm{C} \quad 2.304760 \mathrm{E}-05$ \$Cr-nat

$29000.50 \mathrm{C} \quad 2.081160 \mathrm{E}-05$ \$Cu-nat

$5010.70 \mathrm{C} \quad 3.670657 \mathrm{E}-06$ \$ B-10

$92234.70 \mathrm{C} \quad 5.845600 \mathrm{E}-06$ \$U-234

$92235.70 \mathrm{C} \quad 4.151488 \mathrm{E}-04 \quad$ \$U-235

$92236.70 \mathrm{C} \quad 1.605094 \mathrm{E}-05$ \$U-236

$92237.70 \mathrm{C} \quad 1.453746 \mathrm{E}-07 \quad \$ \mathrm{U}-237$

$92238.70 \mathrm{C} \quad 3.054891 \mathrm{E}-05 \quad \mathrm{SU}-238$

$93237.70 \mathrm{C} \quad 2.204448 \mathrm{E}-07$ \$Np237

$94239.70 \mathrm{C} \quad 4.246852 \mathrm{E}-07$ \$Pu239

$94240.70 \mathrm{C} \quad 3.643572 \mathrm{E}-08$ \$Pu240

$94241.70 \mathrm{C} \quad 1.275155 \mathrm{E}-08$ \$Pu241

$36083.70 \mathrm{C} \quad 2.957309 \mathrm{E}-07$ \$Kr-83

$42095.70 \mathrm{C} \quad 1.155466 \mathrm{E}-07$ \$Mo-95

$44101.70 \mathrm{C} \quad 2.927350 \mathrm{E}-06$ \$Ru101

$45103.70 \mathrm{C} \quad 4.143219 \mathrm{E}-07$ \$Rh103

$45105.70 \mathrm{C} \quad 7.425954 \mathrm{E}-31$ \$Rh105

$48113.70 \mathrm{C} \quad 7.515376 \mathrm{E}-10 \quad \$ \mathrm{Cd} 113$

$54131.70 \mathrm{C} \quad 1.025512 \mathrm{E}-06 \quad \$ \mathrm{Xe1} 31$

$54133.70 \mathrm{C} \quad 5.861527 \mathrm{E}-31$ \$Xe133

$55133.70 \mathrm{C} \quad 2.706961 \mathrm{E}-06 \quad \$ \mathrm{Cs} 133$

$54135.70 \mathrm{C} \quad 5.732034 \mathrm{E}-31$ \$Xe135

$57140.70 \mathrm{C} \quad 5.568047 \mathrm{E}-31$ \$La140

$58141.70 \mathrm{C} \quad 5.528633 \mathrm{E}-31$ \$Ce141

$59143.70 \mathrm{C} \quad 5.451170 \mathrm{E}-31$ \$Pr143

$60143.70 \mathrm{C} \quad 1.500125 \mathrm{E}-06 \$ \mathrm{Nd1} 43$

$60145.70 \mathrm{C} \quad 2.192857 \mathrm{E}-06$ \$Nd145

$61147.70 \mathrm{C} \quad 5.302593 \mathrm{E}-31$ \$Pm147

$61149.70 \mathrm{C} \quad 5.231266 \mathrm{E}-31$ \$Pm149

$62149.70 \mathrm{C} \quad 2.671007 \mathrm{E}-08$ \$Sm149

$61151.70 \mathrm{C} \quad 5.161789 \mathrm{E}-31$ \$Pm151

$62151.70 \mathrm{C} \quad 7.951937 \mathrm{E}-08$ \$Sm151

$62152.70 \mathrm{C} \quad 2.699588 \mathrm{E}-07 \$ \mathrm{Sm} 152$

$63153.70 \mathrm{C} \quad 1.142149 \mathrm{E}-07$ \$Eu153

$63155.70 \mathrm{C} \quad 1.314966 \mathrm{E}-08$ \$Eu155

$64157.70 \mathrm{C} \quad 1.473473 \mathrm{E}-10$ \$Gd157

lwtr.10t

ATR Element No. = 11

Radial Zone No. = 1 
Axial Zone No. $=2$

C Neutron Cross Sections $=27 \mathrm{C}$

$\mathrm{m} 2212$

Total Number Density $=7.966863 \mathrm{E}-02 \mathrm{a} / \mathrm{b}-\mathrm{cm}$

$\begin{array}{llll}1001.70 \mathrm{C} & 3.393340 \mathrm{E}-02 & \$ \mathrm{H}-1 \\ 8016.70 \mathrm{C} & 1.696670 \mathrm{E}-02 & \$ \mathrm{O}-1\end{array}$

$12000.60 \mathrm{C} \quad 2.176490 \mathrm{E}-04$ \$Mg-nat

$13027.70 \mathrm{C} \quad 2.793720 \mathrm{E}-02$ \$Al-27

$14000.60 \mathrm{C} \quad 1.130110 \mathrm{E}-04$ \$Si-nat

$24000.50 \mathrm{C} \quad 2.304760 \mathrm{E}-05$ \$Cr-nat

$29000.50 \mathrm{C} \quad 2.081160 \mathrm{E}-05$ \$Cu-nat

$5010.70 \mathrm{C} \quad 3.670657 \mathrm{E}-06$ \$ B-10

$92234.70 \mathrm{C} \quad 5.586855 \mathrm{E}-06 \quad \$ \mathrm{U}-234$

$92235.70 \mathrm{C} \quad 3.742606 \mathrm{E}-04 \quad \$ \mathrm{U}-235$

$92236.70 \mathrm{C} \quad 2.327413 \mathrm{E}-05$ \$U-236

$92237.70 \mathrm{C} \quad 2.299703 \mathrm{E}-07 \quad \$ \mathrm{U}-237$

$92238.70 \mathrm{C} \quad 3.018292 \mathrm{E}-05$ \$U-238

$93237.70 \mathrm{C} \quad 3.487250 \mathrm{E}-07$ \$Np237

$94239.70 \mathrm{C} \quad 6.718159 \mathrm{E}-07$ \$Pu239

$94240.70 \mathrm{C} \quad 5.763821 \mathrm{E}-08$ \$Pu240

$94241.70 \mathrm{C} \quad 2.017187 \mathrm{E}-08$ \$Pu241

$36083.70 \mathrm{C} \quad 4.678211 \mathrm{E}-07$ \$Kr-83

$42095.70 \mathrm{C} \quad 1.827849 \mathrm{E}-07$ \$Mo-95

$44101.70 \mathrm{C} \quad 4.630819 \mathrm{E}-06$ \$Ru101

$45103.70 \mathrm{C} \quad 6.554220 \mathrm{E}-07$ \$Rh103

$45105.70 \mathrm{C} \quad 1.174723 \mathrm{E}-30$ \$Rh105

$48113.70 \mathrm{C} \quad 1.188869 \mathrm{E}-09$ \$Cd113

$54131.70 \mathrm{C} \quad 1.622272 \mathrm{E}-06$ \$Xe131

$54133.70 \mathrm{C} \quad 9.272438 \mathrm{E}-31$ \$Xe133

$55133.70 \mathrm{C} \quad 4.282182 \mathrm{E}-06 \quad \$ \mathrm{Cs} 133$

$54135.70 \mathrm{C} \quad 9.067592 \mathrm{E}-31$ \$Xe135

$57140.70 \mathrm{C} \quad 8.808179 \mathrm{E}-31$ \$La140

$58141.70 \mathrm{C} \quad 8.745828 \mathrm{E}-31$ \$Ce141

$59143.70 \mathrm{C} \quad 8.623289 \mathrm{E}-31 \quad \$ \mathrm{Pr} 143$

$60143.70 \mathrm{C} \quad 2.373070 \mathrm{E}-06$ \$Nd143

$60145.70 \mathrm{C} \quad 3.468913 \mathrm{E}-06 \$ \mathrm{Nd1} 45$

$61147.70 \mathrm{C} \quad 8.388252 \mathrm{E}-31$ \$Pm147

$61149.70 \mathrm{C} \quad 8.275419 \mathrm{E}-31$ \$Pm149

$62149.70 \mathrm{C} \quad 4.225307 \mathrm{E}-08$ \$Sm149

$61151.70 \mathrm{C} \quad 8.165512 \mathrm{E}-31$ \$Pm151

$62151.70 \mathrm{C} \quad 1.257929 \mathrm{E}-07$ \$Sm151

$62152.70 \mathrm{C} \quad 4.270519 \mathrm{E}-07$ \$Sm152

$63153.70 \mathrm{C} \quad 1.806783 \mathrm{E}-07$ \$Eu153

$63155.70 \mathrm{C} \quad 2.080164 \mathrm{E}-08 \quad \$ \mathrm{Eu} 155$

mt2212 lwtr.10t

$64157.70 \mathrm{C} \quad 2.330909 \mathrm{E}-10$ \$Gd157

c

C

C

$\mathrm{C}$

C

C

C

m2213

ATR Element No. = 11

Radial Zone No. = 1

Axial Zone No. = 3

Neutron Cross Sections $=27 \mathrm{C}$

Total Number Density $=7.964972 \mathrm{E}-02 \mathrm{a} / \mathrm{b}-\mathrm{cm}$
$1001.70 \mathrm{C} \quad 3.393340 \mathrm{E}-02$ \$ $\mathrm{H}-1$
$8016.70 \mathrm{C} \quad 1.696670 \mathrm{E}-02 \$ \mathrm{O}-16$
$12000.60 \mathrm{C} \quad 2.176490 \mathrm{E}-04$ \$Mg-nat
$13027.70 \mathrm{C} \quad 2.793720 \mathrm{E}-02$ \$Al-27
$14000.60 \mathrm{C} \quad 1.130110 \mathrm{E}-04$ \$Si-nat
$24000.50 \mathrm{C} \quad 2.304760 \mathrm{E}-05$ \$Cr-nat
$29000.50 \mathrm{C} \quad 2.081160 \mathrm{E}-05$ \$Cu-nat
$5010.70 \mathrm{C} \quad 3.670657 \mathrm{E}-06$ \$ B 10
$92234.70 \mathrm{C} \quad 5.405670 \mathrm{E}-06$ \$U-234
$92235.70 \mathrm{C} \quad 3.456287 \mathrm{E}-04 \quad \$ \mathrm{U}-235$
$92236.70 \mathrm{C} \quad 2.833216 \mathrm{E}-05 \quad \$ \mathrm{U}-236$
$92237.70 \mathrm{C} \quad 2.892082 \mathrm{E}-07 \quad \$ \mathrm{U}-237$
$92238.70 \mathrm{C} \quad 2.992663 \mathrm{E}-05 \quad \$ \mathrm{U}-238$
$93237.70 \mathrm{C} \quad 4.385530 \mathrm{E}-07$ \$Np237
$94239.70 \mathrm{C} \quad 8.448689 \mathrm{E}-07$ \$Pu239 


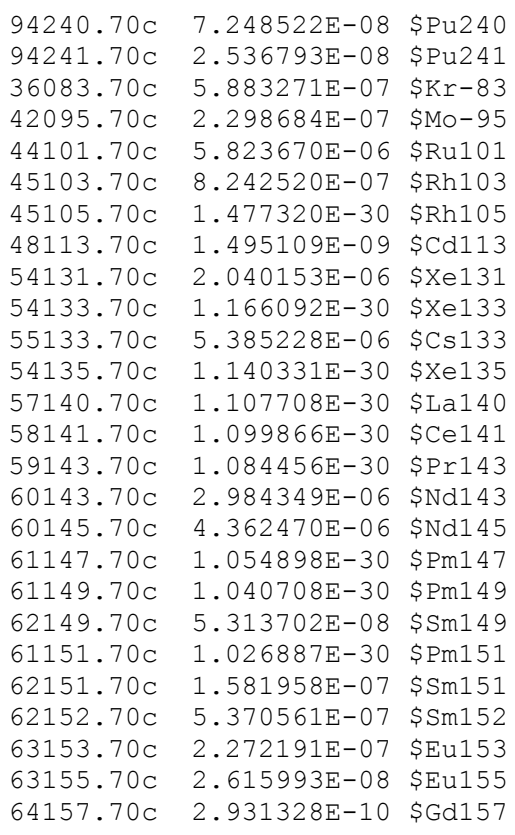

mt2213 lwtr.10t

$\mathrm{C}$

$\mathrm{C}$

C ATR Element No. = 11

C Radial Zone No. = 1

C Axial Zone No. = 4

C Neutron Cross Sections $=27 \mathrm{C}$

c Total Number Density $=7.964210 \mathrm{E}-02 \mathrm{a} / \mathrm{b}-\mathrm{cm}$

m2214

\begin{tabular}{|c|c|c|}
\hline $1001.70 \mathrm{C}$ & $3.393340 \mathrm{E}-02$ & $\$ \mathrm{H}-1$ \\
\hline $8016.70 \mathrm{c}$ & $1.696670 \mathrm{E}-02$ & $\$ 0-16$ \\
\hline $12000.60 \mathrm{c}$ & $2.176490 \mathrm{E}-04$ & \$Mg-nat \\
\hline $13027.70 \mathrm{C}$ & $2.793720 \mathrm{E}-02$ & $\$ A l-27$ \\
\hline $14000.60 \mathrm{C}$ & $1.130110 \mathrm{E}-04$ & \$Si-nat \\
\hline $24000.50 \mathrm{c}$ & $2.304760 \mathrm{E}-05$ & \$Cr-nat \\
\hline $29000.50 \mathrm{C}$ & $2.081160 \mathrm{E}-05$ & \$Cu-nat \\
\hline $5010.70 \mathrm{c}$ & $3.670657 \mathrm{E}-06$ & $\$ B-10$ \\
\hline $92234.70 \mathrm{C}$ & $5.332763 E-06$ & $\$ U-234$ \\
\hline $92235.70 \mathrm{C}$ & $3.341061 E-04$ & $\$ U-235$ \\
\hline $92236.70 \mathrm{C}$ & $3.036801 E-05$ & $\$ U-236$ \\
\hline $92237.70 \mathrm{C}$ & $3.130513 E-07$ & $\$ U-237$ \\
\hline $92238.70 \mathrm{C}$ & $2.982357 \mathrm{E}-05$ & $\$ U-238$ \\
\hline $93237.70 \mathrm{c}$ & $4.747085 E-07$ & $\$ N p 237$ \\
\hline $94239.70 \mathrm{C}$ & $9.145222 \mathrm{E}-07$ & $\$ P u 239$ \\
\hline $94240.70 \mathrm{C}$ & $7.846110 \mathrm{E}-08$ & $\$ P u 240$ \\
\hline $94241.70 \mathrm{C}$ & $2.745933 \mathrm{E}-08$ & \$Pu241 \\
\hline $36083.70 \mathrm{c}$ & $6.368303 E-07$ & $\$ K r-83$ \\
\hline $42095.70 \mathrm{C}$ & $2.488194 \mathrm{E}-07$ & $\$ M o-95$ \\
\hline $44101.70 \mathrm{C}$ & $6.303790 E-06$ & \$Ru101 \\
\hline $45103.70 \mathrm{C}$ & $8.922056 \mathrm{E}-07$ & \$Rh103 \\
\hline $45105.70 \mathrm{C}$ & $1.599114 \mathrm{E}-30$ & \$Rh105 \\
\hline $48113.70 \mathrm{C}$ & $1.618370 \mathrm{E}-09$ & $\$ \mathrm{Cd} 113$ \\
\hline $54131.70 \mathrm{C}$ & $2.208349 E-06$ & \$Xe131 \\
\hline $54133.70 \mathrm{C}$ & $1.262228 \mathrm{E}-30$ & \$Xe133 \\
\hline $55133.70 \mathrm{C}$ & $5.829201 E-06$ & $\$ \mathrm{Cs} 133$ \\
\hline $54135.70 \mathrm{C}$ & $1.234343 E-30$ & $\$ x e 135$ \\
\hline $57140.70 \mathrm{C}$ & $1.199030 \mathrm{E}-30$ & \$La140 \\
\hline $58141.70 \mathrm{C}$ & $1.190542 \mathrm{E}-30$ & $\$ C e 141$ \\
\hline $59143.70 \mathrm{C}$ & $1.173861 \mathrm{E}-30$ & $\$ \operatorname{Pr} 143$ \\
\hline $60143.70 \mathrm{C}$ & $3.230387 \mathrm{E}-06$ & $\$ N d 143$ \\
\hline $60145.70 \mathrm{C}$ & $4.722123 E-06$ & $\$ N d 145$ \\
\hline $61147.70 \mathrm{C}$ & $1.141867 \mathrm{E}-30$ & $\$ P \operatorname{Pm} 147$ \\
\hline 61 & $1.126507 \mathrm{E}-30$ & $\$ P \operatorname{Pm} 149$ \\
\hline
\end{tabular}


$62149.70 \mathrm{C} \quad 5.751778 \mathrm{E}-08 \quad \$ \mathrm{Sm} 149$

$61151.70 \mathrm{C} \quad 1.111546 \mathrm{E}-30 \quad \$ \mathrm{Pm} 151$

$62151.70 \mathrm{C} \quad 1.712379 \mathrm{E}-07$ \$Sm151

$62152.70 \mathrm{C} \quad 5.813325 \mathrm{E}-07$ \$Sm152

$63153.70 \mathrm{C} \quad 2.459517 \mathrm{E}-07$ \$Eu153

$63155.70 \mathrm{C} \quad 2.831662 \mathrm{E}-08$ \$Eu155

$\begin{array}{llll}64157.70 \mathrm{C} \quad 3.172995 \mathrm{E}-10 & \text { \$Gd157 }\end{array}$

C

C

C

C

C

C

C

m2215 mt2215

C

C

C

C

C

C

C

C

$\mathrm{C}$

m2216
ATR Element No. = 11

Radial Zone No. = 1

Axial Zone No. = 5

Neutron Cross Sections $=27 \mathrm{C}$

Total Number Density $=7.965256 \mathrm{E}-02 \mathrm{a} / \mathrm{b}-\mathrm{cm}$

lwtr.10t

\begin{tabular}{|c|c|c|}
\hline $1001.70 \mathrm{c}$ & $3.393340 E-02$ & $\$ \mathrm{H}-1$ \\
\hline $8016.70 \mathrm{c}$ & $1.696670 \mathrm{E}-02$ & $\$ 0-16$ \\
\hline $000.60 \mathrm{c}$ & $2.176490 \mathrm{E}-04$ & $\$ M g-n a t$ \\
\hline $7.70 \mathrm{C}$ & $2.793720 \mathrm{E}-02$ & $\$ A I-27$ \\
\hline $00.60 \mathrm{c}$ & $1.130110 \mathrm{E}-04$ & \$Si-nat \\
\hline .50 & $2.304760 \mathrm{E}-05$ & $\$ C r-n a t$ \\
\hline $0.50 \mathrm{c}$ & $2.081160 \mathrm{E}-05$ & \$Cu-nat \\
\hline 10.70 & $3.670657 \mathrm{E}-06$ & $\$ B-10$ \\
\hline 7.70 & $5.433172 \mathrm{E}-06$ & $\$ U-234$ \\
\hline .70 & $3.499747 \mathrm{E}-04$ & $\$ U-235$ \\
\hline .70 & $2.756441 E-05$ & $\$ U-236$ \\
\hline .70 & $2.802166 \mathrm{E}-07$ & $\$ U-237$ \\
\hline .70 & $2.996553 E-05$ & $\$ U-238$ \\
\hline .70 & $4.249182 \mathrm{E}-07$ & $\$ N p 237$ \\
\hline $.70 \mathrm{C}$ & $8.186016 \mathrm{E}-07$ & \$Pu239 \\
\hline .70 & $7.023162 \mathrm{E}-08$ & $\$ P u 240$ \\
\hline $.70 \mathrm{C}$ & $2.457923 E-08$ & \$Pu 241 \\
\hline 7 & $5.700358 \mathrm{E}-07$ & $\$ K r-83$ \\
\hline $5.70 \mathrm{C}$ & $2.227217 \mathrm{E}-07$ & $\$ M \circ-95$ \\
\hline $70 \Omega$ & $5.642610 \mathrm{E}-06$ & \$Ru101 \\
\hline $.70 \mathrm{C}$ & $7.986257 \mathrm{E}-07$ & \$Rh103 \\
\hline 70 & $1.431389 \mathrm{E}-30$ & \$Rh105 \\
\hline $3.70 \mathrm{C}$ & $1.448625 E-09$ & $\$ \mathrm{Cd} 113$ \\
\hline $.70 \mathrm{c}$ & $1.976724 \mathrm{E}-06$ & $\$ x e 131$ \\
\hline 0 & $1.129838 \mathrm{E}-30$ & \$Xe133 \\
\hline 70 & $00 E-06$ & $\$ \mathrm{Cs} 133$ \\
\hline 5 & $878 E-30$ & \$Xe135 \\
\hline 57 & $9 E-30$ & \$La140 \\
\hline 5 & $671 E-30$ & $\$ \mathrm{Ce} 141$ \\
\hline & $740 E-30$ & \$Pr 143 \\
\hline 60 & $2.891564 E-06$ & $\$ N d 143$ \\
\hline & $5838 E-06$ & $\$ N d 145$ \\
\hline 61 & $1.022101 E-30$ & \$Pm1 47 \\
\hline & $352 E-30$ & $\$ \operatorname{Pm} 149$ \\
\hline $.70 \mathrm{c}$ & $5.148498 \mathrm{E}-08$ & $\$ S m 149$ \\
\hline & $9.949601 E-31$ & \$Pm151 \\
\hline .70 & $1.532775 E-07$ & \$Sm151 \\
\hline & $5.203588 E-07$ & $\$ S m 152$ \\
\hline .70 & $2.201548 E-07$ & \$Eu153 \\
\hline & $2.534661 \mathrm{E}-08$ & \$Eu155 \\
\hline .70 & $2.840191 E-10$ & \$Gd1 57 \\
\hline
\end{tabular}

$64157.70 \mathrm{C} 2.840191 \mathrm{E}-10$ \$ 2.157

1wtr. 10 t

ATR Element No. = 11

Radial Zone No. = 1

Axial Zone No. = 6

Neutron Cross Sections $=27 \mathrm{C}$

Total Number Density $=7.967413 \mathrm{E}-02 \mathrm{a} / \mathrm{b}-\mathrm{cm}$

$1001.70 \mathrm{C} \quad 3.393340 \mathrm{E}-02 \$ \mathrm{H}-1$ 


\begin{tabular}{|c|c|c|}
\hline $\mathrm{Oc}_{\mathrm{c}}$ & -02 & $0-16$ \\
\hline $12000.60 \mathrm{c}$ & $2.176490 \mathrm{E}-04$ & $\$ M g-n a t$ \\
\hline $13027.70 \mathrm{c}$ & $2.793720 \mathrm{E}-02$ & $\$ A 1-27$ \\
\hline $14000.60 \mathrm{c}$ & $1.130110 \mathrm{E}-04$ & \$Si-nat \\
\hline $24000.50 \mathrm{c}$ & $2.304760 \mathrm{E}-05$ & $\$ \mathrm{Cr}$-nat \\
\hline $29000.50 \mathrm{c}$ & $2.081160 \mathrm{E}-05$ & \$Cu-nat \\
\hline $5010.70 \mathrm{c}$ & $3.670657 \mathrm{E}-06$ & $\$ B-10$ \\
\hline $92234.70 \mathrm{C}$ & $5.639757 \mathrm{E}-06$ & $\$ U-234$ \\
\hline $92235.70 \mathrm{C}$ & $3.826204 \mathrm{E}-04$ & $\$ \mathrm{U}-235$ \\
\hline $92236.70 \mathrm{c}$ & $2.179732 \mathrm{E}-05$ & $\$ U-236$ \\
\hline $92237.70 \mathrm{c}$ & $2.126743 \mathrm{E}-07$ & $\$ U-237$ \\
\hline $92238.70 \mathrm{c}$ & $3.025774 \mathrm{E}-05$ & $\$ \mathrm{U}-238$ \\
\hline $93237.70 \mathrm{C}$ & $3.224976 \mathrm{E}-07$ & \$Np237 \\
\hline $94239.70 \mathrm{C}$ & $6.212892 \mathrm{E}-07$ & \$Pu239 \\
\hline $94240.70 \mathrm{C}$ & $5.330328 \mathrm{E}-08$ & \$Pu240 \\
\hline $94241.70 \mathrm{C}$ & $1.865475 \mathrm{E}-08$ & \$Pu241 \\
\hline $36083.70 \mathrm{c}$ & $4.326367 \mathrm{E}-07$ & $\$ K r-83$ \\
\hline $42095.70 \mathrm{c}$ & $1.690378 \mathrm{E}-07$ & $\$ M o-95$ \\
\hline $44101.70 \mathrm{c}$ & $4.282538 \mathrm{E}-06$ & \$Ru101 \\
\hline $45103.70 \mathrm{c}$ & $6.061282 \mathrm{E}-07$ & \$Rh103 \\
\hline $45105.70 \mathrm{c}$ & $1.086373 \mathrm{E}-30$ & \$Rh105 \\
\hline $48113.70 \mathrm{C}$ & $1.099455 \mathrm{E}-09$ & $\$ C d 113$ \\
\hline $54131.70 \mathrm{c}$ & $1.500262 \mathrm{E}-06$ & $\$ \mathrm{Xe131}$ \\
\hline $54133.70 \mathrm{C}$ & $8.575064 \mathrm{E}-31$ & $\$ \mathrm{Xe133}$ \\
\hline $55133.70 \mathrm{C}$ & $3.960122 \mathrm{E}-06$ & $\$ C s 133$ \\
\hline $54135.70 \mathrm{C}$ & $8.385625 \mathrm{E}-31$ & \$Xe135 \\
\hline $57140.70 \mathrm{c}$ & $8.145722 \mathrm{E}-31$ & \$La140 \\
\hline $58141.70 \mathrm{C}$ & $8.088060 \mathrm{E}-31$ & $\$ C e 141$ \\
\hline $59143.70 \mathrm{C}$ & $7.974737 \mathrm{E}-31$ & $\$ \operatorname{Pr} 143$ \\
\hline $60143.70 \mathrm{c}$ & $2.194593 \mathrm{E}-06$ & \$Nd143 \\
\hline $60145.70 \mathrm{c}$ & $3.208019 \mathrm{E}-06$ & \$Nd14 5 \\
\hline $61147.70 \mathrm{c}$ & $7.757377 \mathrm{E}-31$ & $\$ \operatorname{Pm} 147$ \\
\hline $61149.70 \mathrm{c}$ & $7.653031 \mathrm{E}-31$ & $\$ \operatorname{Pm} 149$ \\
\hline $62149.70 \mathrm{C}$ & $3.907524 \mathrm{E}-08$ & $\$$ Sm1 49 \\
\hline $61151.70 \mathrm{c}$ & $7.551389 \mathrm{E}-31$ & \$Pm151 \\
\hline $62151.70 \mathrm{C}$ & $1.163321 \mathrm{E}-07$ & $\$$ Sm151 \\
\hline $62152.70 \mathrm{c}$ & $3.949336 \mathrm{E}-07$ & $\$$ Sm152 \\
\hline $63153.70 \mathrm{c}$ & $1.670896 \mathrm{E}-07$ & \$Eu153 \\
\hline $63155.70 \mathrm{c}$ & $1.923716 \mathrm{E}-08$ & \$Eu155 \\
\hline & $.155603 \mathrm{E}-10$ & 1 \\
\hline
\end{tabular}

c

c

C

C

C

C

C

m2217 
$45103.70 \mathrm{C} \quad 2.808665 \mathrm{E}-07$ \$Rh103

$45105.70 \mathrm{C} \quad 5.034013 \mathrm{E}-31$ \$Rh105

$48113.70 \mathrm{C} \quad 5.094631 \mathrm{E}-10$ \$Cd113

$54131.70 \mathrm{C} \quad 6.951886 \mathrm{E}-07$ \$Xe131

$54133.70 \mathrm{C} \quad 3.973496 \mathrm{E}-31$ \$Xe133

$55133.70 \mathrm{C} \quad 1.835034 \mathrm{E}-06$ \$Cs133

$54135.70 \mathrm{C} \quad 3.885714 \mathrm{E}-31$ \$Xe135

$57140.70 \mathrm{C} \quad 3.774548 \mathrm{E}-31$ \$La140

$58141.70 \mathrm{C} \quad 3.747829 \mathrm{E}-31$ \$Ce141

$59143.70 \mathrm{C} \quad 3.695318 \mathrm{E}-31 \quad \$ \operatorname{Pr} 143$

$60143.70 \mathrm{C} \quad 1.016926 \mathrm{E}-06 \$ \mathrm{Nd1} 43$

$60145.70 \mathrm{C} \quad 1.486525 \mathrm{E}-06 \$ \mathrm{Nd1} 45$

$61147.70 \mathrm{C} \quad 3.594598 \mathrm{E}-31$ Pm147

$61149.70 \mathrm{C} \quad 3.546246 \mathrm{E}-31$ \$Pm149

$62149.70 \mathrm{C} \quad 1.810661 \mathrm{E}-08$ \$Sm149

$61151.70 \mathrm{C} \quad 3.499148 \mathrm{E}-31$ \$Pm151

$62151.70 \mathrm{C} \quad 5.390573 \mathrm{E}-08$ \$Sm151

$62152.70 \mathrm{C} \quad 1.830036 \mathrm{E}-07$ \$Sm152

$63153.70 \mathrm{C} \quad 7.742563 \mathrm{E}-08$ \$Eu153

$63155.70 \mathrm{C} \quad 8.914077 \mathrm{E}-09$ \$Eu155

mt2217 lwtr.10t

C

C

$\mathrm{C}$

C

C

m2218

$64157.70 \mathrm{C} \quad 9.988591 \mathrm{E}-11$ \$Gd157

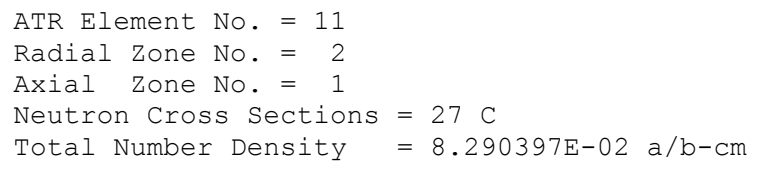

\begin{tabular}{|c|c|c|}
\hline 001. & $3.920790 E-02$ & $\$ \mathrm{H}-1$ \\
\hline $8016.70 \mathrm{C}$ & $1.960390 \mathrm{E}-02$ & $\$ 0-16$ \\
\hline $2000.60 \mathrm{c}$ & $1.660990 \mathrm{E}-04$ & $\$ M g-n a t$ \\
\hline $3027.70 \mathrm{c}$ & $2.315370 E-02$ & $\$ A I-27$ \\
\hline $4000.60 \mathrm{c}$ & $8.624450 \mathrm{E}-05$ & \$Si-nat \\
\hline $00.50 \mathrm{C}$ & $1.552830 \mathrm{E}-05$ & \$Cr-nat \\
\hline $9000.50 \mathrm{c}$ & $1.588240 \mathrm{E}-05$ & $\$ \mathrm{Cu}-\mathrm{nat}$ \\
\hline $0.70 \mathrm{C}$ & $1.714619 \mathrm{E}-28$ & $\$ B-10$ \\
\hline $4.70 \mathrm{C}$ & $7.928286 \mathrm{E}-06$ & $\$ U-234$ \\
\hline $.70 \mathrm{C}$ & $5.768793 E-04$ & $\$ U-235$ \\
\hline $.70 \mathrm{C}$ & $1.689922 \mathrm{E}-05$ & $\$ U-236$ \\
\hline $.70 \mathrm{C}$ & $1.412864 \mathrm{E}-07$ & $\$ U-237$ \\
\hline $.70 \mathrm{C}$ & $4.082754 \mathrm{E}-05$ & $\$ U-238$ \\
\hline $.70 \mathrm{C}$ & $2.142456 \mathrm{E}-07$ & $\$ N p 237$ \\
\hline $.70 \mathrm{C}$ & $4.127423 E-07$ & \$Pu239 \\
\hline $0.70 \mathrm{c}$ & $3.541108 \mathrm{E}-08$ & \$Pu240 \\
\hline $.70 \mathrm{C}$ & $1.239295 \mathrm{E}-08$ & \$Pu241 \\
\hline & $4144 \mathrm{E}-07$ & $\$ K r-83$ \\
\hline $.70 \mathrm{C}$ & $1.122972 \mathrm{E}-07$ & $\$ M \circ-95$ \\
\hline & 2.8 & \$Ru101 \\
\hline $70 \mathrm{C}$ & $4 E-07$ & $\$ R h 103$ \\
\hline & & \$Rh105 \\
\hline $.70 \mathrm{c}$ & $30 E-10$ & $\$ \mathrm{Cd} 113$ \\
\hline & $9.966724 \mathrm{E}-07$ & $\$ x e 131$ \\
\hline $.70 \mathrm{C}$ & 5.6966 & $\$ x e 133$ \\
\hline$C$ & $2.630836 \mathrm{E}-06$ & $\$ C$ s 133 \\
\hline $\mathrm{C}$ & $0 E-31$ & $\$ x e 135$ \\
\hline & $5 E-31$ & \$La140 \\
\hline C & $9 E-31$ & $\$ \mathrm{Ce} 141$ \\
\hline $.70 \mathrm{C}$ & $5.297875 E-31$ & $\$ \operatorname{Pr} 143$ \\
\hline C & $9 E-06$ & $\$ N d 143$ \\
\hline $.70 \mathrm{C}$ & 2.1311 & $\$ N d 145$ \\
\hline $.70 \mathrm{C}$ & 5.1534 & $\$ \operatorname{Pm} 147$ \\
\hline $.70 \mathrm{C}$ & $5.084154 \mathrm{E}-31$ & \$Pm1 49 \\
\hline $.70 \mathrm{C}$ & $2.595894 \mathrm{E}-08$ & $\$ \operatorname{Sm} 149$ \\
\hline .70 & $5.016631 \mathrm{E}-31$ & $\$ P m 151$ \\
\hline 62 & $7.728314 \mathrm{E}-08$ & $\$ S m 151$ \\
\hline & $71 \mathrm{E}-07$ & \$Sm152 \\
\hline .7 & $1.110030 \mathrm{E}-07$ & $\$$ Eu153 \\
\hline
\end{tabular}




\begin{tabular}{|c|c|c|c|}
\hline & $\begin{array}{l}63155.70 \mathrm{C} \\
64157.70 \mathrm{C}\end{array}$ & $\begin{array}{l}1.277986 \mathrm{E}-08 \\
1.432037 \mathrm{E}-10\end{array}$ & $\begin{array}{l}\$ \text { Eu155 } \\
\$ \text { Gd157 }\end{array}$ \\
\hline mt2218 & \multicolumn{3}{|c|}{ lwtr.10t } \\
\hline \multicolumn{4}{|l|}{ c } \\
\hline \multicolumn{4}{|l|}{ c } \\
\hline \multirow{2}{*}{\multicolumn{4}{|c|}{$\begin{array}{l}\mathrm{C} \\
\mathrm{C}\end{array}$}} \\
\hline & & & \\
\hline $\mathrm{c}$ & ATR Element No. = & 11 & \\
\hline $\mathrm{C}$ & Radial Zone No. = & 2 & \\
\hline C & Axial Zone No. = & 2 & \\
\hline c & \multicolumn{3}{|c|}{ Neutron Cross Sections $=27 \mathrm{C}$} \\
\hline \multirow{2}{*}{\multicolumn{4}{|c|}{$=8.287613 \mathrm{E}-02 \mathrm{a} / \mathrm{b}-\mathrm{cm}$}} \\
\hline & & & \\
\hline & $8016.70 \mathrm{c}$ & $1.960390 \mathrm{E}-02$ & $\$ 0-16$ \\
\hline & $12000.60 \mathrm{c}$ & $1.660990 \mathrm{E}-04$ & $\$ M g-n a t$ \\
\hline & $13027.70 \mathrm{c}$ & $2.315370 \mathrm{E}-02$ & $\$ A l-27$ \\
\hline & $14000.60 \mathrm{c}$ & $8.624450 \mathrm{E}-05$ & $\$$ Si-nat \\
\hline & $24000.50 \mathrm{c}$ & $1.552830 \mathrm{E}-05$ & $\$ \mathrm{Cr}$-nat \\
\hline & $29000.50 \mathrm{c}$ & $1.588240 \mathrm{E}-05$ & $\$ \mathrm{Cu}-$ nat \\
\hline & $5010.70 \mathrm{c}$ & $1.714619 \mathrm{E}-28$ & $\$ B-10$ \\
\hline & $92234.70 \mathrm{c}$ & $7.661645 \mathrm{E}-06$ & $\$ \mathrm{U}-234$ \\
\hline & $92235.70 \mathrm{C}$ & $5.347432 \mathrm{E}-04$ & $\$ U-235$ \\
\hline & $92236.70 \mathrm{c}$ & $2.434286 \mathrm{E}-05$ & $\$ \mathrm{U}-236$ \\
\hline & $92237.70 \mathrm{c}$ & $2.284639 \mathrm{E}-07$ & $\$ \mathrm{U}-237$ \\
\hline & $92238.70 \mathrm{c}$ & $4.045038 \mathrm{E}-05$ & $\$ \mathrm{U}-238$ \\
\hline & $93237.70 \mathrm{C}$ & $3.464408 \mathrm{E}-07$ & $\$ \mathrm{~Np} 237$ \\
\hline & $94239.70 \mathrm{C}$ & $6.674154 \mathrm{E}-07$ & \$Pu239 \\
\hline & $94240.70 \mathrm{c}$ & $5.726066 \mathrm{E}-08$ & $\$$ Pu240 \\
\hline & $94241.70 \mathrm{c}$ & $2.003974 \mathrm{E}-08$ & \$Pu241 \\
\hline & $36083.70 \mathrm{c}$ & $4.647568 \mathrm{E}-07$ & $\$ \mathrm{Kr}-83$ \\
\hline & $42095.70 \mathrm{C}$ & $1.815876 \mathrm{E}-07$ & $\$ M \circ-95$ \\
\hline & $44101.70 \mathrm{C}$ & $4.600486 \mathrm{E}-06$ & \$Ru101 \\
\hline & $45103.70 \mathrm{c}$ & $6.511288 \mathrm{E}-07$ & $\$ R h 103$ \\
\hline & $45105.70 \mathrm{c}$ & $1.167028 \mathrm{E}-30$ & \$Rh105 \\
\hline & $48113.70 \mathrm{c}$ & $1.181081 \mathrm{E}-09$ & $\$ C d 113$ \\
\hline & $54131.70 \mathrm{c}$ & $1.611646 \mathrm{E}-06$ & $\$ x e 131$ \\
\hline & $54133.70 \mathrm{c}$ & $9.211702 \mathrm{E}-31$ & $\$$ Xe133 \\
\hline & $55133.70 \mathrm{C}$ & $4.254133 \mathrm{E}-06$ & $\$ C s 133$ \\
\hline & $54135.70 \mathrm{c}$ & $9.008198 \mathrm{E}-31$ & $\$$ Xe135 \\
\hline & $57140.70 \mathrm{c}$ & $8.750483 \mathrm{E}-31$ & $\$ L a 140$ \\
\hline & $58141.70 \mathrm{c}$ & $8.688541 \mathrm{E}-31$ & $\$ \mathrm{Ce} 141$ \\
\hline & $59143.70 \mathrm{c}$ & $8.566805 \mathrm{E}-31$ & $\$ \operatorname{Pr} 143$ \\
\hline & $60143.70 \mathrm{c}$ & $2.357526 \mathrm{E}-06$ & $\$ \mathrm{Nd} 143$ \\
\hline & $60145.70 \mathrm{c}$ & $3.446191 \mathrm{E}-06$ & $\$ \mathrm{Nd1} 45$ \\
\hline & $61147.70 \mathrm{c}$ & $8.333307 \mathrm{E}-31$ & $\$ \operatorname{Pm} 147$ \\
\hline & $61149.70 \mathrm{c}$ & $8.221213 \mathrm{E}-31$ & $\$ \operatorname{Pm} 149$ \\
\hline & $62149.70 \mathrm{C}$ & $4.197630 \mathrm{E}-08$ & $\$ \operatorname{Sm} 149$ \\
\hline & $61151.70 \mathrm{c}$ & $8.112026 \mathrm{E}-31$ & $\$ \operatorname{Pm} 151$ \\
\hline & $62151.70 \mathrm{c}$ & $1.249689 \mathrm{E}-07$ & $\$$ Sm151 \\
\hline & $62152.70 \mathrm{C}$ & $4.242546 \mathrm{E}-07$ & $\$$ Sm152 \\
\hline & $63153.70 \mathrm{c}$ & $1.794948 \mathrm{E}-07$ & \$Eu153 \\
\hline & $63155.70 \mathrm{c}$ & $2.066538 \mathrm{E}-08$ & \$Eu155 \\
\hline & $64157.70 \mathrm{c}$ & $2.315641 \mathrm{E}-10$ & \$Gd157 \\
\hline mt2219 & \multicolumn{3}{|c|}{ lwtr.10t } \\
\hline \multicolumn{4}{|l|}{$\begin{array}{l}\mathrm{C} \\
\mathrm{C}\end{array}$} \\
\hline c & & & \\
\hline \multicolumn{4}{|l|}{$\begin{array}{l}C \\
C\end{array}$} \\
\hline \multicolumn{4}{|l|}{ C } \\
\hline $\mathrm{c}$ & \multicolumn{3}{|c|}{ ATR Element No. $=11$} \\
\hline C & \multicolumn{3}{|c|}{ Radial Zone No. $=2$} \\
\hline $\mathrm{c}$ & Axial Zone No. = & & \\
\hline C & \multicolumn{3}{|c|}{ Neutron Cross Sections $=27 \mathrm{C}$} \\
\hline $\mathrm{C}$ & Total Number Densi & $=8.2854$ & $446 \mathrm{E}-02 \mathrm{a} / \mathrm{b}-\mathrm{cm}$ \\
\hline & $1001.70 \mathrm{c}$ & $3.920790 \mathrm{E}-02$ & $\$ \mathrm{H}-1$ \\
\hline & $8016.70 \mathrm{c}$ & $1.960390 \mathrm{E}-02$ & $\$ 0-16$ \\
\hline & $12000.60 \mathrm{c}$ & $1.660990 \mathrm{E}-04$ & $\$ M g-$ nat \\
\hline & $13027.70 \mathrm{c}$ & $2.315370 \mathrm{E}-02$ & $\$ A I-27$ \\
\hline & $14000.60 \mathrm{c}$ & $8.624450 \mathrm{E}-05$ & \$Si-nat \\
\hline & $24000.50 \mathrm{C}$ & $1.552830 \mathrm{E}-05$ & $\$ C r-n a t$ \\
\hline
\end{tabular}




$$
\begin{aligned}
& 29000.50 \mathrm{C} \quad 1.588240 \mathrm{E}-05 \text { \$Cu-nat } \\
& 5010.70 \mathrm{C} \quad 1.714619 \mathrm{E}-28 \text { \$ B }-10 \\
& 92234.70 \mathrm{C} \quad 7.453874 \mathrm{E}-06 \quad \$ \mathrm{U}-234 \\
& 92235.70 \mathrm{C} \quad 5.019101 \mathrm{E}-04 \quad \$ \mathrm{U}-235 \\
& 92236.70 \mathrm{C} \quad 3.014306 \mathrm{E}-05 \quad \$ \mathrm{U}-236 \\
& 92237.70 \mathrm{C} \quad 2.963940 \mathrm{E}-07 \quad \$ \mathrm{U}-237 \\
& 92238.70 \mathrm{C} \quad 4.015649 \mathrm{E}-05 \quad \$ \mathrm{U}-238 \\
& 93237.70 \mathrm{C} \quad 4.494494 \mathrm{E}-07 \quad \$ \mathrm{~Np} 237 \\
& 94239.70 \mathrm{C} \quad 8.658606 \mathrm{E}-07 \text { \$Pu239 } \\
& 94240.70 \mathrm{C} \quad 7.428621 \mathrm{E}-08 \text { \$Pu240 } \\
& 94241.70 \mathrm{C} \quad 2.599823 \mathrm{E}-08 \text { \$Pu241 } \\
& 36083.70 \mathrm{C} \quad 6.029448 \mathrm{E}-07 \quad \$ \mathrm{Kr}-83 \\
& 42095.70 \mathrm{C} \quad 2.355798 \mathrm{E}-07 \text { \$Mo-95 } \\
& 44101.70 \mathrm{C} \quad 5.968366 \mathrm{E}-06 \text { \$Ru101 } \\
& 45103.70 \mathrm{C} \quad 8.447315 \mathrm{E}-07 \text { \$Rh103 } \\
& 45105.70 \mathrm{C} \quad 1.514025 \mathrm{E}-30 \text { \$Rh105 } \\
& 48113.70 \mathrm{C} \quad 1.532257 \mathrm{E}-09 \text { \$Cd113 } \\
& 54131.70 \mathrm{C} \quad 2.090843 \mathrm{E}-06 \text { \$Xe131 } \\
& 54133.70 \mathrm{C} \quad 1.195065 \mathrm{E}-30 \text { \$Xe133 } \\
& 55133.70 \mathrm{C} \quad 5.519031 \mathrm{E}-06 \quad \$ \mathrm{Cs} 133 \\
& 54135.70 \mathrm{C} \quad 1.168664 \mathrm{E}-30 \quad \$ \mathrm{Xe} 135 \\
& 57140.70 \mathrm{C} \quad 1.135230 \mathrm{E}-30 \text { \$La140 } \\
& 58141.70 \mathrm{C} \quad 1.127194 \mathrm{E}-30 \quad \$ \mathrm{Ce} 141 \\
& 59143.70 \mathrm{C} \quad 1.111401 \mathrm{E}-30 \quad \$ \operatorname{Pr} 143 \\
& 60143.70 \mathrm{C} \quad 3.058499 \mathrm{E}-06 \text { \$Nd143 } \\
& 60145.70 \mathrm{C} \quad 4.470860 \mathrm{E}-06 \$ \mathrm{Nd} 145 \\
& 61147.70 \mathrm{C} \quad 1.081108 \mathrm{E}-30 \$ \mathrm{Pm} 147 \\
& 61149.70 \mathrm{C} \quad 1.066566 \mathrm{E}-30 \quad \$ \mathrm{Pm} 149 \\
& 62149.70 \mathrm{C} \quad 5.445728 \mathrm{E}-08 \quad \$ \mathrm{Sm} 149 \\
& 61151.70 \mathrm{C} \quad 1.052401 \mathrm{E}-30 \text { \$Pm151 } \\
& 62151.70 \mathrm{C} \quad 1.621264 \mathrm{E}-07 \text { \$SM151 } \\
& 62152.70 \mathrm{C} \quad 5.503999 \mathrm{E}-07 \quad \$ \mathrm{Sm} 152 \\
& 63153.70 \mathrm{C} \quad 2.328647 \mathrm{E}-07 \text { \$Eu153 } \\
& 63155.70 \mathrm{C} \quad 2.680990 \mathrm{E}-08 \text { \$Eu155 }
\end{aligned}
$$


$55133.70 \mathrm{C} \quad 6.072065 \mathrm{E}-06 \quad \$ \mathrm{Cs} 133$

$54135.70 \mathrm{C} \quad 1.285770 \mathrm{E}-30 \quad \$ \mathrm{Xe} 135$

$57140.70 \mathrm{C} \quad 1.248985 \mathrm{E}-30$ \$La140

$58141.70 \mathrm{C} \quad 1.240144 \mathrm{E}-30 \$ \mathrm{Ce} 141$

$59143.70 \mathrm{C} \quad 1.222768 \mathrm{E}-30 \quad \$ \operatorname{Pr} 143$

$60143.70 \mathrm{C} \quad 3.364975 \mathrm{E}-06 \quad \$ \mathrm{Nd1} 43$

$60145.70 \mathrm{C} \quad 4.918862 \mathrm{E}-06$ \$Nd145

$61147.70 \mathrm{C} \quad 1.189441 \mathrm{E}-30 \quad \$ \mathrm{Pm} 147$

$61149.70 \mathrm{C} \quad 1.173441 \mathrm{E}-30 \quad \$ \operatorname{Pm} 149$

$62149.70 \mathrm{C} \quad 5.991416 \mathrm{E}-08 \quad \$ \mathrm{Sm} 149$

$61151.70 \mathrm{C} \quad 1.157856 \mathrm{E}-30$ \$Pm151

$62151.70 \mathrm{C} \quad 1.783723 \mathrm{E}-07$ \$Sm151

$62152.70 \mathrm{C} \quad 6.055526 \mathrm{E}-07 \quad \$ \mathrm{Sm} 152$

$63153.70 \mathrm{C} \quad 2.561988 \mathrm{E}-07$ \$Eu153

$63155.70 \mathrm{c} \quad 2.949638 \mathrm{E}-08$ \$Eu155

$\begin{array}{llll}64157.70 \mathrm{C} & 3.305191 \mathrm{E}-10 \quad \$ \text { Gd157 }\end{array}$

c

C

C

C

C

m2222

mt 2222

C

C
ATR Element No. $=11$
Radial Zone No. $=2$
Axial Zone No. $=5$
Neutron Cross Sections $=27 \mathrm{C}$
Total Number Density $=8.285798 \mathrm{E}-02 \mathrm{a} / \mathrm{b}-\mathrm{cm}$

$1001.70 \mathrm{C} \quad 3.920790 \mathrm{E}-02$ \$ H-1

$8016.70 \mathrm{C} \quad 1.960390 \mathrm{E}-02 \$ 0-16$

$12000.60 \mathrm{C} \quad 1.660990 \mathrm{E}-04 \mathrm{\$ Mg}-\mathrm{nat}$

$13027.70 \mathrm{C} \quad 2.315370 \mathrm{E}-02$ \$Al-27

$14000.60 \mathrm{C} \quad 8.624450 \mathrm{E}-05$ \$Si-nat

$24000.50 \mathrm{C} \quad 1.552830 \mathrm{E}-05$ \$Cr-nat

$29000.50 \mathrm{C} \quad 1.588240 \mathrm{E}-05$ \$Cu-nat

$5010.70 \mathrm{C} \quad 1.714619 \mathrm{E}-28$ \$ B-10

$92234.70 \mathrm{C} \quad 7.487749 \mathrm{E}-06$ \$U-234

$92235.70 \mathrm{C} \quad 5.072631 \mathrm{E}-04$ \$U-235

$92236.70 \mathrm{C} \quad 2.919742 \mathrm{E}-05$ \$U-236

$92237.70 \mathrm{C} \quad 2.853189 \mathrm{E}-07$ \$U-237

$92238.70 \mathrm{C} \quad 4.020440 \mathrm{E}-05$ \$U-238

$93237.70 \mathrm{C} \quad 4.326552 \mathrm{E}-07 \quad \$ \mathrm{~Np} 237$

$94239.70 \mathrm{C} \quad 8.335069 \mathrm{E}-07$ \$Pu239

$94240.70 \mathrm{C} \quad 7.151042 \mathrm{E}-08$ \$Pu240

$94241.70 \mathrm{C} \quad 2.502678 \mathrm{E}-08$ \$Pu 241

$36083.70 \mathrm{C} \quad 5.804151 \mathrm{E}-07$ \$Kr-83

$42095.70 \mathrm{C} \quad 2.267771 \mathrm{E}-07$ \$Mo-95

$44101.70 \mathrm{C} \quad 5.745353 \mathrm{E}-06$ \$Ru101

$45103.70 \mathrm{C} \quad 8.131673 \mathrm{E}-07$ \$Rh103

$45105.70 \mathrm{C} \quad 1.457452 \mathrm{E}-30$ \$Rh105

$48113.70 \mathrm{C} \quad 1.475002 \mathrm{E}-09$ \$Cd113

$54131.70 \mathrm{C} \quad 2.012717 \mathrm{E}-06 \quad \$ \mathrm{Xe1} 131$

$54133.70 \mathrm{C} \quad 1.150411 \mathrm{E}-30$ \$Xe133

$55133.70 \mathrm{C} \quad 5.312807 \mathrm{E}-06 \$ \mathrm{Cs} 133$

$54135.70 \mathrm{C} \quad 1.124996 \mathrm{E}-30$ \$Xe135

$57140.70 \mathrm{C} \quad 1.092811 \mathrm{E}-30$ \$La140

$58141.70 \mathrm{C} \quad 1.085075 \mathrm{E}-30$ \$Ce141

$59143.70 \mathrm{C} \quad 1.069872 \mathrm{E}-30 \quad \$ \operatorname{Pr} 143$

$60143.70 \mathrm{C} \quad 2.944214 \mathrm{E}-06 \$ \mathrm{Nd} 143$

$60145.70 \mathrm{C} \quad 4.303802 \mathrm{E}-06 \$ \mathrm{Nd1} 45$

$61147.70 \mathrm{C} \quad 1.040712 \mathrm{E}-30 \quad \$ \mathrm{Pm} 147$

$61149.70 \mathrm{C} \quad 1.026713 \mathrm{E}-30 \$ \mathrm{Pm} 149$

$62149.70 \mathrm{C} \quad 5.242243 \mathrm{E}-08$ \$Sm149

$61151.70 \mathrm{C} \quad 1.013077 \mathrm{E}-30 \quad \$ \mathrm{Pm} 151$

$62151.70 \mathrm{C} \quad 1.560684 \mathrm{E}-07$ \$Sm151

$62152.70 \mathrm{C} \quad 5.298336 \mathrm{E}-07$ \$Sm152

$63153.70 \mathrm{C} \quad 2.241634 \mathrm{E}-07$ \$Eu153

$63155.70 \mathrm{C} \quad 2.580812 \mathrm{E}-08$ \$Eu155

$64157.70 \mathrm{C} \quad 2.891907 \mathrm{E}-10$ \$Gd157

lwtr.10t 
C

C

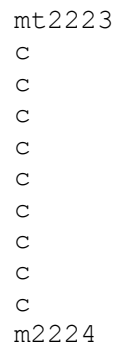

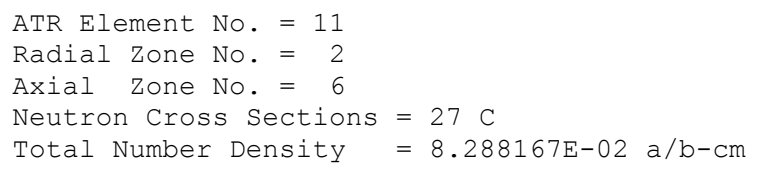




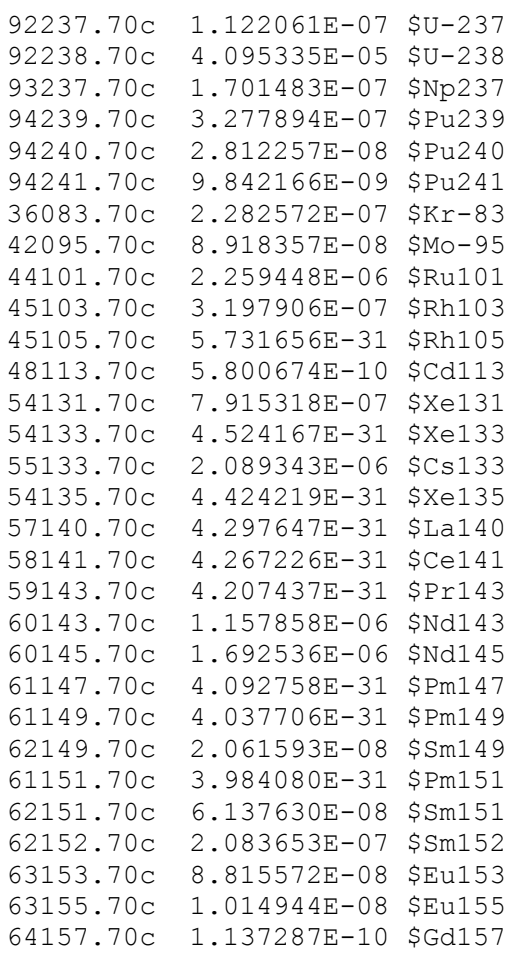
mt2224 lwtr.10t

c

C

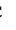

$\mathrm{C}$

m2225

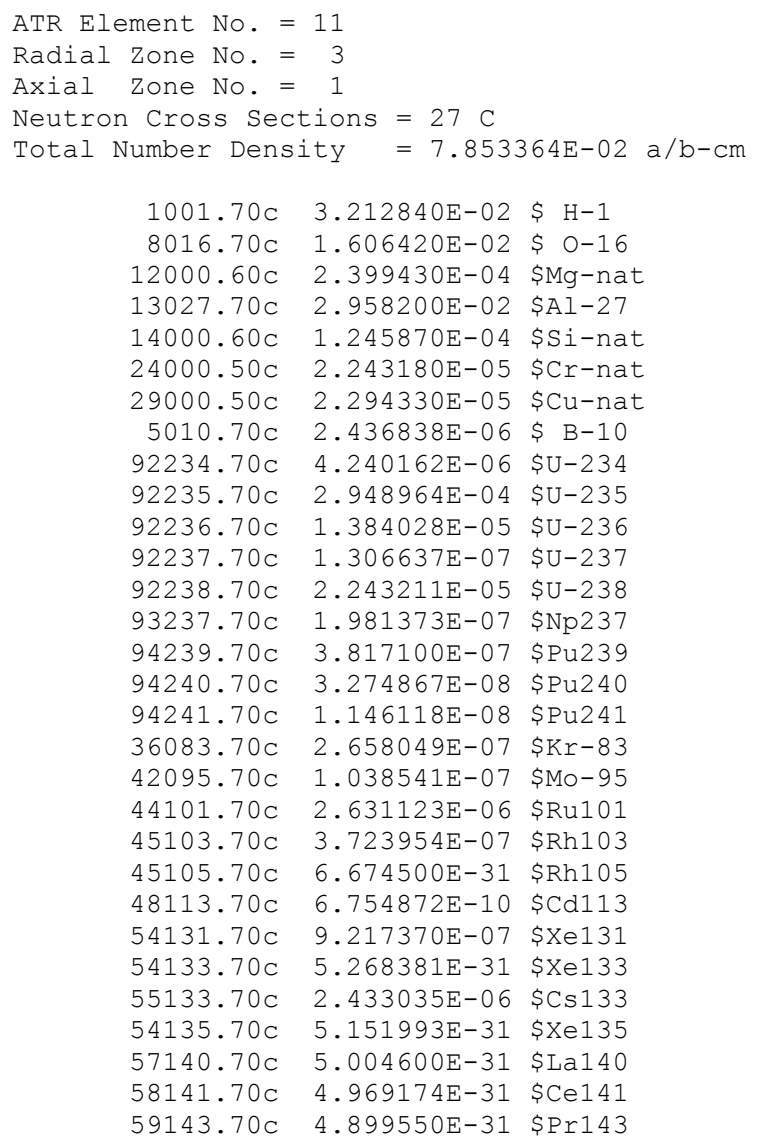


$60143.70 \mathrm{C} \quad 1.348323 \mathrm{E}-06 \$ \mathrm{Nd} 143$

$60145.70 \mathrm{C} \quad 1.970955 \mathrm{E}-06 \$ \mathrm{Nd145}$

$61147.70 \mathrm{C} \quad 4.766008 \mathrm{E}-31$ \$Pm147

$61149.70 \mathrm{C} \quad 4.701899 \mathrm{E}-31 \$ \mathrm{Pm} 149$

$62149.70 \mathrm{C} \quad 2.400720 \mathrm{E}-08$ \$Sm149

$61151.70 \mathrm{c} \quad 4.639452 \mathrm{E}-31$ \$Pm151

$62151.70 \mathrm{C} \quad 7.147256 \mathrm{E}-08 \quad \$ \mathrm{Sm} 151$

$62152.70 \mathrm{C} \quad 2.426408 \mathrm{E}-07 \quad \$ \mathrm{Sm} 152$

$63153.70 \mathrm{C} \quad 1.026572 \mathrm{E}-07$ \$Eu153

$63155.70 \mathrm{C} \quad 1.181900 \mathrm{E}-08 \quad \$ \mathrm{Eu} 155$

mt2225 lwtr.10t

$64157.70 \mathrm{C} \quad 1.324368 \mathrm{E}-10$ \$Gd157

$\mathrm{c}$

C

C

ATR Element No. = 11

Radial Zone No. $=3$

Axial Zone No. = 2

Neutron Cross Sections $=27 \mathrm{C}$

Total Number Density $=7.850948 \mathrm{E}-02 \mathrm{a} / \mathrm{b}-\mathrm{cm}$

$1001.70 \mathrm{C} \quad 3.212840 \mathrm{E}-02 \$ \mathrm{H}-1$

$8016.70 \mathrm{C} \quad 1.606420 \mathrm{E}-02$ \$ O-16

$12000.60 \mathrm{C} \quad 2.399430 \mathrm{E}-04$ \$Mg-nat

$13027.70 \mathrm{C} \quad 2.958200 \mathrm{E}-02$ \$Al-27

$14000.60 \mathrm{C} \quad 1.245870 \mathrm{E}-04$ \$Si-nat

$24000.50 \mathrm{C} \quad 2.243180 \mathrm{E}-05$ \$Cr-nat

$29000.50 \mathrm{C} \quad 2.294330 \mathrm{E}-05$ \$Cu-nat

$5010.70 \mathrm{C} \quad 2.436838 \mathrm{E}-06$ \$ -10

$92234.70 \mathrm{C} \quad 4.009083 \mathrm{E}-06$ \$U-234

$92235.70 \mathrm{C} \quad 2.583801 \mathrm{E}-04 \quad \$ \mathrm{U}-235$

$92236.70 \mathrm{C} \quad 2.029114 \mathrm{E}-05 \quad \mathrm{SU}-236$

$92237.70 \mathrm{C} \quad 2.062141 \mathrm{E}-07 \quad \$ \mathrm{U}-237$

$92238.70 \mathrm{C} \quad 2.210525 \mathrm{E}-05$ \$U-238

$93237.70 \mathrm{C} \quad 3.127014 \mathrm{E}-07$ \$Np237

$94239.70 \mathrm{C} \quad 6.024168 \mathrm{E}-07$ \$Pu239

$94240.70 \mathrm{C} \quad 5.168413 \mathrm{E}-08$ \$Pu240

$94241.70 \mathrm{C} \quad 1.808810 \mathrm{E}-08$ \$Pu241

$36083.70 \mathrm{C} \quad 4.194948 \mathrm{E}-07$ \$Kr-83

$42095.70 \mathrm{C} \quad 1.639030 \mathrm{E}-07$ \$Mo-95

$44101.70 \mathrm{C} \quad 4.152452 \mathrm{E}-06$ \$Ru101

$45103.70 \mathrm{C} \quad 5.877163 \mathrm{E}-07$ \$Rh103

$45105.70 \mathrm{C} \quad 1.053373 \mathrm{E}-30$ \$Rh105

$48113.70 \mathrm{C} \quad 1.066057 \mathrm{E}-09 \$ \mathrm{Cd} 113$

$54131.70 \mathrm{C} \quad 1.454690 \mathrm{E}-06 \quad \$ \mathrm{Xe1} 31$

$54133.70 \mathrm{C} \quad 8.314588 \mathrm{E}-31$ \$Xe133

$55133.70 \mathrm{C} \quad 3.839829 \mathrm{E}-06 \quad \$ \mathrm{Cs} 133$

$54135.70 \mathrm{C} \quad 8.130903 \mathrm{E}-31$ \$Xe135

$57140.70 \mathrm{C} \quad 7.898288 \mathrm{E}-31$ \$La140

$58141.70 \mathrm{C} \quad 7.842376 \mathrm{E}-31$ \$Ce141

$59143.70 \mathrm{C} \quad 7.732496 \mathrm{E}-31$ \$Pr143

$60143.70 \mathrm{C} \quad 2.127930 \mathrm{E}-06 \$ \mathrm{Nd} 143$

$60145.70 \mathrm{C} \quad 3.110571 \mathrm{E}-06 \$ \mathrm{Nd1} 45$

$61147.70 \mathrm{C} \quad 7.521739 \mathrm{E}-31$ \$Pm147

$61149.70 \mathrm{C} \quad 7.420562 \mathrm{E}-31$ \$Pm149

$62149.70 \mathrm{C} \quad 3.788829 \mathrm{E}-08$ \$Sm149

$61151.70 \mathrm{C} \quad 7.322008 \mathrm{E}-31$ \$Pm151

$62151.70 \mathrm{C} \quad 1.127984 \mathrm{E}-07$ \$Sm151

$62152.70 \mathrm{C} \quad 3.829371 \mathrm{E}-07$ \$Sm152

$63153.70 \mathrm{C} \quad 1.620141 \mathrm{E}-07$ \$Eu153

$63155.70 \mathrm{C} \quad 1.865281 \mathrm{E}-08$ \$Eu155

mt2226

$\mathrm{C}$

$\mathrm{C}$

C

lwtr.10t

ATR Element No. = 11

Radial Zone No. $=3$

Axial Zone No. $=3$ 
C

m2227

mt 2227

C

C

C

C

C

C

C

C

m2228

Neutron Cross Sections $=27 \mathrm{C}$

Total Number Density $=7.849169 \mathrm{E}-02 \mathrm{a} / \mathrm{b}-\mathrm{cm}$

\begin{tabular}{|c|c|c|}
\hline $1001.70 \mathrm{C}$ & $3.212840 \mathrm{E}-02$ & $\$ \mathrm{H}-1$ \\
\hline $8016.70 \mathrm{c}$ & $1.606420 \mathrm{E}-02$ & $\$ 0-16$ \\
\hline $12000.60 \mathrm{c}$ & $2.399430 \mathrm{E}-04$ & \$Mg-nat \\
\hline $13027.70 \mathrm{C}$ & $2.958200 \mathrm{E}-02$ & $\$ A 1-27$ \\
\hline $14000.60 \mathrm{C}$ & $1.245870 \mathrm{E}-04$ & $\$$ Si-nat \\
\hline $24000.50 \mathrm{C}$ & $2.243180 \mathrm{E}-05$ & $\$ C r-n a t$ \\
\hline $29000.50 \mathrm{C}$ & $2.294330 \mathrm{E}-05$ & \$Cu-nat \\
\hline $5010.70 \mathrm{C}$ & $2.436838 E-06$ & $\$ B-10$ \\
\hline $92234.70 \mathrm{C}$ & $3.838504 \mathrm{E}-06$ & $\$ U-234$ \\
\hline $92235.70 \mathrm{C}$ & $2.314243 E-04$ & $\$ U-235$ \\
\hline $92236.70 \mathrm{C}$ & $2.505306 \mathrm{E}-05$ & $\$ U-236$ \\
\hline $92237.70 \mathrm{C}$ & $2.619842 \mathrm{E}-07$ & $\$ U-237$ \\
\hline $92238.70 \mathrm{C}$ & $2.186397 \mathrm{E}-05$ & $\$ U-238$ \\
\hline $93237.70 \mathrm{C}$ & $3.972707 \mathrm{E}-07$ & $\$ N p 237$ \\
\hline $94239.70 \mathrm{C}$ & $7.653389 \mathrm{E}-07$ & \$Pu239 \\
\hline $94240.70 \mathrm{C}$ & $6.566197 E-08$ & \$Pu 240 \\
\hline $94241.70 \mathrm{c}$ & $2.297998 E-08$ & \$Pu241 \\
\hline $36083.70 \mathrm{C}$ & $5.329462 E-07$ & $\$ K r-83$ \\
\hline $42095.70 \mathrm{C}$ & $2.082302 \mathrm{E}-07$ & $\$ M \circ-95$ \\
\hline $44101.70 \mathrm{C}$ & $5.275472 E-06$ & \$Ru101 \\
\hline $45103.70 \mathrm{C}$ & $7.466628 \mathrm{E}-07$ & \$Rh103 \\
\hline $45105.70 \mathrm{C}$ & $1.338255 E-30$ & \$Rh105 \\
\hline $48113.70 \mathrm{C}$ & $1.354370 E-09$ & $\$ \mathrm{Cd} 113$ \\
\hline $54131.70 \mathrm{C}$ & $1.848108 E-06$ & $\$ \mathrm{Xe1} 31$ \\
\hline $54133.70 \mathrm{C}$ & $1.056325 \mathrm{E}-30$ & \$xe133 \\
\hline $55133.70 \mathrm{C}$ & $4.878301 \mathrm{E}-06$ & $\$ \mathrm{Cs} 133$ \\
\hline $54135.70 \mathrm{C}$ & $1.032988 \mathrm{E}-30$ & $\$ x e 135$ \\
\hline $57140.70 \mathrm{C}$ & $1.003436 \mathrm{E}-30$ & \$La140 \\
\hline $58141.70 \mathrm{C}$ & $9.963327 E-31$ & $\$ \mathrm{Ce} 141$ \\
\hline $59143.70 \mathrm{C}$ & $9.823730 \mathrm{E}-31$ & $\$ \operatorname{Pr} 143$ \\
\hline $60143.70 \mathrm{c}$ & $2.703423 E-06$ & $\$ N d 143$ \\
\hline $60145.70 \mathrm{C}$ & $3.951818 \mathrm{E}-06$ & $\$ N d 145$ \\
\hline $61147.70 \mathrm{C}$ & $9.555974 \mathrm{E}-31$ & $\$ \operatorname{Pm} 147$ \\
\hline $61149.70 \mathrm{C}$ & $9.427434 \mathrm{E}-31$ & $\$ P \operatorname{Pm} 149$ \\
\hline $62149.70 \mathrm{C}$ & $4.813509 \mathrm{E}-08$ & $\$ \operatorname{Sm} 149$ \\
\hline $61151.70 \mathrm{C}$ & $9.302227 E-31$ & $\$ P \operatorname{Pm} 151$ \\
\hline $62151.70 \mathrm{c}$ & $1.433044 \mathrm{E}-07$ & $\$$ Sm1 51 \\
\hline $62152.70 \mathrm{C}$ & $4.865015 E-07$ & $\$ \mathrm{Sm} 152$ \\
\hline $63153.70 \mathrm{c}$ & $2.058304 \mathrm{E}-07$ & \$Eu153 \\
\hline $63155.70 \mathrm{C}$ & $2.369742 \mathrm{E}-08$ & \$Eu155 \\
\hline $64157.70 \mathrm{C}$ & $2.655393 \mathrm{E}-10$ & \$Gd1 57 \\
\hline
\end{tabular}

lwtr. 10 t

ATR Element No. $=11$

Radial Zone No. $=3$

Axial Zone No. = 4

Neutron Cross Sections $=27 \mathrm{C}$

Total Number Density $=7.848465 \mathrm{E}-02 \mathrm{a} / \mathrm{b}-\mathrm{cm}$

$1001.70 \mathrm{C} \quad 3.212840 \mathrm{E}-02 \$ \mathrm{H}-1$

$8016.70 \mathrm{C} \quad 1.606420 \mathrm{E}-02 \$ 0-16$

$12000.60 \mathrm{C} \quad 2.399430 \mathrm{E}-04$ \$Mg-nat

$13027.70 \mathrm{C} \quad 2.958200 \mathrm{E}-02$ \$Al-27

$14000.60 \mathrm{C} \quad 1.245870 \mathrm{E}-04$ \$Si-nat

$24000.50 \mathrm{C} \quad 2.243180 \mathrm{E}-05$ \$Cr-nat

$29000.50 \mathrm{C} \quad 2.294330 \mathrm{E}-05$ \$Cu-nat

$5010.70 \mathrm{C} \quad 2.436838 \mathrm{E}-06$ \$ B-10

$92234.70 \mathrm{C} \quad 3.771083 \mathrm{E}-06 \quad \$ \mathrm{U}-234$

$92235.70 \mathrm{C} \quad 2.207700 \mathrm{E}-04$ \$U-235

$92236.70 \mathrm{C} \quad 2.693526 \mathrm{E}-05 \quad \$ \mathrm{U}-236$

$92237.70 \mathrm{C} \quad 2.840278 \mathrm{E}-07$ \$U-237

$92238.70 \mathrm{C} \quad 2.176861 \mathrm{E}-05 \mathrm{\$ U}-238$

$93237.70 \mathrm{C} \quad 4.306975 \mathrm{E}-07$ \$Np237

$94239.70 \mathrm{C} \quad 8.297353 \mathrm{E}-07$ \$Pu239

$94240.70 \mathrm{C} \quad 7.118684 \mathrm{E}-08$ \$Pu 240 
$94241.70 \mathrm{C} \quad 2.491353 \mathrm{E}-08 \quad \$ \mathrm{Pu} 241$

$36083.70 \mathrm{C} \quad 5.777887 \mathrm{E}-07$ \$Kr-83

$42095.70 \mathrm{C} \quad 2.257509 \mathrm{E}-07$ \$Mo-95

$44101.70 \mathrm{C} \quad 5.719355 \mathrm{E}-06$ \$Ru101

$45103.70 \mathrm{C} \quad 8.094877 \mathrm{E}-07$ \$Rh103

$45105.70 \mathrm{C} \quad 1.450857 \mathrm{E}-30$ \$Rh105

$48113.70 \mathrm{C} \quad 1.468328 \mathrm{E}-09$ \$Cd113

$54131.70 \mathrm{C} \quad 2.003609 \mathrm{E}-06$ \$Xe131

$54133.70 \mathrm{C} \quad 1.145205 \mathrm{E}-30$ \$Xe133

$55133.70 \mathrm{C} \quad 5.288767 \mathrm{E}-06 \quad \$ \mathrm{Cs} 133$

$54135.70 \mathrm{C} \quad 1.119905 \mathrm{E}-30$ \$Xe135

$57140.70 \mathrm{C} \quad 1.087866 \mathrm{E}-30$ \$La140

$58141.70 \mathrm{C} \quad 1.080165 \mathrm{E}-30 \quad \$ \mathrm{Ce} 141$

$59143.70 \mathrm{C} \quad 1.065031 \mathrm{E}-30 \quad \$ \mathrm{Pr} 143$

$60143.70 \mathrm{C} \quad 2.930892 \mathrm{E}-06 \$ \mathrm{Nd1} 43$

$60145.70 \mathrm{C} \quad 4.284327 \mathrm{E}-06 \$ \mathrm{Nd} 145$

$61147.70 \mathrm{C} \quad 1.036002 \mathrm{E}-30 \$ \mathrm{Pm} 147$

$61149.70 \mathrm{C} \quad 1.022067 \mathrm{E}-30 \$ \mathrm{Pm} 149$

$62149.70 \mathrm{C} \quad 5.218521 \mathrm{E}-08$ \$Sm149

$61151.70 \mathrm{C} \quad 1.008493 \mathrm{E}-30$ \$Pm151

$62151.70 \mathrm{C} \quad 1.553622 \mathrm{E}-07$ \$Sm151

$62152.70 \mathrm{C} \quad 5.274362 \mathrm{E}-07$ \$Sm152

$63153.70 \mathrm{C} \quad 2.231491 \mathrm{E}-07$ \$Eu153

$63155.70 \mathrm{C} \quad 2.569134 \mathrm{E}-08$ \$Eu155

mt2228 lwtr.10t

c

$\mathrm{C}$

$\mathrm{C}$

C

$\mathrm{C}$

C

$64157.70 \mathrm{C} \quad 2.878821 \mathrm{E}-10 \quad \$ \mathrm{Gd} 157$

m2229

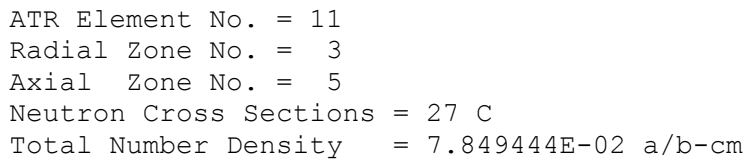

\begin{tabular}{|c|c|c|}
\hline $1001.70 \mathrm{c}$ & 3.212840E-02 & $\$ \mathrm{H}-1$ \\
\hline $8016.70 \mathrm{C}$ & $1.606420 \mathrm{E}-02$ & $\$ 0-16$ \\
\hline $2000.60 \mathrm{c}$ & $2.399430 \mathrm{E}-04$ & $\$ M g-n a t$ \\
\hline $27.70 \mathrm{c}$ & $2.958200 \mathrm{E}-02$ & $\$ A l-27$ \\
\hline $.60 \mathrm{C}$ & $1.245870 \mathrm{E}-04$ & \$Si-nat \\
\hline $00.50 \mathrm{c}$ & $2.243180 \mathrm{E}-05$ & $\$ C r-n a t$ \\
\hline & $2.294330 \mathrm{E}-05$ & $\$ \mathrm{Cu}-$ nat \\
\hline $.70 \mathrm{C}$ & $2.436838 E-06$ & $\$ B-10$ \\
\hline $.70 \mathrm{C}$ & $3.864822 \mathrm{E}-06$ & $\$ U-234$ \\
\hline $.70 \mathrm{C}$ & $2.355833 \mathrm{E}-04$ & $\$ U-235$ \\
\hline $.70 \mathrm{C}$ & $2.431836 \mathrm{E}-05$ & $\$ U-236$ \\
\hline $.70 \mathrm{C}$ & $2.533796 \mathrm{E}-07$ & $\$ U-237$ \\
\hline $.70 \mathrm{C}$ & $2.190120 \mathrm{E}-05$ & $\$ U-238$ \\
\hline $.70 \mathrm{C}$ & $27 E-07$ & \$Np237 \\
\hline $.70 \mathrm{c}$ & $7.402020 \mathrm{E}-07$ & \$Pu239 \\
\hline $.70 \mathrm{C}$ & $6 E-08$ & \$Pu240 \\
\hline $.70 \mathrm{C}$ & $2 E-08$ & \$Pu241 \\
\hline $.70 \mathrm{C}$ & $20 E-07$ & $\$ K r-83$ \\
\hline & $\mathrm{E}-07$ & $\$ M o-95$ \\
\hline $0 \mathrm{c}$ & $3 E-06$ & \$Ru101 \\
\hline & 7.2 & \$Rh103 \\
\hline Dc & $E-30$ & \$Rh105 \\
\hline & $E-09$ & $\$ \mathrm{Cd} 113$ \\
\hline C & $8 E-06$ & $\$ \mathrm{Xe1} 31$ \\
\hline 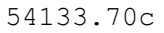 & $1 E-30$ & \$Xe133 \\
\hline C & $3 E-06$ & $\$ C s 133$ \\
\hline & $3 E-31$ & $\$ x e 135$ \\
\hline C & $8 E-31$ & \$La140 \\
\hline & $1 E-31$ & $\$ \mathrm{Ce} 141$ \\
\hline 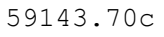 & $8 E-31$ & \$Pr143 \\
\hline & $631 E-06$ & $\$ N d 143$ \\
\hline 60 & $23 E-06$ & $\$ \mathrm{Nd} 145$ \\
\hline & $17 E-31$ & $\$ \operatorname{Pm} 147$ \\
\hline & $7 E-31$ & $\$ \operatorname{Pm} 149$ \\
\hline & $12 E-08$ & \$Sm149 \\
\hline
\end{tabular}




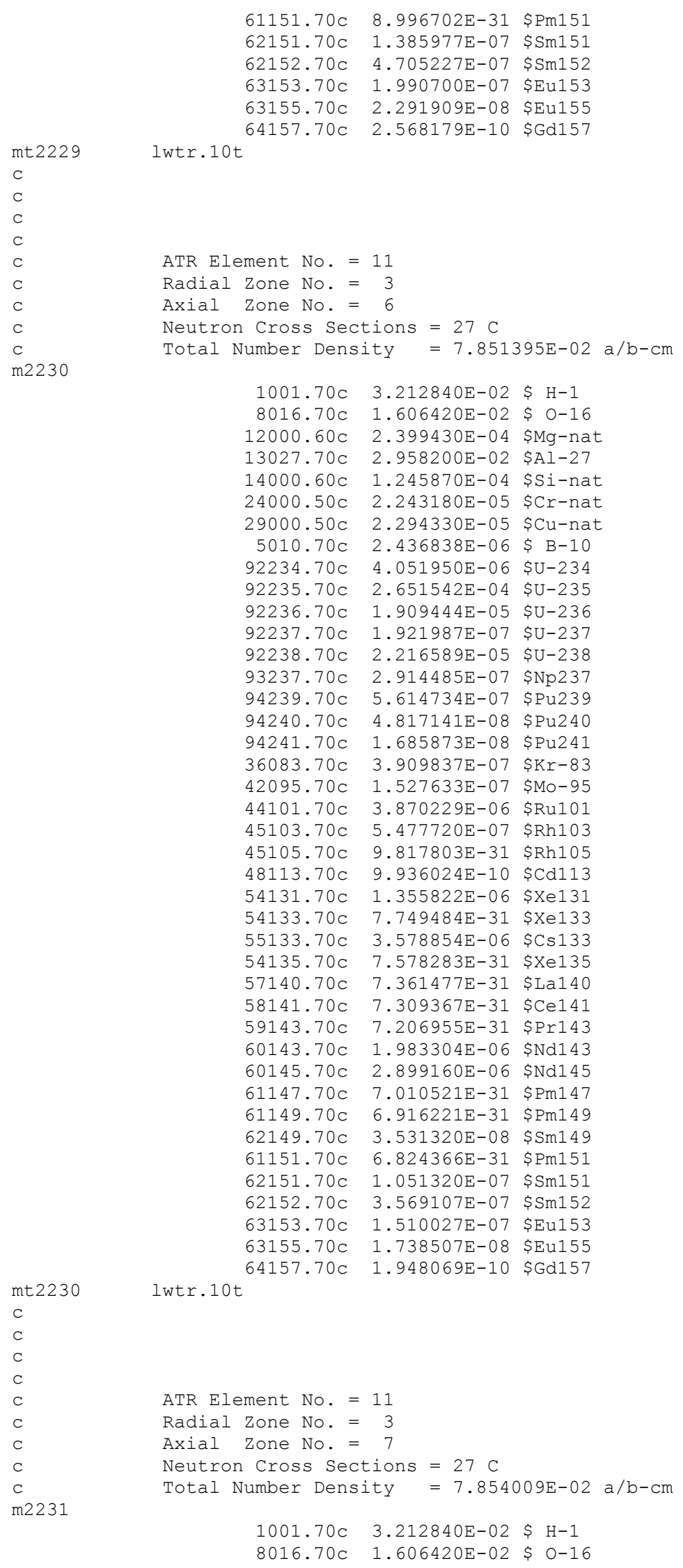




$$
\begin{aligned}
& 12000.60 \mathrm{C} \quad 2.399430 \mathrm{E}-04 \text { \$Mg-nat } \\
& 13027.70 \mathrm{C} \quad 2.958200 \mathrm{E}-02 \text { \$Al-27 } \\
& 14000.60 \mathrm{C} \quad 1.245870 \mathrm{E}-04 \text { \$Si-nat } \\
& 24000.50 \mathrm{C} \quad 2.243180 \mathrm{E}-05 \text { \$Cr-nat } \\
& 29000.50 \mathrm{C} \quad 2.294330 \mathrm{E}-05 \text { \$Cu-nat } \\
& 5010.70 \mathrm{C} \quad 2.436838 \mathrm{E}-06 \text { \$ B-10 } \\
& 92234.70 \mathrm{C} \quad 4.302437 \mathrm{E}-06 \quad \$ \mathrm{U}-234 \\
& 92235.70 \mathrm{C} \quad 3.047374 \mathrm{E}-04 \quad \$ \mathrm{U}-235 \\
& 92236.70 \mathrm{C} \quad 1.210178 \mathrm{E}-05 \quad \$ \mathrm{U}-236 \\
& 92237.70 \mathrm{C} \quad 1.103030 \mathrm{E}-07 \text { \$U-237 } \\
& 92238.70 \mathrm{C} \quad 2.252020 \mathrm{E}-05 \text { \$U-238 } \\
& 93237.70 \mathrm{C} \quad 1.672626 \mathrm{E}-07 \text { \$Np237 } \\
& 94239.70 \mathrm{C} \quad 3.222301 \mathrm{E}-07 \text { \$Pu239 } \\
& 94240.70 \mathrm{C} \quad 2.764561 \mathrm{E}-08 \text { \$Pu240 } \\
& 94241.70 \mathrm{C} \quad 9.675241 \mathrm{E}-09 \text { \$Pu241 } \\
& 36083.70 \mathrm{C} \quad 2.243859 \mathrm{E}-07 \text { \$Kr-83 } \\
& 42095.70 \mathrm{C} \quad 8.767099 \mathrm{E}-08 \text { \$Mo-95 } \\
& 44101.70 \mathrm{C} \quad 2.221128 \mathrm{E}-06 \text { \$Ru101 } \\
& 45103.70 \mathrm{C} \quad 3.143668 \mathrm{E}-07 \text { \$Rh103 } \\
& 45105.70 \mathrm{C} \quad 5.634446 \mathrm{E}-31 \text { \$Rh105 } \\
& 48113.70 \mathrm{C} \quad 5.702293 \mathrm{E}-10 \quad \$ \mathrm{Cd} 113 \\
& 54131.70 \mathrm{C} \quad 7.781073 \mathrm{E}-07 \text { \$Xe131 } \\
& 54133.70 \mathrm{C} \quad 4.447436 \mathrm{E}-31 \text { \$Xe133 } \\
& 55133.70 \mathrm{C} \quad 2.053907 \mathrm{E}-06 \quad \$ \mathrm{Cs} 133 \\
& 54135.70 \mathrm{C} \quad 4.349183 \mathrm{E}-31 \text { \$Xe135 } \\
& 57140.70 \mathrm{C} \quad 4.224758 \mathrm{E}-31 \text { \$La140 } \\
& 58141.70 \mathrm{C} \quad 4.194852 \mathrm{E}-31 \text { \$Ce141 } \\
& 59143.70 \mathrm{C} \quad 4.136078 \mathrm{E}-31 \quad \$ \operatorname{Pr} 143 \\
& 60143.70 \mathrm{C} \quad 1.138220 \mathrm{E}-06 \$ \mathrm{Nd} 143 \\
& 60145.70 \mathrm{C} \quad 1.663831 \mathrm{E}-06 \text { \$Nd145 } \\
& 61147.70 \mathrm{C} \quad 4.023344 \mathrm{E}-31 \text { \$Pm147 } \\
& 61149.70 \mathrm{C} \quad 3.969225 \mathrm{E}-31 \quad \$ \mathrm{Pm} 149 \\
& 62149.70 \mathrm{C} \quad 2.026627 \mathrm{E}-08 \quad \$ \mathrm{Sm} 149 \\
& 61151.70 \mathrm{C} \quad 3.916509 \mathrm{E}-31 \text { \$Pm151 } \\
& 62151.70 \mathrm{C} \quad 6.033534 \mathrm{E}-08 \quad \$ \mathrm{Sm} 151 \\
& 62152.70 \mathrm{C} \quad 2.048313 \mathrm{E}-07 \$ \mathrm{Sm} 152 \\
& 63153.70 \mathrm{C} \quad 8.666058 \mathrm{E}-08 \text { \$Eu153 } \\
& 63155.70 \mathrm{C} \quad 9.977306 \mathrm{E}-09 \text { \$Eu155 }
\end{aligned}
$$

mt2231 lwtr.10t

C

C

C

C

C

C

C

C

m2232

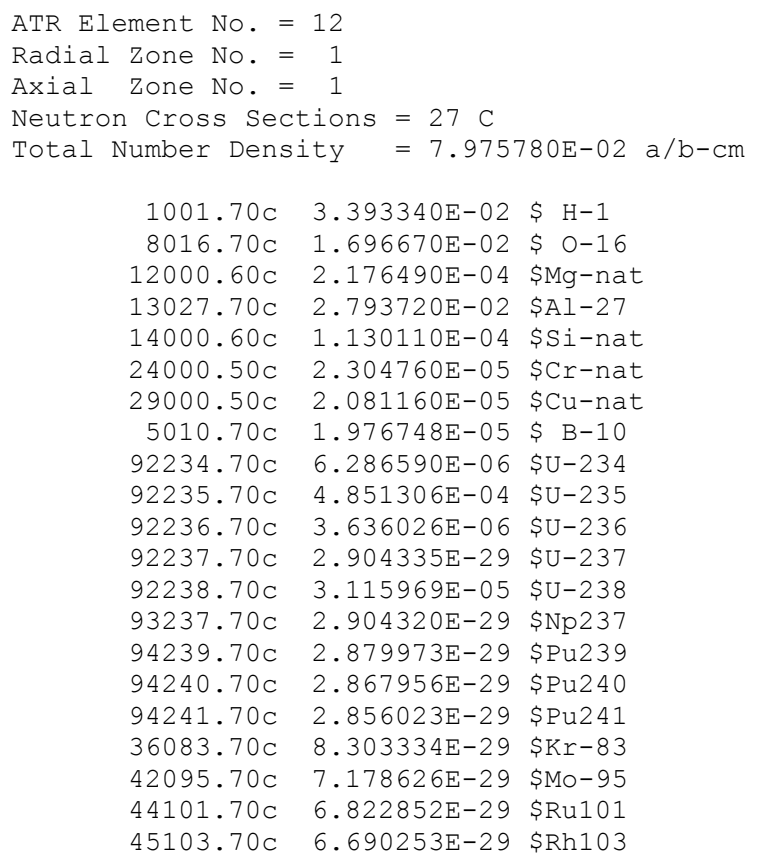




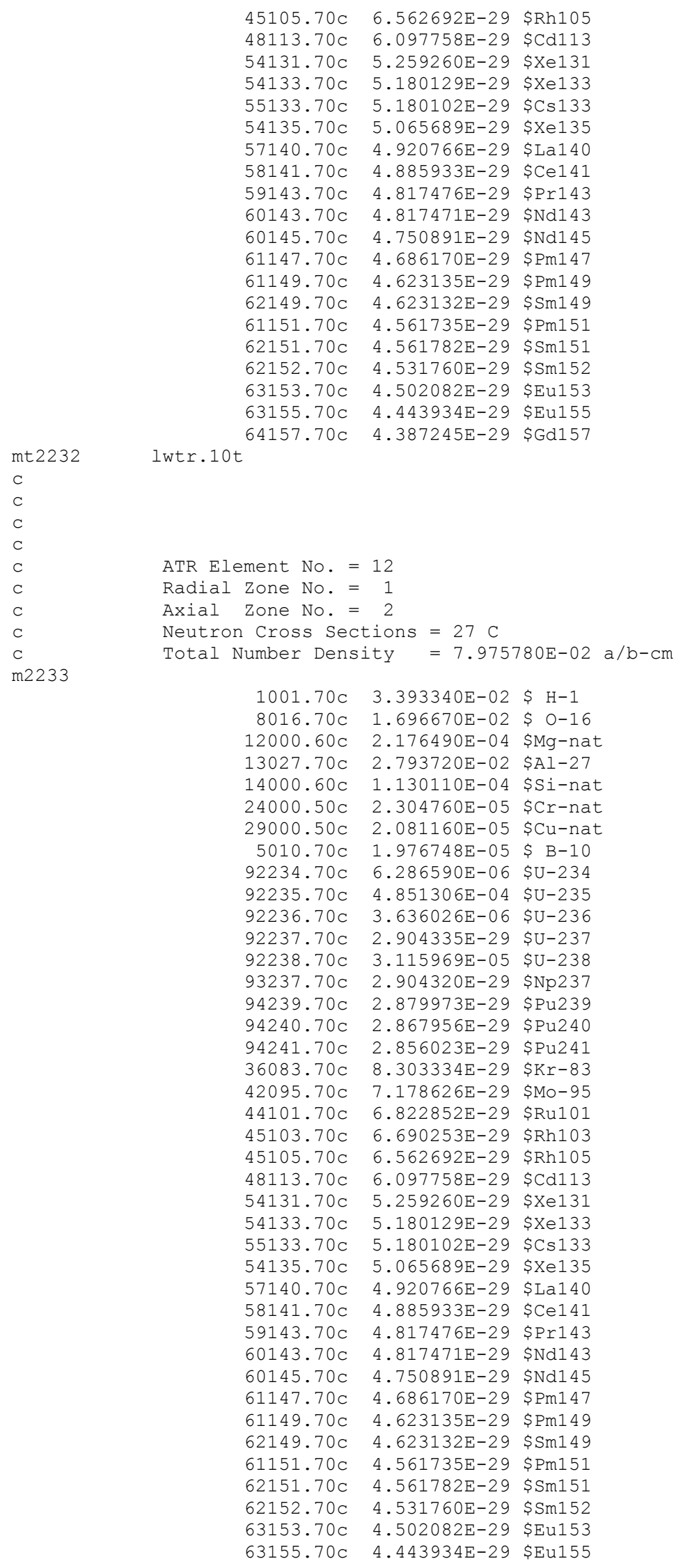


$64157.70 \mathrm{C} \quad 4.387245 \mathrm{E}-29 \$ \mathrm{Gd157}$

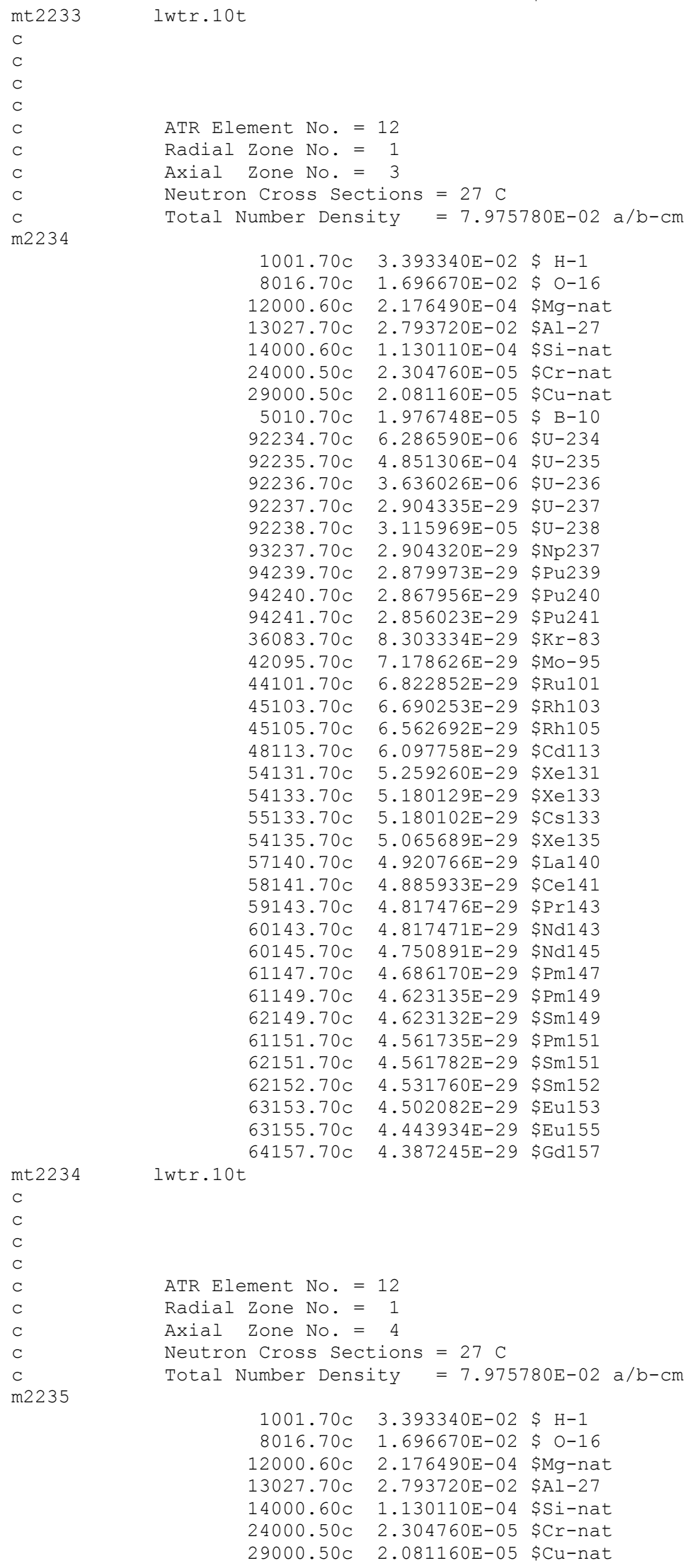




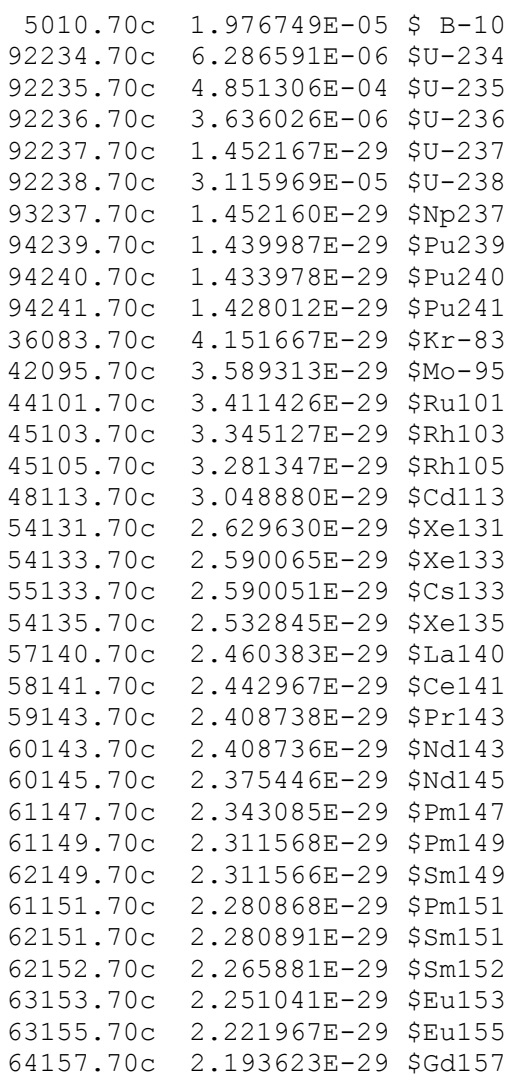

mt2235 lwtr.10t

c

C

C

C

C

$\mathrm{C}$

C

m2236

ATR Element No. $=12$

Radial Zone No. = 1

Axial Zone No. = 5

Neutron Cross Sections $=27 \mathrm{C}$

Total Number Density $=7.975780 \mathrm{E}-02 \mathrm{a} / \mathrm{b}-\mathrm{cm}$

$1001.70 \mathrm{C} \quad 3.393340 \mathrm{E}-02$ \$ $\mathrm{H}-1$

$8016.70 \mathrm{C} \quad 1.696670 \mathrm{E}-02$ \$ O-16

$12000.60 \mathrm{C} \quad 2.176490 \mathrm{E}-04$ \$Mg-nat

$13027.70 \mathrm{C} \quad 2.793720 \mathrm{E}-02$ \$Al-27

$14000.60 \mathrm{C} \quad 1.130110 \mathrm{E}-04$ \$Si-nat

$24000.50 \mathrm{C} \quad 2.304760 \mathrm{E}-05$ \$Cr-nat

$29000.50 \mathrm{C} \quad 2.081160 \mathrm{E}-05$ \$Cu-nat

$5010.70 \mathrm{C} \quad 1.976748 \mathrm{E}-05$ \$ B-10

$92234.70 \mathrm{C} \quad 6.286590 \mathrm{E}-06 \quad \$ \mathrm{U}-234$

$92235.70 \mathrm{C} \quad 4.851306 \mathrm{E}-04 \quad \$ \mathrm{U}-235$

$92236.70 \mathrm{C} \quad 3.636026 \mathrm{E}-06$ \$U-236

$92237.70 \mathrm{C} \quad 2.904335 \mathrm{E}-29$ \$U-237

$92238.70 \mathrm{C} \quad 3.115969 \mathrm{E}-05 \mathrm{\$ U}-238$

$93237.70 \mathrm{C} \quad 2.904320 \mathrm{E}-29 \quad \$ N p 237$

$94239.70 \mathrm{C} \quad 2.879973 \mathrm{E}-29$ \$Pu239

$94240.70 \mathrm{C} \quad 2.867956 \mathrm{E}-29$ \$Pu240

$94241.70 \mathrm{C} \quad 2.856023 \mathrm{E}-29$ \$Pu241

$36083.70 \mathrm{C} \quad 8.303334 \mathrm{E}-29$ \$Kr-83

$42095.70 \mathrm{C} \quad 7.178626 \mathrm{E}-29$ \$Mo-95

$44101.70 \mathrm{C} \quad 6.822852 \mathrm{E}-29$ \$Ru101

$45103.70 \mathrm{C} \quad 6.690253 \mathrm{E}-29$ \$Rh103

$45105.70 \mathrm{C} \quad 6.562692 \mathrm{E}-29$ \$Rh105

$48113.70 \mathrm{C} \quad 6.097758 \mathrm{E}-29 \quad \$ \mathrm{Cd} 113$

$54131.70 \mathrm{C} \quad 5.259260 \mathrm{E}-29 \$ \mathrm{Xe} 131$

$54133.70 \mathrm{C} \quad 5.180129 \mathrm{E}-29$ \$Xe133

$55133.70 \mathrm{C} \quad 5.180102 \mathrm{E}-29 \$ \mathrm{Cs} 133$ 


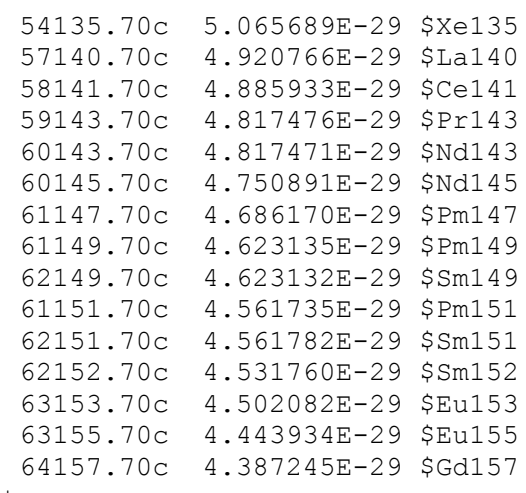

mt2236 lwtr.10t

$\mathrm{C}$

$\mathrm{C}$

C ATR Element No. = 12

C Radial Zone No. = 1

C Axial Zone No. = 6

C Neutron Cross Sections $=27 \mathrm{C}$

c Total Number Density $=7.975780 \mathrm{E}-02 \mathrm{a} / \mathrm{b}-\mathrm{cm}$

m2237

$\begin{array}{lll}1001.70 \mathrm{C} & 3.393340 \mathrm{E}-02 & \$ \mathrm{H}-1 \\ 8016.70 \mathrm{C} & 1.696670 \mathrm{E}-02 & \$ \mathrm{O}-1\end{array}$

$12000.60 \mathrm{C} \quad 2.176490 \mathrm{E}-04$ \$Mg-nat

$13027.70 \mathrm{C} \quad 2.793720 \mathrm{E}-02$ \$Al-27

$14000.60 \mathrm{C} \quad 1.130110 \mathrm{E}-04$ \$Si-nat

$24000.50 \mathrm{C} \quad 2.304760 \mathrm{E}-05$ \$Cr-nat

$29000.50 \mathrm{C} \quad 2.081160 \mathrm{E}-05$ \$Cu-nat

$5010.70 \mathrm{C} \quad 1.976748 \mathrm{E}-05$ \$ B-10

$92234.70 \mathrm{C} \quad 6.286590 \mathrm{E}-06 \quad \$ \mathrm{U}-234$

$92235.70 \mathrm{C} \quad 4.851306 \mathrm{E}-04$ \$U-235

$92236.70 \mathrm{C} \quad 3.636026 \mathrm{E}-06 \quad \$ \mathrm{U}-236$

$92237.70 \mathrm{C} \quad 2.904335 \mathrm{E}-29 \quad \$ \mathrm{U}-237$

$92238.70 \mathrm{C} \quad 3.115969 \mathrm{E}-05 \quad \$ \mathrm{U}-238$

$93237.70 \mathrm{C} \quad 2.904320 \mathrm{E}-29 \quad \$ N p 237$

$94239.70 \mathrm{C} \quad 2.879973 \mathrm{E}-29$ \$Pu239

$94240.70 \mathrm{C} \quad 2.867956 \mathrm{E}-29$ \$Pu240

$94241.70 \mathrm{C} \quad 2.856023 \mathrm{E}-29$ \$Pu241

$36083.70 \mathrm{C} \quad 8.303334 \mathrm{E}-29$ \$Kr-83

$42095.70 \mathrm{C} \quad 7.178626 \mathrm{E}-29$ \$Mo-95

$44101.70 \mathrm{C} \quad 6.822852 \mathrm{E}-29$ \$Ru101

$45103.70 \mathrm{C} \quad 6.690253 \mathrm{E}-29$ \$Rh103

$45105.70 \mathrm{C} \quad 6.562692 \mathrm{E}-29 \$ \mathrm{Rh} 105$

$48113.70 \mathrm{C} \quad 6.097758 \mathrm{E}-29 \$ \mathrm{Cd} 113$

$54131.70 \mathrm{C} \quad 5.259260 \mathrm{E}-29$ \$Xe131

$54133.70 \mathrm{C} \quad 5.180129 \mathrm{E}-29$ \$Xe133

$55133.70 \mathrm{C} \quad 5.180102 \mathrm{E}-29 \$ \mathrm{Cs} 133$

$54135.70 \mathrm{C} \quad 5.065689 \mathrm{E}-29 \$ \mathrm{Xe135}$

$57140.70 \mathrm{C} \quad 4.920766 \mathrm{E}-29$ \$La140

$58141.70 \mathrm{C} \quad 4.885933 \mathrm{E}-29 \$ \mathrm{Ce} 141$

$59143.70 \mathrm{C} \quad 4.817476 \mathrm{E}-29 \$ \mathrm{Pr} 143$

$60143.70 \mathrm{C} \quad 4.817471 \mathrm{E}-29 \$ \mathrm{Nd} 143$

$60145.70 \mathrm{C} \quad 4.750891 \mathrm{E}-29 \$ \mathrm{Nd} 145$

$61147.70 \mathrm{C} \quad 4.686170 \mathrm{E}-29 \$ \mathrm{Pm} 147$

$61149.70 \mathrm{C} \quad 4.623135 \mathrm{E}-29 \$ \mathrm{Pm} 149$

$62149.70 \mathrm{C} \quad 4.623132 \mathrm{E}-29$ \$Sm149

$61151.70 \mathrm{C} \quad 4.561735 \mathrm{E}-29 \$ \mathrm{Pm} 151$

$62151.70 \mathrm{C} \quad 4.561782 \mathrm{E}-29 \$ \mathrm{Sm} 151$

$62152.70 \mathrm{C} \quad 4.531760 \mathrm{E}-29 \quad \$ \mathrm{Sm} 152$

$63153.70 \mathrm{C} \quad 4.502082 \mathrm{E}-29$ \$Eu153

$63155.70 \mathrm{C} \quad 4.443934 \mathrm{E}-29$ \$Eu155

mt2237 lwtr.10t

$64157.70 \mathrm{C} \quad 4.387245 \mathrm{E}-29$ \$Gd157

c

C

C 
C

C

ATR Element No. $=12$

Radial Zone No. = 1

Axial Zone No. $=7$

Neutron Cross Sections $=27 \mathrm{C}$

Total Number Density $=7.975780 \mathrm{E}-02 \mathrm{a} / \mathrm{b}-\mathrm{cm}$

$$
\begin{aligned}
& 1001.70 \mathrm{C} \quad 3.393340 \mathrm{E}-02 \text { \$ } \mathrm{H}-1 \\
& 8016.70 \mathrm{C} \quad 1.696670 \mathrm{E}-02 \text { \$ } 0-16 \\
& 12000.60 \mathrm{C} \quad 2.176490 \mathrm{E}-04 \text { \$Mg-nat } \\
& 13027.70 \mathrm{C} \quad 2.793720 \mathrm{E}-02 \text { \$Al-27 } \\
& 14000.60 \mathrm{C} \quad 1.130110 \mathrm{E}-04 \text { \$Si-nat } \\
& 24000.50 \mathrm{C} \quad 2.304760 \mathrm{E}-05 \text { \$Cr-nat } \\
& 29000.50 \mathrm{C} \quad 2.081160 \mathrm{E}-05 \text { \$Cu-nat } \\
& 5010.70 \mathrm{C} \quad 1.976748 \mathrm{E}-05 \text { \$ B-10 } \\
& 92234.70 \mathrm{C} \quad 6.286590 \mathrm{E}-06 \text { \$U-234 } \\
& 92235.70 \mathrm{C} \quad 4.851306 \mathrm{E}-04 \text { \$U-235 } \\
& 92236.70 \mathrm{C} \quad 3.636026 \mathrm{E}-06 \quad \$ \mathrm{U}-236 \\
& 92237.70 \mathrm{C} \quad 2.904335 \mathrm{E}-29 \text { \$U-237 } \\
& 92238.70 \mathrm{C} \quad 3.115969 \mathrm{E}-05 \quad \$ \mathrm{U}-238 \\
& 93237.70 \mathrm{C} \quad 2.904320 \mathrm{E}-29 \quad \$ \mathrm{~Np} 237 \\
& 94239.70 \mathrm{C} \quad 2.879973 \mathrm{E}-29 \text { \$Pu239 } \\
& 94240.70 \mathrm{C} \quad 2.867956 \mathrm{E}-29 \text { \$Pu } 240 \\
& 94241.70 \mathrm{C} \quad 2.856023 \mathrm{E}-29 \text { \$Pu241 } \\
& 36083.70 \mathrm{C} \quad 8.303334 \mathrm{E}-29 \$ \mathrm{Kr}-83 \\
& 42095.70 \mathrm{C} \quad 7.178626 \mathrm{E}-29 \text { \$Mo-95 } \\
& 44101.70 \mathrm{C} \quad 6.822852 \mathrm{E}-29 \text { \$Ru101 } \\
& 45103.70 \mathrm{C} \quad 6.690253 \mathrm{E}-29 \text { \$Rh103 } \\
& 45105.70 \mathrm{C} \quad 6.562692 \mathrm{E}-29 \text { \$Rh105 } \\
& 48113.70 \mathrm{C} \quad 6.097758 \mathrm{E}-29 \$ \mathrm{Cd} 113 \\
& 54131.70 \mathrm{C} \quad 5.259260 \mathrm{E}-29 \$ \mathrm{Xe131} \\
& 54133.70 \mathrm{C} \quad 5.180129 \mathrm{E}-29 \$ \mathrm{Xe133} \\
& 55133.70 \mathrm{C} \quad 5.180102 \mathrm{E}-29 \$ \mathrm{Cs} 133 \\
& 54135.70 \mathrm{C} \quad 5.065689 \mathrm{E}-29 \text { \$Xe135 } \\
& 57140.70 \mathrm{C} \quad 4.920766 \mathrm{E}-29 \text { \$La140 } \\
& 58141.70 \mathrm{C} \quad 4.885933 \mathrm{E}-29 \text { \$Ce141 } \\
& 59143.70 \mathrm{C} \quad 4.817476 \mathrm{E}-29 \text { \$Pr143 } \\
& 60143.70 \mathrm{C} \quad 4.817471 \mathrm{E}-29 \$ \mathrm{Nd} 143 \\
& 60145.70 \mathrm{C} \quad 4.750891 \mathrm{E}-29 \text { \$Nd145 } \\
& 61147.70 \mathrm{C} \quad 4.686170 \mathrm{E}-29 \$ \mathrm{Pm} 147 \\
& 61149.70 \mathrm{C} \quad 4.623135 \mathrm{E}-29 \$ \mathrm{Pm} 149 \\
& 62149.70 \mathrm{C} \quad 4.623132 \mathrm{E}-29 \$ \mathrm{Sm} 149 \\
& 61151.70 \mathrm{C} \quad 4.561735 \mathrm{E}-29 \$ \mathrm{Pm} 151 \\
& 62151.70 \mathrm{C} \quad 4.561782 \mathrm{E}-29 \$ \mathrm{Sm} 151 \\
& 62152.70 \mathrm{C} \quad 4.531760 \mathrm{E}-29 \text { \$Sm152 } \\
& 63153.70 \mathrm{C} \quad 4.502082 \mathrm{E}-29 \text { \$Eu153 } \\
& 63155.70 \mathrm{C} \quad 4.443934 \mathrm{E}-29 \text { \$Eu155 }
\end{aligned}
$$

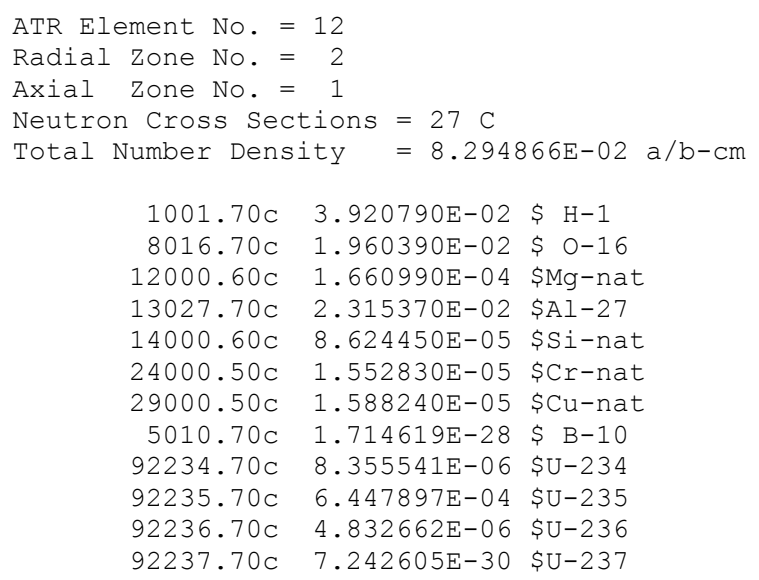


$92238.70 \mathrm{C} \quad 4.141451 \mathrm{E}-05 \quad \$ \mathrm{U}-238$

$93237.70 \mathrm{C} \quad 7.242569 \mathrm{E}-30 \quad \$ \mathrm{~Np} 237$

$94239.70 \mathrm{C} \quad 7.181854 \mathrm{E}-30$ \$Pu239

$94240.70 \mathrm{C} \quad 7.151887 \mathrm{E}-30$ \$Pu 240

$94241.70 \mathrm{C} \quad 7.122128 \mathrm{E}-30$ \$Pu241

$36083.70 \mathrm{C} \quad 2.070621 \mathrm{E}-29$ \$Kr-83

$42095.70 \mathrm{C} \quad 1.790150 \mathrm{E}-29$ \$Mo-95

$44101.70 \mathrm{C} \quad 1.701430 \mathrm{E}-29$ \$Ru101

$45103.70 \mathrm{C} \quad 1.668363 \mathrm{E}-29$ \$Rh103

$45105.70 \mathrm{C} \quad 1.636554 \mathrm{E}-29$ \$Rh105

$48113.70 \mathrm{C} \quad 1.520612 \mathrm{E}-29$ \$Cd113

$54131.70 \mathrm{C} \quad 1.311513 \mathrm{E}-29$ \$Xe131

$54133.70 \mathrm{C} \quad 1.291780 \mathrm{E}-29$ \$Xe133

$55133.70 \mathrm{C} \quad 1.291774 \mathrm{E}-29 \quad \$ \mathrm{Cs} 133$

$54135.70 \mathrm{C} \quad 1.263242 \mathrm{E}-29$ \$Xe135

$57140.70 \mathrm{C} \quad 1.227103 \mathrm{E}-29$ \$La140

$58141.70 \mathrm{C} \quad 1.218416 \mathrm{E}-29 \$ \mathrm{Ce} 141$

$59143.70 \mathrm{C} \quad 1.201345 \mathrm{E}-29 \$ \mathrm{Pr} 143$

$60143.70 \mathrm{C} \quad 1.201344 \mathrm{E}-29 \$ \mathrm{Nd} 143$

$60145.70 \mathrm{C} \quad 1.184740 \mathrm{E}-29 \$ \mathrm{Nd} 145$

$61147.70 \mathrm{C} \quad 1.168601 \mathrm{E}-29 \$ \mathrm{Pm} 147$

$61149.70 \mathrm{C} \quad 1.152882 \mathrm{E}-29 \$ \mathrm{Pm} 149$

$62149.70 \mathrm{C} \quad 1.152881 \mathrm{E}-29 \quad \$ \mathrm{Sm} 149$

$61151.70 \mathrm{C} \quad 1.137570 \mathrm{E}-29$ \$Pm151

$62151.70 \mathrm{C} \quad 1.137582 \mathrm{E}-29 \$ \mathrm{Sm} 151$

$62152.70 \mathrm{C} \quad 1.130095 \mathrm{E}-29$ \$Sm152

$63153.70 \mathrm{C} \quad 1.122694 \mathrm{E}-29$ \$Eu153

$63155.70 \mathrm{C} \quad 1.108194 \mathrm{E}-29$ \$Eu155

mt2239 lwtr.10t

$64157.70 \mathrm{C} \quad 1.094057 \mathrm{E}-29 \$ \mathrm{Gd} 157$

c

C

C

C

$\mathrm{C}$

C

$\mathrm{C}$

m2240

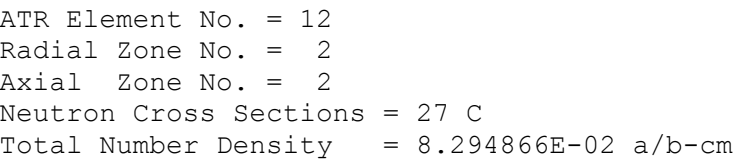

\begin{tabular}{|c|c|c|}
\hline $1001.70 \mathrm{c}$ & $3.920790 \mathrm{E}-02$ & $\$ \mathrm{H}-1$ \\
\hline $8016.70 \mathrm{c}$ & $1.960390 \mathrm{E}-02$ & $\$ 0-16$ \\
\hline $2000.60 \mathrm{c}$ & $1.660990 \mathrm{E}-04$ & $\$ M g-n a t$ \\
\hline $3027.70 \mathrm{c}$ & $2.315370 \mathrm{E}-02$ & $\$ A I-27$ \\
\hline $000.60 \mathrm{c}$ & $8.624450 \mathrm{E}-05$ & \$Si-nat \\
\hline $00.50 \mathrm{c}$ & $1.552830 \mathrm{E}-05$ & $\$ C r-n a t$ \\
\hline $.50 \mathrm{C}$ & $1.588240 \mathrm{E}-05$ & $\$ \mathrm{Cu}-\mathrm{nat}$ \\
\hline$c$ & $519 E-28$ & $\$ B-10$ \\
\hline $.70 \mathrm{C}$ & $8.355541 \mathrm{E}-06$ & $\$ U-234$ \\
\hline $70 c$ & $97 E-04$ & $\$ U-235$ \\
\hline $.70 \mathrm{C}$ & $4.832662 \mathrm{E}-06$ & $\$ U-236$ \\
\hline $.70 \mathrm{C}$ & 7. $242605 \mathrm{E}-30$ & $\$ U-237$ \\
\hline 7 & $1 E-05$ & $\$ U-238$ \\
\hline $.70 \mathrm{C}$ & $569 \mathrm{E}-30$ & $\$ N p 237$ \\
\hline & $E-30$ & \$Pu239 \\
\hline $\mathrm{C}$ & $87 E-30$ & \$Pu240 \\
\hline$c$ & $28 E-30$ & \$Pu2 41 \\
\hline$C$ & $1 E-29$ & $\$ K r-83$ \\
\hline & $50 E-29$ & $\$ \mathrm{Mo}-95$ \\
\hline $\mathrm{C}$ & $0 E-29$ & \$Ru101 \\
\hline & $63 E-29$ & \$Rh103 \\
\hline C & $4 E-29$ & $\$ R h 105$ \\
\hline $.70 \mathrm{C}$ & $1.520612 \mathrm{E}-29$ & $\$ C d 113$ \\
\hline .7 & $3 E-29$ & $\$ x e 131$ \\
\hline $.70 \mathrm{C}$ & 1.291780E-29 & $\$ x e 133$ \\
\hline .7 & $4 E-29$ & $\$ C$ S133 \\
\hline .7 & 1. $263242 \mathrm{E}-29$ & $\$ x e 135$ \\
\hline .7 & $3 E-29$ & $\$$ La140 \\
\hline & $16 \mathrm{E}-29$ & $\$ \mathrm{Ce} 141$ \\
\hline 7 & $5 E-29$ & $\$ \operatorname{Pr} 143$ \\
\hline 7 & $44 E-29$ & $\$ \mathrm{Nd} 143$ \\
\hline
\end{tabular}


$60145.70 \mathrm{C} \quad 1.184740 \mathrm{E}-29 \$ \mathrm{Nd} 145$

$61147.70 \mathrm{C} \quad 1.168601 \mathrm{E}-29 \$ \mathrm{Pm} 147$

$61149.70 \mathrm{C} \quad 1.152882 \mathrm{E}-29$ \$Pm149

$62149.70 \mathrm{C} \quad 1.152881 \mathrm{E}-29 \$ \mathrm{Sm} 149$

$61151.70 \mathrm{C} \quad 1.137570 \mathrm{E}-29 \$ \mathrm{Pm} 151$

$62151.70 \mathrm{C} \quad 1.137582 \mathrm{E}-29$ \$Sm151

$62152.70 \mathrm{C} \quad 1.130095 \mathrm{E}-29 \$ \mathrm{Sm} 152$

$63153.70 \mathrm{C} \quad 1.122694 \mathrm{E}-29 \$ \mathrm{Eu} 153$

$63155.70 \mathrm{C} \quad 1.108194 \mathrm{E}-29$ \$Eu155

mt2240 lwtr.10t

$64157.70 \mathrm{C} \quad 1.094057 \mathrm{E}-29$ \$Gd157

C

C

$\mathrm{C}$

C

$\mathrm{C}$

m2241

$\mathrm{c}$

$\mathrm{C}$

C

ATR Element No. $=12$

Radial Zone No. $=2$

Axial Zone No. $=3$

Neutron Cross Sections $=27 \mathrm{C}$

Total Number Density $=8.294866 \mathrm{E}-02 \mathrm{a} / \mathrm{b}-\mathrm{cm}$

$1001.70 \mathrm{C} \quad 3.920790 \mathrm{E}-02$ \$ H-1

$8016.70 \mathrm{C} \quad 1.960390 \mathrm{E}-02$ \$ O-16

$12000.60 \mathrm{c} \quad 1.660990 \mathrm{E}-04 \$ \mathrm{Mg}-$ nat

$13027.70 \mathrm{C} \quad 2.315370 \mathrm{E}-02$ \$Al-27

$14000.60 \mathrm{c} \quad 8.624450 \mathrm{E}-05$ \$Si-nat

$24000.50 \mathrm{C} \quad 1.552830 \mathrm{E}-05$ \$Cr-nat

$29000.50 \mathrm{C} \quad 1.588240 \mathrm{E}-05$ \$Cu-nat

$5010.70 \mathrm{C} \quad 1.714619 \mathrm{E}-28$ \$ B-10

$92234.70 \mathrm{C} \quad 8.355541 \mathrm{E}-06 \quad \$ \mathrm{U}-234$

$92235.70 \mathrm{C} \quad 6.447897 \mathrm{E}-04 \quad \$ \mathrm{U}-235$

$92236.70 \mathrm{C} \quad 4.832662 \mathrm{E}-06 \quad \$ \mathrm{U}-236$

$92237.70 \mathrm{C} \quad 7.242605 \mathrm{E}-30 \quad \$ \mathrm{U}-237$

$92238.70 \mathrm{C} \quad 4.141451 \mathrm{E}-05$ \$U-238

$93237.70 \mathrm{C} \quad 7.242569 \mathrm{E}-30 \quad \$ \mathrm{~Np} 237$

$94239.70 \mathrm{C} \quad 7.181854 \mathrm{E}-30$ \$Pu239

$94240.70 \mathrm{C} \quad 7.151887 \mathrm{E}-30$ \$Pu 240

$94241.70 \mathrm{C} \quad 7.122128 \mathrm{E}-30$ \$Pu241

$36083.70 \mathrm{C} \quad 2.070621 \mathrm{E}-29$ \$Kr-83

$42095.70 \mathrm{C} \quad 1.790150 \mathrm{E}-29$ \$Mo-95

$44101.70 \mathrm{C} \quad 1.701430 \mathrm{E}-29$ \$Ru101

$45103.70 \mathrm{C} \quad 1.668363 \mathrm{E}-29$ \$Rh103

$45105.70 \mathrm{C} \quad 1.636554 \mathrm{E}-29 \$ \mathrm{Rh} 105$

$48113.70 \mathrm{C} \quad 1.520612 \mathrm{E}-29 \$ \mathrm{Cd} 113$

$54131.70 \mathrm{C} \quad 1.311513 \mathrm{E}-29$ \$Xe131

$54133.70 \mathrm{C} \quad 1.291780 \mathrm{E}-29$ \$Xe133

$55133.70 \mathrm{C} \quad 1.291774 \mathrm{E}-29 \$ \mathrm{Cs} 133$

$54135.70 \mathrm{C} \quad 1.263242 \mathrm{E}-29$ \$Xe135

$57140.70 \mathrm{C} \quad 1.227103 \mathrm{E}-29$ La140

$58141.70 \mathrm{C} \quad 1.218416 \mathrm{E}-29$ \$Ce141

$59143.70 \mathrm{C} \quad 1.201345 \mathrm{E}-29 \$ \mathrm{Pr} 143$

$60143.70 \mathrm{C} \quad 1.201344 \mathrm{E}-29 \$ \mathrm{Nd} 143$

$60145.70 \mathrm{C} \quad 1.184740 \mathrm{E}-29$ \$Nd145

$61147.70 \mathrm{C} \quad 1.168601 \mathrm{E}-29 \$ \mathrm{Pm} 147$

$61149.70 \mathrm{C} \quad 1.152882 \mathrm{E}-29$ \$Pm149

$62149.70 \mathrm{C} \quad 1.152881 \mathrm{E}-29 \$ \mathrm{Sm} 149$

$61151.70 \mathrm{C} \quad 1.137570 \mathrm{E}-29$ \$Pm151

$62151.70 \mathrm{C} \quad 1.137582 \mathrm{E}-29$ \$Sm151

$62152.70 \mathrm{C} \quad 1.130095 \mathrm{E}-29$ \$Sm152

$63153.70 \mathrm{C} \quad 1.122694 \mathrm{E}-29$ \$Eu153

$63155.70 \mathrm{C} \quad 1.108194 \mathrm{E}-29$ \$Eu155

$64157.70 \mathrm{C} \quad 1.094057 \mathrm{E}-29 \$ \mathrm{Gd} 157$

lwtr.10t

ATR Element No. $=12$

Radial Zone No. $=2$

Axial Zone No. $=4$

Neutron Cross Sections $=27 \mathrm{C}$ 
C

m2242

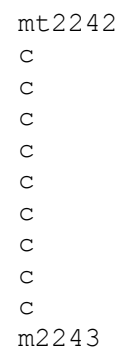

Total Number Density $=8.294866 \mathrm{E}-02 \mathrm{a} / \mathrm{b}-\mathrm{cm}$
$1001.70 \mathrm{C} \quad 3.920790 \mathrm{E}-02 \$ \mathrm{H}-1$

$8016.70 \mathrm{C} \quad 1.960390 \mathrm{E}-02$ \$ $0-16$

$12000.60 \mathrm{C} \quad 1.660990 \mathrm{E}-04 \$ \mathrm{Mg}-\mathrm{nat}$

$13027.70 \mathrm{C} \quad 2.315370 \mathrm{E}-02$ \$Al-27

$14000.60 \mathrm{C} \quad 8.624450 \mathrm{E}-05$ \$Si-nat

$24000.50 \mathrm{C} \quad 1.552830 \mathrm{E}-05$ \$Cr-nat

$29000.50 \mathrm{C} \quad 1.588240 \mathrm{E}-05$ \$Cu-nat

$5010.70 \mathrm{C} \quad 8.573098 \mathrm{E}-29 \$ \mathrm{~B}-10$

$92234.70 \mathrm{C} \quad 8.355538 \mathrm{E}-06 \$ \mathrm{U}-234$

$92235.70 \mathrm{C} \quad 6.447894 \mathrm{E}-04 \quad \$ \mathrm{U}-235$

$92236.70 \mathrm{C} \quad 4.832660 \mathrm{E}-06 \quad \$ \mathrm{U}-236$

$92237.70 \mathrm{C} \quad 3.621303 \mathrm{E}-30$ \$U-237

$92238.70 \mathrm{C} \quad 4.141449 \mathrm{E}-05$ \$U-238

$93237.70 \mathrm{C} \quad 3.621285 \mathrm{E}-30 \quad \$ \mathrm{~Np} 237$

$94239.70 \mathrm{C} \quad 3.590928 \mathrm{E}-30$ \$Pu239

$94240.70 \mathrm{C} \quad 3.575944 \mathrm{E}-30 \quad \$ \mathrm{Pu} 240$

$94241.70 \mathrm{C} \quad 3.561065 \mathrm{E}-30$ \$Pu241

$36083.70 \mathrm{C} \quad 1.035311 \mathrm{E}-29 \$ \mathrm{Kr}-83$

$42095.70 \mathrm{C} \quad 8.950752 \mathrm{E}-30$ \$Mo-95

$44101.70 \mathrm{C} \quad 8.507152 \mathrm{E}-30$ \$Ru101

$45103.70 \mathrm{C} \quad 8.341818 \mathrm{E}-30$ \$Rh103

$45105.70 \mathrm{C} \quad 8.182769 \mathrm{E}-30$ \$Rh105

$48113.70 \mathrm{C} \quad 7.603060 \mathrm{E}-30 \$ \mathrm{Cd} 113$

$54131.70 \mathrm{C} \quad 6.557568 \mathrm{E}-30 \quad \$ \mathrm{Xe131}$

$54133.70 \mathrm{C} \quad 6.458903 \mathrm{E}-30$ \$Xe133

$55133.70 \mathrm{C} \quad 6.458870 \mathrm{E}-30 \quad \$ \mathrm{Cs} 133$

$54135.70 \mathrm{C} \quad 6.316213 \mathrm{E}-30$ \$Xe135

$57140.70 \mathrm{C} \quad 6.135514 \mathrm{E}-30$ \$La140

$58141.70 \mathrm{C} \quad 6.092082 \mathrm{E}-30 \quad \$ \mathrm{Ce} 141$

$59143.70 \mathrm{C} \quad 6.006725 \mathrm{E}-30 \quad \$ \operatorname{Pr} 143$

$60143.70 \mathrm{C} \quad 6.006719 \mathrm{E}-30 \$ \mathrm{Nd} 143$

$60145.70 \mathrm{C} \quad 5.923703 \mathrm{E}-30 \$ \mathrm{Nd} 145$

$61147.70 \mathrm{C} \quad 5.843005 \mathrm{E}-30 \quad \$ \mathrm{Pm} 147$

$61149.70 \mathrm{C} \quad 5.764410 \mathrm{E}-30 \$ \mathrm{Pm} 149$

$62149.70 \mathrm{C} \quad 5.764405 \mathrm{E}-30 \$ \mathrm{Sm} 149$

$61151.70 \mathrm{C} \quad 5.687851 \mathrm{E}-30 \quad \$ \mathrm{Pm} 151$

$62151.70 \mathrm{C} \quad 5.687911 \mathrm{E}-30$ \$Sm151

$62152.70 \mathrm{C} \quad 5.650478 \mathrm{E}-30 \$ \mathrm{Sm} 152$

$63153.70 \mathrm{C} \quad 5.613472 \mathrm{E}-30 \quad \$ \mathrm{Eu} 153$

$63155.70 \mathrm{C} \quad 5.540969 \mathrm{E}-30$ \$Eu155

lwtr. 10 t

ATR Element No. $=12$

Radial Zone No. $=2$

Axial Zone No. $=5$

Neutron Cross Sections $=27 \mathrm{C}$

Total Number Density $=8.294866 \mathrm{E}-02 \mathrm{a} / \mathrm{b}-\mathrm{cm}$
$1001.70 \mathrm{C} \quad 3.920790 \mathrm{E}-02$ \$ $\mathrm{H}-1$
$8016.70 \mathrm{C} \quad 1.960390 \mathrm{E}-02 \$ 0-16$
$12000.60 \mathrm{C} \quad 1.660990 \mathrm{E}-04$ \$Mg-nat
$13027.70 \mathrm{C} \quad 2.315370 \mathrm{E}-02$ \$Al-27
$14000.60 \mathrm{C} \quad 8.624450 \mathrm{E}-05$ \$Si-nat
$24000.50 \mathrm{C} \quad 1.552830 \mathrm{E}-05$ \$Cr-nat
$29000.50 \mathrm{C} \quad 1.588240 \mathrm{E}-05 \mathrm{\$ Cu}-\mathrm{nat}$
$5010.70 \mathrm{C} \quad 1.714619 \mathrm{E}-28$ \$ B-10
$92234.70 \mathrm{C} \quad 8.355541 \mathrm{E}-06 \quad \$ \mathrm{U}-234$
$92235.70 \mathrm{C} \quad 6.447897 \mathrm{E}-04 \quad \$ \mathrm{U}-235$
$92236.70 \mathrm{C} \quad 4.832662 \mathrm{E}-06$ \$U-236
$92237.70 \mathrm{C} \quad 7.242605 \mathrm{E}-30 \quad \mathrm{SU}-237$
$92238.70 \mathrm{C} \quad 4.141451 \mathrm{E}-05$ \$U-238
$93237.70 \mathrm{C} \quad 7.242569 \mathrm{E}-30 \quad \$ \mathrm{~Np} 237$
$94239.70 \mathrm{C} \quad 7.181854 \mathrm{E}-30$ \$Pu239
$94240.70 \mathrm{C} \quad 7.151887 \mathrm{E}-30$ \$Pu 240
$94241.70 \mathrm{C} \quad 7.122128 \mathrm{E}-30$ \$Pu 241 


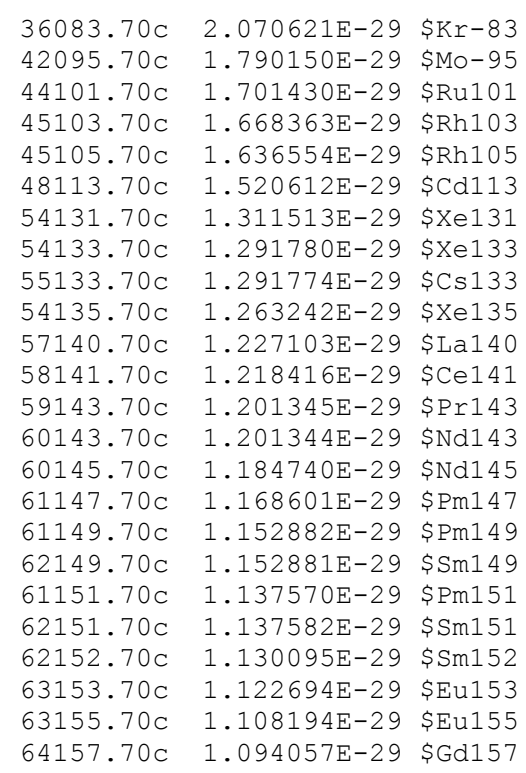

mt224
C
C
C
C
C
C
C
C
C
m2244 lwtr.10t

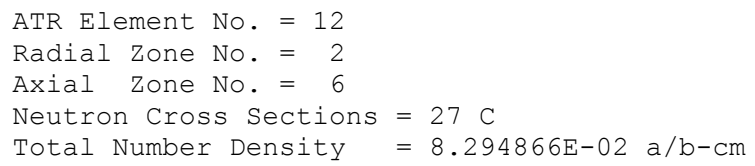


$62151.70 \mathrm{C} \quad 1.137582 \mathrm{E}-29 \$ \mathrm{Sm} 151$

$62152.70 \mathrm{C} \quad 1.130095 \mathrm{E}-29$ \$Sm152

$63153.70 \mathrm{C} \quad 1.122694 \mathrm{E}-29$ \$Eu153

$63155.70 \mathrm{C} \quad 1.108194 \mathrm{E}-29$ \$Eu155

mt2244 1 wtr. $10 t$

$64157.70 \mathrm{C} \quad 1.094057 \mathrm{E}-29 \$ \mathrm{Gd} 157$

c

C

C

C

$\mathrm{C}$

m2245

mt2245

C

$\mathrm{C}$

$\mathrm{C}$

C

C

C

C

c

m2246

lwtr.10t

ATR Element No. $=12$

Radial Zone No. $=2$

Axial Zone No. $=7$

Neutron Cross Sections $=27 \mathrm{C}$

Total Number Density $=8.294866 \mathrm{E}-02 \mathrm{a} / \mathrm{b}-\mathrm{cm}$

\begin{abstract}
$1001.70 \mathrm{C} \quad 3.920790 \mathrm{E}-02 \$ \mathrm{H}-1$
$8016.70 \mathrm{C} \quad 1.960390 \mathrm{E}-02 \$ 0-16$

$12000.60 \mathrm{C} \quad 1.660990 \mathrm{E}-04 \$ \mathrm{Mg}-\mathrm{nat}$

$13027.70 \mathrm{C} \quad 2.315370 \mathrm{E}-02$ \$Al-27

$14000.60 \mathrm{C} \quad 8.624450 \mathrm{E}-05$ \$Si-nat

$24000.50 \mathrm{C} \quad 1.552830 \mathrm{E}-05$ \$Cr-nat

$29000.50 \mathrm{C} \quad 1.588240 \mathrm{E}-05$ \$Cu-nat

$5010.70 \mathrm{C} \quad 1.714619 \mathrm{E}-28$ \$ B 10

$92234.70 \mathrm{C} \quad 8.355541 \mathrm{E}-06 \$ \mathrm{U}-234$

$92235.70 \mathrm{C} \quad 6.447897 \mathrm{E}-04 \quad \$ \mathrm{U}-235$

$92236.70 \mathrm{C} \quad 4.832662 \mathrm{E}-06 \quad \$ \mathrm{U}-236$

$92237.70 \mathrm{C} \quad 7.242605 \mathrm{E}-30 \quad \mathrm{SU}-237$

$92238.70 \mathrm{C} \quad 4.141451 \mathrm{E}-05$ \$U-238

$93237.70 \mathrm{C} \quad 7.242569 \mathrm{E}-30 \quad \$ \mathrm{~Np} 237$

$94239.70 \mathrm{C} \quad 7.181854 \mathrm{E}-30$ \$Pu239

$94240.70 \mathrm{C} \quad 7.151887 \mathrm{E}-30$ \$Pu240

$94241.70 \mathrm{C} \quad 7.122128 \mathrm{E}-30$ \$Pu241

$36083.70 \mathrm{C} \quad 2.070621 \mathrm{E}-29 \$ \mathrm{Kr}-83$

$42095.70 \mathrm{C} \quad 1.790150 \mathrm{E}-29$ \$Mo-95

$44101.70 \mathrm{C} \quad 1.701430 \mathrm{E}-29$ \$Ru101

$45103.70 \mathrm{C} \quad 1.668363 \mathrm{E}-29$ \$Rh103

$45105.70 \mathrm{C} \quad 1.636554 \mathrm{E}-29 \$ \mathrm{Rh} 105$

$48113.70 \mathrm{C} \quad 1.520612 \mathrm{E}-29$ \$Cd113

$54131.70 \mathrm{C} \quad 1.311513 \mathrm{E}-29$ \$Xe131

$54133.70 \mathrm{C} \quad 1.291780 \mathrm{E}-29$ \$Xe133

$55133.70 \mathrm{C} \quad 1.291774 \mathrm{E}-29$ \$Cs133

$54135.70 \mathrm{C} \quad 1.263242 \mathrm{E}-29 \$ \mathrm{Xe} 135$

$57140.70 \mathrm{C} \quad 1.227103 \mathrm{E}-29$ \$La140

$58141.70 \mathrm{C} \quad 1.218416 \mathrm{E}-29$ \$Ce141

$59143.70 \mathrm{C} \quad 1.201345 \mathrm{E}-29$ \$Pr143

$60143.70 \mathrm{C} \quad 1.201344 \mathrm{E}-29 \$ \mathrm{Nd} 143$

$60145.70 \mathrm{C} \quad 1.184740 \mathrm{E}-29$ \$Nd145

$61147.70 \mathrm{C} \quad 1.168601 \mathrm{E}-29 \$ \mathrm{Pm} 147$

$61149.70 \mathrm{C} \quad 1.152882 \mathrm{E}-29 \$ \mathrm{Pm} 149$

$62149.70 \mathrm{C} \quad 1.152881 \mathrm{E}-29 \$ \mathrm{Sm} 149$

$61151.70 \mathrm{C} \quad 1.137570 \mathrm{E}-29$ \$Pm151

$62151.70 \mathrm{C} \quad 1.137582 \mathrm{E}-29$ \$Sm151

$62152.70 \mathrm{C} \quad 1.130095 \mathrm{E}-29 \$ \mathrm{Sm} 152$

$63153.70 \mathrm{C} \quad 1.122694 \mathrm{E}-29$ \$Eu153

$63155.70 \mathrm{C} \quad 1.108194 \mathrm{E}-29$ \$Eu155
\end{abstract}

$64157.70 \mathrm{C} \quad 1.094057 \mathrm{E}-29$ \$Gd157

lwtr. 10 t

ATR Element No. $=12$

Radial Zone No. = 3

Axial Zone No. $=1$

Neutron Cross Sections $=27 \mathrm{C}$

Total Number Density $=7.870346 \mathrm{E}-02 \mathrm{a} / \mathrm{b}-\mathrm{cm}$

$1001.70 \mathrm{C} \quad 3.212840 \mathrm{E}-02 \$ \mathrm{H}-1$

$8016.70 \mathrm{C} \quad 1.606420 \mathrm{E}-02 \$ 0-16$

$12000.60 \mathrm{C} \quad 2.399430 \mathrm{E}-04$ \$Mg-nat 


\begin{tabular}{|c|c|c|c|}
\hline & & & \\
\hline & $13027.70 \mathrm{c}$ & $2.958200 \mathrm{E}-02$ & $\$ A 1-27$ \\
\hline & $14000.60 \mathrm{c}$ & $1.245870 \mathrm{E}-04$ & \$Si-nat \\
\hline & $24000.50 c$ & $2.243180 \mathrm{E}-05$ & $\$ C r-n a t$ \\
\hline & $29000.50 c$ & $2.294330 \mathrm{E}-05$ & $\$ \mathrm{Cu}-$ nat \\
\hline & $5010.70 \mathrm{c}$ & $1.966257 \mathrm{E}-05$ & $\$ B-10$ \\
\hline & $92234.70 \mathrm{c}$ & $5.964864 \mathrm{E}-06$ & $\$ U-234$ \\
\hline & $92235.70 \mathrm{c}$ & $4.603032 \mathrm{E}-04$ & $\$ U-235$ \\
\hline & $92236.70 \mathrm{c}$ & $3.449947 \mathrm{E}-06$ & $\$ \mathrm{U}-236$ \\
\hline & $92237.70 \mathrm{c}$ & $1.545203 \mathrm{E}-29$ & $\$ \mathrm{U}-237$ \\
\hline & $92238.70 \mathrm{C}$ & $2.956504 \mathrm{E}-05$ & $\$ U-238$ \\
\hline & $93237.70 \mathrm{c}$ & $1.545195 \mathrm{E}-29$ & $\$ N p 237$ \\
\hline & $94239.70 \mathrm{c}$ & $1.532242 \mathrm{E}-29$ & \$Pu239 \\
\hline & $94240.70 \mathrm{c}$ & $1.525848 \mathrm{E}-29$ & \$Pu240 \\
\hline & $94241.70 \mathrm{c}$ & $1.519499 \mathrm{E}-29$ & \$Pu241 \\
\hline & $36083.70 \mathrm{c}$ & $4.417651 \mathrm{E}-29$ & $\$ \mathrm{Kr}-83$ \\
\hline & $42095.70 \mathrm{c}$ & $3.819269 \mathrm{E}-29$ & $\$ M o-95$ \\
\hline & $44101.70 \mathrm{c}$ & $3.629985 E-29$ & $\$ R u 101$ \\
\hline & $45103.70 \mathrm{c}$ & $3.559438 \mathrm{E}-29$ & \$Rh103 \\
\hline & $45105.70 \mathrm{C}$ & $3.491572 E-29$ & \$Rh105 \\
\hline & $48113.70 \mathrm{c}$ & $3.244211 \mathrm{E}-29$ & $\$ C d 113$ \\
\hline & $54131.70 \mathrm{c}$ & $2.798102 \mathrm{E}-29$ & $\$ x e 131$ \\
\hline & $54133.70 \mathrm{c}$ & $2.756002 \mathrm{E}-29$ & $\$ x e 133$ \\
\hline & $55133.70 \mathrm{C}$ & $2.755987 E-29$ & $\$ \mathrm{Cs} 133$ \\
\hline & $54135.70 \mathrm{c}$ & $2.695116 \mathrm{E}-29$ & $\$ X e 135$ \\
\hline & $57140.70 \mathrm{c}$ & $2.618012 \mathrm{E}-29$ & $\$ L a 140$ \\
\hline & $58141.70 \mathrm{c}$ & $2.599480 \mathrm{E}-29$ & $\$ C e 141$ \\
\hline & $59143.70 \mathrm{c}$ & $2.563058 \mathrm{E}-29$ & $\$ \operatorname{Pr} 143$ \\
\hline & $60143.70 \mathrm{c}$ & $2.563055 \mathrm{E}-29$ & $\$ N d 143$ \\
\hline & $60145.70 \mathrm{c}$ & $2.527633 \mathrm{E}-29$ & $\$ \mathrm{Nd1} 45$ \\
\hline & $61147.70 \mathrm{c}$ & $2.493199 \mathrm{E}-29$ & $\$ \operatorname{Pm} 147$ \\
\hline & $61149.70 \mathrm{c}$ & $2.459663 \mathrm{E}-29$ & $\$ \operatorname{Pm} 149$ \\
\hline & $62149.70 \mathrm{c}$ & $2.459661 \mathrm{E}-29$ & $\$$ Sm1 49 \\
\hline & $61151.70 \mathrm{C}$ & $2.426995 E-29$ & $\$ \operatorname{Pm} 151$ \\
\hline & $62151.70 \mathrm{c}$ & $2.427021 \mathrm{E}-29$ & $\$$ Sm151 \\
\hline & $62152.70 \mathrm{c}$ & $2.411048 \mathrm{E}-29$ & $\$$ Sm152 \\
\hline & $63153.70 \mathrm{c}$ & $2.395258 \mathrm{E}-29$ & \$Eu153 \\
\hline & $63155.70 \mathrm{c}$ & $2.364321 \mathrm{E}-29$ & \$Eu155 \\
\hline & $64157.70 \mathrm{c}$ & $2.334161 \mathrm{E}-29$ & \$Gd157 \\
\hline lwtr.10 & & & \\
\hline ATR El & ement No. = & 12 & \\
\hline Radial & Zone No. = & 3 & \\
\hline Axial & Zone No. = & 2 & \\
\hline Neutro & n Cross Sec & tions $=27 \mathrm{C}$ & \\
\hline & Number Dens: & ity $=7.8703$ & $346 \mathrm{E}-02 \mathrm{a} / \mathrm{b}-\mathrm{cm}$ \\
\hline & $1001.70 \mathrm{c}$ & $3.212840 \mathrm{E}-02$ & $\$ \mathrm{H}-1$ \\
\hline & $8016.70 \mathrm{C}$ & $1.606420 \mathrm{E}-02$ & $\$ 0-16$ \\
\hline & $12000.60 \mathrm{c}$ & $2.399430 \mathrm{E}-04$ & $\$ M g-n a t$ \\
\hline & $13027.70 \mathrm{c}$ & $2.958200 \mathrm{E}-02$ & $\$ A l-27$ \\
\hline & $14000.60 \mathrm{c}$ & $1.245870 \mathrm{E}-04$ & \$Si-nat \\
\hline & $24000.50 c$ & $2.243180 \mathrm{E}-05$ & $\$ C r-n a t$ \\
\hline & $29000.50 c$ & $2.294330 \mathrm{E}-05$ & $\$ \mathrm{Cu}-$ nat \\
\hline & $5010.70 \mathrm{c}$ & $1.966257 \mathrm{E}-05$ & $\$ B-10$ \\
\hline & $92234.70 \mathrm{c}$ & $5.964864 \mathrm{E}-06$ & $\$ U-234$ \\
\hline & $92235.70 \mathrm{c}$ & $4.603032 \mathrm{E}-04$ & $\$ U-235$ \\
\hline & $92236.70 \mathrm{c}$ & $3.449947 \mathrm{E}-06$ & $\$ U-236$ \\
\hline & $92237.70 \mathrm{c}$ & $1.545203 \mathrm{E}-29$ & $\$ U-237$ \\
\hline & $92238.70 \mathrm{c}$ & $2.956504 \mathrm{E}-05$ & $\$ U-238$ \\
\hline & $93237.70 \mathrm{C}$ & $1.545195 \mathrm{E}-29$ & $\$ \mathrm{~Np} 237$ \\
\hline & $94239.70 \mathrm{C}$ & $1.532242 \mathrm{E}-29$ & \$Pu239 \\
\hline & $94240.70 \mathrm{c}$ & $1.525848 \mathrm{E}-29$ & $\$ P u 240$ \\
\hline & $94241.70 \mathrm{c}$ & $1.519499 \mathrm{E}-29$ & \$Pu241 \\
\hline & $36083.70 \mathrm{c}$ & $4.417651 \mathrm{E}-29$ & $\$ K r-83$ \\
\hline & $42095.70 \mathrm{C}$ & $3.819269 \mathrm{E}-29$ & $\$ M o-95$ \\
\hline & $44101.70 \mathrm{C}$ & $3.629985 \mathrm{E}-29$ & \$Ru101 \\
\hline & $45103.70 \mathrm{c}$ & $3.559438 \mathrm{E}-29$ & \$Rh103 \\
\hline & $45105.70 c$ & $3.491572 \mathrm{E}-29$ & \$Rh105 \\
\hline
\end{tabular}

mt2246 lwtr.10t

C

C

C

C

C

C

C m2247 


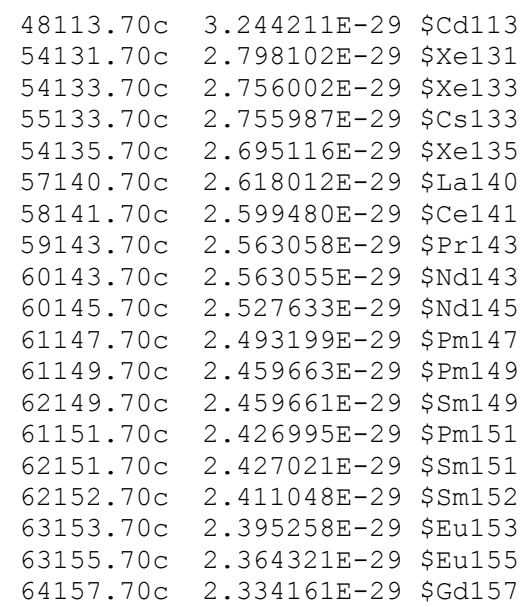

mt2247 lwtr.10t

C

C

C

C

C ATR Element No. $=12$

C Radial Zone No. = 3

C Axial Zone No. = 3

C Neutron Cross Sections $=27 \mathrm{C}$

C Total Number Density $=7.870346 \mathrm{E}-02 \mathrm{a} / \mathrm{b}-\mathrm{cm}$

m2248

\begin{tabular}{|c|c|c|}
\hline $1001.70 \mathrm{C}$ & $3.212840 \mathrm{E}-02$ & $\$ \mathrm{H}-1$ \\
\hline $8016.70 \mathrm{C}$ & $1.606420 \mathrm{E}-02$ & $\$ 0-16$ \\
\hline $12000.60 \mathrm{c}$ & $2.399430 E-04$ & $\$ M g-n a t$ \\
\hline $13027.70 \mathrm{C}$ & $2.958200 \mathrm{E}-02$ & $\$ A l-27$ \\
\hline $14000.60 \mathrm{C}$ & $1.245870 \mathrm{E}-04$ & \$Si-nat \\
\hline $24000.50 \mathrm{c}$ & $2.243180 \mathrm{E}-05$ & $\$ C r-n a t$ \\
\hline $29000.50 \mathrm{c}$ & $2.294330 \mathrm{E}-05$ & $\$ \mathrm{Cu}-\mathrm{nat}$ \\
\hline $5010.70 \mathrm{C}$ & $1.966257 \mathrm{E}-05$ & $\$ B-10$ \\
\hline $92234.70 \mathrm{C}$ & $5.964864 \mathrm{E}-06$ & $\$ U-234$ \\
\hline $92235.70 \mathrm{C}$ & $4.603032 \mathrm{E}-04$ & $\$ U-235$ \\
\hline $92236.70 \mathrm{C}$ & $3.449947 \mathrm{E}-06$ & $\$ U-236$ \\
\hline $92237.70 \mathrm{c}$ & $1.545203 \mathrm{E}-29$ & $\$ U-237$ \\
\hline $92238.70 \mathrm{c}$ & $2.956504 \mathrm{E}-05$ & $\$ U-238$ \\
\hline $93237.70 \mathrm{C}$ & $1.545195 \mathrm{E}-29$ & $\$ N p 237$ \\
\hline $94239.70 \mathrm{C}$ & $1.532242 \mathrm{E}-29$ & \$Pu239 \\
\hline $94240.70 \mathrm{C}$ & $1.525848 \mathrm{E}-29$ & $\$ \mathrm{Pu} 240$ \\
\hline $94241.70 \mathrm{C}$ & $1.519499 \mathrm{E}-29$ & \$Pu241 \\
\hline $36083.70 \mathrm{c}$ & $4.417651 \mathrm{E}-29$ & $\$ K r-83$ \\
\hline $42095.70 \mathrm{C}$ & $3.819269 \mathrm{E}-29$ & $\$ M \circ-95$ \\
\hline $44101.70 \mathrm{C}$ & $3.629985 E-29$ & \$Ru101 \\
\hline $45103.70 \mathrm{c}$ & $3.559438 E-29$ & \$Rh103 \\
\hline $45105.70 \mathrm{C}$ & $3.491572 \mathrm{E}-29$ & \$Rh105 \\
\hline $48113.70 \mathrm{C}$ & $3.244211 \mathrm{E}-29$ & $\$ \mathrm{Cd} 113$ \\
\hline $54131.70 \mathrm{C}$ & $2.798102 \mathrm{E}-29$ & $\$ x e 131$ \\
\hline $54133.70 \mathrm{C}$ & $2.756002 \mathrm{E}-29$ & $\$ x e 133$ \\
\hline $55133.70 \mathrm{C}$ & $2.755987 \mathrm{E}-29$ & $\$ \mathrm{Cs} 133$ \\
\hline $54135.70 \mathrm{C}$ & $2.695116 \mathrm{E}-29$ & $\$ x e 135$ \\
\hline $57140.70 \mathrm{C}$ & $2.618012 \mathrm{E}-29$ & \$La140 \\
\hline $58141.70 \mathrm{C}$ & $2.599480 \mathrm{E}-29$ & $\$ \mathrm{Ce} 141$ \\
\hline $59143.70 \mathrm{C}$ & $2.563058 \mathrm{E}-29$ & $\$ \operatorname{Pr} 143$ \\
\hline $60143.70 \mathrm{C}$ & $2.563055 E-29$ & $\$ N d 143$ \\
\hline $60145.70 \mathrm{C}$ & $2.527633 E-29$ & $\$ N d 145$ \\
\hline $61147.70 \mathrm{C}$ & $2.493199 \mathrm{E}-29$ & $\$ \operatorname{Pm} 147$ \\
\hline $61149.70 \mathrm{C}$ & $2.459663 E-29$ & $\$ \operatorname{Pm} 149$ \\
\hline $62149.70 \mathrm{C}$ & $2.459661 \mathrm{E}-29$ & $\$ S m 149$ \\
\hline $61151.70 \mathrm{C}$ & $2.426995 E-29$ & \$Pm151 \\
\hline $62151.70 \mathrm{c}$ & $2.427021 \mathrm{E}-29$ & $\$ \mathrm{Sm} 151$ \\
\hline $62152.70 \mathrm{C}$ & $2.411048 \mathrm{E}-29$ & $\$$ Sm152 \\
\hline $63153.70 \mathrm{c}$ & $2.395258 \mathrm{E}-29$ & \$Eu153 \\
\hline $63155.70 \mathrm{C}$ & $2.364321 \mathrm{E}-29$ & \$Eu155 \\
\hline $64157.70 \mathrm{C}$ & $2.334161 \mathrm{E}-29$ & $\$$ Gd1 57 \\
\hline
\end{tabular}




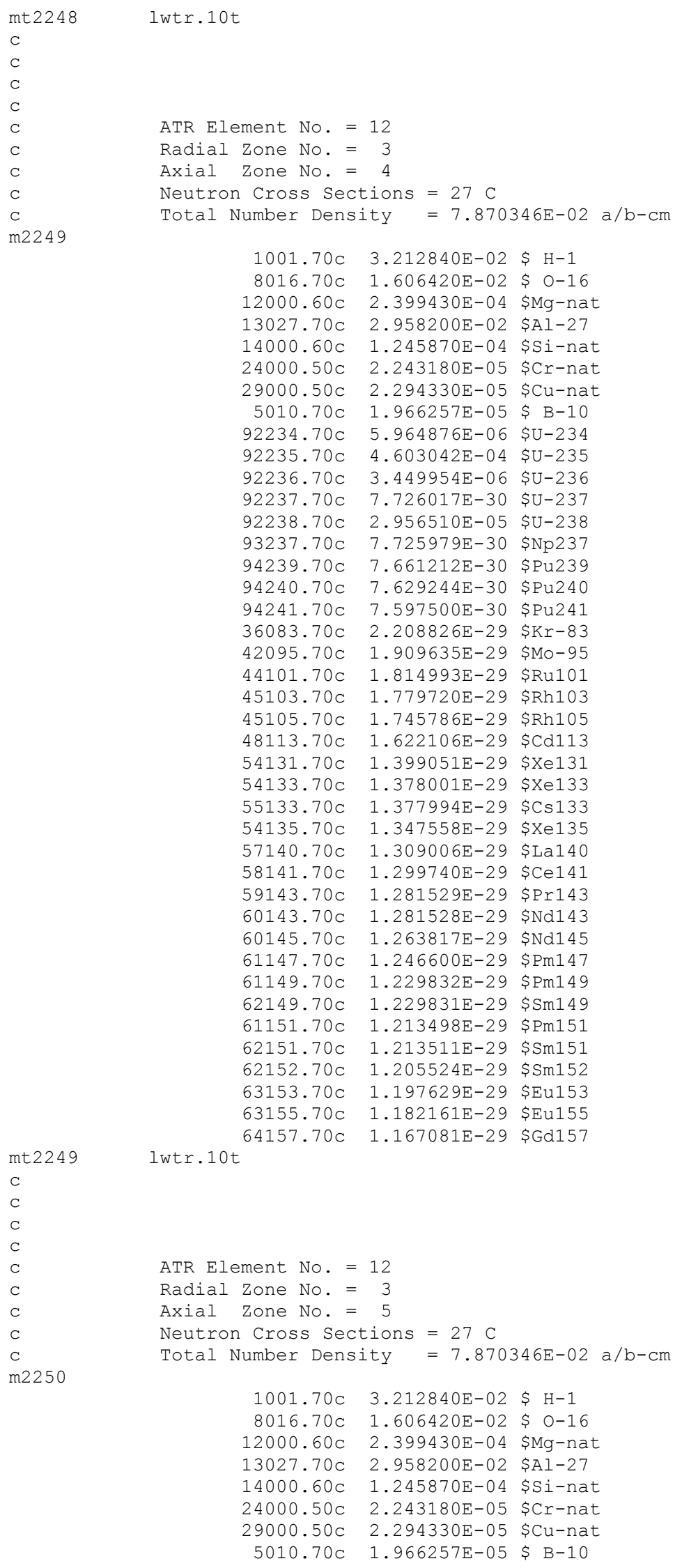




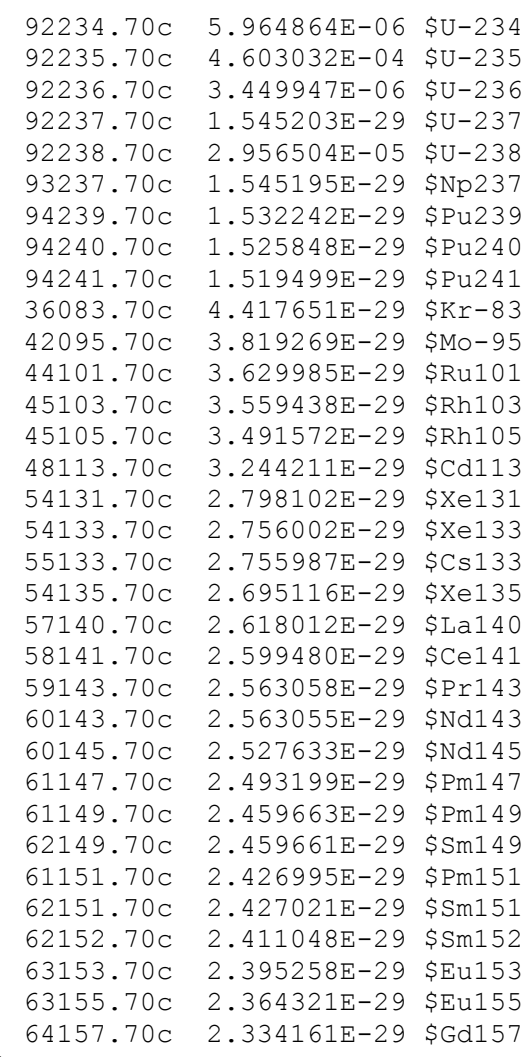

mt2250 lwtr.10t

$\mathrm{C}$

C

C

C 
$57140.70 \mathrm{C} \quad 2.618012 \mathrm{E}-29$ \$La140

$58141.70 \mathrm{C} \quad 2.599480 \mathrm{E}-29$ \$Ce141

$59143.70 \mathrm{C} \quad 2.563058 \mathrm{E}-29 \quad \$ \mathrm{Pr} 143$

$60143.70 \mathrm{C} \quad 2.563055 \mathrm{E}-29 \$ \mathrm{Nd} 143$

$60145.70 \mathrm{C} \quad 2.527633 \mathrm{E}-29 \$ \mathrm{Nd} 145$

$61147.70 \mathrm{C} \quad 2.493199 \mathrm{E}-29 \$ \mathrm{Pm} 147$

$61149.70 \mathrm{C} \quad 2.459663 \mathrm{E}-29 \$ \mathrm{Pm} 149$

$62149.70 \mathrm{C} \quad 2.459661 \mathrm{E}-29 \quad \$ \mathrm{Sm} 149$

$61151.70 \mathrm{C} \quad 2.426995 \mathrm{E}-29$ \$Pm151

$62151.70 \mathrm{C} \quad 2.427021 \mathrm{E}-29$ \$Sm151

$62152.70 \mathrm{C} \quad 2.411048 \mathrm{E}-29 \$ \mathrm{Sm} 152$

$63153.70 \mathrm{C} \quad 2.395258 \mathrm{E}-29$ \$Eu153

$63155.70 \mathrm{C} \quad 2.364321 \mathrm{E}-29$ \$Eu155

mt2251 lwtr.10t

C

C

C

C

$\mathrm{C}$

C

C

C

m2252

$64157.70 \mathrm{C} \quad 2.334161 \mathrm{E}-29 \$ \mathrm{Gd} 157$

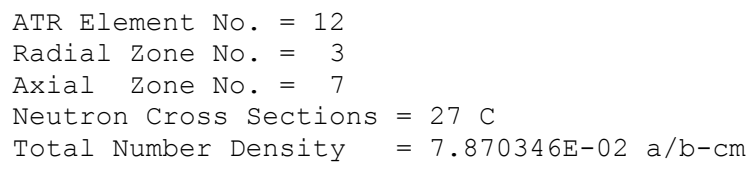




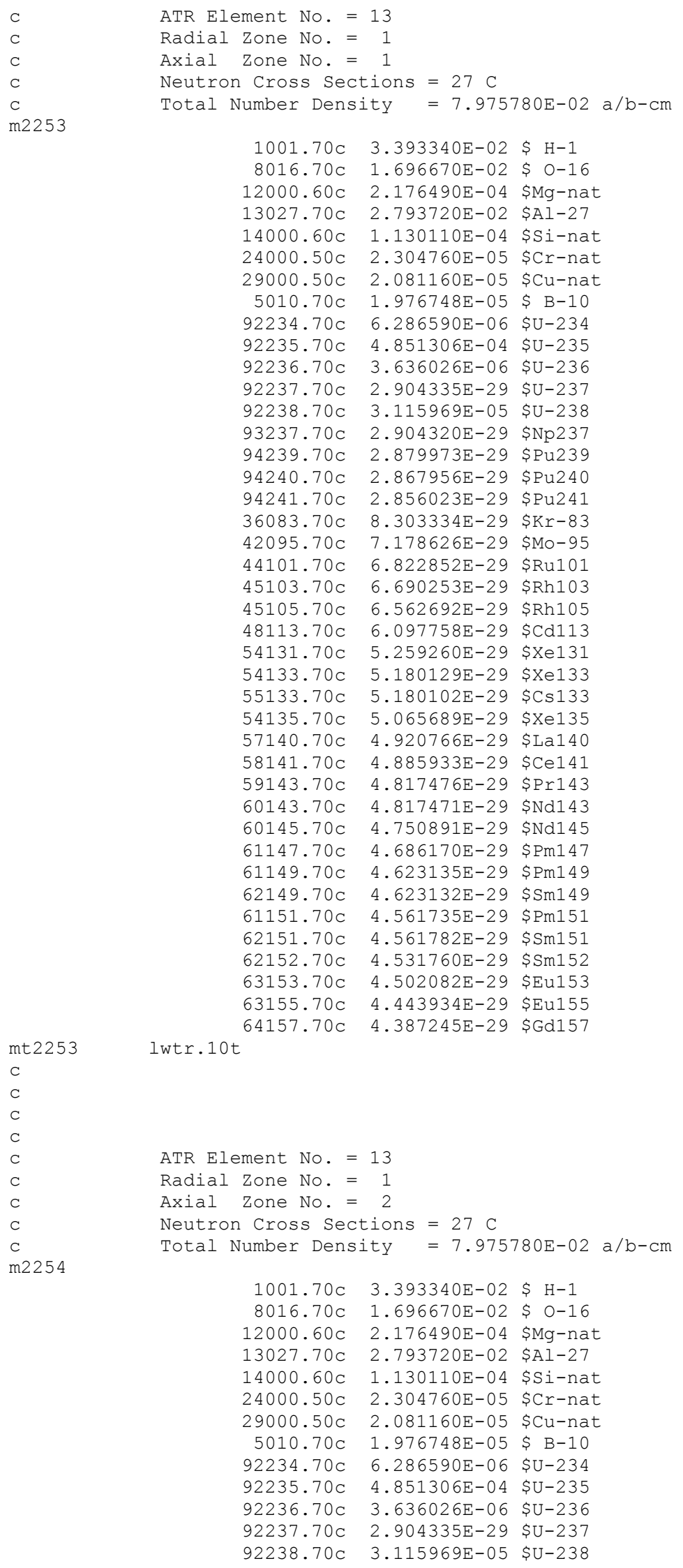




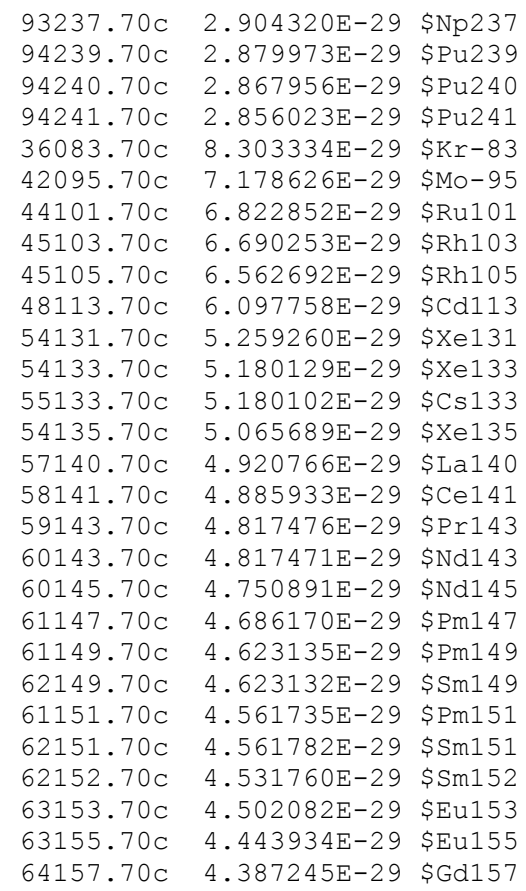

mt2254 lwtr.10t

$\mathrm{C}$

$\mathrm{C}$

C

$\mathrm{C}$

C ATR Element No. = 13

Radial Zone No. = 1

C Axial Zone No. = 3

C Neutron Cross Sections $=27 \mathrm{C}$

c Total Number Density $=7.975780 \mathrm{E}-02 \mathrm{a} / \mathrm{b}-\mathrm{cm}$

m2255

\begin{tabular}{|c|c|c|}
\hline $1001.70 \mathrm{c}$ & $3.393340 E-02$ & $\$ \mathrm{H}-1$ \\
\hline $8016.70 \mathrm{c}$ & $1.696670 \mathrm{E}-02$ & $\$ 0-16$ \\
\hline $12000.60 \mathrm{c}$ & $2.176490 \mathrm{E}-04$ & \$Mg-nat \\
\hline $13027.70 \mathrm{c}$ & $2.793720 \mathrm{E}-02$ & $\$ A l-27$ \\
\hline $14000.60 \mathrm{c}$ & $1.130110 \mathrm{E}-04$ & \$Si-nat \\
\hline $24000.50 \mathrm{c}$ & $2.304760 E-05$ & $\$ C r-n a t$ \\
\hline $29000.50 \mathrm{C}$ & $2.081160 \mathrm{E}-05$ & \$Cu-nat \\
\hline $5010.70 \mathrm{c}$ & $1.976748 E-05$ & $\$ B-10$ \\
\hline $92234.70 \mathrm{C}$ & $6.286590 \mathrm{E}-06$ & \$U-234 \\
\hline $92235.70 \mathrm{C}$ & $4.851306 \mathrm{E}-04$ & $\$ \mathrm{U}-235$ \\
\hline $92236.70 \mathrm{C}$ & $3.636026 \mathrm{E}-06$ & $\$ \mathrm{U}-236$ \\
\hline $92237.70 \mathrm{C}$ & $2.904335 E-29$ & $\$ \mathrm{U}-237$ \\
\hline $92238.70 \mathrm{C}$ & $3.115969 \mathrm{E}-05$ & $\$ \mathrm{U}-238$ \\
\hline $93237.70 \mathrm{C}$ & $2.904320 E-29$ & $\$ N p 237$ \\
\hline $94239.70 \mathrm{C}$ & $2.879973 E-29$ & \$Pu239 \\
\hline $94240.70 \mathrm{C}$ & $2.867956 \mathrm{E}-29$ & $\$ P u 240$ \\
\hline $94241.70 \mathrm{C}$ & $2.856023 E-29$ & \$Pu241 \\
\hline $36083.70 \mathrm{c}$ & $8.303334 \mathrm{E}-29$ & $\$ K r-83$ \\
\hline $42095.70 \mathrm{C}$ & $7.178626 \mathrm{E}-29$ & $\$ M o-95$ \\
\hline $44101.70 \mathrm{C}$ & $6.822852 \mathrm{E}-29$ & \$Ru101 \\
\hline $45103.70 \mathrm{C}$ & $6.690253 E-29$ & \$Rh103 \\
\hline $45105.70 \mathrm{C}$ & $6.562692 \mathrm{E}-29$ & \$Rh105 \\
\hline $48113.70 \mathrm{C}$ & $6.097758 E-29$ & $\$ \mathrm{Cd} 113$ \\
\hline $54131.70 \mathrm{C}$ & $5.259260 \mathrm{E}-29$ & \$Xe131 \\
\hline $54133.70 \mathrm{C}$ & $5.180129 \mathrm{E}-29$ & \$Xe133 \\
\hline $55133.70 \mathrm{c}$ & $5.180102 E-29$ & $\$ \mathrm{Cs} 133$ \\
\hline $54135.70 \mathrm{C}$ & $5.065689 \mathrm{E}-29$ & $\$ \mathrm{Xe1} 35$ \\
\hline $57140.70 \mathrm{C}$ & $4.920766 E-29$ & \$La140 \\
\hline $58141.70 \mathrm{C}$ & $4.885933 E-29$ & $\$ C e 141$ \\
\hline $59143.70 \mathrm{C}$ & $4.817476 E-29$ & $\$ \operatorname{Pr} 143$ \\
\hline $60143.70 \mathrm{c}$ & $4.817471 E-29$ & $\$ N d 143$ \\
\hline $60145.70 \mathrm{c}$ & $4.750891 E-29$ & $\$ N d 145$ \\
\hline
\end{tabular}


$61147.70 \mathrm{C} \quad 4.686170 \mathrm{E}-29 \$ \mathrm{Pm} 147$

$61149.70 \mathrm{C} \quad 4.623135 \mathrm{E}-29 \$ \mathrm{Pm} 149$

$62149.70 \mathrm{C} \quad 4.623132 \mathrm{E}-29$ \$Sm149

$61151.70 \mathrm{C} \quad 4.561735 \mathrm{E}-29$ \$Pm151

$62151.70 \mathrm{C} \quad 4.561782 \mathrm{E}-29 \$ \mathrm{Sm} 151$

$62152.70 \mathrm{C} \quad 4.531760 \mathrm{E}-29 \$ \mathrm{Sm} 152$

$63153.70 \mathrm{C} \quad 4.502082 \mathrm{E}-29$ \$Eu153

$63155.70 \mathrm{C} \quad 4.443934 \mathrm{E}-29 \quad \$ \mathrm{Eu} 155$

mt2255 lwtr.10t

$64157.70 \mathrm{C} \quad 4.387245 \mathrm{E}-29$ \$Gd157

c

C

C

C

C

C

$\mathrm{C}$

m2256 mt2256

C

C

C

C

C

C

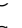

ATR Element No. = 13

Radial Zone No. = 1

Axial Zone No. = 4

Neutron Cross Sections $=27 \mathrm{C}$

Total Number Density $=7.975780 \mathrm{E}-02 \mathrm{a} / \mathrm{b}-\mathrm{cm}$

$\begin{array}{llll}1001.70 \mathrm{C} & 3.393340 \mathrm{E}-02 & \mathrm{~S}-1 \\ 8016.70 \mathrm{C} & 1.696670 \mathrm{E}-02 & \mathrm{~S} & \mathrm{O}-1\end{array}$

$\begin{array}{rrr}8016.70 \mathrm{C} & 1.696670 \mathrm{E}-02 & \$ \mathrm{O}-16 \\ 12000.60 \mathrm{C} & 2.176490 \mathrm{E}-04 & \$ \mathrm{Mg}-\mathrm{nat}\end{array}$

$13027.70 \mathrm{C} \quad 2.793720 \mathrm{E}-02$ \$Al-27

$14000.60 \mathrm{C} \quad 1.130110 \mathrm{E}-04$ \$Si-nat

$24000.50 \mathrm{C} \quad 2.304760 \mathrm{E}-05$ \$Cr-nat

$29000.50 \mathrm{C} \quad 2.081160 \mathrm{E}-05$ \$Cu-nat

$5010.70 \mathrm{C} \quad 1.976749 \mathrm{E}-05$ \$ $\mathrm{B}-10$

$92234.70 \mathrm{C} \quad 6.286591 \mathrm{E}-06 \quad \$ \mathrm{U}-234$

$92235.70 \mathrm{C} \quad 4.851306 \mathrm{E}-04 \quad \$ \mathrm{U}-235$

$92236.70 \mathrm{C} \quad 3.636026 \mathrm{E}-06 \quad \$ \mathrm{U}-236$

$92237.70 \mathrm{C} \quad 1.452167 \mathrm{E}-29 \quad \$ \mathrm{U}-237$

$92238.70 \mathrm{C} \quad 3.115969 \mathrm{E}-05 \quad \mathrm{SU}-238$

$93237.70 \mathrm{C} \quad 1.452160 \mathrm{E}-29 \$ \mathrm{~Np} 237$

$94239.70 \mathrm{C} \quad 1.439987 \mathrm{E}-29$ \$Pu239

$94240.70 \mathrm{C} \quad 1.433978 \mathrm{E}-29$ \$Pu240

$94241.70 \mathrm{C} \quad 1.428012 \mathrm{E}-29$ \$Pu241

$36083.70 \mathrm{C} \quad 4.151667 \mathrm{E}-29$ \$Kr-83

$42095.70 \mathrm{C} \quad 3.589313 \mathrm{E}-29$ \$Mo-95

$44101.70 \mathrm{C} \quad 3.411426 \mathrm{E}-29$ \$Ru101

$45103.70 \mathrm{C} \quad 3.345127 \mathrm{E}-29 \$ \mathrm{Rh} 103$

$45105.70 \mathrm{C} \quad 3.281347 \mathrm{E}-29$ \$Rh105

$48113.70 \mathrm{C} \quad 3.048880 \mathrm{E}-29 \$ \mathrm{Cd} 113$

$54131.70 \mathrm{C} \quad 2.629630 \mathrm{E}-29$ \$Xe131

$54133.70 \mathrm{C} \quad 2.590065 \mathrm{E}-29 \$ \mathrm{Xe} 133$

$55133.70 \mathrm{C} \quad 2.590051 \mathrm{E}-29$ \$Cs133

$54135.70 \mathrm{C} \quad 2.532845 \mathrm{E}-29$ \$Xe135

$57140.70 \mathrm{C} \quad 2.460383 \mathrm{E}-29$ \$La140

$58141.70 \mathrm{C} \quad 2.442967 \mathrm{E}-29$ Se141

$59143.70 \mathrm{C} \quad 2.408738 \mathrm{E}-29$ \$Pr143

$60143.70 \mathrm{C} \quad 2.408736 \mathrm{E}-29 \$ \mathrm{Nd} 143$

$60145.70 \mathrm{C} \quad 2.375446 \mathrm{E}-29 \$ \mathrm{Nd} 145$

$61147.70 \mathrm{C} \quad 2.343085 \mathrm{E}-29$ \$Pm147

$61149.70 \mathrm{C} \quad 2.311568 \mathrm{E}-29 \$ \mathrm{Pm} 149$

$62149.70 \mathrm{C} \quad 2.311566 \mathrm{E}-29 \quad \$ \mathrm{Sm} 149$

$61151.70 \mathrm{C} \quad 2.280868 \mathrm{E}-29$ \$Pm151

$62151.70 \mathrm{C} \quad 2.280891 \mathrm{E}-29$ \$Sm151

$62152.70 \mathrm{C} \quad 2.265881 \mathrm{E}-29$ \$Sm152

$63153.70 \mathrm{C} \quad 2.251041 \mathrm{E}-29$ \$Eu153

$63155.70 \mathrm{C} \quad 2.221967 \mathrm{E}-29$ \$Eu155

lwtr.10t

$64157.70 \mathrm{C} \quad 2.193623 \mathrm{E}-29$ \$Gd157

ATR Element No. = 13

Radial Zone No. $=1$

Axial Zone No. = 5

Neutron Cross Sections $=27 \mathrm{C}$

Total Number Density $=7.975780 \mathrm{E}-02 \mathrm{a} / \mathrm{b}-\mathrm{cm}$ 


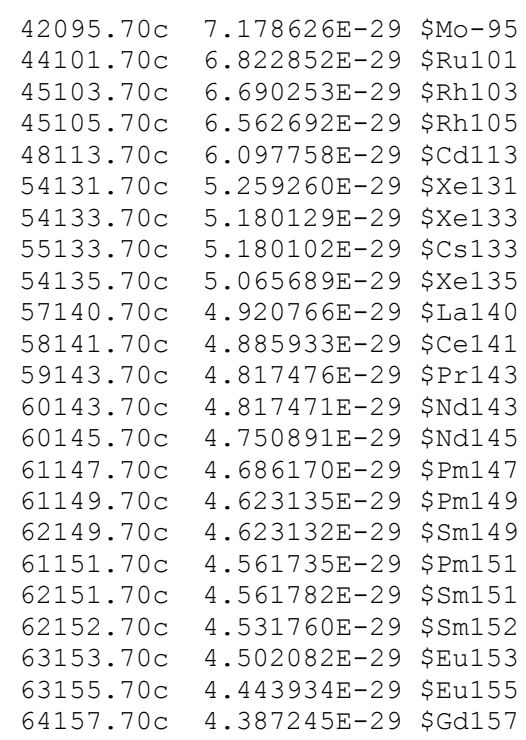

mt2258 lwtr.10t

c

C

C

C

C

C

C

m2259

ATR Element No. = 13

Radial Zone No. $=1$

Axial Zone No. $=7$

Neutron Cross Sections $=27 \mathrm{C}$

Total Number Density $=7.975780 \mathrm{E}-02 \mathrm{a} / \mathrm{b}-\mathrm{cm}$

$1001.70 \mathrm{C} \quad 3.393340 \mathrm{E}-02$ \$ $\mathrm{H}-1$

$8016.70 \mathrm{C} \quad 1.696670 \mathrm{E}-02$ \$ O-16

$12000.60 \mathrm{C} \quad 2.176490 \mathrm{E}-04$ \$Mg-nat

$13027.70 \mathrm{C} \quad 2.793720 \mathrm{E}-02$ \$Al-27

$14000.60 \mathrm{C} \quad 1.130110 \mathrm{E}-04$ \$Si-nat

$24000.50 \mathrm{C} \quad 2.304760 \mathrm{E}-05$ \$Cr-nat

$29000.50 \mathrm{C} \quad 2.081160 \mathrm{E}-05$ \$Cu-nat

$5010.70 \mathrm{C} \quad 1.976748 \mathrm{E}-05$ \$ B-10

$92234.70 \mathrm{C} \quad 6.286590 \mathrm{E}-06 \quad \$ \mathrm{U}-234$

$92235.70 \mathrm{C} \quad 4.851306 \mathrm{E}-04 \quad \$ \mathrm{U}-235$

$92236.70 \mathrm{C} \quad 3.636026 \mathrm{E}-06$ \$U-236

$92237.70 \mathrm{C} \quad 2.904335 \mathrm{E}-29 \quad \$ \mathrm{U}-237$

$92238.70 \mathrm{C} \quad 3.115969 \mathrm{E}-05$ \$U-238

$93237.70 \mathrm{C} \quad 2.904320 \mathrm{E}-29 \quad \$ N p 237$

$94239.70 \mathrm{C} \quad 2.879973 \mathrm{E}-29$ \$Pu239

$94240.70 \mathrm{C} \quad 2.867956 \mathrm{E}-29$ \$Pu240

$94241.70 \mathrm{C} \quad 2.856023 \mathrm{E}-29$ \$Pu 241

$36083.70 \mathrm{C} \quad 8.303334 \mathrm{E}-29$ \$Kr-83

$42095.70 \mathrm{C} \quad 7.178626 \mathrm{E}-29$ \$Mo-95

$44101.70 \mathrm{C} \quad 6.822852 \mathrm{E}-29$ \$Ru101

$45103.70 \mathrm{C} \quad 6.690253 \mathrm{E}-29$ \$Rh103

$45105.70 \mathrm{C} \quad 6.562692 \mathrm{E}-29 \$ \mathrm{Rh} 105$

$48113.70 \mathrm{C} \quad 6.097758 \mathrm{E}-29$ \$Cd113

$54131.70 \mathrm{C} \quad 5.259260 \mathrm{E}-29$ \$Xe131

$54133.70 \mathrm{C} \quad 5.180129 \mathrm{E}-29$ \$Xe133

$55133.70 \mathrm{C} \quad 5.180102 \mathrm{E}-29 \$ \mathrm{Cs} 133$

$54135.70 \mathrm{C} \quad 5.065689 \mathrm{E}-29$ \$Xe135

$57140.70 \mathrm{C} \quad 4.920766 \mathrm{E}-29$ \$La140

$58141.70 \mathrm{C} \quad 4.885933 \mathrm{E}-29$ \$Ce141

$59143.70 \mathrm{C} \quad 4.817476 \mathrm{E}-29 \quad \$ \mathrm{Pr} 143$

$60143.70 \mathrm{C} \quad 4.817471 \mathrm{E}-29 \$ \mathrm{Nd1} 43$

$60145.70 \mathrm{C} \quad 4.750891 \mathrm{E}-29$ \$Nd145

$61147.70 \mathrm{C} \quad 4.686170 \mathrm{E}-29 \$ \mathrm{Pm} 147$

$61149.70 \mathrm{C} \quad 4.623135 \mathrm{E}-29 \quad \$ \mathrm{Pm} 149$

$62149.70 \mathrm{C} \quad 4.623132 \mathrm{E}-29 \$ \mathrm{Sm} 149$

$61151.70 \mathrm{C} \quad 4.561735 \mathrm{E}-29$ \$Pm151

$62151.70 \mathrm{C} \quad 4.561782 \mathrm{E}-29 \$ \mathrm{Sm} 151$ 


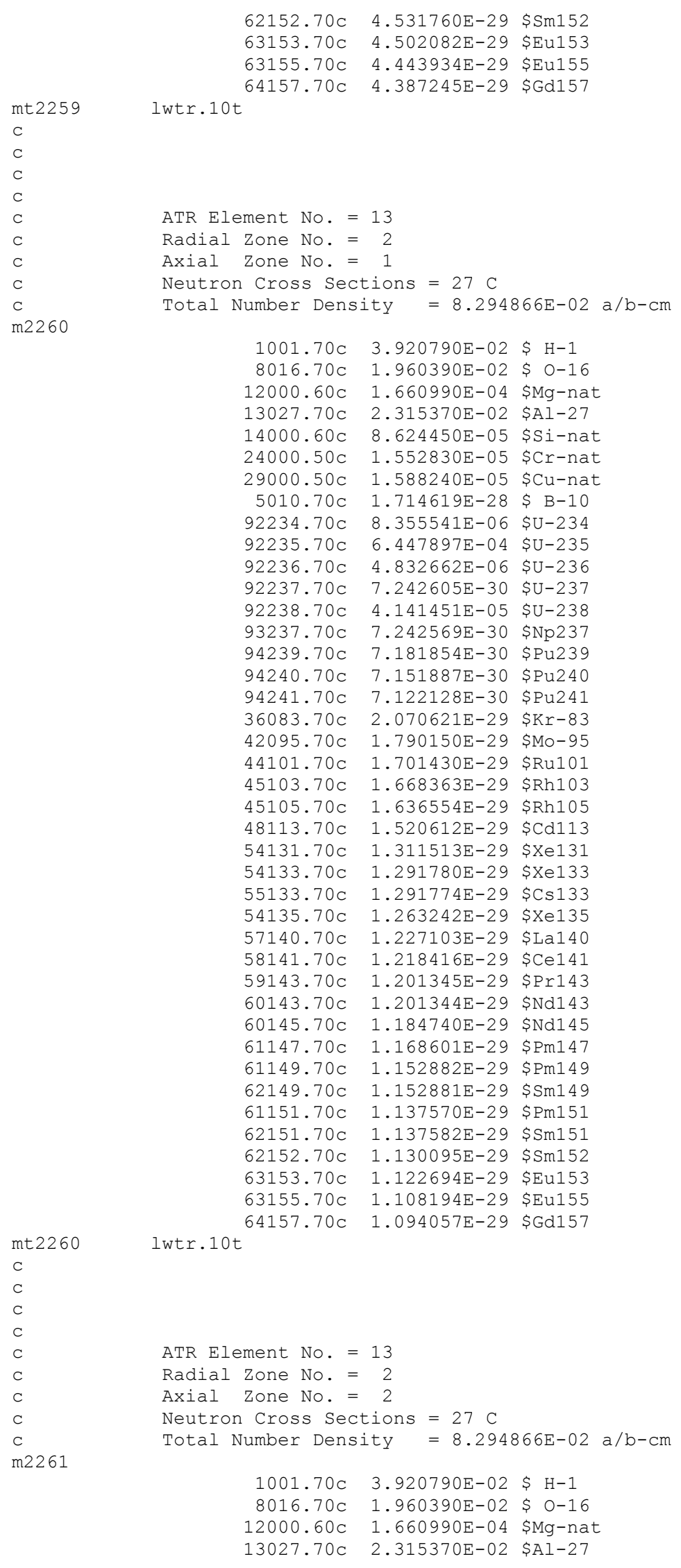




\begin{tabular}{|c|c|c|}
\hline $14000.60 \mathrm{C}$ & $8.624450 \mathrm{E}-05$ & \$Si-nat \\
\hline $24000.50 \mathrm{c}$ & $1.552830 \mathrm{E}-05$ & $\$ C r-n a t$ \\
\hline $9000.50 \mathrm{c}$ & $1.588240 \mathrm{E}-05$ & $\$ \mathrm{Cu}-\mathrm{nat}$ \\
\hline $5010.70 \mathrm{c}$ & $1.714619 \mathrm{E}-28$ & $\$ B-10$ \\
\hline $2234.70 \mathrm{C}$ & $8.355541 \mathrm{E}-06$ & $\$ U-234$ \\
\hline $92235.70 \mathrm{C}$ & $6.447897 \mathrm{E}-04$ & $\$ U-235$ \\
\hline $92236.70 \mathrm{c}$ & $4.832662 \mathrm{E}-06$ & $\$ \mathrm{U}-236$ \\
\hline $92237.70 \mathrm{C}$ & $7.242605 \mathrm{E}-30$ & $\$ U-237$ \\
\hline $2238.70 c$ & $4.141451 \mathrm{E}-05$ & $\$ U-238$ \\
\hline $237.70 \mathrm{c}$ & $7.242569 \mathrm{E}-30$ & $\$ N p 237$ \\
\hline $4239.70 \mathrm{C}$ & $7.181854 \mathrm{E}-30$ & \$Pu239 \\
\hline $94240.70 \mathrm{c}$ & $7.151887 \mathrm{E}-30$ & \$Pu240 \\
\hline $94241.70 \mathrm{C}$ & $7.122128 \mathrm{E}-30$ & \$Pu2 41 \\
\hline $36083.70 \mathrm{c}$ & $2.070621 \mathrm{E}-29$ & $\$ K r-83$ \\
\hline $42095.70 \mathrm{C}$ & $1.790150 \mathrm{E}-29$ & \$Mo-95 \\
\hline $44101.70 \mathrm{C}$ & $1.701430 \mathrm{E}-29$ & \$Ru101 \\
\hline $45103.70 \mathrm{C}$ & $1.668363 \mathrm{E}-29$ & \$Rh103 \\
\hline $45105.70 \mathrm{c}$ & $1.636554 \mathrm{E}-29$ & \$Rh105 \\
\hline $48113.70 \mathrm{C}$ & $1.520612 \mathrm{E}-29$ & $\$ C d 113$ \\
\hline $54131.70 \mathrm{C}$ & $1.311513 \mathrm{E}-29$ & $\$$ Xe131 \\
\hline $54133.70 \mathrm{C}$ & $1.291780 \mathrm{E}-29$ & $\$ \mathrm{Xe133}$ \\
\hline $55133.70 \mathrm{C}$ & $1.291774 \mathrm{E}-29$ & $\$ C s 133$ \\
\hline $54135.70 \mathrm{C}$ & $1.263242 \mathrm{E}-29$ & $\$$ Xe135 \\
\hline $57140.70 \mathrm{C}$ & $1.227103 \mathrm{E}-29$ & \$La140 \\
\hline $58141.70 \mathrm{C}$ & $1.218416 \mathrm{E}-29$ & $\$ C e 141$ \\
\hline $59143.70 \mathrm{C}$ & $1.201345 \mathrm{E}-29$ & $\$ \operatorname{Pr} 143$ \\
\hline $60143.70 \mathrm{c}$ & $1.201344 \mathrm{E}-29$ & $\$ \mathrm{Nd} 143$ \\
\hline $60145.70 \mathrm{c}$ & $1.184740 \mathrm{E}-29$ & $\$ N d 145$ \\
\hline $61147.70 \mathrm{c}$ & $1.168601 \mathrm{E}-29$ & \$Pm1 47 \\
\hline $61149.70 \mathrm{C}$ & $1.152882 \mathrm{E}-29$ & $\$ \operatorname{Pm} 149$ \\
\hline $62149.70 \mathrm{c}$ & $1.152881 \mathrm{E}-29$ & $\$ \operatorname{Sm} 149$ \\
\hline $61151.70 \mathrm{C}$ & $1.137570 \mathrm{E}-29$ & \$Pm151 \\
\hline $62151.70 \mathrm{C}$ & $1.137582 \mathrm{E}-29$ & \$Sm151 \\
\hline $62152.70 \mathrm{c}$ & $1.130095 \mathrm{E}-29$ & \$Sm152 \\
\hline $63153.70 \mathrm{c}$ & $1.122694 \mathrm{E}-29$ & \$Eu153 \\
\hline $63155.70 \mathrm{C}$ & $1.108194 \mathrm{E}-29$ & \$Eu155 \\
\hline 64157.7 & $1.094057 \mathrm{E}-29$ & \$Gd157 \\
\hline
\end{tabular}

mt2261 lwtr.10t

c

C

C

C

C ATR Element No. $=13$

C Radial Zone No. = 2

C Axial Zone No. = 3

C Neutron Cross Sections $=27 \mathrm{C}$

c Total Number Density $=8.294866 \mathrm{E}-02 \mathrm{a} / \mathrm{b}-\mathrm{cm}$

m2262

$\begin{array}{rll}1001.70 \mathrm{C} & 3.920790 \mathrm{E}-02 & \text { \$ }-1 \\ 8016.70 \mathrm{C} & 1.960390 \mathrm{E}-02 & \text { \$ }-16 \\ 12000.60 \mathrm{C} & 1.660990 \mathrm{E}-04 & \text { \$Mg-nat } \\ 13027.70 \mathrm{C} & 2.315370 \mathrm{E}-02 & \text { \$Al-27 } \\ 14000.60 \mathrm{C} & 8.624450 \mathrm{E}-05 & \text { \$Si-nat } \\ 24000.50 \mathrm{C} & 1.552830 \mathrm{E}-05 & \text { \$Cr-nat } \\ 29000.50 \mathrm{C} & 1.588240 \mathrm{E}-05 & \text { \$Cu-nat } \\ 5010.70 \mathrm{C} & 1.714619 \mathrm{E}-28 & \text { \$ }-10 \\ 92234.70 \mathrm{C} & 8.355541 \mathrm{E}-06 & \text { \$U-234 } \\ 92235.70 \mathrm{C} & 6.447897 \mathrm{E}-04 & \text { \$U-235 } \\ 92236.70 \mathrm{C} & 4.832662 \mathrm{E}-06 & \text { \$U-236 } \\ 92237.70 \mathrm{C} & 7.242605 \mathrm{E}-30 & \text { \$U-237 } \\ 92238.70 \mathrm{C} & 4.141451 \mathrm{E}-05 & \text { \$U-238 } \\ 93237.70 \mathrm{C} & 7.242569 \mathrm{E}-30 & \text { \$Np237 } \\ 94239.70 \mathrm{C} & 7.181854 \mathrm{E}-30 & \text { \$Pu239 } \\ 94240.70 \mathrm{C} & 7.151887 \mathrm{E}-30 & \text { \$Pu240 } \\ 94241.70 \mathrm{C} & 7.122128 \mathrm{E}-30 & \text { \$Pu241 } \\ 36083.70 \mathrm{C} & 2.070621 \mathrm{E}-29 & \text { \$Kr-83 } \\ 42095.70 \mathrm{C} & 1.790150 \mathrm{E}-29 & \text { \$Mo-95 } \\ 44101.70 \mathrm{C} & 1.701430 \mathrm{E}-29 & \text { \$Ru101 } \\ 45103.70 \mathrm{C} & 1.668363 \mathrm{E}-29 & \text { \$Rh103 } \\ 45105.70 \mathrm{C} & 1.636554 \mathrm{E}-29 & \text { \$Rh105 } \\ 48113.70 \mathrm{C} & 1.520612 \mathrm{E}-29 & \text { \$Cd113 }\end{array}$



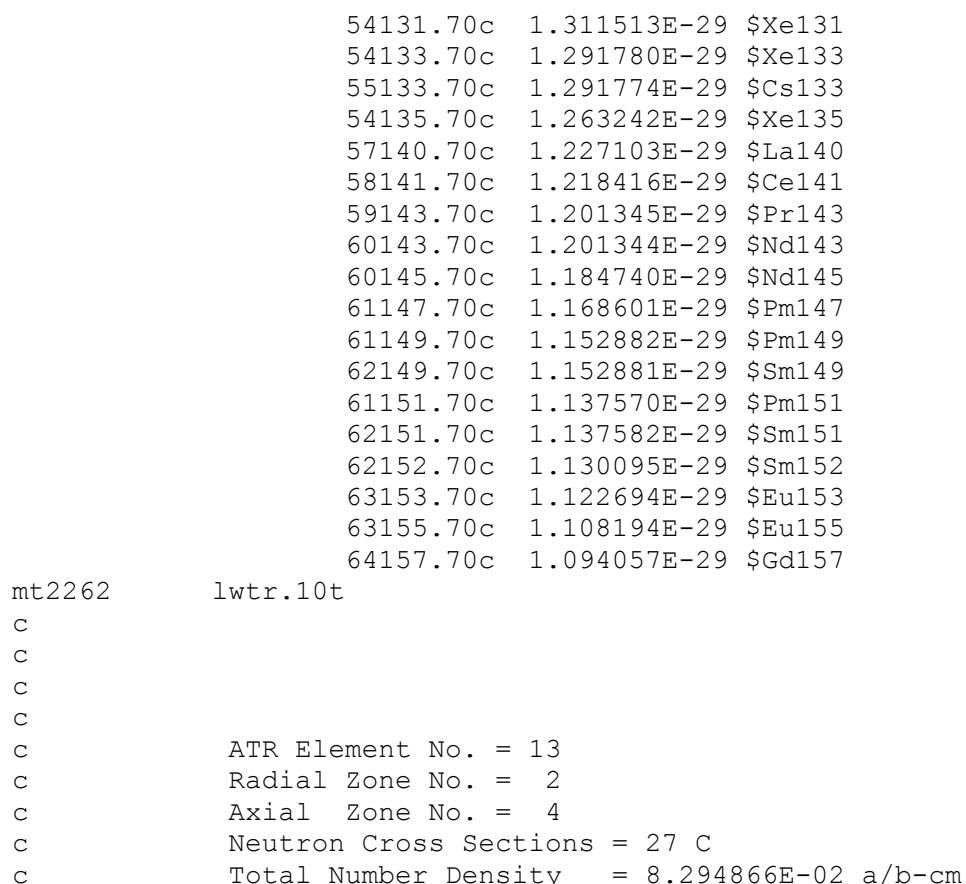
C
ATR Element No. = 13

Radial Zone No. = 2

Axial Zone No. = 5

Neutron Cross Sections $=27 \mathrm{C}$

Total Number Density $=8.294866 \mathrm{E}-02 \mathrm{a} / \mathrm{b}-\mathrm{cm}$

$8016.70 \mathrm{C} \quad 1.960390 \mathrm{E}-02$ \$ $0-16$

$12000.60 \mathrm{C} \quad 1.660990 \mathrm{E}-04$ \$Mg-nat

$13027.70 \mathrm{C} \quad 2.315370 \mathrm{E}-02$ \$Al-27

$14000.60 \mathrm{C} \quad 8.624450 \mathrm{E}-05$ \$Si-nat

$24000.50 \mathrm{C} \quad 1.552830 \mathrm{E}-05$ \$Cr-nat

$29000.50 \mathrm{C} \quad 1.588240 \mathrm{E}-05$ \$Cu-nat

$5010.70 \mathrm{C} \quad 1.714619 \mathrm{E}-28$ \$ B -10

$92234.70 \mathrm{C} \quad 8.355541 \mathrm{E}-06$ \$U-234

$92235.70 \mathrm{C} \quad 6.447897 \mathrm{E}-04 \quad \$ \mathrm{U}-235$

$92236.70 \mathrm{C} \quad 4.832662 \mathrm{E}-06 \quad \$ \mathrm{U}-236$

$92237.70 \mathrm{C} \quad 7.242605 \mathrm{E}-30$ \$U-237

$92238.70 \mathrm{C} \quad 4.141451 \mathrm{E}-05$ \$U-238

$93237.70 \mathrm{C} \quad 7.242569 \mathrm{E}-30 \quad \$ \mathrm{~Np} 237$

$94239.70 \mathrm{C} \quad 7.181854 \mathrm{E}-30$ \$Pu239

$94240.70 \mathrm{C} \quad 7.151887 \mathrm{E}-30$ \$Pu240

$94241.70 \mathrm{C} \quad 7.122128 \mathrm{E}-30$ \$Pu241

$36083.70 \mathrm{C} \quad 2.070621 \mathrm{E}-29 \mathrm{SKr}-83$

$42095.70 \mathrm{C} \quad 1.790150 \mathrm{E}-29$ \$Mo-95

$44101.70 \mathrm{C} \quad 1.701430 \mathrm{E}-29$ \$Ru101

$45103.70 \mathrm{C} \quad 1.668363 \mathrm{E}-29$ \$Rh103

$45105.70 \mathrm{C} \quad 1.636554 \mathrm{E}-29$ \$Rh105

$48113.70 \mathrm{C} \quad 1.520612 \mathrm{E}-29$ \$Cd113

$54131.70 \mathrm{C} \quad 1.311513 \mathrm{E}-29$ \$Xe131

$54133.70 \mathrm{C} \quad 1.291780 \mathrm{E}-29$ \$Xe133

$55133.70 \mathrm{C} \quad 1.291774 \mathrm{E}-29$ \$Cs133

$54135.70 \mathrm{C} \quad 1.263242 \mathrm{E}-29$ \$Xe135

$57140.70 \mathrm{C} \quad 1.227103 \mathrm{E}-29$ \$La140

$58141.70 \mathrm{C} \quad 1.218416 \mathrm{E}-29$ \$Ce141

$59143.70 \mathrm{C} \quad 1.201345 \mathrm{E}-29 \$ \operatorname{Pr} 143$

$60143.70 \mathrm{C} \quad 1.201344 \mathrm{E}-29 \$ \mathrm{Nd} 143$

$60145.70 \mathrm{C} \quad 1.184740 \mathrm{E}-29$ \$Nd145

$61147.70 \mathrm{C} \quad 1.168601 \mathrm{E}-29 \$ \mathrm{Pm} 147$

$61149.70 \mathrm{C} \quad 1.152882 \mathrm{E}-29$ \$Pm149

$62149.70 \mathrm{C} \quad 1.152881 \mathrm{E}-29$ \$Sm149

$61151.70 \mathrm{C} \quad 1.137570 \mathrm{E}-29$ \$Pm151

$62151.70 \mathrm{C} \quad 1.137582 \mathrm{E}-29 \$ \mathrm{Sm} 151$

$62152.70 \mathrm{C} \quad 1.130095 \mathrm{E}-29$ \$Sm152

$63153.70 \mathrm{C} \quad 1.122694 \mathrm{E}-29$ \$Eu153

$63155.70 \mathrm{C} \quad 1.108194 \mathrm{E}-29$ \$Eu155

$64157.70 \mathrm{C} \quad 1.094057 \mathrm{E}-29 \$ \mathrm{Gd} 157$

lwtr.10t

ATR Element No. $=13$

Radial Zone No. $=2$

Axial Zone No. $=6$

Neutron Cross Sections $=27 \mathrm{C}$

Total Number Density $=8.294866 \mathrm{E}-02 \mathrm{a} / \mathrm{b}-\mathrm{cm}$

$1001.70 \mathrm{C} \quad 3.920790 \mathrm{E}-02 \$ \mathrm{H}-1$

$8016.70 \mathrm{C} \quad 1.960390 \mathrm{E}-02 \$ 0-16$

$12000.60 \mathrm{C} \quad 1.660990 \mathrm{E}-04$ \$Mg-nat

$13027.70 \mathrm{C} \quad 2.315370 \mathrm{E}-02$ \$Al-27

$14000.60 \mathrm{C} \quad 8.624450 \mathrm{E}-05$ \$Si-nat

$24000.50 \mathrm{C} \quad 1.552830 \mathrm{E}-05$ \$Cr-nat

$29000.50 \mathrm{C} \quad 1.588240 \mathrm{E}-05$ \$Cu-nat

$5010.70 \mathrm{C} \quad 1.714619 \mathrm{E}-28$ \$ B-10

$92234.70 \mathrm{C} \quad 8.355541 \mathrm{E}-06$ \$U-234 
$92235.70 \mathrm{C} \quad 6.447897 \mathrm{E}-04 \quad \$ \mathrm{U}-235$

$92236.70 \mathrm{C} \quad 4.832662 \mathrm{E}-06 \quad \$ \mathrm{U}-236$

$92237.70 \mathrm{C} \quad 7.242605 \mathrm{E}-30 \quad \$ \mathrm{U}-237$

$92238.70 \mathrm{C} \quad 4.141451 \mathrm{E}-05 \mathrm{\$ U}-238$

$93237.70 \mathrm{C} \quad 7.242569 \mathrm{E}-30 \quad \$ \mathrm{~Np} 237$

$94239.70 \mathrm{C} \quad 7.181854 \mathrm{E}-30$ \$Pu239

$94240.70 \mathrm{C} \quad 7.151887 \mathrm{E}-30$ \$Pu2 40

$94241.70 \mathrm{C} \quad 7.122128 \mathrm{E}-30$ \$Pu 241

$36083.70 \mathrm{C} \quad 2.070621 \mathrm{E}-29$ \$Kr-83

$42095.70 \mathrm{C} \quad 1.790150 \mathrm{E}-29$ \$Mo-95

$44101.70 \mathrm{C} \quad 1.701430 \mathrm{E}-29$ \$Ru101

$45103.70 \mathrm{C} \quad 1.668363 \mathrm{E}-29$ \$Rh103

$45105.70 \mathrm{C} \quad 1.636554 \mathrm{E}-29$ \$Rh105

$48113.70 \mathrm{C} \quad 1.520612 \mathrm{E}-29$ \$Cd113

$54131.70 \mathrm{C} \quad 1.311513 \mathrm{E}-29$ \$Xe131

$54133.70 \mathrm{C} \quad 1.291780 \mathrm{E}-29$ \$Xe133

$55133.70 \mathrm{C} \quad 1.291774 \mathrm{E}-29 \quad \$ \mathrm{Cs} 133$

$54135.70 \mathrm{C} \quad 1.263242 \mathrm{E}-29$ \$Xe135

$57140.70 \mathrm{C} \quad 1.227103 \mathrm{E}-29$ \$La140

$58141.70 \mathrm{C} \quad 1.218416 \mathrm{E}-29 \$ \mathrm{Ce} 141$

$59143.70 \mathrm{C} \quad 1.201345 \mathrm{E}-29 \$ \operatorname{Pr} 143$

$60143.70 \mathrm{C} \quad 1.201344 \mathrm{E}-29 \$ \mathrm{Nd} 143$

$60145.70 \mathrm{C} \quad 1.184740 \mathrm{E}-29 \$ \mathrm{Nd1} 45$

$61147.70 \mathrm{C} \quad 1.168601 \mathrm{E}-29$ \$Pm147

$61149.70 \mathrm{C} \quad 1.152882 \mathrm{E}-29 \$ \mathrm{Pm} 149$

$62149.70 \mathrm{C} \quad 1.152881 \mathrm{E}-29$ \$Sm149

$61151.70 \mathrm{C} \quad 1.137570 \mathrm{E}-29$ \$Pm151

$62151.70 \mathrm{C} \quad 1.137582 \mathrm{E}-29 \quad \$ \mathrm{Sm} 151$

$62152.70 \mathrm{C} \quad 1.130095 \mathrm{E}-29 \$ \mathrm{Sm} 152$

$63153.70 \mathrm{C} \quad 1.122694 \mathrm{E}-29$ \$Eu153

$63155.70 \mathrm{C} \quad 1.108194 \mathrm{E}-29$ \$Eu155

mt2265 lwtr.10t

$64157.70 \mathrm{C} \quad 1.094057 \mathrm{E}-29$ \$Gd157

C

C

$\mathrm{C}$

m2266

ATR Element No. = 13

Radial Zone No. = 2

Axial Zone No. = 7

Neutron Cross Sections $=27 \mathrm{C}$

Total Number Density $=8.294866 \mathrm{E}-02 \mathrm{a} / \mathrm{b}-\mathrm{cm}$

$1001.70 \mathrm{C} \quad 3.920790 \mathrm{E}-02$ \$ H-1

$8016.70 \mathrm{C} \quad 1.960390 \mathrm{E}-02$ \$ O-16

$12000.60 \mathrm{C} \quad 1.660990 \mathrm{E}-04$ \$Mg-nat

$13027.70 \mathrm{C} \quad 2.315370 \mathrm{E}-02$ \$Al-27

$14000.60 \mathrm{C} \quad 8.624450 \mathrm{E}-05$ \$Si-nat

$24000.50 \mathrm{C} \quad 1.552830 \mathrm{E}-05$ \$Cr-nat

$29000.50 \mathrm{C} \quad 1.588240 \mathrm{E}-05$ \$Cu-nat

$5010.70 \mathrm{C} \quad 1.714619 \mathrm{E}-28$ \$ B-10

$92234.70 \mathrm{C} \quad 8.355541 \mathrm{E}-06 \quad \$ \mathrm{U}-234$

$92235.70 \mathrm{C} \quad 6.447897 \mathrm{E}-04 \quad \mathrm{SU}-235$

$92236.70 \mathrm{C} \quad 4.832662 \mathrm{E}-06 \quad \$ \mathrm{U}-236$

$92237.70 \mathrm{C} \quad 7.242605 \mathrm{E}-30 \quad \$ \mathrm{U}-237$

$92238.70 \mathrm{C} \quad 4.141451 \mathrm{E}-05$ \$U-238

$93237.70 \mathrm{C} \quad 7.242569 \mathrm{E}-30 \quad \$ \mathrm{~Np} 237$

$94239.70 \mathrm{C} \quad 7.181854 \mathrm{E}-30$ \$Pu239

$94240.70 \mathrm{C} \quad 7.151887 \mathrm{E}-30$ \$Pu240

$94241.70 \mathrm{C} \quad 7.122128 \mathrm{E}-30 \$ \mathrm{Pu} 241$

$36083.70 \mathrm{C} \quad 2.070621 \mathrm{E}-29$ \$Kr-83

$42095.70 \mathrm{C} \quad 1.790150 \mathrm{E}-29$ \$Mo-95

$44101.70 \mathrm{C} \quad 1.701430 \mathrm{E}-29$ \$Ru101

$45103.70 \mathrm{C} \quad 1.668363 \mathrm{E}-29$ \$Rh103

$45105.70 \mathrm{C} \quad 1.636554 \mathrm{E}-29$ \$Rh105

$48113.70 \mathrm{C} \quad 1.520612 \mathrm{E}-29$ \$Cd113

$54131.70 \mathrm{C} \quad 1.311513 \mathrm{E}-29$ \$Xe131

$54133.70 \mathrm{C} \quad 1.291780 \mathrm{E}-29$ \$Xe133

$55133.70 \mathrm{C} \quad 1.291774 \mathrm{E}-29 \$ \mathrm{Cs} 133$

$54135.70 \mathrm{C} \quad 1.263242 \mathrm{E}-29$ \$Xe135

$57140.70 \mathrm{C} \quad 1.227103 \mathrm{E}-29$ \$La140 


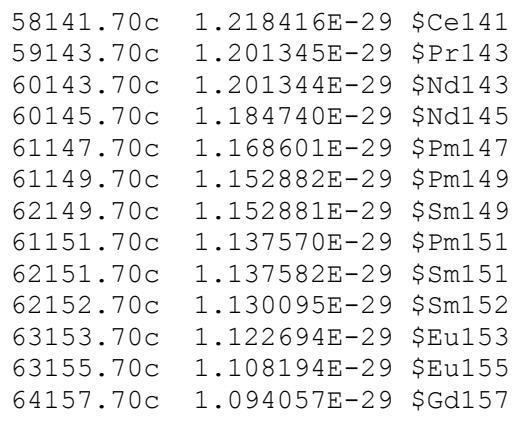

mt2266
C
C
C
C
C
C
C
C
C
m2267

lwtr.10t

ATR Element No. = 13

Radial Zone No. $=3$

Axial Zone No. = 1

Neutron Cross Sections $=27 \mathrm{C}$

Total Number Density $=7.870346 \mathrm{E}-02 \mathrm{a} / \mathrm{b}-\mathrm{cm}$

$1001.70 \mathrm{C} \quad 3.212840 \mathrm{E}-02 \$ \mathrm{H}-1$

$8016.70 \mathrm{C} \quad 1.606420 \mathrm{E}-02 \$ 0-16$

$12000.60 \mathrm{C} \quad 2.399430 \mathrm{E}-04$ \$Mg-nat

$13027.70 \mathrm{C} \quad 2.958200 \mathrm{E}-02 \quad \$ \mathrm{Al}-27$

$14000.60 \mathrm{C} \quad 1.245870 \mathrm{E}-04$ \$Si-nat

$24000.50 \mathrm{C} \quad 2.243180 \mathrm{E}-05$ \$Cr-nat

$29000.50 \mathrm{C} \quad 2.294330 \mathrm{E}-05$ \$Cu-nat

$5010.70 \mathrm{C} \quad 1.966257 \mathrm{E}-05 \$ \mathrm{~B}-10$

$92234.70 \mathrm{C} \quad 5.964864 \mathrm{E}-06 \quad \$ \mathrm{U}-234$

$92235.70 \mathrm{C} \quad 4.603032 \mathrm{E}-04 \quad \$ \mathrm{U}-235$

$92236.70 \mathrm{C} \quad 3.449947 \mathrm{E}-06 \quad \$ \mathrm{U}-236$

$92237.70 \mathrm{C} \quad 1.545203 \mathrm{E}-29 \quad \$ \mathrm{U}-237$

$92238.70 \mathrm{C} \quad 2.956504 \mathrm{E}-05 \quad \$ \mathrm{U}-238$

$93237.70 \mathrm{C} \quad 1.545195 \mathrm{E}-29$ \$Np237

$94239.70 \mathrm{C} \quad 1.532242 \mathrm{E}-29$ \$Pu239

$94240.70 \mathrm{C} \quad 1.525848 \mathrm{E}-29$ \$Pu240

$94241.70 \mathrm{C} \quad 1.519499 \mathrm{E}-29$ \$Pu241

$36083.70 \mathrm{C} \quad 4.417651 \mathrm{E}-29 \quad \$ \mathrm{Kr}-83$

$42095.70 \mathrm{C} \quad 3.819269 \mathrm{E}-29$ \$Mo-95

$44101.70 \mathrm{C} \quad 3.629985 \mathrm{E}-29$ \$Ru101

$45103.70 \mathrm{C} \quad 3.559438 \mathrm{E}-29$ \$Rh103

$45105.70 \mathrm{C} \quad 3.491572 \mathrm{E}-29$ \$Rh105

$48113.70 \mathrm{C} \quad 3.244211 \mathrm{E}-29$ \$Cd113

$54131.70 \mathrm{C} \quad 2.798102 \mathrm{E}-29$ \$Xe131

$54133.70 \mathrm{C} \quad 2.756002 \mathrm{E}-29$ \$Xe133

$55133.70 \mathrm{C} \quad 2.755987 \mathrm{E}-29 \quad \$ \mathrm{Cs} 133$

$54135.70 \mathrm{C} \quad 2.695116 \mathrm{E}-29$ Xe135

$57140.70 \mathrm{C} \quad 2.618012 \mathrm{E}-29$ \$La140

$58141.70 \mathrm{C} \quad 2.599480 \mathrm{E}-29$ \$Ce141

$59143.70 \mathrm{C} \quad 2.563058 \mathrm{E}-29$ \$Pr143

$60143.70 \mathrm{C} \quad 2.563055 \mathrm{E}-29 \$ \mathrm{Nd} 143$

$60145.70 \mathrm{C} \quad 2.527633 \mathrm{E}-29$ \$Nd145

$61147.70 \mathrm{C} \quad 2.493199 \mathrm{E}-29 \quad \$ \mathrm{Pm} 147$

$61149.70 \mathrm{C} \quad 2.459663 \mathrm{E}-29 \$ \mathrm{Pm} 149$

$62149.70 \mathrm{C} \quad 2.459661 \mathrm{E}-29$ \$Sm149

$61151.70 \mathrm{C} \quad 2.426995 \mathrm{E}-29$ \$Pm151

$62151.70 \mathrm{C} \quad 2.427021 \mathrm{E}-29$ \$Sm151

$62152.70 \mathrm{C} \quad 2.411048 \mathrm{E}-29 \$ \mathrm{Sm} 152$

$63153.70 \mathrm{C} \quad 2.395258 \mathrm{E}-29$ \$Eu153

$63155.70 \mathrm{C} \quad 2.364321 \mathrm{E}-29$ \$Eu155

mt2267 lwtr.10t

$64157.70 \mathrm{C} \quad 2.334161 \mathrm{E}-29$ \$Gd157

$\mathrm{C}$

C

C

c

ATR Element No. = 13 


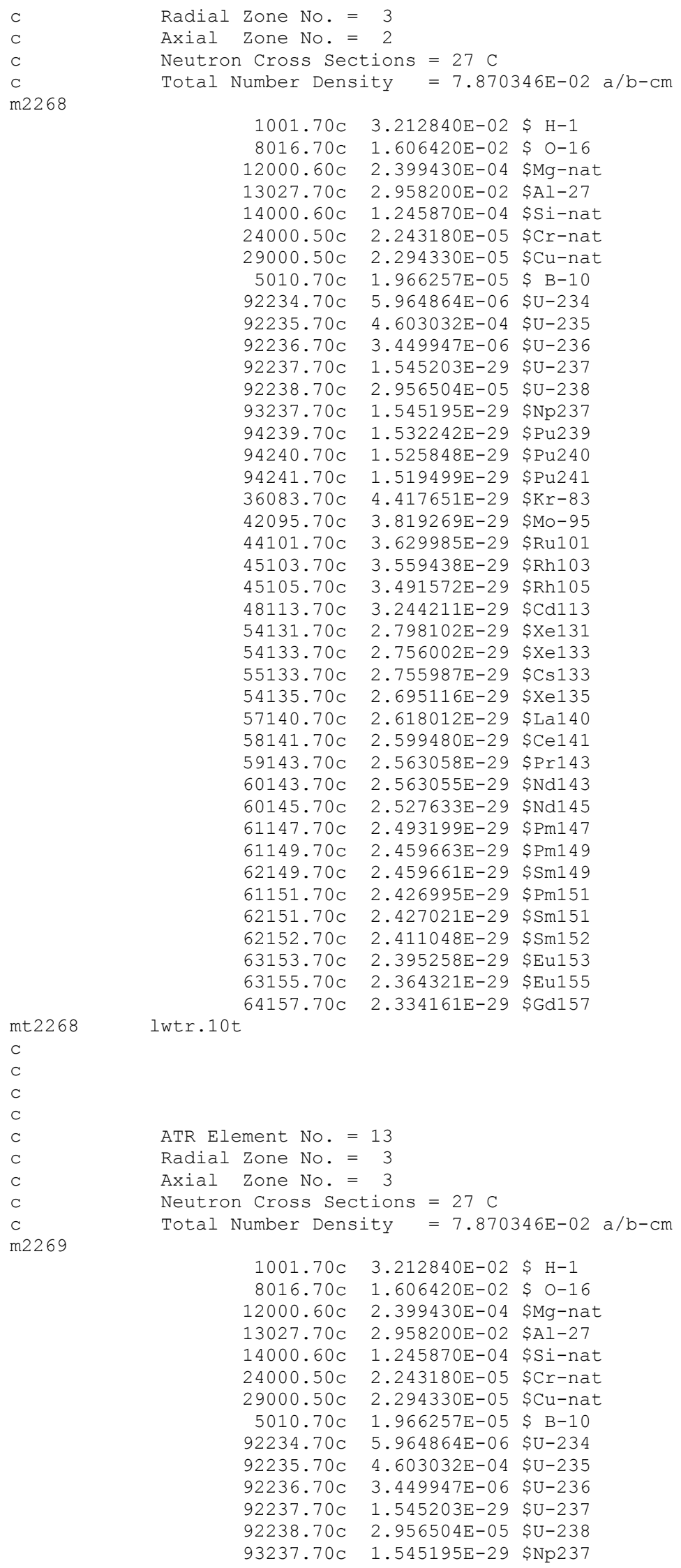


$94239.70 \mathrm{C} \quad 1.532242 \mathrm{E}-29 \$ \mathrm{Pu} 239$

$94240.70 \mathrm{C} \quad 1.525848 \mathrm{E}-29$ \$Pu240

$94241.70 \mathrm{C} \quad 1.519499 \mathrm{E}-29$ \$Pu241

$36083.70 \mathrm{C} \quad 4.417651 \mathrm{E}-29 \$ \mathrm{Kr}-83$

$42095.70 \mathrm{C} \quad 3.819269 \mathrm{E}-29$ \$Mo-95

$44101.70 \mathrm{C} \quad 3.629985 \mathrm{E}-29$ \$Ru101

$45103.70 \mathrm{C} \quad 3.559438 \mathrm{E}-29 \$ \mathrm{Rh} 103$

$45105.70 \mathrm{C} \quad 3.491572 \mathrm{E}-29$ \$Rh105

$48113.70 \mathrm{C} \quad 3.244211 \mathrm{E}-29 \$ \mathrm{Cd} 113$

$54131.70 \mathrm{C} \quad 2.798102 \mathrm{E}-29$ \$Xe131

$54133.70 \mathrm{C} \quad 2.756002 \mathrm{E}-29$ \$Xe133

$55133.70 \mathrm{C} \quad 2.755987 \mathrm{E}-29 \$ \mathrm{Cs} 133$

$54135.70 \mathrm{C} \quad 2.695116 \mathrm{E}-29$ \$Xe135

$57140.70 \mathrm{C} \quad 2.618012 \mathrm{E}-29$ \$La140

$58141.70 \mathrm{C} \quad 2.599480 \mathrm{E}-29$ Se141

$59143.70 \mathrm{C} \quad 2.563058 \mathrm{E}-29 \quad \$ \mathrm{Pr} 143$

$60143.70 \mathrm{C} \quad 2.563055 \mathrm{E}-29 \$ \mathrm{Nd} 143$

$60145.70 \mathrm{C} \quad 2.527633 \mathrm{E}-29 \$ \mathrm{Nd} 145$

$61147.70 \mathrm{C} \quad 2.493199 \mathrm{E}-29 \quad \$ \mathrm{Pm} 147$

$61149.70 \mathrm{C} \quad 2.459663 \mathrm{E}-29 \$ \mathrm{Pm} 149$

$62149.70 \mathrm{C} \quad 2.459661 \mathrm{E}-29 \quad \$ \mathrm{Sm} 149$

$61151.70 \mathrm{C} \quad 2.426995 \mathrm{E}-29$ \$Pm151

$62151.70 \mathrm{C} \quad 2.427021 \mathrm{E}-29 \quad \$ \mathrm{Sm} 151$

$62152.70 \mathrm{C} \quad 2.411048 \mathrm{E}-29 \$ \mathrm{Sm} 152$

$63153.70 \mathrm{C} \quad 2.395258 \mathrm{E}-29 \$ \mathrm{Eu} 153$

$63155.70 \mathrm{C} \quad 2.364321 \mathrm{E}-29$ \$Eu155

mt2269 lwtr.10t

$64157.70 \mathrm{C} \quad 2.334161 \mathrm{E}-29 \$ \mathrm{Gd} 157$

c

$\mathrm{C}$

$\mathrm{C}$

C

$\mathrm{C}$

C

m2270

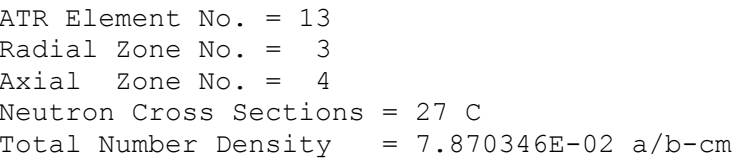

\begin{tabular}{|c|c|c|}
\hline $1001.70 \mathrm{c}$ & $3.212840 \mathrm{E}-02$ & $\$ \mathrm{H}-1$ \\
\hline $8016.70 \mathrm{C}$ & $1.606420 \mathrm{E}-02$ & $\$ 0-16$ \\
\hline $2000.60 \mathrm{c}$ & $2.399430 \mathrm{E}-04$ & $\$ M g-n a t$ \\
\hline $027.70 \mathrm{C}$ & $2.958200 \mathrm{E}-02$ & $\$ A 1-27$ \\
\hline $000.60 \mathrm{C}$ & $1.245870 \mathrm{E}-04$ & \$Si-nat \\
\hline $4000.50 \mathrm{c}$ & $2.243180 \mathrm{E}-05$ & \$Cr-nat \\
\hline $000.50 \mathrm{C}$ & $2.294330 \mathrm{E}-05$ & $\$ \mathrm{Cu}-\mathrm{nat}$ \\
\hline$C$ & $5257 E-05$ & $\$ B-10$ \\
\hline $.70 \mathrm{C}$ & $5.964876 \mathrm{E}-06$ & $\$ U-234$ \\
\hline $.70 \mathrm{C}$ & $4.603042 E-04$ & $\$ U-235$ \\
\hline $.70 \mathrm{C}$ & $3.449954 \mathrm{E}-06$ & $\$ U-236$ \\
\hline $.70 \mathrm{C}$ & $7.726017 E-30$ & $\$ U-237$ \\
\hline $70 \mathrm{C}$ & $2.956510 \mathrm{E}-05$ & $\$ U-238$ \\
\hline $.70 \mathrm{C}$ & $7.725979 E-30$ & $\$ \mathrm{~Np} 237$ \\
\hline$C$ & $2 E-30$ & $\$ P u 239$ \\
\hline $.70 \mathrm{C}$ & 7. $629244 \mathrm{E}-30$ & $\$ \mathrm{Pu} 240$ \\
\hline c & $0 E-30$ & \$Pu241 \\
\hline $.70 \mathrm{C}$ & $2.208826 \mathrm{E}-29$ & $\$ K r-83$ \\
\hline$c$ & 1. $909635 \mathrm{E}-29$ & $\$ M o-95$ \\
\hline $.70 \mathrm{C}$ & $3 E-29$ & \$Ru101 \\
\hline .7 & $1.779720 \mathrm{E}-29$ & $\$ R h 103$ \\
\hline $.70 \mathrm{C}$ & $6 E-29$ & \$Rh105 \\
\hline & $1.622106 \mathrm{E}-29$ & $\$ \mathrm{Cd} 113$ \\
\hline C & $1.399051 \mathrm{E}-29$ & $\$ x e 131$ \\
\hline $.70 \mathrm{C}$ & $1.378001 \mathrm{E}-29$ & $\$ x e 133$ \\
\hline $.70 \mathrm{C}$ & $994 E-29$ & $\$ C$ s 133 \\
\hline $.70 \mathrm{C}$ & $1.347558 \mathrm{E}-29$ & \$Xe135 \\
\hline $.70 \mathrm{C}$ & $006 E-29$ & $\$ \mathrm{La} 140$ \\
\hline $.70 \mathrm{C}$ & 1.299740E-29 & $\$ C e 141$ \\
\hline 59 & 1.281529E-29 & $\$ \operatorname{Pr} 143$ \\
\hline & 1.281528E-29 & $\$ N d 143$ \\
\hline 601 & $1.263817 \mathrm{E}-29$ & $\$ N d 145$ \\
\hline 611 & $1.246600 \mathrm{E}-29$ & $\$ P m 147$ \\
\hline
\end{tabular}


$61149.70 \mathrm{C} \quad 1.229832 \mathrm{E}-29 \$ \mathrm{Pm} 149$

$62149.70 \mathrm{C} \quad 1.229831 \mathrm{E}-29 \$ \mathrm{Sm} 149$

$61151.70 \mathrm{C} \quad 1.213498 \mathrm{E}-29$ \$Pm151

$62151.70 \mathrm{C} \quad 1.213511 \mathrm{E}-29 \$ \mathrm{Sm} 151$

$62152.70 \mathrm{C} \quad 1.205524 \mathrm{E}-29 \$ \mathrm{Sm} 152$

$63153.70 \mathrm{C} \quad 1.197629 \mathrm{E}-29$ \$Eu153

$63155.70 \mathrm{C} \quad 1.182161 \mathrm{E}-29$ \$Eu155

mt2270 lwtr.10t

$64157.70 \mathrm{C} \quad 1.167081 \mathrm{E}-29$ \$Gd157

C

$\mathrm{C}$

C

C ATR Element No. = 13

C Radial Zone No. = 3

C Axial Zone No. = 5

C Neutron Cross Sections $=27 \mathrm{C}$

C Total Number Density $=7.870346 \mathrm{E}-02 \mathrm{a} / \mathrm{b}-\mathrm{cm}$

m2271

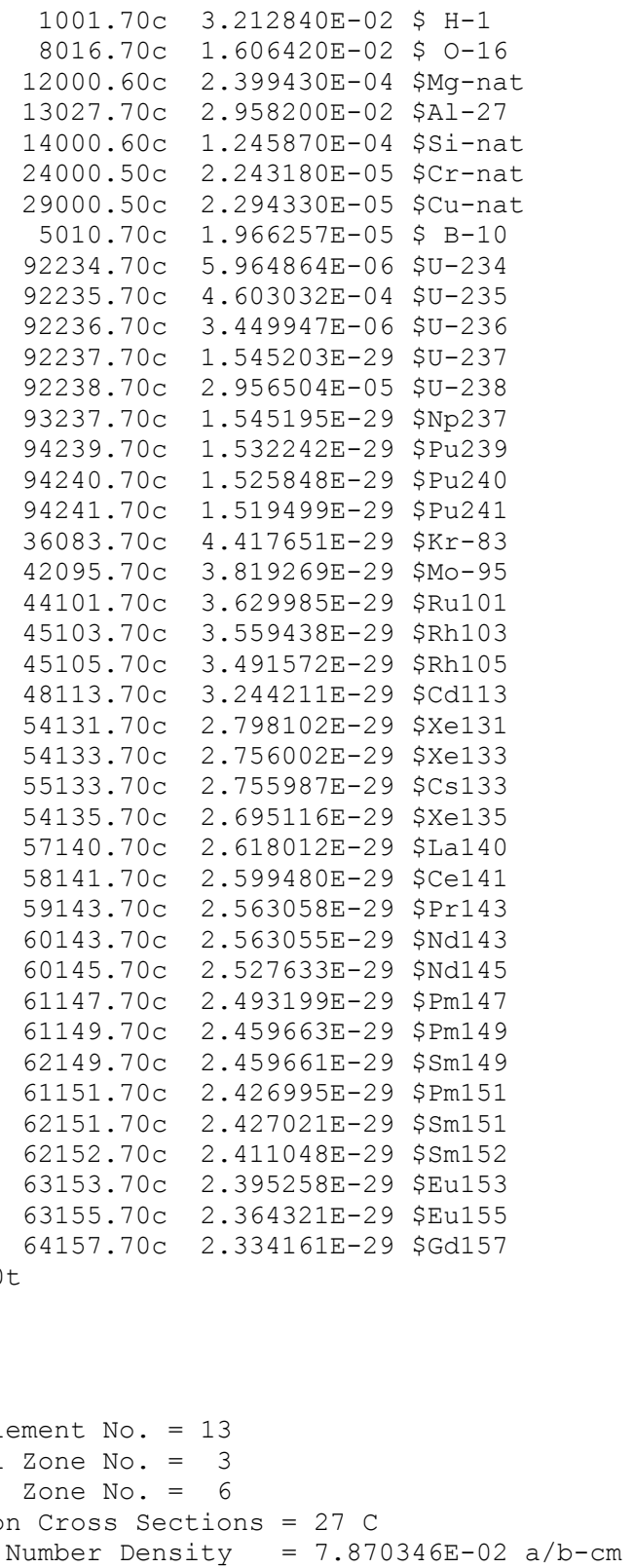

mt2271 lwtr.10t

c

C

C

C

$\mathrm{C}$
ATR Element NO= $=13$

Radial Zone No. $=3$

Axial Zone No. =

Neutron Cross Sections $=27 \mathrm{C}$

Total Number Density $=7.870346 \mathrm{E}-02 \mathrm{a} / \mathrm{b}-\mathrm{cm}$ 


\begin{tabular}{|c|c|c|}
\hline $1001.70 \mathrm{C}$ & $3.212840 \mathrm{E}-02$ & $\$ H-1$ \\
\hline $8016.70 c$ & $1.606420 \mathrm{E}-02$ & $\$ 0-16$ \\
\hline $12000.60 \mathrm{c}$ & $2.399430 \mathrm{E}-04$ & \$Mg-nat \\
\hline $13027.70 \mathrm{C}$ & $2.958200 \mathrm{E}-02$ & $\$ A 1-27$ \\
\hline $14000.60 \mathrm{c}$ & $1.245870 \mathrm{E}-04$ & \$Si-nat \\
\hline $24000.50 \mathrm{C}$ & $2.243180 \mathrm{E}-05$ & $\$ \mathrm{Cr}-\mathrm{nat}$ \\
\hline $29000.50 \mathrm{C}$ & $2.294330 \mathrm{E}-05$ & $\$ \mathrm{Cu}-\mathrm{nat}$ \\
\hline $5010.70 \mathrm{C}$ & $1.966257 \mathrm{E}-05$ & $\$ B-10$ \\
\hline $92234.70 \mathrm{C}$ & $5.964864 \mathrm{E}-06$ & $\$ U-234$ \\
\hline $92235.70 \mathrm{C}$ & $4.603032 \mathrm{E}-04$ & $\$ U-235$ \\
\hline $92236.70 \mathrm{C}$ & $3.449947 \mathrm{E}-06$ & $\$ U-236$ \\
\hline $92237.70 \mathrm{C}$ & $1.545203 \mathrm{E}-29$ & $\$ U-237$ \\
\hline $92238.70 \mathrm{c}$ & $2.956504 \mathrm{E}-05$ & $\$ U-238$ \\
\hline $93237.70 \mathrm{c}$ & $1.545195 \mathrm{E}-29$ & \$Np237 \\
\hline $94239.70 \mathrm{C}$ & $1.532242 \mathrm{E}-29$ & \$Pu239 \\
\hline $94240.70 \mathrm{C}$ & $1.525848 \mathrm{E}-29$ & \$Pu240 \\
\hline $94241.70 \mathrm{C}$ & $1.519499 \mathrm{E}-29$ & \$Pu241 \\
\hline $36083.70 \mathrm{c}$ & $4.417651 \mathrm{E}-29$ & $\$ K r-83$ \\
\hline $42095.70 \mathrm{c}$ & $3.819269 \mathrm{E}-29$ & $\$ M o-95$ \\
\hline $44101.70 \mathrm{C}$ & $3.629985 \mathrm{E}-29$ & \$Ru101 \\
\hline $45103.70 \mathrm{C}$ & $3.559438 \mathrm{E}-29$ & \$Rh103 \\
\hline $45105.70 \mathrm{C}$ & $3.491572 \mathrm{E}-29$ & \$Rh105 \\
\hline $48113.70 c$ & $3.244211 \mathrm{E}-29$ & $\$ \mathrm{Cd} 113$ \\
\hline $54131.70 \mathrm{C}$ & $2.798102 \mathrm{E}-29$ & $\$$ Xe131 \\
\hline $54133.70 \mathrm{c}$ & $2.756002 \mathrm{E}-29$ & $\$ \mathrm{Xe133}$ \\
\hline $55133.70 \mathrm{C}$ & $2.755987 \mathrm{E}-29$ & $\$ C s 133$ \\
\hline $54135.70 \mathrm{C}$ & $2.695116 \mathrm{E}-29$ & $\$$ Xe135 \\
\hline $57140.70 \mathrm{C}$ & $2.618012 \mathrm{E}-29$ & \$La140 \\
\hline $58141.70 \mathrm{C}$ & $2.599480 \mathrm{E}-29$ & $\$ C e 141$ \\
\hline $59143.70 \mathrm{c}$ & $2.563058 \mathrm{E}-29$ & $\$ \operatorname{Pr} 143$ \\
\hline $60143.70 \mathrm{c}$ & $2.563055 \mathrm{E}-29$ & $\$ N d 143$ \\
\hline $60145.70 \mathrm{c}$ & $2.527633 \mathrm{E}-29$ & $\$ N d 145$ \\
\hline $61147.70 \mathrm{C}$ & $2.493199 \mathrm{E}-29$ & $\$ \operatorname{Pm} 147$ \\
\hline $61149.70 \mathrm{c}$ & $2.459663 \mathrm{E}-29$ & $\$ P m 149$ \\
\hline $62149.70 \mathrm{C}$ & $2.459661 \mathrm{E}-29$ & $\$$ Sm149 \\
\hline $61151.70 \mathrm{C}$ & $2.426995 \mathrm{E}-29$ & \$Pm151 \\
\hline $62151.70 \mathrm{C}$ & $2.427021 \mathrm{E}-29$ & $\$$ Sm151 \\
\hline $62152.70 \mathrm{C}$ & $2.411048 \mathrm{E}-29$ & $\$$ Sm152 \\
\hline $63153.70 \mathrm{c}$ & $2.395258 \mathrm{E}-29$ & \$Eu153 \\
\hline $63155.70 \mathrm{C}$ & $2.364321 \mathrm{E}-29$ & \$Eu155 \\
\hline 64157.7 & $2.334161 \mathrm{E}-29$ & \$Gd157 \\
\hline
\end{tabular}




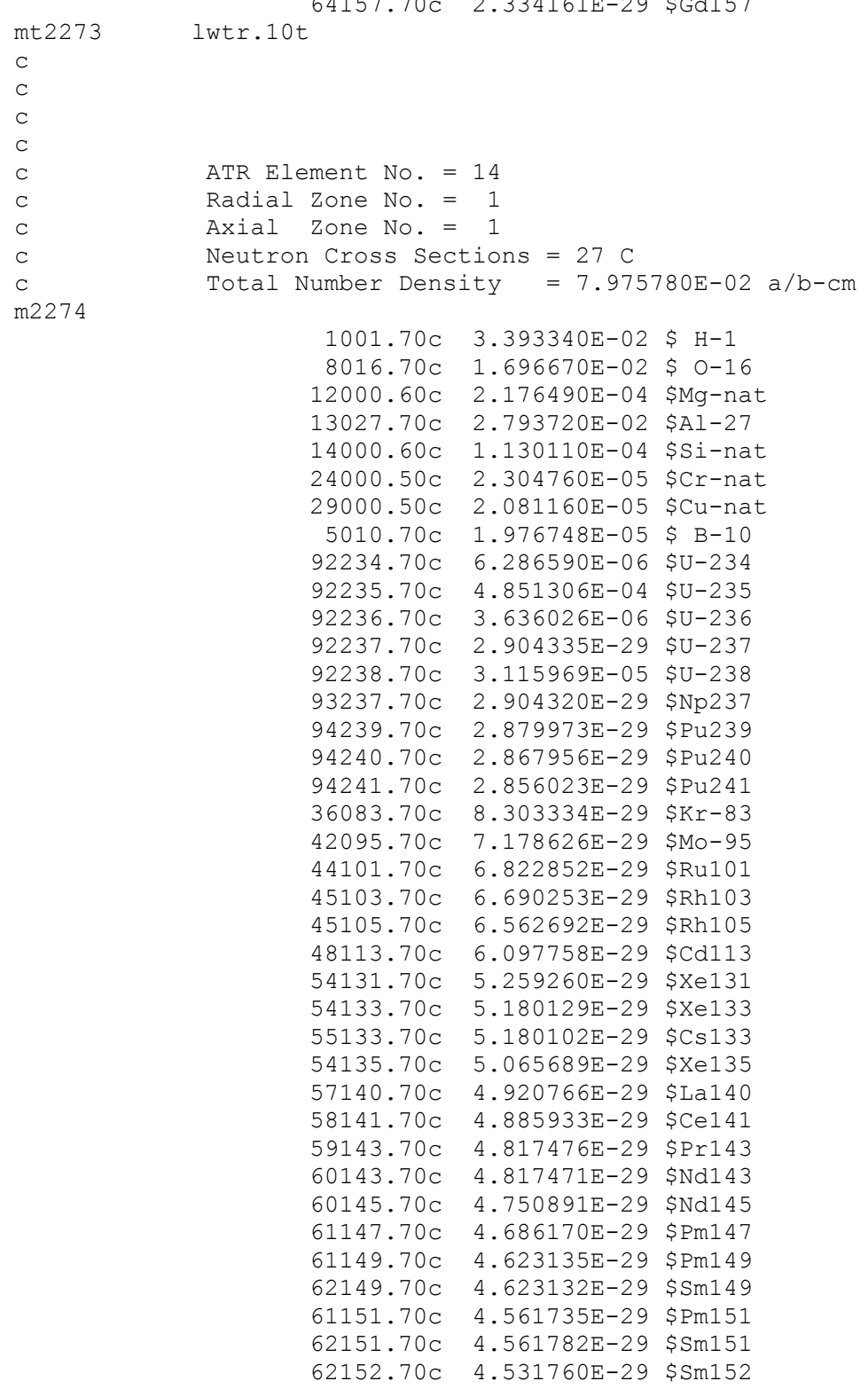

$44101.70 \mathrm{C} \quad 3.629985 \mathrm{E}-29$ \$Ru101

$45103.70 \mathrm{C} \quad 3.559438 \mathrm{E}-29$ \$Rh103

$45105.70 \mathrm{C} \quad 3.491572 \mathrm{E}-29$ \$Rh105

$48113.70 \mathrm{C} \quad 3.244211 \mathrm{E}-29$ \$Cd113

$54131.70 \mathrm{C} \quad 2.798102 \mathrm{E}-29$ SXe131

$54133.70 \mathrm{C} \quad 2.756002 \mathrm{E}-29$ \$Xe133

$55133.70 \mathrm{C} \quad 2.755987 \mathrm{E}-29 \$ \mathrm{Cs} 133$

$54135.70 \mathrm{C} \quad 2.695116 \mathrm{E}-29$ \$Xe135

$57140.70 \mathrm{C} \quad 2.618012 \mathrm{E}-29$ \$La140

$58141.70 \mathrm{C} \quad 2.599480 \mathrm{E}-29$ \$Ce141

$59143.70 \mathrm{C} \quad 2.563058 \mathrm{E}-29 \$ \operatorname{Pr} 143$

$60143.70 \mathrm{C} \quad 2.563055 \mathrm{E}-29 \$ \mathrm{Nd1} 43$

$60145.70 \mathrm{C} \quad 2.527633 \mathrm{E}-29 \$ \mathrm{Nd} 145$

$61147.70 \mathrm{C} \quad 2.493199 \mathrm{E}-29 \$ \mathrm{Pm} 147$

$61149.70 \mathrm{C} \quad 2.459663 \mathrm{E}-29$ \$Pm149

$62149.70 \mathrm{C} \quad 2.459661 \mathrm{E}-29$ \$Sm149

$61151.70 \mathrm{C} \quad 2.426995 \mathrm{E}-29 \$ \mathrm{Pm} 151$

$62151.70 \mathrm{C} \quad 2.427021 \mathrm{E}-29$ \$Sm151

$62152.70 \mathrm{C} \quad 2.411048 \mathrm{E}-29 \$ \mathrm{Sm} 152$

$63153.70 \mathrm{C} \quad 2.395258 \mathrm{E}-29$ \$Eu153

$63155.70 \mathrm{C} \quad 2.364321 \mathrm{E}-29$ \$Eu155

$64157.70 \mathrm{C} \quad 2.334161 \mathrm{E}-29$ \$Gd157

$=14$

Axial Zone No. = 1

Neutron Cross Sections $=27 \mathrm{C}$

$29000.50 \mathrm{C} \quad 2.081160 \mathrm{E}-05$ \$Cu-nat

1.976748E-05 \$ B-10

$6.286590 \mathrm{E}-06$ \$U-234

$93237.70 \mathrm{C} \quad 2.904320 \mathrm{E}-29 \quad \$ \mathrm{~Np} 237$

$94239.70 \mathrm{C} \quad 2.879973 \mathrm{E}-29$ \$Pu239

$94240.70 \mathrm{C} \quad 2.867956 \mathrm{E}-29$ \$Pu240

1. $70 \mathrm{C}-2.856023 \mathrm{E}-29$ \$Pu241

$42095.70 \mathrm{C} \quad 7.178626 \mathrm{E}-29$ \$Mo-95

$44101.70 \mathrm{C} \quad 6.822852 \mathrm{E}-29$ \$Ru101

$54135.70 \mathrm{C} \quad 5.065689 \mathrm{E}-29$ \$Xe135

4. $920766 \mathrm{E}-29$ SLa140

$4.885933 \mathrm{E}-29$ \$Ce141

$60145.70 \mathrm{C} \quad 4.750891 \mathrm{E}-29$ \$Nd145

$61147.70 \mathrm{C} \quad 4.686170 \mathrm{E}-29 \$ \mathrm{Pm} 147$

$61149.70 \mathrm{C} \quad 4.623135 \mathrm{E}-29$ \$Pm149

62149.70 $423132 \mathrm{E}-29$ \$Sm149

$62151.70 \mathrm{C}$ 
$63153.70 \mathrm{C} \quad 4.502082 \mathrm{E}-29 \$ \mathrm{Eu} 153$

$63155.70 \mathrm{C} \quad 4.443934 \mathrm{E}-29$ \$Eu155

$64157.70 \mathrm{C} \quad 4.387245 \mathrm{E}-29$ \$Gd157

mt2274
C
C
c
c
C
c
C
C
C
m2275

lwtr.10t

ATR Element No. $=14$

Radial Zone No. $=1$

Axial Zone No. $=2$

Neutron Cross Sections $=27 \mathrm{C}$

Total Number Density $=7.975780 \mathrm{E}-02 \mathrm{a} / \mathrm{b}-\mathrm{cm}$

\begin{tabular}{|c|c|c|}
\hline $1001.70 \mathrm{C}$ & $3.393340 E-02$ & $\$ \mathrm{H}-1$ \\
\hline $8016.70 \mathrm{c}$ & $1.696670 \mathrm{E}-02$ & $\$ 0-16$ \\
\hline $2000.60 \mathrm{c}$ & $2.176490 \mathrm{E}-04$ & \$Mg-nat \\
\hline $3027.70 c$ & $2.793720 \mathrm{E}-02$ & $\$ A I-27$ \\
\hline $0.60 \mathrm{c}$ & $1.130110 \mathrm{E}-04$ & \$Si-nat \\
\hline & $2.304760 \mathrm{E}-05$ & $\$ \mathrm{Cr}-\mathrm{nat}$ \\
\hline 0.5 & $2.081160 \mathrm{E}-05$ & \$Cu-nat \\
\hline & $1.976748 \mathrm{E}-05$ & $\$ B-10$ \\
\hline 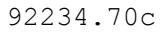 & $6.286590 \mathrm{E}-06$ & $\$ U-234$ \\
\hline & $4.851306 \mathrm{E}-04$ & $\$ U-235$ \\
\hline 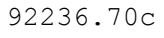 & $3.636026 \mathrm{E}-06$ & $\$ U-236$ \\
\hline & $2.904335 E-29$ & $\$ U-237$ \\
\hline .7 & $3.115969 \mathrm{E}-05$ & $\$ U-238$ \\
\hline & $2.904320 \mathrm{E}-29$ & $\$ N p 237$ \\
\hline$\cdot$ & $2.879973 E-29$ & \$Pu239 \\
\hline & $2.867956 \mathrm{E}-29$ & \$Pu240 \\
\hline .10 & $2.856023 E-29$ & \$Pu 241 \\
\hline & $8.303334 \mathrm{E}-29$ & $\$ \mathrm{Kr}-83$ \\
\hline $5.70 \mathrm{C}$ & $7.178626 \mathrm{E}-29$ & $\$ M \circ-95$ \\
\hline 7 & $6.822852 \mathrm{E}-29$ & \$Ru101 \\
\hline$\cdot$ & $6.690253 E-29$ & \$Rh103 \\
\hline- & $6.562692 \mathrm{E}-29$ & \$Rh105 \\
\hline$\cdot$ & $6.097758 E-29$ & $\$ \mathrm{Cd} 113$ \\
\hline $70 \mathrm{C}$ & $5.259260 \mathrm{E}-29$ & $\$ x e 131$ \\
\hline$\cdot$ & $5.180129 \mathrm{E}-29$ & $\$ x e 133$ \\
\hline 7 & $2 E-29$ & $\$ \mathrm{Cs} 133$ \\
\hline • & $689 E-29$ & $\$ x e 135$ \\
\hline 7 & $6 E-29$ & \$La140 \\
\hline .7 & $4.885933 E-29$ & $\$ C e 141$ \\
\hline 7 & $4.817476 \mathrm{E}-29$ & $\$ \operatorname{Pr} 143$ \\
\hline .7 & $4.817471 E-29$ & $\$ N d 143$ \\
\hline- & $891 E-29$ & $\$ N d 145$ \\
\hline .7 & $4.686170 \mathrm{E}-29$ & $\$ \operatorname{Pm} 147$ \\
\hline .7 & $4.623135 E-29$ & $\$ P \operatorname{Pm} 149$ \\
\hline .7 & $4.623132 E-29$ & \$Sm149 \\
\hline 6 & $4.561735 E-29$ & $\$ P \operatorname{Pm} 151$ \\
\hline .7 & $4.561782 \mathrm{E}-29$ & $\$$ Sm1 51 \\
\hline & $4.531760 \mathrm{E}-29$ & $\$$ Sm1 52 \\
\hline 63 & $4.502082 E-29$ & \$Eu153 \\
\hline & $934 E-29$ & \$Eu155 \\
\hline . & $245 E-2$ & \$Gd157 \\
\hline
\end{tabular}

mt2275 lwtr.10t

$\mathrm{C}$

C

C

C

c

C

C

m2276

ATR Element No. = 14

Radial Zone No. = 1

Axial Zone No. $=3$

Neutron Cross Sections $=27 \mathrm{C}$

Total Number Density $=7.975780 \mathrm{E}-02 \mathrm{a} / \mathrm{b}-\mathrm{cm}$

$1001.70 \mathrm{C} \quad 3.393340 \mathrm{E}-02 \$ \mathrm{H}-1$

$8016.70 \mathrm{C} \quad 1.696670 \mathrm{E}-02$ \$ O-16

$12000.60 \mathrm{C} \quad 2.176490 \mathrm{E}-04$ \$Mg-nat

$13027.70 \mathrm{C} \quad 2.793720 \mathrm{E}-02$ \$Al-27

$14000.60 \mathrm{C} \quad 1.130110 \mathrm{E}-04$ \$Si-nat 


\begin{tabular}{|c|c|c|}
\hline $24000.50 \mathrm{C}$ & $2.304760 \mathrm{E}-05$ & \$Cr-nat \\
\hline $9000.50 \mathrm{C}$ & $2.081160 \mathrm{E}-05$ & $\$ \mathrm{Cu}-\mathrm{nat}$ \\
\hline $5010.70 c$ & $1.976748 \mathrm{E}-05$ & $\$ B-10$ \\
\hline $2234.70 \mathrm{c}$ & $6.286590 \mathrm{E}-06$ & $\$ \mathrm{U}-234$ \\
\hline $2235.70 \mathrm{c}$ & $4.851306 \mathrm{E}-04$ & $\$ \mathrm{U}-235$ \\
\hline $92236.70 \mathrm{C}$ & $3.636026 \mathrm{E}-06$ & $\$ U-236$ \\
\hline $2237.70 \mathrm{C}$ & $2.904335 \mathrm{E}-29$ & $\$ U-237$ \\
\hline 2238. & $3.115969 \mathrm{E}-05$ & $\$ \mathrm{U}-238$ \\
\hline 237. & $2.904320 \mathrm{E}-29$ & \$Np237 \\
\hline $4239.70 \mathrm{C}$ & $2.879973 \mathrm{E}-29$ & \$Pu239 \\
\hline $4240.70 \mathrm{C}$ & $2.867956 \mathrm{E}-29$ & $\$ P u 240$ \\
\hline $94241.70 \mathrm{C}$ & $2.856023 \mathrm{E}-29$ & \$Pu241 \\
\hline $36083.70 \mathrm{c}$ & $8.303334 \mathrm{E}-29$ & $\$ K r-83$ \\
\hline $2095.70 c$ & $7.178626 \mathrm{E}-29$ & $\$ M o-95$ \\
\hline $44101.70 \mathrm{C}$ & $6.822852 \mathrm{E}-29$ & \$Ru101 \\
\hline $45103.70 \mathrm{C}$ & $6.690253 \mathrm{E}-29$ & \$Rh103 \\
\hline $45105.70 \mathrm{c}$ & $6.562692 \mathrm{E}-29$ & \$Rh105 \\
\hline $48113.70 \mathrm{C}$ & $6.097758 \mathrm{E}-29$ & $\$ \mathrm{Cd} 113$ \\
\hline $54131.70 \mathrm{C}$ & $5.259260 \mathrm{E}-29$ & $\$$ Xe131 \\
\hline $54133.70 \mathrm{C}$ & $5.180129 \mathrm{E}-29$ & $\$ \mathrm{Xe133}$ \\
\hline $5133.70 c$ & $5.180102 \mathrm{E}-29$ & $\$ \mathrm{Cs} 133$ \\
\hline 54135. & $5.065689 \mathrm{E}-29$ & $\$ \mathrm{Xe135}$ \\
\hline $57140.70 \mathrm{C}$ & $4.920766 \mathrm{E}-29$ & \$La140 \\
\hline $58141.70 \mathrm{c}$ & $4.885933 \mathrm{E}-29$ & $\$ C e 141$ \\
\hline $59143.70 \mathrm{C}$ & $4.817476 \mathrm{E}-29$ & $\$ \operatorname{Pr} 143$ \\
\hline 60143.70 & $4.817471 \mathrm{E}-29$ & $\$ \mathrm{Nd} 143$ \\
\hline 60145.70 & $4.750891 \mathrm{E}-29$ & $\$ N d 145$ \\
\hline $61147.70 \mathrm{C}$ & $4.686170 \mathrm{E}-29$ & $\$ \operatorname{Pm} 147$ \\
\hline $61149.70 \mathrm{c}$ & $4.623135 \mathrm{E}-29$ & \$Pm1 49 \\
\hline $62149.70 \mathrm{c}$ & $4.623132 \mathrm{E}-29$ & $\$$ Sm1 49 \\
\hline $61151.70 c$ & $4.561735 \mathrm{E}-29$ & \$Pm151 \\
\hline 62151.70 & $4.561782 \mathrm{E}-29$ & \$Sm151 \\
\hline $62152.70 c$ & $4.531760 \mathrm{E}-29$ & \$Sm152 \\
\hline $63153.70 \mathrm{c}$ & $4.502082 \mathrm{E}-29$ & \$Eu153 \\
\hline 3155.70 & $4.443934 \mathrm{E}-29$ & \$Eu155 \\
\hline$\$ 157.70$ & $4.387245 \mathrm{E}-29$ & $\$$ Gd157 \\
\hline
\end{tabular}

$\begin{array}{ll}\text { mt2276 lwtr.10t } \\ \mathrm{C} \\ \mathrm{C} \\ \mathrm{C} & \\ \mathrm{C} & \text { ATR Element No. }=14 \\ \mathrm{C} & \text { Radial Zone No. }=1 \\ \mathrm{C} & \text { Axial Zone No. }=4 \\ \mathrm{C} & \text { Neutron Cross Sections }=27 \mathrm{C} \\ \mathrm{C} & \text { Total Number Density }=7.975780 \mathrm{E}-02 \mathrm{a} / \mathrm{b}-\mathrm{cm} \\ \mathrm{C} & \end{array}$

\begin{tabular}{|c|c|c|}
\hline $1001.70 \mathrm{C}$ & $3.393340 \mathrm{E}-02$ & $\$ \mathrm{H}-1$ \\
\hline $8016.70 \mathrm{c}$ & $1.696670 \mathrm{E}-02$ & $\$ 0-16$ \\
\hline $12000.60 \mathrm{c}$ & $2.176490 \mathrm{E}-04$ & $\$ M g-n a t$ \\
\hline $13027.70 \mathrm{C}$ & $2.793720 \mathrm{E}-02$ & $\$ A I-27$ \\
\hline $14000.60 \mathrm{c}$ & $1.130110 \mathrm{E}-04$ & \$Si-nat \\
\hline $24000.50 \mathrm{C}$ & $2.304760 \mathrm{E}-05$ & $\$ C r-n a t$ \\
\hline $29000.50 c$ & $2.081160 \mathrm{E}-05$ & \$Cu-nat \\
\hline 5010.70 & $1.976749 \mathrm{E}-05$ & $\$ B-10$ \\
\hline $92234.70 c$ & $6.286591 \mathrm{E}-06$ & $\$ U-234$ \\
\hline $92235.70 \mathrm{c}$ & $4.851306 \mathrm{E}-04$ & $\$ U-235$ \\
\hline $92236.70 \mathrm{c}$ & $3.636026 \mathrm{E}-06$ & $\$ U-236$ \\
\hline 92237.7 & $1.452167 \mathrm{E}-29$ & $\$ U-237$ \\
\hline 92238.700 & $3.115969 \mathrm{E}-05$ & $\$ U-238$ \\
\hline $93237.70 c$ & $1.452160 \mathrm{E}-29$ & \$Np237 \\
\hline 94239. & $1.439987 \mathrm{E}-29$ & \$Pu239 \\
\hline 94240 . & $1.433978 \mathrm{E}-29$ & \$Pu240 \\
\hline $94241.70 c$ & $1.428012 \mathrm{E}-29$ & \$Pu241 \\
\hline $36083.70 c$ & $4.151667 \mathrm{E}-29$ & $\$ K r-83$ \\
\hline 42095.70 & $3.589313 \mathrm{E}-29$ & $\$ M o-95$ \\
\hline 44101.70 & $3.411426 \mathrm{E}-29$ & \$Ru101 \\
\hline $45103.70 \mathrm{c}$ & $3.345127 \mathrm{E}-29$ & $\$ R h 103$ \\
\hline $45105.70 c$ & $3.281347 \mathrm{E}-29$ & \$Rh105 \\
\hline 48113.7 & $3.048880 \mathrm{E}-29$ & $\$ C d 113$ \\
\hline 54131.7 & $2.629630 \mathrm{E}-29$ & $\$ X e 131$ \\
\hline
\end{tabular}




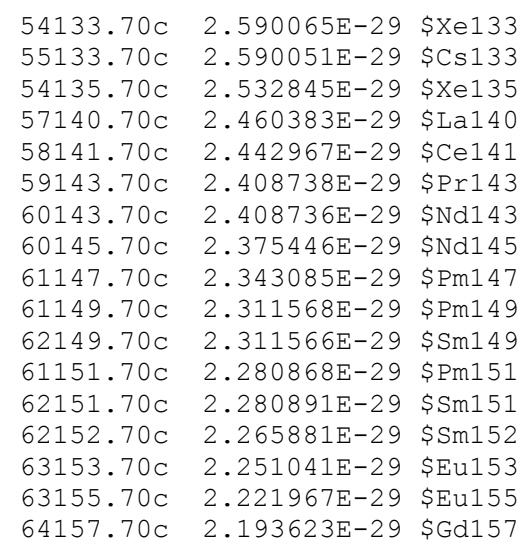

mt2277 lwtr.10t

$\mathrm{c}$

C

C

C

C ATR Element No. = 14

C Radial Zone No. = 1

C Axial Zone No. = 5

C Neutron Cross Sections $=27 \mathrm{C}$

c Total Number Density $=7.975780 \mathrm{E}-02 \mathrm{a} / \mathrm{b}-\mathrm{cm}$

m2278

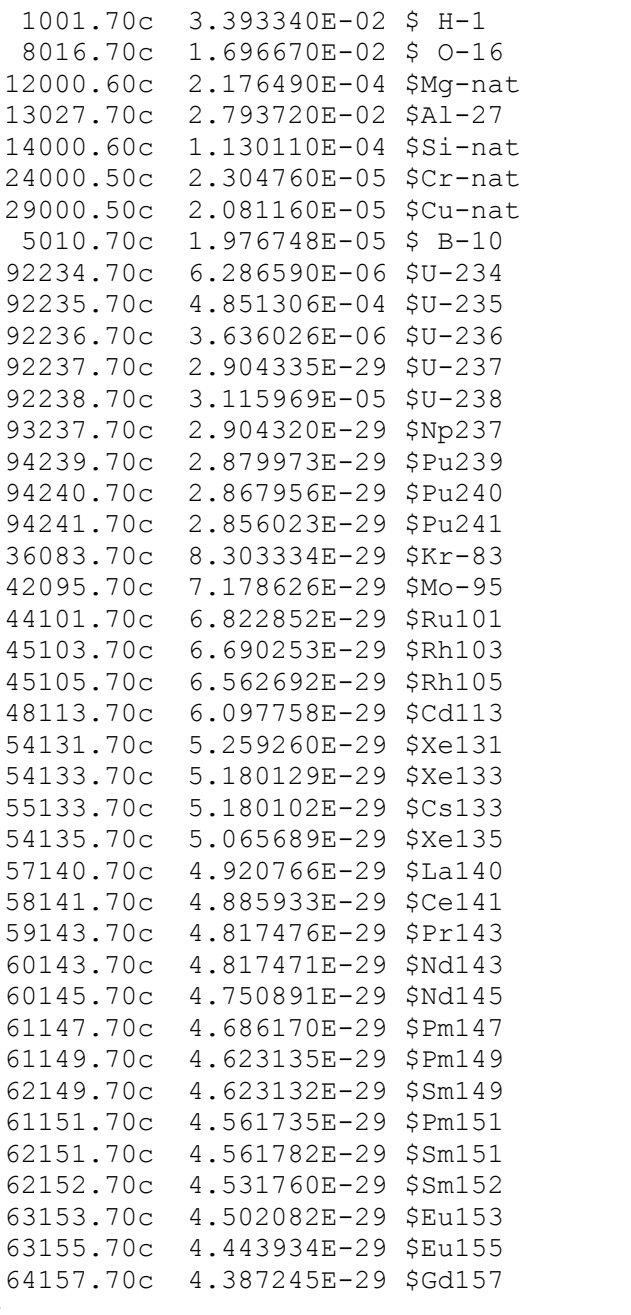

mt2278 lwtr.10t

$\mathrm{C}$ 
C ATR Element No. = 14

lwtr. 10 t

ATR Element No. = 14

Radial Zone No. $=1$

Axial Zone No. = 7
Radial Zone No. $=1$

Axial Zone No. $=6$

Neutron Cross Sections $=27 \mathrm{C}$

Total Number Density $=7.975780 \mathrm{E}-02 \mathrm{a} / \mathrm{b}-\mathrm{cm}$

\begin{tabular}{|c|c|c|}
\hline $1001.70 \mathrm{C}$ & $3.393340 E-02$ & $\$ \mathrm{H}-1$ \\
\hline $8016.70 \mathrm{C}$ & $1.696670 \mathrm{E}-02$ & $\$ 0-16$ \\
\hline $000.60 \mathrm{c}$ & $2.176490 \mathrm{E}-04$ & $\$ M g-n a t$ \\
\hline $27.70 \mathrm{C}$ & $2.793720 \mathrm{E}-02$ & $\$ A I-27$ \\
\hline $4000.60 \mathrm{c}$ & $1.130110 \mathrm{E}-04$ & \$Si-nat \\
\hline 4000.50 & $2.304760 \mathrm{E}-05$ & \$Cr-nat \\
\hline 9000.50 & $2.081160 \mathrm{E}-05$ & $\$ \mathrm{Cu}-\mathrm{nat}$ \\
\hline 10.70 & $1.976748 \mathrm{E}-05$ & $\$ B-10$ \\
\hline 34.700 & $6.286590 \mathrm{E}-06$ & $\$ U-234$ \\
\hline & $4.851306 \mathrm{E}-04$ & $\$ U-235$ \\
\hline .70 & $3.636026 \mathrm{E}-06$ & $\$ U-236$ \\
\hline & $2.904335 E-29$ & $\$ U-237$ \\
\hline .7 & $3.115969 \mathrm{E}-05$ & $\$ U-238$ \\
\hline & $2.904320 E-29$ & $\$ \mathrm{~Np} 237$ \\
\hline $.70 \mathrm{C}$ & $2.879973 E-29$ & \$Pu239 \\
\hline & $2.867956 E-29$ & \$Pu 240 \\
\hline $.70 \mathrm{C}$ & $2.856023 E-29$ & \$Pu241 \\
\hline & $8.303334 \mathrm{E}-29$ & $\$ \mathrm{Kr}-83$ \\
\hline $.10 \mathrm{C}$ & $7.178626 \mathrm{E}-29$ & $\$ M o-95$ \\
\hline 70 & $6.822852 \mathrm{E}-29$ & \$Ru101 \\
\hline .10 & $6.690253 E-29$ & \$Rh103 \\
\hline $70 \mathrm{c}$ & $6.562692 \mathrm{E}-29$ & \$Rh105 \\
\hline$\cdot$ & $6.097758 \mathrm{E}-29$ & $\$ \mathrm{Cd} 113$ \\
\hline 7 & $5.259260 \mathrm{E}-29$ & $\$ x e 131$ \\
\hline$\cdot$ & $5.180129 \mathrm{E}-29$ & \$Xe133 \\
\hline- & $2 E-29$ & $\$ C s 133$ \\
\hline .7 & $9 E-29$ & $\$ x e 135$ \\
\hline 7 & $6 E-29$ & $\$ \mathrm{La} 140$ \\
\hline .7 & $33 E-29$ & $\$ C e 141$ \\
\hline 7 & $6 E-29$ & $\$ \operatorname{Pr} 143$ \\
\hline 60 & $471 E-29$ & $\$ N d 143$ \\
\hline 60 & $91 E-29$ & $\$ N d 145$ \\
\hline .7 & $4.686170 \mathrm{E}-29$ & $\$ \operatorname{Pm} 147$ \\
\hline 61 & $35 E-29$ & $\$ P m 149$ \\
\hline .7 & $4.623132 E-29$ & \$Sm1 49 \\
\hline 7 & $5 E-29$ & \$Pm151 \\
\hline .7 & $4.561782 \mathrm{E}-29$ & \$Sm1 51 \\
\hline & $0 E-29$ & $\$ \operatorname{Sm} 152$ \\
\hline 7 & $4.502082 E-29$ & \$Eu153 \\
\hline & $A E-29$ & \$Eu155 \\
\hline .10 & 4.38 & $\$$ Gd1 57 \\
\hline
\end{tabular}

.387245E-29 \$Gd157

Neutron Cross Sections $=27 \mathrm{C}$

Total Number Density $=7.975780 \mathrm{E}-02 \mathrm{a} / \mathrm{b}-\mathrm{cm}$

$1001.70 \mathrm{C} \quad 3.393340 \mathrm{E}-02$ \$ $\mathrm{H}-1$

$8016.70 \mathrm{C} \quad 1.696670 \mathrm{E}-02 \$ \mathrm{O}-16$

$12000.60 \mathrm{C} \quad 2.176490 \mathrm{E}-04$ \$Mg-nat

$13027.70 \mathrm{C} \quad 2.793720 \mathrm{E}-02$ \$Al-27

$14000.60 \mathrm{c} \quad 1.130110 \mathrm{E}-04$ \$Si-nat

$24000.50 \mathrm{C} \quad 2.304760 \mathrm{E}-05$ \$Cr-nat

$29000.50 \mathrm{C} \quad 2.081160 \mathrm{E}-05$ \$Cu-nat

$5010.70 \mathrm{C} \quad 1.976748 \mathrm{E}-05$ \$ B-10

$92234.70 \mathrm{C} \quad 6.286590 \mathrm{E}-06$ \$U-234

$92235.70 \mathrm{C} \quad 4.851306 \mathrm{E}-04$ \$U-235 


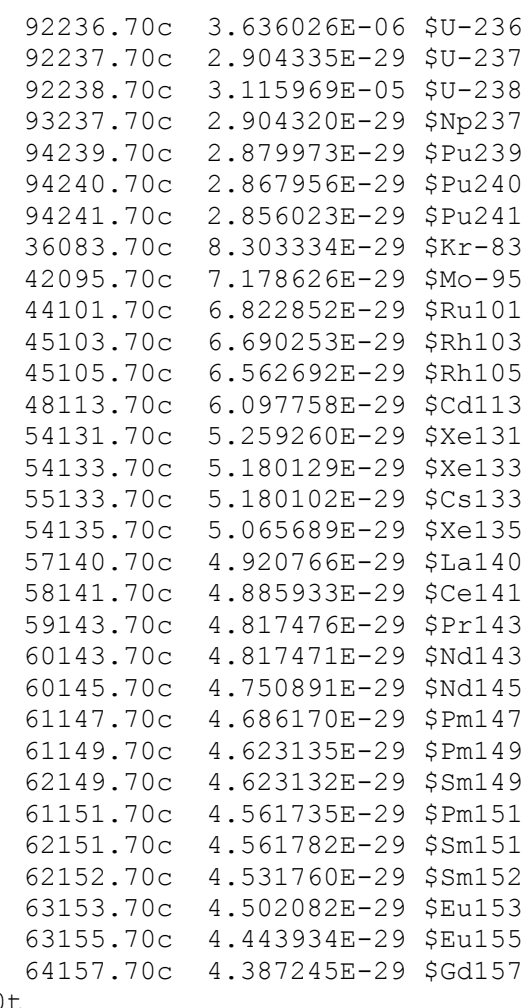

$\begin{array}{ll}\text { mt2280 lwtr.10t } \\ \mathrm{C} \\ \mathrm{C} \\ \mathrm{C} \\ \mathrm{C} & \text { ATR Element No. }=14 \\ \mathrm{C} & \text { Radial Zone No. }=2 \\ \mathrm{C} & \text { Axial Zone No. }=1 \\ \mathrm{C} & \text { Neutron Cross Sections }=27 \mathrm{C} \\ \mathrm{C} & \text { Total Number Density }=8.294866 \mathrm{E}-02 \mathrm{a} / \mathrm{b}-\mathrm{cm} \\ \mathrm{C} & \end{array}$

\begin{tabular}{|c|c|c|}
\hline $1001.70 \mathrm{C}$ & $3.920790 \mathrm{E}-02$ & $\$ \mathrm{H}-1$ \\
\hline $8016.70 \mathrm{c}$ & $1.960390 \mathrm{E}-02$ & $\$ 0-16$ \\
\hline $12000.60 \mathrm{c}$ & $1.660990 \mathrm{E}-04$ & \$Mg-nat \\
\hline $13027.70 \mathrm{C}$ & $2.315370 \mathrm{E}-02$ & $\$ A l-27$ \\
\hline $14000.60 \mathrm{C}$ & $8.624450 \mathrm{E}-05$ & \$Si-nat \\
\hline $24000.50 \mathrm{C}$ & $1.552830 \mathrm{E}-05$ & \$Cr-nat \\
\hline $29000.50 \mathrm{C}$ & $1.588240 \mathrm{E}-05$ & $\$ \mathrm{Cu}-\mathrm{nat}$ \\
\hline $5010.70 \mathrm{C}$ & $1.714619 \mathrm{E}-28$ & $\$ B-10$ \\
\hline $92234.70 \mathrm{C}$ & $8.355541 \mathrm{E}-06$ & $\$ U-234$ \\
\hline $92235.70 \mathrm{C}$ & $6.447897 \mathrm{E}-04$ & $\$ U-235$ \\
\hline $92236.70 \mathrm{C}$ & $4.832662 \mathrm{E}-06$ & $\$ U-236$ \\
\hline $92237.70 \mathrm{C}$ & $7.242605 \mathrm{E}-30$ & $\$ U-237$ \\
\hline $92238.70 \mathrm{C}$ & $4.141451 \mathrm{E}-05$ & $\$ U-238$ \\
\hline $93237.70 \mathrm{C}$ & $7.242569 \mathrm{E}-30$ & \$Np237 \\
\hline $94239.70 \mathrm{C}$ & $7.181854 \mathrm{E}-30$ & \$Pu239 \\
\hline $94240.70 \mathrm{C}$ & $7.151887 \mathrm{E}-30$ & \$Pu240 \\
\hline $94241.70 \mathrm{C}$ & $7.122128 \mathrm{E}-30$ & \$Pu241 \\
\hline $36083.70 \mathrm{c}$ & $2.070621 \mathrm{E}-29$ & $\$ K r-83$ \\
\hline $42095.70 \mathrm{C}$ & $1.790150 \mathrm{E}-29$ & $\$ M o-95$ \\
\hline $44101.70 \mathrm{C}$ & $1.701430 \mathrm{E}-29$ & \$Ru101 \\
\hline $45103.70 \mathrm{C}$ & $1.668363 \mathrm{E}-29$ & \$Rh103 \\
\hline $45105.70 \mathrm{C}$ & $1.636554 \mathrm{E}-29$ & \$Rh105 \\
\hline $48113.70 \mathrm{C}$ & $1.520612 \mathrm{E}-29$ & $\$ \mathrm{Cd} 113$ \\
\hline $54131.70 \mathrm{C}$ & $1.311513 \mathrm{E}-29$ & $\$ X e 131$ \\
\hline $54133.70 \mathrm{C}$ & $1.291780 \mathrm{E}-29$ & \$Xe133 \\
\hline $55133.70 \mathrm{C}$ & $1.291774 \mathrm{E}-29$ & $\$ \mathrm{Cs} 133$ \\
\hline $54135.70 \mathrm{c}$ & $1.263242 \mathrm{E}-29$ & \$Xe135 \\
\hline $57140.70 \mathrm{C}$ & $1.227103 E-29$ & \$La140 \\
\hline $58141.70 \mathrm{C}$ & $1.218416 \mathrm{E}-29$ & $\$ \mathrm{Ce} 141$ \\
\hline
\end{tabular}


$59143.70 \mathrm{C} \quad 1.201345 \mathrm{E}-29 \$ \operatorname{Pr} 143$

$60143.70 \mathrm{C} \quad 1.201344 \mathrm{E}-29$ \$Nd143

$60145.70 \mathrm{C} \quad 1.184740 \mathrm{E}-29$ \$Nd145

$61147.70 \mathrm{C} \quad 1.168601 \mathrm{E}-29$ \$Pm147

$61149.70 \mathrm{C} \quad 1.152882 \mathrm{E}-29 \$ \mathrm{Pm} 149$

$62149.70 \mathrm{C} \quad 1.152881 \mathrm{E}-29 \quad \$ \mathrm{Sm} 149$

$61151.70 \mathrm{C} \quad 1.137570 \mathrm{E}-29 \$ \mathrm{Pm} 151$

$62151.70 \mathrm{C} \quad 1.137582 \mathrm{E}-29$ \$Sm151

$62152.70 \mathrm{C} \quad 1.130095 \mathrm{E}-29$ \$Sm152

$63153.70 \mathrm{C} \quad 1.122694 \mathrm{E}-29$ \$Eu153

$63155.70 \mathrm{C} \quad 1.108194 \mathrm{E}-29$ \$Eu155

mt2281 lwtr.10t

$64157.70 \mathrm{C} \quad 1.094057 \mathrm{E}-29$ \$Gd157

$\mathrm{C}$

C

C

C

$\mathrm{C}$

m2282

mt 2282

c

C

C

C

C
ATR Element No. $=14$

Radial Zone No. $=2$

Axial Zone No. $=2$

Neutron Cross Sections $=27 \mathrm{C}$

Total Number Density $=8.294866 \mathrm{E}-02 \mathrm{a} / \mathrm{b}-\mathrm{cm}$

$1001.70 \mathrm{C} \quad 3.920790 \mathrm{E}-02$ \$ $\mathrm{H}-1$

$8016.70 \mathrm{C} \quad 1.960390 \mathrm{E}-02$ \$ $0-16$

$12000.60 \mathrm{C} \quad 1.660990 \mathrm{E}-04$ \$Mg-nat

$13027.70 \mathrm{C} \quad 2.315370 \mathrm{E}-02$ \$Al-27

$14000.60 \mathrm{C} \quad 8.624450 \mathrm{E}-05$ \$Si-nat

$24000.50 \mathrm{C} \quad 1.552830 \mathrm{E}-05$ \$Cr-nat

$29000.50 \mathrm{C} \quad 1.588240 \mathrm{E}-05 \mathrm{\$ Cu}-\mathrm{nat}$

$5010.70 \mathrm{C} \quad 1.714619 \mathrm{E}-28$ \$ B-10

$92234.70 \mathrm{C} \quad 8.355541 \mathrm{E}-06 \quad \$ \mathrm{U}-234$

$92235.70 \mathrm{C} \quad 6.447897 \mathrm{E}-04 \quad \$ \mathrm{U}-235$

$92236.70 \mathrm{C} \quad 4.832662 \mathrm{E}-06 \quad \$ \mathrm{U}-236$

$92237.70 \mathrm{C} \quad 7.242605 \mathrm{E}-30 \quad \$ \mathrm{U}-237$

$92238.70 \mathrm{C} \quad 4.141451 \mathrm{E}-05$ \$U-238

$93237.70 \mathrm{C} \quad 7.242569 \mathrm{E}-30 \quad \$ \mathrm{~Np} 237$

$94239.70 \mathrm{C} \quad 7.181854 \mathrm{E}-30$ \$Pu239

$94240.70 \mathrm{C} \quad 7.151887 \mathrm{E}-30$ \$Pu240

$94241.70 \mathrm{C} \quad 7.122128 \mathrm{E}-30$ \$Pu 241

$36083.70 \mathrm{C} \quad 2.070621 \mathrm{E}-29$ \$Kr-83

$42095.70 \mathrm{C} \quad 1.790150 \mathrm{E}-29$ \$Mo-95

$44101.70 \mathrm{C} \quad 1.701430 \mathrm{E}-29$ \$Ru101

$45103.70 \mathrm{C} \quad 1.668363 \mathrm{E}-29$ \$Rh103

$45105.70 \mathrm{C} \quad 1.636554 \mathrm{E}-29$ \$Rh105

$48113.70 \mathrm{C} \quad 1.520612 \mathrm{E}-29$ \$Cd113

$54131.70 \mathrm{C} \quad 1.311513 \mathrm{E}-29$ \$Xe131

$54133.70 \mathrm{C} \quad 1.291780 \mathrm{E}-29 \quad \$ \mathrm{Xe} 133$

$55133.70 \mathrm{C} \quad 1.291774 \mathrm{E}-29 \$ \mathrm{Cs} 133$

$54135.70 \mathrm{C} \quad 1.263242 \mathrm{E}-29$ \$Xe135

$57140.70 \mathrm{C} \quad 1.227103 \mathrm{E}-29$ \$La140

$58141.70 \mathrm{C} \quad 1.218416 \mathrm{E}-29$ \$Ce141

$59143.70 \mathrm{C} \quad 1.201345 \mathrm{E}-29 \$ \mathrm{Pr} 143$

$60143.70 \mathrm{C} \quad 1.201344 \mathrm{E}-29 \$ \mathrm{Nd1} 43$

$60145.70 \mathrm{C} \quad 1.184740 \mathrm{E}-29$ \$Nd145

$61147.70 \mathrm{C} \quad 1.168601 \mathrm{E}-29 \$ \mathrm{Pm} 147$

$61149.70 \mathrm{C} \quad 1.152882 \mathrm{E}-29 \$ \mathrm{Pm} 149$

$62149.70 \mathrm{C} \quad 1.152881 \mathrm{E}-29$ \$Sm149

$61151.70 \mathrm{C} \quad 1.137570 \mathrm{E}-29$ \$Pm151

$62151.70 \mathrm{C} \quad 1.137582 \mathrm{E}-29$ \$Sm151

$62152.70 \mathrm{C} \quad 1.130095 \mathrm{E}-29 \$ \mathrm{Sm} 152$

$63153.70 \mathrm{C} \quad 1.122694 \mathrm{E}-29$ \$Eu153

$63155.70 \mathrm{C} \quad 1.108194 \mathrm{E}-29$ \$Eu155

$64157.70 \mathrm{C} \quad 1.094057 \mathrm{E}-29$ \$Gd157

lwtr.10t

ATR Element No. = 14

Radial Zone No. $=2$ 


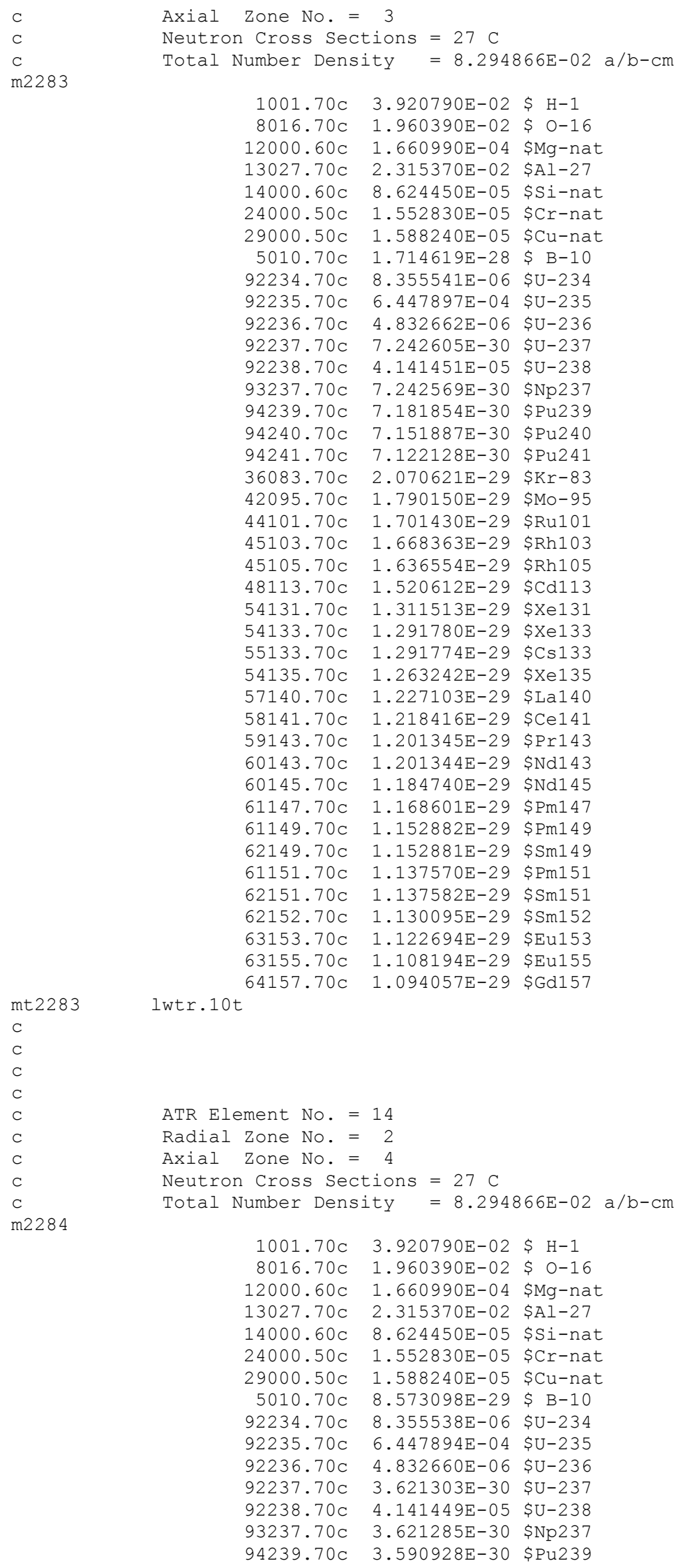




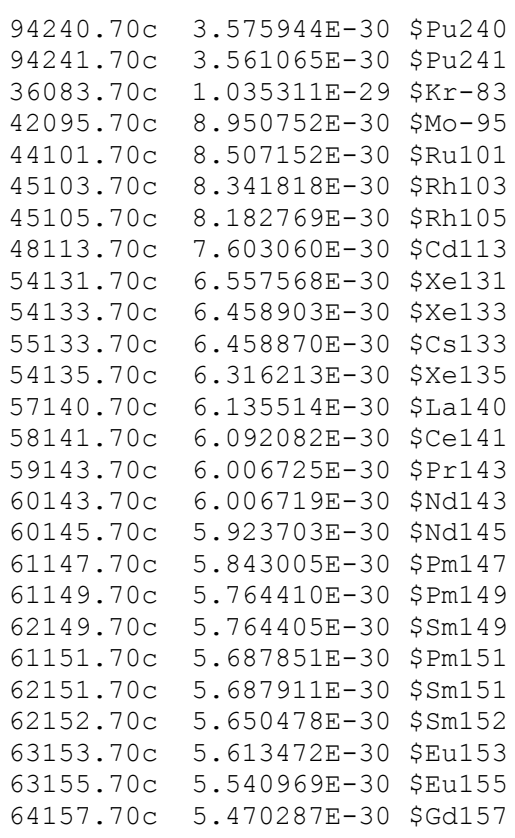

mt2284 lwtr.10t

$\mathrm{C}$

$\mathrm{C}$

C ATR Element No. = 14

C Radial Zone No. $=2$

C Axial Zone No. = 5

C Neutron Cross Sections $=27 \mathrm{C}$

C Total Number Density $=8.294866 \mathrm{E}-02 \mathrm{a} / \mathrm{b}-\mathrm{cm}$

m2285

\begin{tabular}{|c|c|c|}
\hline $1001.70 \mathrm{c}$ & $3.920790 E-02$ & $\$ \mathrm{H}-1$ \\
\hline $8016.70 \mathrm{c}$ & $1.960390 \mathrm{E}-02$ & $\$ 0-16$ \\
\hline $12000.60 \mathrm{c}$ & $1.660990 \mathrm{E}-04$ & $\$ M g-n a t$ \\
\hline $13027.70 \mathrm{C}$ & $2.315370 \mathrm{E}-02$ & $\$ A I-27$ \\
\hline $14000.60 \mathrm{c}$ & $8.624450 \mathrm{E}-05$ & \$Si-nat \\
\hline $24000.50 \mathrm{c}$ & $1.552830 \mathrm{E}-05$ & $\$ C r-n a t$ \\
\hline $29000.50 \mathrm{C}$ & $1.588240 \mathrm{E}-05$ & \$Cu-nat \\
\hline $5010.70 \mathrm{c}$ & $1.714619 \mathrm{E}-28$ & $\$ B-10$ \\
\hline $92234.70 \mathrm{C}$ & $8.355541 \mathrm{E}-06$ & $\$ U-234$ \\
\hline $92235.70 \mathrm{C}$ & $6.447897 \mathrm{E}-04$ & $\$ U-235$ \\
\hline $92236.70 \mathrm{C}$ & $4.832662 \mathrm{E}-06$ & $\$ U-236$ \\
\hline $92237.70 \mathrm{C}$ & $7.242605 \mathrm{E}-30$ & $\$ U-237$ \\
\hline $92238.70 \mathrm{C}$ & $4.141451 E-05$ & $\$ U-238$ \\
\hline $93237.70 \mathrm{C}$ & $7.242569 \mathrm{E}-30$ & $\$ N p 237$ \\
\hline $94239.70 \mathrm{C}$ & $7.181854 \mathrm{E}-30$ & \$Pu239 \\
\hline $94240.70 \mathrm{C}$ & $7.151887 \mathrm{E}-30$ & $\$ P u 240$ \\
\hline $94241.70 \mathrm{C}$ & $7.122128 \mathrm{E}-30$ & \$Pu241 \\
\hline $36083.70 \mathrm{c}$ & $2.070621 E-29$ & $\$ K r-83$ \\
\hline $42095.70 \mathrm{C}$ & $1.790150 \mathrm{E}-29$ & $\$ M o-95$ \\
\hline $44101.70 \mathrm{C}$ & $1.701430 \mathrm{E}-29$ & \$Ru101 \\
\hline $45103.70 \mathrm{C}$ & $1.668363 \mathrm{E}-29$ & \$Rh103 \\
\hline $45105.70 \mathrm{C}$ & $1.636554 \mathrm{E}-29$ & \$Rh105 \\
\hline $48113.70 \mathrm{C}$ & $1.520612 \mathrm{E}-29$ & $\$ \mathrm{Cd} 113$ \\
\hline $54131.70 \mathrm{C}$ & 1. $311513 E-29$ & \$Xe131 \\
\hline $54133.70 \mathrm{C}$ & $1.291780 \mathrm{E}-29$ & $\$ x e 133$ \\
\hline $55133.70 \mathrm{C}$ & 1.291774E-29 & $\$ \mathrm{Cs} 133$ \\
\hline $54135.70 \mathrm{C}$ & 1. $263242 \mathrm{E}-29$ & \$Xe135 \\
\hline $57140.70 \mathrm{C}$ & $1.227103 E-29$ & \$La1 40 \\
\hline $58141.70 \mathrm{C}$ & 1.218416E-29 & $\$ \mathrm{Ce} 141$ \\
\hline $59143.70 \mathrm{C}$ & $1.201345 E-29$ & $\$ \operatorname{Pr} 143$ \\
\hline $60143.70 \mathrm{c}$ & $1.201344 \mathrm{E}-29$ & $\$ N d 143$ \\
\hline $60145.70 \mathrm{c}$ & $1.184740 \mathrm{E}-29$ & $\$ N d 145$ \\
\hline $61147.70 \mathrm{c}$ & $1.168601 \mathrm{E}-29$ & \$Pm1 47 \\
\hline 61149.70 & $1.152882 \mathrm{E}-29$ & $\$ \operatorname{Pm} 149$ \\
\hline
\end{tabular}


$62149.70 \mathrm{C} \quad 1.152881 \mathrm{E}-29 \$ \mathrm{Sm} 149$

$61151.70 \mathrm{C} \quad 1.137570 \mathrm{E}-29$ \$Pm151

$62151.70 \mathrm{C} \quad 1.137582 \mathrm{E}-29$ \$Sm151

$62152.70 \mathrm{C} \quad 1.130095 \mathrm{E}-29 \$ \mathrm{Sm} 152$

$63153.70 \mathrm{C} \quad 1.122694 \mathrm{E}-29$ \$Eu153

$63155.70 \mathrm{C} \quad 1.108194 \mathrm{E}-29$ \$Eu155

$\begin{array}{ll}\text { mt2285 } 64157.70 \mathrm{C} \quad 1.094057 \mathrm{E}-29 \text { \$Gd157 } \\ \mathrm{C} & \\ \mathrm{C} & \\ \mathrm{C} & \text { Awtr.10t Element No. }=14 \\ \mathrm{C} & \text { Radial Zone No. }=2 \\ \mathrm{C} & \text { Axial Zone No. }=6 \\ \mathrm{C} & \text { Neutron Cross Sections }=27 \mathrm{C} \\ \mathrm{C} & \text { Total Number Density }=8.294866 \mathrm{E}-02 \mathrm{a} / \mathrm{b}-\mathrm{cm} \\ \mathrm{C} & \end{array}$

m2286

mt2286

$\mathrm{C}$

C

C

C

C

$\mathrm{C}$

C

C

C

m2287

\begin{abstract}
$1001.70 \mathrm{C} \quad 3.920790 \mathrm{E}-02 \$ \mathrm{H}-1$
$8016.70 \mathrm{C} \quad 1.960390 \mathrm{E}-02$ \$ $0-16$

$12000.60 \mathrm{C} \quad 1.660990 \mathrm{E}-04$ \$Mg-nat

$13027.70 \mathrm{C} \quad 2.315370 \mathrm{E}-02 \quad \$ \mathrm{Al}-27$

$14000.60 \mathrm{C} \quad 8.624450 \mathrm{E}-05$ \$Si-nat

$24000.50 \mathrm{C} \quad 1.552830 \mathrm{E}-05$ \$Cr-nat

$29000.50 \mathrm{C} \quad 1.588240 \mathrm{E}-05$ \$Cu-nat

$5010.70 \mathrm{C} \quad 1.714619 \mathrm{E}-28$ \$ B-10

$92234.70 \mathrm{C} \quad 8.355541 \mathrm{E}-06 \quad \$ \mathrm{U}-234$

$92235.70 \mathrm{C} \quad 6.447897 \mathrm{E}-04 \quad \$ \mathrm{U}-235$

$92236.70 \mathrm{C} \quad 4.832662 \mathrm{E}-06$ \$U-236

$92237.70 \mathrm{C} \quad 7.242605 \mathrm{E}-30 \quad \$ \mathrm{U}-237$

$92238.70 \mathrm{C} \quad 4.141451 \mathrm{E}-05 \mathrm{\$ U}-238$

$93237.70 \mathrm{C} \quad 7.242569 \mathrm{E}-30 \quad \$ \mathrm{~Np} 237$

$94239.70 \mathrm{C} \quad 7.181854 \mathrm{E}-30$ \$Pu239

$94240.70 \mathrm{C} \quad 7.151887 \mathrm{E}-30$ \$Pu240

$94241.70 \mathrm{C} \quad 7.122128 \mathrm{E}-30$ \$Pu241

$36083.70 \mathrm{C} \quad 2.070621 \mathrm{E}-29 \$ \mathrm{Kr}-83$

$42095.70 \mathrm{C} \quad 1.790150 \mathrm{E}-29$ \$Mo-95

$44101.70 \mathrm{C} \quad 1.701430 \mathrm{E}-29$ \$Ru101

$45103.70 \mathrm{C} \quad 1.668363 \mathrm{E}-29$ \$Rh103

$45105.70 \mathrm{C} \quad 1.636554 \mathrm{E}-29$ \$Rh105

$48113.70 \mathrm{C} \quad 1.520612 \mathrm{E}-29$ \$Cd113

$54131.70 \mathrm{C} \quad 1.311513 \mathrm{E}-29$ \$Xe131

$54133.70 \mathrm{c} \quad 1.291780 \mathrm{E}-29$ \$Xe133

$55133.70 \mathrm{C} \quad 1.291774 \mathrm{E}-29$ \$Cs133

$54135.70 \mathrm{C} \quad 1.263242 \mathrm{E}-29$ \$Xe135

$57140.70 \mathrm{C} \quad 1.227103 \mathrm{E}-29$ \$La140

$58141.70 \mathrm{C} \quad 1.218416 \mathrm{E}-29$ \$Ce141

$59143.70 \mathrm{C} \quad 1.201345 \mathrm{E}-29 \quad \$ \operatorname{Pr} 143$

$60143.70 \mathrm{C} \quad 1.201344 \mathrm{E}-29 \$ \mathrm{Nd} 143$

$60145.70 \mathrm{C} \quad 1.184740 \mathrm{E}-29$ \$Nd145

$61147.70 \mathrm{C} \quad 1.168601 \mathrm{E}-29 \$ \mathrm{Pm} 147$

$61149.70 \mathrm{C} \quad 1.152882 \mathrm{E}-29$ \$Pm149

$62149.70 \mathrm{C} \quad 1.152881 \mathrm{E}-29 \quad \$ \mathrm{Sm} 149$

$61151.70 \mathrm{C} \quad 1.137570 \mathrm{E}-29$ \$Pm151

$62151.70 \mathrm{C} \quad 1.137582 \mathrm{E}-29$ \$Sm151

$62152.70 \mathrm{C} \quad 1.130095 \mathrm{E}-29 \$ \mathrm{Sm} 152$

$63153.70 \mathrm{C} \quad 1.122694 \mathrm{E}-29$ \$Eu153

$63155.70 \mathrm{C} \quad 1.108194 \mathrm{E}-29$ \$Eu155

$64157.70 \mathrm{C} \quad 1.094057 \mathrm{E}-29$ \$Gd157
\end{abstract}

lwtr.10t

ATR Element No. $=14$

Radial Zone No. $=2$

Axial Zone No. = 7

Neutron Cross Sections $=27 \mathrm{C}$

Total Number Density $=8.294866 \mathrm{E}-02 \mathrm{a} / \mathrm{b}-\mathrm{cm}$

$1001.70 \mathrm{C} \quad 3.920790 \mathrm{E}-02 \$ \mathrm{H}-1$ 


\begin{tabular}{|c|c|c|}
\hline $\mathrm{Oc}_{\mathrm{c}}$ & -02 & $0-16$ \\
\hline $12000.60 \mathrm{c}$ & $1.660990 \mathrm{E}-04$ & $\$ M g-n a t$ \\
\hline $13027.70 \mathrm{c}$ & $2.315370 \mathrm{E}-02$ & $\$ A 1-27$ \\
\hline $14000.60 \mathrm{c}$ & $8.624450 \mathrm{E}-05$ & \$Si-nat \\
\hline $24000.50 \mathrm{c}$ & $1.552830 \mathrm{E}-05$ & $\$ \mathrm{Cr}$-nat \\
\hline $29000.50 \mathrm{c}$ & $1.588240 \mathrm{E}-05$ & \$Cu-nat \\
\hline $5010.70 \mathrm{c}$ & $1.714619 \mathrm{E}-28$ & $\$ B-10$ \\
\hline $92234.70 \mathrm{C}$ & $8.355541 \mathrm{E}-06$ & $\$ U-234$ \\
\hline $92235.70 \mathrm{C}$ & $6.447897 \mathrm{E}-04$ & $\$ \mathrm{U}-235$ \\
\hline $92236.70 \mathrm{c}$ & $4.832662 \mathrm{E}-06$ & $\$ U-236$ \\
\hline $92237.70 \mathrm{c}$ & $7.242605 \mathrm{E}-30$ & $\$ U-237$ \\
\hline $92238.70 \mathrm{c}$ & $4.141451 \mathrm{E}-05$ & $\$ \mathrm{U}-238$ \\
\hline $93237.70 \mathrm{C}$ & $7.242569 \mathrm{E}-30$ & \$Np237 \\
\hline $94239.70 \mathrm{C}$ & $7.181854 \mathrm{E}-30$ & \$Pu239 \\
\hline $94240.70 \mathrm{C}$ & $7.151887 \mathrm{E}-30$ & \$Pu240 \\
\hline $94241.70 \mathrm{C}$ & $7.122128 \mathrm{E}-30$ & \$Pu241 \\
\hline $36083.70 \mathrm{c}$ & $2.070621 \mathrm{E}-29$ & $\$ K r-83$ \\
\hline $42095.70 \mathrm{c}$ & $1.790150 \mathrm{E}-29$ & \$Mo-95 \\
\hline $44101.70 \mathrm{C}$ & $1.701430 \mathrm{E}-29$ & \$Ru101 \\
\hline $45103.70 \mathrm{c}$ & $1.668363 \mathrm{E}-29$ & $\$ R h 103$ \\
\hline $45105.70 \mathrm{c}$ & $1.636554 \mathrm{E}-29$ & \$Rh105 \\
\hline $48113.70 \mathrm{C}$ & $1.520612 \mathrm{E}-29$ & $\$ \mathrm{Cd} 113$ \\
\hline $54131.70 \mathrm{c}$ & 1. $311513 \mathrm{E}-29$ & $\$ \mathrm{Xe131}$ \\
\hline $54133.70 \mathrm{C}$ & $1.291780 \mathrm{E}-29$ & $\$ \mathrm{Xe133}$ \\
\hline $55133.70 \mathrm{C}$ & $1.291774 \mathrm{E}-29$ & $\$ C s 133$ \\
\hline $54135.70 \mathrm{C}$ & $1.263242 \mathrm{E}-29$ & \$Xe135 \\
\hline $57140.70 \mathrm{c}$ & $1.227103 \mathrm{E}-29$ & $\$$ La140 \\
\hline $58141.70 \mathrm{C}$ & $1.218416 \mathrm{E}-29$ & $\$ C e 141$ \\
\hline $59143.70 \mathrm{C}$ & $1.201345 \mathrm{E}-29$ & $\$ \operatorname{Pr} 143$ \\
\hline $60143.70 \mathrm{c}$ & $1.201344 \mathrm{E}-29$ & \$Nd143 \\
\hline $60145.70 \mathrm{c}$ & $1.184740 \mathrm{E}-29$ & \$Nd14 5 \\
\hline $61147.70 \mathrm{c}$ & $1.168601 \mathrm{E}-29$ & $\$ \operatorname{Pm} 147$ \\
\hline $61149.70 \mathrm{c}$ & $1.152882 \mathrm{E}-29$ & $\$ \operatorname{Pm} 149$ \\
\hline $62149.70 \mathrm{C}$ & $1.152881 \mathrm{E}-29$ & $\$$ Sm1 49 \\
\hline $61151.70 \mathrm{c}$ & $1.137570 \mathrm{E}-29$ & \$Pm151 \\
\hline $62151.70 \mathrm{C}$ & $1.137582 \mathrm{E}-29$ & $\$$ Sm151 \\
\hline $62152.70 \mathrm{c}$ & $1.130095 \mathrm{E}-29$ & $\$$ Sm152 \\
\hline $63153.70 \mathrm{c}$ & $1.122694 \mathrm{E}-29$ & \$Eu153 \\
\hline $63155.70 \mathrm{c}$ & $1.108194 \mathrm{E}-29$ & \$Eu155 \\
\hline & $1.094057 \mathrm{E}-29$ & ונד. \\
\hline
\end{tabular}


$45103.70 \mathrm{C} \quad 3.559438 \mathrm{E}-29 \$ \mathrm{Rh} 103$

$45105.70 \mathrm{C} \quad 3.491572 \mathrm{E}-29$ \$Rh105

$48113.70 \mathrm{C} \quad 3.244211 \mathrm{E}-29$ \$Cd113

$54131.70 \mathrm{C} \quad 2.798102 \mathrm{E}-29$ \$Xe131

$54133.70 \mathrm{C} \quad 2.756002 \mathrm{E}-29 \$ \mathrm{Xe133}$

$55133.70 \mathrm{C} \quad 2.755987 \mathrm{E}-29 \quad \$ \mathrm{Cs} 133$

$54135.70 \mathrm{C} \quad 2.695116 \mathrm{E}-29 \$ \mathrm{Xe} 135$

$57140.70 \mathrm{C} \quad 2.618012 \mathrm{E}-29$ \$La140

$58141.70 \mathrm{C} \quad 2.599480 \mathrm{E}-29$ \$Ce141

$59143.70 \mathrm{C} \quad 2.563058 \mathrm{E}-29 \$ \mathrm{Pr} 143$

$60143.70 \mathrm{C} \quad 2.563055 \mathrm{E}-29 \$ \mathrm{Nd1} 43$

$60145.70 \mathrm{C} \quad 2.527633 \mathrm{E}-29 \$ \mathrm{Nd1} 45$

$61147.70 \mathrm{C} \quad 2.493199 \mathrm{E}-29 \$ \mathrm{Pm} 147$

$61149.70 \mathrm{C} \quad 2.459663 \mathrm{E}-29 \quad \$ \mathrm{Pm} 149$

$62149.70 \mathrm{C} \quad 2.459661 \mathrm{E}-29 \$ \mathrm{Sm} 149$

$61151.70 \mathrm{C} \quad 2.426995 \mathrm{E}-29 \$ \mathrm{Pm} 151$

$62151.70 \mathrm{C} \quad 2.427021 \mathrm{E}-29$ \$Sm151

$62152.70 \mathrm{C} \quad 2.411048 \mathrm{E}-29$ \$Sm152

$63153.70 \mathrm{C} \quad 2.395258 \mathrm{E}-29 \quad \$ \mathrm{Eu} 153$

$63155.70 \mathrm{C} \quad 2.364321 \mathrm{E}-29$ \$Eu155

mt2288

C

C

C

$\mathrm{C}$

m2289

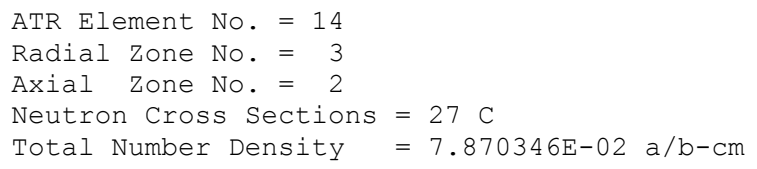


$63155.70 \mathrm{C} \quad 2.364321 \mathrm{E}-29 \$ \mathrm{Eu} 155$

$64157.70 \mathrm{C} \quad 2.334161 \mathrm{E}-29$ \$Gd157

mt2289
C
C
C
C
C
C
C
C
C
m2290

lwtr.10t

ATR Element No. = 14

Radial Zone No. $=3$

Axial Zone No. $=3$

Neutron Cross Sections $=27 \mathrm{C}$

Total Number Density $=7.870346 \mathrm{E}-02 \mathrm{a} / \mathrm{b}-\mathrm{cm}$

$1001.70 \mathrm{C} \quad 3.212840 \mathrm{E}-02$ \$ $\mathrm{H}-1$

$8016.70 \mathrm{C} \quad 1.606420 \mathrm{E}-02$ \$ $0-16$

$12000.60 \mathrm{C} \quad 2.399430 \mathrm{E}-04$ \$Mg-nat

$13027.70 \mathrm{C} \quad 2.958200 \mathrm{E}-02$ \$Al-27

$14000.60 \mathrm{C} \quad 1.245870 \mathrm{E}-04$ \$Si-nat

$24000.50 \mathrm{C} \quad 2.243180 \mathrm{E}-05$ \$Cr-nat

$29000.50 \mathrm{C} \quad 2.294330 \mathrm{E}-05$ \$Cu-nat

$5010.70 \mathrm{C} \quad 1.966257 \mathrm{E}-05 \$ \mathrm{~B}-10$

$92234.70 \mathrm{C} \quad 5.964864 \mathrm{E}-06 \quad \$ \mathrm{U}-234$

$92235.70 \mathrm{C} \quad 4.603032 \mathrm{E}-04 \quad \$ \mathrm{U}-235$

$92236.70 \mathrm{C} \quad 3.449947 \mathrm{E}-06 \quad \$ \mathrm{U}-236$

$92237.70 \mathrm{C} \quad 1.545203 \mathrm{E}-29$ \$U-237

$92238.70 \mathrm{C} \quad 2.956504 \mathrm{E}-05 \quad \$ \mathrm{U}-238$

$93237.70 \mathrm{C} \quad 1.545195 \mathrm{E}-29 \$ \mathrm{~Np} 237$

$94239.70 \mathrm{C} \quad 1.532242 \mathrm{E}-29$ \$Pu239

$94240.70 \mathrm{C} \quad 1.525848 \mathrm{E}-29$ \$Pu240

$94241.70 \mathrm{C} \quad 1.519499 \mathrm{E}-29$ \$Pu241

$36083.70 \mathrm{C} \quad 4.417651 \mathrm{E}-29$ \$Kr-83

$42095.70 \mathrm{C} \quad 3.819269 \mathrm{E}-29$ \$Mo-95

$44101.70 \mathrm{C} \quad 3.629985 \mathrm{E}-29$ \$Ru101

$45103.70 \mathrm{C} \quad 3.559438 \mathrm{E}-29$ \$Rh103

$45105.70 \mathrm{C} \quad 3.491572 \mathrm{E}-29$ \$Rh105

$48113.70 \mathrm{C} \quad 3.244211 \mathrm{E}-29 \$ \mathrm{Cd} 113$

$54131.70 \mathrm{C} \quad 2.798102 \mathrm{E}-29$ \$Xe131

$54133.70 \mathrm{C} \quad 2.756002 \mathrm{E}-29$ \$Xe133

$55133.70 \mathrm{C} \quad 2.755987 \mathrm{E}-29 \$ \mathrm{Cs} 133$

$54135.70 \mathrm{C} \quad 2.695116 \mathrm{E}-29$ \$Xe135

$57140.70 \mathrm{C} \quad 2.618012 \mathrm{E}-29$ \$La140

$58141.70 \mathrm{C} \quad 2.599480 \mathrm{E}-29$ Se141

$59143.70 \mathrm{C} \quad 2.563058 \mathrm{E}-29 \$ \mathrm{Pr} 143$

$60143.70 \mathrm{C} \quad 2.563055 \mathrm{E}-29 \$ \mathrm{Nd} 143$

$60145.70 \mathrm{C} \quad 2.527633 \mathrm{E}-29 \$ \mathrm{Nd} 145$

$61147.70 \mathrm{C} \quad 2.493199 \mathrm{E}-29 \$ \mathrm{Pm} 147$

$61149.70 \mathrm{C} \quad 2.459663 \mathrm{E}-29 \$ \mathrm{Pm} 149$

$62149.70 \mathrm{C} \quad 2.459661 \mathrm{E}-29$ \$Sm149

$61151.70 \mathrm{C} \quad 2.426995 \mathrm{E}-29$ \$Pm151

$62151.70 \mathrm{C} \quad 2.427021 \mathrm{E}-29$ \$Sm151

$62152.70 \mathrm{C} \quad 2.411048 \mathrm{E}-29$ \$Sm152

$63153.70 \mathrm{C} \quad 2.395258 \mathrm{E}-29$ \$Eu153

$63155.70 \mathrm{C} \quad 2.364321 \mathrm{E}-29$ \$Eu155

mt2290 lwtr.10t

$64157.70 \mathrm{C} \quad 2.334161 \mathrm{E}-29 \$ \mathrm{Gd} 157$

c

C

C

$\mathrm{C}$

C

$\mathrm{C}$

C

m2291

ATR Element No. $=14$

Radial Zone No. $=3$

Axial Zone No. = 4

Neutron Cross Sections $=27 \mathrm{C}$

Total Number Density $=7.870346 \mathrm{E}-02 \mathrm{a} / \mathrm{b}-\mathrm{cm}$
$1001.70 \mathrm{C} \quad 3.212840 \mathrm{E}-02$ \$ $\mathrm{H}-1$
$8016.70 \mathrm{C} \quad 1.606420 \mathrm{E}-02 \$ 0-16$
$12000.60 \mathrm{C} \quad 2.399430 \mathrm{E}-04$ \$Mg-nat
$13027.70 \mathrm{C} \quad 2.958200 \mathrm{E}-02$ \$Al-27
$14000.60 \mathrm{C} \quad 1.245870 \mathrm{E}-04$ \$Si-nat
$24000.50 \mathrm{C} \quad 2.243180 \mathrm{E}-05$ \$Cr-nat 


\begin{tabular}{|c|c|c|}
\hline $29000.50 \mathrm{C}$ & $2.294330 E-05$ & $\$ \mathrm{Cu}-\mathrm{nat}$ \\
\hline $5010.70 \mathrm{C}$ & $1.966257 \mathrm{E}-05$ & $\$ B-10$ \\
\hline $92234.70 \mathrm{C}$ & $5.964876 \mathrm{E}-06$ & $\$ U-234$ \\
\hline $92235.70 \mathrm{c}$ & $4.603042 E-04$ & $\$ \mathrm{U}-235$ \\
\hline $92236.70 \mathrm{c}$ & $3.449954 \mathrm{E}-06$ & $\$ \mathrm{U}-236$ \\
\hline $92237.70 \mathrm{c}$ & $7.726017 \mathrm{E}-30$ & $\$ U-237$ \\
\hline $92238.70 \mathrm{C}$ & $2.956510 \mathrm{E}-05$ & $\$ \mathrm{U}-238$ \\
\hline $93237.70 \mathrm{C}$ & $7.725979 E-30$ & $\$ N p 237$ \\
\hline $4239.70 \mathrm{C}$ & $7.661212 \mathrm{E}-30$ & \$Pu239 \\
\hline 4240.70 & $7.629244 \mathrm{E}-30$ & \$Pu240 \\
\hline $94241.70 \mathrm{c}$ & $7.597500 \mathrm{E}-30$ & \$Pu241 \\
\hline $36083.70 \mathrm{c}$ & $2.208826 \mathrm{E}-29$ & $\$ K r-83$ \\
\hline $2095.70 \mathrm{c}$ & $1.909635 \mathrm{E}-29$ & $\$ M o-95$ \\
\hline $44101.70 \mathrm{C}$ & $1.814993 \mathrm{E}-29$ & \$Ru101 \\
\hline $45103.70 c$ & $1.779720 \mathrm{E}-29$ & \$Rh103 \\
\hline $45105.70 c$ & $1.745786 \mathrm{E}-29$ & \$Rh105 \\
\hline $8113.70 c$ & $1.622106 \mathrm{E}-29$ & $\$ C d 113$ \\
\hline $54131.70 \mathrm{c}$ & $1.399051 \mathrm{E}-29$ & $\$ \mathrm{Xe131}$ \\
\hline $54133.70 \mathrm{c}$ & $1.378001 \mathrm{E}-29$ & $\$ \mathrm{Xe133}$ \\
\hline $55133.70 c$ & $1.377994 \mathrm{E}-29$ & $\$ C s 133$ \\
\hline $54135.70 c$ & $1.347558 \mathrm{E}-29$ & $\$ X e 135$ \\
\hline 57140.70 & $1.309006 \mathrm{E}-29$ & $\$$ La140 \\
\hline $58141.70 \mathrm{C}$ & $1.299740 \mathrm{E}-29$ & $\$ C e 141$ \\
\hline $59143.70 \mathrm{c}$ & $1.281529 \mathrm{E}-29$ & $\$ \operatorname{Pr} 143$ \\
\hline $60143.70 \mathrm{c}$ & $1.281528 \mathrm{E}-29$ & $\$ N d 143$ \\
\hline 60145.70 & $1.263817 \mathrm{E}-29$ & $\$ N d 145$ \\
\hline 61147.70 & $1.246600 \mathrm{E}-29$ & $\$ \operatorname{Pm} 147$ \\
\hline 61149.70 & $1.229832 \mathrm{E}-29$ & $\$ \operatorname{Pm} 149$ \\
\hline $62149.70 \mathrm{c}$ & $1.229831 \mathrm{E}-29$ & \$Sm149 \\
\hline 61151.70 & $1.213498 \mathrm{E}-29$ & \$Pm151 \\
\hline 2151.7 & $1.213511 \mathrm{E}-29$ & $\$$ Sm151 \\
\hline 62152.7 & $1.205524 \mathrm{E}-29$ & $\$$ Sm152 \\
\hline . & $1.197629 \mathrm{E}-29$ & \$Eu153 \\
\hline 3155.7 & $1.182161 \mathrm{E}-29$ & \$Eu155 \\
\hline & $1.167081 \mathrm{E}-29$ & $\$$ Gd157 \\
\hline
\end{tabular}

mt2291 lwtr.10t

C

C

C

C ATR Element No. = 14

C Radial Zone No. = 3

C Axial Zone No. = 5

C Neutron Cross Sections $=27 \mathrm{C}$

c Total Number Density $=7.870346 \mathrm{E}-02 \mathrm{a} / \mathrm{b}-\mathrm{cm}$

m2292

\begin{tabular}{|c|c|c|}
\hline $1001.70 \mathrm{C}$ & $3.212840 \mathrm{E}-02$ & $\$ \mathrm{H}-1$ \\
\hline $8016.70 \mathrm{c}$ & $1.606420 \mathrm{E}-02$ & $\$ 0-16$ \\
\hline $12000.60 \mathrm{c}$ & $2.399430 \mathrm{E}-04$ & $\$ M g-n a t$ \\
\hline $13027.70 \mathrm{c}$ & $2.958200 \mathrm{E}-02$ & $\$ A l-27$ \\
\hline $14000.60 \mathrm{c}$ & $1.245870 \mathrm{E}-04$ & \$Si-nat \\
\hline $24000.50 \mathrm{c}$ & $2.243180 \mathrm{E}-05$ & \$Cr-nat \\
\hline $29000.50 \mathrm{c}$ & $2.294330 \mathrm{E}-05$ & $\$ \mathrm{Cu}-\mathrm{nat}$ \\
\hline $5010.70 \mathrm{C}$ & $1.966257 \mathrm{E}-05$ & $\$ B-10$ \\
\hline $92234.70 \mathrm{C}$ & $5.964864 \mathrm{E}-06$ & $\$ U-234$ \\
\hline $92235.70 \mathrm{C}$ & $4.603032 \mathrm{E}-04$ & $\$ U-235$ \\
\hline $92236.70 \mathrm{c}$ & $3.449947 \mathrm{E}-06$ & $\$ U-236$ \\
\hline $92237.70 \mathrm{C}$ & $1.545203 \mathrm{E}-29$ & $\$ U-237$ \\
\hline $92238.70 \mathrm{c}$ & $2.956504 \mathrm{E}-05$ & $\$ U-238$ \\
\hline $93237.70 \mathrm{c}$ & $1.545195 \mathrm{E}-29$ & $\$ N p 237$ \\
\hline $94239.70 \mathrm{C}$ & $1.532242 \mathrm{E}-29$ & \$Pu239 \\
\hline $94240.70 \mathrm{c}$ & $1.525848 \mathrm{E}-29$ & $\$ \mathrm{Pu} 240$ \\
\hline $94241.70 \mathrm{c}$ & $1.519499 \mathrm{E}-29$ & \$Pu241 \\
\hline $36083.70 \mathrm{c}$ & $4.417651 \mathrm{E}-29$ & $\$ K r-83$ \\
\hline $42095.70 \mathrm{c}$ & $3.819269 \mathrm{E}-29$ & $\$ M o-95$ \\
\hline $44101.70 \mathrm{c}$ & $3.629985 E-29$ & \$Ru101 \\
\hline $45103.70 \mathrm{c}$ & $3.559438 \mathrm{E}-29$ & $\$ R h 103$ \\
\hline $45105.70 \mathrm{c}$ & $3.491572 \mathrm{E}-29$ & \$Rh105 \\
\hline $48113.70 \mathrm{c}$ & $3.244211 E-29$ & $\$ \mathrm{Cd} 113$ \\
\hline $54131.70 \mathrm{c}$ & $2.798102 \mathrm{E}-29$ & \$Xe131 \\
\hline $54133.70 \mathrm{C}$ & $2.756002 \mathrm{E}-29$ & $\$ x e 133$ \\
\hline
\end{tabular}




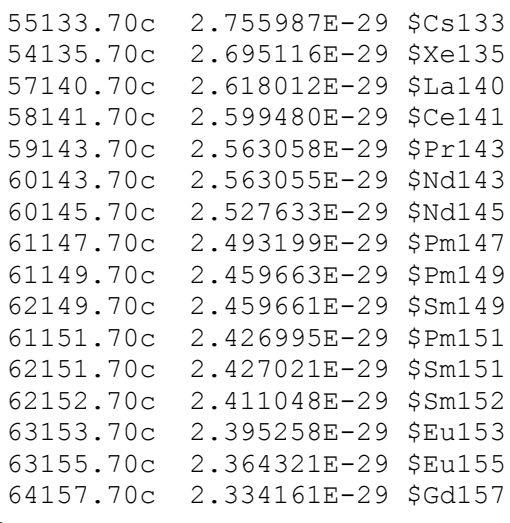

mt2292 lwtr.10t

c

C

$\mathrm{C}$

$\mathrm{C}$

C

C

C

m2293

mt2293

$\mathrm{C}$

C
ATR Element No. $=14$
Radial Zone No. $=3$
Axial Zone No. = 6
Neutron Cross Sections $=27 \mathrm{C}$
Total Number Density $=7.870346 \mathrm{E}-02 \mathrm{a} / \mathrm{b}-\mathrm{cm}$

$1001.70 \mathrm{C} \quad 3.212840 \mathrm{E}-02$ \$ $\mathrm{H}-1$

$8016.70 \mathrm{C} \quad 1.606420 \mathrm{E}-02 \$ 0-16$

$12000.60 \mathrm{c} \quad 2.399430 \mathrm{E}-04 \$ \mathrm{Mg}-$ nat

$13027.70 \mathrm{C} \quad 2.958200 \mathrm{E}-02$ \$Al-27

$14000.60 \mathrm{C} \quad 1.245870 \mathrm{E}-04$ \$Si-nat

$24000.50 \mathrm{C} \quad 2.243180 \mathrm{E}-05$ \$Cr-nat

$29000.50 \mathrm{C} \quad 2.294330 \mathrm{E}-05$ \$Cu-nat

$5010.70 \mathrm{C} \quad 1.966257 \mathrm{E}-05$ \$ B-10

$92234.70 \mathrm{C} \quad 5.964864 \mathrm{E}-06$ \$U-234

$92235.70 \mathrm{C} \quad 4.603032 \mathrm{E}-04 \quad \$ \mathrm{U}-235$

$92236.70 \mathrm{C} \quad 3.449947 \mathrm{E}-06 \quad \$ \mathrm{U}-236$

$92237.70 \mathrm{C} \quad 1.545203 \mathrm{E}-29$ \$U-237

$92238.70 \mathrm{C} \quad 2.956504 \mathrm{E}-05$ \$U-238

$93237.70 \mathrm{C} \quad 1.545195 \mathrm{E}-29 \$ \mathrm{~Np} 237$

$94239.70 \mathrm{C} \quad 1.532242 \mathrm{E}-29$ \$Pu239

$94240.70 \mathrm{C} \quad 1.525848 \mathrm{E}-29$ \$Pu240

$94241.70 \mathrm{C} \quad 1.519499 \mathrm{E}-29$ \$Pu241

$36083.70 \mathrm{C} \quad 4.417651 \mathrm{E}-29$ \$Kr-83

$42095.70 \mathrm{C} \quad 3.819269 \mathrm{E}-29$ \$Mo-95

$44101.70 \mathrm{C} \quad 3.629985 \mathrm{E}-29$ \$Ru101

$45103.70 \mathrm{C} \quad 3.559438 \mathrm{E}-29$ \$Rh103

$45105.70 \mathrm{C} \quad 3.491572 \mathrm{E}-29$ \$Rh105

$48113.70 \mathrm{C} \quad 3.244211 \mathrm{E}-29$ \$Cd113

$54131.70 \mathrm{C} \quad 2.798102 \mathrm{E}-29$ \$Xe131

$54133.70 \mathrm{C} \quad 2.756002 \mathrm{E}-29$ \$Xe133

$55133.70 \mathrm{C} \quad 2.755987 \mathrm{E}-29 \$ \mathrm{Cs} 133$

$54135.70 \mathrm{C} \quad 2.695116 \mathrm{E}-29$ \$Xe135

$57140.70 \mathrm{C} \quad 2.618012 \mathrm{E}-29$ \$La140

$58141.70 \mathrm{C} \quad 2.599480 \mathrm{E}-29$ \$Ce141

$59143.70 \mathrm{C} \quad 2.563058 \mathrm{E}-29$ \$Pr143

$60143.70 \mathrm{C} \quad 2.563055 \mathrm{E}-29 \$ \mathrm{Nd} 143$

$60145.70 \mathrm{C} \quad 2.527633 \mathrm{E}-29$ \$Nd145

$61147.70 \mathrm{C} \quad 2.493199 \mathrm{E}-29 \$ \mathrm{Pm} 147$

$61149.70 \mathrm{C} \quad 2.459663 \mathrm{E}-29$ \$Pm149

$62149.70 \mathrm{C} \quad 2.459661 \mathrm{E}-29 \$ \mathrm{Sm} 149$

$61151.70 \mathrm{C} \quad 2.426995 \mathrm{E}-29 \$ \mathrm{Pm} 151$

$62151.70 \mathrm{C} \quad 2.427021 \mathrm{E}-29$ \$Sm151

$62152.70 \mathrm{C} \quad 2.411048 \mathrm{E}-29$ \$Sm152

$63153.70 \mathrm{C} \quad 2.395258 \mathrm{E}-29$ \$Eu153

$63155.70 \mathrm{C} \quad 2.364321 \mathrm{E}-29$ \$Eu155

$64157.70 \mathrm{C} \quad 2.334161 \mathrm{E}-29$ \$Gd157

lwtr.10t 
C

C
ATR Element No. = 14

Radial Zone No. $=3$

Axial Zone No. $=7$

Neutron Cross Sections $=27 \mathrm{C}$

Total Number Density $=7.870346 \mathrm{E}-02 \mathrm{a} / \mathrm{b}-\mathrm{cm}$

$1001.70 \mathrm{C} \quad 3.212840 \mathrm{E}-02 \$ \mathrm{H}-1$

$8016.70 \mathrm{C} \quad 1.606420 \mathrm{E}-02 \$ 0-16$

$12000.60 \mathrm{C} \quad 2.399430 \mathrm{E}-04$ \$Mg-nat

$13027.70 \mathrm{C} \quad 2.958200 \mathrm{E}-02$ \$Al-27

$14000.60 \mathrm{C} \quad 1.245870 \mathrm{E}-04 \mathrm{SSi-nat}$

$24000.50 \mathrm{C} \quad 2.243180 \mathrm{E}-05$ \$Cr-nat

$29000.50 \mathrm{C} \quad 2.294330 \mathrm{E}-05$ \$Cu-nat

$5010.70 \mathrm{C} \quad 1.966257 \mathrm{E}-05$ \$ B -10

$92234.70 \mathrm{C} \quad 5.964864 \mathrm{E}-06$ \$U-234

$92235.70 \mathrm{C} \quad 4.603032 \mathrm{E}-04 \quad \$ \mathrm{U}-235$

$92236.70 \mathrm{C} \quad 3.449947 \mathrm{E}-06 \quad \$ \mathrm{U}-236$

$92237.70 \mathrm{C} \quad 1.545203 \mathrm{E}-29 \$ \mathrm{U}-237$

$92238.70 \mathrm{C} \quad 2.956504 \mathrm{E}-05 \quad \$ \mathrm{U}-238$

$93237.70 \mathrm{C} \quad 1.545195 \mathrm{E}-29 \$ \mathrm{~Np} 237$

$94239.70 \mathrm{C} \quad 1.532242 \mathrm{E}-29$ \$Pu239

$94240.70 \mathrm{C} \quad 1.525848 \mathrm{E}-29$ \$Pu240

$94241.70 \mathrm{C} \quad 1.519499 \mathrm{E}-29$ \$Pu241

$36083.70 \mathrm{C} \quad 4.417651 \mathrm{E}-29$ \$Kr-83

$42095.70 \mathrm{C} \quad 3.819269 \mathrm{E}-29$ \$Mo-95

$44101.70 \mathrm{C} \quad 3.629985 \mathrm{E}-29$ \$Ru101

$45103.70 \mathrm{C} \quad 3.559438 \mathrm{E}-29$ \$Rh103

$45105.70 \mathrm{C} \quad 3.491572 \mathrm{E}-29$ \$Rh105

$48113.70 \mathrm{C} \quad 3.244211 \mathrm{E}-29 \$ \mathrm{Cd} 113$

$54131.70 \mathrm{C} \quad 2.798102 \mathrm{E}-29$ \$Xe131

$54133.70 \mathrm{C} \quad 2.756002 \mathrm{E}-29 \$ \mathrm{Xe133}$

$55133.70 \mathrm{C} \quad 2.755987 \mathrm{E}-29 \quad \$ \mathrm{Cs} 133$

$54135.70 \mathrm{C} \quad 2.695116 \mathrm{E}-29$ \$Xe135

$57140.70 \mathrm{C} \quad 2.618012 \mathrm{E}-29$ \$La140

$58141.70 \mathrm{C} \quad 2.599480 \mathrm{E}-29$ \$Ce141

$59143.70 \mathrm{C} \quad 2.563058 \mathrm{E}-29 \$ \operatorname{Pr} 143$

$60143.70 \mathrm{C} \quad 2.563055 \mathrm{E}-29$ \$Nd143

$60145.70 \mathrm{C} \quad 2.527633 \mathrm{E}-29 \$ \mathrm{Nd} 145$

$61147.70 \mathrm{C} \quad 2.493199 \mathrm{E}-29 \$ \mathrm{Pm} 147$

$61149.70 \mathrm{C} \quad 2.459663 \mathrm{E}-29 \$ \mathrm{Pm} 149$

$62149.70 \mathrm{C} \quad 2.459661 \mathrm{E}-29 \$ \mathrm{Sm} 149$

$61151.70 \mathrm{C} \quad 2.426995 \mathrm{E}-29$ \$Pm151

$62151.70 \mathrm{C} \quad 2.427021 \mathrm{E}-29 \$ \mathrm{Sm} 151$

$62152.70 \mathrm{C} \quad 2.411048 \mathrm{E}-29$ \$Sm152

$63153.70 \mathrm{C} \quad 2.395258 \mathrm{E}-29 \$ \mathrm{Eu} 153$

$63155.70 \mathrm{C} \quad 2.364321 \mathrm{E}-29$ \$Eu155

lwtr. $10 t$

$64157.70 \mathrm{C} \quad 2.334161 \mathrm{E}-29$ \$Gd157

ATR Element No. = 15

Radial Zone No. = 1

Axial Zone No. = 1

Neutron Cross Sections $=27 \mathrm{C}$

Total Number Density $=7.975780 \mathrm{E}-02 \mathrm{a} / \mathrm{b}-\mathrm{cm}$

$1001.70 \mathrm{C} \quad 3.393340 \mathrm{E}-02$ \$ $\mathrm{H}-1$

$8016.70 \mathrm{C} \quad 1.696670 \mathrm{E}-02 \$ 0-16$

$12000.60 \mathrm{C} \quad 2.176490 \mathrm{E}-04 \quad \$ \mathrm{Mg}-$ nat

$13027.70 \mathrm{C} \quad 2.793720 \mathrm{E}-02$ \$Al-27

$14000.60 \mathrm{C} \quad 1.130110 \mathrm{E}-04$ \$Si-nat

$24000.50 \mathrm{C} \quad 2.304760 \mathrm{E}-05$ \$Cr-nat

$29000.50 \mathrm{C} \quad 2.081160 \mathrm{E}-05$ \$Cu-nat

$5010.70 \mathrm{C} \quad 1.976748 \mathrm{E}-05$ \$ B-10

$92234.70 \mathrm{C} \quad 6.286590 \mathrm{E}-06 \quad \$ \mathrm{U}-234$

$92235.70 \mathrm{C} \quad 4.851306 \mathrm{E}-04 \quad \$ \mathrm{U}-235$

$92236.70 \mathrm{C} \quad 3.636026 \mathrm{E}-06$ \$U-236 


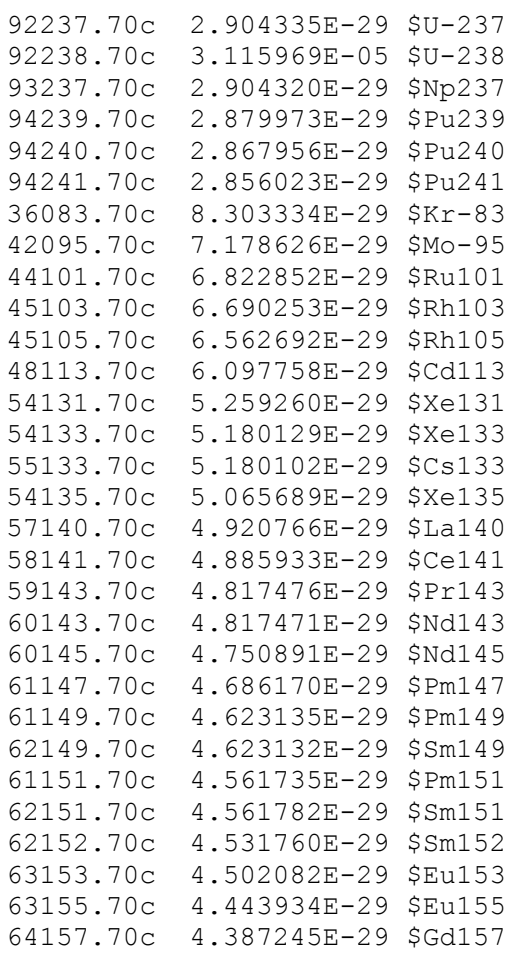
mt2295 lwtr.10t

$\mathrm{C}$

C

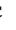

m2296

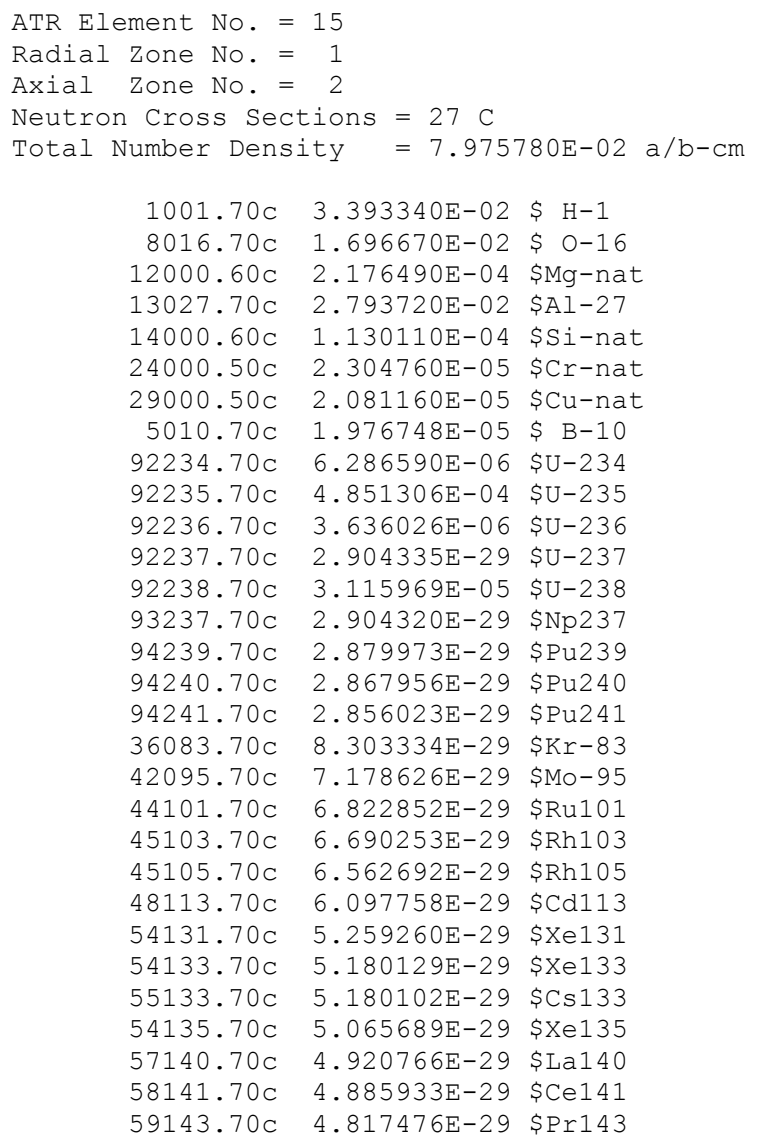


$60143.70 \mathrm{C} \quad 4.817471 \mathrm{E}-29 \$ \mathrm{Nd1} 43$

$60145.70 \mathrm{C} \quad 4.750891 \mathrm{E}-29 \$ \mathrm{Nd} 145$

$61147.70 \mathrm{C} \quad 4.686170 \mathrm{E}-29 \$ \mathrm{Pm} 147$

$61149.70 \mathrm{C} \quad 4.623135 \mathrm{E}-29 \$ \mathrm{Pm} 149$

$62149.70 \mathrm{C} \quad 4.623132 \mathrm{E}-29 \$ \mathrm{Sm} 149$

$61151.70 \mathrm{c} \quad 4.561735 \mathrm{E}-29$ \$Pm151

$62151.70 \mathrm{C} \quad 4.561782 \mathrm{E}-29 \$ \mathrm{Sm} 151$

$62152.70 \mathrm{C} \quad 4.531760 \mathrm{E}-29 \$ \mathrm{Sm} 152$

$63153.70 \mathrm{C} \quad 4.502082 \mathrm{E}-29$ \$Eu153

$63155.70 \mathrm{C} \quad 4.443934 \mathrm{E}-29 \quad \$ \mathrm{Eu} 155$

mt2296 lwtr.10t

$64157.70 \mathrm{C} \quad 4.387245 \mathrm{E}-29$ \$Gd157

C

C

ATR Element No. $=15$

Radial Zone No. $=1$

Axial Zone No. $=3$

Neutron Cross Sections $=27 \mathrm{C}$

Total Number Density $=7.975780 \mathrm{E}-02 \mathrm{a} / \mathrm{b}-\mathrm{cm}$

$1001.70 \mathrm{C} \quad 3.393340 \mathrm{E}-02$ \$ H-1

$8016.70 \mathrm{C} \quad 1.696670 \mathrm{E}-02$ \$ O-16

$12000.60 \mathrm{C} \quad 2.176490 \mathrm{E}-04$ \$Mg-nat

$13027.70 \mathrm{C} \quad 2.793720 \mathrm{E}-02$ \$Al-27

$14000.60 \mathrm{C} \quad 1.130110 \mathrm{E}-04$ \$Si-nat

$24000.50 \mathrm{C} \quad 2.304760 \mathrm{E}-05$ \$Cr-nat

$29000.50 \mathrm{C} \quad 2.081160 \mathrm{E}-05$ \$Cu-nat

$5010.70 \mathrm{C} \quad 1.976748 \mathrm{E}-05$ \$ -10

$92234.70 \mathrm{C} \quad 6.286590 \mathrm{E}-06$ \$U-234

$92235.70 \mathrm{C} \quad 4.851306 \mathrm{E}-04 \quad \$ \mathrm{U}-235$

$92236.70 \mathrm{C} \quad 3.636026 \mathrm{E}-06 \quad \$ \mathrm{U}-236$

$92237.70 \mathrm{C} \quad 2.904335 \mathrm{E}-29 \quad \$ \mathrm{U}-237$

$92238.70 \mathrm{C} \quad 3.115969 \mathrm{E}-05$ \$U-238

$93237.70 \mathrm{C} \quad 2.904320 \mathrm{E}-29 \$ \mathrm{~Np} 237$

$94239.70 \mathrm{C} \quad 2.879973 \mathrm{E}-29$ \$Pu239

$94240.70 \mathrm{C} \quad 2.867956 \mathrm{E}-29$ \$Pu 240

$94241.70 \mathrm{C} \quad 2.856023 \mathrm{E}-29$ \$Pu241

$36083.70 \mathrm{C} \quad 8.303334 \mathrm{E}-29 \$ \mathrm{Kr}-83$

$42095.70 \mathrm{C} \quad 7.178626 \mathrm{E}-29$ \$Mo-95

$44101.70 \mathrm{C} \quad 6.822852 \mathrm{E}-29$ \$Ru101

$45103.70 \mathrm{C} \quad 6.690253 \mathrm{E}-29$ \$Rh103

$45105.70 \mathrm{C} \quad 6.562692 \mathrm{E}-29$ \$Rh105

$48113.70 \mathrm{C} \quad 6.097758 \mathrm{E}-29 \$ \mathrm{Cd} 113$

$54131.70 \mathrm{C} \quad 5.259260 \mathrm{E}-29$ Se131

$54133.70 \mathrm{C} \quad 5.180129 \mathrm{E}-29 \$ \mathrm{Xe133}$

$55133.70 \mathrm{C} \quad 5.180102 \mathrm{E}-29 \mathrm{SCs} 133$

$54135.70 \mathrm{C} \quad 5.065689 \mathrm{E}-29 \$ \mathrm{Xe1} 35$

$57140.70 \mathrm{C} \quad 4.920766 \mathrm{E}-29$ \$La140

$58141.70 \mathrm{C} \quad 4.885933 \mathrm{E}-29 \$ \mathrm{Ce} 141$

$59143.70 \mathrm{C} \quad 4.817476 \mathrm{E}-29 \quad \$ \operatorname{Pr} 143$

$60143.70 \mathrm{C} \quad 4.817471 \mathrm{E}-29 \$ \mathrm{Nd} 143$

$60145.70 \mathrm{C} \quad 4.750891 \mathrm{E}-29 \$ \mathrm{Nd} 145$

$61147.70 \mathrm{C} \quad 4.686170 \mathrm{E}-29$ \$Pm147

$61149.70 \mathrm{C} \quad 4.623135 \mathrm{E}-29 \$ \mathrm{Pm} 149$

$62149.70 \mathrm{C} \quad 4.623132 \mathrm{E}-29$ \$Sm149

$61151.70 \mathrm{C} \quad 4.561735 \mathrm{E}-29$ \$Pm151

$62151.70 \mathrm{C} \quad 4.561782 \mathrm{E}-29 \$ \mathrm{Sm} 151$

$62152.70 \mathrm{C} \quad 4.531760 \mathrm{E}-29$ \$Sm152

$63153.70 \mathrm{C} \quad 4.502082 \mathrm{E}-29$ \$Eu153

$63155.70 \mathrm{C} \quad 4.443934 \mathrm{E}-29$ \$Eu155

mt2297 lwtr.10t

$64157.70 \mathrm{C} \quad 4.387245 \mathrm{E}-29$ \$Gd157

c

$\mathrm{C}$

C

C

C

ATR Element No. = 15

Radial Zone No. $=1$

Axial Zone No. = 4 


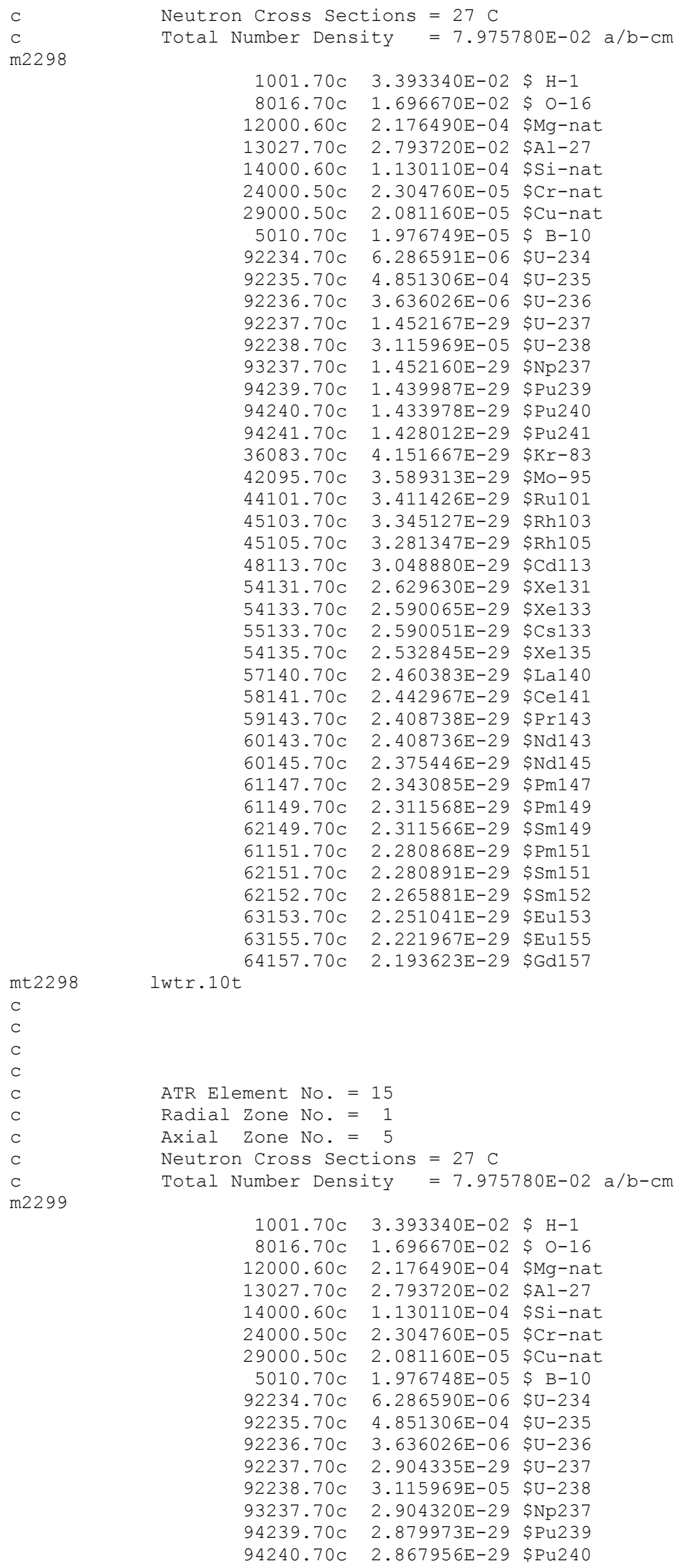




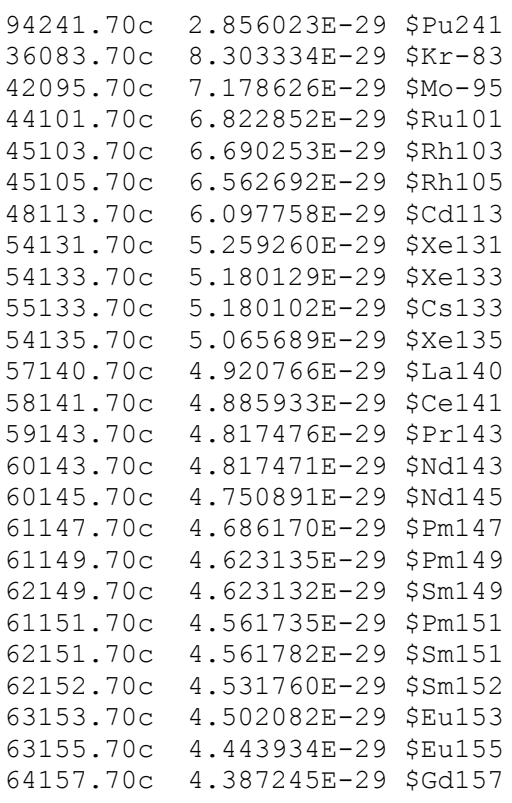

mt2299 lwtr.10t

c

C

C

C

C

m2300

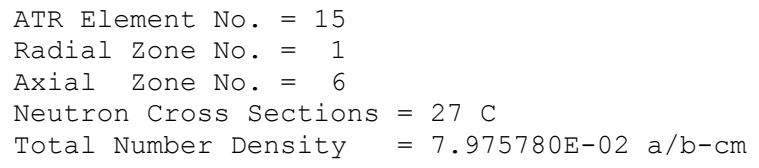
$1001.70 \mathrm{C} \quad 3.393340 \mathrm{E}-02$ \$ $\mathrm{H}-1$
$8016.70 \mathrm{C} \quad 1.696670 \mathrm{E}-02 \$ 0-16$
$12000.60 \mathrm{C} \quad 2.176490 \mathrm{E}-04$ \$Mg-nat
$13027.70 \mathrm{C} \quad 2.793720 \mathrm{E}-02$ \$Al-27
$14000.60 \mathrm{C} \quad 1.130110 \mathrm{E}-04$ \$Si-nat
$24000.50 \mathrm{C} \quad 2.304760 \mathrm{E}-05$ \$Cr-nat
$29000.50 \mathrm{C} \quad 2.081160 \mathrm{E}-05$ \$Cu-nat
$5010.70 \mathrm{C} \quad 1.976748 \mathrm{E}-05$ \$ $\mathrm{B}-10$
$92234.70 \mathrm{C} \quad 6.286590 \mathrm{E}-06 \quad \$ \mathrm{U}-234$
$92235.70 \mathrm{C} \quad 4.851306 \mathrm{E}-04 \quad \$ \mathrm{U}-235$
$92236.70 \mathrm{C} \quad 3.636026 \mathrm{E}-06 \quad \$ \mathrm{U}-236$
$92237.70 \mathrm{C} \quad 2.904335 \mathrm{E}-29 \quad \$ \mathrm{U}-237$
$92238.70 \mathrm{C} \quad 3.115969 \mathrm{E}-05 \mathrm{\$ U}-238$
$93237.70 \mathrm{C} \quad 2.904320 \mathrm{E}-29 \$ \mathrm{~Np} 237$
$94239.70 \mathrm{C} \quad 2.879973 \mathrm{E}-29$ \$Pu239
$94240.70 \mathrm{C} \quad 2.867956 \mathrm{E}-29$ \$Pu240
$94241.70 \mathrm{C} \quad 2.856023 \mathrm{E}-29$ \$Pu241
$36083.70 \mathrm{C} \quad 8.303334 \mathrm{E}-29 \$ \mathrm{Kr}-83$
$42095.70 \mathrm{C} \quad 7.178626 \mathrm{E}-29$ \$Mo-95
$44101.70 \mathrm{C} \quad 6.822852 \mathrm{E}-29$ \$Ru101
$45103.70 \mathrm{C} \quad 6.690253 \mathrm{E}-29$ \$Rh103
$45105.70 \mathrm{C} \quad 6.562692 \mathrm{E}-29$ \$Rh105
$48113.70 \mathrm{C} \quad 6.097758 \mathrm{E}-29 \$ \mathrm{Cd} 113$
$54131.70 \mathrm{C} \quad 5.259260 \mathrm{E}-29 \$ \mathrm{Xe} 131$
$54133.70 \mathrm{C} \quad 5.180129 \mathrm{E}-29$ \$Xe133
$55133.70 \mathrm{C} \quad 5.180102 \mathrm{E}-29 \$ \mathrm{Cs} 133$
$54135.70 \mathrm{C} \quad 5.065689 \mathrm{E}-29$ Se135
$57140.70 \mathrm{C} \quad 4.920766 \mathrm{E}-29$ La140
$58141.70 \mathrm{C} \quad 4.885933 \mathrm{E}-29$ Se141
$59143.70 \mathrm{C} \quad 4.817476 \mathrm{E}-29 \quad \$ \mathrm{Pr} 143$
$60143.70 \mathrm{C} \quad 4.817471 \mathrm{E}-29 \$ \mathrm{Nd} 143$
$60145.70 \mathrm{C} \quad 4.750891 \mathrm{E}-29$ \$Nd145
$61147.70 \mathrm{C} \quad 4.686170 \mathrm{E}-29 \$ \mathrm{Pm} 147$
$61149.70 \mathrm{C} \quad 4.623135 \mathrm{E}-29 \$ \mathrm{Pm} 149$
$62149.70 \mathrm{C} \quad 4.623132 \mathrm{E}-29 \$ \mathrm{Sm} 149$ 


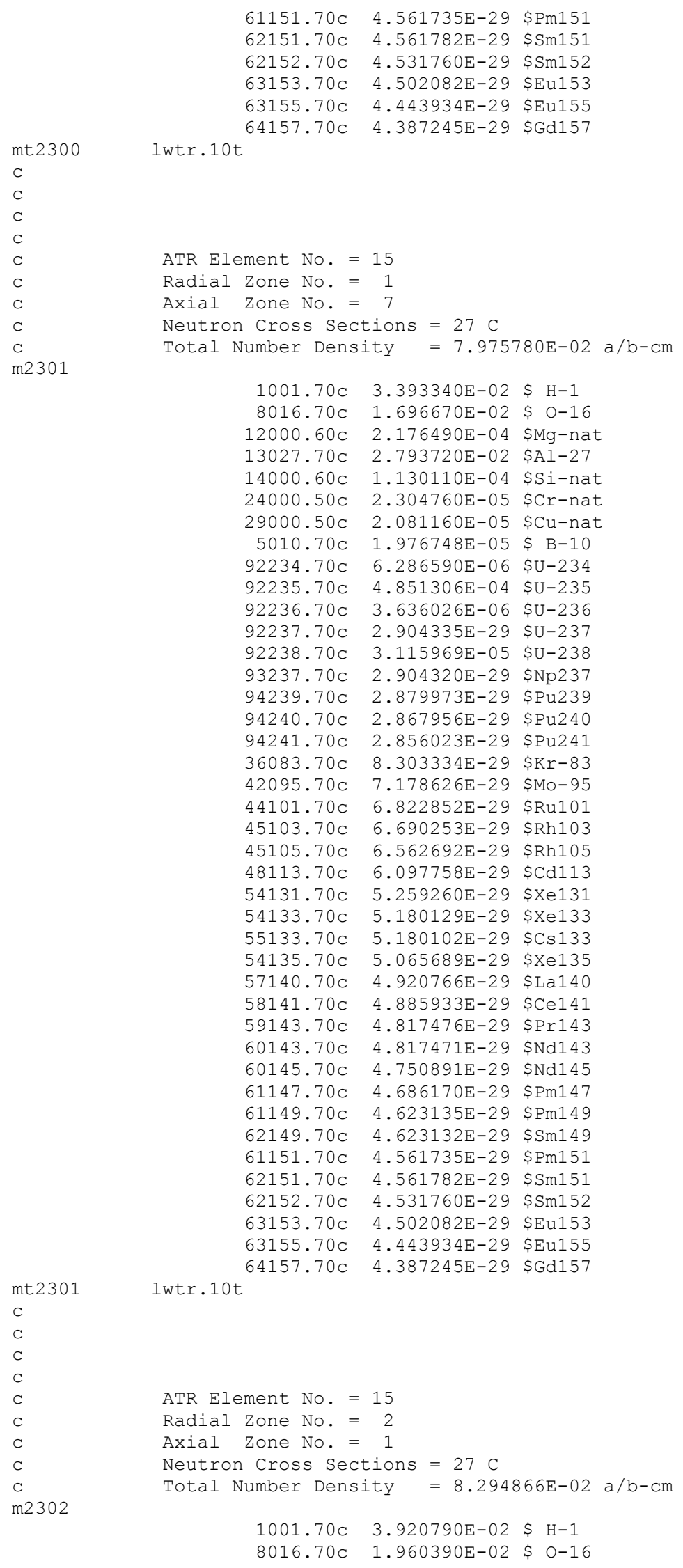




$$
\begin{aligned}
& 12000.60 \mathrm{C} \quad 1.660990 \mathrm{E}-04 \text { \$Mg-nat } \\
& 13027.70 \mathrm{C} \quad 2.315370 \mathrm{E}-02 \text { \$Al-27 } \\
& 14000.60 \mathrm{C} \quad 8.624450 \mathrm{E}-05 \text { \$Si-nat } \\
& 24000.50 \mathrm{C} \quad 1.552830 \mathrm{E}-05 \mathrm{\$ Cr}-\mathrm{nat} \\
& 29000.50 \mathrm{C} \quad 1.588240 \mathrm{E}-05 \text { \$Cu-nat } \\
& 5010.70 \mathrm{C} \quad 1.714619 \mathrm{E}-28 \text { \$ B-10 } \\
& 92234.70 \mathrm{C} \quad 8.355541 \mathrm{E}-06 \quad \$ \mathrm{U}-234 \\
& 92235.70 \mathrm{C} \quad 6.447897 \mathrm{E}-04 \quad \$ \mathrm{U}-235 \\
& 92236.70 \mathrm{C} \quad 4.832662 \mathrm{E}-06 \text { \$U-236 } \\
& 92237.70 \mathrm{C} \quad 7.242605 \mathrm{E}-30 \quad \$ \mathrm{U}-237 \\
& 92238.70 \mathrm{C} \quad 4.141451 \mathrm{E}-05 \quad \$ \mathrm{U}-238 \\
& 93237.70 \mathrm{C} \quad 7.242569 \mathrm{E}-30 \quad \$ \mathrm{~Np} 237 \\
& 94239.70 \mathrm{C} \quad 7.181854 \mathrm{E}-30 \text { \$Pu239 } \\
& 94240.70 \mathrm{C} \quad 7.151887 \mathrm{E}-30 \text { \$Pu240 } \\
& 94241.70 \mathrm{C} \quad 7.122128 \mathrm{E}-30 \text { \$Pu241 } \\
& 36083.70 \mathrm{C} \quad 2.070621 \mathrm{E}-29 \$ \mathrm{Kr}-83 \\
& 42095.70 \mathrm{C} \quad 1.790150 \mathrm{E}-29 \text { \$Mo-95 } \\
& 44101.70 \mathrm{C} \quad 1.701430 \mathrm{E}-29 \text { \$Ru101 } \\
& 45103.70 \mathrm{C} \quad 1.668363 \mathrm{E}-29 \text { \$Rh103 } \\
& 45105.70 \mathrm{C} \quad 1.636554 \mathrm{E}-29 \text { \$Rh105 } \\
& 48113.70 \mathrm{C} \quad 1.520612 \mathrm{E}-29 \text { \$Cd113 } \\
& 54131.70 \mathrm{C} \quad 1.311513 \mathrm{E}-29 \text { \$Xe131 } \\
& 54133.70 \mathrm{C} \quad 1.291780 \mathrm{E}-29 \text { \$Xe133 } \\
& 55133.70 \mathrm{C} \quad 1.291774 \mathrm{E}-29 \quad \$ \mathrm{Cs} 133 \\
& 54135.70 \mathrm{C} \quad 1.263242 \mathrm{E}-29 \text { \$Xe135 } \\
& 57140.70 \mathrm{C} \quad 1.227103 \mathrm{E}-29 \text { \$La140 } \\
& 58141.70 \mathrm{C} \quad 1.218416 \mathrm{E}-29 \text { \$Ce141 } \\
& 59143.70 \mathrm{C} \quad 1.201345 \mathrm{E}-29 \quad \$ \operatorname{Pr} 143 \\
& 60143.70 \mathrm{C} \quad 1.201344 \mathrm{E}-29 \$ \mathrm{Nd} 143 \\
& 60145.70 \mathrm{C} \quad 1.184740 \mathrm{E}-29 \text { \$Nd145 } \\
& 61147.70 \mathrm{C} \quad 1.168601 \mathrm{E}-29 \$ \mathrm{Pm} 147 \\
& 61149.70 \mathrm{C} \quad 1.152882 \mathrm{E}-29 \$ \mathrm{Pm} 149 \\
& 62149.70 \mathrm{C} \quad 1.152881 \mathrm{E}-29 \text { \$Sm149 } \\
& 61151.70 \mathrm{C} \quad 1.137570 \mathrm{E}-29 \text { \$Pm151 } \\
& 62151.70 \mathrm{C} \quad 1.137582 \mathrm{E}-29 \text { \$Sm151 } \\
& 62152.70 \mathrm{C} \quad 1.130095 \mathrm{E}-29 \$ \mathrm{Sm} 152 \\
& 63153.70 \mathrm{C} \quad 1.122694 \mathrm{E}-29 \text { \$Eu153 } \\
& 63155.70 \mathrm{C} \quad 1.108194 \mathrm{E}-29 \text { \$Eu155 } \\
& \text { mt2302 lwtr.10t } \\
& 64157.70 \mathrm{C} \quad 1.094057 \mathrm{E}-29 \text { \$Gd157 }
\end{aligned}
$$

$\begin{array}{rll}1001.70 \mathrm{C} & 3.920790 \mathrm{E}-02 & \text { \$ } \mathrm{H}-1 \\ 8016.70 \mathrm{C} & 1.960390 \mathrm{E}-02 & \text { \$ O-16 } \\ 12000.60 \mathrm{C} & 1.660990 \mathrm{E}-04 & \text { \$Mg-nat } \\ 13027.70 \mathrm{C} & 2.315370 \mathrm{E}-02 & \text { \$Al-27 } \\ 14000.60 \mathrm{C} & 8.624450 \mathrm{E}-05 & \text { \$Si-nat } \\ 24000.50 \mathrm{C} & 1.552830 \mathrm{E}-05 & \text { \$Cr-nat } \\ 29000.50 \mathrm{C} & 1.588240 \mathrm{E}-05 & \text { \$Cu-nat } \\ 5010.70 \mathrm{C} & 1.714619 \mathrm{E}-28 & \text { \$B-10 } \\ 92234.70 \mathrm{C} & 8.355541 \mathrm{E}-06 & \text { \$U-234 } \\ 92235.70 \mathrm{C} & 6.447897 \mathrm{E}-04 & \text { \$U-235 } \\ 92236.70 \mathrm{C} & 4.832662 \mathrm{E}-06 & \text { \$U-236 } \\ 92237.70 \mathrm{C} & 7.242605 \mathrm{E}-30 & \text { \$U-237 } \\ 92238.70 \mathrm{C} & 4.141451 \mathrm{E}-05 & \text { \$U-238 } \\ 93237.70 \mathrm{C} & 7.242569 \mathrm{E}-30 & \text { \$Np237 } \\ 94239.70 \mathrm{C} & 7.181854 \mathrm{E}-30 & \text { \$Pu239 } \\ 94240.70 \mathrm{C} & 7.151887 \mathrm{E}-30 & \text { \$Pu240 } \\ 94241.70 \mathrm{C} & 7.122128 \mathrm{E}-30 & \text { \$Pu241 } \\ 36083.70 \mathrm{C} & 2.070621 \mathrm{E}-29 & \text { \$Kr-83 } \\ 42095.70 \mathrm{C} & 1.790150 \mathrm{E}-29 & \text { \$Mo-95 } \\ 44101.70 \mathrm{C} & 1.701430 \mathrm{E}-29 & \text { \$Ru101 } \\ 45103.70 \mathrm{C} & 1.668363 \mathrm{E}-29 & \text { \$Rh103 }\end{array}$




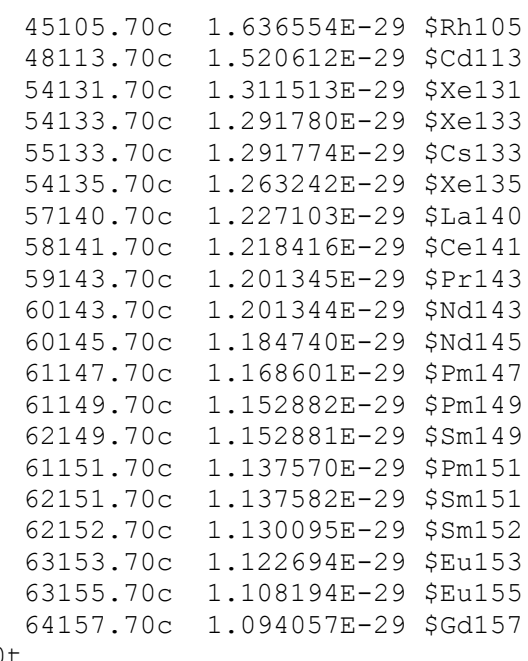

\begin{tabular}{|c|c|}
\hline \multirow{2}{*}{\multicolumn{2}{|c|}{$m t 2303$}} \\
\hline & \\
\hline C & \\
\hline C & \\
\hline C & \\
\hline C & \\
\hline C & ATR Element No. $=15$ \\
\hline C & Radial Zone No. $=2$ \\
\hline C & Axial Zone No. $=3$ \\
\hline C & Neutron Cross Sections $=27 \mathrm{C}$ \\
\hline C & Total Number Density $=8.294866 \mathrm{E}-02 \mathrm{a} / \mathrm{b}-\mathrm{cm}$ \\
\hline
\end{tabular}

\begin{tabular}{|c|c|c|}
\hline $1001.70 \mathrm{C}$ & $3.920790 \mathrm{E}-02$ & $\$ \mathrm{H}-1$ \\
\hline $8016.70 \mathrm{C}$ & $1.960390 \mathrm{E}-02$ & $\$ 0-16$ \\
\hline $2000.60 \mathrm{c}$ & 1. $660990 \mathrm{E}-04$ & $\$ M g-n a t$ \\
\hline $3027.70 \mathrm{c}$ & $2.315370 \mathrm{E}-02$ & $\$ A l-27$ \\
\hline $00.60 \mathrm{C}$ & $8.624450 \mathrm{E}-05$ & \$Si-nat \\
\hline $000.50 \mathrm{C}$ & $1.552830 \mathrm{E}-05$ & \$Cr-nat \\
\hline & $1.588240 \mathrm{E}-05$ & $\$ \mathrm{Cu}-$ nat \\
\hline $.70 \mathrm{C}$ & $1.714619 \mathrm{E}-28$ & $\$ B-10$ \\
\hline $4.70 \mathrm{C}$ & $8.355541 E-06$ & $\$ U-234$ \\
\hline $.70 \mathrm{C}$ & $6.447897 \mathrm{E}-04$ & $\$ U-235$ \\
\hline $.70 \mathrm{C}$ & $4.832662 E-06$ & $\$ U-236$ \\
\hline $.70 \mathrm{C}$ & 7. $242605 \mathrm{E}-30$ & $\$ U-237$ \\
\hline $.70 \mathrm{c}$ & $4.141451 E-05$ & $\$ U-238$ \\
\hline $.70 \mathrm{C}$ & $7.242569 \mathrm{E}-30$ & $\$ N p 237$ \\
\hline $.70 \mathrm{C}$ & $7.181854 \mathrm{E}-30$ & $\$ P u 239$ \\
\hline $.70 \mathrm{C}$ & $887 E-30$ & $\$ P u 240$ \\
\hline $.70 \mathrm{c}$ & $128 E-30$ & $\$ P u 241$ \\
\hline $.70 \mathrm{C}$ & $21 E-29$ & $\$ K r-83$ \\
\hline $.70 \mathrm{c}$ & $1.790150 \mathrm{E}-29$ & $\$ M o-95$ \\
\hline $.70 \mathrm{C}$ & $30 E-29$ & \$Ru101 \\
\hline & $3 E-29$ & $\$ R h 103$ \\
\hline $.70 \mathrm{C}$ & $4 E-29$ & $\$ R h 105$ \\
\hline & $2 E-29$ & $\$ \mathrm{Cd} 113$ \\
\hline $.70 \mathrm{C}$ & $3 E-29$ & $\$ x e 131$ \\
\hline & $0 E-29$ & $\$ x e 133$ \\
\hline $.70 \mathrm{C}$ & $74 E-29$ & $\$ \mathrm{Cs} 133$ \\
\hline & $42 E-29$ & $\$ x e 135$ \\
\hline $.70 \mathrm{C}$ & $3 E-29$ & $\$ \mathrm{La} 140$ \\
\hline$c$ & $6 E-29$ & $\$ C e 141$ \\
\hline $.70 \mathrm{C}$ & $45 E-29$ & $\$ \operatorname{Pr} 143$ \\
\hline & $1.201344 \mathrm{E}-29$ & $\$ N d 143$ \\
\hline $.70 \mathrm{C}$ & $40 E-29$ & $\$ N d 145$ \\
\hline $.70 \mathrm{C}$ & $1.168601 \mathrm{E}-29$ & $\$ \operatorname{Pm} 147$ \\
\hline $.70 \mathrm{C}$ & $1.152882 \mathrm{E}-29$ & $\$ P m 149$ \\
\hline $.70 \mathrm{C}$ & $1.152881 \mathrm{E}-29$ & $\$ S m 149$ \\
\hline $.70 \mathrm{C}$ & $1.137570 \mathrm{E}-29$ & $\$ P m 151$ \\
\hline $.70 \mathrm{C}$ & $1.137582 \mathrm{E}-29$ & $\$ S m 151$ \\
\hline $.70 \mathrm{C}$ & $1.130095 \mathrm{E}-29$ & \$Sm152 \\
\hline & $594 E-29$ & \$Eu153 \\
\hline 631 & $1.108194 \mathrm{E}-29$ & \$Eu155 \\
\hline
\end{tabular}


$64157.70 \mathrm{C} \quad 1.094057 \mathrm{E}-29 \$ \mathrm{Gd} 157$

mt2304
c
c
c
c
c
c
c
c
c
m2305

lwtr.10t

ATR Element No. $=15$

Radial Zone No. $=2$

Axial Zone No. = 4

Neutron Cross Sections $=27 \mathrm{C}$

Total Number Density $=8.294866 \mathrm{E}-02 \mathrm{a} / \mathrm{b}-\mathrm{cm}$

\begin{abstract}
$1001.70 \mathrm{C} \quad 3.920790 \mathrm{E}-02$ \$ $\mathrm{H}-1$
$8016.70 \mathrm{C} \quad 1.960390 \mathrm{E}-02$ \$ O-16

$12000.60 \mathrm{C} \quad 1.660990 \mathrm{E}-04$ \$Mg-nat

$13027.70 \mathrm{C} \quad 2.315370 \mathrm{E}-02$ \$Al-27

$14000.60 \mathrm{C} \quad 8.624450 \mathrm{E}-05$ \$Si-nat

$24000.50 \mathrm{C} \quad 1.552830 \mathrm{E}-05$ \$Cr-nat

$29000.50 \mathrm{C} \quad 1.588240 \mathrm{E}-05$ \$Cu-nat

$5010.70 \mathrm{C} \quad 8.573098 \mathrm{E}-29$ \$ B-10

$92234.70 \mathrm{C} \quad 8.355538 \mathrm{E}-06 \quad \$ \mathrm{U}-234$

$92235.70 \mathrm{C} \quad 6.447894 \mathrm{E}-04 \quad \$ \mathrm{U}-235$

$92236.70 \mathrm{C} \quad 4.832660 \mathrm{E}-06 \quad \$ \mathrm{U}-236$

$92237.70 \mathrm{C} \quad 3.621303 \mathrm{E}-30$ \$U-237

$92238.70 \mathrm{C} \quad 4.141449 \mathrm{E}-05 \mathrm{\$ U}-238$

$93237.70 \mathrm{C} \quad 3.621285 \mathrm{E}-30$ \$Np237

$94239.70 \mathrm{C} \quad 3.590928 \mathrm{E}-30 \quad \$ \mathrm{Pu} 239$

$94240.70 \mathrm{C} \quad 3.575944 \mathrm{E}-30$ \$Pu240

$94241.70 \mathrm{C} \quad 3.561065 \mathrm{E}-30$ \$Pu241

$36083.70 \mathrm{C} \quad 1.035311 \mathrm{E}-29$ \$Kr-83

$42095.70 \mathrm{C} \quad 8.950752 \mathrm{E}-30 \quad \$ M o-95$

$44101.70 \mathrm{C} \quad 8.507152 \mathrm{E}-30$ \$Ru101

$45103.70 \mathrm{C} \quad 8.341818 \mathrm{E}-30$ \$Rh103

$45105.70 \mathrm{C} \quad 8.182769 \mathrm{E}-30$ \$Rh105

$48113.70 \mathrm{C} \quad 7.603060 \mathrm{E}-30 \quad \$ \mathrm{Cd} 113$

$54131.70 \mathrm{C} \quad 6.557568 \mathrm{E}-30 \quad \$ \mathrm{Xe1} 131$

$54133.70 \mathrm{C} \quad 6.458903 \mathrm{E}-30$ \$Xe133

$55133.70 \mathrm{C} \quad 6.458870 \mathrm{E}-30 \quad \$ \mathrm{Cs} 133$

$54135.70 \mathrm{c} \quad 6.316213 \mathrm{E}-30 \quad \$ \mathrm{Xe135}$

$57140.70 \mathrm{C} \quad 6.135514 \mathrm{E}-30$ \$La140

$58141.70 \mathrm{C} \quad 6.092082 \mathrm{E}-30 \quad \$ \mathrm{Ce} 141$

$59143.70 \mathrm{C} \quad 6.006725 \mathrm{E}-30 \quad \$ \operatorname{Pr} 143$

$60143.70 \mathrm{C} \quad 6.006719 \mathrm{E}-30 \quad \$ N d 143$

$60145.70 \mathrm{C} \quad 5.923703 \mathrm{E}-30 \quad \$ \mathrm{Nd} 145$

$61147.70 \mathrm{C} \quad 5.843005 \mathrm{E}-30 \quad \$ \mathrm{Pm} 147$

$61149.70 \mathrm{C} \quad 5.764410 \mathrm{E}-30 \$ \mathrm{Pm} 149$

$62149.70 \mathrm{C} \quad 5.764405 \mathrm{E}-30 \quad \$ \mathrm{Sm} 149$

$61151.70 \mathrm{C} \quad 5.687851 \mathrm{E}-30$ \$Pm151

$62151.70 \mathrm{C} \quad 5.687911 \mathrm{E}-30 \$ \mathrm{Sm} 151$

$62152.70 \mathrm{C} \quad 5.650478 \mathrm{E}-30$ \$Sm152

$63153.70 \mathrm{C} \quad 5.613472 \mathrm{E}-30$ \$Eu153

$63155.70 \mathrm{C} \quad 5.540969 \mathrm{E}-30$ \$Eu155

$64157.70 \mathrm{C} \quad 5.470287 \mathrm{E}-30$ \$Gd157
\end{abstract}

mt2305 lwtr.10t

c

C

C

C

C

C

C

C

m2306

ATR Element No. $=15$

Radial Zone No. $=2$

Axial Zone No. = 5

Neutron Cross Sections $=27 \mathrm{C}$

Total Number Density $=8.294866 \mathrm{E}-02 \mathrm{a} / \mathrm{b}-\mathrm{cm}$

$1001.70 \mathrm{C} \quad 3.920790 \mathrm{E}-02 \$ \mathrm{H}-1$

$8016.70 \mathrm{C} \quad 1.960390 \mathrm{E}-02 \$ 0-16$

$12000.60 \mathrm{C} \quad 1.660990 \mathrm{E}-04$ \$Mg-nat

$13027.70 \mathrm{C} \quad 2.315370 \mathrm{E}-02$ \$Al-27

$14000.60 \mathrm{C} \quad 8.624450 \mathrm{E}-05$ \$Si-nat

$24000.50 \mathrm{C} \quad 1.552830 \mathrm{E}-05$ \$Cr-nat

$29000.50 \mathrm{C} \quad 1.588240 \mathrm{E}-05$ \$Cu-nat 


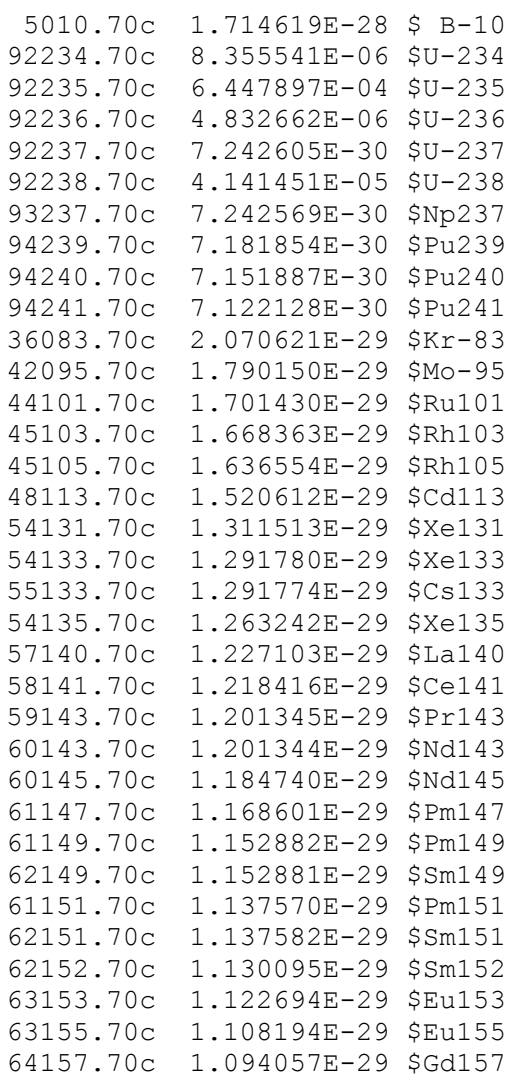

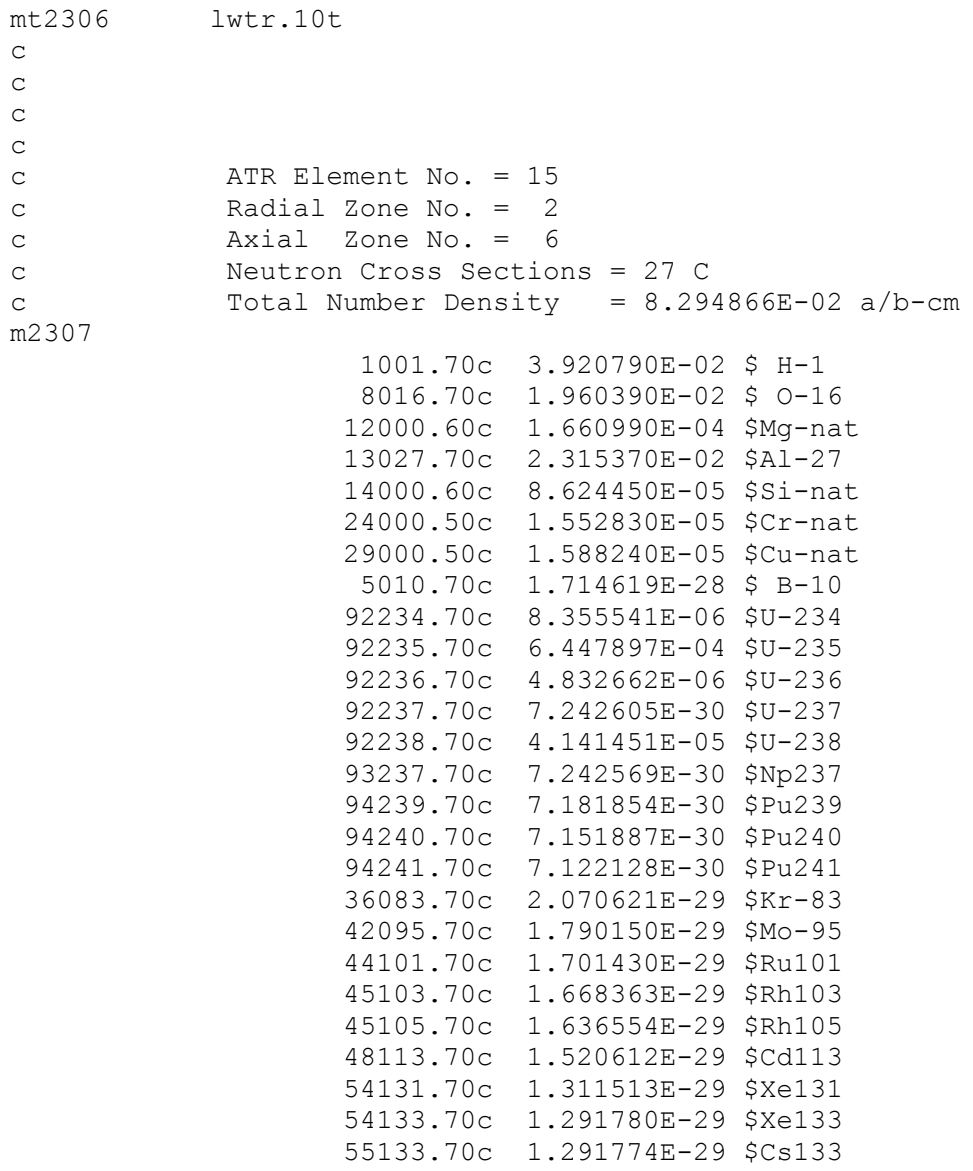




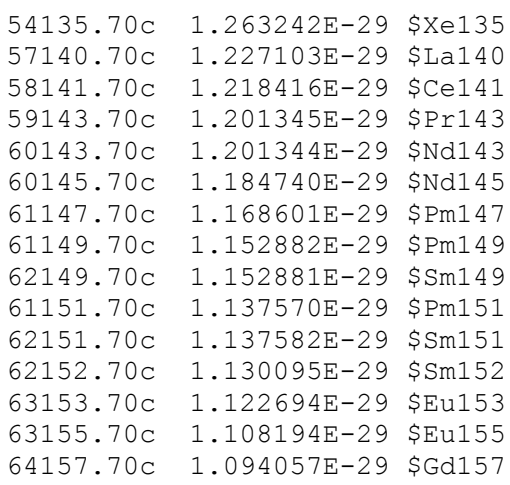

mt2307 lwtr.10t

C

$\mathrm{C}$

$\mathrm{C}$

C ATR Element No. = 15

C Radial Zone No. $=2$

C Axial Zone No. = 7

C Neutron Cross Sections $=27 \mathrm{C}$

C Total Number Density $=8.294866 \mathrm{E}-02 \mathrm{a} / \mathrm{b}-\mathrm{cm}$

m2308

$1001.70 \mathrm{C} \quad 3.920790 \mathrm{E}-02 \$ \mathrm{H}-1$

$8016.70 \mathrm{C} \quad 1.960390 \mathrm{E}-02 \$ 0-16$

$12000.60 \mathrm{C} \quad 1.660990 \mathrm{E}-04$ \$Mg-nat

$13027.70 \mathrm{C} \quad 2.315370 \mathrm{E}-02$ \$Al-27

$14000.60 \mathrm{C} \quad 8.624450 \mathrm{E}-05$ \$Si-nat

$24000.50 \mathrm{C} \quad 1.552830 \mathrm{E}-05$ \$Cr-nat

$29000.50 \mathrm{C} \quad 1.588240 \mathrm{E}-05$ \$Cu-nat

$5010.70 \mathrm{C} \quad 1.714619 \mathrm{E}-28$ \$ B 10

$92234.70 \mathrm{C} \quad 8.355541 \mathrm{E}-06 \quad \$ \mathrm{U}-234$

$92235.70 \mathrm{C} \quad 6.447897 \mathrm{E}-04 \quad \$ \mathrm{U}-235$

$92236.70 \mathrm{C} \quad 4.832662 \mathrm{E}-06 \quad \$ \mathrm{U}-236$

$92237.70 \mathrm{C} \quad 7.242605 \mathrm{E}-30 \quad \$ \mathrm{U}-237$

$92238.70 \mathrm{C} \quad 4.141451 \mathrm{E}-05$ \$U-238

$93237.70 \mathrm{C} \quad 7.242569 \mathrm{E}-30 \quad \$ \mathrm{~Np} 237$

$94239.70 \mathrm{C} \quad 7.181854 \mathrm{E}-30$ \$Pu239

$94240.70 \mathrm{C} \quad 7.151887 \mathrm{E}-30$ \$Pu 240

$94241.70 \mathrm{C} \quad 7.122128 \mathrm{E}-30$ \$Pu241

$36083.70 \mathrm{C} \quad 2.070621 \mathrm{E}-29$ \$Kr-83

$42095.70 \mathrm{C} \quad 1.790150 \mathrm{E}-29$ \$Mo-95

$44101.70 \mathrm{C} \quad 1.701430 \mathrm{E}-29$ \$Ru101

$45103.70 \mathrm{C} \quad 1.668363 \mathrm{E}-29$ \$Rh103

$45105.70 \mathrm{C} \quad 1.636554 \mathrm{E}-29$ \$Rh105

$48113.70 \mathrm{C} \quad 1.520612 \mathrm{E}-29$ \$Cd113

$54131.70 \mathrm{C} \quad 1.311513 \mathrm{E}-29$ \$Xe131

$54133.70 \mathrm{C} \quad 1.291780 \mathrm{E}-29$ \$Xe133

$55133.70 \mathrm{C} \quad 1.291774 \mathrm{E}-29$ \$Cs133

$54135.70 \mathrm{C} \quad 1.263242 \mathrm{E}-29$ \$Xe135

$57140.70 \mathrm{C} \quad 1.227103 \mathrm{E}-29$ \$La140

$58141.70 \mathrm{C} \quad 1.218416 \mathrm{E}-29 \$ \mathrm{Ce} 141$

$59143.70 \mathrm{C} \quad 1.201345 \mathrm{E}-29 \$ \mathrm{Pr} 143$

$60143.70 \mathrm{C} \quad 1.201344 \mathrm{E}-29$ \$Nd143

$60145.70 \mathrm{C} \quad 1.184740 \mathrm{E}-29 \$ \mathrm{Nd} 145$

$61147.70 \mathrm{C} \quad 1.168601 \mathrm{E}-29 \$ \mathrm{Pm} 147$

$61149.70 \mathrm{C} \quad 1.152882 \mathrm{E}-29 \$ \mathrm{Pm} 149$

$62149.70 \mathrm{C} \quad 1.152881 \mathrm{E}-29 \$ \mathrm{Sm} 149$

$61151.70 \mathrm{C} \quad 1.137570 \mathrm{E}-29 \$ \mathrm{Pm} 151$

$62151.70 \mathrm{C} \quad 1.137582 \mathrm{E}-29$ \$Sm151

$62152.70 \mathrm{C} \quad 1.130095 \mathrm{E}-29$ \$Sm152

$63153.70 \mathrm{C} \quad 1.122694 \mathrm{E}-29 \$ \mathrm{Eu} 153$

$63155.70 \mathrm{C} \quad 1.108194 \mathrm{E}-29$ \$Eu155

mt2308 lwtr.10t

$64157.70 \mathrm{C} \quad 1.094057 \mathrm{E}-29$ \$Gd157

c 
C

C
ATR Element No. $=15$

Radial Zone No. = 3

Axial Zone No. = 1

Neutron Cross Sections $=27 \mathrm{C}$

Total Number Density $=7.870346 \mathrm{E}-02 \mathrm{a} / \mathrm{b}-\mathrm{cm}$

lwtr. 10 t

\begin{tabular}{|c|c|c|}
\hline $1001.70 \mathrm{C}$ & 3.2128 & $\$ \mathrm{H}$ \\
\hline $8016.70 \mathrm{c}$ & $1.606420 \mathrm{E}-02$ & $\$ 0-16$ \\
\hline $12000.60 \mathrm{c}$ & $2.399430 \mathrm{E}-04$ & \$Mg-nat \\
\hline $3027.70 \mathrm{C}$ & $2.958200 E-02$ & $\$ A 1-27$ \\
\hline $0.60 \mathrm{c}$ & $1.245870 \mathrm{E}-04$ & \$Si-nat \\
\hline .50 & $2.243180 E-05$ & \$Cr-nat \\
\hline .50 & $2.294330 E-05$ & \$Cu-nat \\
\hline .70 & $1.966257 E-05$ & $\$ B-10$ \\
\hline .70 & $5.964864 \mathrm{E}-06$ & SU-234 \\
\hline 1 & $4.603032 E-04$ & $\$ U-235$ \\
\hline & $3.449947 E-06$ & $\$ U-236$ \\
\hline .70 & $1.545203 E-29$ & $\$ U-237$ \\
\hline 7 & $2.956504 \mathrm{E}-05$ & $\$ \mathrm{U}-238$ \\
\hline .70 & $1.545195 E-29$ & $\$ N p 237$ \\
\hline 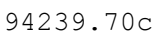 & $1.532242 \mathrm{E}-29$ & $\$ \mathrm{Pu} 239$ \\
\hline .70 & $1.525848 \mathrm{E}-29$ & \$Pu 240 \\
\hline 7 & $1.519499 \mathrm{E}-29$ & $\$ \mathrm{Pu} 241$ \\
\hline . 1 & $4.417651 E-29$ & $\$ K r-83$ \\
\hline-78 & $3.819269 E-29$ & $\$ M o-95$ \\
\hline .70 & $3.629985 E-29$ & \$Ru101 \\
\hline 7 & $3.559438 E-29$ & \$Rh103 \\
\hline 1 & $3.491572 \mathrm{E}-29$ & \$Rh105 \\
\hline & $E-29$ & $\$ \mathrm{Cd} 113$ \\
\hline 54 & $2.798102 E-29$ & \$xe131 \\
\hline & $02 E-29$ & $\$ x e 133$ \\
\hline 55 & $2.755987 E-29$ & $\$ \mathrm{Cs} 133$ \\
\hline & $2.695116 \mathrm{E}-29$ & $\$ x e 135$ \\
\hline .70 & $2.618012 E-29$ & \$La140 \\
\hline 58 & $2.599480 \mathrm{E}-29$ & $\$ \mathrm{Ce} 141$ \\
\hline 59 & $2.563058 \mathrm{E}-29$ & $\$ \operatorname{Pr} 143$ \\
\hline 60 & $2.563055 E-29$ & $\$ N d 143$ \\
\hline 60 & $2.527633 E-29$ & $\$ N d 145$ \\
\hline 61 & $2.493199 \mathrm{E}-29$ & $\$ \operatorname{Pm} 147$ \\
\hline 61 & $2.459663 E-29$ & $\$ P m 149$ \\
\hline 62 & $2.459661 \mathrm{E}-29$ & $\$ \operatorname{Sm} 149$ \\
\hline .70 & $2.426995 E-29$ & $\$ P \operatorname{Pm} 151$ \\
\hline 62 & $2.427021 E-29$ & $\$$ Sm151 \\
\hline .70 & $2.411048 E-29$ & $\$ S \mathrm{Sm} 152$ \\
\hline 63 & $2.395258 E-29$ & \$Eu153 \\
\hline .7 & $2.364321 E-29$ & \$Eu155 \\
\hline .7 & $61 E-29$ & $\$$ Gd157 \\
\hline
\end{tabular}

$64157.70 \mathrm{C} \quad 2.334161 \mathrm{E}-29 \$ \mathrm{Gd} 157$

ATR Element No. $=15$

Radial Zone No. $=3$

Axial Zone No. = 2

Neutron Cross Sections $=27 \mathrm{C}$

Total Number Density $=7.870346 \mathrm{E}-02 \mathrm{a} / \mathrm{b}-\mathrm{cm}$

$1001.70 \mathrm{C} \quad 3.212840 \mathrm{E}-02$ \$ $\mathrm{H}-1$

$8016.70 \mathrm{C} \quad 1.606420 \mathrm{E}-02 \$ 0-16$

$12000.60 \mathrm{C} \quad 2.399430 \mathrm{E}-04$ \$Mg-nat

$13027.70 \mathrm{C} \quad 2.958200 \mathrm{E}-02$ \$Al-27

$14000.60 \mathrm{C} \quad 1.245870 \mathrm{E}-04$ \$Si-nat

$24000.50 \mathrm{C} \quad 2.243180 \mathrm{E}-05$ \$Cr-nat

$29000.50 \mathrm{C} \quad 2.294330 \mathrm{E}-05$ \$Cu-nat

$5010.70 \mathrm{C} \quad 1.966257 \mathrm{E}-05$ \$ B-10

$92234.70 \mathrm{C} \quad 5.964864 \mathrm{E}-06 \quad \$ \mathrm{U}-234$

$92235.70 \mathrm{C} \quad 4.603032 \mathrm{E}-04 \quad \$ \mathrm{U}-235$

$92236.70 \mathrm{C} \quad 3.449947 \mathrm{E}-06 \quad \$ \mathrm{U}-236$

$92237.70 \mathrm{C} \quad 1.545203 \mathrm{E}-29$ \$U-237 
mt2310 lwtr.10t

c

C

$\mathrm{C}$

C

C

C

C

m2311

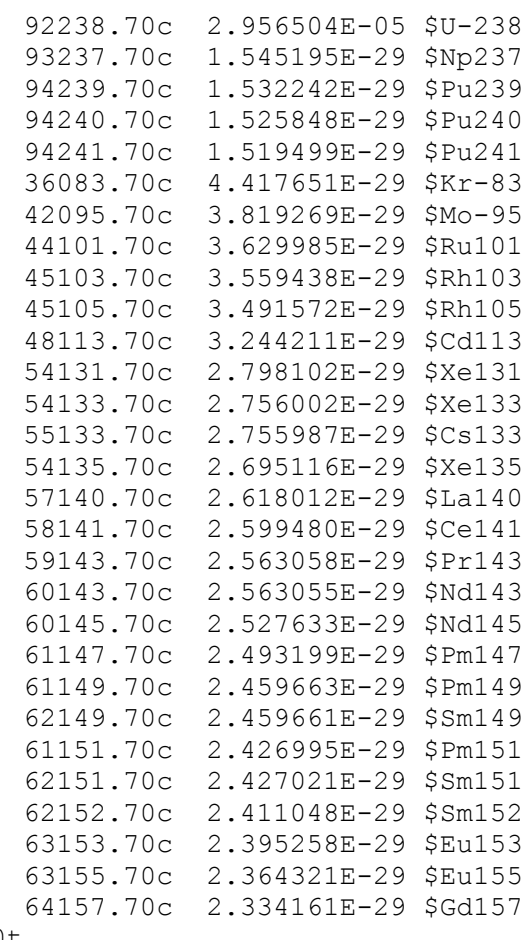

$92238.70 \mathrm{C} \quad 2.956504 \mathrm{E}-05 \quad \$ \mathrm{U}-238$

$93237.70 \mathrm{C} \quad 1.545195 \mathrm{E}-29$ \$Np237

$94241.70 \mathrm{C} \quad 1.519499 \mathrm{E}-29$ \$Pu241

$36083.70 \mathrm{C} \quad 4.417651 \mathrm{E}-29$ \$Kr -83

$42095.70 \mathrm{C} \quad 3.819269 \mathrm{E}-29$ \$Mo-95

4.629985E-29 \$Ru101

$45103.70 \mathrm{C} \quad 3.559438 \mathrm{E}-29$ \$Rh103

3.491572E-29 \$Rh105

$48113.70 \mathrm{C} \quad 3.244211 \mathrm{E}-29$ \$Cd113

.798102E-29 \$Xe131

$55133.70 \mathrm{C} \quad 2.755987 \mathrm{E}-29 \$ \mathrm{Cs} 133$

$54135.70 \mathrm{C} \quad 2.695116 \mathrm{E}-29$ \$Xe135

$57140.70 \mathrm{C} \quad 2.618012 \mathrm{E}-29$ \$La140

$58141.70 \mathrm{C} \quad 2.599480 \mathrm{E}-29$ \$Ce141

$59143.70 \mathrm{C} \quad 2.563058 \mathrm{E}-29 \$ \operatorname{Pr} 143$

2.563055E-29 \$Nd143

$61147.70 \mathrm{C} \quad 2.493199 \mathrm{E}-29$ \$Pm1 47

$61149.70 \mathrm{C} \quad 2.459663 \mathrm{E}-29$ \$Pm149

$62149.70 \mathrm{C} \quad 2.459661 \mathrm{E}-29 \$ \mathrm{Sm} 149$

$61151.70 \mathrm{C} \quad 2.426995 \mathrm{E}-29$ \$Pm151

$62151.70 \mathrm{C} \quad 2.427021 \mathrm{E}-29 \$ \mathrm{Sm} 151$

$62152.70 \mathrm{C} \quad 2.411048 \mathrm{E}-29 \$ \mathrm{Sm} 152$

$64157.70 \mathrm{C} \quad 2.334161 \mathrm{E}-29 \$ \mathrm{Gd} 157$

ATR Element No. $=15$

Radial Zone No. $=3$

Axial Zone No. $=3$

Neutron Cross Sections $=27 \mathrm{C}$

Total Number Density $=7.870346 \mathrm{E}-02 \mathrm{a} / \mathrm{b}-\mathrm{cm}$ 


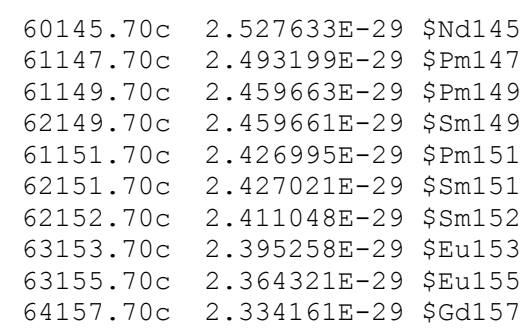

mt2311
C
C
c
C
C
C
c
C
C
m2312

lwtr. $10 t$

$63155.70 \mathrm{C} \quad 2.364321 \mathrm{E}-29$ \$Eu155

ATR Element No. $=15$

Radial Zone No. $=3$

Axial Zone No. = 4

Neutron Cross Sections $=27 \mathrm{C}$

Total Number Density $=7.870346 \mathrm{E}-02 \mathrm{a} / \mathrm{b}-\mathrm{cm}$

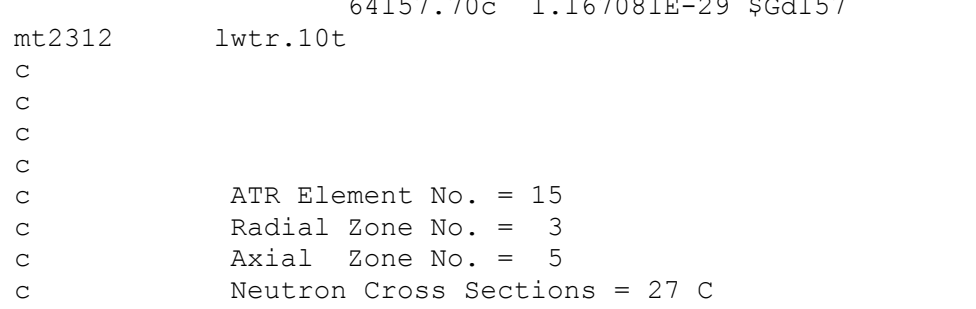


$1001.70 \mathrm{C} \quad 3.212840 \mathrm{E}-02 \$ \mathrm{H}-1$ 


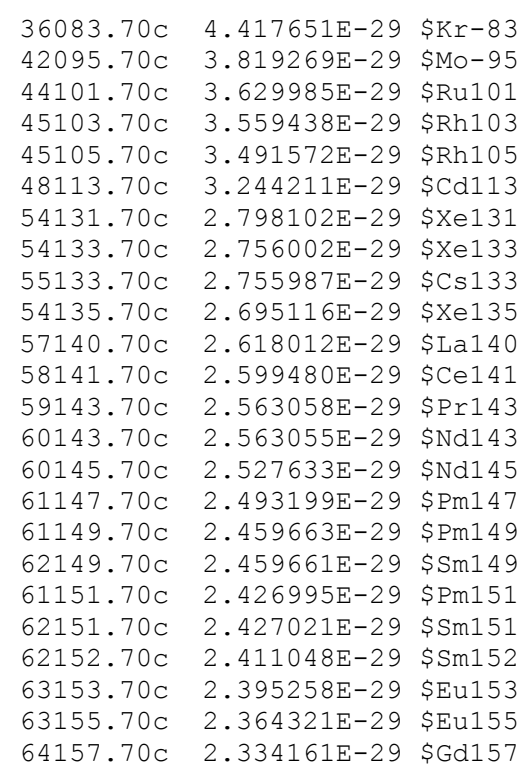

mt2314
C
C
C
C
C
C
C
C
C
m2315

lwtr.10t

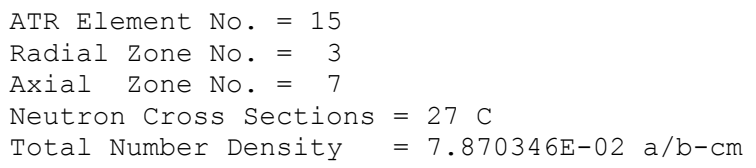

\begin{tabular}{|c|c|c|}
\hline $1001.70 \mathrm{c}$ & 3.212840E-02 & $\$ \mathrm{H}-1$ \\
\hline $8016.70 \mathrm{c}$ & $1.606420 \mathrm{E}-02$ & $\$ 0-16$ \\
\hline $2000.60 \mathrm{c}$ & $2.399430 \mathrm{E}-04$ & $\$ M g-n a t$ \\
\hline $27.70 \mathrm{C}$ & $2.958200 \mathrm{E}-02$ & $\$ A l-27$ \\
\hline $0.60 \mathrm{c}$ & $1.245870 \mathrm{E}-04$ & \$Si-nat \\
\hline $00.50 \mathrm{c}$ & $2.243180 \mathrm{E}-05$ & \$Cr-nat \\
\hline & $2.294330 \mathrm{E}-05$ & $\$ \mathrm{Cu}-$ nat \\
\hline $0 \mathrm{C}$ & $1.966257 \mathrm{E}-05$ & $\$ B-10$ \\
\hline $70 \mathrm{c}$ & $5.964864 \mathrm{E}-06$ & $\$ U-234$ \\
\hline 7 & $4.603032 E-04$ & $\$ \mathrm{U}-235$ \\
\hline & $3.449947 \mathrm{E}-06$ & $\$ U-236$ \\
\hline $.70 \mathrm{C}$ & $1.545203 E-29$ & $\$ U-237$ \\
\hline 7 & $2.956504 \mathrm{E}-05$ & $\$ U-238$ \\
\hline $.70 \mathrm{C}$ & $1.545195 \mathrm{E}-29$ & $\$ \mathrm{~Np} 237$ \\
\hline $.70 \mathrm{C}$ & $1.532242 \mathrm{E}-29$ & \$Pu239 \\
\hline $.70 \mathrm{C}$ & 1. $525848 E-29$ & \$Pu240 \\
\hline $.70 \mathrm{C}$ & $1.519499 \mathrm{E}-29$ & \$Pu241 \\
\hline $.70 \mathrm{C}$ & 4.4176 & $\$ K r-83$ \\
\hline $70 \mathrm{c}$ & $E-29$ & $\$ M o-95$ \\
\hline $.70 \mathrm{C}$ & $5 E-29$ & \$Ru101 \\
\hline $.70 \mathrm{C}$ & $3 E-29$ & \$Rh103 \\
\hline $0 \mathrm{C}$ & 3.4915 & \$Rh105 \\
\hline $\mathrm{C}$ & $E-29$ & $\$ C d 113$ \\
\hline $.70 \mathrm{C}$ & $2 E-29$ & \$Xe131 \\
\hline C & $2 E-29$ & $\$ x e 133$ \\
\hline $.70 \mathrm{C}$ & $2.755987 \mathrm{E}-29$ & $\$ \mathrm{Cs} 133$ \\
\hline C & $2.695116 \mathrm{E}-29$ & $\$ \mathrm{Xe1} 35$ \\
\hline .7 & $2 E-29$ & \$La140 \\
\hline C & $E-29$ & $\$ C e 141$ \\
\hline .7 & $58 E-29$ & $\$ \operatorname{Pr} 143$ \\
\hline 60 & $5 E-29$ & $\$ N d 143$ \\
\hline 60 & $2.527633 E-29$ & $\$ N d 145$ \\
\hline & $9 E-29$ & $\$ \operatorname{Pm} 147$ \\
\hline 61 & $663 E-29$ & \$Pm14 9 \\
\hline & $1 E-29$ & \$Sm149 \\
\hline & $995 E-29$ & \$Pm1 51 \\
\hline
\end{tabular}




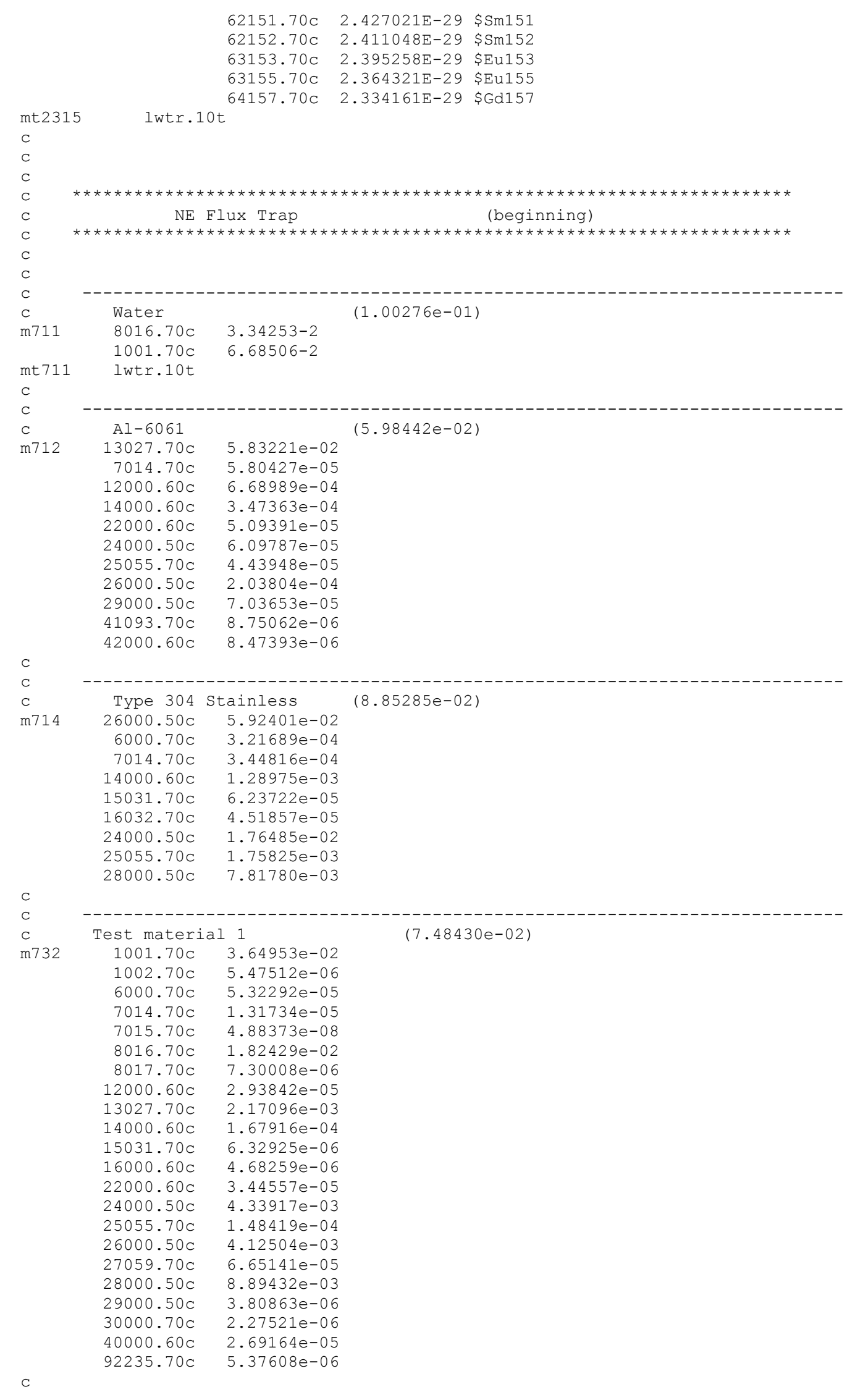




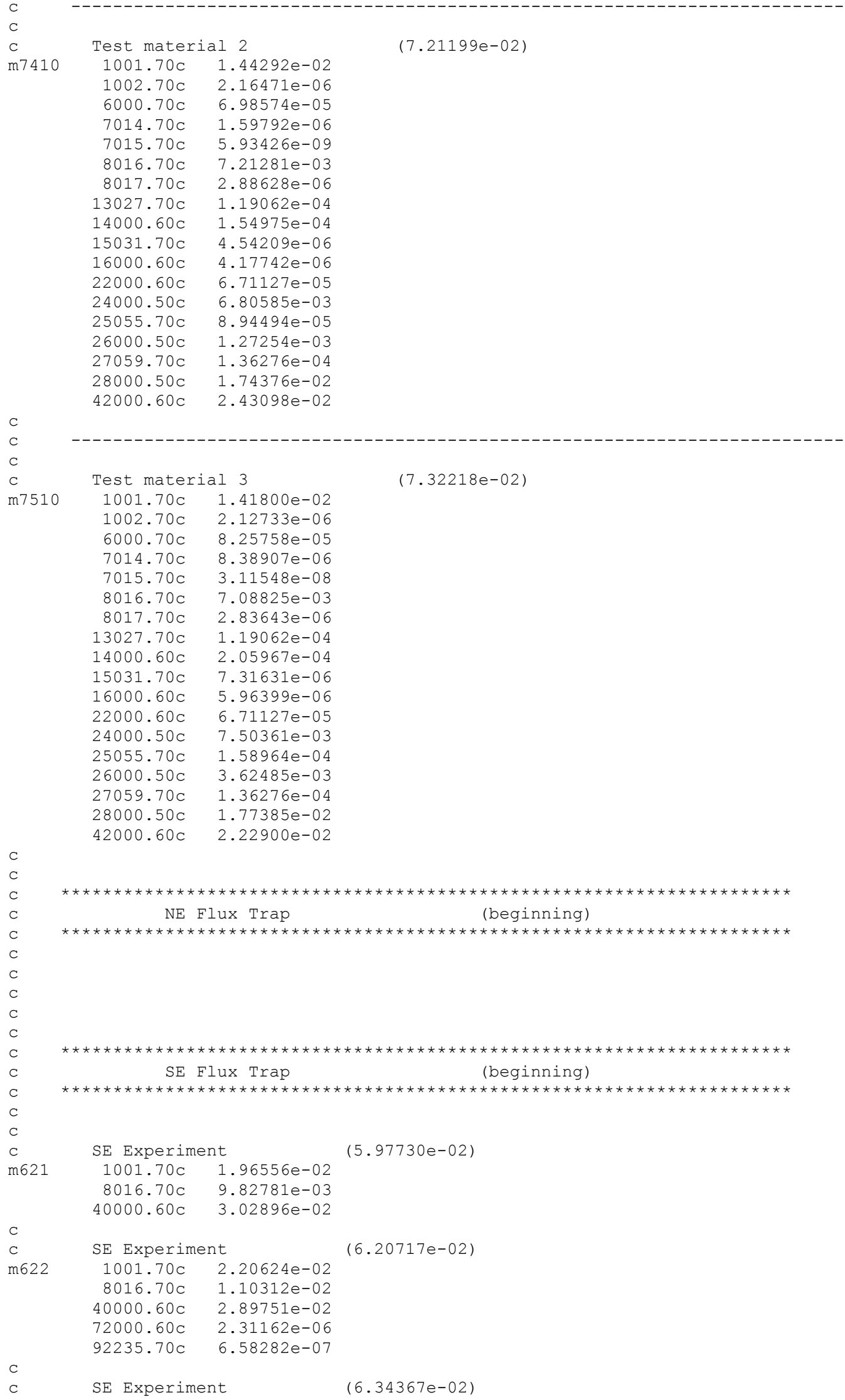




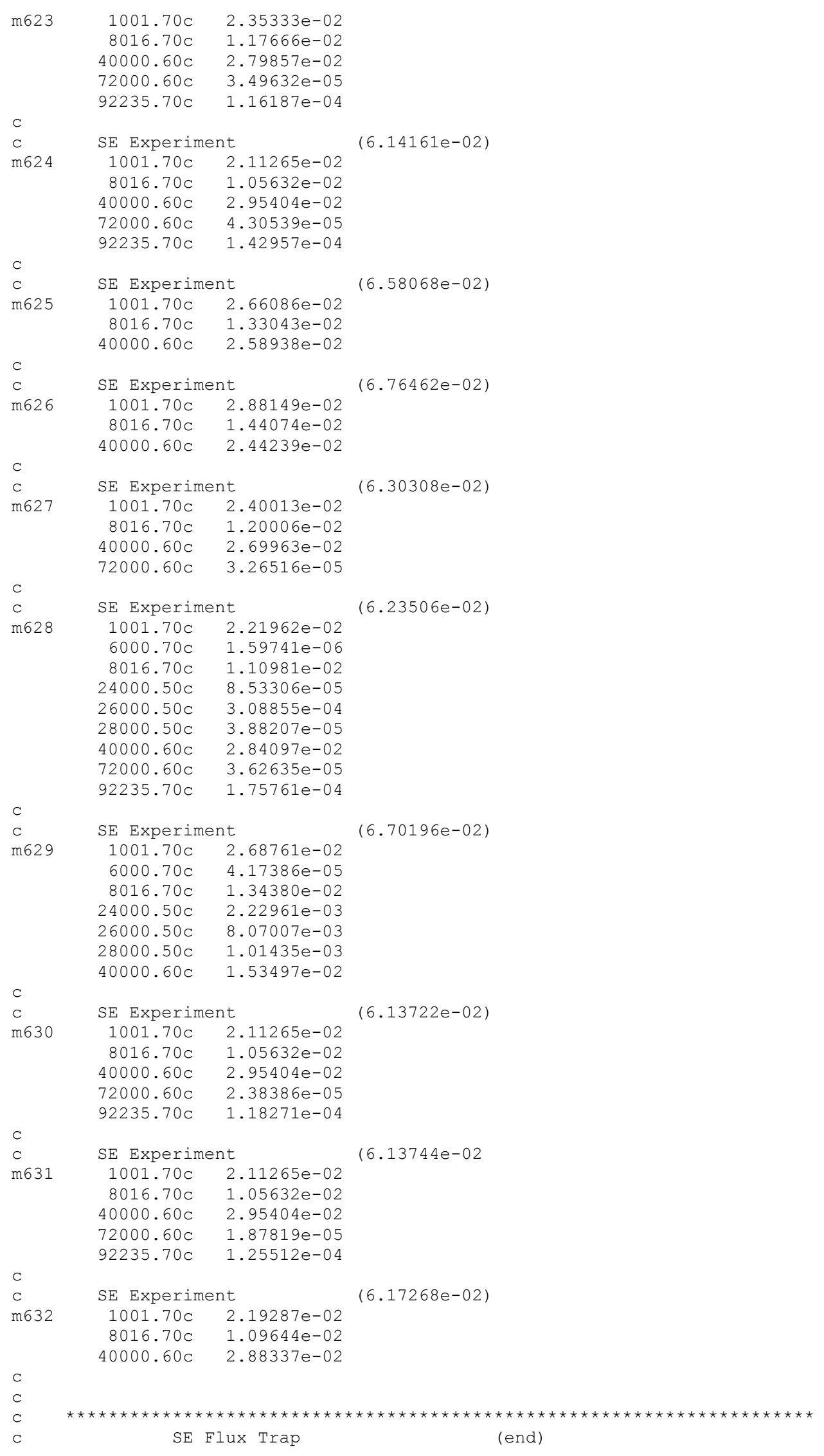




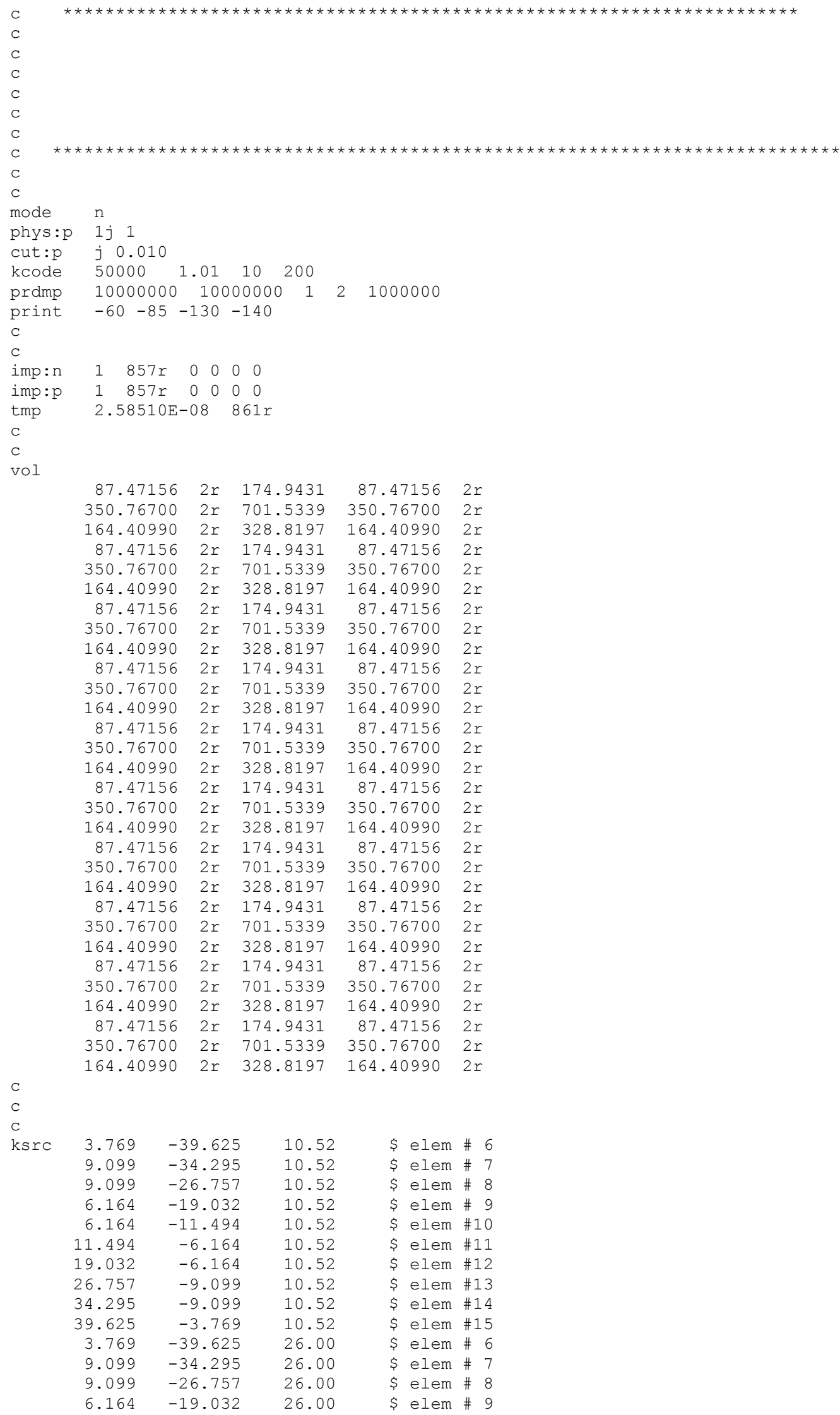




\begin{tabular}{|c|c|c|c|c|c|}
\hline 6.164 & -11.494 & 26.00 & $\$$ & elem & $\# 10$ \\
\hline 11.494 & -6.164 & 26.00 & $\$$ & elem & \#11 \\
\hline 19.032 & -6.164 & 26.00 & $\$$ & elem & \#12 \\
\hline 26.757 & -9.099 & 26.00 & $\$$ & elem & \#13 \\
\hline 34.295 & -9.099 & 26.00 & $\$$ & elem & \#14 \\
\hline 39.625 & -3.769 & 26.00 & $\$$ & elem & \#15 \\
\hline 3.769 & -39.625 & 41.00 & $\$$ & elem & \# \\
\hline 9.099 & -34.295 & 41.00 & $\$$ & elem & \# \\
\hline 9.099 & -26.757 & 41.00 & $\$$ & elem & \# \\
\hline 6.164 & -19.032 & 41.00 & $\$$ & elem & \# \\
\hline 6.164 & -11.494 & 41.00 & $\$$ & elem & $\# 10$ \\
\hline 11.494 & -6.164 & 41.00 & $\$$ & elem & \#11 \\
\hline 19.032 & -6.164 & 41.00 & $\$$ & elem & $\# 12$ \\
\hline 26.757 & -9.099 & 41.00 & $\$$ & elem & \#13 \\
\hline 34.295 & -9.099 & 41.00 & $\$$ & elem & \#14 \\
\hline 39.625 & -3.769 & 41.00 & $\$$ & elem & \#15 \\
\hline 3.769 & -39.625 & 63.50 & $\$$ & elem & \# \\
\hline 9.099 & -34.295 & 63.50 & $\$$ & elem & \# \\
\hline 9.099 & -26.757 & 63.50 & $\$$ & elem & \# \\
\hline 6.164 & -19.032 & 63.50 & $\$$ & elem & \# \\
\hline 6.164 & -11.494 & 63.50 & $\$$ & elem & $\# 10$ \\
\hline 11.494 & -6.164 & 63.50 & $\$$ & elem & \#11 \\
\hline 19.032 & -6.164 & 63.50 & $\$$ & elem & $\# 12$ \\
\hline 26.757 & -9.099 & 63.50 & $\$$ & elem & \#13 \\
\hline 34.295 & -9.099 & 63.50 & $\$$ & elem & $\# 14$ \\
\hline 39.625 & -3.769 & 63.50 & $\$$ & elem & \#15 \\
\hline 3.769 & -39.625 & 86.00 & $\$$ & elem & \# \\
\hline 9.099 & -34.295 & 86.00 & $\$$ & elem & \# \\
\hline 9.099 & -26.757 & 86.00 & $\$$ & elem & \# \\
\hline 6.164 & -19.032 & 86.00 & $\$$ & elem & \# \\
\hline 6.164 & -11.494 & 86.00 & $\$$ & elem & $\# 10$ \\
\hline 11.494 & -6.164 & 86.00 & $\$$ & elem & \#11 \\
\hline 19.032 & -6.164 & 86.00 & $\$$ & elem & \#12 \\
\hline 26.757 & -9.099 & 86.00 & $\$$ & elem & \#13 \\
\hline 34.295 & -9.099 & 86.00 & $\$$ & elem & \#14 \\
\hline 39.625 & -3.769 & 86.00 & $\$$ & elem & \#15 \\
\hline 3.769 & -39.625 & 101.00 & $\$$ & elem & \# \\
\hline 9.099 & -34.295 & 101.00 & $\$$ & elem & \# \\
\hline 9.099 & -26.757 & 101.00 & $\$$ & elem & \# \\
\hline 6.164 & -19.032 & 101.00 & $\$$ & elem & \# \\
\hline 6.164 & -11.494 & 101.00 & $\$$ & elem & \#10 \\
\hline 11.494 & -6.164 & 101.00 & $\$$ & elem & \#11 \\
\hline 19.032 & -6.164 & 101.00 & $\$$ & elem & \#12 \\
\hline 26.757 & -9.099 & 101.00 & $\$$ & elem & \#13 \\
\hline 34.295 & -9.099 & 101.00 & $\$$ & elem & \#14 \\
\hline 39.625 & -3.769 & 101.00 & $\$$ & elem & \#15 \\
\hline 3.769 & -39.625 & 116.48 & $\$$ & elem & \# \\
\hline 9.099 & -34.295 & 116.48 & $\$$ & elem & \# \\
\hline 9.099 & -26.757 & 116.48 & $\$$ & elem & \# \\
\hline 6.164 & -19.032 & 116.48 & $\$$ & elem & \# \\
\hline 6.164 & -11.494 & 116.48 & $\$$ & elem & $\# 10$ \\
\hline 11.494 & -6.164 & 116.48 & $\$$ & elem & \#11 \\
\hline 19.032 & -6.164 & 116.48 & $\$$ & elem & \#12 \\
\hline 26.757 & -9.099 & 116.48 & $\$$ & elem & \#13 \\
\hline 34.295 & -9.099 & 116.48 & $\$$ & elem & \#14 \\
\hline & -3.769 & 116.48 & $\$$ & elem & \#1 \\
\hline
\end{tabular}

